\title{
Reactivity and Functionalization of Naphthalene and Anthracene Complexes of $\left\{\mathrm{TpW}(\mathrm{NO})\left(\mathrm{PMe}_{3}\right)\right\}$
}

\author{
Laura Jessica Strausberg \\ Baltimore, Maryland
}

B.A., Hollins University, 2008

A Dissertation presented to the Graduate Faculty

of the University of Virginia in Candidacy for the Degree of

Doctor of Philosophy

Department of Chemistry

University of Virginia

July, 2013 


\begin{abstract}
Chapter 1 introduces the organic chemistry of aromatic hydrocarbons, with attention paid to regiochemical outcomes of organic reactions. The binding of naphthalene and anthracene to metal complexes is discussed, along with organic transformations they undergo as a result of their complexation. The previous work on osmium and rhenium complexes of naphthalene from the Harman group is explored. Finally, some spectroscopic techniques for exploring the chemistry of $\left\{\mathrm{TpW}(\mathrm{NO})\left(\mathrm{PMe}_{3}\right)\right\}$ complexes of naphthalene and anthracene are introduced.
\end{abstract}

Chapter 2 discusses the highly distorted allyl complexes formed from $\left\{\mathrm{TpW}(\mathrm{NO})\left(\mathrm{PMe}_{3}\right)\right\}$ and the exploration of their origin. Attempts at stereoselectively deprotonating these cationic complexes is also discussed.

Chapter 3 describes our study of $\operatorname{TpW}(\mathrm{NO})\left(\mathrm{PMe}_{3}\right)\left(3,4-\eta^{2}\right.$-naphthalene)'s ability to undergo a Diels-Alder reaction with $N$-methylmaleimide. A solvent study suggested that this reaction proceeds by a concerted mechanism. To probe the mechanism further, we synthesized a series of methylated and methoxylated naphthalene complexes and measured their rates of reaction with $\mathrm{N}$-methylmaleimide compared to the parent complex. We found that 1 substitution on the naphthalene increased the rate of cycloaddition, even if the substituent was in the unbound ring, while 2-substitution slowed the reaction rate when in the bound ring. This information is consistent with a concerted mechanism, as a 2-substituted product would be less able to isomerize to form the active isomer for the cycloaddition to occur.

Chapter 4 discusses tandem electrophile and nucleophile additions to $\operatorname{TpW}(\mathrm{NO})\left(\mathrm{PMe}_{3}\right)\left(3,4-\eta^{2}\right.$-naphthalene) and $\operatorname{TpW}(\mathrm{NO})\left(\mathrm{PMe}_{3}\right)\left(3,4-\eta^{2}\right.$-anthracene), where the electrophile is not carbon-based. Addition of a proton to each complex yields a complex that undergoes Friedel-Crafts-type additions to aromatic nucleophiles. Decomplexation conditions 
were developed for several of these. $\operatorname{TpW}(\mathrm{NO})\left(\mathrm{PMe}_{3}\right)\left(3,4-\eta^{2}\right.$-anthracene) also reacts with $\mathrm{N}$ bromosuccinimide to yield a cationic species to which a variety of nucleophiles added. Loss of bromide allows for addition of a second equivalent of nucleophile.

Chapter 5 discusses reactions of carbon electrophiles with the naphthalene and anthracene complexes. $\operatorname{TpW}(\mathrm{NO})\left(\mathrm{PMe}_{3}\right)\left(3,4-\eta^{2}\right.$-anthracene) reacts with acetal reagents to generate cationic ylidene species. Nucleophiles could be added to both $\mathrm{C} 2$ on the anthracene ring and to the benzyl carbon, depending on the nucleophile. Decomplexation conditions were developed for several complexes, including 1-substituted anthracenes. Attempts a oxidizing a substituted anthracene to anthraquinone is briefly addressed.

Chapter 6 considers the implications of the work described here on generating new functional molecules from aromatic hydrocarbons. 


\section{Acknowledgements}

Chapter 3 is a published work and has been reproduced in accordance with Section II.1 of the American Chemical Society Journal Publishing Agreement. Chapters 2 and 4 derive from published works, some text of which has also been reproduced here. The authors of these publications are given below, with thanks.

Chapter 2: Harrison, D. P.; Nichols-Nielander, A. C.; Zottig, V. E.; Strausberg, L.; Salomon, R. J.; Trindle, C. O.; Sabat, M.; Gunnoe, T. B.; lovan, D. A.; Myers, W. H.; Harman, W. D.; “Hyperdistorted Tungsten Allyl Complexes and Their Stereoselective Deprotonation to Form Dihapto-Coordinated Dienes" Organometallics 2011, 30, 2587.

Chapter 3: Strausberg, L.; Li, M.; Harrison, D. P.; Myers, W. H.; Sabat, M.; Harman, W. D.; "Exploiting the o-Quinodimethane Nature of Naphthalene: Cycloaddition Reactions with $\eta^{2}$ Coordinated Tungsten- Naphthalene Complexes" Organometallics 2013, 32, 915.

Chapter 4: Pienkos, J. A.; Zottig, V. E.; lovan, D. A.; Li, M.; Harrison, D. P.; Sabat, M.; Salomon, R. J.; Strausberg, L.; Teran, V. A.; Myers, W. H.; Harman, W. D.; “Friedel-Crafts Ring-Coupling Reactions Promoted by Tungsten Dearomatization Agent" Organometallics 2013, 32, 691. 


\section{Graduation Beer}

This beer was conceived of and brewed for my graduation, and it was enjoyed on 22 July 2013 by many wonderful people, including my parents, my sister, Allison, my advisor, Dean, Dan and Vic, who taught me many things about chemistry, Monica, who worked with me in lab and is a wonderful friend, my most excellent labmates, Bri, Ben, Jared, Jeff, Phil, and Andrew, and finally, Bill Myers, of Richmond. This recipe was reproduced from notes I made and memory. May or may not be reproducible.

$1 / 3$ ounce lavendar

1/3 ounce German Hallertau hops

4 ounces Bohemian Pilsner malt

1 pound extra light dried malt extract (DME)

4 ounces light DME

1) The pilsner malt was steeped in 1.5 quarts sterilized water at $160-170{ }^{\circ} \mathrm{C}$ for 30 minutes.

2) The grains were removed and the DME was carefully stirred into the water, adding an extra cup of sterile water, if needed. Once dissolved, the mixture was boiled for 15 minutes (watch out for foaming).

3) The hops were added and boiling continued another 30 minutes.

4) The lavender was added and boiling continued another 5 minutes.

5) The mixture was allowed to cool a little before being added to a gallon carboy containing 2 quarts chilled sterile water. Sterile water was added to bring the volume to one gallon.

6) When the temperature of the wort was below $100{ }^{\circ} \mathrm{C}$, yeast ( 3 grams) in several tablespoons warm water was added to the carboy. The carboy was sealed with a stopped with a tube leading to a container with water.

7) Fermentation occurred! As per the observation of carbon production.

8) The wort was racked into a second carboy after about 9 days. Fermentation continued for about two weeks. My apartment was probably kept at $75-76{ }^{\circ} \mathrm{C}$ during this time.

9) Some sugar (maybe 1/4 cup?) was dissolved in 1.5 cups water and boiled for 15 minutes.

10) The sugar water was transferred to a glass carboy and the beer racked over it and mixed well.

11) The beer was siphoned into bottles, capped, and kept three weeks at $75-76^{\circ} \mathrm{C}$.

12) The beer was enjoyed at graduation. 


\section{Table of Contents}

Abstract

Acknowledgements $\quad$ iv

Table of Contents $\quad$ v

List of Figures viii

List of Schemes $\quad$ xi

List of Tables $\quad$ XV

List of Abbreviations $\quad$ xvi

Chapter 1: Introduction to the Organic and Organometallic

\section{Chemistry of Aromatic Hydrocarbons}

$\begin{array}{ll}\text { Properties of aromatic hydrocarbons } & 2\end{array}$

Organic reactions of aromatic hydrocarbons $\quad 5$

$\begin{array}{ll}\text { Organometallic complexes of aromatic hydrocarbons and their reactions } & 11\end{array}$

$\begin{array}{ll}\text { Osmium and rhenium complexes of naphthalene } & 15\end{array}$

$\begin{array}{ll}\text { Tungsten complexes of naphthalene and anthracene } & 21\end{array}$

$\begin{array}{ll}\text { References } & 23\end{array}$

Chapter 2: Hyper-Distorted Tungsten Allyl Complexes and Their Stereoselective Deprotonation to Form Dihapto-Coordinated Dienes

$\begin{array}{ll}\text { Introduction } & 27\end{array}$

Structural and Spectroscopic Analysis of TpW(NO)(PMe $\left.\mathrm{PM}_{3}\right)(\pi$-allyl) Complexes 29

$\begin{array}{lll}\text { Calculations } & 33\end{array}$ 
Stereoselective Preparations of $\eta^{2}$-Diene Complexes 39

$\begin{array}{ll}\text { Conclusion } & 41\end{array}$

$\begin{array}{ll}\text { Experimental Section } & 41\end{array}$

$\begin{array}{ll}\text { References } & 53\end{array}$

Chapter 3: Exploiting the o-Quinodimethane Nature of Naphthalene:

Cycloaddition Reactions with $\eta^{2}$-Coordinated Tungsten- Naphthalene Complexes

$\begin{array}{ll}\text { Introduction } & 56\end{array}$

$\begin{array}{ll}\text { Results and Discussion } & 58\end{array}$

$\begin{array}{ll}\text { Conclusion } & 74\end{array}$

$\begin{array}{ll}\text { Experimental Section } & 75\end{array}$

$\begin{array}{ll}\text { References } & 93\end{array}$

Chapter 4: Tandem Electrophile-Nucleophile Additions to Naphthalene and

Anthracene Complexes

$\begin{array}{ll}\text { Introduction } & 97\end{array}$

$\begin{array}{ll}\text { Results and Discussion } & 98\end{array}$

Tandem Additions to $\mathrm{TpW}(\mathrm{NO})\left(\mathrm{PMe}_{3}\right)\left(3,4-\eta^{2}\right.$-anthracene) 103

$\begin{array}{ll}\text { Conclusion } & 114\end{array}$

$\begin{array}{ll}\text { Experimental Section } & 114\end{array}$

$\begin{array}{ll}\text { References } & 132\end{array}$

\section{Chapter 5: Acetal Additions to Anthracene Complexes}

Introduction 
$\begin{array}{ll}\text { Results } & 136\end{array}$

$\begin{array}{lr}\text { Discussion } & 149\end{array}$

$\begin{array}{ll}\text { Ylidene Formation } & 153\end{array}$

$\begin{array}{ll}\text { Benzyl vs. Ring Addition } & 155\end{array}$

$\begin{array}{ll}\text { Irradiation } & 157\end{array}$

$\begin{array}{lr}\text { Anthraquinones } & 159\end{array}$

$\begin{array}{lr}\text { Conclusion } & 160\end{array}$

$\begin{array}{ll}\text { Experimental Section } & 160\end{array}$

$\begin{array}{ll}\text { References } & 184\end{array}$

Chapter 6: Concluding Remarks 187 


\section{List of Figures}

\section{Chapter 1:}

Figure 1: Structures of naphthalene and anthracene 2

Figure 2: Hydrogenation enthalpies of alkenes and benzene 3

$\begin{array}{ll}\text { Figure 3: Resonance contributors of anthracene } & 4\end{array}$

$\begin{array}{ll}\text { Figure 4: } & 4\end{array}$

Figure 5: Structures of 2,6-dimethylnapthalene, 2,7-dimethylnaphthalene, $\begin{array}{ll}\text { and 1-(p-tolyl)-2-methylbutane } & 7\end{array}$

Figure 6: $\eta^{6}$-Arene complex fragments $\quad 11$

$\begin{array}{ll}\text { Figure 7: Schematic of } \eta^{2} \text { bonding } & 15\end{array}$

Figure 8: Figures of osmium-naphthalene and anthracene complexes $\quad 16$

Figure 9: $\operatorname{Tp} \operatorname{Re}(\mathrm{CO})(\mathrm{L})(\pi$-ligand), with naphthalene isomers $\quad 19$

Figure 10: Isomerization pathways for $\operatorname{TpRe}(\operatorname{CO})(L)\left(3,4-\eta^{2}\right.$-naphthalene) 19

Figure 11: $\mathrm{TpW}(\mathrm{NO})\left(\mathrm{PMe}_{3}\right)\left(3,4-\eta^{2}\right.$-naphthalene) 22

\section{Chapter 2:}

Figure 1: ORTEPs of 1, 2, and $3 \quad 29$

$\begin{array}{ll}\text { Figure 2: ORTEP of } 6 & 30\end{array}$

Figure 3: ORTEPs of 7 and $8 \quad 32$

Figure 4: LUMO of 1, calculated from Gaussian 35

Figure 5: Reaction coordinate diagram for allyl cation isomerization in the complex

$$
\left[\mathrm{W}\left(\mathrm{NH}_{3}\right)_{5-\mathrm{n}}(\mathrm{NO})_{\mathrm{n}}\left(\mathrm{C}_{3} \mathrm{H}_{5}\right)\right]^{(\mathrm{n}+1)+} \text {, where } \mathrm{n}=0,1
$$

Figure 6: Molecular orbitals for the allyl complex $\left[\mathrm{W}\left(\mathrm{NH}_{3}\right)_{4}(\mathrm{NO})\left(\mathrm{C}_{3} \mathrm{H}_{5}\right)\right]^{2+} \quad 36$ 
Figure 7: Schematic representation of metal-allyl orbital mixing 37

Figure 8: Schematic representation of $\eta^{3} \rightarrow \eta^{2}$ distortions of the allyl ligand 38

Figure 9: $\mathrm{HOMO}$ of the fragment $\left\{\mathrm{TpW}(\mathrm{NO})\left(\mathrm{PMe}_{3}\right)\right\}$

\section{Chapter 3:}

Figure 1: Isomerization of a dihapto-coordinated rhenium naphthalene complex

Figure 2: The cycloaddition reaction of a dihapto-coordinated naphthalene complex

Figure 3: Dihapto-coordinated naphthalene complexes 2a-8

Figure 4: Cycloaddition of substituted naphthalene complexes $\quad 64$

Figure 5: ORTEP drawing of 11b 64

Figure 6: Decomplexation of cycloadducts $\quad 66$

Figure 7: Isomerization mechanisms for $\eta^{2}$-naphthalene complexes 66

Figure 8: Proposed mechanism for cycloaddition for $\eta^{2}$-naphthalene complexes and transition state and 2,3- $\eta^{2}$ intermediate (calculated) 68

Figure 9: Substituent effects for the isomerization to the purported $o$-quinodimethane Intermediate

Figure 10: Interfacial and intrafacial isomerizations required to form $\mathbf{1 2}$ and $\mathbf{1 3}$ with observed stereochemistry

Figure 11: Attempted cycloaddition of an $\eta^{2}$-anthracene complex at the A ring

Figure 12: Alternate mechanism (disfavored) involving ionic intermediates

\section{Chapter 4:}

Figure 1: ORTEP of 3

Figure 2: Gaussian structure of $\mathbf{2 1}$ 


\section{Chapter 5:}

Figure 1: ORTEP of 12

Figure 2: Acetal reagents added to 4

Figure 3: LUMO of 5, optimized in Gaussian

Figure 4: $\mathrm{HOMO}$ and LUMO of 1

Figure 5: HOMO and LUMO of phenol-derivative complex

\section{Chapter 6:}




\section{List of Schemes}

Chapter 1:

Scheme 1

$\begin{array}{ll}\text { Scheme } 2 & 6\end{array}$

$\begin{array}{ll}\text { Scheme } 3 & 7\end{array}$

$\begin{array}{lr}\text { Scheme } 4 & 8\end{array}$

$\begin{array}{ll}\text { Scheme } 5 & 8\end{array}$

$\begin{array}{ll}\text { Scheme } 6 & 9\end{array}$

$\begin{array}{lr}\text { Scheme } 7 & 9\end{array}$

$\begin{array}{ll}\text { Scheme } 8 & 10\end{array}$

$\begin{array}{lr}\text { Scheme } 9 & 10\end{array}$

$\begin{array}{ll}\text { Scheme } 10 & 11\end{array}$

Scheme $11 \quad 12$

\begin{tabular}{l|l} 
Scheme 12 & 13
\end{tabular}

$\begin{array}{ll}\text { Scheme } 13 & 13\end{array}$

$\begin{array}{ll}\text { Scheme } 14 & 13\end{array}$

$\begin{array}{ll}\text { Scheme } 15 & 14\end{array}$

$\begin{array}{ll}\text { Scheme } 16 & 14\end{array}$

$\begin{array}{ll}\text { Scheme } 17 & 15\end{array}$

$\begin{array}{lr}\text { Scheme } 18 & 16\end{array}$

$\begin{array}{ll}\text { Scheme } 19 & 17\end{array}$

$\begin{array}{ll}\text { Scheme } 20 & 17\end{array}$

$\begin{array}{ll}\text { Scheme } 21 & 18\end{array}$ 
Scheme 23

Scheme 24

Chapter 2:

\begin{tabular}{l|l} 
Scheme 1 & 27
\end{tabular}

$\begin{array}{ll}\text { Scheme } 2 & 28\end{array}$

$\begin{array}{ll}\text { Scheme } 3 & 30\end{array}$

$\begin{array}{ll}\text { Scheme } 4 & 30\end{array}$

$\begin{array}{ll}\text { Scheme } 5 & 31\end{array}$

$\begin{array}{ll}\text { Scheme } 6 & 33\end{array}$

$\begin{array}{ll}\text { Scheme } 7 & 40\end{array}$

$\begin{array}{ll}\text { Scheme } 8 & 41\end{array}$

Chapter 4:

$\begin{array}{ll}\text { Scheme } 1 & 97\end{array}$

$\begin{array}{ll}\text { Scheme } 2 & 99\end{array}$

$\begin{array}{ll}\text { Scheme } 3 & 100\end{array}$

$\begin{array}{ll}\text { Scheme } 4 & 101\end{array}$

$\begin{array}{ll}\text { Scheme } 5 & 102\end{array}$

$\begin{array}{ll}\text { Scheme } 6 & 103\end{array}$

$\begin{array}{lr}\text { Scheme } 7 & 104\end{array}$

$\begin{array}{lr}\text { Scheme } 8 & 105\end{array}$

$\begin{array}{lr}\text { Scheme } 9 & 106\end{array}$ 
Scheme 11

Scheme 12

Scheme 13

Scheme 14

Scheme 15

Scheme 16

Scheme 17

Scheme 18

Chapter 5:

Scheme 1

Scheme 2

138

Scheme 3

Scheme 4

Scheme 5

Scheme 6

Scheme 7

Scheme 8

Scheme 9

Scheme 10

Scheme 11

Scheme 12 
Scheme 14

Scheme 15

Scheme 16

154

Scheme 17

Chapter 6: 


\section{List of Tables}

\section{Chapter 1:}

Table 1: Bond lengths of carbon-carbon bonds in naphthalene and anthracene

Table 2: $\operatorname{TpRe}(\mathrm{CO})(\mathrm{L})\left(3,4-\eta^{2}\right.$-naphthalene) isomer ratios, by ligand

\section{Chapter 2:}

Table 1: Experimental and [calculated] bond lengths for $\eta^{3}$-allyl and $\eta^{2}$-alkene complexes of the form $\mathrm{TpW}(\mathrm{NO})\left(\mathrm{PMe}_{3}\right)(\mathrm{L})$. Calculated values are in brackets $(\Delta=(\mathrm{W}-\mathrm{C} 1)-(\mathrm{W}-\mathrm{C} 3))$

\section{Chapter 3:}

Table 1: Solvent Effects on Cycloaddition of 1 with $N$-methylmaleimide

Table 2: Rate of Cycloadduct Formation $\left(25^{\circ} \mathrm{C}\right)$ for Naphthalene Complexes of $\{\mathrm{TpW}(\mathrm{NO})(\mathrm{PMe})\}$

Table 3 


\section{List of Abbreviations}

\begin{tabular}{|c|c|}
\hline aq & Aqueous \\
\hline br & Broad \\
\hline CAN & Ceric Ammonium Nitrate \\
\hline COSY & Correlation Spectroscopy \\
\hline CV & Cyclic Voltammetry \\
\hline DBU & 1,8-Diazabicyclo[5.4.0]undec-7-ene \\
\hline DCM & Dichloromethane \\
\hline DDQ & 2,3-dichloro-5,6-dicyano- $p$-quinone \\
\hline DFT & Density functional theory \\
\hline DMA & $\mathrm{N}, \mathrm{N}$-Dimethylacetamide \\
\hline DME & 1,2-Dimethoxyethane \\
\hline DMF & Dimethyformamide \\
\hline DMM & Dimethoxymethane \\
\hline DMSO & Dimethyl sulfoxide \\
\hline DPhAT & Diphenylammonium Triflate \\
\hline ee & Enantiomeric excess \\
\hline ESI-MS & Electrospray lonization Mass Spectrometry \\
\hline EtOAc & Ethyl Acetate \\
\hline GC-MS & Gas Chromatography- Mass Spectrometry \\
\hline HATR & Horizontal Attenuated Total Reflectance \\
\hline HMBC & Heteronuclear Multiple Bond Coherence Spectroscopy \\
\hline MS & High-Resolution Mass Spectroscopy \\
\hline $\mathrm{QC}$ & Heteronuclear Single Quantum Correlation Spectroscc \\
\hline
\end{tabular}




\begin{tabular}{|c|c|}
\hline IR & Infrared \\
\hline LRMS & Low-Resolution Mass Spectroscopy \\
\hline$m C P B A$ & $m$-Chloroperbenzoic Acid \\
\hline Melm & $N$-Methylimidazole \\
\hline MVK & Methyl Vinyl Ketone \\
\hline NBS & $N$-Bromosuccinimide \\
\hline NCS & $N$-Chlorosuccinimide \\
\hline NHE & Normal Hydrogen Electrode \\
\hline NMR & Nuclear Magnetic Resonance \\
\hline NOE & Nuclear Overhauser Effect \\
\hline NOESY & Nuclear Overhauser Effect Spectroscopy \\
\hline ORTEP & Oak Ridge Thermal Ellipsoid Program \\
\hline OTf & Trifluoromethanesulfonate or triflate \\
\hline Pov-Ray & The Persistence of Vision Raytracer \\
\hline ppm & Parts Per Million \\
\hline QM & Quinone methide \\
\hline sat & Saturated \\
\hline ТВAH & Tetrabutylammonium Hexafluorphosphate \\
\hline TEA & Triethylamine \\
\hline THF & Tetrahydrofuran \\
\hline TLC & Thin layer chromatography \\
\hline TMS & Trimethylsilyl \\
\hline Tp & Hydridotris(pyrazolyl)borate \\
\hline
\end{tabular}


Chapter 1

Introduction to the Organic and Organometallic Chemistry of Aromatic Hydrocarbons 


\section{Properties of Aromatic Hydrocarbons}

Aromatic hydrocarbons are naturally occurring compounds found in coal tar and petroleum deposits, and can also be produced during combustion processes, such as in forest fires. ${ }^{1}$ These compounds find many industrial uses, and thus are an important subject of study for chemists and engineers. For example, nearly a half-million tons of naphthalene was produced worldwide in 2000 , with phthalic anhydride production for making plastics a major source of demand. ${ }^{2}$ Naphthalene is also used in producing dyes, resins, and decalin for solvent applications. $^{2}$

Naphthalene is composed of two six-membered carbon rings fused together by two carbons, and has a formula of $\mathrm{C}_{10} \mathrm{H}_{8}$. The carbon skeleton is planar, allowing an atomic p-orbital on each carbon to mix and creating a delocalized system of electrons over the entirety of the molecule, known as a $\pi$ system. The analogous three-ring system in which the rings are linear is called anthracene, and has a formula of $\mathrm{C}_{14} \mathrm{H}_{10}$.

Figure 1 - Structures of naphthalene and anthracene<smiles>c1ccc2ccccc2c1</smiles><smiles>c1ccc2cc3ccccc3cc2c1</smiles>

While naphthalene and anthracene can both be represented as a series of alternating single and double bonds, they are both aromatic molecules whose chemistry differs significantly from that of alkenes. Their aromatic character can be distinguished from several features that may not be obvious from their skeleton structures. First, they possess resonance stabilization energy. One convenient way of measuring this is by comparing the hydrogenation energy of an aromatic molecule, for example, benzene, with the expected hydrogenation energy of a similar molecule in which no special interactions between bonds are expected, like cyclohexatriene (Figure 2). ${ }^{3}$ If the heat of hydrogenation of cyclohexene is $28.6 \mathrm{kcal} / \mathrm{mol}$, the heat of hydrogenation of cyclohexatriene should be three times that, or $85.8 \mathrm{kcal} / \mathrm{mol}$. The actual heat 
of hydrogenation of benzene is $49.8 \mathrm{kcal} / \mathrm{mol}$, giving a stabilization energy of $36 \mathrm{kcal} / \mathrm{mol}$ of energy for benzene. ${ }^{3}$

Figure 2 - Hydrogenation enthalpies of alkenes and benzene

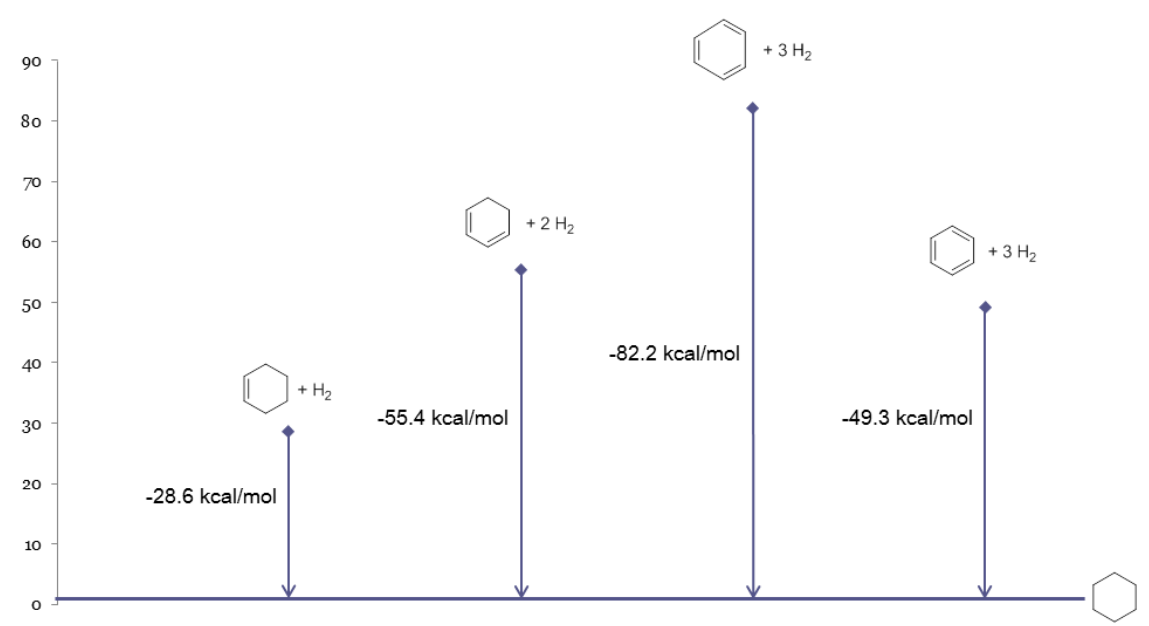

Similarly, the resonance stabilization energies of naphthalene and anthracene are approximately 61 and $84 \mathrm{kcal} / \mathrm{mol}$, respectively. ${ }^{4}$ While the stabilization energies increase as the molecule size increases, the stabilization energy per ring actually decreases slightly from 36 $\mathrm{kcal} / \mathrm{mol}$ for benzene, to $30.5 \mathrm{kcal} / \mathrm{mol}$ for naphthalene, to $28 \mathrm{kcal} / \mathrm{mol}$ for anthracene. This decrease in stabilization energy has important consequences for the reactivity of naphthalene and anthracene, as will be discussed later.

Another physical feature of naphthalene and anthracene that is representative of their aromatic character is the bond lengths observed between carbon atoms. A carbon-carbon single bond has a bond length of approximately $1.52 \AA$, a double bond is approximately $1.34 \AA$, and a carbon-carbon bond in benzene is approximately $1.39 \AA$. While all of the carbon-carbon bonds in benzene are identical, this is not true for naphthalene and anthracene. One way in which we can consider the difference in bond lengths for these compounds is via Clar's method, ${ }^{5}$ in which $\pi$ electrons are assigned to rings exclusively in such a way that the number of sextets is maximized. In Figure 1, above, it is clear that only one ring in each structure may have a sextet; 
the remaining $\pi$ electrons are assigned in diene configurations. While resonance structures can be drawn for each molecule, certain bonds are drawn as double bonds more often than other bonds. Anthracene's resonance contributors are below (Figure 3). One bond, indicated by its 12 numbering, is represented as a double bound in three of the four resonance contributors for anthrancene, so we may hypothesize that it is shorter than the 2-3 bond, which is represented as a single bond in three of the four structures.

Figure 3 - Resonance contributors of anthracene<smiles>Ic1c2ccccc2cc2c(CSc3cccc4cc5ccccc5cc34)cccc12</smiles>

While the bond lengths for naphthalene and anthracene, given below, are all intermediate between a double and single bond, differentiation of lengths is observed. ${ }^{6}$ While none of the bond lengths is greatly different from the length observed in benzene, the additional ring in anthracene is accompanied by slightly greater differentiation of bond lengths, suggesting greater localization of electron density (Table 1, Figure 4). This characteristic provides clues into the expected reactivity of these molecules.

Figure 4<smiles>c1ccc2ccccc2c1</smiles><smiles>c1ccc2cc3ccccc3cc2c1</smiles>

Table 1 - Bond lengths of carbon-carbon bonds in naphthalene and anthracene ${ }^{6}$

\begin{tabular}{|l|l|}
\hline Naphthalene & Anthracene \\
\hline a: $1.422 \AA$ & a: $1.444 \AA$ \\
\hline b: $1.371 \AA$ & b: $1.375 \AA$ \\
\hline c: $1.412 \AA$ & c: $1.418 \AA$ \\
\hline d: $1.420 \AA$ & d: $1.433 \AA$ \\
\hline & e: $1.405 \AA$ \\
\hline
\end{tabular}


Aromaticity is also evident in the ring current observed in NMR spectroscopy. ${ }^{3}$ The delocalized $\pi$ electrons in a ring system produce a magnetic field, detectable during NMR experiments. Nuclei that lie above or below the plane of the aromatic ring will be shielded by the induced magnetic field and appear upfield in NMR spectra. Nuclei that lie in the plane will be deshielded by the induced magnetic field, and appear downfield in the NMR spectra, relative to comparable compounds. For example, the alkene protons of cyclohexene have a resonance at $\sim 5.7 \mathrm{ppm}$ in deuterated chloroform, relative to tetramethylsilane, in the ${ }^{1} \mathrm{H}$ NMR spectrum. The protons on benzene appear at $\sim 7.3 \mathrm{ppm}$ in deuterated chloroform, significantly downfield.

\section{Organic Reactions of Aromatic Hydrocarbons}

Aromatic hydrocarbons are highly resistant to addition reactions. This may seem surprising, as the concentration of electron density in the $\pi$ system appears to make it an ideal target of electrophiles. In fact, addition of an electrophile results in an arenium ion. Once this addition occurs, elimination of the proton is generally fast, yielding a rearomatized compound with a new substituent (Scheme 1). The regiochemistry observed for any particular reaction depends on the identity of the electrophile, the solvent and temperature conditions, and any other conditions such as a catalyst. Nevertheless, electrophilic substitution reactions for naphthalene and anthracene follow general regiochemical trends.

Scheme 1

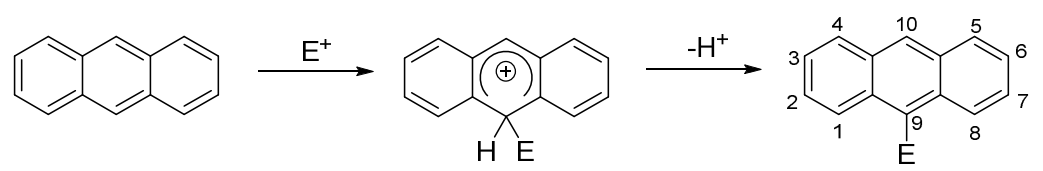

In anthracene, electrophilic attack occurs preferentially in the middle ring, as the resultant carbocation can be conceived of as incorporating two intact benzene rings in its structure. If the attack occurred at a terminal ring, the "aromatic portion" of the carbocation 
would be a naphthalene fragment. The stabilization energy of two benzene rings is greater than that of one naphthalene fragment, making this the more favorable carbocation and substitution on the middle ring the preferred product in most cases. ${ }^{3}$

In naphthalene, initial addition of electrophiles typically occurs at the $\alpha$ carbon, or C1. This is kinetically preferred because the arenium ion benefits from both allylic and benzylic stabilization in the transition state. ${ }^{3}$ Isomerization gives the thermodynamically more stable addition at the $\beta$ carbon, or C2 (Scheme 2).

Scheme 2

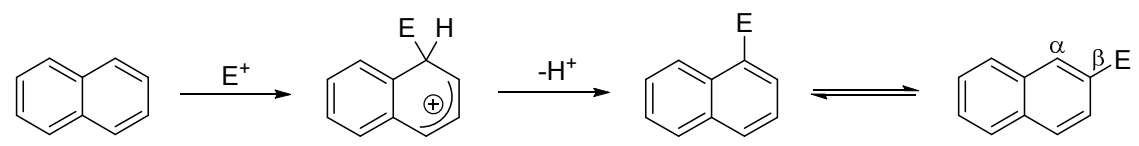

A study of Friedel-Crafts alkylations of naphthalene performed by Olah et al., yielded useful insights into the preference for $\alpha$ vs. $\beta$ substitution. ${ }^{7,8}$ Alpha substitution predominated early in the reaction when the alkyl group being added was a methyl or ethyl group. When run in carbon disulfide solvent, equilibration with the $\beta$ product was rapid. Equilibration was much slower in nitromethane solvent. When the alkyl group was an isopropyl group, the $\beta$ product predominated in carbon disulfide, but the $\alpha$ product was preferred in nitromethane. The preference for the $\beta$ product was even greater when the alkyl group was a tert-butyl group, but the $\alpha$ product was observed in high excess at $0{ }^{\circ} \mathrm{C}$. Equilibration of the $\alpha$ and $\beta$ products was fairly rapidly for the isopropyl and tert-butyl groups, with $\beta$ substitution being highly favored. Steric repulsion from the hydrogen on the adjacent naphthalene ring would increase the favorability of the $\beta$ product when the alkyl group is large. ${ }^{3}$ The authors posited that carbocation stability could facilitate intramolecular isomerization prior to deprotonation (Scheme 3). ${ }^{7}$ 
Scheme 3

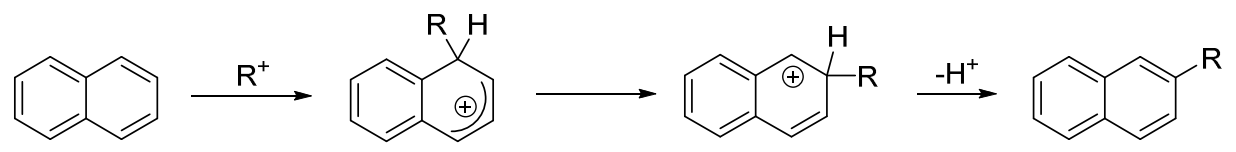

Alkylation of naphthalene is of considerable commercial interest. Naphthalene-2,6dicarboxylic acid is a precursor of poly(ethylene naphthalate), a plastic with such diverse applications as films, fibers, adhesives, and liquid crystalline polymers. ${ }^{9}$ Naphthalene-2,6dicarboxylic acid is produced from 2,6-dimethylnaphthalene, which can be synthesized by several methods, each with shortcomings. Sequential Friedel-Crafts reactions with aluminum trichloride and alkyl halides are favorable, given that an alkylated naphthalene is more electron rich, and thus more activated to electrophilic attack. However, Olah's work showed that while $\beta$ substitution is preferred, it is by no means exclusively observed. ${ }^{7}$ Thus, Friedel-Crafts methods would produce a number of mono- and di-substituted products from which the desired 2,6dimethylnaphthalene would have to be isolated. The current industrial method for producing 2,6-dimethylnaphthalene utilizes 1-(p-tolyl)-2-methylbutane in a dehydrocyclization reaction, which also produces numerous byproducts and suffers from poor yields (Figure 5). ${ }^{10}$ One area of growth for naphthalene alkylation is the use of zeolite catalysts. Zeolites offer microporous structures that, because of their shape, can influence product selectivity, as well as providing a surface support for reactions. ${ }^{11}$ However, they are not selective for 2,6-dimethylnaphthalene over 2,7-dimethylnaphthalene, and larger alkyl groups have proven more difficult to convert to the dicarboxylic acid. ${ }^{11,12}$

Figure 5 - Structures of 2,6-dimethylnapthalene, 2,7-dimethylnaphthalene, and 1-(p-tolyl)-2-methylbutane<smiles>Cc1ccc2cc(C)ccc2c1</smiles><smiles>Cc1ccc2ccc(C)cc2c1</smiles><smiles>CCC(C)Cc1ccc(C)cc1</smiles> 
While addition reactions to naphthalene are rarely observed because of the high energetic cost of breaking aromaticity, addition reactions of anthracene are more favorable. The arenium ion in Scheme 1 shows that addition occurs at the center ring, forming a carbocation that is sufficiently stable to make addition of a nucleophile possible. Nucleophilic addition at the center ring gives a product with two aromatic benzene rings, the resonance stabilization from which is competitive with the stabilization that would be achieved if the arenium ion deprotonated to give a substituted anthracene. Thus, electrophilic-nucleophilic additions can be achieved under various conditions to give 9,10-dihydroanthracene products. One report found that 9,10-dihydro-9,10-dimethoxyanthracene could be generated from bromine in basic methanol solutions, where a second equivalent of methanol displaced the bromine that had added electrophilicly (Scheme 4). ${ }^{13} \quad \mathrm{~A}$ more straightforward example was observed in the nitration of anthracene in the presence of hydrochloric acid (Scheme 5). ${ }^{14}$

Scheme 4

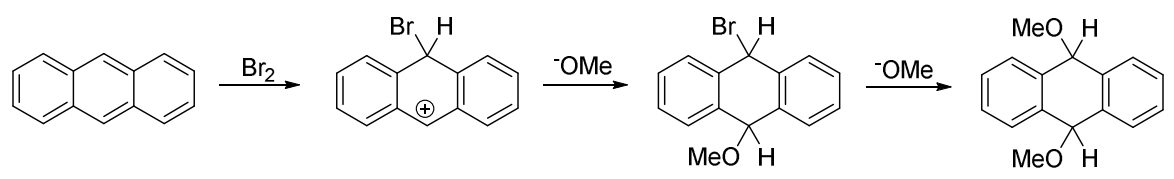

Scheme 5

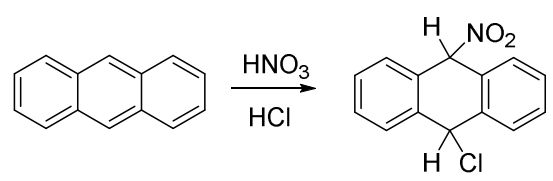

Functionalization of naphthalene and anthracene need not be initiated by electrophilic addition, however. For example, anthracene can be reduced by lithium aluminum hydride in refluxing diglyme to form 9,10-dihydroanthracene (Scheme 6). ${ }^{15}$ Interestingly, these conditions lead to the formation of 9,9,10,10-tetramethylanthracene over time due to the decomposition of diglyme. 
Scheme 6

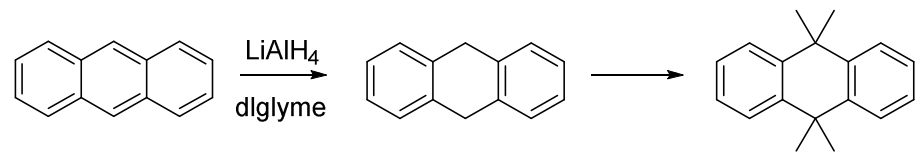

Hydrogenation of anthracene is also possible using heterogeneous catalysts such as activated carbon, where both 9,10-dihydroanthracene (favored) and 1,2,3,4tetrahydroanthracene are observed..$^{16}$ 1,2,3,4-Tetrahydroanthracene was the major product of hydrogenation with the homogeneous catalyst [RuH2(H2)2(PCy3)2], although 1,2,3,4,5,6,7,8octahydroanthracene was also formed after prolonged reaction times. ${ }^{17}$ Naphthalene can be similarly hydrogenated over activated carbon $^{16}$ and by transition metals on carbon support. ${ }^{18}$ Both arenes can be hydrogenated by heterogeneous metals, but these reactions are not always selective for particular bonds or rings, and over-hydrogenation can be problematic. ${ }^{4,19}$

It is possible to reduce naphthalene under Birch conditions, where lithium metal in liquid ammonia forms 1,4-dihydronaphthalene (Scheme 7). ${ }^{20}$ This product can then convert to the thermodynamically favored 1,2-dihydronaphthalene. Naphthalene can be methylated under similar conditions, where methyl bromide is added after the naphthalene has been reductively lithiated. ${ }^{21}$ Interestingly, switching from lithium to sodium in this reaction gives the 1,4-dimethylnaphthalene product, where the methyl groups are cis. $^{21}$ Naphthalene and anthracene can be arylated under similar conditions, but these reactions result in the formation of multiple products. ${ }^{20}$

\section{Scheme 7}
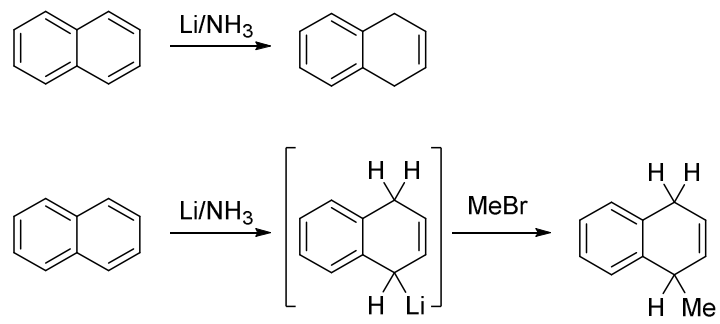
Anthracene can react with alkyl lithium reagents to 9-alkylated anions, which can be quenched with water to given 9-alkyl-9,10-dihydroanthracene, or can react with an alkyl halide to given a 9,10-dialkylated-9,10-dihydroanthracene (Scheme 8$)^{22-24}$

Scheme 8

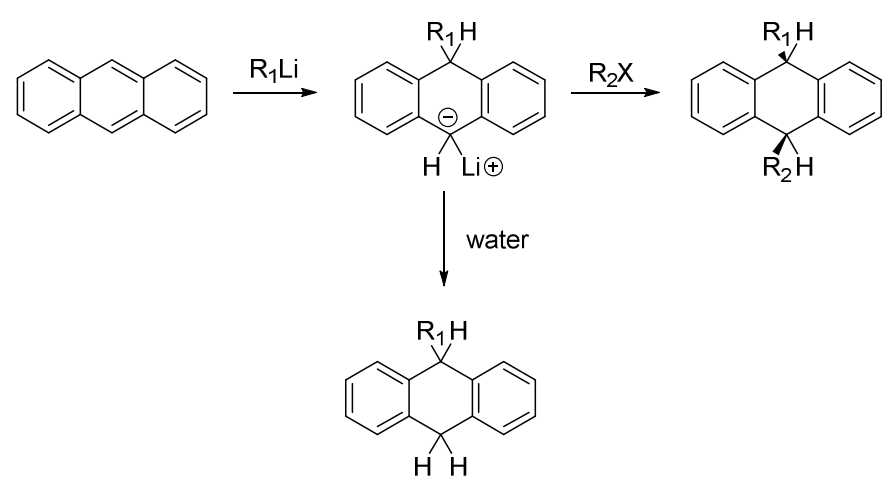

There are examples of naphthalene and anthracene undergoing Diels-Alder cycloadditions, although this reaction is much easier for anthracene as the addition occurs at the middle ring, generating two benzene rings in the final product (Scheme 9). ${ }^{25-27}$ Diels-Alder reactions of naphthalene are discussed in greater detail in Chapter 3.

\section{Scheme 9}

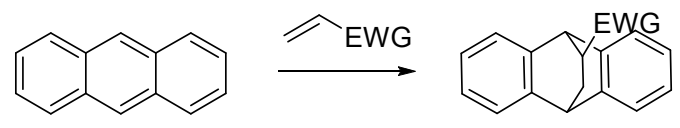

Naphthalene and anthracene undergo oxidation reactions with a variety of oxidants. Oxidation of anthracene to 9,10-anthraquinone is a reaction of particular interest, as compounds with anthraquinone cores have a much broader utility than corresponding anthracenes (Scheme 10). ${ }^{2}$ Methods have been developed for this oxidation using ruthenium, ${ }^{28}$ iridium, rhodium, and palladium salts and hydrogen peroxide, ${ }^{29}$ hypervalent iodine reagents, ${ }^{30}$ nitric acid and acetic acid in air, ${ }^{31}$ and vanadyl acetylacetonate and hydrogen peroxide. ${ }^{32}$ One report detailed the synthesis of 1,4-dihydroanthracene from anthracene via a series of brominations and oxidations. ${ }^{33}$ 


\section{Scheme 10}

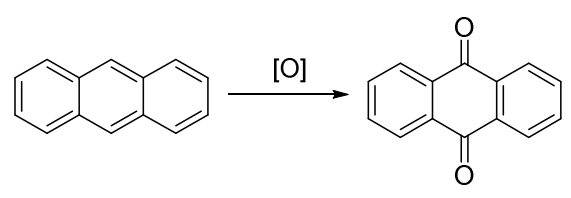

\section{Organometallic complexes of aromatic hydrocarbons and their reactions}

A rich chemistry has been developed for transition metal complexes of polycyclic aromatic hydrocarbons. Transition metal complexes, with their d-orbitals available for bonding, and aromatic molecules, with multiple cites of potential attachment, are able to combine in many ways, forming complexes in which the metal is bound to one through six carbons in an arene ring. ${ }^{34}$ Chemistry has been most fully developed for complexes in which the arene is bound through all six carbons (hexa-hapto, or $\eta^{6}$ ), in which the metal fragment is frequently a metal tricarbonyl species or metal cyclopentadienyl species (Figure 6). ${ }^{35}$

Figure $6-\eta^{6}$-Arene complex fragments

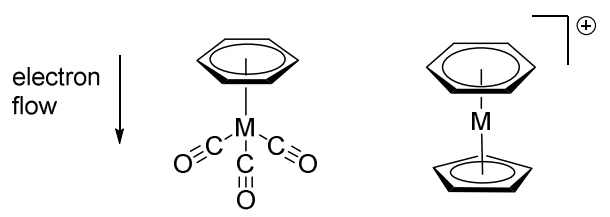

The reactivity of an arene is drastically modified by its attachment to a metal tricarbonyl or cyclopentadienyl fragment. The metals in these fragments are relatively electron poor and form bonds with arenes by withdrawing electron density from their $\pi$ systems. This makes the protons on the arene ring significantly more acidic and amenable to deprotonation. ${ }^{36}$ It also makes the arenes much more susceptible to nucleophilic attack. ${ }^{37}$ Naphthalene and anthracene can form complexes with metal tricarbonyl and cyclopentadienyl fragments, but these ligands tend to be labile as they are able to adopt $\eta^{4}$ and $\eta^{2}$ binding modes as the naphthalene slips off the metal, increasing aromaticity in the unbound portion of the system. ${ }^{35,38}$ For example $\left(\eta^{6}\right.$ - 
naphthalene) $\mathrm{Cr}(\mathrm{CO})_{3}$ can be used as a starting material for the substitution of other arenes on the chromium fragment, ${ }^{38}$ including a report of styrene (Scheme 11). ${ }^{39}$ Anthracene also undergoes ready displacement from ruthenium cyclopentadienyl complexes in the presence of a coordinating solvent. ${ }^{40}$

\section{Scheme 11}

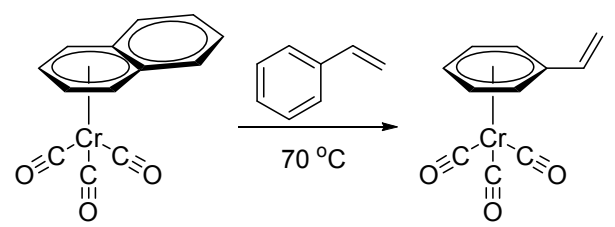

Naphthalene and anthracene complexes of Lewis acidic metal fragments can still undergo substitution and addition reactions similar to those described in the preceding section. Performing such reactions (and similar ones) on complexed arenes offers greater control over isomer distribution of products as well as complementary routes to desired products. ${ }^{36}$ One such route is the lithiation of $\left(\eta^{6}\right.$-naphthalene $) \mathrm{Cr}(\mathrm{CO})_{3}$ at the $\beta$ carbon with a bulky lithium amide base, followed by the addition of methyl iodide as an electrophile (Scheme 12$).^{38}$ This reaction generates 2-methylnaphthalene, the major product of the Friedel-Crafts reaction of naphthalene and methyl iodide. However in the Friedel-Crafts process, about $25 \%$ of the product is 1-methylnapthalene when the isomers are at equilibrium under the reaction conditions. ${ }^{7}$ The relative electron deficiency of the bound ring of $\left(\eta^{6}\right.$-naphthalene $) \mathrm{Cr}(\mathrm{CO})_{3}$ offers another route to alkylation using carbon nucleophiles. In one report, lithium methylpropionitrile added to $\left(\eta^{6}\right.$-naphthalene $) \mathrm{Cr}(\mathrm{CO})_{3}$ at $-65{ }^{\circ} \mathrm{C}$ yielded a mixture of $\alpha$ and $\beta$ isomers, while the same reaction at $0{ }^{\circ} \mathrm{C}$ yielded the $\alpha$ product exclusively (Scheme 13). ${ }^{41}$ The thermodynamic product in this reaction is the opposite of what would be expected from an organic alkylation. 


\section{Scheme 12}

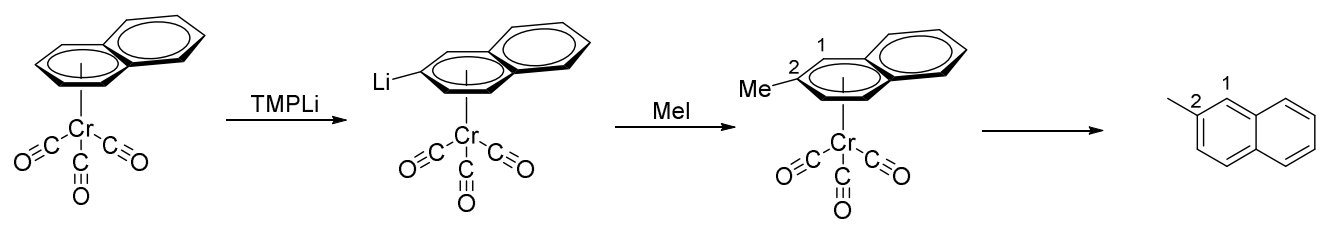

\section{Scheme 13}

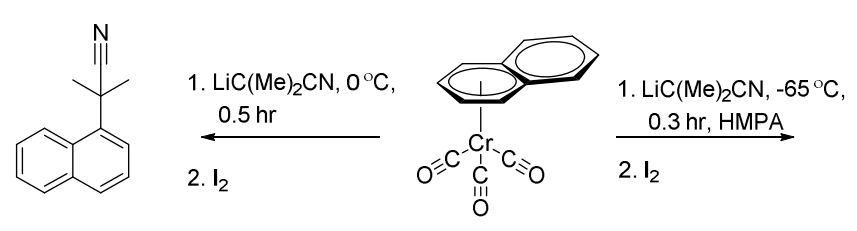

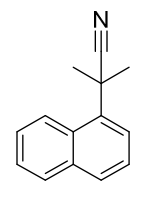

$42 \%$

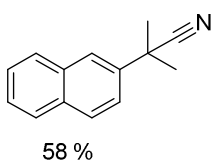

There are many examples of complexes of substituted naphthalenes that can be functionalized to dearomatized products, including those of chromium, ${ }^{42}$ platinum, ${ }^{43}$ and palladium. ${ }^{44-46}$ One study of $\left(\eta^{6}-5\right.$-methoxynaphthalene $) \mathrm{Cr}(\mathrm{CO})_{3}$ added a carbon nucleophile followed a carbon electrophile and $\mathrm{CO}$ insertion to give a 1,2-addition product with trans stereochemistry (Scheme 14). ${ }^{42}$

Scheme 14

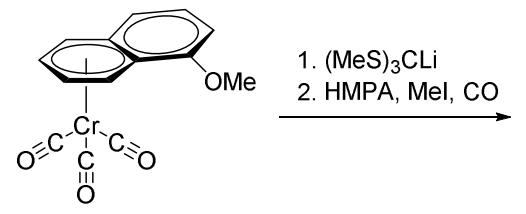

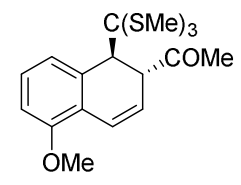

$86 \%$

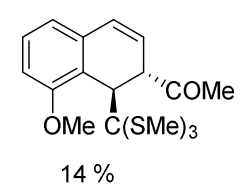

$14 \%$

The reactions of naphthalenes with palladium complexes are interesting in that they are catalytic with respect to the palladium, which does not bind the arene $\eta^{6}$ but rather inserts into a carbon-chloride bond to form an $\eta^{3}$ allyl species. ${ }^{45,46}$ This exo allyl structure requires that aromaticity be broken in the bound naphthalene ring, facilitating a cross-coupling reaction to give a 1,2-dihydronaphthalene (Scheme 15$).{ }^{46}$ The $\eta^{3}$ allyl fragment can also undergo addition reactions to generate 1,2-dihydronaphthalenes or 1,4-dihydronaphthalenes, depending on the bulkiness of the incoming nucleophile (Scheme 16). ${ }^{45}$ 


\section{Scheme 15}

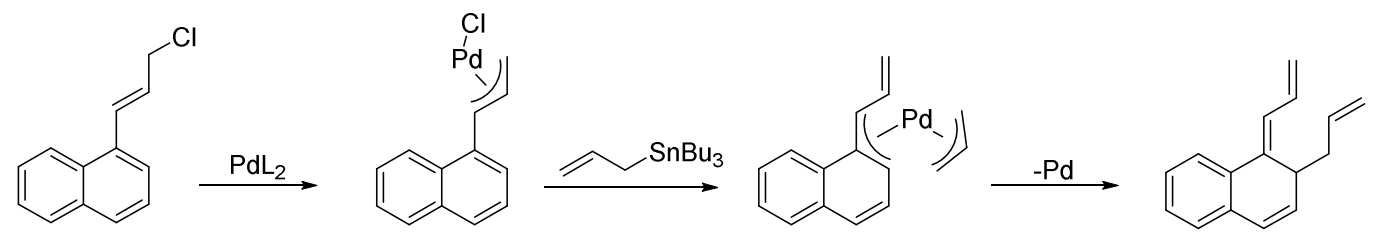

\section{Scheme 16}

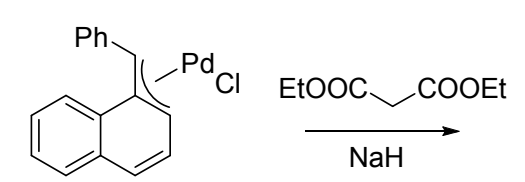<smiles>CCOC(=O)C(C(=O)OCC)C1C=Cc2ccccc2C1=CPc1ccccc1</smiles>

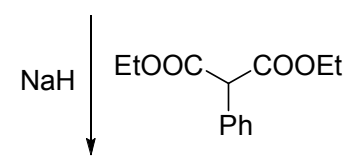

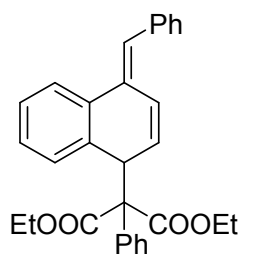

The $\eta^{3}$ naphthalene palladium model was further shown to be useful in producing aminated naphthalenes, where the primary or secondary amine attachment is para to the alkyl chain to which the palladium initially binds (Scheme 17). ${ }^{47}$ As in the case of the 1,4dihydronaphthalene shown above, the palladium is thought to isomerize from its initial binding site (where it inserted into the carbon-chloride bond) so that it is situated inside the naphthalene ring. The amine was then added in the presence of sodium hydride, generating a product similar to the 1,4-dihydronaphthalene above. Proton transfer yielded the paraaminated product. This reaction was found to be general for 9-chloromethylanthracene, as well, albeit in lower yields. 


\section{Scheme 17}

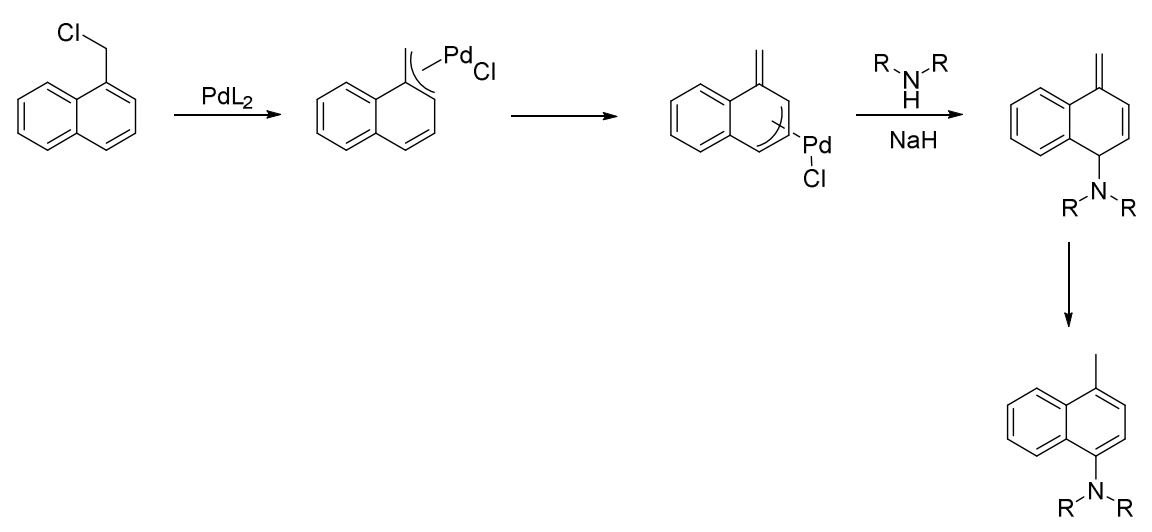

\section{Osmium and rhenium complexes of naphthalene}

A complementary strategy to hydrocarbon functionalization utilizes metal complexes that, instead of being electron deficient, are very electron rich. These complexes bind arenes $\eta^{2}$, or via one electron pair from the arene $\pi$ system. The metal-arene bond is composed of two interactions: the $\sigma$ donation of the electron pair from the arene to the metal complex, and the $\pi$ donation of electron density from the metal d-orbitals into the $\pi^{*}$ orbital of the arene (backbonding). This interaction has two consequences that are crucial to the success of $\eta^{2}$ dearomatization chemistry. First, the metal acts as a protecting group for the bond to which it is bound, preventing reactions from occurring there and acting as a point of reference when predicting regio- and stereochemical outcomes of products. Second, the donation of electron density into the arene $\pi$ system makes the arene more electron rich while localizing the electron density into discrete bonds (Figure 7). In this way, the arene may appear (and react) more like a diene than an arene. Thus, these electron rich complexes should exhibit patterns of reactivity that differ from those of the electron deficient metal complexes, but also of the free arene.

Figure 7 - Schematic of $\eta^{2}$ bonding

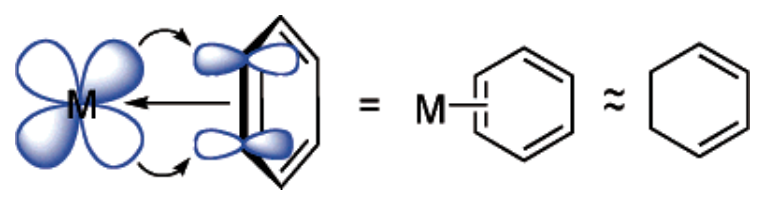


The first electron rich, or $\pi$ basic, metal fragment to be widely used in arene functionalization was an osmium complex developed by W. Dean Harman, $\left\{\left[\mathrm{Os}\left(\mathrm{NH}_{3}\right)_{5}\right]^{2+}(\mathrm{OTf}\right.$ )$\left._{2}\right\}^{48,49}$ This osmium fragment could form complexes with naphthalene and anthracene, in which the metal is bound to the terminal ring (Figure 8 ). ${ }^{50,51}$ Individual resonances could be observed for each proton in the ${ }^{1} \mathrm{H}$ NMR spectra of these complexes, a feature not observed in the parent $\left[\mathrm{Os}\left(\mathrm{NH}_{3}\right)_{5}\left(\eta^{2} \text {-benzene }\right)\right]^{2+}(\mathrm{OTf})_{2}$ complex. While the metal fragment can isomerize, such that it is attached to different $\pi$ bonds in the arene, this process is too slow to be observed on the NMR time scale for naphthalene and anthracene.

Figure 8 - Figures of osmium-naphthalene and anthracene complexes

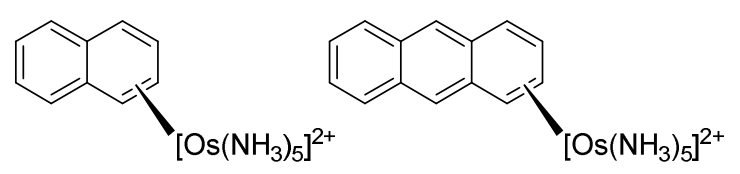

The unique reactivity observed from these arene-osmium complexes is aptly illustrated by their behavior upon addition of a simple electrophile, a proton. Typically, a proton that added to naphthalene or anthracene is quickly deprotonated, although arenium ions are observable spectroscopically under certain conditions. ${ }^{4}$ When a proton adds to $\left[\mathrm{Os}\left(\mathrm{NH}_{3}\right)_{5}\left(\mathrm{\eta}^{2}-\right.\right.$ naphthalene $)]^{2+}(\mathrm{OTf})_{2}$ or $\left[\mathrm{Os}\left(\mathrm{NH}_{3}\right)_{5}\left(\eta^{2} \text {-anthracene }\right)\right]^{2+}(\mathrm{OTf})_{2}$ it forms an arenium complex that is stable in solution for up to three hours at room temperature (Scheme 18). ${ }^{51}$ The electronrichness of the osmium fragment is able to stabilize allylic arenium ligand, 1, opening a pathway to further elaboration.

Scheme 18

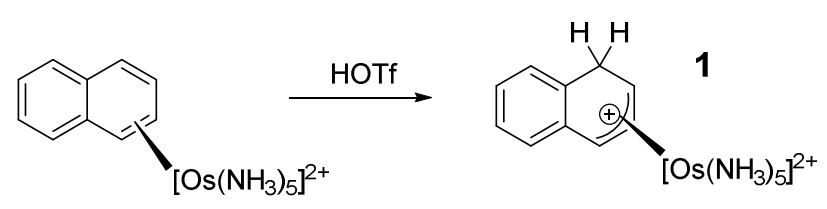


Mark Winemiller found that 1 underwent nucleophilic addition at $-40{ }^{\circ} \mathrm{C}$ to give $1,4-$ dihydronaphthalene products, using triethylsilane and 1-methoxy-2-methyl-1-(trimethylsiloxy) propene (MMTP) as nucleophiles (Scheme 19). ${ }^{52}$

Scheme 19
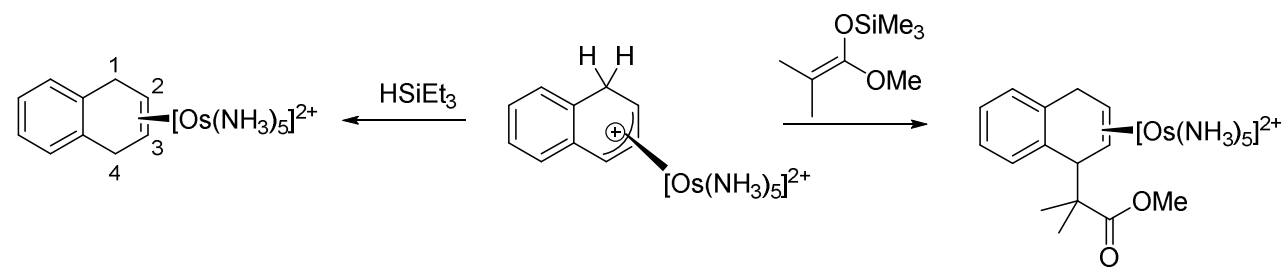

The relative acidity of $1\left(p K_{a}=-8.2\right)$ limited the range of nucleophiles which could be added to $1 .{ }^{51}$ Carbon electrophiles were found to generate more stable arenium complexes with $\left[\mathrm{Os}\left(\mathrm{NH}_{3}\right)_{5}\left(\eta^{2} \text {-naphthalene }\right)\right]^{2+}\left(\mathrm{OTf}^{-}\right)_{2}$ owing to the unfavorableness of eliminating a carbocation and the apparent unfavorableness of eliminating the ipso proton instead. Winemiller reported naphthalene complexes in which dimethoxymethane, methyl vinyl ketone, and tert-butyl alcohol all successfully added as carbocations. Nucleophiles added to these arenium complexes to give 1,4 -addition products. $^{52}$ A couple of examples are given below (Scheme 20).

\section{Scheme 20}

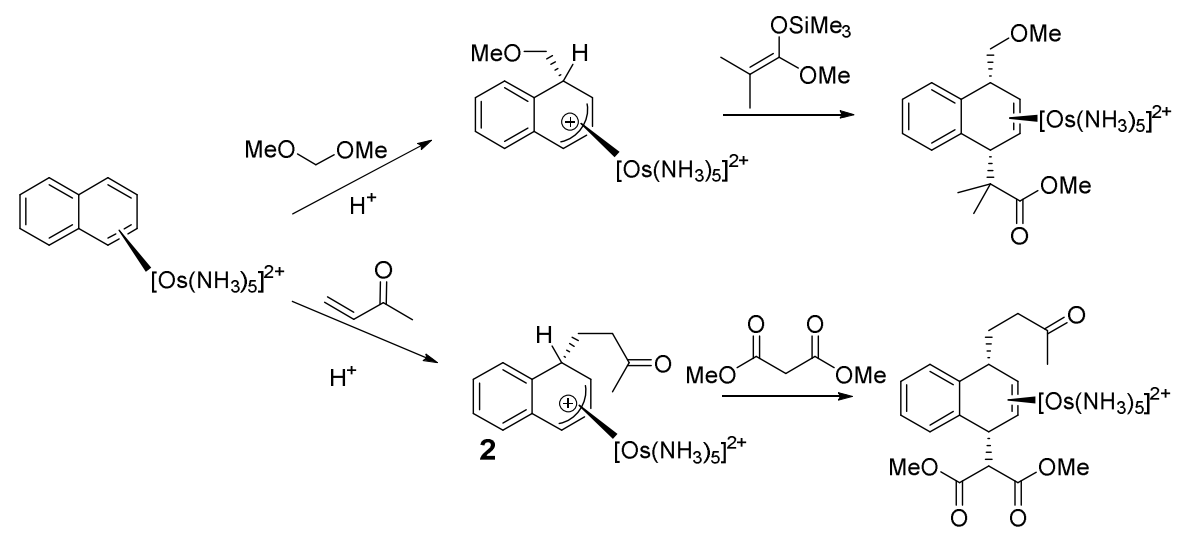

In all examples of tandem electrophile-nucleophile additions to $\left[\mathrm{Os}\left(\mathrm{NH}_{3}\right)_{5}\left(\eta^{2}-\right.\right.$ naphthalene) $]^{2+}(\mathrm{OTf})_{2}$ the addition gave 1,4 -stereochemistry. 1,2-Stereochemistry might be 
expected to be preferred, as in this case the double bond to which the metal is bound is also in conjugation with unbound ring, allowing electron density from the metal to be shared over a larger $\pi$ system. The authors rationalized the observed stereochemistry by considering that C4 should be more cationic than $\mathrm{C} 2$, as placement of the positive charge on C4 allows it to be delocalized over the adjacent ring $\pi$ system. ${ }^{52}$ Under kinetically-controlled conditions, nucleophiles would attack at C4. Oxidation of the metal with silver triflate allowed the functionalized organics to be isolated.

In addition to the tandem additions described above, the MVK-derived arenium, 2, also underwent cyclization reactions in acidic solution. ${ }^{53}$ The ketone tail proved to be enolizable, generating a nucleophile that could attack at $\mathrm{C} 2$ to give a tricyclic species, which again could be isolated via oxidation of the metal with silver triflate (Scheme 21).

\section{Scheme 21}

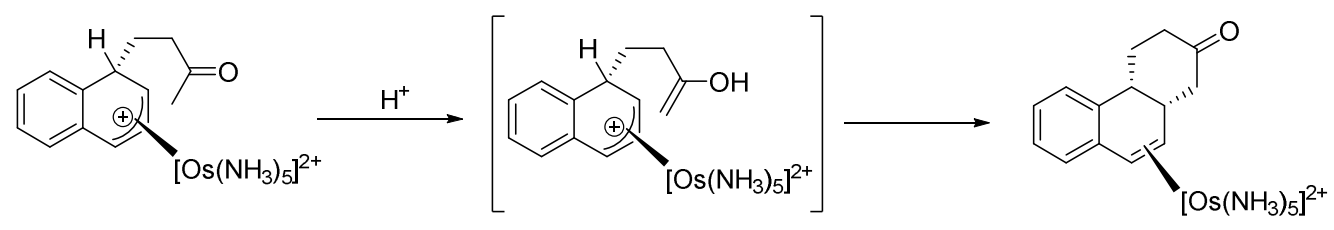

While the $\left\{\left[\mathrm{Os}\left(\mathrm{NH}_{3}\right)_{5}\right]^{2+}(\mathrm{OTf})_{2}\right\}$ fragment expanded scope of reactivity possible with naphthalene, there were several shortcomings of this system that led Harman et al. to develop other dearomatization agents. ${ }^{48}$ Specifically, the osmium fragment is achiral, inhibiting stereoselective and enantioselective functionalization. The expense and toxicity of osmium were also motivations for developing new complexes.

The second generation dearomatization agents were complexes of rhenium, $\{\operatorname{TpRe}(\mathrm{CO})(\mathrm{L})\}$ (Figure 9). These complexes were chiral at the rhenium center, ${ }^{54}$ with the added benefit of bearing a tunable ancillary ligand, $\mathrm{L}$, where $\mathrm{L}$ may be $t$-BuNC, $\mathrm{PMe}_{3}, \mathrm{~N}$ methylimidazole (Melm), pyridine, or some other $\sigma$ donor. ${ }^{55}$ This ancillary ligand allowed the electronics of the complex to be tuned, altering the chemistry possible on the arene ligand. The 
chiral environment around the metal center allowed for different bonds and faces of the naphthalene ring to be differentiated, and two diastereomers of rhenium naphthalene complexes are observed for most ancillary ligands, in which the unbound ring is distal to the ancillary ligand (A) or proximal to it (B) (Figure 9). ${ }^{56}$ The ratio of $\mathbf{A}$ and $\mathbf{B}$ is variable with the identity of the ancillary ligand; some of them are given in Table 2. The interconversion of $\mathbf{A}$ and B is observable on the NMR time scale, and isomerization rate constants were elucidated for several rhenium naphthalene complexes (and complexes of other ligands). ${ }^{56}$ Two distinct isomerization pathways were observable: an interfacial pathway, where the metal migrates from one side of the bound bond to the other, and an intrafacial pathway, where the metal migrates from one double bond to the other in the bound ring (Figure 10).

Figure $9-\operatorname{TpRe}(\mathrm{CO})(\mathrm{L})(\pi$-ligand), with naphthalene isomers
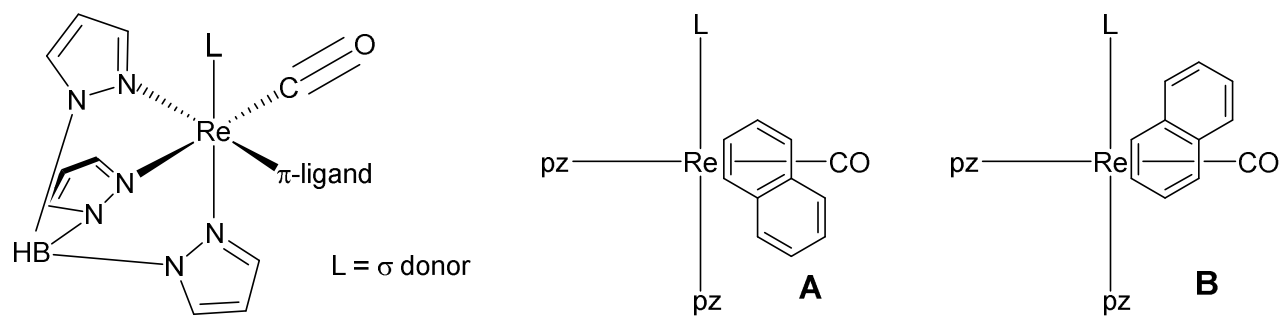

Table 2 - TpRe(CO)(L)(3,4- $\eta^{2}$-naphthalene) isomer ratios, by ligand

\begin{tabular}{|lc|}
\hline Ancillary ligand, $\mathrm{L}$ & $\mathrm{B}: \mathrm{A}$ \\
\hline$t$-BuNC & $1.2: 1$ \\
$\mathrm{PMe}_{3}$ & $<1 / 19$ \\
Pyridine & $3: 1$ \\
Melm & $4.5: 1$ \\
\hline
\end{tabular}

Figure 10 - Isomerization pathways for $\operatorname{TpRe}(\mathrm{CO})(L)\left(3,4-\eta^{2}\right.$-naphthalene)
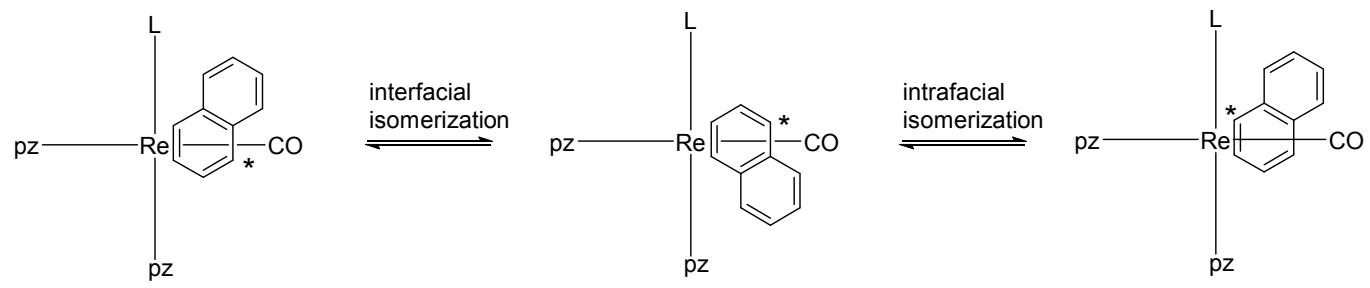
The variety of rhenium naphthalene complexes brought with it a variety of reaction outcomes. While the isomer ratio of the neutral naphthalene complexes is low in some cases, addition of a proton to form an arenium complex provided much greater isomer differentiation. ${ }^{57}$ This isomer differentiation led to different regiochemical outcomes. For example, when MMTP added to a naphthalenium complex where the ancillary ligand was $\mathrm{PMe}_{3}$, the product was a 1,4-dihydronaphthalene (Scheme 22). When the ancillary ligand was $N$ methylimidazole, the product was a 1,2-dihydronaphthalene. ${ }^{57}$

\section{Scheme 22}
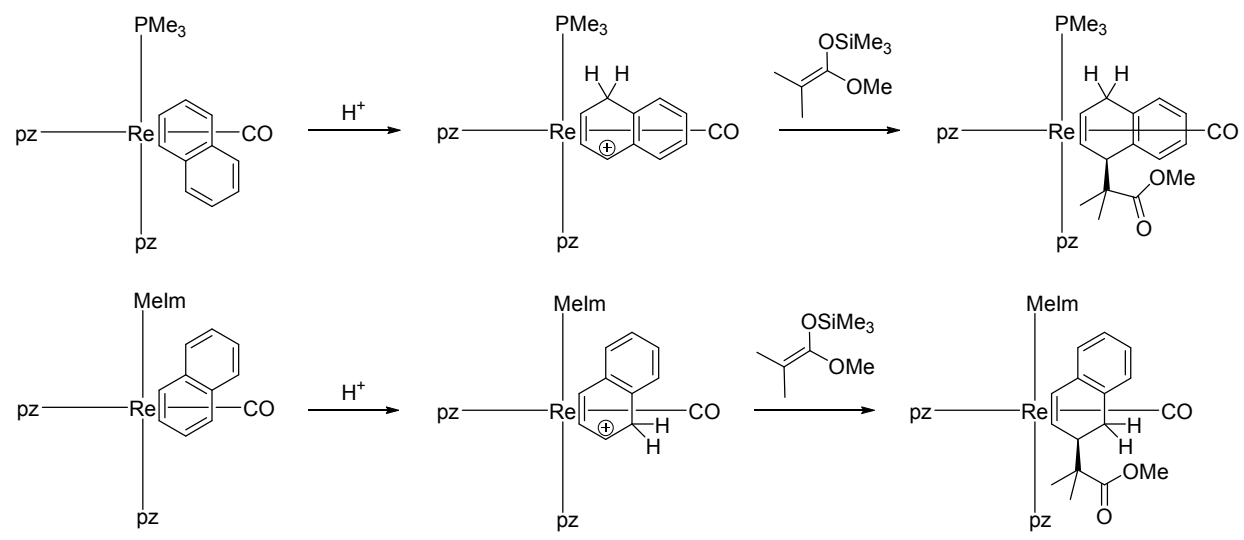

Carbon electrophiles easily added to the rhenium naphthalene complexes, and the resulting naphthalenium complexes reacted with nucleophiles to give 1,4-dihydronaphthalene products. ${ }^{58}$ The preference for 1,4-addition may be due to the steric effects of the carbon electrophiles.

The intrafacial isomerization observed between forms A and B of the complexes likely proceeds via a 2,3- $\eta^{2}$-naphthalene intermediate (Scheme 23). As the aromaticity of unbound ring is disrupted in this form, and the entire unbound portion of the naphthalene fragment resembles an o-quinone dimethide structure, dienophiles were tested with the rhenium complexes to see if they would undergo Diels-Alder cycloadditions. 
Scheme 23

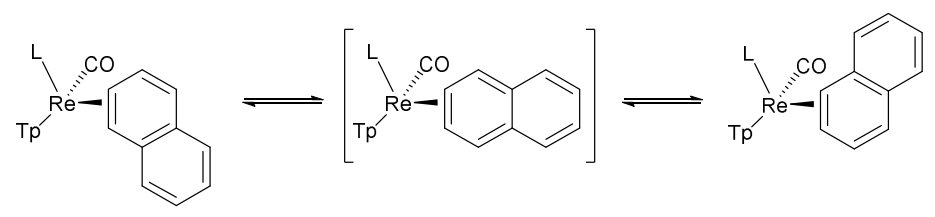

Despite the expected accessibility of the 2,3- $\eta^{2}$-naphthalene intermediate, no cycloaddition was observed when TpRe(CO)(Melm)(3,4- $\eta^{2}$-naphthalene) was combined with $N$ methylmaleimide, a powerful dienophile. A cycloadduct was observed when $\operatorname{TpRe}(\mathrm{CO})(\mathrm{Melm})\left(3,4-\eta^{2}\right.$-naphthalene) reacted with methyl acrylate (Scheme 24$) .{ }^{58}$ However, in this reaction no metal complex could be isolated with the cycloadduct intact. The cycloadduct could be isolated as a free organic after oxidation of the metal with silver triflate. This result suggests that the cycloaddition does not proceed via a concerted, Diels-Alder-like mechanism, but rather, step-wise.

\section{Scheme 24}

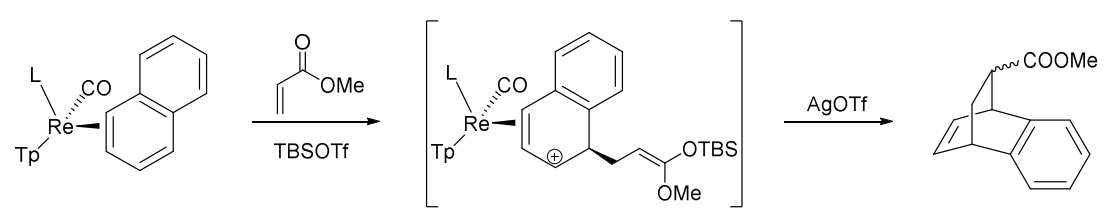

\section{Tungsten complexes of naphthalene and anthracene}

While the rhenium dearomatization agents made many improvements on the earlier osmium pentammine system, they were not without flaws. The synthesis of the complexes were cumbersome and could not be easily scaled-up and the rhenium metal, while cheaper than osmium, was expensive. ${ }^{48}$ Lessons learned from the development of the rhenium complexes could be applied to the development of tungsten complexes, which were desirable for several reasons. They were expected to be more electron rich than either the osmium or rhenium system, owing to tungsten's position in the periodic table relative to those metals. This electron 
richness would potentially open up new avenues of reactivity. Tungsten is also less expensive than osmium or rhenium, which would broaden a tungsten complex's utility to other synthetic groups as $\eta^{2}$ dearomatization methodology expands beyond the Harman group.

Early work by Peter Graham and Kevin Welch on the chemistry of $\left\{\mathrm{TpW}(\mathrm{NO})\left(\mathrm{PMe}_{3}\right)\right\}$ showed that this dearomatization agent also formed a stable complex with naphthalene. ${ }^{59}$ Like its rhenium precursor, it is a chiral metal fragment, but unlike its rhenium precursor, it can be isolated as a single naphthalene diastereomer (Figure 11). The complex, $\mathrm{TpW}(\mathrm{NO})\left(\mathrm{PMe}_{3}\right)\left(3,4-\eta^{2}-\right.$ naphthalene), was found to be thermally stable over several weeks of heating in solution. ${ }^{60}$ This feature would be potentially useful as the reactivity of the complex was probed.

Figure $11-\mathrm{TpW}(\mathrm{NO})\left(\mathrm{PMe}_{3}\right)\left(3,4-\eta^{2}-\right.$-naphthalene $)$

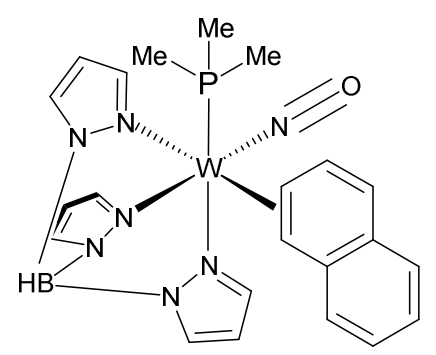

One feature of $\mathrm{TpW}(\mathrm{NO})\left(\mathrm{PMe}_{3}\right)\left(3,4-\eta^{2}\right.$-naphthalene) that proved to be very useful as the chemistry of the complex was probed was the $\mathrm{PMe}_{3}$ ligand. $\mathrm{As}^{31} \mathrm{P}$ is an NMR active nucleus and in $100 \%$ abundance, early characterization of this complex and others, performed by Kevin Welch, used ${ }^{31} \mathrm{P}$ NMR spectroscopy as a means of characterization of the complexes. ${ }^{60}$ This study did not find any strong correlation between ${ }^{31} \mathrm{P}$ NMR chemical shifts and complex type, but did find a correlation between ${ }^{183} \mathrm{~W}-{ }^{31} \mathrm{P}$ coupling constants for the chemical shifts and complex type. Complexes in which an arene or other $\pi$ ligand was bound $\eta^{2}$ to the metal had ${ }^{183} \mathrm{~W}-{ }^{31} \mathrm{P}$ coupling constants between 255 and $314 \mathrm{~Hz}$. The coupling tended to be on the smaller end for complexes that were less electron rich. The ${ }^{183} \mathrm{~W}-{ }^{31} \mathrm{P}$ coupling constant for $\mathrm{TpW}(\mathrm{NO})\left(\mathrm{PMe}_{3}\right)\left(3,4-\eta^{2}\right.$-naphthalene) was measure as $297 \mathrm{~Hz}{ }^{60}$ 
${ }^{31} \mathrm{P}$ NMR spectroscopy proved extremely useful over the course of the work described in the following chapters, as it allowed us to get information about the progression and outcome of a reaction without needing to perform a work-up of the reaction. As $\left\{\mathrm{TpW}(\mathrm{NO})\left(\mathrm{PMe}_{3}\right)\right\}$ complexes have only one phosphine ligand, counting the number of signals in a ${ }^{31} \mathrm{P} N M R$ spectrum tells us how many complexes are present, including starting material, intermediates, and products. The ${ }^{183} \mathrm{~W}-{ }^{31} \mathrm{P}$ coupling constant gives us some information about the nature of those complexes, for example, whether they are neutral or cationic. Cationic complexes have smaller ${ }^{183} \mathrm{~W}_{-}{ }^{31} \mathrm{P}$ coupling constants. Monitoring reactions via ${ }^{31} \mathrm{P}$ NMR spectroscopy saved us time and materials by allowing us to pursue reactions that we could be reasonably sure had worked before working them up.

\section{References}

(1) Grimmer, G. Environmental Carcinogens: Polycyclic Aromatic Hydrocarbons; CRC Press: Boca Raton, FL, 1983.

(2) International Agency for Research on Cancer, S. IARC Monographs on the Evaluation of Carcinogenic Risks to Humans, Volume 82 : Some Traditional Herbal Medicines, Some Mycotoxins, Naphthalene and Styrene; World Health Organization (WHO): Albany, NY, USA, 2002.

(3) Carey, F. A. S., Richard J. Advanced Organic Chemistry, Part A: Structure and Mechanisms; Springer: New York, 2007; Vol. 5.

(4) Harvey, R. G. Polycyclic Aromatic Hydrocarbons; Wiley-VCH: New York, 1997.

(5) Clar, E. The Aromatic Sextet; Wiley: New York, 1972.

(6) Herndon, W. C. Journal of the American Chemical Society 1974, 96, 7605.

(7) Olah, G. A.; Olah, J. A. Journal of the American Chemical Society 1976, 98, 1839.

(8) Olah, G. A. Friedel-Crafts and Related Reactions; Wiley-Interscience: New York, 1970.

(9) Smith, K.; Roberts, S. D. Catalysis Today 2000, 60, 227.

(10) Vahteristo, K.; Sahala, K.-M.; Koskimies, S. Industrial \& Engineering Chemistry Research 2010, 49, 4018.

(11) Smith, K.; Al-Khalaf, A. K. H.; El-Hiti, G. A.; Pattisson, S. Green Chemistry 2012, $14,1103$.

(12) Fraenkel, D.; Cherniavsky, M.; Ittah, B.; Levy, M. Journal of catalysis 1986, 101, 273.

(13) Jang, K. S.; Shin, H. Y.; Chi, D. Y. Tetrahedron 2008, 64, 5666.

(14) C. E. Braun, C. D. C., C. Merritt, J. E. Rousseau Organic Syntheses 1965, IV, 711. 
(15) Granoth, I.; Segall, Y.; Leader, H.; Alkabets, R. The Journal of Organic Chemistry $1976,41,3682$.

(16) Sun, L.-B.; Zong, Z.-M.; Kou, J.-H.; Zhang, L.-F.; Ni, Z.-H.; Yu, G.-Y.; Chen, H.; Wei, X.-Y.; Lee, C. W. Energy \& Fuels 2004, 18, 1500.

(17) Borowski, A. F.; Sabo-Etienne, S.; Chaudret, B. Journal of Molecular Catalysis A: Chemical 2001, 174, 69.

(18) Hiyoshi, N.; Osada, M.; Rode, C. V.; Sato, O.; Shirai, M. Applied Catalysis A: General 2007, 331, 1.

(19) Fu, P. P.; Lee, H. M.; Harvey, R. G. The Journal of Organic Chemistry 1980, 45, 2797.

(20) Rossi, R. A.; Camusso, C. C.; Madoery, O. D. The Journal of Organic Chemistry 1974, 39, 3254.

(21) Rabideau, P. W.; Harvey, R. G. Tetrahedron Letters 1970, 11, 4139.

(22) Nicholls, D.; Szware, M. Journal of the American Chemical Society 1966, 88, 5757.

(23) Harvey, R. G.; Davis, C. C. The Journal of Organic Chemistry 1969, 34, 3607.

(24) Zieger, H. E.; Gelbaum, L. T. The Journal of Organic Chemistry 1972, 37, 1012.

(25) Sanyal, A.; Snyder, J. K. Organic Letters 2000, 2, 2527.

(26) Bentolila, A.; Totre, J.; Zozulia, I.; Levin-Elad, M.; Domb, A. J. Macromolecules $2013,46,4822$.

(27) Krenske, E. H.; Houk, K. N. Accounts of Chemical Research 2012, 46, 979.

(28) Tandon, P. K.; Baboo, R.; Singh, A. K.; Gayatri Appl. Organomet. Chem. 2006, 20, 20.

(29) Tandon, P. K.; Gayatri; Sahgal, S.; Srivastava, M.; Singh, S. B. Appl. Organomet. Chem. 2007, 21, 135.

(30) Zhdankin, V. V. The Journal of Organic Chemistry 2011, 76, 1185.

(31) Rodríguez, F.; Blanco, M. D.; Adrados, L. F.; Burillo, J. C.; Tijero, J. F. Tetrahedron Letters 1989, 30, 2417.

(32) Charleton, K. D. M.; Prokopchuk, E. M. Journal of Chemical Education 2011, 88, 1155.

(33) Akar, K. B.; Cakmak, O.; Buyukgungor, O.; Sahin, E. Beilstein J. Org. Chem. 2011, 7, 1036.

(34) Transition Metal Arene pi-Complexes in Organic Synthesis and Catalysis; Kuendig, E. P., Ed.; Springer: New York, 2004; Vol. 7.

(35) Kuendig, E. P. Topics in Organometallic Chemistry 2004, 7, 3.

(36) Morley, J. A.; Woolsey, N. F. The Journal of Organic Chemistry 1992, 57, 6487.

(37) Martin F. Semmelhack, A. C. Topics in Organometallic Chemistry 2004, 7, 43.

(38) Kuendig, E. P.; Desobry, V.; Grivet, C.; Rudolph, B.; Spichiger, S. Organometallics 1987, 6, 1173.

(39) Kundig, E. P.; Perret, C.; Spichiger, S.; Bernardinelli, G. Journal of Organometallic Chemistry 1985, 286, 183.

(40) McNair, A. M.; Mann, K. R. Inorganic Chemistry 1986, 25, 2519.

(41) Kuendig, E. P.; Desobry, V.; Simmons, D. P.; Wenger, E. Journal of the American Chemical Society 1989, 111, 1804.

(42) Kuendig, E. P.; Inage, M.; Bernardinelli, G. Organometallics 1991, 10, 2921.

(43) Shibuya, T.; Noguchi, K.; Tanaka, K. Angewandte Chemie International Edition 2012, 51, 6219. 
(44) Peng, B.; Feng, X.; Zhang, X.; Zhang, S.; Bao, M. The Journal of Organic Chemistry 2010, 75, 2619.

(45) Peng, B.; Zhang, S.; Yu, X.; Feng, X.; Bao, M. Organic Letters 2011, 13, 5402.

(46) Lu, S.; Xu, Z.; Bao, M.; Yamamoto, Y. Angewandte Chemie International Edition 2008, 47, 4366.

(47) Zhang, S.; Wang, Y.; Feng, X.; Bao, M. Journal of the American Chemical Society 2012, 134, 5492.

(48) Keane, J. M.; Harman, W. D. Organometallics 2005, 24, 1786.

(49) Harman, W. D.; Taube, H. Journal of the American Chemical Society 1987, 109, 1883. 7906.

(50) Harman, W. D.; Taube, H. Journal of the American Chemical Society 1988, 110,

(51) Winemiller, M. D.; Kopach, M. E.; Harman, W. D. Journal of the American Chemical Society 1997, 119, 2096.

(52) Winemiller, M. D.; Harman, W. D. Journal of the American Chemical Society $1998,120,7835$.

(53) Winemiller, M. D.; Harman, W. D. The Journal of Organic Chemistry 2000, 65, 1249.

(54) Meiere, S. H.; Valahovic, M. T.; Harman, W. D. Journal of the American Chemical Society 2002, 124, 15099.

(55) Meiere, S. H.; Brooks, B. C.; Gunnoe, T. B.; Carrig, E. H.; Sabat, M.; Harman, W. D. Organometallics 2001, 20, 3661.

(56) Brooks, B. C.; Meiere, S. H.; Friedman, L. A.; Carrig, E. H.; Gunnoe, T. B.; Harman, W. D. Journal of the American Chemical Society 2001, 123, 3541.

(57) Valahovic, M. T.; Gunnoe, T. B.; Sabat, M.; Harman, W. D. Journal of the American Chemical Society 2002, 124, 3309.

(58) Ding, F.; Valahovic, M. T.; Keane, J. M.; Anstey, M. R.; Sabat, M.; Trindle, C. O.; Harman, W. D. The Journal of Organic Chemistry 2004, 69, 2257.

(59) Ha, Y.; Dilsky, S.; Graham, P. M.; Liu, W.; Reichart, T. M.; Sabat, M.; Keane, J. M.; Harman, W. D. Organometallics 2006, 25, 5184.

(60) Welch, K. D.; Harrison, D. P.; Lis, E. C.; Liu, W.; Salomon, R. J.; Harman, W. D.; Myers, W. H. Organometallics 2007, 26, 2791. 
Chapter 2

Hyper-Distorted Tungsten Allyl Complexes and Their Stereoselective Deprotonation to Form Dihapto-Coordinated Dienes 
One remarkable feature observed in arene complexes of $\left\{\mathrm{TpW}(\mathrm{NO})\left(\mathrm{PMe}_{3}\right)\right\}$ and their rhenium and osmium precursors is the high degree of regio- and stereoselectivity with which their tandem additions typically proceed. When arene complexes of $\left\{\mathrm{TpW}(\mathrm{NO})\left(\mathrm{PMe}_{3}\right)\right\}$ are protonated, the resulting allyls are highly asymmetric in terms of the bonding experienced by each carbon with the tungsten atom. In many cases this asymmetry influences the regioselectivity of the products formed. In other cases, the product opposite of what allylic asymmetry would predict is observed. We sought to better understand the origin of this asymmetry by studying a number of model complexes, the hydrocarbon models of which will be presented here.

We also hoped to exploit the asymmetry of similar allylic complexes to produce single disastereomers of $\eta^{2}$-1,3-diene complexes via asymmetric deprotonation. Previously, we have demonstrated that dihapto-coordinated cyclic 1,3-dienes can undergo both 1,2- and 1,4tandem addition reactions of electrophiles followed by nucleophiles. While both addition reactions occur to the face of the diene opposite to that which is coordinated, ${ }^{1-5}$ the ability to control the absolute stereochemistry of this reaction sequence ultimately relies not only on access to an enantio-enriched, chiral $\pi$-base $\left(M^{*}\right),{ }^{6-8}$ but also on being able to obtain a single coordination diastereomer of the diene precursor.

\section{Scheme 1}

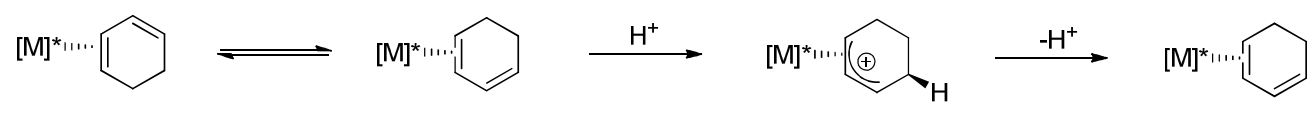

When 1,3-dienes and similar compounds bind to $\left\{\mathrm{TpW}(\mathrm{NO})\left(\mathrm{PMe}_{3}\right)\right\}$, two diastereomers typically result, limiting their potential usefulness as synthons (Scheme 2). Thus, alternative methods to produce single diastereomers are desirable. 
Scheme 2

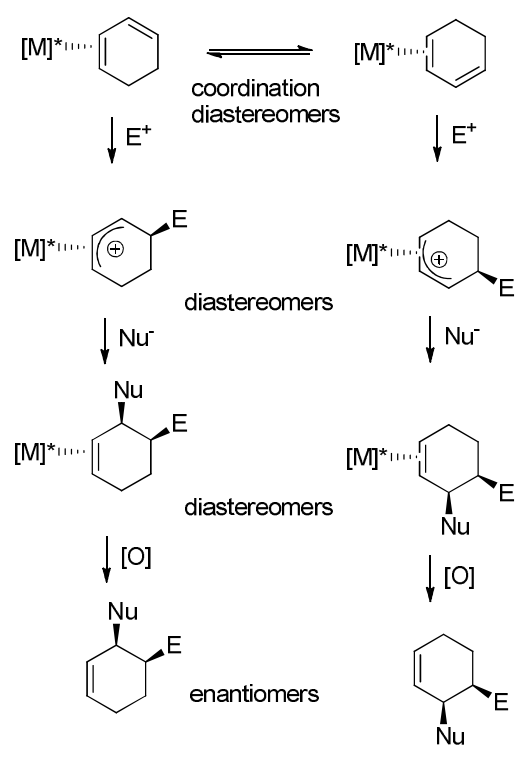

\section{Structural and Spectroscopic Analysis of TpW(NO)(PMe $)(\pi$-allyl) Complexes}

Given the prominent role of $\pi$-allyl intermediates in $\eta^{2}$-aromatic chemistry, ${ }^{9,10}$ and their potential as synthons for $\eta^{2}$-dienes (vide supra), we sought to understand better the structural and spectroscopic features of the $\mathrm{TpW}(\mathrm{NO})\left(\mathrm{PMe}_{3}\right)(\pi$-allyl) complexes, particularly those of hydrocarbons. X-ray data were obtained from single crystals $\left[\mathrm{TpW}(\mathrm{NO})\left(\mathrm{PMe}_{3}\right)\left(\pi-\mathrm{C}_{6} \mathrm{H}_{9}\right)\right] \mathrm{OTf}, \mathbf{1}$, with $\left[\mathrm{TpW}(\mathrm{NO})\left(\mathrm{PMe}_{3}\right)\left(\pi-\mathrm{C}_{7} \mathrm{H}_{10} \mathrm{NO}\right)\right] \mathrm{OTf}, \mathbf{2}$, and $\mathrm{TpW}(\mathrm{NO})\left(\mathrm{PMe}_{3}\right)\left(\eta^{2}-\mathrm{C}_{7} \mathrm{H}_{9} \mathrm{NO}\right), \mathbf{3}$, a pyridine-derived alkene given for comparison (Figure 1). Complex 1 was synthesized from the combination of 1,3-cyclohexadiene and $\mathrm{TpW}(\mathrm{NO})\left(\mathrm{PMe}_{3}\right)\left(\eta^{2}\right.$-benzene), followed by protonation with triflic acid (HOTf). Complex 3 has been previously synthesized from $\mathrm{TpW}(\mathrm{NO})\left(\mathrm{PMe}_{3}\right)\left(\eta^{2}\right.$-benzene) and pyridine-borane. ${ }^{11}$ Complex 2 can be obtained by protonation of $\mathbf{3}$. Syntheses of these three complexes were developed by Dan Harrison. 
Figure 1 - ORTEP diagrams (30\% probability) for allyl complexes $\left[T p W(N O)\left(P M e_{3}\right)\left(\pi-C_{6} H_{9}\right)\right] O T f, \quad 1$, and $\left[\mathrm{TpW}(\mathrm{NO})\left(\mathrm{PMe}_{3}\right)\left(\pi-\mathrm{C}_{7} \mathrm{H}_{10} \mathrm{NO}\right)\right] \mathrm{OTf}, 2$, showing $\eta^{3} \rightarrow \eta^{2}$ distortion (OTf omitted), and the dihydropyridine complex $\mathrm{TpW}(\mathrm{NO})\left(\mathrm{PMe}_{3}\right)\left(\eta^{2}-\mathrm{C}_{7} \mathrm{H}_{9} \mathrm{NO}\right), \mathbf{3}$, for comparison.
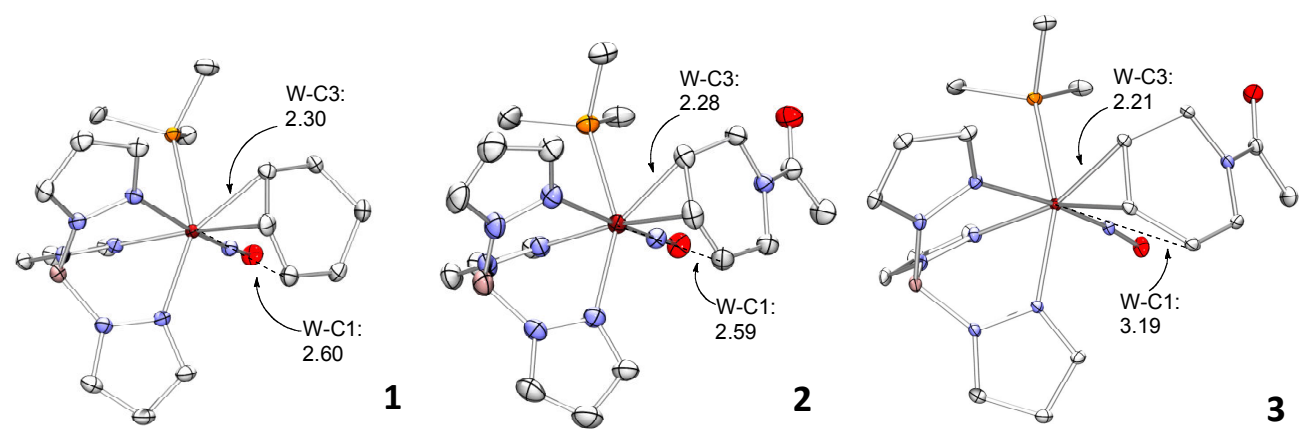

The resulting molecular structure determinations reveal that $\mathrm{C} 2$ and $\mathrm{C} 3$ are nearly equidistant from the tungsten center (ranging from 2.28-2.31 $\AA$ ), with the W-C1 distance markedly elongated (2.60 ̊ for $1 ; 2.59 \AA$ for 2$).{ }^{1} \mathrm{H}$ and ${ }^{13} \mathrm{C}$ NMR spectroscopic data also indicate a stark contrast between the two terminal carbons of the allyl ligand [e.g., for $1:\left({ }^{1} \mathrm{H},{ }^{13} \mathrm{C} \mathrm{ppm}\right)$ C3: $4.38,70.0 ;$ C1: $6.59,138.3]$, which suggested a buildup of positive charge at the C1 terminus. Similar structural features were observed for the analogous cyclohexadienyl molybdenum system, in which the Mo-C1 bond was determined to be $2.64 \AA$ ( $c f .2 .33 \AA$ for $\mathrm{C} 2$ and $\mathrm{C} 3$ ). ${ }^{9}$ In Figure $1 \mathrm{~W}$-C distances of the $\pi$-allyl complexes $(\mathbf{1}, \mathbf{2})$ are compared to the limiting case of the $\eta^{2}$-bound dihydropyridine complex, 3, in which bonding to the allylic carbon would be considered non-existent.

The naphthalenium complex, $\left[\mathrm{TpW}(\mathrm{NO})\left(\mathrm{PMe}_{3}\right)\left(\pi-\mathrm{C}_{10} \mathrm{H}_{9}\right)\right] \mathrm{OTf}, 4$, could be synthesized from the parent $\operatorname{TpW}(\mathrm{NO})\left(\mathrm{PMe}_{3}\right)\left(3,4-\eta^{2} \text {-naphthalene }\right)^{12}$ via protonation with HOTf and precipitation in ether. Attempts to grow crystals of $\mathbf{4}$ were unsuccessful, owing to the instability of the protonated complex. The complex was sufficiently stable, however, to allow for the collection of NMR spectroscopy data. The asymmetry observed in the NMR data for 1 was also observed in 4, where $\mathrm{H} 3$ was observed at $4.95 \mathrm{ppm}\left({ }^{13} \mathrm{C}: 72.2 \mathrm{ppm}\right)$, while $\mathrm{H} 3$ was observed at $6.71 \mathrm{ppm}\left({ }^{13} \mathrm{C}: 130.5 \mathrm{ppm}\right)$. Asymmetry was also observed in the anthracenium analog, 5, for 
which $\mathrm{H} 3$ was observed at $4.69 \mathrm{ppm}\left({ }^{13} \mathrm{C}: 69.1 \mathrm{ppm}\right)$ and $\mathrm{H} 1$ was observed at $7.30 \mathrm{ppm}\left({ }^{13} \mathrm{C}\right.$ : $137.1 \mathrm{ppm}$ ) (Scheme 3). Complex 5 was synthesized from protonation of $\mathrm{TpW}(\mathrm{NO})\left(\mathrm{PMe}_{3}\right)(3,4-$ $\eta^{2}$-anthracene), which has been previously reported. ${ }^{13}$ While it is difficult to compare the degree of asymmetry present in $\mathbf{4}$ or $\mathbf{5}$ to other allyl complexes using only NMR spectroscopy, the spectroscopy data is consistent with $\mathbf{1}$ in that the allyl terminus distal to the phosphine is more deshielded than the proximal terminus.

Scheme 3

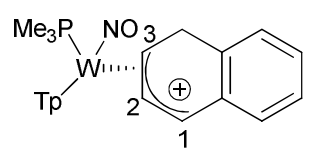

4

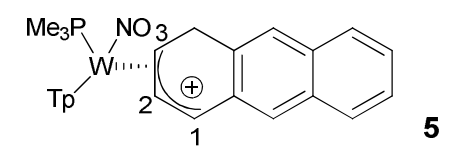

A crystal suitable for X-ray diffraction was grown from a sample of $\left[\mathrm{TpW}(\mathrm{NO})\left(\mathrm{PMe}_{3}\right)(\pi-\right.$ $\left.\mathrm{C}_{7} \mathrm{H}_{9}\right)$ ]OTf, 6 (Figure 2). The complex was synthesized from $\mathrm{TpW}(\mathrm{NO})\left(\mathrm{PMe}_{3}\right)\left(\eta^{2}\right.$-benzene) and 1,3,5-cycloheptatriene, followed by protonation with HOTf. The neutral complex exists as a mixture of three isomers (Scheme 4), but the allyl complex 6 can be isolated as a single isomer.

Scheme 4

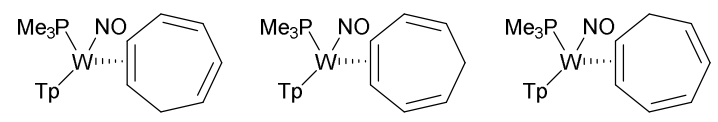

This system contains an extended $\pi$ system along the cycloheptyl ring, making it difficult to compare its structural data directly to that of other allyl systems. One interesting structural feature of 6 is that its C6-C7 bond length is $1.32 \AA$, which is consistent with a C-C bond order of 2.0 and is shorter than any of the C2-C3 (1.39 $\AA)$, C1-C2 (1.40 $\AA)$, and C7-C1 (1.46 $)$ bond lengths. The $\mathrm{W}-\mathrm{C}$ bond length distortion for $\mathrm{W}-\mathrm{C} 3$ and $\mathrm{W}-\mathrm{C} 1$ is not as pronounced as in $\mathbf{1}$. The dihedral angle measured over $\mathrm{C} 2-\mathrm{C} 1-\mathrm{C} 7-\mathrm{C} 6$ is $11^{\circ}$, which is not sufficiently distorted to prevent conjugation between the alkene and allyl, but this has not affected the W-C1 bond length. 
Figure 2 - ORTEP diagrams (30\% probability) for allyl complex $\left[\mathrm{TpW}(\mathrm{NO})\left(\mathrm{PMe}_{3}\right)\left(\pi-\mathrm{C}_{7} \mathrm{H}_{9}\right)\right] \mathrm{OTf}, 6$

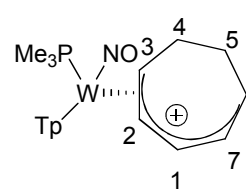

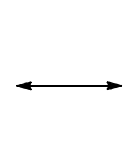

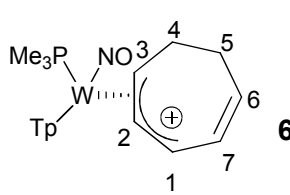

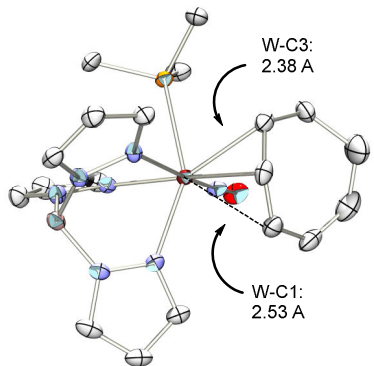

While other groups have documented " $\sigma-\pi$ " or " $\eta^{3} \rightarrow \eta^{2}$ " $\pi$-allyl complexes, ${ }^{14-18}$ differences between the $\mathrm{M}-\mathrm{C}$ bond lengths of the terminal allylic carbons in these species tend to be less than $0.2 \AA$ (Scheme 5). Yet, Legzdins et al. have observed more significant deviations in complexes of the form $\mathrm{Cp} * \mathrm{~W}(\mathrm{NO})(\mathrm{R})\left(\pi-\mathrm{CH}_{2} \mathrm{CHC}(\mathrm{Me})_{2}\right) \cdot{ }^{15,18}$ The authors attribute the unusually large distortions (e.g., $\mathrm{R}=\mathrm{CH}_{2} \mathrm{TMS}, \Delta=0.69 \AA$ ) to steric factors. ${ }^{18}$ The $\sigma-\pi$ distortion formalism describes the metal as forming a sigma bond with one terminal carbon (C3), and a dative bond with the remaining two carbons of the allyl ligand $(C 2=C 1)$. Of course, the closely related $\left\{\mathrm{TpW}(\mathrm{NO})\left(\mathrm{PMe}_{3}\right)\right\}$ systems could also be described this way.

\section{Scheme 5}

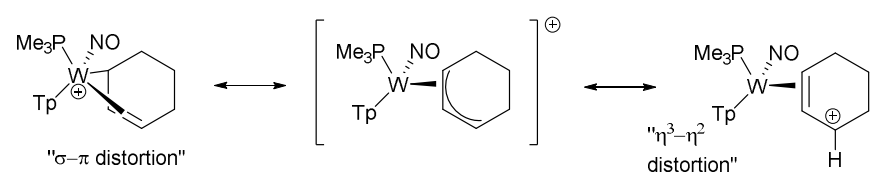

However, the alternative representation of a $W(0)$ center with an $\eta^{2}$-distorted allyl cation is appealing as it readily provides a foundation for the observed structural and spectroscopic features. For example, the W-C2 (2.30 A) and W-C3 (2.31 A) bond lengths of 1 or 2 are virtually identical, and are only $\sim 0.05 \AA$ longer than typical alkene complexes of the form $\operatorname{TpW}(\mathrm{NO})\left(\mathrm{PMe}_{3}\right)(\mathrm{L}) \quad$ (e.g., $\mathrm{L}=$ cyclopentene) or for the diene complex 3 (Figure 1); the C2-C3 bond distance of $1.43 \AA$ is also in the range of typical alkene complexes of the $\left\{\mathrm{TpW}(\mathrm{NO})\left(\mathrm{PMe}_{3}\right)\right\}$ system. ${ }^{4,7,19}$ Moreover, in the $\eta^{2}$-distortion description of the allylic bonding, all carbon atoms maintain their designation as being $\mathrm{sp}^{2}$ hybridized, meaning that all atoms can participate in 
extended conjugated systems in addition to $\pi$ bonding with the tungsten metal. This extended conjugation may be useful in stabilizing the naphthalene and anthracene-derived allyls in $\mathbf{4}$ and 5, in which C1 appears to be more cationic than in 1 . At the same time, the relative lack of distortion to the W-C1 bond length observed in $\mathbf{6}$ compared to 1 may indicate a limitation to the stabilizing effect that an extended conjugation will have for hydrocarbon systems. The double bond character observed between $\mathrm{C} 6$ and $\mathrm{C} 7$ also speaks against extended $\pi$ systems being a significant contributor to $\pi$ allyl complex distortion.

A study of allyl complexes $\left[\mathrm{TpW}(\mathrm{NO})\left(\mathrm{PMe}_{3}\right)\left(\pi-\mathrm{C}_{3} \mathrm{H}_{5}\right)\right] \mathrm{OTf}, 7$, and $\left[\mathrm{TpW}(\mathrm{NO})\left(\mathrm{PMe}_{3}\right)(\pi-\right.$ $\left.\mathrm{C}_{6} \mathrm{H}_{11}\right)$ ]OTf, 8, both developed by Dan Harrison, found that substituents on the allyl do have an effect on the degree of $\eta^{3} \rightarrow \eta^{2}$ distortion observed (Figure 3). For 7 , which has no substituents on the allyl, the difference between W-C3 and W-C1 is only $0.16 \AA$, which is comparable to allyl distortions seen in other complexes. ${ }^{14,16,17}$ For $\mathbf{8}$, which has two methyl groups on the C1 terminus (and another on $\mathrm{C} 2$ ), the W-C1 bond length is $2.91 \AA$, or $0.70 \AA$ longer than $\mathrm{W}-\mathrm{C} 3$, and approaching a length at which C1 might not be considered bound to the tungsten at all. Additional evidence is found in the increased back-donation to the nitrosyl ligand as the number of alkyl substituents for $\mathrm{C} 1$ increases from 0 to $2\left(v_{\mathrm{NO}}=1647 \mathrm{~cm}^{-1}(\mathbf{7}), 1635 \mathrm{~cm}^{-1}(\mathbf{1}), 1624 \mathrm{~cm}^{-1}\right.$ (8)).

Figure 3 - ORTEP diagrams (30\% probability) of $\left[\mathrm{TpW}(\mathrm{NO})\left(\mathrm{PMe}_{3}\right)\left(\pi-\mathrm{C}_{3} \mathrm{H}_{5}\right)\right] \mathrm{OTf}, \mathbf{7}$, and $\left[\mathrm{TpW}(\mathrm{NO})\left(\mathrm{PMe}_{3}\right)\left(\pi-\mathrm{C}_{6} \mathrm{H}_{11}\right)\right] \mathrm{OTf}, \mathbf{8}$.
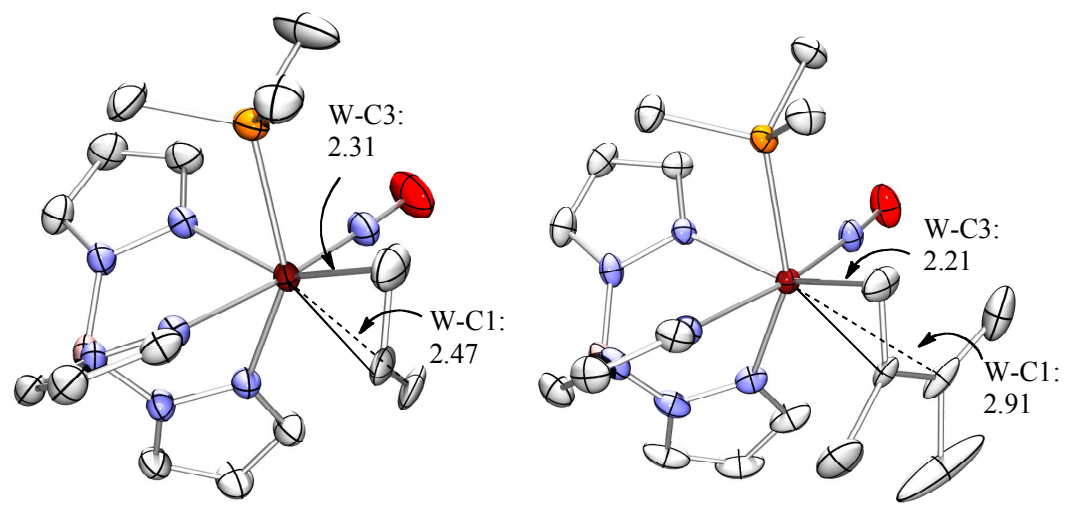
A particularly interesting illustration of the ability of alkyl groups to enhance $\eta^{3} \rightarrow \eta^{2}$ allyl distortion through hyperconjugation was earlier documented in protonation studies of alkylated benzenes bound to pentaammineosmium(II). ${ }^{21}$ Whereas protonation of benzene in the complex $\left[\mathrm{Os}\left(\mathrm{NH}_{3}\right)_{5}\left(\eta^{2} \text {-benzene }\right)\right]^{2+}$ results in an $\eta^{3}$-benzenium complex, 9, with carbon resonances typical of symmetrical allyl species of this metal fragment, protonation of the analogous $m$-xylene complex $\left(-40^{\circ} \mathrm{C}\right)$ forms complex 10 with carbon resonances reminiscent of the type of $\eta^{2}$-allyl systems described herein (Scheme 6). ${ }^{21}$

Scheme 6

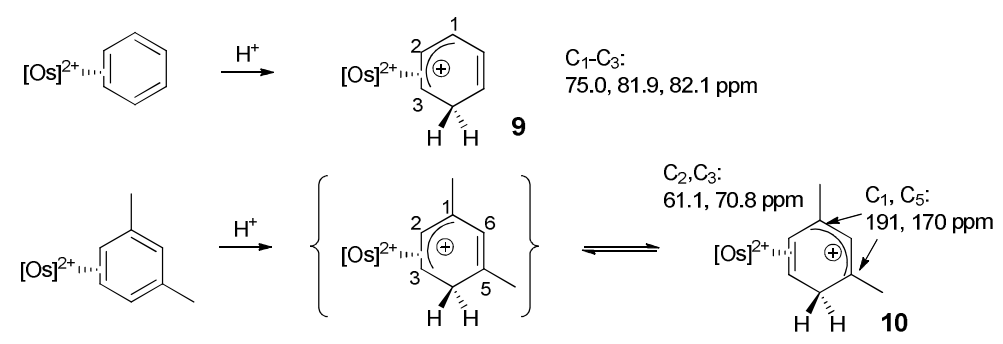

$[\mathrm{Os}]=\left(\mathrm{NH}_{3}\right)_{5} \mathrm{OS}$

\section{Calculations}

In their pioneering studies, Hoffman, Faller, et al. $^{22}$ and later Curtis and Eisenstein, ${ }^{23}$ described how orbital interactions can be used to rationalize regiochemical preferences of nucleophilic addition to asymmetric Mo(II) allyl(-1) complexes. Templeton, Pregosin, et al. ${ }^{17}$ investigated similar orbital interactions in order to understand allyl orientations for the related system $\left\{\operatorname{TpW}^{\prime \prime}(\mathrm{CO})_{2}(\pi\right.$-allyl) $\}$. To better understand the origins of this $\eta^{3} \rightarrow \eta^{2}$ distortion for the $\left\{\mathrm{TpW}(\mathrm{NO})\left(\mathrm{PMe}_{3}\right)\right\}$ complexes reported herein, Dan Harrison and Carl Trindle developed a series of DFT studies using the B3LYP method using a "hybrid" basis set with the LANL2DZ pseudopotential and basis set on $\mathrm{W}$ and $6-31 \mathrm{G}(\mathrm{d})$ on all other atoms. As Table 1 shows, computed bond lengths in the B3LYP/hybrid model are in semi-quantitative agreement with those derived from X-ray analysis. Specifically, the shortest M-C bonds are to C3 in all cases, 
most markedly so for the $\mathrm{C}_{6} \mathrm{H}_{11}{ }^{+}$ligand. The calculated W-C1 distances are considerably longer than what is indicated by X-ray data, especially for the substituted allyl ions. The W-C3 and W-C2 distances support the notion that the $\eta^{2}$ coordination is unsymmetrical. This asymmetry is slightly overestimated in the computed structures. In all cases, W-C and C-C bond lengths for the bound allyl fragment of the calculated compounds are semi-quantitatively reproduced (Table 1) for both endo and exo isomers. The most serious errors are the over-estimates of the W-C distances, by up to $0.12 \AA$ (4\%).

Table 1 - Experimental and [calculated] bond lengths for $\eta^{3}$-allyl and $\eta^{2}$-alkene complexes of the form $\mathrm{TpW}(\mathrm{NO})\left(\mathrm{PMe}_{3}\right)(\mathrm{L})$. Calculated values are in brackets $(\Delta=(\mathrm{W}-\mathrm{C} 1)-(\mathrm{W}-\mathrm{C} 3))$.

\begin{tabular}{|l|l|l|l|l|l|l|}
\hline Compound, $(\mathrm{L})$ & $\mathbf{W}-\mathrm{C1}, \AA$ & $\mathbf{W}-\mathrm{C} 2, \AA$ & $\mathbf{W}-\mathrm{C} 3, \AA$ & $\Delta, \AA$ & $\mathbf{C} 1-\mathrm{C} 2, \AA$ & $\mathbf{C} 2-\mathrm{C} 3, \AA$ \\
\hline exo-7 $\left(\mathrm{C}_{3} \mathrm{H}_{5}{ }^{+}\right)$ & 2.47 & 2.38 & 2.31 & 0.16 & 1.32 & 1.43 \\
& {$[2.53]$} & {$[2.38]$} & {$[2.33]$} & {$[0.20]$} & {$[1.38]$} & {$[1.42]$} \\
\hline exo-1 $\left(\mathrm{C}_{6} \mathrm{H}_{9}{ }^{+}\right)$ & 2.60 & 2.30 & 2.31 & 0.29 & 1.38 & 1.43 \\
& {$[2.72]$} & {$[2.33]$} & {$[2.31]$} & {$[0.41]$} & {$[1.38]$} & {$[1.44]$} \\
\hline exo-2 $\left(\mathrm{C}_{7} \mathrm{H}_{10} \mathrm{NO}^{+}\right)$ & 2.59 & 2.29 & 2.28 & 0.31 & 1.36 & 1.43 \\
& {$[2.72]$} & {$[2.34]$} & {$[2.28]$} & {$[0.44]$} & {$[1.38]$} & {$[1.44]$} \\
\hline exo-8 $\left(\mathrm{C}_{6} \mathrm{H}_{11}{ }^{+}\right)$ & 2.91 & 2.40 & 2.21 & 0.70 & 1.39 & 1.47 \\
& {$[3.01]$} & {$[2.45]$} & {$[2.21]$} & {$[0.80]$} & {$[1.39]$} & {$[1.47]$} \\
\hline exo-4 $\left(\mathrm{C}_{10} \mathrm{H}_{9}{ }^{+}\right)$ & {$[2.90]$} & {$[2.35]$} & {$[2.27]$} & {$[0.63]$} & {$[1.39]$} & {$[1.45]$} \\
\hline exo-5 $\left(\mathrm{C}_{14} \mathrm{H}_{11}{ }^{+}\right)$ & {$[2.96]$} & {$[2.37]$} & {$[2.21]$} & {$[0.75]$} & {$[1.36]$} & {$[1.46]$} \\
\hline exo-6 $\left(\mathrm{C}_{7} \mathrm{H}_{9}{ }^{+}\right)$ & 2.53 & 2.34 & 2.38 & 0.15 & 1.40 & 1.39 \\
& {$[2.75]$} & {$[2.37]$} & {$[2.33]$} & {$[0.42]$} & {$[1.40]$} & {$[1.43]$} \\
\hline exo-3 $\left(\mathrm{C}_{7} \mathrm{H}_{9} \mathrm{NO}\right)$ & 3.19 & 2.22 & 2.21 & 0.98 & 1.46 & 1.45 \\
& {$[3.23]$} & {$[2.25]$} & {$[2.24]$} & {$[0.99]$} & {$[1.47]$} & {$[1.45]$} \\
\hline \hline
\end{tabular}

The isomer of 8 with an exo orientation of the $\mathrm{C}_{6} \mathrm{H}_{11}{ }^{+}$ligand is calculated to be favored by $1-2 \mathrm{kcal} / \mathrm{mol}$ over the endo isomer, and exo and endo isomers differ by a similar amount for the unsubstituted allyl complex 7. In contrast, the exo-1 isomer is favored over endo-1 by about $10 \mathrm{kcal} / \mathrm{mol}$. Inspection of the computed structure of endo-1 reveals a large steric repulsion between one of the Tp pyrazole rings and that of the cyclohexane-based ligand. Supporting the notion that the allyl complexes have carbocation character at $\mathrm{C} 1$, all of the DFT calculations 
suggest substantial $2 p$ character localized on C1 of the LUMO. The LUMO of exo-7 is shown in Figure 4 as an example.

Figure 4 - LUMO for the complex $\left[\mathrm{TpW}(\mathrm{NO})\left(\mathrm{PMe}_{3}\right)\left(\text { exo- } \mathrm{C}_{3} \mathrm{H}_{5}\right)\right]^{+}(7)$ showing the large contribution from the $2 \mathrm{p}$ orbital of $\mathrm{C} 1$.

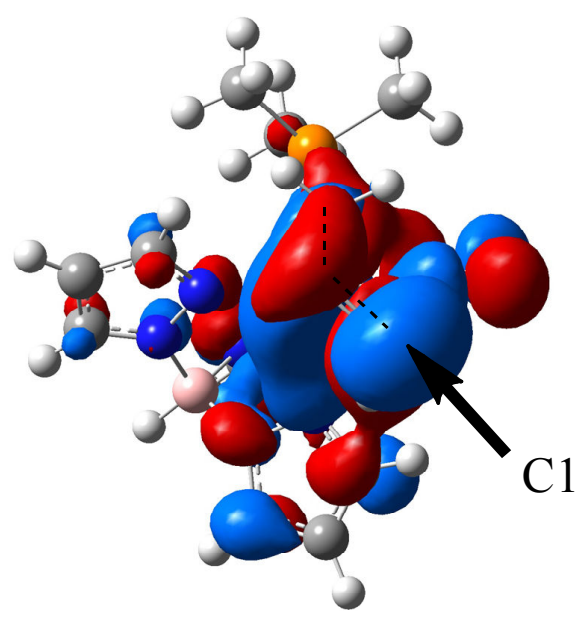

In order to better understand what causes the $\eta^{3} \rightarrow \eta^{2}$ distortion for complexes of $\left\{\mathrm{TpW}(\mathrm{NO})\left(\mathrm{PMe}_{3}\right)\right\}$, we first considered the hypothetical complex $\left[\mathrm{W}\left(\mathrm{NH}_{3}\right)_{5}\left(\pi-\mathrm{C}_{3} \mathrm{H}_{5}\right)\right]^{+}$, which is isoelectronic to the previously reported $\left[\mathrm{Os}\left(\mathrm{NH}_{3}\right)_{5}(\pi \text {-allyl) }]^{3+}\right.$ systems. ${ }^{24}$ DFT calculations reveal that, as was observed for the osmium species, ${ }^{24}$ the tungsten allyl complex is completely symmetrical, with W-C1 and W-C3 bond lengths of $2.23 \AA$, somewhat longer than the calculated W-C2 bond length of $2.16 \AA$. When a nitrosyl ligand replaces one of the cis ammines, its strong backbonding interaction with the metal drives two of the $\pi$-symmetry tungsten orbitals lower in energy, leaving only the $d_{x y}$ (where the W-NO bond is along the $z$ axis) to interact with the allyl fragment. For cis- $\left\{\mathrm{W}\left(\mathrm{NH}_{3}\right)_{4}(\mathrm{NO})\left(\pi-\mathrm{C}_{3} \mathrm{H}_{5}\right)\right\}^{2+}$, the considerable $\eta^{3} \rightarrow \eta^{2}$ distortion $(\mathrm{W}-\mathrm{C} 3=2.34 \AA$, $\mathrm{W}-\mathrm{C} 1=2.57 \AA$ ) cannot be attributed to asymmetry in the ligand set. In fact, the symmetryconstrained $C_{s}$ allyl complex is a transition state with a kinetic barrier lying $1.1 \mathrm{kcal} / \mathrm{mol}$ above the two symmetrically equivalent distorted forms (Figure 5). 
Figure 5 - Reaction coordinate diagram for allyl cation isomerization in the complex $\left[\mathrm{W}\left(\mathrm{NH}_{3}\right)_{5-n}\left(\mathrm{NO}_{n}\left(\mathrm{C}_{3} \mathrm{H}_{5}\right)\right]^{(\mathrm{n}+1)+}\right.$, where $n=0,1$.

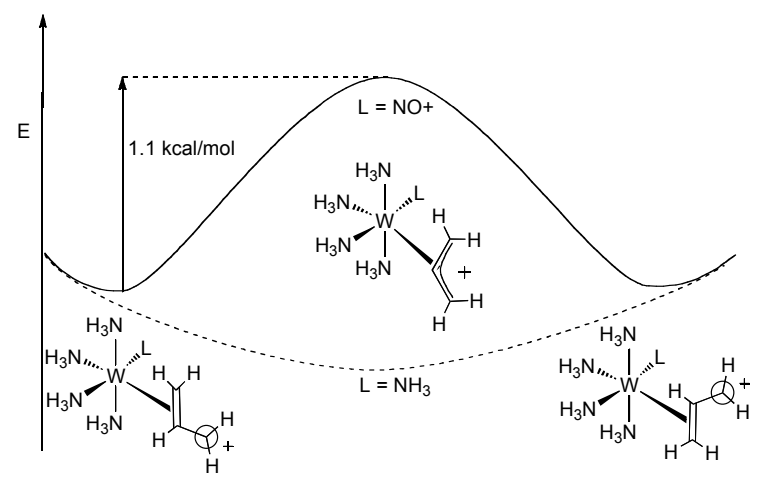

As with the $\left\{\mathrm{TpW}(\mathrm{NO})\left(\mathrm{PMe}_{3}\right)\right\}$ analog, the LUMO of the complex $\left[\mathrm{W}\left(\mathrm{NH}_{3}\right)_{4}(\mathrm{NO})(\pi-\right.$ $\left.\left.\mathrm{C}_{3} \mathrm{H}_{5}\right)\right]^{2+}$ has a large $2 p$ component at the terminal carbon (C1) farthest from the metal (Figure 6). The highest occupied and subjacent MOs are dominated by W-NO local $\pi$ interactions, and the HOMO-2 displays the strong mixing of the high energy $W d_{x y}$ atomic orbital (AO) with the $\pi$ nonbonding orbital of the allyl cation. Significantly, the allyl distortion allows a stabilizing admixture of the allyl $\pi^{*}$ with the $W d_{x y} A O$ in accordance with Figure 7 . When the $C_{3} \mathrm{H}_{5}{ }^{+}$allyl ligand in Figure 7 is replaced with $\mathrm{C}_{6} \mathrm{H}_{9}{ }^{+}$and $\mathrm{L}=\mathrm{NO}^{+}$, the isomerization barrier is calculated to be 5.7 $\mathrm{kcal} / \mathrm{mol}$. For comparison, $\left[\mathrm{TpW}(\mathrm{NO})\left(\mathrm{PMe}_{3}\right)\left(\mathrm{C}_{6} \mathrm{H}_{9}\right)\right]^{+}$is calculated to have a transition state for this isomerization of $6.2 \mathrm{kcal} / \mathrm{mol}$ with an isomerization energy of $3.7 \mathrm{kcal} / \mathrm{mol}$.

Figure 6 - Molecular orbitals for the allyl complex $\left[\mathrm{W}\left(\mathrm{NH}_{3}\right)_{4}(\mathrm{NO})\left(\mathrm{C}_{3} \mathrm{H}_{5}\right)\right]^{2+}$.

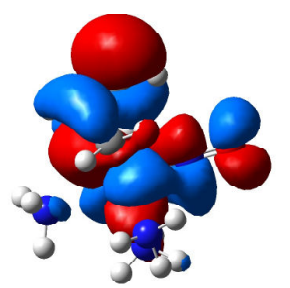

LUMO

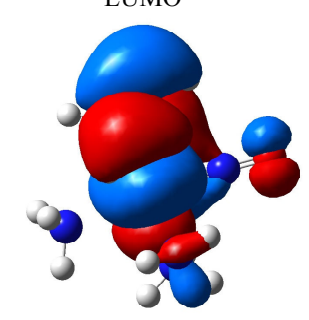

HOMO-2

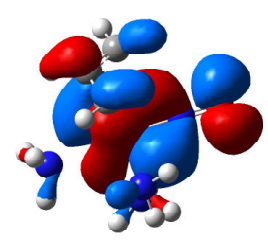

HOMO-1

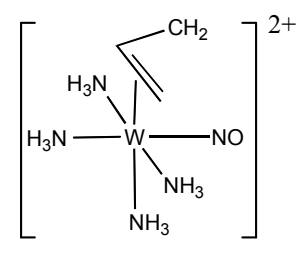


Figure 7 - Schematic representation of the mixing of the HOMO of $\left\{\mathrm{W}\left(\mathrm{NH}_{3}\right)_{4}(\mathrm{NO})\right\}^{+}\left(\mathrm{d}_{\mathrm{xy}}\right)$ with the non-bonding $\left(\pi_{\mathrm{nb}}\right)$ and antibonding $\left(\pi^{*}\right)$ orbitals of $\mathrm{C}_{3} \mathrm{H}_{5}^{+}$. Better overlap of $\mathrm{d}_{x y}$ and $\pi^{*}$ is achieved by partial rotation (moving $\mathrm{C} 1$ toward NO).

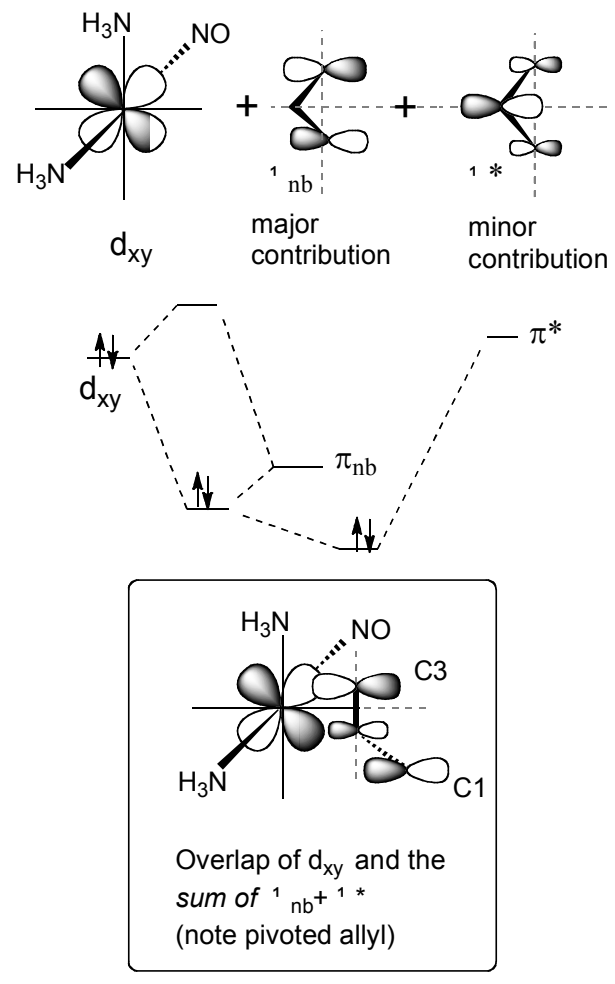

We now consider why the allyl ligand in $\left\{\mathrm{TpW}(\mathrm{NO})\left(\mathrm{PMe}_{3}\right)\left(\pi-\mathrm{C}_{3} \mathrm{H}_{5}\right)\right\}^{+}$distorts in such a way as to place the electrophilic methylene (C1) distal rather than proximal to $\mathrm{PMe}_{3}$ ligand. Inspection of the $\mathrm{HOMO}$ for the fragment $\left\{\mathrm{TpW}(\mathrm{NO})\left(\mathrm{PMe}_{3}\right)\right\}$ reveals a significant interaction of a $\pi$-orbital of the pyrazole ligand trans to $\mathrm{PMe}_{3}$ with the tungsten $\mathrm{d}_{\mathrm{xy}}$ orbital (Figure 8; W-NO is the $z$ axis). The HOMO is represented by the antibonding combination of these orbitals. The result of this $\pi^{*}$ interaction is a distortion of the $d_{x y}$ orbital, causing the major lobes to extend toward the $\mathrm{PMe}_{3}$ (Figure 9). Optimal overlap is achieved when the $\pi$ non-bonding orbital of the allyl fragment twists in such a way as to maximize overlap of one of these major lobes with a terminal carbon. Thus, the W-C1 bond is weakened in order to achieve better overlap with C2 and C3 (Figure 8). The cost of such a distortion is to lessen the interaction of the allyl $\pi^{*}$ orbital with $d_{x z}$. However, as the $d_{x z}$ orbital has been stabilized by its interaction with the nitrosyl, the interaction of the $d_{x z}$ and allyl $\pi^{*}$ orbitals is inconsequential. 
Figure 8 - Overlap of the allyl $\pi_{\mathrm{nb}}$ and $\pi^{*}$ orbital combination with the asymmetric HOMO $\left(\mathrm{d}_{\mathrm{xy}}\right)$ of $\left\{\mathrm{TpW}(\mathrm{NO})\left(\mathrm{PMe} \mathrm{C}_{3}\right)\right\}$ for the two possible $\eta^{3} \rightarrow \eta^{2}$ distortions of the allyl ligand.

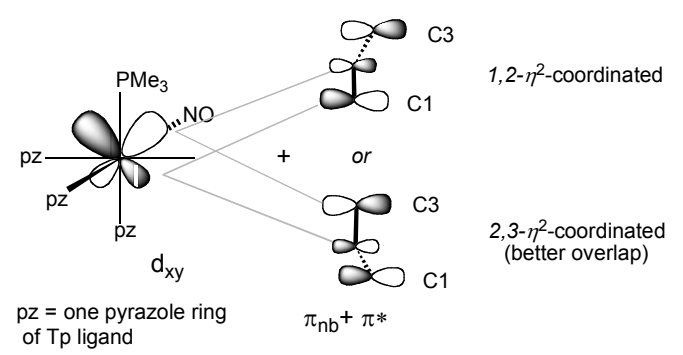

Figure $9-\mathrm{HOMO}$ of the fragment $\left\{\mathrm{TpW}(\mathrm{NO})\left(\mathrm{PMe}_{3}\right)\right\}$ showing the participation of the pyrazole ring trans to the phosphine (note the asymmetric $d_{x y}$ orbital). The $\pi$ orbital of the heterocycle distorts and raises the energy of the HOMO.
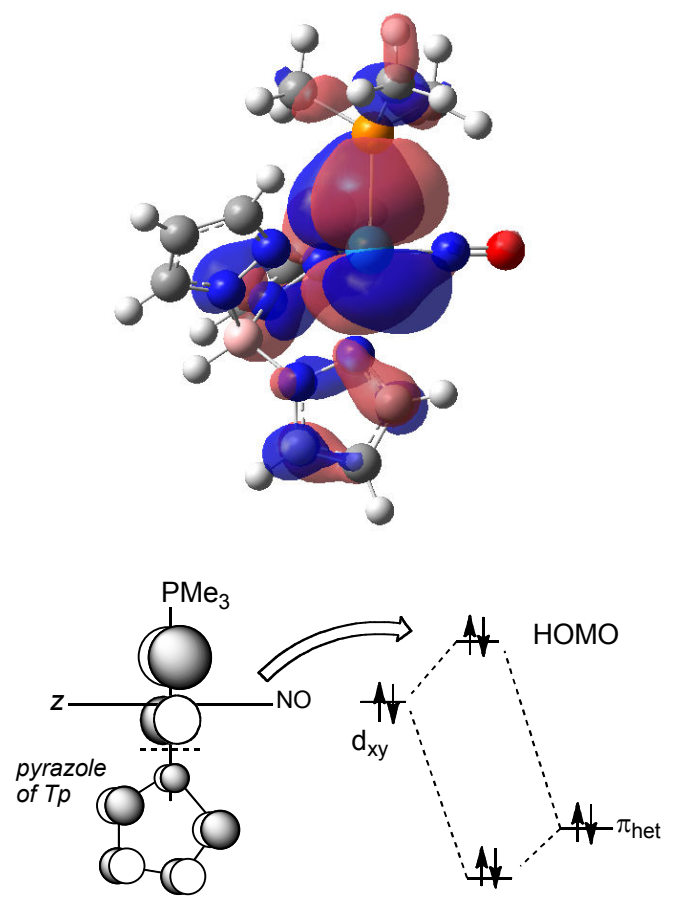

When we replaced an ammine of the theoretical allyl complex $\left[\mathrm{W}\left(\mathrm{NH}_{3}\right)_{4}(\mathrm{NO})\left(\pi-\mathrm{C}_{6} \mathrm{H}_{9}\right)\right]^{2+}$ with $\mathrm{PMe}_{3}$ (cis to both the allyl and $\mathrm{NO}$ ), the difference between the distal and proximal distorted allyl isomers is only $0.2 \mathrm{kcal} / \mathrm{mol}(\mathrm{TS}=4.9 \mathrm{kcal} / \mathrm{mol})$. However, if three ammines of $\left[\mathrm{W}\left(\mathrm{NH}_{3}\right)_{4}(\mathrm{NO})\left(\mathrm{C}_{6} \mathrm{H}_{9}\right)\right]^{2+}$ are replaced with Tp, the difference between distorted allyls becomes 4.1 $\mathrm{kcal} / \mathrm{mol}$. For comparison, this isomerization energy is calculated to be $3.7 \mathrm{kcal} / \mathrm{mol}$ for the $\left\{\mathrm{TpW}\left(\mathrm{PMe}_{3}\right)(\mathrm{NO})\left(\pi-\mathrm{C}_{6} \mathrm{H}_{9}\right)\right\}^{+}$system $(\mathrm{TS}=6.2 \mathrm{kcal} / \mathrm{mol})$. Thus, while the $\mathrm{NO}^{+}$is primarily 
responsible for the $\eta^{3} \rightarrow \eta^{2}$ distortion in these $\left\{\mathrm{TpW}(\mathrm{NO})\left(\mathrm{PMe}_{3}\right)\right\}$ systems, it is the difference between the imidazole arm of $\mathrm{Tp}$ and $\mathrm{PMe}_{3}$, that determines the direction of the allyl distortion.

\section{Stereoselective Preparation of $\eta^{2}$-Diene Complexes}

Examples of $\pi$-allyl complexes undergoing deprotonation to form diene complexes are rare, ${ }^{25}$ especially for cases in which the resulting diene is dihapto-coordinated. However, the allyl complex $\left[\mathrm{TpMo}(\mathrm{NO})(\mathrm{Melm})\left(\pi-\mathrm{C}_{6} \mathrm{H}_{9}\right)\right]^{+}, \mathbf{1 6}$, has been reported to readily undergo deprotonation $\left(\mathrm{pK}_{\mathrm{a}} \sim 2\right)$ to form the corresponding $\eta^{2}$-diene complex, 17, as a mixture of coordination diastereomers. ${ }^{5,26}$ We chose for our initial studies to pursue the tungsten analog $\left[\mathrm{TpW}(\mathrm{NO})\left(\mathrm{PMe}_{3}\right)\left(\pi-\mathrm{C}_{6} \mathrm{H}_{9}\right)\right]^{+}, \mathbf{1}$, because of its anticipated improved kinetic stability compared to 16. ${ }^{4,12,27}$

Complex 1 could be prepared from $\operatorname{TpW}(\mathrm{NO})\left(\mathrm{PMe}_{3}\right)\left(\pi-\mathrm{C}_{6} \mathrm{H}_{8}\right), \mathbf{1 1}$, which exists as a mixture (1.1:1) of two coordination diastereomers (11d, 11p). Treatment of $\mathbf{1 1}$ with triflic acid yielded 1. As with its molybdenum congener $16,{ }^{26}$ the acidity of allyl complex 1 could be estimated ( $\mathrm{pK}_{\mathrm{a}}$ (DMSO) of $\sim 7.0$ ) by observing the reaction of 4 with various acids. Treating 1 with various amine bases (e.g., 1,8-diaza[5.4.0]bicycloundecene (DBU), N,N-diisopropylethylamine (DIEA), 2,6-lutidine, morpholine, aniline) gave dienes $\mathbf{1 1 d}$ and $\mathbf{1 1 p}$ in varying ratios (Scheme 4). In general, weaker bases gave lower coordination diastereomer ratios (cdr) and stronger bases led to an increased amount of $\mathbf{1 1 d}(>10: 1)$. The reaction with the non-nucleophilic base $\mathrm{NaH}$ also delivered diene 11, but in a modest cdr of only 4:1 (d:p). Ultimately, we settled on reaction conditions that incorporated DBU, which gave the highest isolated yield of $91 \%(d r=10: 1)$. NOE experiments confirmed that the uncoordinated diene in 11d is distal to the $\mathrm{PMe}_{3}$ group (Scheme 7). Allowing a $\mathrm{CDCl}_{3}$ solution of $\mathbf{1 1 d}$ to stand at ambient conditions in the presence of $\mathrm{DBUH}^{+}$ 
forms an equilibrium mixture of $1.1: 1$ (11d : 11p) over a period of several days $(\Delta G=0.2$ $\mathrm{kcal} / \mathrm{mol})$.

Scheme 7

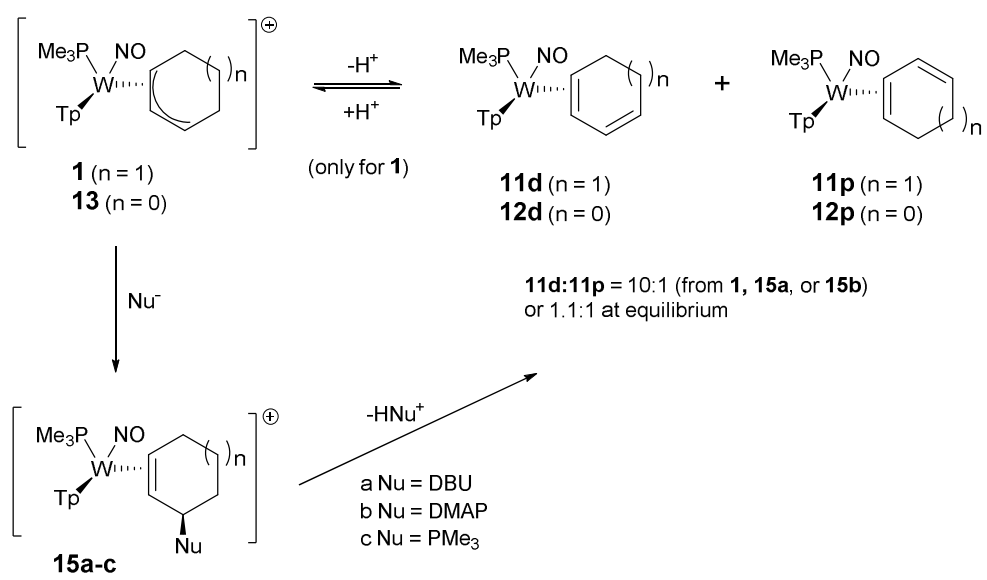

Interestingly, ${ }^{31} \mathrm{P}$ and ${ }^{1} \mathrm{H}$ NMR data revealed that immediately after the addition of either DBU or DMAP to allyl 1, a third compound was present in the crude reaction mixture, which converted to $\mathbf{1 1 d}$ and $\mathbf{1 1 p}$ over several days. We speculate that these intermediates (15a, 15b) are likely to be the addition products shown in Scheme 4, but facile elimination prevented their full characterization. In contrast, the addition of $\mathrm{PMe}_{3}$ to allyl $\mathbf{1}$ generated phosphonium complex $\mathbf{1 5 c}$ stereoselectively, which was isolated and fully characterized.

Cyclopentadiene also forms a complex with $\left\{\mathrm{TpW}(\mathrm{NO})\left(\mathrm{PMe}_{3}\right)\right\}$ in which the diene is dihapto-coordinated, $12 .{ }^{28}$ As with the cyclohexadiene analogs, the initially formed coordination diastereomer ratio of the isolated mixture is low (2.3:1). Protonation of this mixture produces a single diastereomer of the allyl complex 13. However, the reaction of $\mathbf{1 3}$ with any of the bases mentioned above failed to return any of the cyclopentadiene complex 12 (see Scheme 7). Rather, ${ }^{31} \mathrm{P}$ NMR spectroscopic data ( $\mathrm{d}$ and $J_{\mathrm{WP}}$ ) are consistent with nucleophilic addition products similar to $15 \mathrm{a}-\mathrm{c}$, which failed to eliminate even when exposed to $1 \mathrm{M} \mathrm{NaOH}(\mathrm{aq})$. Their identities were not pursued further. Addition of triethylamine to $\mathbf{1 3}$ led to decomposition of the complex. 
Attempts were made to selectively deprotonate the cycloheptadienyl complex, $\mathbf{6}$, using DBU, triethylamine, and sodium methoxide. The amine bases yielded mixtures of each of the three possible products in low diastereomeric ratios. The sodium methoxide yielded a product with spectroscopic similarities to $15 \mathrm{a}-\mathrm{c}$, which was determined to be 14 by COSY experiments (Scheme 8). The poor diastereomeric ratios observed after deprotonation may signal the metal complex's remote influence is weaker as the $\pi$-system becomes larger. In any case, triene complexes from 6 were not further pursued.

\section{Scheme 8}
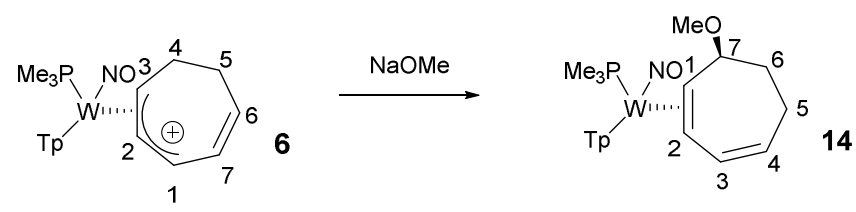

\section{Conclusion}

We have prepared series of $\pi$-allyl complexes of tungsten that show an unusually large degree of $\eta^{3} \rightarrow \eta^{2}$ distortion. DFT calculations for several of these allyl complexes reproduce the general distortions observed and indicate a significant buildup of positive charge at C1 along with a large component of the $2 p$ orbital at this carbon. The presence of a single powerful $\pi$-acid $\left(\mathrm{NO}^{+}\right)$in the fragment $\left\{\mathrm{TpW}(\mathrm{NO})\left(\mathrm{PMe}_{3}\right)\right\}$ results in a single high-energy $\mathrm{d}_{\pi}$ orbital (orthogonal to the nitrosyl), and its interaction with both the $\pi_{\mathrm{nb}}$ and allyl $\pi^{*}$ orbitals is thought to cause the observed distortion of the $\pi$-allyl ligand. In the case of cyclohexyl allyls, this allyl distortion can be utilized to prepare stereoselectively $\eta^{2}$-1,3-diene complexes.

\section{Experimental Section.}

All chemical shifts are reported in $\mathrm{ppm}$ with proton and carbon shifts referenced to tetramethylsilane (TMS). Phosphorus NMR signals are referenced to $85 \% \mathrm{H}_{3} \mathrm{PO}_{4}(\delta=0.00)$ using 
a triphenylphosphate external standard $(\delta=-16.58)$. Coupling constants $(J)$ are reported in hertz $(\mathrm{Hz})$. Infrared spectra (IR) were recorded as a glaze using a Horizontal Attenuated Total Reflectance (HATR) accessory (Pike Industries). Cyclic voltammetry data were aquired at ambient temperature $\left(\sim 25^{\circ} \mathrm{C}\right)$, under nitrogen at $100 \mathrm{mV} / \mathrm{s}$ in a standard three-electrode cell with a glassy carbon working electrode, $\mathrm{N}, \mathrm{N}$-dimethylacetamide (DMA) or acetonitrile (MeCN) solvent (unless otherwise specified), and tetrabutylammonium hexaflurophosphate (TBAH) electrolyte (approx. 0.5 M). All potentials are reported versus NHE (Normal Hydrogen Electrode) using cobaltocenium hexafluorophosphate $\left(E_{1 / 2}=-0.78 \mathrm{~V}\right)$, ferrocene $\left(E_{1 / 2}=+0.55 \mathrm{~V}\right)$, or decamethylferrocene $\left(E_{1 / 2}=+0.04 \mathrm{~V}\right)$ as an internal standard. The peak-to-peak separation was less than $100 \mathrm{mV}$ for all reversible couples. Unless otherwise noted, all synthetic reactions were performed in a glovebox under a dry nitrogen atmosphere. $\mathrm{CH}_{2} \mathrm{Cl}_{2}$ and benzene were purified by passage through a column packed with activated alumina. Other solvents and liquid reagents were thoroughly purged with dry nitrogen prior to use. Deuterated solvents were used as received from Cambridge Isotopes. Coordination diastereomers are described as either proximal (p) or distal (d) based on the proximity of a defining feature (e.g., the "carbocationic" center of an allyl ligand) to the $\mathrm{PMe}_{3}$ ligand. Synthesis of compounds $\mathrm{TpW}(\mathrm{NO})\left(\mathrm{PMe}_{3}\right)\left(\eta^{2}\right.$-benzene) ${ }^{12} \mathbf{2}^{35}$ $\mathbf{3}^{27}$, and $\mathbf{1 3},{ }^{28}$ have been previously reported. Synthesis of compounds $\mathbf{4}$ and $\mathbf{5}$ are given in

\section{Chapter 4.}

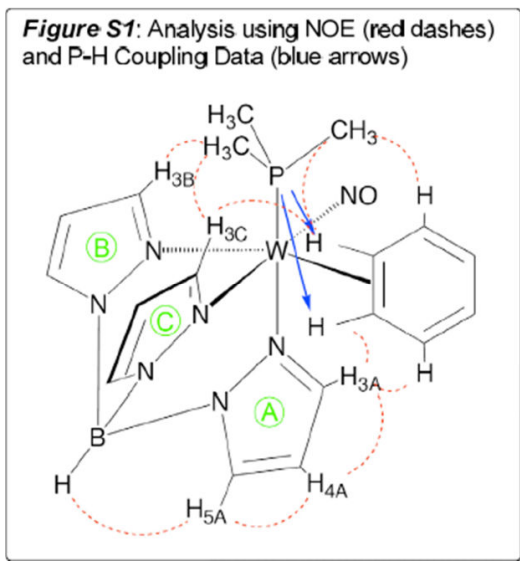


DFT Calculations. ${ }^{27}$ Initial structures were built in Spartan $^{27 a}$ and optimized with the extended version of the PM3 semi-empirical method available in that package, or in GAUSSVIEW (5.0.8) with the PM6 semi-empirical method in GAUSSIAN 09. ${ }^{27 b, c}$ These structures were refined stepwise in Spartan and Gaussian using B3LYP and a series of basis functions incorporating LANL2 pseudopotentials and associated basis functions provided in those packages or directly from the PM6 structures. The most demanding calculations reported here put the LANL2DZ pseudopotential and its basis only on the $W$ atom, and used the $6-31 G(d)$ basis for all other atoms.

For transition state structures, vibrational analysis revealed the presence of a single imaginary frequency. In all other cases, vibrational analyses verified that optimized structures were located at local minima, with the presence of only real frequencies. Many of the systems calculated herein have very soft vibrational modes. This has the consequence that in many cases reports from vibrational calculations showed small violations of the convergence criteria on the predicted root-mean-square and/or maximum displacement, for structures which had satisfied all convergence criteria in the optimization step. This unsatisfactory behavior can be remedied by reoptimization, computing the force matrix at each optimization step, and using the UltraFine grid for numerical integrations. Our spot checks showed that structures and zero point vibrational energies were unchanged by this expensive refinement. For this reason we believe that the structures, calculated energies, and zero-point energy values computed with default convergence criteria (FinGrid) for optimization, are reliable for comparisons reported here.

$\left[\mathrm{TpW}(\mathrm{NO})\left(\mathrm{PMe}_{3}\right)\left(2,3-\eta^{2}\right.\right.$-cyclohexan-2-en-1-ylium)][OTf] (1) 


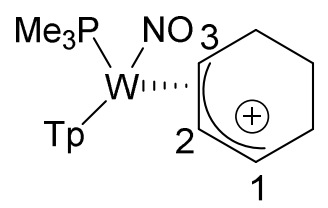

1,3-Cyclohexadiene (1.05 g, $13.1 \mathrm{mmol})$ was added to an oven-dried test tube containing a heterogeneous yellow solution of $\mathrm{TpW}(\mathrm{NO})\left(\mathrm{PMe}_{3}\right)\left(\eta^{2}\right.$-benzene) $(0.525 \mathrm{~g}, 0.904 \mathrm{mmol})$ in $\mathrm{DME}$ (2.71 g). The solution was added to a $60{ }^{\circ} \mathrm{C}$ oil bath and allowed to stir. Upon warming the solution became a brown-slightly purple homogeneous solution. After $1.5 \mathrm{~h}$, the solution was removed from the warm bath and allowed to cool for 5 minutes. A solution of HOTf $(0.135 \mathrm{~g}$, $0.900 \mathrm{mmol}$ ) in $\mathrm{MeCN}(0.695 \mathrm{~g})$ was added to the solution to make a yellow solution that precipitated a yellow solid from the shortly thereafter. After $2 \mathrm{~h} 15 \mathrm{~min}$, the yellow precipitate was collected on a $15 \mathrm{~mL}$ medium porosity fritted funnel, washed with $\sim 4 \times 0.2 \mathrm{~g} \mathrm{DME}$, and placed under vacuum (0.403 g, $0.550 \mathrm{mmol}, 61 \%$ yield).

${ }^{1} \mathrm{H}$ NMR $\left(\mathrm{CD}_{3} \mathrm{CN}, \delta\right): 8.41(\mathrm{~d}, J=2.0,1 \mathrm{H}, \mathrm{PzB} 3), 8.13(\mathrm{~d}, J=2.0,1 \mathrm{H}, \mathrm{PzA} 3), 8.03(\mathrm{~d}, J=2.0,1 \mathrm{H}$, PzB5), 7.97 (d, $J=2.0,1 \mathrm{H}, \mathrm{PzC}$ ), 7.96 (d, $J=2.0,1 \mathrm{H}, \mathrm{PzC}$ ), 7.81 (d, $J=2.0,1 \mathrm{H}, \mathrm{PzA} 5), 6.59$ (m, $1 \mathrm{H}, \mathrm{H} 1), 6.55(\mathrm{t}, J=2.0,1 \mathrm{H}, \mathrm{PzC} 4), 6.53(\mathrm{t}, J=2.0,1 \mathrm{H}, \mathrm{PzB} 4), 6.34(\mathrm{t}, J=2.0,1 \mathrm{H}, \mathrm{PzA} 4), 5.13(\mathrm{t}, J=$ 7.4, $1 \mathrm{H}, \mathrm{H} 2), 4.38\left(\mathrm{dtt}, J=7.4,1.6,{ }^{3} \mathrm{JPH}_{\mathrm{PH}}=14.5,1 \mathrm{H}, \mathrm{H} 3\right), 3.34(\mathrm{~m}, 1 \mathrm{H}, \mathrm{H} 6), 3.26\left(\mathrm{~m}, 2 \mathrm{H}, \mathrm{H} 6^{\prime} / \mathrm{H} 4\right)$, 2.47 (dddd, $\left.J=15.4,10.8,6.4,1.4,1 \mathrm{H}, \mathrm{H}^{\prime}\right), 1.59(\mathrm{~m}, 1 \mathrm{H}, \mathrm{H} 5), 1.33\left(\mathrm{~m}, 1 \mathrm{H}, \mathrm{H} 5^{\prime}\right), 1.20\left(\mathrm{~d}, J_{\mathrm{PH}}=9.8\right.$ 9H, $\mathrm{PMe}_{3}$ ). ${ }^{13} \mathrm{C}$ NMR $\left(\mathrm{CD}_{3} \mathrm{CN}, \delta\right): 142.3$ (PzA3), 146.4 (PzB3), 143.2 (PzC3), 139.6 (PzA5), 139.5 (PzB5/PzC5), 138.3 (C1), 109.5/109.0 (PzB4/PzC4), 108.1 (PzA4), 103.8 (C2, d, $\left.{ }^{2} J_{P C}=3.5\right), 70.0$ $\left(C 3, d, J_{P C}=12.6\right), 27.1(C 4), 27.0(C 6), 26.9(C 5), 13.4\left(\mathrm{PMe}_{3}, \mathrm{~d},{ }^{1} J_{\mathrm{PC}}=32.7\right) .{ }^{31} \mathrm{P} \mathrm{NMR}\left(\mathrm{CDCl}_{3}, \delta\right):-$ $7.85\left(J_{\mathrm{WP}}=273\right)$. IR: $v_{\mathrm{BH}}=2522 \mathrm{~cm}^{-1}, v_{\mathrm{NO}}=1635 \mathrm{~cm}^{-1} . \mathrm{CV}(\mathrm{MeCN}): E_{\mathrm{p}, \mathrm{a}}=+1.83 \mathrm{~V}, E_{\mathrm{p}, \mathrm{c}}=-0.95 . \mathrm{ESI}-$ MS: obs'd (\%), calc'd (\%), ppm (M-OTf) ${ }^{+}: 582.1675$ (89.7), 582.1672 (86.8), 0.5; 583.1697 (63.2), 583.1698 (79.3), 0.1; 584.1698 (100), 584.1695 (100), 0.5; 585.1759 (52.2), 585.1739 (40.1), 3.3; 
586.1731 (100), 586.1728 (84.9), 0.5. Anal. Calc'd for $\mathrm{C}_{19} \mathrm{H}_{30} \mathrm{BF}_{3} \mathrm{~N}_{7} \mathrm{O}_{4} \mathrm{PSW}: \mathrm{C}, 31.04 ; \mathrm{H}, 4.11 ; \mathrm{N}$, 13.34. Found: C, 31.26; H, 3.90; N, 13.33 .

\section{$\left[\mathrm{TpW}(\mathrm{NO})\left(\mathrm{PMe}_{3}\right)\left(2,3-\eta^{2}\right.\right.$-cycloheptan-2-en-1-ylium)][OTf] (6)}

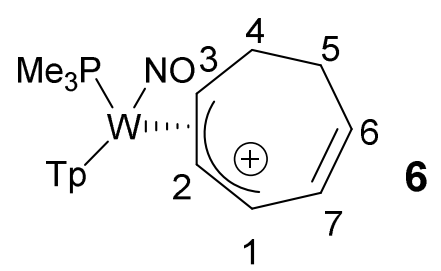

In a glovebox, $\mathrm{TpW}(\mathrm{NO})\left(\mathrm{PMe}_{3}\right)\left(\eta^{2}\right.$-benzene) $(1.006 \mathrm{~g}, 1.731 \mathrm{mmol})$ was dissolved in DME (10.10 g). 1,3,5-cycloheptatriene ( $4.11 \mathrm{~g}, 44.6 \mathrm{mmol})$ was added and the solution stirred 16 hours. The solution was precipitated over $200 \mathrm{~mL}$ stirring hexanes to give a light brown solid. The precipitate was filtered over a $60-\mathrm{mL} M$ fritted funnel leaving a yellow filtrate. The filtrate was evaporated to dryness, redissolved in minimal DME, and transferred to a vial. A triflic acid solution (260 mg in $331 \mathrm{mg} \mathrm{MeCN}$ ) was added to the vial and the solution was allowed to stand 24 hours. The solution was precipitated over $200 \mathrm{~mL}$ stirring ether as a yellow solid, which was filtered over a 60-mL M fritted funnel to give 6 (384 mg, 29.8\%).

${ }^{1} \mathrm{H}$ NMR (acetonitrile- $\left.d_{3}, \delta\right)$, major species: $8.28(1 \mathrm{H}, \mathrm{d}(J=1.6), \mathrm{Tp}), 8.10(1 \mathrm{H}, \mathrm{d}(J=1.8), \mathrm{Tp}), 8.06$ $(1 \mathrm{H}, \mathrm{d}(J=1.9), T p), 7.87(1 \mathrm{H}, \mathrm{d}(J=1.9), T p), 7.86(1 \mathrm{H},(J=2.0), T p), 7.71(1 \mathrm{H}, \mathrm{d}(J=2.2), 6.56(1 \mathrm{H}$, $t(J=4.2), T p), 6.53\left(1 \mathrm{H}, \mathrm{m}, \mathrm{H}_{4}\right), 6.48(1 \mathrm{H}, \mathrm{t}(J=4.6), T p), 6.34\left(1 \mathrm{H}, \mathrm{m}, \mathrm{H}_{5}\right), 6.32(1 \mathrm{H}, \mathrm{t}(J=4.4), T p)$, $6.13\left(1 \mathrm{H}, \mathrm{t}(J=14.0), \mathrm{H}_{3}\right), 5.21\left(1 \mathrm{H}, \mathrm{t}(J=18.3), \mathrm{H}_{2}\right), 4.87\left(1 \mathrm{H}, \mathrm{dt}\left(J_{\mathrm{d}}=15.5, J_{\mathrm{t}}=16.7\right), \mathrm{H}_{1}\right), 3.13(1 \mathrm{H}, \mathrm{m}$, geminal proton), $3.00(1 \mathrm{H}, \mathrm{t}(J=27.5)$, geminal proton $), 2.58(1 \mathrm{H}, \mathrm{t}(J=26.1)$, geminal proton), 2.34 $\left(1 \mathrm{H}, \mathrm{m}\right.$, geminal proton). ${ }^{13} \mathrm{C}$ NMR (acetonitrile- $\left.d_{3}, \delta\right)$, major species: $146.60(1 \mathrm{C}, \mathrm{s}, \mathrm{Tp}), 144.95$ (1C, s, Tp), 142.77 (1C, s, Tp), 138.25 (4C, 2 Tp, 2 ring), 126.87 (1C, s), 124.35 (1C, s), 109.09 (1C, s), 108.90 (1C, s, Tp), 108.61 (1C, s, Tp), 107.33 (1C, s, Tp), 77.44 (1C, d, J = 12), 36.51 (1C, s), $30.30(1 \mathrm{C}, \mathrm{s}), 13.77\left(3 \mathrm{C}, \mathrm{d}(J=32.8), \mathrm{PMe}_{3}\right) .{ }^{31} \mathrm{P}$ NMR (acetonitrile- $\left.d_{3}, \delta\right):-5.70\left(J_{\mathrm{WP}}=269\right),-8.63 . \mathrm{CV}$ : DMA, TBAH, $100 \mathrm{mV} / \mathrm{s}$, vs. NHE, $E_{p, c}=-0.80$ V. HRMS: obs'd (\%), calc'd (\%), ppm, (M) ${ }^{+}: 594.1664$ 
(86.9), 594.1672 (86.2), 1.3; 595.1688 (90), 595.1698 (79.6), 1.7; 596.1687 (100), 596.1696

(100), 1.4; 597.1751 (58.7), 597.1739 (40.8), 2; 598.1749 (100), 598.1728 (84.6), 3.5.

\section{$\left[\mathrm{TpW}(\mathrm{NO})\left(\mathrm{PMe}_{3}\right)\left(2,3-\eta^{2}\right.\right.$-propan-1-ylium)][OTf] (7)}

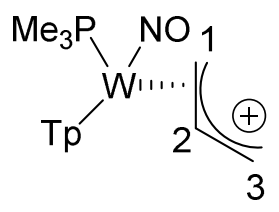

Diallyl ether $(0.92 \mathrm{~g}, 9.4 \mathrm{mmol})$ was added to a flame-dried test tube containing $\operatorname{TpW}(\mathrm{NO})\left(\mathrm{PMe}_{3}\right)\left(\eta^{2}\right.$-benzene) $(0.506 \mathrm{~g}, 0.871 \mathrm{mmol})$ in DME $(2.62 \mathrm{~g})$ to make a homogeneous yellow solution that was allowed to stir in a $57^{\circ} \mathrm{C}$ oil bath. After $1.5 \mathrm{~h}$, the dark purple-brown solution was removed from the warm bath. A solution of $\mathrm{HOTf}(0.127 \mathrm{~g}, 0.846 \mathrm{mmol})$ in $\mathrm{MeCN}$ $(0.640 \mathrm{~g})$ was added to the reaction solution to make a dark yellow solution. The reaction solution was placed in a $0{ }^{\circ} \mathrm{C}$ cold bath overnight. After $15 \mathrm{~h}$, the solution was removed from the cold bath and added to $100 \mathrm{~mL}$ of stirring $\mathrm{Et}_{2} \mathrm{O}$. The tan-yellow precipitate was then collected on a $30 \mathrm{~mL}$ medium porosity fritted funnel, washed with $2 \times 15 \mathrm{~mL} \mathrm{Et} \mathrm{O}_{2} \mathrm{O}$ and placed under vacuum (0.298 g, $0.430 \mathrm{mmol}, 49 \%$ yield).

${ }^{1} \mathrm{H}$ NMR $\left(\mathrm{CD}_{3} \mathrm{CN}, \delta\right): 8.4(\mathrm{~d}, J=2.0,1 \mathrm{H}, \operatorname{PzB} 3(\mathrm{exo})), 8.16(\mathrm{~d}, J=2.0,1 \mathrm{H}, \operatorname{PzA} 3(\mathrm{exo})), 8.1(\mathrm{~s}(\mathrm{br}), 2 \mathrm{H}$, 2 Tp's), 8.08 (d, J = 2.0, 1H, PzB3(endo)), 8.00/7.99 (m, 2H, PzC5(exo)/Tp), 7.98 (d, J = 2.0, 1H, PzA3(endo)), 7.89 (m, 2H, PzC3(exo)/Tp), 7.81 (d, J = 2.0, 1H, PzA5(exo)), 7.71 (d, $J=2.0,1 \mathrm{H}$, PzC3(endo)), 6.55/6.54/6.52/6.50 (t, $J=2.0,4 \mathrm{H}, \mathrm{PzC}($ endo)/3 Tp4's), $6.38(\mathrm{t}, J=2.0,1 \mathrm{H}$, PzC4(exo)), $6.35(t, J=2.0,1 \mathrm{H}, \mathrm{PzA} 4(\mathrm{exo})), 1.24\left(\mathrm{~d}, J=10.4,9 \mathrm{H}, \mathrm{PMe}_{3}(\mathrm{exo})\right), 1.21(\mathrm{~d}, J=10.1,9 \mathrm{H}$, $\mathrm{PMe}_{3}$ (endo)), Exo Isomer: 5.36 (ddddd, $J=14.6,13.3,8.3,8.0,{ }^{3} J_{\mathrm{PH}}=1.8,1 \mathrm{H}, \mathrm{H} 3$ ), 5.05 (ddd, $J=$ $\left.14.6,1.0,{ }^{3} J_{\mathrm{PH}}=2.6,1 \mathrm{H}, \mathrm{H} 5\right), 4.83\left(\mathrm{dddd}, J=8.3,2.9,1.1,{ }^{3} \mathrm{~J}_{\mathrm{PH}}=1.2,1 \mathrm{H}, \mathrm{H} 4\right), 3.83(\mathrm{dddd}, J=8.0$, 2.9, 2.9, ${ }^{3} J_{\mathrm{PH}}=13.4,1 \mathrm{H}, \mathrm{H} 2$ ), 2.57 (ddddd, $J=13.3,2.9,1.1,1.0,{ }^{3} J_{\mathrm{PH}}=8.8,1 \mathrm{H}, \mathrm{H} 1$ ), Endo Isomer: $6.33\left(\right.$ ddddd $, J=14.3,10.5,7.8,7.4,{ }^{3} J_{\mathrm{PH}}=1.1,1 \mathrm{H}, \mathrm{H} 3$ ), 4.53 (ddddd, $J=7.8,3.2,1.2,0.8,{ }^{3} J_{\mathrm{PH}}=$ 
$1.0,1 \mathrm{H}, \mathrm{H} 4$ ), 3.70 (dddd, $J=7.4,3.6,3.2,{ }^{3} J_{\mathrm{PH}}=14.3,1 \mathrm{H}, \mathrm{H} 2$ ), 3.60 (dddd, $J=14.3,1.2,0.5,{ }^{3} J_{\mathrm{PH}}=$ 1.9, $1 \mathrm{H}, \mathrm{H} 5$ ), 2.35 (ddddd, $\left.J=10.5,3.6,0.8,0.5,{ }^{3} J_{\mathrm{PH}}=9.4,1 \mathrm{H}, \mathrm{H} 1\right) .{ }^{13} \mathrm{C} N M R\left(\mathrm{CD}_{3} \mathrm{CN}, \delta\right): 149.0$ (PzA3(exo)), 147.9 (PzA3(endo)), $146.9\left(\mathrm{~d}, 4 \mathrm{~J}_{\mathrm{PC}}=2.4, \mathrm{PzB} 3(\mathrm{exo})\right), 145.3\left(\mathrm{~d},{ }^{4} J_{\mathrm{PC}}=2.4, \mathrm{PzB} 3(\right.$ endo $\left.)\right)$, $144.4 \quad$ (PzC3(endo)), $144.1 \quad$ (РzC3(exo)), $140.5 / 140.1 / 139.8 / 139.2 \quad(6 \quad$ Tp5's), 109.4/108.8/108.6/108.4/108.1 (6 Tp4's), 13.7 (d, $\left.J_{\mathrm{PC}}=33.8, \mathrm{PMe}_{3}(\mathrm{exo})\right), 13.0$ (d, $J_{\mathrm{PC}}=33.8$, $\mathrm{PMe}_{3}\left(\right.$ endo)), Allyl ligand signals for the Exo Isomer: $115.1\left(\mathrm{~d}, J_{\mathrm{PC}}=5.8, \mathrm{C} 3\right), 100.9\left(\mathrm{~d}, J_{\mathrm{PC}}=2.4\right.$, $C 2), 60.7\left(d, J_{P C}=11.3, C 1\right)$. Allyl ligand signals for the Endo Isomer: $120.6\left(d, J_{P C}=5.8, C 3\right), 100.1$ $\left(d, J_{\mathrm{PC}}=1.8, \mathrm{C} 2\right), 62.3\left(\mathrm{~d}, J_{\mathrm{PC}}=12.5, \mathrm{C} 1\right) .{ }^{31} \mathrm{P} \mathrm{NMR}\left(\mathrm{CD}_{3} \mathrm{CN}, \delta\right):-2.91\left(J_{\mathrm{WP}}=252\right),-7.40\left(J_{\mathrm{WP}}=256\right)$. IR: $v_{B H}=2515 \mathrm{~cm}^{-1}, v_{N O}=1647 \mathrm{~cm}^{-1} . C V(M e C N): E_{p, a}=+2.07$ V,$E_{p, c}=-1.08$ V. ESI-MS: obs'd (\%), calc'd (\%), ppm, (M-OTf) : 542.1376 (84.4), 542.1359 (88.4), 3.2; 543.1397 (64.4), 543.1384 (78.5), 2.3; 544.1392 (100), 544.1381 (100), 1.9; 545.1441 (32.2), 545.1427 (37.8), 2.5; 546.1428 (87.2), 546.1414 (85.9), 2.6.

\section{$\left[\mathrm{TpW}(\mathrm{NO})\left(\mathrm{PMe}_{3}\right)\left(1,2-\eta^{2}\right.\right.$-(2,3-dimethylbut-3-en-2-ylium)][OTf] (8)}

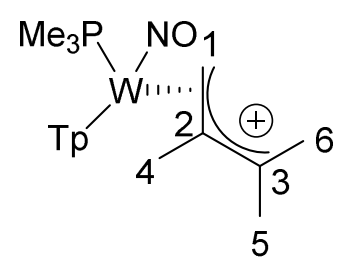

In a flame dried test tube, 2,3-dimethylbutadiene $(0.91 \mathrm{~g}, 11.1 \mathrm{mmol})$ was added to a homogeneous yellow solution of $\operatorname{TpW}(\mathrm{NO})\left(\mathrm{PMe}_{3}\right)\left(\eta^{2}\right.$-benzene) $(0.501 \mathrm{~g}, 0.862 \mathrm{mmol})$ in DME (2.62 g). The tube was added to a $57{ }^{\circ} \mathrm{C}$ oil bath and allowed to stir. The dark purple-brown solution was removed from the warm bath after $1.5 \mathrm{~h}$. An HOTf $(0.130 \mathrm{~g}, 0.866 \mathrm{mmol})$ in MeCN $(0.62 \mathrm{~g})$ was added to the solution to make a dark yellow solution. After 45 minutes the solution became heterogeneous. The solution was allowed to stir for an additional $15 \mathrm{~h}$ and the yellow precipitate was collected on a $15 \mathrm{~mL}$ medium porosity fritted funnel. The precipitate was washed with 3x0.3 g DME and placed under vacuum (0.298 g, $0.405 \mathrm{mmol}, 47 \%$ yield). 
${ }^{1} \mathrm{H}$ NMR $\left(\mathrm{CD}_{3} \mathrm{CN}, \delta\right)$ : Endo: $8.28(\mathrm{~d}, J=2.0,1 \mathrm{H}, \mathrm{PzC} 3), 8.18(\mathrm{~d}, J=2.0,1 \mathrm{H}, \mathrm{PzB} 3), 8.04(\mathrm{~d}, J=2.0$, 1H, PzC5), 7.92 (d, $J=2.0,1 \mathrm{H}, \mathrm{PzA} 5), 7.87$ (d, $J=2.0,1 \mathrm{H}, \mathrm{PzB} 5), 7.67$ (d, $J=2.0,1 \mathrm{H}, \mathrm{PzA}$ ), 6.55 (t, $J=2.0,1 \mathrm{H}, \mathrm{PzC} 4), 6.40$ (m(overlap), 2H, PzB4), 6.35 (t, $J=2.0,1 \mathrm{H}, \mathrm{PzA} 4), 3.36$ (dd, $J=7.2,{ }^{3} \mathrm{JH}_{\mathrm{PH}}$ $=10.9,1 \mathrm{H}, \mathrm{H} 1), 3.12\left(\mathrm{dd}, J=7.2 \mathrm{~s}^{3} \mathrm{JH}_{\mathrm{PH}}=10.1,1 \mathrm{H}, \mathrm{H} 1^{\prime}\right), 2.25(\mathrm{~s}, 3 \mathrm{H}, \mathrm{H} 6), 1.92(\mathrm{~s}, 3 \mathrm{H}, \mathrm{H} 5) 1.34(\mathrm{~s}$, $3 \mathrm{H}, \mathrm{H} 4), 1.27\left(\mathrm{~d}, J_{\mathrm{PH}}=9.8,9 \mathrm{H}, \mathrm{PMe}_{3}\right) . \mathrm{ExO}: 8.07(\mathrm{~m}, 2 \mathrm{H}, \mathrm{PzC} 5 / \mathrm{PzC} 3), 7.99(\mathrm{~d}, J=2.0,1 \mathrm{H}, \mathrm{PzA} 5)$, $7.93(\mathrm{~d}, J=2.0,1 \mathrm{H}, \mathrm{PzB} 3), 7.91$ (d, $J=2.0,1 \mathrm{H}, \mathrm{PzB} 5), 7.83$ (d, $J=2.0,1 \mathrm{H}, \mathrm{PzA} 3), 6.53(\mathrm{t}, J=2.0$, $1 \mathrm{H}, \mathrm{PzC} 4), 6.4$ (m(overlap), $1 \mathrm{H}, \mathrm{PzA} 4), 6.38(\mathrm{t}, J=2.0,1 \mathrm{H}, \mathrm{PzB} 4), 3.71\left(\mathrm{dd}, J=6.3,{ }^{3} J_{\mathrm{PH}}=15.2,1 \mathrm{H}\right.$, $\mathrm{H} 1), 2.80\left(\mathrm{dd}, J=6.3,{ }^{3} J_{\mathrm{PH}}=5.7,1 \mathrm{H}, \mathrm{H} 1^{\prime}\right), 2.38(\mathrm{~s}, 3 \mathrm{H}, \mathrm{H} 6), 1.78(\mathrm{~s}, 3 \mathrm{H}, \mathrm{H} 5), 1.28\left(\mathrm{~d}, J_{\mathrm{PH}}=9.8,9 \mathrm{H}\right.$, $\left.\mathrm{PMe}_{3}\right), 1.00$ (s, 3H, H4). ${ }^{13} \mathrm{C}$ NMR (CD $\left.\mathrm{CN}, \delta\right):$ Endo: 176.8 (C3), 147.9 (PzA3), 145.8 (d, $J=2.4$, PzB3), 145.1 (PzC3), 140.7 (PzA5), 139.6/139.5 (PzB5/PzC5), 122.2 (q, J JF $=321 \mathrm{~Hz}$, Triflate), 108.7 (PzB4), 108.4 (PzC4), 108.2 (PzA4), $102.3\left(\mathrm{~d}, J_{\mathrm{PC}}=3.8, \mathrm{C} 2\right), 65.2\left(\mathrm{~d}, J_{\mathrm{PC}}=14.2, \mathrm{C} 1\right), 29.1$ (C5),

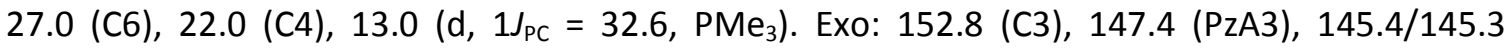
(PzB3/PzC3), 141.5 (PzA5), 139.9/139.8 (PzB5/PzC5), $112.6\left(\mathrm{~d}, J_{\mathrm{PC}}=4.6, \mathrm{C} 2\right), 108.8$ (PzC4), 108.6/108.5 (PzA4/BzB4), $65.2\left(d, J_{P C}=14.2, C 1\right), 27.4(C 5), 24.5(C 4), 24(C 6), 13.2\left(d,{ }^{1} J_{P C}=32.8\right.$, $\left.\mathrm{PMe}_{3}\right) .{ }^{31} \mathrm{P}$ NMR $\left(\mathrm{CD}_{3} \mathrm{CN}, \delta\right):-4.09\left(J_{\mathrm{WP}}=259\right),-7.35\left(J_{\mathrm{WP}}=258\right) . \mathrm{IR}: v_{\mathrm{BH}}=2511 \mathrm{~cm}^{-1}, v_{\mathrm{NO}}=1624$ $\mathrm{cm}^{-1} . \mathrm{CV}(\mathrm{MeCN}): E_{\mathrm{p}, \mathrm{a}}=+1.93 \mathrm{~V}, E_{1 / 2}=-0.78 \mathrm{~V}, E_{1 / 2}=-1.66 \mathrm{~V} . \mathrm{ESI}-\mathrm{MS}:$ obs'd (\%), calc'd (\%), ppm, $M^{+}: 584.1828$ (71.7), 584.1828 (86.8), 0.1; 585.1858 (65.7), 585.1854 (79.3), 0.6; 586.1863 (100), 586.1852 (100), 1.9; 587.1899 (33), 587.1896 (40.1), 0.5; 588.1903 (62.3), 588.1884 (84.9), 3.1. Anal. Calc'd for $\mathrm{C}_{19} \mathrm{H}_{30} \mathrm{BF}_{3} \mathrm{~N}_{7} \mathrm{O}_{4}$ PSW: $\mathrm{C}, 31.04 ; \mathrm{H}, 4.11 ; \mathrm{N}, 13.34$; Found: $\mathrm{C}, 31.17 ; \mathrm{H}, 4.29 ; \mathrm{N}$, 13.50.

$\operatorname{TpW}(\mathrm{NO})\left(\mathrm{PMe}_{3}\right)\left(1,2-\eta^{2}\right.$-cyclohexa-1,3-diene) (11p, 11d)

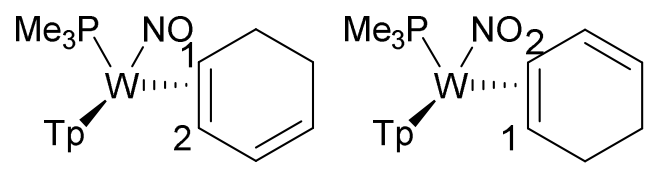


1,3-Cyclohexadiene $(0.261 \mathrm{~g}, 3.3 \mathrm{mmol})$ was added to a homogeneous yellow solution of $\mathrm{TpW}(\mathrm{NO})\left(\mathrm{PMe}_{3}\right)\left(\eta^{2}\right.$-benzene) $(0.151 \mathrm{~g}, 0.260 \mathrm{mmol})$ in DME $(3.0 \mathrm{~g})$, and allowed to stir at room temperature for 20 hours. The resulting dark brown solution was precipitated over a stirring mixture of $20 \mathrm{~mL}$ ether and $50 \mathrm{~mL}$ hexanes. The brown/purple precipitate was filtered over a 30 $\mathrm{mL}$ fine-porosity fritted funnel and discarded. The yellow filtrate was evaporated to dryness and dissolved in minimal DME. About $0.5 \mathrm{~mL}$ acetonitrile was added to the solution, which stirred ten minutes and evaporated to dryness. $1 \mathrm{~mL}$ acetonitrile was triturated with the residue; ether was added dropwise to encourage precipitation. Majority of solvent was evaporated, but did not give precipitation. Residue was dissolved in minimal DME and precipitated dropwise over $40 \mathrm{~mL}$ stirring water. A pale tan solid was filtered over a $15 \mathrm{~mL}$ fine-porosity fritted funnel and stored overnight in a desiccator to give $0.054 \mathrm{~g}(0.093 \mathrm{mmol}, 36 \%$ yield $)$.

${ }^{1} \mathrm{H}$ NMR $\left(\mathrm{CDCl}_{3}, \delta\right): 8.25(\mathrm{~d}, J=2.0,1 \mathrm{H}, \operatorname{PzA} 3(\mathrm{~d})), 8.08(\mathrm{~m}, 1 \mathrm{H}, \operatorname{PzB} 3(\mathrm{p}+\mathrm{d})), 8.05(\mathrm{~d}, J=2.0,1 \mathrm{H}$, $\operatorname{PzA}(p)), 7.70(d, J=2.0,1 \mathrm{H}, \operatorname{PzB} 5(p)), 7.68(\mathrm{~d}, J=2.0,1 \mathrm{H}, \operatorname{PzB} 5(\mathrm{~d})), 7.61(\mathrm{~d}, J=2.0,1 \mathrm{H}, \operatorname{PzA} 5(\mathrm{p}))$, $7.57(\mathrm{~d}, J=2.0,1 \mathrm{H}, \operatorname{PzA} 5(\mathrm{~d})), 7.34(\mathrm{~d}, J=2.0,1 \mathrm{H}, \operatorname{PzC}(\mathrm{d})), 7.28(\mathrm{~d}, J=2.0,1 \mathrm{H}, \operatorname{PzC}(\mathrm{p})), 6.29(\mathrm{t}, J$ $=2.0,1 \mathrm{H}, \operatorname{PzB} 4(\mathrm{~d})), 6.27(\mathrm{t}, J=2.0,1 \mathrm{H}, \operatorname{PzB} 4(\mathrm{p})), 6.21(\mathrm{t}, J=2.0,1 \mathrm{H}, \operatorname{PzA} 4(\mathrm{p})), 6.18(\mathrm{t}, J=2.0,1 \mathrm{H}$, $\operatorname{PzA} 4(d)), 6.17 / 6.16(t, J=2.0,1 \mathrm{H}, \mathrm{PzC} 4(\mathrm{p}+\mathrm{d})), 1.28\left(\mathrm{~d}, J=8.3,9 \mathrm{H}, \mathrm{PMe}_{3}(\mathrm{p})\right), 1.27(\mathrm{~d}, J=8.2,9 \mathrm{H}$, $\mathrm{PMe}_{3}(\mathrm{~d})$ ), Distal Diene (d-Major isomer): 6.68 (ddd, $J=9.0,5.6,2.9,1 \mathrm{H}, \mathrm{H} 3$ ), 5.23 (ddd, $J=9.0$, 6.6, 2.0,1H, H4), $3.65(m, 1 H, H 6), 2.66\left(m, 1 H, H 1 / H 6^{\prime}\right), 2.45$ (m, 1H, H5), $1.94\left(m, 1 H, H 5^{\prime}\right), 1.68$ $(\mathrm{m}, 1 \mathrm{H}, \mathrm{H} 2)$, Proximal Diene (p-minor isomer): 6.48 (ddd, $J=9.0,4.8,2.7,1 \mathrm{H}, \mathrm{H} 3), 5.24(\mathrm{~m}, 1 \mathrm{H}$, $\mathrm{H} 4), 3.33\left(\mathrm{~m}, 1 \mathrm{H}, \mathrm{H} 6\right.$ (anti)), 2.89 (ddd, $\left.J=10.3,5.0,{ }^{3} J_{\mathrm{PH}}=14.2,1 \mathrm{H}, \mathrm{H} 2\right), 2.66(\mathrm{~m}, 1 \mathrm{H}, \mathrm{H} 6(\mathrm{syn}))$, $2.45(\mathrm{~m}, 1 \mathrm{H}, \mathrm{H} 5), 1.95\left(\mathrm{~m}, 1 \mathrm{H}, \mathrm{H} 5^{\prime}\right), 1.38(\mathrm{~d}, J=10.1,1 \mathrm{H}, \mathrm{H} 1) .{ }^{13} \mathrm{CNMR}\left(\mathrm{CDCl}_{3}, \delta\right): 144.5(\mathrm{PzA} 3(\mathrm{~d}))$, 143.4/143.3 (PzB3(p,d)), 142.1 (PzA3(p)), 140.2/140.1 (PzC3(p,d)), 136.4 (Tp5), 136.2 (Tp5), 135.6 (2 Tp5's), 135.2 (Tp5), 134.8 (Tp5), 106.3 (Tp4), 106.1 (Tp4), 105.7 (Tp4), 105.5 (3 Tp4's), $14.0\left(\mathrm{~d},{ }^{1} J_{\mathrm{PC}}=27.8, \mathrm{PMe}_{3}(\mathrm{p})\right), 13.4\left(\mathrm{~d},{ }^{1} J_{\mathrm{PC}}=27.8, \mathrm{PMe}_{3}(\mathrm{~d})\right)$, Distal Diene: $133.4(\mathrm{C} 3), 120.8(\mathrm{C} 4)$, 
$56.6\left(\mathrm{~d}, J_{\mathrm{PC}}=11.9, \mathrm{C} 1\right), 50.8(\mathrm{C} 2), 23.5(\mathrm{C} 5), 21.6(\mathrm{C} 6)$, Proximal Diene: 130.8 (C3), 120.8 (C4), $55.4(C 1), 50.8\left(d, J_{P C}=8.4, C 2\right), 26.5(C 6), 23.5(C 5) .{ }^{31} \mathrm{P} N M R:\left(C D C l_{3}, \delta\right):-9.75\left(J_{W P}=286 \mathrm{~Hz}\right),-$ $11.94\left(J_{\mathrm{WP}}=284 \mathrm{~Hz}\right)$. IR: $v_{\mathrm{BH}}=2488 \mathrm{~cm}^{-1}, v_{\mathrm{NO}}=1554 \mathrm{~cm}^{-1} . \mathrm{CV}(\mathrm{DMA}): E_{\mathrm{p}, \mathrm{a}}=+0.44$ V. ESI-MS: obs'd (\%), calc'd (\%), ppm, (M+H) $)^{+}: 594.1664$ (86.9), 594.1672 (86.2), 1.3; 595.1688 (90), 595.1698 (79.6), 1.7; 596.1687 (100), 596.1696 (100), 1.4; 597.1751 (45.4), 597.1739 (40.8), 2.0; 598.1749 (77.5), 598.1728 (84.6), 3.5.

\section{$\mathrm{TpW}(\mathrm{NO})\left(\mathrm{PMe}_{3}\right)\left(1,2-\eta^{2}\right.$-cyclopenta-1,3-diene) (12)}

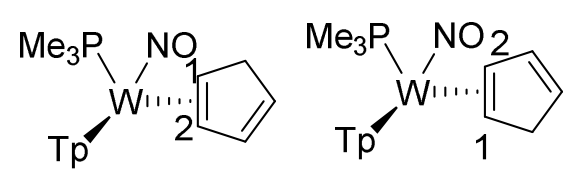

To a flame-dried test tube with a stir bar was added $\operatorname{TpW}(\mathrm{NO})\left(\mathrm{PMe}_{3}\right)\left(\eta^{2}\right.$-benzene) $(0.100 \mathrm{~g}$, $0.172 \mathrm{mmol}$ ) and DME (2.0 g), to give a homogeneous yellow solution, to which cyclopentadiene (0.212 g, $3.207 \mathrm{mmol}$ ) was added. The test tube was placed in a $67^{\circ} \mathrm{C}$ oil bath for 16 hours. A dark brown solution was cooled to room temperature and precipitated over a mixture of $37 \mathrm{~mL}$ hexanes and $13 \mathrm{~mL}$ ether. A brown/purple precipitate was filtered using a $15 \mathrm{~mL}$ fine-porosity fritted funnel and discarded. The light yellow filtrate was evaporated to dryness and dissolved in minimal dichloromethane for transfer to a 4 dram vial with stirbar. The dichloromethane was evaporated and $2 \mathrm{~mL}$ ether added to the vial and allowed to stir overnight. An orange liquid was carefully pipeted out of the vial, leaving a pale tan solid. One $\mathrm{mL}$ of hexanes and 5 drops ether was added to the vial and allowed to stir overnight. One $\mathrm{mL}$ of hexanes was added to the vial and the pale tan solid was filtered over a $2 \mathrm{~mL}$ fine-porosity fritted funnel to give $0.058 \mathrm{~g}(0.102$ $\mathrm{mmol}, 60 \%$ yield).

${ }^{1} \mathrm{H} \mathrm{NMR}\left(\mathrm{CDCl}_{3}, \delta\right): 8.42(\mathrm{~d}, J=1.7,1 \mathrm{H}, \operatorname{PzA} 3(\mathrm{p})), 8.25(\mathrm{~d}, J=1.7,1 \mathrm{H}, \operatorname{PzA} 3(\mathrm{~d})), 8.05(\mathrm{~d}, J=1.8,1 \mathrm{H}$, PzB3(p)), $8.04(d, J=2.0,1 \mathrm{H}, \mathrm{PzB} 3(\mathrm{~d})), 7.72(\mathrm{~m}, 1 \mathrm{H}, \mathrm{PzB} 5(\mathrm{p}+\mathrm{d})), 7.67(\mathrm{~m}, 1 \mathrm{H}, \mathrm{PzC}(\mathrm{p}+\mathrm{d})), 7.61(\mathrm{~d}$, $J=2.0,1 \mathrm{H}, \operatorname{PzA} 5(\mathrm{p})), 7.59(\mathrm{~d}, 1 \mathrm{H}, \operatorname{PzA} 5(\mathrm{~d})), 7.28(\mathrm{~m}, 1 \mathrm{H}, \operatorname{PzC} 5(\mathrm{p}+\mathrm{d})), 6.29(\mathrm{~m}, 1 \mathrm{H}, \mathrm{PzB} 4(\mathrm{p}+\mathrm{d})), 6.23$ 
$(m, 1 H, P z A 4(p+d)), 6.15(m, 1 H, P z C 4(p+d)), 1.31\left(d, J=7.5,9 H, P_{3}(p)\right), 1.27(d, J=7.7,9 H$, $\mathrm{PMe}_{3}(\mathrm{~d})$ ), Proximal diene (p - Major isomer): $6.41(\mathrm{dd}, J=4.8,1.9,1 \mathrm{H}, \mathrm{H} 3), 5.37(\mathrm{~m}, 1 \mathrm{H}, \mathrm{H} 4)$, $4.50(\mathrm{~m}, 1 \mathrm{H}, \mathrm{H} 5), 3.95\left(\mathrm{~m}, 1 \mathrm{H}, \mathrm{H} 5^{\prime}\right), 3.72(\mathrm{dd}, \mathrm{J}=12.5,7.3,1 \mathrm{H}, \mathrm{H} 2), 2.15(\mathrm{~m}, 1 \mathrm{H}, \mathrm{H} 1)$, Distal diene (d-minor isomer): $6.68(\mathrm{dd}, J=5.0,2.1,1 \mathrm{H}, \mathrm{H} 3), 5.30(\mathrm{~m}, 1 \mathrm{H}, \mathrm{H} 4), 4.54(\mathrm{~m}, 1 \mathrm{H}, \mathrm{H} 5), 3.54(\mathrm{~m}, 1 \mathrm{H}$, $\left.\mathrm{H} 5^{\prime}\right), 3.40(\mathrm{dt}, J=14.0,7.0,1 \mathrm{H}, \mathrm{H} 1), 2.44(\mathrm{dd}, J=7.4,2.3,1 \mathrm{H}, \mathrm{H} 2) .{ }^{13} \mathrm{C} \mathrm{NMR}\left(\mathrm{CDCl}_{3}, \delta\right): 144.4(\mathrm{Tp})$, 144.3 (Tp), 143.9 (Tp), 141.2 (Tp), 140.9 (Tp), 140.6 (Tp), 136.3 (Tp), 136.2 (Tp), 135.8 (Tp), 135.7 (Tp), 135.1 (Tp), 134.8 (Tp), 106.4 (Tp), 106.3 (Tp), 105.9 (Tp), 105.7 (Tp), 105.6 (Tp), 105.5 (Tp), $14.5\left(\mathrm{~d}, J_{\mathrm{P}-\mathrm{C}}=27.5,3 \mathrm{C}, \mathrm{PMe}_{3}\right), 14.1\left(\mathrm{~d}, J_{\mathrm{PC}}=27.1,3 \mathrm{C}, \mathrm{PMe}_{3}\right)$, Proximal diene: $136.4(\mathrm{C} 3), 123.0$ (C4), 67.9 (C2), 57.3 (C1), 43.0 (C5), Distal diene: 138.0 (C3), 121.9 (C4), 66.4 (C2), 58.6 (C1), 43.2 (C5). ${ }^{31} \mathrm{P}$ NMR $\left(\mathrm{CDCl}_{3}, \delta\right):-11.11\left(J_{\mathrm{WP}}=280\right),-11.88\left(J_{\mathrm{WP}}=290\right) . \mathrm{IR}: \mathrm{v}_{\mathrm{BH}}=2484 \mathrm{~cm}^{-1}, \mathrm{v}_{\mathrm{NO}}=1554 \mathrm{~cm}^{-}$ ${ }^{1}$. CV (DMA): $E_{p, a}=+0.36$ V. HRMS: Overlapping signals for hydride loss $(M-H)+$ and protonation $(\mathrm{M}+\mathrm{H})+$ complicated the spectrum and caused overlapping $M / Z$ peaks to not fit within the acceptable 5 ppm difference from that of the calculated. ESI-MS $\left((M-H)^{+}\right)$: obs'd $(\%)$, calc'd $(\%)$, ppm: 566.1355 (61.8), 566.1359 (87.3), 0.7; 567.139 (56.2), 567.1385 (79.1), 0.9; 568.1419 (100), 568.1382 (100), 6.5; 569.1484 (45.2), 569.1427 (39.3), 10.0; 570.1451 (89.3), 570.1415 (85.2), 6.3. ESI-MS ((M+H) $\left.)^{+}\right): 568.1419$ (112), 568.1515 (87.3), 16.9; 569.1484 (50.7), 569.1541 (79.1), 10.1; 570.1451 (100), 570.1538 (100), 15.4; 571.1514 (22.7), 571.1583 (39.3), 12.2; 572.1573 (36.2), $572.1571(85.2), 0.3$.

\section{$\left[\mathrm{TpW}(\mathrm{NO})\left(\mathrm{PMe}_{3}\right)\left(1,2-\eta^{2}-(\mathrm{S})-7-\right.\right.$ methoxycyclohepta-1,3-diene) (14)}

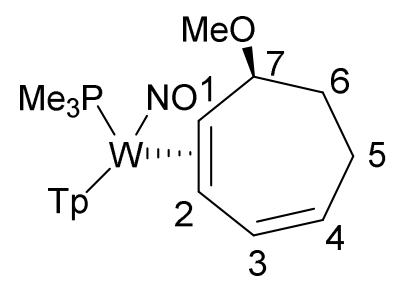

NaOMe (12 mg, $0.222 \mathrm{mmol})$ was dissolved in $\mathrm{MeOH}(1.083 \mathrm{~g})$ and added to 6 (20 mg, 0.032 $\mathrm{mmol}$ ) and allowed to stand overnight. The reaction was diluted with $5 \mathrm{~mL} \mathrm{DCM}$ and extracted 
$3 x$ with $2 \mathrm{~mL}$ portions of saturated aqueous $\mathrm{NaHCO}_{3}$. The aqueous layers were backextracted with $2 \mathrm{~mL} \mathrm{DCM}$. The organic layers were dried over $\mathrm{MgSO}_{4}$, filtered over a Celite plug and evaporated in vacuo to give 14, on which NMR data was collected.

${ }^{1} \mathrm{H} \mathrm{NMR}\left(\mathrm{CDCl}_{3}, \delta\right): 8.22(\mathrm{~d}, J=2.0,1 \mathrm{H}, \mathrm{Tp}), 8.02(\mathrm{~d}, J=2.0,1 \mathrm{H}, \mathrm{Tp}), 7.71(\mathrm{~d}, J=2.0,1 \mathrm{H}, \mathrm{Tp}), 7.69$ $(\mathrm{d}, J=2.0,1 \mathrm{H}, \mathrm{Tp}), 7.61(\mathrm{~d}, J=2.0,1 \mathrm{H}, \mathrm{Tp}), 7.32(\mathrm{~d}, J=2.0,1 \mathrm{H}, \mathrm{Tp}), 6.28(\mathrm{t}, J=2.0,1 \mathrm{H}, \mathrm{Tp}), 6.21$ (m, $1 \mathrm{H}, \mathrm{H} 3), 6.20(\mathrm{t}, J=2.0,1 \mathrm{H}, \mathrm{Tp}), 6.18(\mathrm{t}, J=2.0,1 \mathrm{H}, \mathrm{Tp}), 5.12(\mathrm{dt}, J=4.4,11.7,1 \mathrm{H}, \mathrm{H} 4), 4.33$ (dd, $J=4.8,7.6,1 \mathrm{H}, \mathrm{H} 7), 3.48(\mathrm{~s}, 3 \mathrm{H}, \mathrm{OMe}), 2.97(\mathrm{~m}, 1 \mathrm{H}, \mathrm{H} 1), 2.76(\mathrm{~m}, 1 \mathrm{H}, \mathrm{H} 5), 2.53\left(\mathrm{~m}, 1 \mathrm{H}, \mathrm{H} 5^{\prime}\right)$, $2.45(\mathrm{~m}, 1 \mathrm{H}, \mathrm{H} 6), 2.03\left(\mathrm{~m}, 1 \mathrm{H}, \mathrm{H} 6^{\prime}\right), 1.35(\mathrm{dd}, J=5.2,10.7,1 \mathrm{H}, \mathrm{H} 2), 1.20\left(\mathrm{~d}, J=8.2,9 \mathrm{H}, \mathrm{PMe}_{3}\right) .{ }^{31} \mathrm{P}$ $\operatorname{NMR}\left(\mathrm{CDCl}_{3}, \delta\right):-10.73\left(J_{\mathrm{WP}}=281\right)$.

$\left[\mathrm{TpW}(\mathrm{NO})\left(\mathrm{PMe}_{3}\right)\left(2,3-\eta^{2}-(\right.\right.$ cyclohex-2-en-1-yltrimethylphosphonium)](OTf) (15c)

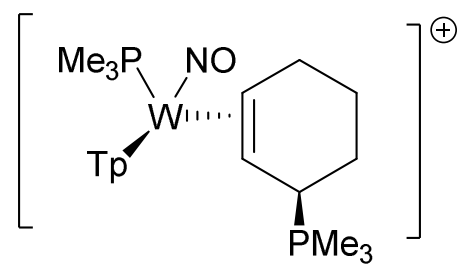

$\mathrm{PMe}_{3}(0.037 \mathrm{~g}, 0.473 \mathrm{mmol})$ was added to a heterogeneous yellow solution of $3(0.036 \mathrm{~g}, 0.049$ mmol) in $\mathrm{CHCl}_{3}(2.01 \mathrm{~g})$ to become homogeneous and pale yellow. After two minutes, the stirring reaction solution was diluted with $20 \mathrm{~mL} \mathrm{Et}{ }_{2} \mathrm{O}$ to precipitate a white solid that was collected on a $15 \mathrm{~mL}$ medium porosity fritted funnel. The white residue on the reaction flask was dissolved in $1 \mathrm{~mL} \mathrm{CHCl}$ and precipitated with $20 \mathrm{~mL} \mathrm{Et}_{2} \mathrm{O}$. The solid was collected on the same $15 \mathrm{~mL}$ medium porosity fritted funnel, washed with $2 \times 7 \mathrm{~mL} \mathrm{Et}{ }_{2} \mathrm{O}$ and placed under vacuum (0.022 g, $0.027 \mathrm{mmol}, 55 \%$ yield).

${ }^{1} \mathrm{H}$ NMR $\left(\mathrm{CDCl}_{3}, \delta\right): 8.00(\mathrm{~d}, J=2.0,1 \mathrm{H}, \mathrm{PzB} 3), 7.98(\mathrm{~d}, J=2.0,1 \mathrm{H}, \mathrm{PzA} 3), 7.74(\mathrm{~d}, J=2.0,1 \mathrm{H}$, PzC5), 7.70 (d, $J=2.0,1 \mathrm{H}, \mathrm{PzB} 5), 7.68$ (d, $J=2.0,1 \mathrm{H}, \mathrm{PzA} 5), 7.29$ (d, $J=2.0,1 \mathrm{H}, \mathrm{PzC}$ ), 6.30+6.29 $(\mathrm{t}, J=2.0,2 \mathrm{H}, \mathrm{PzB} 4 / \mathrm{PzC} 4), 6.22(\mathrm{t}, J=2.0,1 \mathrm{H}, \mathrm{PzA} 4), 3.60\left(\mathrm{ddd},{ }^{2} J_{\mathrm{PH}}=8.9, J=6.7,6.7,1 \mathrm{H}, \mathrm{H} 1\right)$, $3.24(\mathrm{~m}, 1 \mathrm{H}, \mathrm{H} 4), 2.77\left(\mathrm{~m}, 2 \mathrm{H}, \mathrm{H} 3+\mathrm{H} 4{ }^{\prime}\right), 2.07(\mathrm{~m}, 1 \mathrm{H}, \mathrm{H} 6), 1.87(\mathrm{~m}, 1 \mathrm{H}, \mathrm{H} 5), 1.64\left(\mathrm{~d}, 2 \mathrm{~J}_{\mathrm{PH}}=13.3\right.$, 
$\left.\left.9 \mathrm{H}, \mathrm{C}-\mathrm{PMe}_{3}\right), 1.60\left(\mathrm{~m}, 1 \mathrm{H}, \mathrm{H} 6^{\prime}\right), 1.5\left(\mathrm{~m}, 1 \mathrm{H}, \mathrm{H} 5^{\prime}\right), 1.22\left(\mathrm{~d},{ }^{2} J_{\mathrm{PH}}=8.3,9 \mathrm{H}, \mathrm{W}-\mathrm{PMe}\right)_{3}\right), 0.62\left(\mathrm{dd},{ }^{3} J_{\mathrm{PH}}=\right.$ 22.9, $J=11.1,1 \mathrm{H}, \mathrm{H} 2) .{ }^{13} \mathrm{C} \mathrm{NMR}\left(\mathrm{CDCl}_{3}, \delta\right): 143.3$ (PzB3), 141.9 (PzA3), 140.1 (PzC3), 137.5 (PzA5), 137.1 (PzC5), 136.6 (PzB5), $129.9\left(\mathrm{q}, 1 J_{\mathrm{FC}}=320\right.$, triflate), 107.0/106.7/106.6 $(\mathrm{PzA} 4 / \mathrm{PzB} 4 / \mathrm{PzC} 4), 49.7\left(\mathrm{~d},{ }^{2} J_{\mathrm{PC}}=11.3, \mathrm{C} 3\right), 45.4\left(\mathrm{~d}, J_{\mathrm{PC}}=3.0, \mathrm{C} 2\right), 33.4\left(\mathrm{~d},{ }^{1} J_{\mathrm{PC}}=42.6, \mathrm{C} 1\right), 29.1(\mathrm{~d}$, $\left.J_{\mathrm{PC}}=3.3, \mathrm{C} 4\right), 23.1\left(\mathrm{~d},{ }^{2} J_{\mathrm{PC}}=3.6, \mathrm{C} 6\right), 21.4\left(\mathrm{~d},{ }^{3} J_{\mathrm{PC}}=9.5, \mathrm{C} 5\right), 13.8\left(\mathrm{~d},{ }^{1} J_{\mathrm{PC}}=28.3, \mathrm{~W}-\mathrm{PMe}_{3}\right), 6.9(\mathrm{~d}$, $\left.\left.{ }^{1} J_{\mathrm{PC}}=53.5, \mathrm{C}-\mathrm{PMe}\right)_{3}\right){ }^{31} \mathrm{P} \mathrm{NMR}\left(\mathrm{CDCl}_{3}, \delta\right):-10.04\left(J_{\mathrm{WP}}=286\right), 35.41\left(\mathrm{C}-\mathrm{PMe}_{3}\right) \cdot \mathrm{IR}: \mathrm{V}_{\mathrm{BH}}=2481 \mathrm{~cm}^{-1}$, $\mathrm{v}_{\mathrm{NO}}=1542 \mathrm{~cm}^{-1} . \mathrm{CV}(\mathrm{MeCN}): E_{\mathrm{p}, \mathrm{a}}=+0.54 \mathrm{~V} . \mathrm{ESI}-\mathrm{MS}$ : obs'd (\%), calc'd (\%), ppm, $\mathrm{M}^{+}: 658.2128$ (81.8), 658.2114 (85.1), 2.1; 659.2139 (95.5), 659.214 (80.1), 0.1; 660.2156 (100), 660.2138 (100), 2.7; 661.2296 (31.8), 661.2180 (42.3), 17.5; 662.2158 (72.7), 662.2171 (84), 1.9.

\section{References}

(1) Winemiller, M. D.; Harman, W. D. J. Am. Chem. Soc. 1998, 120, 7835.

(2) Winemiller, M. D.; Harman, W. D. J. Org. Chem. 2000, 65, 1249.

(3) Valahovic, M. T.; Gunnoe, T. B.; Sabat, M.; Harman, W. D. J. Am. Chem. Soc. 2002, 124, 3309.

(4) Todd, M. A.; Sabat, M.; Myers, W. H.; Smith, T. M.; Harman, W. D. Journal of the American Chemical Society 2008, 130, 6906.

(5) Liu, W.; You, F.; Mocella, C. J.; Harman, W. D. J. Am. Chem. Soc. 2006, 128, 1426.

(6) Meiere, S. H.; Valahovic, M. T.; Harman, W. D. J. Am. Chem. Soc. 2002, 124, 15099.

(7) Graham, P. M.; Delafuente, D. A.; Liu, W.; Myers, W. H.; Sabat, M.; Harman, W. D. J. Am. Chem. Soc. 2005, ASAP (7/05).

(8) Ding, F.; Valahovic, M. T.; Keane, J. M.; Anstey, M. R.; Sabat, M.; Trindle, C. O.; Harman, W. D. J. Org. Chem. 2004, 69, 2257.

(9) Meiere, S. H.; Brooks, B. C.; Gunnoe, T. B.; Sabat, M.; Harman, W. D. Organometallics 2001, 20, 1038.

(10) Keane, J. M.; Harman, W. D. Organomet. 2005, 24, 1786.

(11) Harrison, D. P.; Welch, K. D.; Nichols-Nielander, A. C.; Sabat, M.; Myers, W. H.; Harman, W. D. Journal of the American Chemical Society 2008, 130, 16844.

(12) Welch, K. D.; Harrison, D. P.; Lis, E. C.; Liu, W.; Salomon, R. J.; Harman, W. D.; Myers, W. H. Organometallics 2007, 26, 2791.

(13) Strausberg, L.; Li, M.; Harrison, D. P.; Myers, W. H.; Sabat, M.; Harman, W. D. Organometallics 2013, 32, 915.

(14) Madrahimov, S. T.; Markovic, D.; Hartwig, J. F. Journal of the American Chemical Society 2009, 131, 7228.

(15) Tsang, J. Y. K.; Buschhaus, M. S. A.; Fujita-Takayama, C.; Patrick, B. O.; Legzdins, P. Organometallics 2008, 27, 1634. 
(16) Villanueva, L. A.; Ward, Y. D.; Lachicotte, R.; Liebeskind, L. S. Organometallics 1996, 15, 4190.

(17) Frohnapfel, D. S.; White, P. S.; Templeton, J. L.; Ruegger, H.; Pregosin, P. S. Organometallics 1997, 16, 3737.

(18) Ng, S. H. K.; Adams, C. S.; Hayton, T. W.; Legzdins, P.; Patrick, B. O. Journal of the American Chemical Society 2003, 125, 15210.

(19) Myers, W. H.; Welch, K. D.; Graham, P. M.; Keller, A.; Sabat, M.; Trindle, C. O.; Harman, W. D. Organometallics 2005, in press.

(20) Coombs, T. C.; Huang, W.; Garnier-Amblard, E. C.; Liebeskind, L. Organometallics 2010, ASAP DOI: 10.1021/om100305f. 2096.

(21) Winemiller, W. D.; Kopach, M. E.; Harman, W. D. J. Am. Chem. Soc. 1997, 119,

(22) Schilling, B. E. R.; Hoffmann, R.; Faller, J. W. Journal of the American Chemical Society 1979, 101, 592.

(23) Curtis, M. D.; Eisenstein, O. Organometallics 1984, 3, 887.

(24) Spera, M. L.; Chin, R. M.; Winemiller, M. D.; Lopez, K. W.; Sabat, M.; Harman, W. D. Organometallics 1996, 15, 5447.

(25) Kirchner, K.; Mereiter, K.; Umfahrer, A.; Schmid, R. Organometallics 1994, 13, 1886.

(26) Mocella, C. J.; Delafuente, D. A.; Keane, J. M.; Warner, G. R.; Friedman, L. A.; Sabat, M.; Harman, W. D. Organometallics 2004, 23, 3772.

(27) Harrison, D. P.; Welch, K. D.; Nichols-Nielander, A. C.; Sabat, M.; Myers, W. H.; Harman, W. D. Journal of the American Chemical Society 2008, 130, 16844.

(28) Lis, E. C.; Delafuente, D. A.; Lin, Y.; Mocella, C. J.; Todd, M. A.; Liu, W.; Sabat, M.; Myers, W. H.; Harman, W. D. Organometallics 2006, 25, 5051.

(27a) Shao, Y. et al., Phys. Chem. Chem. Phys., 2006, 8, 3172

(27b) http://www.gaussian.com/g_tech/gv5ref/gv5ref_toc.htm

(27c) Gaussian 09, Revision A.1, Frisch, M. J. et al. Gaussian, Inc., Wallingford CT, 2009. 


\section{Chapter 3}

Exploiting the $o$-Quinodimethane Nature of Naphthalene: Cycloaddition Reactions with $\eta^{2}$-Coordinated Tungsten- Naphthalene Complexes 
Diels-Alder reactions comprise an important class of synthetic transformations for the stereoselective generation of cyclic molecules. A number of natural products and pharmaceuticals include a Diels-Alder step in their synthesis, ${ }^{1}$ (e.g., taxol (anti-cancer). ${ }^{2}$ The in situ generation of highly reactive dienes has become a popular strategy for effecting cyclization with otherwise unreactive dienophiles. In particular, oquinodimethanes are versatile reagents for Diels-Alder reactions (Eq 1), owing to the aromatic stabilization that accompanies formation of the cycloadduct.

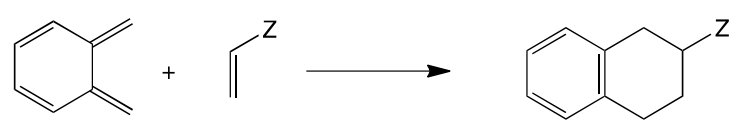

Eqn 1

Several routes for generating o-quinodimethanes have been developed, including photochemical means, ${ }^{3}$ and extrusion of small molecules such as dinitrogen or carbon dioxide from benzo derivatives. ${ }^{2}$

While much less reactive than o-quinodimethanes, anthracenes have shown analogous reactivity (Eqn 2). When these systems undergo cycloaddition with highly activated dienophiles, bonding occurs at the middle ring, thereby producing two isolated benzene $\pi$ systems:

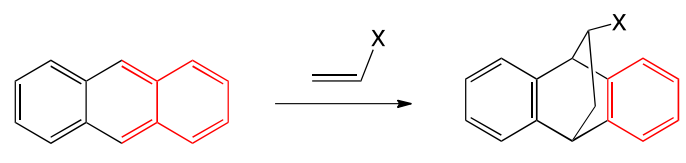

Eqn 2

In contrast to anthracene, naphthalene's predilection toward Diels-Alder cycloaddition reactions is greatly reduced, even with highly activated dienophiles. ${ }^{3}$ However, we believed that a $2,3-\eta^{2}$-naphthalene metal complex might produce a 
naphthalene-based $\pi$ system that mimics the cycloaddition tendencies of an $o$ quinodimethane or anthracene $\pi$ system:

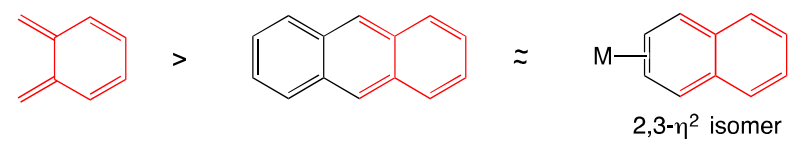

Eqn 3

While several examples of dihapto-coordinated naphthalene complexes have been reported, ${ }^{4-7}$ a 2,3- $\eta^{2}$ species has not been observed, owing to a much stronger thermodynamic preference for a 1,2 binding mode (vide infra). However, isomerization studies of the rhenium complexes $\operatorname{TpRe}(\mathrm{CO})(\mathrm{L})\left(\eta^{2}\right.$-naphthalene) $\left(\mathrm{L}={ }^{\mathrm{t}} \mathrm{BuNC}, \mathrm{PMe}_{3}, \mathrm{py}\right.$, Melm) ${ }^{6}$ showed that the two $1,2-\eta^{2}$ naphthalene stereoisomers could be interconverted by an intrafacial isomerization process (ring-walk; vide infra), and this observation suggests that a C2-C3 bound intermediate is kinetically accessible (Figure 1). ${ }^{6}$

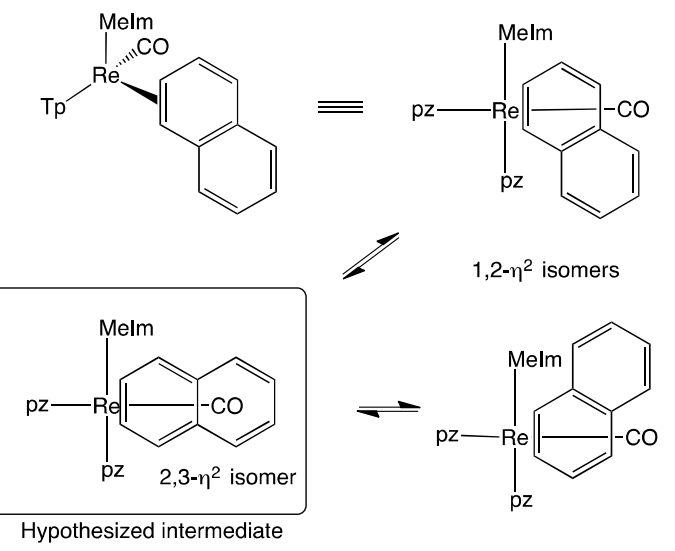

Figure 1. Isomerization of a dihapto-coordinated rhenium naphthalene complex. Unfortunately, a Diels-Alder reaction was not observed when TpRe(Melm)(CO) $\left(\eta^{2}\right.$-naphthalene) was combined with various dienophiles. ${ }^{8}$ Since $\left\{\mathrm{TpW}(\mathrm{NO})\left(\mathrm{PMe}_{3}\right)\right\}$ has been shown to be a stronger $\pi$ base than the analogous rhenium systems, ${ }^{9}$ we hypothesized that this fragment would form more reactive $2,3-\eta^{2}$ - 
naphthalene intermediates. Thus, in this study we set out to determine whether it would be possible to effect Diels-Alder cycloaddition reactions with naphthalenes by coordinating the latter to a tungsten $\pi$-base.

\section{Results and Discussion}

Naphthalene complexes 1-8 were prepared from the benzene precursor $\operatorname{TpW}(\mathrm{NO})\left(\mathrm{PMe}_{3}\right)\left(\eta^{2}\right.$-benzene) and naphthalene (1), 1-methylnaphthalene (2), 1methoxynaphthalene (3), 2-methoxynaphthalene (4), 1,4-dimethoxynaphthalene (5), 1,4-dimethylnaphthalene (6), 1,8-dimethylnaphthalene (7), and 2,6dimethylnaphthalene (8). ${ }^{9}$ When $\operatorname{TpW}(\mathrm{NO})\left(\mathrm{PMe}_{3}\right)\left(\eta^{2}\right.$-naphthalene), $\mathbf{1}$, was combined with approximately ten equivalents $(0.43 \mathrm{M})$ of $N$-methylmaleimide in a $3: 1(\mathrm{w} / \mathrm{w})$ mixture of $\mathrm{CHCl}_{3}$ and $n-\mathrm{BuOH}$ under an inert atmosphere and at room temperature, a cycloadduct slowly formed ( $t_{1 / 2} \sim$ nine days). The half-life was derived from a plot of In(conc 1 ) as a function of time, assuming pseudo-first order conditions. The plot can be seen at the end of the chapter (p. 75). Subsequent reactions were carried out at elevated temperatures $\left(\sim 50{ }^{\circ} \mathrm{C}\right)$ to shorten reaction times. Using $2 \mathrm{D}$ NMR techniques, ${ }^{10}$ we were able to assign the major product as the endo cycloadduct product, 9 (Figure 2).

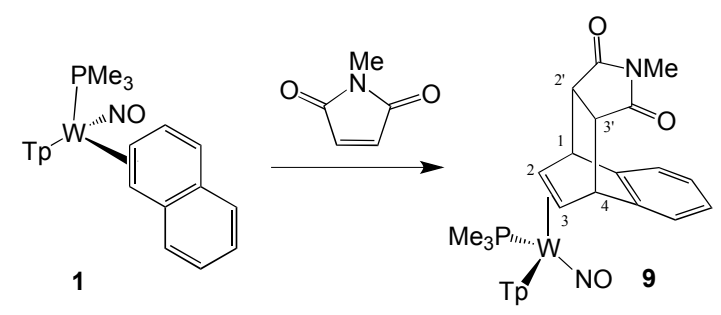

Figure 2. The cycloaddition reaction of a dihapto-coordinated naphthalene complex. 
The tungsten-bound methine hydrogen $(\mathrm{H} 2)$ proximal to the $\mathrm{PMe}_{3}$ was identified by its strong coupling to ${ }^{31} \mathrm{P}$ and NOESY interaction with the $\mathrm{PMe}_{3}$ protons. ${ }^{11}$ Proton $\mathrm{H} 3$ (distal to $\mathrm{PMe}_{3}$ ) is characteristically shifted upfield ( $\delta 1.57 \mathrm{ppm}$ ) due to the anisotropic environment between the pyrazole rings, and can be identified by its coupling to $\mathrm{H} 2 .^{11}$ The bridgehead protons, $\mathrm{H} 1$ and $\mathrm{H} 4$, overlap at 4.23 ppm, and both $\mathrm{H} 2$ and $\mathrm{H} 3$ display a COSY interaction with this region of the spectrum. The maleimide proton signals for $\mathrm{H}^{\prime}$ (3.39 ppm) and $\mathrm{H}^{\prime}$ (3.24 ppm) couple to those of the bridgehead protons in the COSY spectrum and also display NOE interactions with $\mathrm{H} 2$ and $\mathrm{H} 3$, respectively. These $\mathrm{H} 2-\mathrm{H} 2^{\prime}$ and $\mathrm{H} 3-\mathrm{H}^{\prime}$ NOE interactions allow the conclusive assignment of endo stereochemistry, shown in Figure 2. There is a minor product from the reaction of the naphthalene complex 1 and $\mathrm{N}$-methylmaleimide that comprises $6 \%$ of the crude reaction mixture, but our inability to isolate this component precludes its identification. Proton and ${ }^{31} \mathrm{P}$ data are inconsistent with this minor product being an electrophilic substitution at $\mathrm{C} 1$. However, an exo cycloadduct remains a possibility.

In order to gain insight into the reaction mechanism, the rate of cycloaddition was explored as a function of solvent. Addition of an alcohol as a co-solvent was found to accelerate the reaction, but the rate decreased in polar aprotic solvents (Table 1). ${ }^{12}$ Repeating the reaction in the presence of 2,6-lutidine decreased the reaction rate modestly, and this observation discounted the possibility that trace amounts of an adventitious Lewis acid were somehow effecting the reaction. Deliberate addition of Lewis acids such as $\mathrm{AlCl}_{3}$, LiOTf, $\mathrm{Zn}(\mathrm{OTf})_{2}$, and $\mathrm{ZnCl}_{2} \quad(\sim 1$ eq) resulted in the decomposition of $\mathbf{1}$ into an unrecognizable mixture of products. 
Table 1. Solvent Effects on Cycloaddition of 1 with $N$-methylmaleimide

\begin{tabular}{|crrc|}
\hline Solvent & $\begin{array}{c}\text { Dienophile } \\
\text { Concentration }\end{array}$ & $\mathbf{t}_{\mathbf{1 / 2}}$ (days) $^{\mathbf{b}}$ & $\begin{array}{c}\text { Relative } \\
\text { Rate }\end{array}$ \\
\hline $\begin{array}{c}\mathrm{CHCl}_{3} \\
\mathrm{CHCl}_{3} / n-\end{array}$ & $0.337 \mathrm{M}$ & 3.41 & 12.0 \\
$\mathrm{BuOH}^{\mathrm{a}}$ & $0.341 \mathrm{M}$ & 1.43 & 28.6 \\
$1,2-\mathrm{DCE}$ & $0.348 \mathrm{M}$ & 14.3 & 2.9 \\
$\mathrm{DMF}$ & $0.339 \mathrm{M}$ & 40.9 & 1.0 \\
$\mathrm{THF}$ & $0.337 \mathrm{M}$ & 34.8 & 1.2 \\
Acetone & $0.405 \mathrm{M}$ & 18.2 & 2.2 \\
EtOAC & $0.339 \mathrm{M}$ & 22.7 & 1.8 \\
\hline
\end{tabular}

${ }^{\mathrm{a}} 3: 1 \mathrm{v} / \mathrm{v} \mathrm{CH}_{3} \mathrm{Cl}: n$-BuOH ${ }^{\mathrm{b}}$ assuming pseudo-first order conditions

Most of the other dienophiles tested with naphthalene complex 1 were found to be unreactive at $20-50^{\circ} \mathrm{C}$. For example, 2-chloroacrylonitrile, dimethyl maleate, and dimethylacetylene dicarboxylate, all gave no reaction with 1 at $50{ }^{\circ} \mathrm{C}$. Maleic anhydride and 1 yielded a material with similar ${ }^{31} \mathrm{P}$ NMR data to $\mathbf{9}$, but this reaction was plagued by significant decomposition (as determined by the presence of multiple weak signals in the $\left.{ }^{31} \mathrm{P} N M R\right)$. Tetracyanoethylene on the other hand, reacted with 1 to form only paramagnetic materials.

Our attention next turned to the substituted naphthalene complexes $\mathbf{2 a - 8}$ (Figure 3). In addition to the expected inductive effects, we believed that certain isomers of these complexes would experience steric crowding between the arene substituents and the Tp or $\mathrm{PMe}_{3}$ ligands that could destabilize the 1,2- or 3,4- $\eta^{2}$ isomers relative to the purported $2,3-\eta^{2}$ form. 

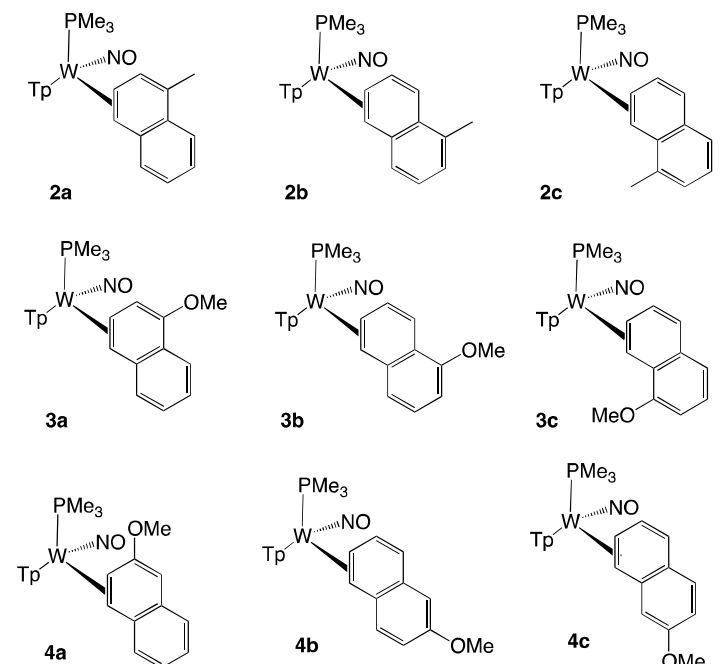

$3 \mathrm{~b}$
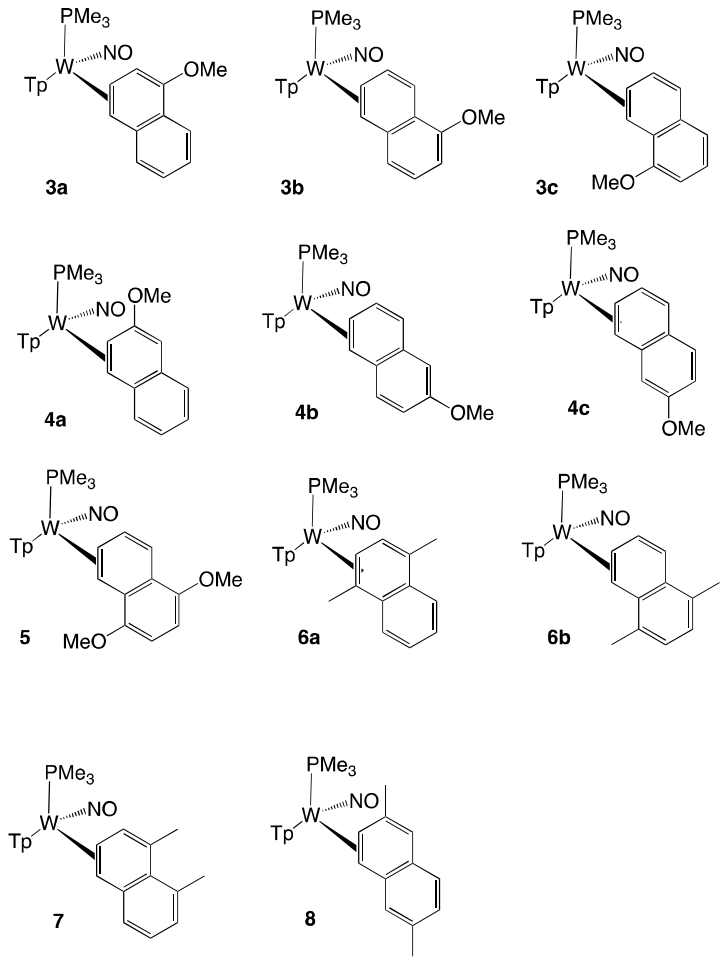

Figure 3. Dihapto-coordinated naphthalene complexes 2a-8.

The 1-methyl-, 1-methoxy-, and 2-methoxynaphthalene compounds were each isolated as a mixture of three isomers $(2: d r=2.0: 1.2: 1) ; \quad 3: d r=3.5: 2.5: 1 ; 4: d r=$ 4.8:2.5:1). Unfortunately, the NMR spectra for $\mathbf{2}$ - $\mathbf{4}$ were too complex to allow the full assignment of all NMR resonances. However, the disubstituted complexes $\mathbf{5}-\mathbf{8}$ were more selective. After allowing for equilibration, 5, 6, and 7 could be isolated as single isomers, while 8 contained only $7 \%$ of a minor isomer. For example, a solution of the 1,4-dimethylnaphthalene complex $(6 \mathbf{a}, \mathbf{6 b})$, evolved over a period of one day from a 3:2 ratio of isomers into $\mathbf{6} \mathbf{b}$ exclusively. The dominant isomers of the disubstituted 
naphthalene complexes $(\mathbf{5}, \mathbf{6 b}, \mathbf{7}$, and $\mathbf{8})$ were each fully characterized using 2D NMR techniques. $^{13}$

Compared to the parent naphthalene complex $\mathbf{1}$, naphthalene complexes 5-7 were all faster to undergo cycloaddition with $N$-methylmaleimide (Table 2). Cycloadducts 9 - 13 (figure 4) were each fully characterized using 2D NMR techniques and an X-ray diffraction molecular structure determination was undertaken on $\mathbf{1 1 b}$ (Figure 5) in order to verify its stereochemistry. In contrast, we found no reaction for the 2,6-dimethylnaphthalene analog 8 with $N$-methylmaleimide or the 2methoxynaphthalene isomer, complexes in which the metal was bound to a 2substituted ring (vide infra). ${ }^{14}$ In cases where mixtures of isomers prevented their complete characterization, COSY and NOESY data still allowed for differentiation of the isomer ("a" designations in Figure 3) in which the metal was bound to the substituted ring from the others $(\mathbf{b}, \mathbf{c})$. When the 1-methoxynaphthalene complex mixture $\mathbf{3}$ was treated with $\mathrm{N}$-methylmaleimide (NMM), one of the isomers (3a) reacted more rapidly than the other two, and all three were significantly more reactive than the parent naphthalene 1 . In contrast, when the same procedure was repeated with the 2methoxy analog, the isomer in which the metal was in the substituted ring 4 a showed no reaction over the course of the experiment. 
Table 2. Rate of Cycloadduct Formation $\left(25^{\circ} \mathrm{C}\right)$ for Naphthalene Complexes of $\left\{\mathrm{TpW}(\mathrm{NO})\left(\mathrm{PMe}_{3}\right)\right\}$

\begin{tabular}{|ccc|}
\hline Complex & $\mathbf{k}\left(\mathbf{M}^{-\mathbf{1}} \mathbf{s}^{-\mathbf{1}}\right)$ & Relative rate \\
\hline $\mathbf{1}$ & $2.00 \times 10^{-6}$ & 1.00 \\
$\mathbf{3 a}$ & $6.19 \times 10^{-5}$ & 30.9 \\
$\mathbf{3 b}+\mathbf{3 c}$ & $\sim 2 \times 10^{-5}$ & $\sim 10$ \\
$\mathbf{4 b}+\mathbf{4 c}$ & $\sim 4 \times 10^{-6}$ & $\sim 2$ \\
$\mathbf{5}$ & $4.26 \times 10^{-5}$ & 21.3 \\
$\mathbf{6 a}$ & $2.19 \times 10^{-4}$ & 110 \\
$\mathbf{6 b}$ & $1.94 \times 10^{-5}$ & 9.74 \\
$\mathbf{7 b}$ & $3.16 \times 10^{-5}$ & 15.8 \\
\hline
\end{tabular}

$n$-Butanol comprised $23+/-1 \%(w / w)$ of reaction solvent. $T=25^{\circ} \mathrm{C}$. The $[N M M]$ ranged from 0.29 to $0.43 \mathrm{M}$.

Finally, in an effort to identify the minor isomer of the expected cycloadduct 11, we combined 6 (2:1 mixture of $6 \mathbf{b}: 6 a)$ with approximately 20 equivalents of $N$ methylmaleimide $(0.86 \mathrm{M})$ in a $3: 1$ mixture of $\mathrm{CHCl} 3: n$-butanol at $25^{\circ} \mathrm{C}$, and stopped the reaction after six hours, once the minor isomer had been completely consumed. The resulting reaction mixture was examined by $N M R$, and was found to contain three major tungsten species: the 1,4-dimethylnaphthalene complex $\mathbf{6 b}$, the cycloadduct (11b) resulting from $\mathbf{6 b}$, and the purported cycloadduct (11a) generated from 6a. After subtracting definitive resonances for $\mathbf{6 b}$ and $\mathbf{1 1 b}$, several signals were unambiguously associated with 11a. These included a doublet for $\mathrm{PMe}_{3}$ at $1.07(9 \mathrm{H})$ which displays an NOE interaction with a singlet at $2.13(3 \mathrm{H}, \mathrm{Me})$ and a multiplet at $2.95\left(\mathrm{H} 2,1 \mathrm{H},{ }^{31} \mathrm{P}\right.$ coupled). The signal at 2.95 also has an NOE interaction with a signal at 1.49 thought to be $\mathrm{H} 3$. In addition a singlet at $1.74(3 \mathrm{H})$ shows a weak NOE interaction with the purported signal for H3. Both methyl groups appear to have a correlation with signals 
overlapping with the free maleimide signal, which we believe are likely $\mathrm{H}^{\prime}$ and $\mathrm{H}^{\prime}$ '.

Together, these data support the assignment of 11a as shown in Figure 4:

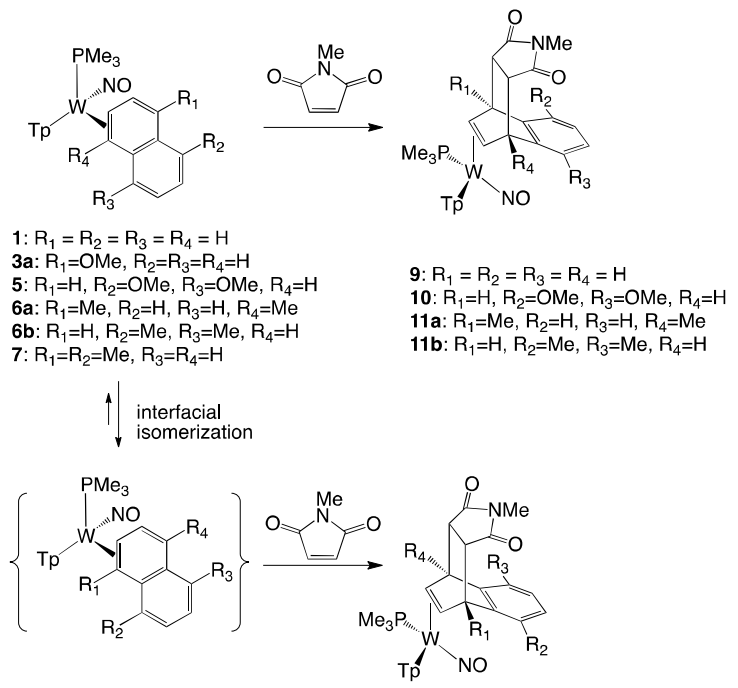

12: $\mathrm{R}_{1}=\mathrm{OMe}, \mathrm{R}_{2}=\mathrm{R}_{3}=\mathrm{R}_{4}=\mathrm{H}$

13: $R_{1}=R_{2}=M e, R_{3}=R_{4}=H$

Figure 4 Cycloaddition of substituted naphthalene complexes (only major isomers shown).

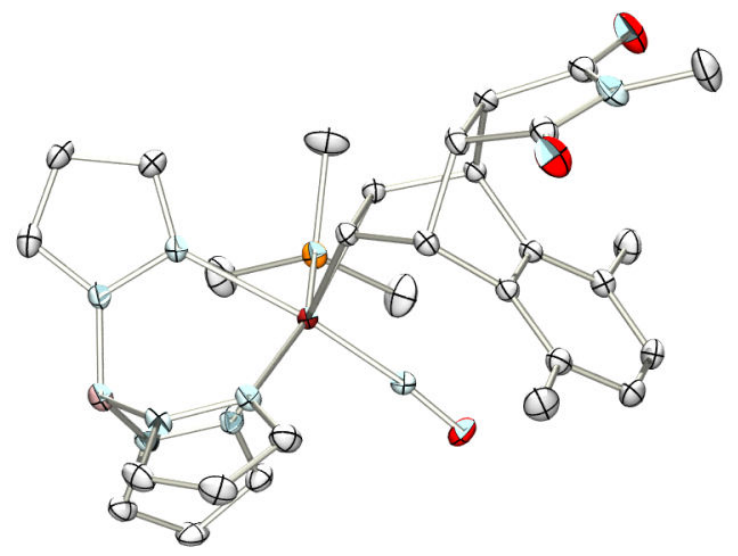

Figure 5. ORTEP drawing of $\mathbf{1 1 b}$ (Half equiv of $\mathrm{CH}_{2} \mathrm{Cl}_{2}$ omitted). 
In most cases, the dominant stereoisomer of the naphthalene complex was directly correlated to that of the product cycloadduct, where cycloaddition occurred to the arene face opposite to metal coordination. However, in two cases (cycloadducts $\mathbf{1 2}$ and 13) NMR analysis revealed that the opposite was true. In these cases, an interfacial isomerization of the naphthalene complex (3a or 7 ) appears to precede the cycloaddition event (Figure 4).

In order to demonstrate the potential utility of this chemistry as a method for the preparation of dihydroethanonaphthalenes under neutral conditions, we explored various reaction conditions for the oxidative decomplexation of these ligands. Ultimately, we found an acceptable procedure where an acetone solution of a cycloadduct complex was combined with an aqueous solution of ceric ammonium nitrate (CAN; one eq). After stirring for 1-2 h, extraction yielded the desired organic product in practically pure form ( $5 \%$ impurities). Thus, cycloadducts $\mathbf{1 4}$ and $\mathbf{1 5}$ were generated from $\mathbf{1 0}$ and $\mathbf{1 1 b}$ respectively (Figure 6). A preparative TLC plate was run for 15, which was isolated in pure form with a $63 \%$ yield. NOESY experiments of 14 and 15 revealed an NOE interaction between the alkene and maleimide protons, confirming retention of endo stereochemistry. 


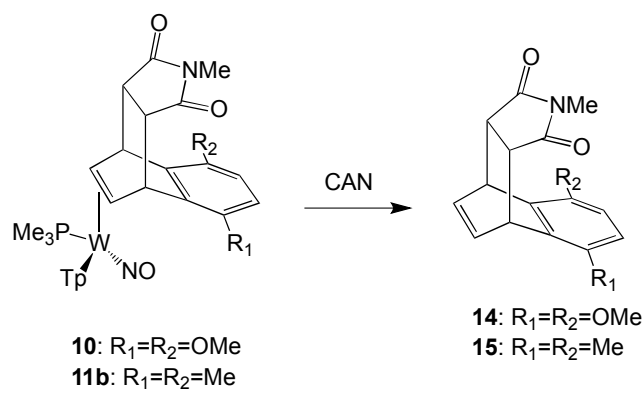

Figure 6. Decomplexation of cycloadducts.

Substituted naphthalene complexes of the form $\operatorname{TpW}(\mathrm{NO})\left(\mathrm{PMe}_{3}\right)\left(\eta^{2}-\right.$ naphthalene) exist as mixtures of structural- and stereoisomers. It will be useful in the discussion that follows to define the different possible forms of isomerization. Herein, intra-ring isomerization refers to the movement of the metal center from one bonding position in a particular ring to another position in the same ring, and can occur via interfacial or intrafacial isomerization mechanisms (Figure 7). For example, this kind of isomerization allows conversion between $\mathbf{3 b}$ and $\mathbf{3 c}$, and $\mathbf{4 b}$ and $\mathbf{4 c}$. Inter-ring isomerization refers to an isomerization in which the metal center migrates from a $\pi$ bond in one ring to one in the other ring. The latter type of isomerization converts $3 a$ to $3 b$ or $3 c, 4 a$ to $4 b$ or $4 c$, and $6 a$ to $6 b$.

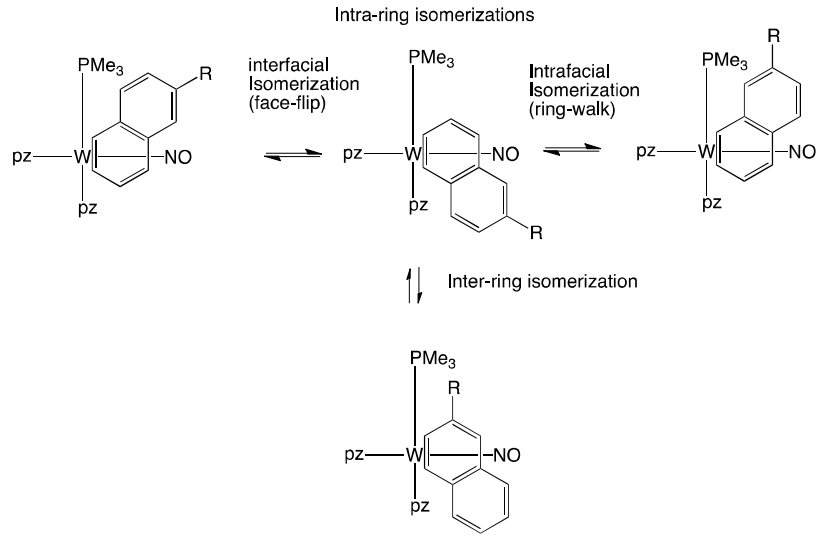

Figure 7. Isomerization mechanisms for $\eta^{2}$-naphthalene complexes. 
Based on changes in the integrated ${ }^{31}$ P NMR signals for the different isomers of the 1-methylnaphthalene complex 2 over time ${ }^{15}$ the intra-ring isomerization specific rate for $\mathbf{2} \mathbf{b} \rightarrow \mathbf{2} \mathbf{c}$ can be determined to be $k_{1}=1.4 \times 10^{-5} \mathrm{~s}^{-1}$ with a specific back-rate of $k_{-1}$ $=2.0 \times 10^{-5} \mathrm{~s}^{-1}\left(\mathrm{~K}_{\mathrm{eq}}=0.70 ; 20^{\circ} \mathrm{C}\right)$ These rates are significantly slower than the rhenium analog (vide supra). ${ }^{16}$ For the analogous complex $\operatorname{TpRe}(\mathrm{CO})\left({ }^{\mathrm{t} B u N C}\right)\left(\eta^{2}\right.$-naphthalene), where the minor isomer is observable, the intrafacial isomerization specific rate is $2.8 \mathrm{~s}^{-1}$ $\left(0^{\circ} \mathrm{C}\right) \cdot{ }^{16}$ Meanwhile, the inter-ring specific rates were found to be for $\mathbf{2 a} \rightarrow(\mathbf{2 b}+\mathbf{2 c}): k_{2}$ $=3.8 \times 10^{-8} \mathrm{~s}^{-1}$, and for $(\mathbf{2 b}+\mathbf{2 c}) \rightarrow \mathbf{2 a}, \mathrm{K}_{-2}=2.1 \times 10^{-8} \mathrm{~s}^{-1}$, where $\mathrm{K}_{2}=([\mathbf{2 b}]+[\mathbf{2 c}]) /[\mathbf{2 a}]=$ 1.8 at $25^{\circ} \mathrm{C}$. For comparison, the inter-ring isomerization rate for $\left[\mathrm{Os}\left(\mathrm{NH}_{3}\right)_{5}(1-\right.$ methylnaphthalene) $]^{2+}$ at $26^{\circ} \mathrm{C}$ is $2.8 \times 10^{-6} \mathrm{~s}^{-1} \cdot{ }^{17}$
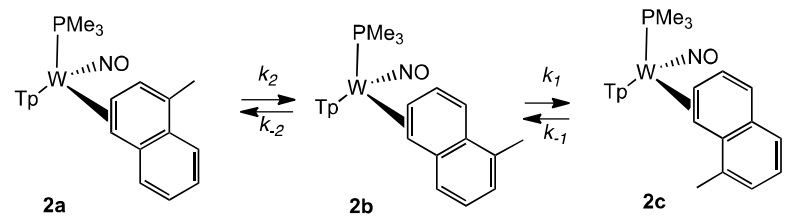

2c

Eqn 4

Two mechanistic pathways were considered for the observed cycloaddition products. For the purported mechanism envisioned at the onset of this study, the naphthalene would first isomerize to its $2,3-\eta^{2}$ intermediate, followed by the concerted cycloaddition of the maleimide (Figure 8). Although the 2,3 isomer is calculated to be $15.2 \mathrm{kcal} / \mathrm{mol}$ higher in energy, with an estimated isomerization barrier of $19 \mathrm{kcal} / \mathrm{mol}$ (DFT calculations; Figure 8), the $2,3-\eta^{2}$ intermediate requires unbound portions of the naphthalene to adopt an o-quinodimethane structure (Figure 8), which is expected to be highly reactive toward cycloaddition. The mild acceleration with alcohols could be 
understood in the context of a concerted mechanism by considering the effect that hydrogen bonding would have in lowering the energy of the dienophile LUMO.
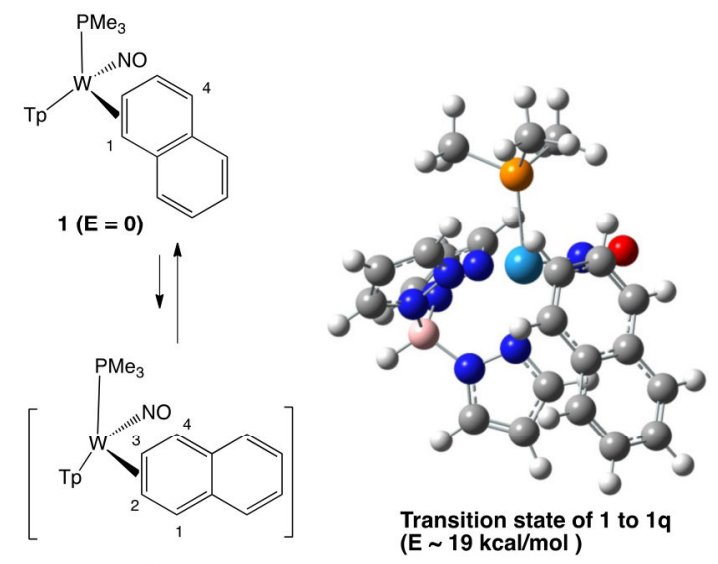

19

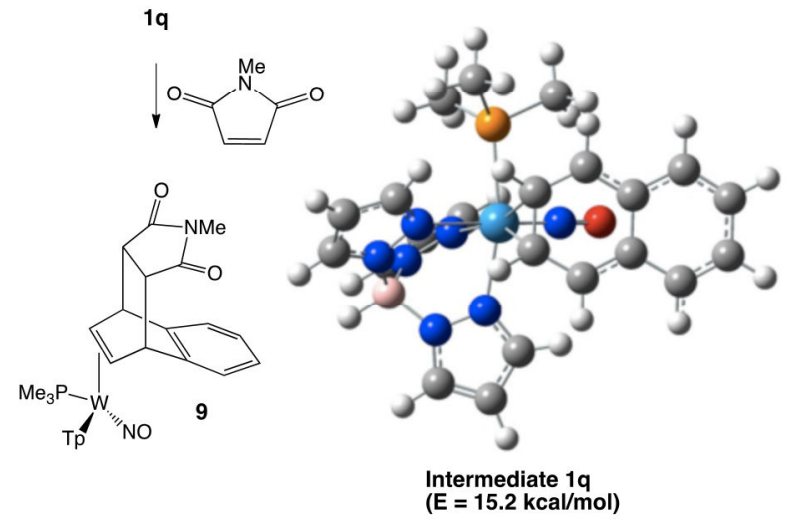

Figure 8. Proposed mechanism for cycloaddition for $\eta^{2}$-naphthalene complexes and transition state and $2,3-\eta^{2}$ intermediate (calculated).

While we were unable to run the reaction at high enough concentrations of NMM to observe a zero-order rate dependence of the maleimide, naphthalene substituent effects supported the notion of a pre-equilibrium step in the mechanism of cyclization reaction. If the reaction mechanism involves such an isomerization step, we expected that substituents at the 1 position (e.g., as in 6a) on the naphthalene ring would be expected to destabilize the $1,2-\eta^{2}$ isomer, enhance the concentration of the intermediate, (Type A in Figure 9) and consequently increase the overall rate of the 
cycloaddition reaction. As a result, the $1,2-\eta^{2} \rightarrow 2,3-\eta^{2}$ isomerization energy would be expected to decrease as a result of destabilization of the former. Alternatively, when adoption of a C2-C3 binding pattern would create steric interaction between the metal and ring substituent such as with $4 a$ or 8 a (Type $C$ in Figure 9), we expect that the 1,2- $\eta^{2}$ $\rightarrow 2,3-\eta^{2}$ isomerization would be increasingly disfavored, and thus lower concentrations of the intermediate and slower overall reaction rates would be expected.

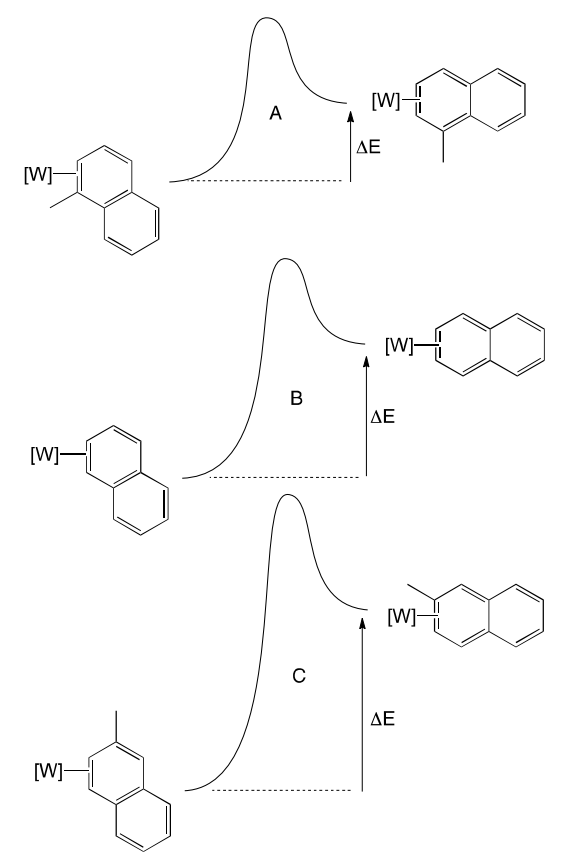

Figure 9. Substituent effects for the isomerization to the purported 0 quinodimethane intermediate. $[\mathrm{W}]=\left\{\mathrm{TpW}(\mathrm{NO})\left(\mathrm{PMe}_{3}\right)\right\}$.

Scenario A in Figure 9 could not be verified with our chosen set of naphthalene complexes, but consider the contrast in reactivities observed for the methoxynaphthalene complexes $\mathbf{3} \mathbf{a}$ and $\mathbf{4 a}$. In the case of $\mathbf{3 a}$, the rate of cycloaddition is approximately 31 times faster than the rate for the parent complex, $\mathbf{1}$. For $\mathbf{3 b}$ and $\mathbf{3 c}$, 
the methoxy group still makes the naphthalene fragment more electron-rich overall, and the rate is approximately ten times faster than 1 . In contrast, for the 2-methoxy analog 4, isomers in which the methoxy group was in the unbound ring (4b and $4 \mathbf{c}$ ) experienced modest rate acceleration for the cycloaddition, about twice that for 1 . However, in 4 a cycloadduct formation is fully inhibited. Likewise, no cycloaddition is observed from 2,6-dimethylnaphthalene complex, 8.

Further support for a quinodimethide intermediate is seen in the observation of exclusive endo stereochemistry in the cycloadducts. If the cycloaddition occurred through a Michael addition reaction, such a high degree of stereoselectivity in the final cycloadduct would not be expected. A surprising observation in this study was that in the generation of cycloadducts 12 and 13, the methyl or methoxy substituent is oriented distal to the $\mathrm{PMe}_{3}$, whereas in their precursors $3 \mathrm{a}$ and 7, these groups were proximal to the phosphine ligand. In other words, in order for the observed cycloadducts to be generated, $\mathbf{3 a}$ and $\mathbf{7}$ would need to pass through an intermediate in which the ring-face was coordinated opposite to the observed coordination in $3 a$ or 7 . Calculations ${ }^{18}$ for the two purported $2,3-\eta^{2}$ coordination diastereomers $\mathbf{3 d}$ and $\mathbf{3 e}$ indicate that the isomer with the methoxy group distal to the $\mathrm{PMe}_{3}(3 e)$ is $0.5 \mathrm{kcal} / \mathrm{mol}$ more stable than with the methoxy group proximal (3d) (figure 10). Assuming that the rate of intra- and interfacial isomerizations are similar, as they are in the case of the earlier reported rhenium systems, ${ }^{16}$ it is reasonable to postulate that the interfacial isomerization (i.e., face-flip) could occur prior to cycloaddition and that the $2,3-\eta^{2}$ isomer may be in equilibrium with the other isomers (Figure 10). Furthermore, an investigation of Diels- 
Alder reactions between maleimides and 2,3- $\eta^{2}$-anisole complexes of $\left\{\mathrm{TpW}(\mathrm{NO})\left(\mathrm{PMe}_{3}\right)\right\}$ revealed a reactivity preference for the stereoisomer in which the OMe group is distal to the $\mathrm{PMe}_{3}{ }^{19}$ By similar reasoning, intermediate $\mathbf{3 e}$ may be not only present in higher concentration, but may be more reactive as well. Similar factors are likely responsible for the observed stereochemistry of the cycloaddition of 1,8-dimethylnaphthalene complex, $\mathbf{7}$, which may pass through $\mathbf{7 d}$ and $\mathbf{7 e}$ prior to reacting.
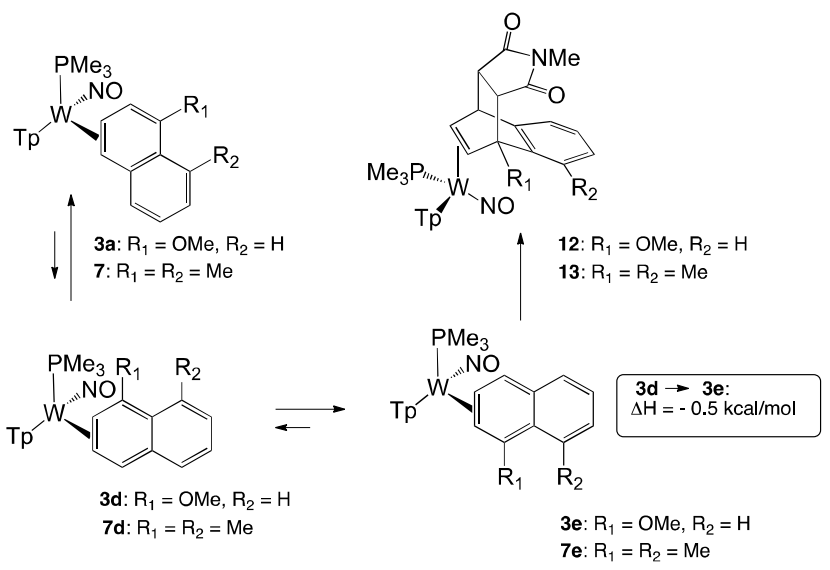

Figure 10. Interfacial and intrafacial isomerizations required to form $\mathbf{1 2}$ and $\mathbf{1 3}$ with observed stereochemistry.

Additional insight into the cycloaddition of $\eta^{2}$-naphthalene complexes and the role of the purported quinodimethane intermediate can be gained by comparing the reaction rate of naphthalene complex 1 with that of its benzene and anthracene (16) analogs (Table 3). For the organic arenes, anthracene is most reactive, followed by naphthalene, with benzene showing virtually no reactivity toward cycloaddition with maleimides at ambient pressures. In contrast, with $\mathrm{TpW}(\mathrm{NO})\left(\mathrm{PMe}_{3}\right)\left(\eta^{2}\right.$-arene $)$ complexes this order is decidedly reversed (Table 3). For the benzene complex cycloaddition readily occurs at ambient temperature and pressure, ${ }^{20}$ as isomerization is not necessary to reveal a reactive diene fragment. Alternatively, the quinodimethide- 
like isomer for anthracene $\left(2,3-\eta^{2}\right.$ isomer; eq 6) is less accessible $\left(\Delta H_{\text {calc }}=22.2\right.$ $\mathrm{kcal} / \mathrm{mol})^{18}$ than the naphthalene analog $(c f .15 .2 \mathrm{kcal} / \mathrm{mol})$, as a result of disruption of aromatic character in more rings, and hence the equilibrium concentration is lower and overall reaction slower to form cycloadduct (17) than with naphthalene. Monitoring a reaction of $\mathbf{1 6}$ under conditions similar to those used in the preparation of naphthalene cycloadduct 9 shows only slight decomposition after a period of weeks, and this establishes an upper limit for the specific rate of the reaction (Table 3).

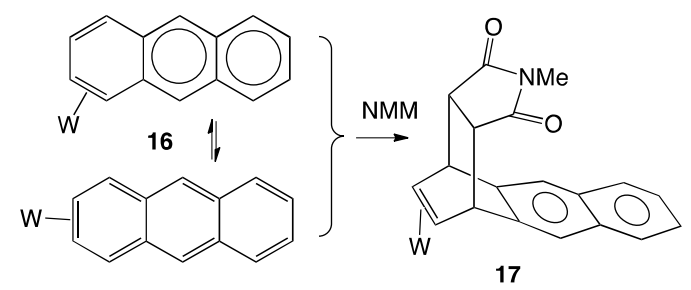

Figure 11. Attempted cycloaddition of an $\eta^{2}$-anthracene complex at the A ring.

Table 3.

\begin{tabular}{|cccc|}
\hline $\begin{array}{c}\text { Arene complex of } \\
\left\{\mathrm{TpW}(\mathrm{NO})\left(\mathrm{PMe}_{3}\right)\right\}\end{array}$ & $\mathbf{k}\left(\mathrm{M}^{-1} \mathbf{s}^{-1}\right)$ & Relative rate & $\begin{array}{c}\text { Isomerization } \\
\text { energy (DFT) } \\
\text { (kcal/mol) }\end{array}$ \\
\hline Benzene & $6.1 * 10^{-3}$ & $3.1 \times 10^{3}$ & 0 \\
Naphthalene & $2.00 * 10^{-6}$ & 1 & 15.2 \\
Anthracene & $<6.14 * 10^{-7}$ & $<0.3$ & 22 \\
\hline
\end{tabular}

In an alternate mechanism, these cyclization reactions could proceed via a stepwise process in which the maleimide acts as a Michael acceptor to generate a zwitterionic intermediate. The resulting enolate could then attack the positive terminus of the allyl to form the tricyclic product (Figure 12). A modification of this mechanism was invoked to explain the formation of a cycloaddition product prepared from $\operatorname{TpRe}(\mathrm{CO})(\mathrm{Melm})\left(\eta^{2}\right.$-naphthalene) and methyl acrylate in the presence of 1 equiv of 
TBSOTf. $^{8}$ In that case, however, ring closure did not occur until the metal was oxidatively removed.

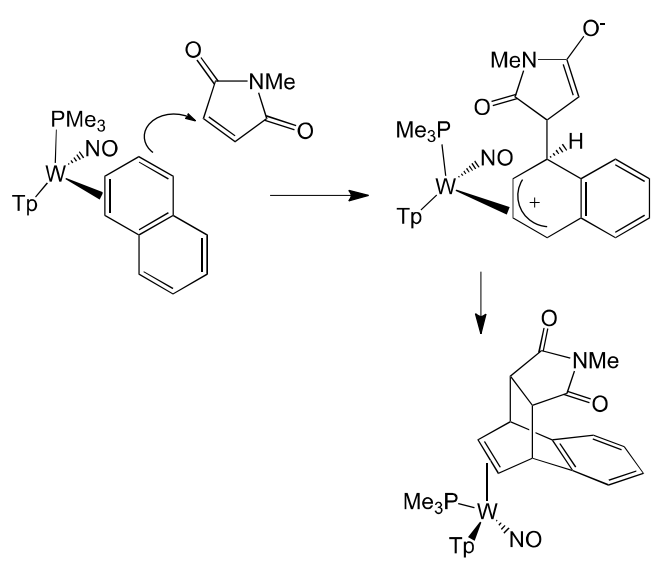

Figure 12. Alternate mechanism (disfavored) involving ionic intermediates.

If the reaction were to occur stepwise with zwitterionic intermediates, one would expect it to be significantly faster in polar solvents, which would help to stabilize the intermediate and associated transition state. However, such was not the case. Using $n$-butanol as a cosolvent only modestly accelerates the reaction, and running the reaction in DMF or THF actually retards the reaction compared to what was observed in chlorinated solvents. Further, the addition of 2,6-lutidine has only a marginal influence on reaction rate. We note that this observation rules out the participation of an unexpected acid impurity. Finally, recall that the 2-methoxynaphthalene isomer $\mathbf{4 a}$ was completely unreactive with $\mathrm{N}$-methylmaleimide, in contrast to its isomers $\mathbf{4 b}$ or $\mathbf{4 c}$. Were a Michael reaction mechanism in play, this isomer should have been the most reactive owing to the $\pi$ donating effect of the methoxy group. An alternative explanation, however, is that the purported cycloadduct derived from $\mathbf{4 a}$ is thermodynamically unstable due to a steric interaction of the methoxy group. 
This paper has focused on the exploitation of a purported highly reactive, accessible quinodimethane-like intermediate from $\mathrm{TpW}(\mathrm{NO})\left(\mathrm{PMe}_{3}\right)\left(\eta^{2}\right.$-naphthalene) to perform stereoselective cycloaddition reactions under mild temperatures and neutral reaction conditions. The complexes can then be demetallated to release the intact cycloadduct. A search for published examples of naphthalene-derived cycloadducts provides few results. Diels-Alder additions of naphthalene with maleimides typically need high $\left(100^{\circ} \mathrm{C}\right)$ temperatures and substantial pressures $(10-12 \mathrm{kbar})$ to proceed, and they suffer from problems of thermal reversibility. ${ }^{21}$ Polymethylnaphthalenes were found to react with maleic anhydride at $110^{\circ} \mathrm{C}$, sometimes giving a mixture of endo and exo products often with poor regiochemistry. ${ }^{22}$

However, one report found that naphthalene would react with $N$ phenylmaleimide at room temperature when gallium(III) chloride was present, generating the endo addition product. $^{23}$ We were curious about the range of applicability of this method for generating Diels-Alder adducts and sought to repeat the synthesis on 1,4-dimethylnaphthalene. Following the described procedure, we generated an adduct consistent with the organic cycloadduct 15, in which the maleimide attached in the ring without the methyl groups. Attempts to repeat the procedure using $\mathrm{N}$-methylmaleimide to generate 15 resulted in a mixture of products.

\section{Conclusion}

In this report we detail a Diels-Alder cycloaddition of $\mathrm{N}$-methylmaleimide to various naphthalenes assisted by the $\pi$-basic complex $\left\{\mathrm{TpW}(\mathrm{NO})\left(\mathrm{PMe}_{3}\right)\right\}$ under mild and 
chemically neutral conditions. Various experimental data support a mechanism in which the metal isomerizes to the $2,3-\eta^{2}$ isomer prior to cyclization, an intermediate that chemically resembles a quinodimethane. Acceleration of this reaction is observed when the naphthalene has electron donor substituents at the alpha carbons, but the reaction is inhibited by a substituent at a beta carbon of the bound ring. Both of these observations are consistent with the modulation of the isomerization energy and hence equilibrium between the $1,2-\eta^{2}$ and $2,3-\eta^{2}$ isomers controlling the reaction rate of cycloaddition.

Chart 1

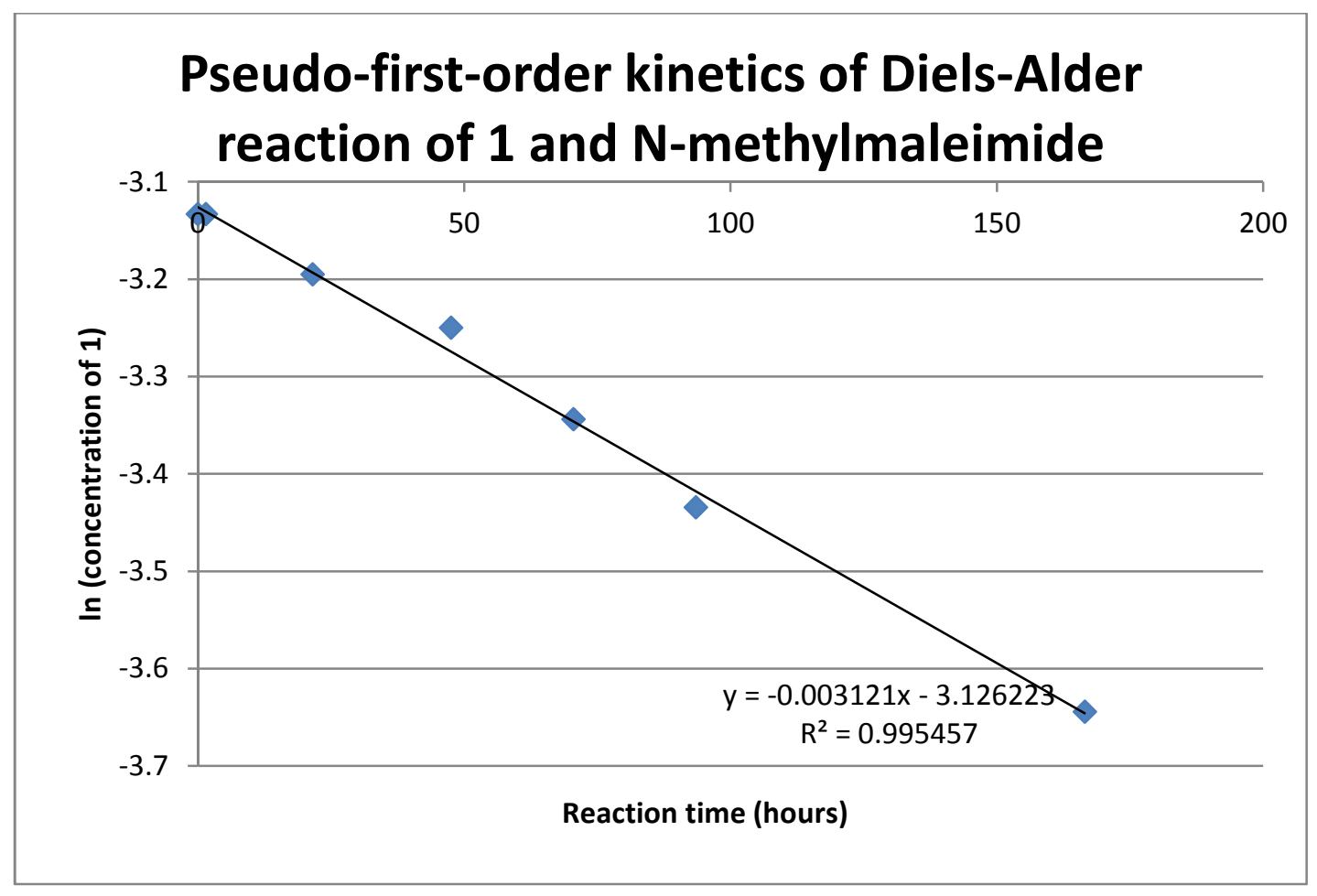

\section{Experimental Section}


General Methods. NMR spectra were obtained on a 300 (Varian INOVA), or 500, 600, or $800 \mathrm{MHz}$ spectrometer (Bruker Avance). All chemical shifts are reported in ppm and proton and carbon shifts are referenced to tetramethylsilane (TMS) utilizing residual ${ }^{1} \mathrm{H}$ or ${ }^{13} \mathrm{C}$ signals of the deuterated solvents as an internal standard. Phosphorus NMR signals are referenced to $85 \% \mathrm{H}_{3} \mathrm{PO}_{4}(\delta=0.00)$ using a triphenylphosphate external standard $(\delta=-16.58)$. Coupling constants $(J)$ are reported in hertz $(\mathrm{Hz})$. Infrared spectra (IR) were recorded on a MIDAC Prospect Series (Model PRS) spectrometer or a Nicolet Avatar 330 FT-IR spectrometer as a glaze on a Horizontal Attenuated Total Reflectance (HATR) accessory (Pike Industries). Electrochemical experiments were performed under a dinitrogen atmosphere using a BAS Epsilon EC-2000 potentiostat. Cyclic voltammetry data was taken at ambient temperature at $100 \mathrm{mV} / \mathrm{s}\left(25^{\circ} \mathrm{C}\right)$ in a standard threeelectrode cell with a glassy carbon working electrode, $N, N$-dimethylacetamide (DMA) or acetonitrile (MeCN) solvent (unless otherwise specified), and tetrabutylammonium hexaflurophosphate (TBAH) electrolyte (approx. $0.5 \mathrm{M})$. All potentials are reported versus NHE (Normal Hydrogen Electrode) using cobaltocenium hexafluorophosphate $\left(E_{1 / 2}=-0.78 \mathrm{~V}\right)$, ferrocene $\left(E_{1 / 2}=+0.55 \mathrm{~V}\right)$, or decamethylferrocene $\left(E_{1 / 2}=+0.04 \mathrm{~V}\right)$ as an internal standard. The peak-to-peak separation was less than $100 \mathrm{mV}$ for all reversible couples. Elemental analyses (EA) were obtained from Atlantic Microlabs. High resolution electrospray ionization mass spectrometry (ESI-MS) analyses were obtained from the University of Illinois at Urbana-Champaign Mass Spectrometry Laboratory or the University of Richmond on a Bruker BioTOF-Q running in ESI mode, from samples dissolved in water/acetonitrile solution containing $0.1 \mathrm{M}$ trifluoroacetic acid then mixed 
with $0.1 \mathrm{M}$ aqueous sodium trifluoroacetate (NaTFA), using $[\mathrm{Na}(\mathrm{NaTFA}) \mathrm{x}]^{+}$clusters as an internal standard. Unless otherwise noted, all synthetic reactions were performed in a glovebox under a dry nitrogen atmosphere. $\mathrm{CHCl}_{3}$ was purified by passage through a column packed with activated alumina. Other solvents and liquid reagents were thoroughly purged with nitrogen prior to use. Deuterated solvents were used as received from Cambridge Isotopes. General Proton Assignments are in accordance with the Figure S1. Protons of Tp and other ligands were assigned using a combination of 2dimensional NMR experiments and phosphorus-proton coupling when unambiguous assignments were possible. When unambiguous assignments were not possible the $\mathrm{Pz}$ protons are labeled as Tp protons. Coordination diastereomers are described by the defining feature's proximity to the $\mathrm{PMe}_{3}$ ligand relative to the $\mathrm{W}-\mathrm{PMe}_{3}$ bond (e.g. the fewer number of bonds from the $\mathrm{PMe}_{3}$ passing through the upper portion of the coordinated ring system to the defining feature dictates the proximal $(P)$ ligand).

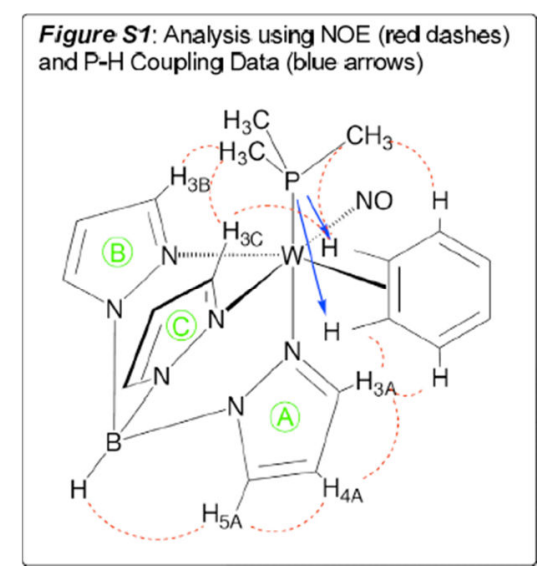

DFT Calculations. Initial structures were built in GAUSSVIEW (5.0.8) with the PM6 semiempirical method in GAUSSIAN 09. These structures were refined stepwise in Gaussian using B3LYP and a series of basis functions incorporating LANL2 pseudopotentials and 
associated basis functions provided in those packages or directly from the PM6 structures. The most demanding calculations reported here put the LANL2DZ pseudopotential and its basis only on the $W$ atom, and used the 6-31G(d) basis for all other atoms.

For transition state structures, vibrational analysis revealed the presence of a single imaginary frequency. In all other cases, vibrational analyses verified that optimized structures were located at local minima, with the presence of only real frequencies.

Synthesis and characterization for $\operatorname{TpW}(\mathrm{NO})\left(\mathrm{PMe}_{3}\right)\left(\eta^{2}\right.$-benzene) and $\mathrm{TpW}(\mathrm{NO})\left(\mathrm{PMe}_{3}\right)\left(\eta^{2}-\right.$ naphthalene) (1) have been previously reported. ${ }^{11}$

\section{$\operatorname{TpW}(\mathrm{NO})\left(\mathrm{PMe}_{3}\right)\left(3,4-\eta^{2}-1-\right.$ methylnaphthalene) (2)}

$\mathrm{TpW}(\mathrm{NO})\left(\mathrm{PMe}_{3}\right)\left(\mathrm{n}^{2}\right.$-benzene) (604 mg, $\left.1.04 \mathrm{mmol}\right)$, 1-methylnaphthalene (801 mg, 5.64 $\mathrm{mmol})$, and DME (2.00 g) were combined in a flame-dried vial and stirred for 20 hours. A yellow-brown precipitate was filtered with a $15 \mathrm{~mL}$ fine-porosity fritted funnel and washed with two portions of ether $(4 \mathrm{~mL}$ ). The solid was dried in vacuo to give 2 (277 $\mathrm{mg}, 40 \%)$.

${ }^{31} \mathrm{P} N M R\left(\mathrm{CDCl}_{3}\right): \delta-13.31\left(J_{W-\mathrm{P}}=300\right),-13.85\left(J_{W-\mathrm{P}}=295\right),-14.03\left(J_{W-P}=298\right) . \mathrm{CV}: \mathrm{E}_{\mathrm{p}, \mathrm{a}}=$ +0.060 V. IR: $v_{\mathrm{NO}}=1568 \mathrm{~cm}^{-1}$

$\operatorname{TpW}(\mathrm{NO})\left(\mathrm{PMe}_{3}\right)\left(3,4-\eta^{2}-1-\right.$ methoxynaphthalene) (3) 
$\mathrm{TpW}(\mathrm{NO})\left(\mathrm{PMe}_{3}\right)\left(\eta^{2}\right.$-benzene) $(511 \mathrm{mg}, 0.880 \mathrm{mmol})$ and 1-methoxynaphthalene (650 $\mathrm{mg}, 4.11 \mathrm{mmol})$ were weighed into a vial and dissolved in DME (1.67 g). The reaction was stirred overnight and a brown precipitate formed. The mixture was diluted with 5 $\mathrm{mL} \mathrm{Et} \mathrm{t}_{2} \mathrm{O}$ and filtered with a $15 \mathrm{~mL}$ fine-porosity fritted funnel. The solid was washed with two $4 \mathrm{~mL}$ portions of $\mathrm{Et}_{2} \mathrm{O}$ and dried in vacuo to give 3 as a yellow-brown solid (259 $\mathrm{mg}, 45 \%)$.

${ }^{1} \mathrm{H}$ NMR $\left(\mathrm{CDCl}_{3}\right): \delta 3 \mathrm{a}: 6.75(\mathrm{~d}, J=7.5,1 \mathrm{H}, \mathrm{H} 5), 6.12(\mathrm{~m}, \mathrm{H} 2), 3.98(\mathrm{~m}, \mathrm{H} 3), 3.88(\mathrm{~s}, 3 \mathrm{H}$, OMe), 2.44 (d, J=9.5, 1H, H4) 1.35 (d, J=8.0, 9H, PMe 3 ); 3b: $6.85(\mathrm{~m}, \mathrm{H} 2), 6.69$ (d, $J=$ 9.5, $1 \mathrm{H}, \mathrm{H} 1), 6.40(\mathrm{~d}, J=7.6,1 \mathrm{H}, \mathrm{H} 5), 3.87(\mathrm{~m}, \mathrm{H} 3), 3.86(\mathrm{~s}, 3 \mathrm{H}, \mathrm{OMe}), 2.53(\mathrm{~d}, J=9.5$, H4), 1.30 (d, J=8.0, 9H, PMe $)_{3}$; 3c: $6.86(\mathrm{~m}, \mathrm{H} 2), 6.60$ (d, J = 8.0, 1H, H6), 6.28 (d, J = 9.2, 1H, H1), $3.91(m, H 3), 3.36(s, 3 H, O M e), 3.00(m, H 4), 1.32\left(d, J=8.0,9 H, \mathrm{PMe}_{3}\right) .{ }^{31} \mathrm{P}$ $\operatorname{NMR}\left(\mathrm{CDCl}_{3}\right): \delta-12.41,-13.21,-13.67 . \mathrm{CV}(\mathrm{DMA}): \mathrm{E}_{\mathrm{p}, \mathrm{a}}=+0.019 \mathrm{~V} . \mathrm{IR}: \mathrm{v}_{\mathrm{NO}}=1566 \mathrm{~cm}^{-1}$ Anal. Calc'd for $\mathrm{C}_{23} \mathrm{H}_{29} \mathrm{BN}_{7} \mathrm{O}_{2} \mathrm{PW}$ : C, 41.78; $\mathrm{H}, 4.42 ; \mathrm{N}, 14.83$; Found: $\mathrm{C}, 40.89 ; \mathrm{H} 4.38 ; \mathrm{N}$, 15.09 .

\section{$\operatorname{TpW}(\mathrm{NO})\left(\mathrm{PMe}_{3}\right)\left(3,4-\eta^{2}-2-\right.$ methoxynaphthalene) (4)}

$\mathrm{TpW}(\mathrm{NO})\left(\mathrm{PMe}_{3}\right)\left(\eta^{2}\right.$-benzene) $(496 \mathrm{mg}, 0.854 \mathrm{mmol})$ and 2-methoxynaphthalene (1.040 $\mathrm{g}, 6.57 \mathrm{mmol})$ were weighed into a vial and dissolved in DME (2.138 g). The reaction was stirred for four days and a light yellow solid precipitated. Ether $(2 \mathrm{~mL})$ was added to the mixture to encourage precipitation. The mixture was filtered with a $15 \mathrm{~mL}$ fineporosity fritted funnel and washed $3 x$ with $1 \mathrm{~mL}$ portions of ether. The product was dried in vacuo to give 4 as a light yellow solid (153 mg, 27\%). 
${ }^{1} \mathrm{H} \mathrm{NMR}\left(\mathrm{CDCl}_{3}\right): \delta$ 4a: $7.23(\mathrm{~m}, 1 \mathrm{H}, \mathrm{H} 8), 6.92(\mathrm{~m}, 1 \mathrm{H}, \mathrm{H} 7), 6.91(\mathrm{~m}, 1 \mathrm{H}, \mathrm{H} 6), 6.57(\mathrm{~m}, 1 \mathrm{H}$, H5), $5.67(\mathrm{~s}, 1 \mathrm{H}, \mathrm{H} 1), 3.93(\mathrm{~m}, 1 \mathrm{H}, \mathrm{H} 3), 3.87(\mathrm{~s}, 3 \mathrm{H}, \mathrm{OMe}), 2.45$ (d, J = 10.0, 1H, H4), 1.27 (d, $\left.J=8.6,9 \mathrm{H}, \mathrm{PMe}_{3}\right)$; 4b: $6.85(\mathrm{~m}, 1 \mathrm{H}, \mathrm{H} 8), 6.73(\mathrm{dd}, J=9.4,4.9,1 \mathrm{H}, \mathrm{H} 2), 6.66(\mathrm{dd}, J=$ 8.4, 2.6, $1 \mathrm{H}, \mathrm{H} 6), 6.63(\mathrm{~d}, J=8.3,1 \mathrm{H}, \mathrm{H} 5), 6.26(\mathrm{~m}, 1 \mathrm{H}, \mathrm{H} 1), 3.85(\mathrm{~m}, 1 \mathrm{H}, \mathrm{H} 3), 3.84(\mathrm{~s}, 3 \mathrm{H}$, OMe), $2.50(\mathrm{~d}, J=8.3,1 \mathrm{H}, \mathrm{H} 4), 1.31\left(\mathrm{~d}, J=8.0,9 \mathrm{H}, \mathrm{PMe}_{3}\right) ; 4 \mathrm{c}: 7.18(\mathrm{~m}, 1 \mathrm{H}, \mathrm{H} 8), 6.73(\mathrm{~m}$, $1 H, H 2), 6.59(m, 1 H, H 7), 6.29(m, 1 H, H 5), 6.26(m, 1 H, H 1), 3.85(m, 1 H, H 3), 3.73(s$, $3 \mathrm{H}, \mathrm{OMe}), 2.48(\mathrm{~d}, J=9.5,1 \mathrm{H}, \mathrm{H} 4), 1.31\left(\mathrm{~d}, J=8.0,9 \mathrm{H}, \mathrm{PMe}_{3}\right) .{ }^{31} \mathrm{P} \mathrm{NMR}\left(\mathrm{CDCl}_{3}\right): \delta-12.36$ $\left(J_{W-P}=290 \mathrm{~Hz}\right),-13.56\left(J_{W-P}=299 \mathrm{~Hz}\right),-13.90\left(J_{W-P}=295 \mathrm{~Hz}\right) . C V(D M A): E_{p, a}=+0.117 \mathrm{~V}$ IR: $v_{\text {NO }}=1566 \mathrm{~cm}^{-1}$. HRMS obs'd (\%), calc'd (\%), ppm, $(M+H)^{+}: 660.1773(67.2), 660.1778$ (83.9), 0.8; 661.1782 (73.9), 661.1804 (80.4), 3.3; 662.1816 (88.3), 662.1803 (100), 2.1; 663.1813 (52), 663.1844 (43.8), 4.6; 664.1833 (100), 664.1835 (83.6), 0.2. Anal. Calc'd for $\mathrm{C}_{23} \mathrm{H}_{29} \mathrm{BN}_{7} \mathrm{O}_{2} \mathrm{PW}: \mathrm{C}, 41.78 ; \mathrm{H}, 4.42 ; \mathrm{N}, 14.83$; Found: $\mathrm{C}, 41.84 ; \mathrm{H} 4.32 ; \mathrm{N}, 14.57$.

\section{$\mathrm{TpW}(\mathrm{NO})\left(\mathrm{PMe}_{3}\right)\left(3,4-\eta^{2}-5,8-\right.$ dimethoxynaphthalene) (5)}

$\mathrm{TpW}(\mathrm{NO})\left(\mathrm{PMe}_{3}\right)\left(\eta^{2}\right.$-benzene) (300 mg, $\left.0.516 \mathrm{mmol}\right)$ and 1,4-dimethoxynaphthalene (218 $\mathrm{mg}, 1.16 \mathrm{mmol}$ ) were weighed into a vial and dissolved in DME (635 mg). The reaction mixture was stirred overnight as a light brown solid precipitated. The mixture was filtered with a $15 \mathrm{~mL}$ fine-porosity fritted funnel and dried in vacuo to give $\mathbf{5}$ as a light brown solid (202 mg, 57\%).

${ }^{1} \mathrm{H} \mathrm{NMR}\left(\mathrm{CDCl}_{3}\right): \delta 7.82(\mathrm{~d}, J=2.0,1 \mathrm{H}, \mathrm{pz3B}), 7.78(\mathrm{~d}, J=2.0,1 \mathrm{H}, \mathrm{pz} 5 \mathrm{C}), 7.69(\mathrm{~d}, J=2.0$ $1 \mathrm{H}, \mathrm{pz} 5 \mathrm{~B}), 7.60(\mathrm{~d}, J=2.0,1 \mathrm{H}, \mathrm{pz} 5 \mathrm{~A}), 7.24(\mathrm{~d}, J=2.0,1 \mathrm{H}, \mathrm{pz} 3 \mathrm{C}), 7.03(\mathrm{~d}, J=2.0,1 \mathrm{H}$, pz3A), $6.87(\mathrm{dd}, J=9.3,4.7,1 \mathrm{H}, \mathrm{H} 2), 6.64(\mathrm{~d}, J=9.4,1 \mathrm{H}, \mathrm{H} 1), 6.49(\mathrm{~d}, J=8.6,1 \mathrm{H}, \mathrm{H} 6)$, 
$6.46(\mathrm{~d}, J=8.7,1 \mathrm{H}, \mathrm{H} 7), 6.25(\mathrm{t}, J=2.0,1 \mathrm{H}, \mathrm{pz} 4 \mathrm{C}), 6.18(\mathrm{t}, J=2.0,1 \mathrm{H}, \mathrm{pz} 4 \mathrm{~B}), 6.02(\mathrm{t}, J=$ 2.0, $1 \mathrm{H}, \mathrm{pz} 4 \mathrm{~A}), 3.95$ (ddd, $J=14.2,9.5,4.8,1 \mathrm{H}, \mathrm{H} 3), 3.83(\mathrm{~s}, 3 \mathrm{H}, \mathrm{H} 11), 3.32(\mathrm{~s}, 3 \mathrm{H}, \mathrm{H} 12)$, $3.01(\mathrm{~d}, J=9.4,1 \mathrm{H}, \mathrm{H} 4), 1.31\left(\mathrm{~d}, J=7.9,9 \mathrm{H}, \mathrm{PMe}_{3}\right) .{ }^{13} \mathrm{C} \mathrm{NMR}\left(\mathrm{CDCl}_{3}\right): \delta 151.20(\mathrm{C} 5)$, 149.26 (C8), 143.79 (pz3B), 142.18 (pz3A), 140.11 (pz3C), 136.28 (pz5C), 135.65 (pz5B), 135.09 (pz5A), 131.21 (C10 or C2), 131.19 (C10 or C2), 121.20 (C9), 112.20 (C1), 106.06 (pz4C), 106.02 (pz4B), 105.34 (C7), 104.71 (C6), 104.60 (pz4A), 59.66 (d, $J=8.2, C 3$ ), 56.82 (C11), 55.88 (C4), 54.44 (C12), 13.65 (d, $\left.J=27.3, \mathrm{PMe}_{3}\right) .{ }^{31} \mathrm{P} \mathrm{NMR}\left(\mathrm{CDCl}_{3}\right): \delta-13.45$ $\left(J_{P-W}=299\right) . C V(D M A): E_{p, a}=+0.007$ V. IR: $V_{N O}=1566 \mathrm{~cm}^{-1}$.

\section{$\operatorname{TpW}(\mathrm{NO})\left(\mathrm{PMe}_{3}\right)\left(3,4-\eta^{2}-5,8-\right.$ dimethylnaphthalene) (6b)}

$\mathrm{TpW}(\mathrm{NO})\left(\mathrm{PMe}_{3}\right)\left(\eta^{2}\right.$-benzene) $(608 \mathrm{mg}, 1.05 \mathrm{mmol})$ and 1,4-dimethylnaphthalene (798 $\mathrm{mg}, 5.12 \mathrm{mmol})$ were weighed into a vial and dissolved in DME (1.99 g). The reaction was stirred overnight and precipitated into $300 \mathrm{~mL}: 120 \mathrm{~mL}$ hexanes:Et ${ }_{2} \mathrm{O}$. A light brown solid was filtered with a medium-porosity fritted funnel and the yellow filtrate was evaporated to a volume of $50 \mathrm{~mL}$. A yellow precipitate was filtered with a mediumporosity fritted funnel, rinsed with $15 \mathrm{~mL}$ hexanes, and dried in vacuo to give $\mathbf{6 b}$ (248 $\mathrm{mg}, 36 \%)$.

${ }^{1} \mathrm{H} \mathrm{NMR}\left(\mathrm{CDCl}_{3}\right): \delta 7.81(\mathrm{~d}, J=2.0,1 \mathrm{H}, \mathrm{pz3B}), 7.79(\mathrm{~d}, J=2.0,1 \mathrm{H}, \mathrm{pz} 5 \mathrm{C}), 7.70(\mathrm{~d}, J=2.0$, $1 \mathrm{H}, \mathrm{pz} 5 \mathrm{~B}), 7.66(\mathrm{~d}, J=2.0,1 \mathrm{H}, \mathrm{pz} 5 \mathrm{~A}), 7.20(\mathrm{~d}, J=2.0,1 \mathrm{H}, \mathrm{pz} 3 \mathrm{C}), 6.85(\mathrm{dd}, J=4.5,9.3,1 \mathrm{H}$, H2), $6.73(\mathrm{~m}, 1 \mathrm{H}, \mathrm{H6}), 6.72(\mathrm{~m}, 1 \mathrm{H}, \mathrm{H7}), 6.49(\mathrm{~d}, J=2.0,1 \mathrm{H}, \mathrm{pz} 3 \mathrm{~A}), 6.48(\mathrm{~d}, J=9.3,1 \mathrm{H}$, $\mathrm{H} 1), 6.26(\mathrm{t}, J=2.0,1 \mathrm{H}, \mathrm{pz} 4 \mathrm{C}), 6.19(\mathrm{t}, J=2.0,1 \mathrm{H}, \mathrm{pz} 4 \mathrm{~B}), 6.02(\mathrm{t}, J=2.0,1 \mathrm{H}, \mathrm{pz} 4 \mathrm{~A}), 4.11$ (ddd, $J=14.5,9.5,4.8,1 \mathrm{H}, \mathrm{H} 3$ ), $2.54(\mathrm{~d}, J=9.4,1 \mathrm{H}, \mathrm{H} 4), 2.45(\mathrm{~s}, 3 \mathrm{H}, \mathrm{H} 12), 1.37$ (d, J = 7.9, 
$\left.9 \mathrm{H}, \mathrm{PMe}_{3}\right), 1.28(\mathrm{~s}, 3 \mathrm{H}, \mathrm{H} 11) .{ }^{13} \mathrm{C} \mathrm{NMR}\left(\mathrm{CDCl}_{3}\right): \delta 143.77$ (pz3B), $142.24(\mathrm{pz} 3 \mathrm{~A}), 140.99$ (C10), 139.87 (pz3C), 136.26 (pz5C), 135.94 (pz5B), 135.47 (pz5A), 132.17 (C5), 130.31 (C8), 130.14 (C2), 128.16 (C9), 125.68 (C6), 124.35 (C7), 115.67 (C1), 106.22 (pz4C), 106.06 (pz4B), 105.58 (pz4A), 59.84 (C4), 58.98 (d, J = 7.9, C3), 19.73 (C12), 18.47 (C11), $13.97\left(\mathrm{~d}, J=27.3, \mathrm{PMe}_{3}\right) .{ }^{31} \mathrm{P} \mathrm{NMR}\left(\mathrm{CDCl}_{3}\right): \delta-13.46\left(J_{\mathrm{P}-\mathrm{W}}=298\right) . \mathrm{CV}: \mathrm{E}_{\mathrm{p}, \mathrm{a}}=+0.065 \mathrm{~V} . \mathrm{IR}:$ $\mathrm{V}_{\mathrm{NO}}=1568 \mathrm{~cm}^{-1}$.

Selected ${ }^{1} \mathrm{H}$ data for $6 a: \delta 6.85(\mathrm{~m}$, under $6 \mathrm{~b} \mathrm{H} 2, \mathrm{H} 2), 3.48(\mathrm{~m}, \mathrm{H} 3), 2.58(\mathrm{~s}, 3 \mathrm{H}, \mathrm{H} 1), 1.32$ $\left(\mathrm{d}, J=8.0,9 \mathrm{H}, \mathrm{PMe}_{3}\right), 1.14(\mathrm{~s}, 3 \mathrm{H}, \mathrm{H} 4)$.

\section{$\mathrm{TpW}(\mathrm{NO})\left(\mathrm{PMe}_{3}\right)\left(3,4-\eta^{2}\right.$-1,8-dimethylnaphthalene) (7)}

$\mathrm{TpW}(\mathrm{NO})\left(\mathrm{PMe}_{3}\right)\left(\mathrm{n}^{2}\right.$-benzene) (391 mg, $\left.0.673 \mathrm{mmol}\right)$ and 1,8-dimethylnaphthalene (328 $\mathrm{mg}, 2.10 \mathrm{mmol})$ were weighed into a vial and dissolved in DME (1.21 g). The reaction was stirred overnight and a brown precipitate formed. The mixture was diluted with 4 $\mathrm{mL} \mathrm{Et} t_{2} \mathrm{O}$ and filtered with a $15 \mathrm{~mL}$ fine-porosity fritted funnel. The solid was washed with $3 \mathrm{~mL} \mathrm{Et}{ }_{2} \mathrm{O}$ and dried in vacuo to give 7 as a brown powder (243 mg, 55\%).

${ }^{1} \mathrm{H}$ NMR $\left(\mathrm{CDCl}_{3}\right): \delta 7.80(\mathrm{~d}, J=2.0,1 \mathrm{H}, \mathrm{pz} 3 \mathrm{~B}), 7.77(\mathrm{~d}, J=2.0,1 \mathrm{H}, \mathrm{pz} 5 \mathrm{C}), 7.70(\mathrm{~d}, J=2.0$, $1 \mathrm{H}, \mathrm{pz} 5 \mathrm{~B}), 7.64(\mathrm{~d}, J=2.0,1 \mathrm{H}, \mathrm{pz} 5 \mathrm{~A}), 7.21(\mathrm{~d}, J=2.0,1 \mathrm{H}, \mathrm{pz} 3 \mathrm{C}), 6.96(\mathrm{~d}, J=2.0,1 \mathrm{H}$, pz3A), $6.89(\mathrm{t}, J=7.6,1 \mathrm{H}, \mathrm{H6}), 6.78(\mathrm{~d}, J=7.6,1 \mathrm{H}, \mathrm{H7}), 6.69(\mathrm{~d}, J=5.1,1 \mathrm{H}, \mathrm{H} 2), 6.57(\mathrm{~d}, J$ $=7.6,1 \mathrm{H}, \mathrm{H} 5), 6.23(\mathrm{t}, J=2.0,1 \mathrm{H}, \mathrm{pz} 4 \mathrm{C}), 6.20(\mathrm{t}, J=2.0,1 \mathrm{H}, \mathrm{pz} 4 \mathrm{~B}), 6.09(\mathrm{t}, J=2.0,1 \mathrm{H}$, pz4A), 3.91 (ddd, $J=14.2,9.4,5.2,1 \mathrm{H}, \mathrm{H} 3), 2.82(\mathrm{~s}, 3 \mathrm{H}, \mathrm{H} 11), 2.71$ (s, 3H, H12), 2.56 (d, J $=9.3,1 \mathrm{H}, \mathrm{H} 4), 1.33\left(\mathrm{~d}, J=8.0,9 \mathrm{H}, \mathrm{PMe}_{3}\right) .{ }^{13} \mathrm{C} \mathrm{NMR}\left(\mathrm{CDCl}_{3}\right): \delta 144.60(\mathrm{C} 10), 143.91$ (pz3B), 143.33 (pz3A), 140.11 (pz3C), 136.40 (pz5C), 135.84 (pz5B), 135.50 (pz5A), 
133.32 (C2), 133.14 (C9), 130.72 (C8), 127.84 (C7), 127.73 (C5), 125.56 (C1), 123.25 (C6), 106.18 (pz4B), 106.03 (pz4C), 104.79 (pz4A), 62.19 (C4), 59.27 (C3), 25.64 (C12), 25.03 (C11), $13.72\left(\mathrm{~d}, J=27.2, \mathrm{PMe}_{3}\right) \cdot{ }^{31} \mathrm{P} \mathrm{NMR}\left(\mathrm{CDCl}_{3}\right): \delta-12.48\left(J_{\mathrm{P}-\mathrm{W}}=297\right) . \mathrm{CV}: \mathrm{E}_{\mathrm{p}, \mathrm{a}}=+0.036$ V. IR: $V_{\mathrm{NO}}=1569 \mathrm{~cm}^{-1}$.

\section{$\mathrm{TpW}(\mathrm{NO})\left(\mathrm{PMe}_{3}\right)\left(3,4-\eta^{2}-2,6-\right.$ dimethylnaphthalene) (8)}

$\mathrm{TpW}(\mathrm{NO})\left(\mathrm{PMe}_{3}\right)\left(\mathrm{n}^{2}\right.$-benzene) (300 mg, $\left.0.516 \mathrm{mmol}\right)$ and 2,6-dimethylnapthalene (392 $\mathrm{mg}, 2.51 \mathrm{mmol}$ ) were weighed into a vial and dissolved in DME (1.015 g). The reaction was stirred for two days and a brown precipitate formed. The mixture was diluted with $3 \mathrm{~mL} \mathrm{Et}{ }_{2} \mathrm{O}$ and filtered with a $15 \mathrm{~mL}$ fine-porosity fritted funnel. The solid was washed with $2 \mathrm{mLEt} \mathrm{E}_{2} \mathrm{O}$ and $0.5 \mathrm{~mL} \mathrm{DME}$ and dried in vacuo to give 8 as a yellow-brown solid (168 mg, 49\%).

${ }^{1} \mathrm{H} \mathrm{NMR}\left(\mathrm{CDCl}_{3}\right): 7.83(\mathrm{~d}, J=2.0,1 \mathrm{H}, \mathrm{pz} 3 \mathrm{~B}), 7.81(\mathrm{~d}, J=2.0,1 \mathrm{H}, \mathrm{pz} 5 \mathrm{C}), 7.72(\mathrm{~d}, J=2.0,1 \mathrm{H}$, pz5B), $7.66(d, J=2.0,1 \mathrm{H}, \mathrm{pz} 5 \mathrm{~A}), 7.17(\mathrm{~d}, J=2.0,1 \mathrm{H}, \mathrm{pz3C}), 7.14(\mathrm{~d}, J=7.6,1 \mathrm{H}, \mathrm{H} 8), 6.76$ $(\mathrm{d}, J=7.6,1 \mathrm{H}, \mathrm{H7}), 6.46(\mathrm{~d}, J=2.0,1 \mathrm{H}, \mathrm{pz} 3 \mathrm{~A}), 6.27(\mathrm{t}, J=2.0,1 \mathrm{H}, \mathrm{pz} 4 \mathrm{C}), 6.23(\mathrm{~s}, 1 \mathrm{H}, \mathrm{H} 1)$, $6.21(\mathrm{~s}, 1 \mathrm{H}, \mathrm{H} 5), 6.21(\mathrm{t}, J=2.0,1 \mathrm{H}, \mathrm{pz} 4 \mathrm{~B}), 6.02(\mathrm{t}, J=2.0,1 \mathrm{H}, \mathrm{pz} 4 \mathrm{~A}), 4.11(\mathrm{dd}, J=9.4$, 13.7, 1H, H3), $2.45(\mathrm{~d}, J=9.8,1 \mathrm{H}, \mathrm{H} 4), 2.27(\mathrm{~s}, 3 \mathrm{H}, \mathrm{H} 11), 2.22(\mathrm{~s}, 3 \mathrm{H}, \mathrm{H} 12), 1.26(\mathrm{~d}, J=$ 7.8, 9H, $\left.\mathrm{PMe}_{3}\right) .{ }^{13} \mathrm{C}$ NMR $\left(\mathrm{CDCl}_{3}\right): 143.52$ (pz3A), 141.07 (C10), 139.87 (pz3C), 139.05 (C2), 136.32 (pz3B, pz5A, pz5B, or pz5C), 136.08 (pz3B, pz5A, pz5B, or pz5C), 135.89 (pz3B, pz5A, pz5B, or pz5C), 131.61 (C6), 129.65 (C5), 128.75 (C9), 124.77 (C8), 123.97 (C7), 118.14 (C1), 106.41 (pz4C), 106.28 (pz4B), 104.64 (pz4A), 64.75 (C4), 61.15 (d, $J=$ 8.2, C3), 25.96 (C11), 21.32 (C12), $14.53\left(\mathrm{~d}, J=27.2, \mathrm{PMe}_{3}\right) .{ }^{31} \mathrm{P} \mathrm{NMR}\left(\mathrm{CDCl}_{3}\right): \delta-14.15\left(J_{\mathrm{P}-}\right.$ 
$w=298),-10.23 . C V(D M A): E_{p, a}=+0.007$ V. IR: $v_{N O}=1566 \mathrm{~cm}^{-1}$. Anal. Calc'd for $\mathrm{C}_{24} \mathrm{H}_{31} \mathrm{BN}_{7} \mathrm{OPW} * 1 / 2$ eq $\mathrm{H}_{2} \mathrm{O}: \mathrm{C}, 43.14 ; \mathrm{H}, 4.83 ; \mathrm{N}, 14.67$; Found: $\mathrm{C}, 42.80 ; \mathrm{H}, 4.79 ; \mathrm{N}$, 14.96 .

$\mathrm{TpW}(\mathrm{NO})\left(\mathrm{PMe}_{3}\right)\left(2,3-\eta^{2}-(3 a R, 4 \mathrm{R}, 9 \mathrm{~S}, 9 \mathrm{aS})-2-m e t h y l-3 a, 4,9,9 a-t e t r a h y d r o-1 H-4,9-\right.$ ethenobenzo[f]isoindole-1,3(2H)-dione) (9)

$1(101 \mathrm{mg}, 0.160 \mathrm{mmol}), \mathrm{N}$-methylmaleimide $(101 \mathrm{mg}, 0.909 \mathrm{mmol})$, and 3:1 w/w $\mathrm{CHCl}_{3} / \mathrm{n}$-butanol $(1.05 \mathrm{~g})$ were combined in a test tube with a stir bar. The dark brown solution was stirred at $50{ }^{\circ} \mathrm{C}$ for 90 hours. The solution was diluted to $2 \mathrm{~mL}$ with $\mathrm{CHCl}_{3}$ and precipitated into $7 \mathrm{~mL} \mathrm{Et}{ }_{2} \mathrm{O}$. The filtrate was evaporated to an orange oil, which was washed with $2 \times 3 \mathrm{mLEt}{ }_{2} \mathrm{O}$. The pale yellow precipitate was collected, dissolved in $5 \mathrm{~mL}$ DCM, and precipitated into $20 \mathrm{~mL}$ hexanes. The filtrate was evaporated to $10 \mathrm{~mL}$, and the precipitate was collected to give 9 as a very pale yellow solid (61.9 mg, 52\%).

${ }^{1} \mathrm{H}\left(\mathrm{CDCl}_{3}\right): \delta 8.03(\mathrm{~d}, J=1.8,1 \mathrm{H}, \mathrm{Pz} 3 \mathrm{~A}), 7.84(\mathrm{~d}, J=1.9,1 \mathrm{H}, \mathrm{Pz} 3 \mathrm{~B}), 7.69(\mathrm{~d}, J=2.2,1 \mathrm{H}$, Pz5C), $7.64(\mathrm{~d}, J=2.3,1 \mathrm{H}, \mathrm{Pz} 5 \mathrm{~B}), 7.53(\mathrm{~d}, J=2.3,1 \mathrm{H}, \mathrm{Pz} 5 \mathrm{~A}), 7.30(\mathrm{~d}, J=2.1,1 \mathrm{H}, \mathrm{Pz} 3 \mathrm{C}$ ), $7.17(\mathrm{~m}, 2 \mathrm{H}, \mathrm{H} 5$ and $\mathrm{H} 6$ or H7), $7.13(\mathrm{~m}, 1 \mathrm{H}, \mathrm{H} 6$ or $\mathrm{H} 7), 7.03(\mathrm{~d}, J=6.9,1 \mathrm{H}, \mathrm{H} 8), 6.23(\mathrm{t}, J$ $=2.2,1 \mathrm{H}, \mathrm{Pz} 4 \mathrm{~A}), 6.19(\mathrm{t}, \mathrm{J}=2.2,1 \mathrm{H}, \mathrm{Pz} 4 \mathrm{C}), 6.19(\mathrm{t}, \mathrm{J}=2.2,1 \mathrm{H}, \mathrm{Pz} 4 \mathrm{~B}), 4.23(\mathrm{~m}, 2 \mathrm{H}, \mathrm{H} 1$ and H4), $3.39\left(\mathrm{dd}, J=3.6,7.9 \mathrm{~Hz}, 1 \mathrm{H}, \mathrm{H2} 2^{\prime}\right), 3.24\left(\mathrm{dd}, J=3.6,7.9,1 \mathrm{H}, \mathrm{H} 3^{\prime}\right), 2.60(\mathrm{~m}, 1 \mathrm{H}, \mathrm{H} 2)$, $2.40\left(\mathrm{~s}, 3 \mathrm{H}, \mathrm{H} 5^{\prime}\right), 1.57(\mathrm{~m}, 1 \mathrm{H}, \mathrm{H} 3), 1.28\left(\mathrm{~d}, \mathrm{~J}=8.2,9 \mathrm{H}, \mathrm{PMe}_{3}\right) \cdot{ }^{13} \mathrm{C}\left(\mathrm{CDCl}_{3}\right): \delta 178.86\left(\mathrm{Cl}^{\prime}\right.$ and $\mathrm{C4}^{\prime}$ ), 144.82 (Pz3A), 142.85 (Pz3B), 140.53 (Pz3C), 138.98 (C10), 137.94 (C9), 136.63 (Pz5C), 135.78 (Pz5B), 134.68 (Pz5A), 127.57 (C6 or C7), 127.10 (C6 or C7), 124.64 (C5 or C8), 124.22 (C5 or C8), 106.26 (Tp), 106.05 (Tp), 105.72 (Tp), 57.49 (C3), 56.69 (d, J = 
15.3, C2), 52.06 (C2' or $\left.C 3^{\prime}\right), 51.76$ (C2' or $\left.C^{\prime}\right), 46.16$ (C4), 44.83 (C1), $23.83\left(\mathrm{C5}^{\prime}\right), 14.23$ $\left(\mathrm{d}, J=27.9, \mathrm{PMe}_{3}\right) \cdot{ }^{31} \mathrm{P}\left(\mathrm{CDCl}_{3}\right): \delta-11.66\left(J_{\mathrm{W}-\mathrm{P}}=267\right) . \mathrm{CV}: \mathrm{E}_{\mathrm{p}, \mathrm{a}}=+0.642 \mathrm{~V} . \mathrm{IR}: \mathrm{V}_{\mathrm{NO}}=1568$ $\mathrm{cm}^{-1}, \mathrm{v}_{\mathrm{CO}}=1693 \mathrm{~cm}^{-1}$. HRMS obs'd (\%), calc'd (\%), ppm, $(\mathrm{M}+\mathrm{Na})^{+}: 763.1848$ (81.4), 763.1813 (81.4), 4.6; 764.1821 (88.6), 764.1838 (81.2), 2.2; 765.1837 (100), 765.1838 (100), 0.1; 766.1856 (55.5), 766.1877 (46.9), 2.7; 767.1882 (81.3), 767.1870 (82.6), 1.6.

\section{$\mathrm{TpW}(\mathrm{NO})\left(\mathrm{PMe}_{3}\right)\left(2,3-\eta^{2}\right.$-5,8-dimethoxy-2-methyl-3a,4,9,9a-tetrahydro-1H-4,9-} ethenobenzo[f]isoindole-1,3(2H)-dione) (10)

5 (225 mg, $0.326 \mathrm{mmol}), N$-methylmaleimide $(78.1 \mathrm{mg}, 0.703 \mathrm{mmol})$, and $3: 1 \mathrm{w} / \mathrm{w}$ $\mathrm{CHCl}_{3} / n$-butanol (3.43 g) were combined in a test tube with a stir bar. The dark brown solution was stirred at $49{ }^{\circ} \mathrm{C}$ for 24 hours. The solution was diluted to $5 \mathrm{~mL}$ with $\mathrm{CHCl}_{3}$ and precipitated into $15 \mathrm{~mL} \mathrm{Et}{ }_{2} \mathrm{O}$. The filtrate was evaporated to a brown oil, which was washed with $2 \times 5 \mathrm{~mL} 3: 1 \mathrm{v} / \mathrm{v} \mathrm{Et}_{2} \mathrm{O} /$ hexanes. The pale brown precipitate was collected, dissolved in $10 \mathrm{~mL} \mathrm{DCM}$, and precipitated into $35 \mathrm{~mL}$ hexanes. The filtrate was evaporated to $15 \mathrm{~mL}$, and the precipitate was collected to give 10 as a tan solid (102.8 $\mathrm{mg}, 39 \%)$.

${ }^{1} \mathrm{H}\left(\mathrm{CDCl}_{3}\right): \delta 8.35(\mathrm{~d}, J=1.8,1 \mathrm{H}, \mathrm{Pz3A}), 7.84(\mathrm{~d}, J=1.9,1 \mathrm{H}, \mathrm{Pz} 3 \mathrm{~B}), 7.67(\mathrm{~d}, J=2.2,1 \mathrm{H}$, Pz5C), $7.63(\mathrm{~d}, J=2.2,1 \mathrm{H}, \mathrm{Pz5B}), 7.52(\mathrm{~d}, J=2.3,1 \mathrm{H}, \mathrm{Pz} 5 \mathrm{~A}), 7.29(\mathrm{~d}, J=1.8,1 \mathrm{H}, \mathrm{Pz3C})$, $6.66(\mathrm{~d}, J=9.0,1 \mathrm{H}, \mathrm{H} 6), 6.63(\mathrm{~d}, J=9.0,1 \mathrm{H}, \mathrm{H} 7), 6.21(\mathrm{t}, J=2.2,1 \mathrm{H}, \mathrm{Pz} 4 \mathrm{~A}), 6.18(\mathrm{t}, J=$ 2.2, 1H, Pz4B), $6.17(\mathrm{t}, J=2.1,1 \mathrm{H}, \mathrm{Pz} 4 \mathrm{C}), 4.75(\mathrm{t}, J=3.6,1 \mathrm{H}, \mathrm{H} 4), 4.73(\mathrm{t}, J=3.6,1 \mathrm{H}, \mathrm{H} 1)$, $3.82(s, 3 H, H 12), 3.77$ (s, 3H, H11), 3.34 (dd, $J=3.6,7.8,1 H, H 2^{\prime}$ ), 3.18 (dd, J = 3.6, 7.8, $\left.1 \mathrm{H}, \mathrm{H} 3^{\prime}\right), 2.54(\mathrm{~m}, 1 \mathrm{H}, \mathrm{H} 2), 2.48\left(\mathrm{~s}, 3 \mathrm{H}, \mathrm{H} 5^{\prime}\right), 1.51(\mathrm{~m}, 1 \mathrm{H}, \mathrm{H} 3), 1.27\left(\mathrm{~d}, J=8.3,9 \mathrm{H}, \mathrm{PMe}_{3}\right)$. 
${ }^{13} \mathrm{C}\left(\mathrm{CDCl}_{3}\right): \delta 178.95$ (C1' and $\left.\mathrm{C4} 4^{\prime}\right), 148.95$ (C5), 148.52 (C8), 145.29 (Pz3A), 142.65 (Pz3B), 140.52 (Pz3C), 136.48 (Pz5C), 135.55 (Pz5B), 134.50 (Pz5A), 128.42 (C10), 127.14 (C9), 110.32 (C6), 109.65 (C7), 106.23 (Pz4A/Pz4B), 106.20 (Pz4A/Pz4B), 105.60 (Pz4C), 56.99 (C3), 56.71 (d, J = 15.2, C2), 56.63 (C12), 56.10 (C11), 51.31 (C2'/C3'), 51.16 (C2'/C3'), 37.92 (C1), $37.04(\mathrm{C} 4), 24.02\left(\mathrm{C}^{\prime}\right), 13.95\left(\mathrm{~d}, J=28.0, \mathrm{PMe}_{3}\right) .{ }^{31} \mathrm{P}$ NMR $\left(\mathrm{CDCl}_{3}\right)$ : $\delta-12.07\left(J_{P-W}=269\right) . C V: E_{p, a}=+0.632$ V. IR: $v_{N O}=1566 \mathrm{~cm}^{-1}, v_{C O}=1697 \mathrm{~cm}^{-1}$. obs'd $(\%)$, HRMS calc'd (\%), ppm, $(\mathrm{M}+\mathrm{Na})^{+}: 823.1999(75.4), 823.2024(80), 3.1 ; 824.202$ (88.2), 824.205 (81.3), 3.6; 825.2052 (100), 825.205 (100), 0.3; 826.2075 (54.6), 826.2088 (48.5), 1.5; 827.2078 (82.6), 827.2082 (82.3), 0.5. Anal. Calc'd for $\mathrm{C}_{29} \mathrm{H}_{36} \mathrm{BN}_{8} \mathrm{O}_{5} \mathrm{PW} * \mathrm{H}_{2} \mathrm{O}$ : C, 42.46; H, 4.67; N, 13.66; Found: C, 42.20; H, 4.57; N, 13.91.

\section{$\mathrm{TpW}(\mathrm{NO})\left(\mathrm{PMe}_{3}\right)\left(2,3-\eta^{2}-2,5,8\right.$-trimethyl-3a,4,9,9a-tetrahydro-1H-4,9-}

\section{ethenobenzo[f]isoindole-1,3(2H)-dione) (11b)}

6b $(82 \mathrm{mg}, 0.124 \mathrm{mmol}), \mathrm{N}$-methylmaleimide $(30.3 \mathrm{mg}, 0.273 \mathrm{mmol})$, and 3:1 w/w $\mathrm{CHCl}_{3} / n$-butanol $(0.605 \mathrm{~g})$ were combined in a vial with a stir bar. The dark brown solution was stirred at $25{ }^{\circ} \mathrm{C}$ for 15 days. The solution was diluted to $2 \mathrm{~mL}$ with $\mathrm{CHCl}_{3}$ and precipitated into $7 \mathrm{~mL} \mathrm{Et}{ }_{2} \mathrm{O}$. The filtrate was evaporated to a brown oil, which was washed with $2 \times 2 \mathrm{mLEt}{ }_{2} \mathrm{O}$. The precipitate was collected to give $11 \mathrm{~b}$ as a peach-colored solid (54.9 mg, 57\%).

${ }^{1} \mathrm{H}\left(\mathrm{CDCl}_{3}\right): \delta 8.20(\mathrm{~d}, J=1.7,1 \mathrm{H}, \mathrm{Pz} 3 \mathrm{~A}), 7.89(\mathrm{~d}, J=1.9,1 \mathrm{H}, \mathrm{Pz} 3 \mathrm{~B}), 7.69(\mathrm{~d}, J=2.2,1 \mathrm{H}$, Pz5C), $7.66(\mathrm{~d}, J=2.3,1 \mathrm{H}, \mathrm{Pz} 5 \mathrm{~B}), 7.54(\mathrm{~d}, J=2.3,1 \mathrm{H}, \mathrm{Pz} 5 \mathrm{~A}), 7.36(\mathrm{~d}, J=1.9,1 \mathrm{H}, \mathrm{Pz} 3 \mathrm{C})$, $6.88(\mathrm{~d}, J=7.9,1 \mathrm{H}, \mathrm{H} 6$ or H7), 6.86 (d, $J=7.9,1 \mathrm{H}, \mathrm{H} 6$ or H7), 6.23 (t, $J=2.2,1 \mathrm{H}, \mathrm{Pz} 4 \mathrm{~A}$ ), 
$6.21(t, J=2.2,1 \mathrm{H}, \mathrm{Pz} 4 \mathrm{~B}), 6.20(\mathrm{t}, J=2.2,1 \mathrm{H}, \mathrm{Pz} 4 \mathrm{C}), 4.60(\mathrm{t}, J=3.6,1 \mathrm{H}, \mathrm{H} 4), 4.57(\mathrm{t}, J=$ 3.6, $1 \mathrm{H}, \mathrm{H} 1), 3.35\left(\mathrm{dd}, J=3.6,7.9,1 \mathrm{H}, \mathrm{H} 2^{\prime}\right), 3.20\left(\mathrm{dd}, J=3.6,7.9,1 \mathrm{H}, \mathrm{H} 3^{\prime}\right), 2.64(\mathrm{~m}, 1 \mathrm{H}$, $H 2), 2.45(s, 3 H, H 12), 2.41\left(s, 3 H, H 5^{\prime}\right), 2.32(s, 3 H, H 11), 1.66(m, 1 H, H 3), 1.19(d, J=$ 8.2, $\left.9 \mathrm{H}, \mathrm{PMe}_{3}\right) .{ }^{13} \mathrm{C}\left(\mathrm{CDCl}_{3}\right): \delta 179.06\left(\mathrm{C}^{\prime}\right.$ or $\left.\mathrm{C} 4^{\prime}\right), 179.03\left(\mathrm{C}^{\prime}\right.$ or $\left.\mathrm{C} 4^{\prime}\right), 144.77(\mathrm{Pz} 3 \mathrm{~A})$, 142.23 (Pz3B), 140.58 (Pz3C), 136.91 (C10), 136.65 (Pz5C), 136.10 (C9), 135.78 (Pz5B), 134.88 (Pz5A), 129.68 (C5), 128.67 (C6 or 7), 128.63 (C8), 128.22 (C6 or C7), 106.55 (Pz4B), 105.97 (Pz4A or Pz4C), 105.82 (Pz4A or Pz4C), 58.24 (d, J = 15.0, C2), 57.53 (C3), 51.58 (C2' or $\mathrm{C3}^{\prime}$ ), 51.53 (C2' or $\mathrm{C3}^{\prime}$ ), 41.65 (C1), 40.66 (C4), 23.89 (C5'), 18.79 (C11 or C12), 18.78 (C11 or C12), $13.82\left(\mathrm{~d}, J=27.9, \mathrm{PMe}_{3}\right) .{ }^{31} \mathrm{P}\left(\mathrm{CDCl}_{3}\right): \delta-12.92\left(\mathrm{JP}_{\mathrm{W}}=267\right) . \mathrm{CV}$ : $E_{p, a}=+0.661$ V. IR: $v_{N O}=1568 \mathrm{~cm}^{-1}, v_{C O}=1692 \mathrm{~cm}^{-1}$. HRMS obs'd (\%), calc'd (\%), ppm, $(\mathrm{M}+\mathrm{Na})^{+}: 791.2115(61), 791.2126(80.3), 1.5 ; 792.212$ (65.9), 792.2151 (81.5), 3.9; 793.2144 (100), 793.2151 (100), 0.9; 794.2193 (78), 794.219 (48.3), 0.4; 795.2162 (100), 795.2183 (82.2), 2.7. Anal. Calc'd for C29H36BN8O3PW • 1/2 eq $\mathrm{H}_{2} \mathrm{O}: \mathrm{C}, 44.70 ; \mathrm{H}, 4.79 ; \mathrm{N}$, 14.38; Found: C, 44.71; H, 4.65; N, 14.08 .

\section{$\mathrm{TpW}(\mathrm{NO})\left(\mathrm{PMe}_{3}\right)\left(2,3-\eta^{2}\right.$-4-methoxy-2-methyl-3a,4,9,9a-tetrahydro-1H-4,9-}

\section{ethenobenzo[f]isoindole-1,3(2H)-dione) (12)}

3 (150 mg, $0.227 \mathrm{mmol}$ ), $\mathrm{N}$-methylmaleimide (34 mg, $0.306 \mathrm{mmol}$ ), and 3:1 w/w $\mathrm{CHCl} 3 / \mathrm{n}$-butanol (10.02 g) were combined in a flame-dried test tube with a stir bar. The dark brown solution was stirred at $55{ }^{\circ} \mathrm{C}$ for 72 hours and removed from the glovebox. The volume was reduced to $3 \mathrm{~mL}$ and the reaction was precipitated in $11 \mathrm{~mL}$ stirring ether. The solid was collected on a $15 \mathrm{~mL}$ medium-porosity fritted funnel and 
discarded. The filtrate was evaporated in vacuo to give a brown oil. The oil was washed with 2 portions of $4 \mathrm{~mL} \mathrm{3:1} \mathrm{Et}_{2} \mathrm{O}$ :hexanes to give a tan precipitate, which was collected on a $15 \mathrm{~mL}$ fine-porosity fritted funnel. A small amount of solid precipitated from the filtrate and was collected separately. The solids were recombined and dissolved in $7 \mathrm{~mL}$ DCM and precipitated into $26 \mathrm{~mL}$ of hexanes. The solid was filtered with a $15 \mathrm{~mL}$ fineporosity fritted funnel and discarded. The filtrate was evaporated to $15 \mathrm{~mL}$ to precipitate a light brown solid, which was collected on a $15 \mathrm{~mL} F$ porosity fritted funnel. The solid was redissolved in $3 \mathrm{~mL} \mathrm{DCM}$ and precipitated into $30 \mathrm{~mL}$ of hexanes. The brown solid was filtered with a $15 \mathrm{~mL}$ fine-porosity fritted funnel and discarded. The filtrate was evaporated to $15 \mathrm{~mL}$ to precipitate a tan solid, which was filtered with a 15 $\mathrm{mL}$ fine-porosity fritted funnel to give $\mathbf{1 2}(59 \mathrm{mg}, 34 \%)$.

${ }^{1} \mathrm{H} \operatorname{NMR}\left(\mathrm{CDCl}_{3}\right): \delta 8.63(\mathrm{~d}, J=2.0,1 \mathrm{H}, \mathrm{pz} 3 \mathrm{~A}), 7.86(\mathrm{~d}, J=2.0,1 \mathrm{H}, \mathrm{pz} 3 \mathrm{~B}), 7.72(\mathrm{~d}, J=2.0$, $1 \mathrm{H}, \mathrm{pz} 5 \mathrm{C}), 7.63(\mathrm{~d}, J=2.0,1 \mathrm{H}, \mathrm{pz} 5 \mathrm{~B}), 7.58(\mathrm{~d}, J=7.9,1 \mathrm{H}, \mathrm{H} 5), 7.48(\mathrm{~d}, J=2.0,1 \mathrm{H}, \mathrm{pz} 5 \mathrm{~A})$, $7.38(\mathrm{~d}, J=2.0,1 \mathrm{H}, \mathrm{pz} 3 \mathrm{C}), 7.25(\mathrm{dt}, J=1.2,7.4,1 \mathrm{H}, \mathrm{H} 6), 7.15(\mathrm{dt}, J=1.2,7.4,1 \mathrm{H}, \mathrm{H} 7$ ), $7.04(\mathrm{~d}, J=7.4,1 \mathrm{H}, \mathrm{H} 8), 6.24(\mathrm{t}, J=2.0,1 \mathrm{H}, \mathrm{pz} 4 \mathrm{C}), 6.17(\mathrm{t}, J=2.0,1 \mathrm{H}, \mathrm{pz} 4 \mathrm{~B}), 6.15(\mathrm{t}, J=$ 2.0, $1 \mathrm{H}, \mathrm{pz} 4 \mathrm{~A}), 4.11(\mathrm{t}, J=3.4,1 \mathrm{H}, \mathrm{H} 1), 3.62\left(\mathrm{~d}, J=8.2,1 \mathrm{H}, \mathrm{H} 3^{\prime}\right), 3.54(\mathrm{dd}, J=3.4,8.2,1 \mathrm{H}$, $\mathrm{H} 2^{\prime}$ ), $3.50\left(\mathrm{~s}, 3 \mathrm{H}, \mathrm{OMe}\right.$ ), 2.66 (ddd, $J=3.2,11.2,14.4,1 \mathrm{H}, \mathrm{H} 2$ ), $2.40\left(\mathrm{~s}, 3 \mathrm{H}, \mathrm{H} 5^{\prime}\right), 1.95$ (dd, $J=2.7,11.2,1 \mathrm{H}, \mathrm{H} 3), 1.22\left(\mathrm{~d}, J=8.4,9 \mathrm{H}, \mathrm{PMe}_{3}\right) \cdot{ }^{13} \mathrm{C} \mathrm{NMR}\left(\mathrm{CDCl}_{3}\right): \delta 178.28\left(\mathrm{C1}^{\prime}\right), 175.63$ (C4'), 148.26 (pz3A), 142.68 (pz3B), 140.41 (pz3C), 140.40 (C10), 136.84 (pz5C), 135.76 (C9), 134.99 (pz5B), 134.63 (pz5A), 127.64 (C6 or C7), 127.11 (C6 or C7), 124.10 (C8), 120.91 (C5), 106.31 (pz4C), 105.79 (pz4B), 105.49 (pz4A), 87.07 (C4), 57.04 (d, J = 16.1, C2), 54.96 (C2'), 52.89 (C3), 50.30 (C11), 49.53 (C3'), 45.12 (C1), 23.84 (C5'), 13.85 (d, J = 
28.2, $\left.\mathrm{PMe}_{3}\right) .{ }^{31} \mathrm{P} N M R\left(\mathrm{CDCl}_{3}\right): \delta-11.23\left(J_{\mathrm{P}-\mathrm{W}}=267\right),-11.79,-11.98 . \mathrm{CV}: \mathrm{E}_{\mathrm{p}, \mathrm{a}}=+0.703 \mathrm{~V} . \mathrm{IR}:$ $v_{\text {NO }}=1570 \mathrm{~cm}^{-1}, v_{\mathrm{CO}}=1693 \mathrm{~cm}^{-1}$. HRMS obs'd (\%), calc'd (\%), ppm, $(\mathrm{M}+\mathrm{Na})^{+}: 793.1882$ (97.3), 793.1919 (80.7), 4.7; 794.1935 (88.8), 794.1944 (81.2), 1.2; 795.1924 (100), 795.1944 (100), 2.5; 796.1978 (49.7), 796.1982 (47.7), 0.5; 797.1959 (89.4), 797.1976 (82.5), 2.1.

$\operatorname{TpW}(\mathrm{NO})\left(\mathrm{PMe}_{3}\right)\left(2,3-\eta^{2}-2,4,5-t r i m e t h y l-3 a, 4,9,9 a-t e t r a h y d r o-1 H-4,9-\right.$ ethenobenzo[f]isoindole-1,3(2H)-dione) (13)

7 (153 mg, $0.232 \mathrm{mmol}$ ), N-methylmaleimide (49.8 mg, $0.448 \mathrm{mmol}$ ), and 3:1 w/w $\mathrm{CHCl}_{3} / n$-butanol (1.01 g) were combined in a vial with a stir bar. The dark brown solution was stirred at $25{ }^{\circ} \mathrm{C}$ for 8 days. The solution was diluted to $2.5 \mathrm{~mL}$ with $\mathrm{CHCl}_{3}$ and precipitated into $10 \mathrm{~mL} \mathrm{Et}{ }_{2} \mathrm{O}$. The filtrate was evaporated to a brown oil, which was washed with $2 \times 4 \mathrm{mLEt}{ }_{2} \mathrm{O}$. The tan precipitate was collected, dissolved in $7 \mathrm{~mL} \mathrm{DCM}$, and precipitated into $30 \mathrm{~mL}$ hexanes. The filtrate was evaporated to $10 \mathrm{~mL}$, and the precipitate was collected to give 13 as a peach-colored solid ( $85.7 \mathrm{mg}, 48 \%)$.

${ }^{1} \mathrm{H}\left(\mathrm{CDCl}_{3}\right): \delta$ (major isomer) $8.02(\mathrm{~d}, J=1.7,1 \mathrm{H}, \mathrm{Pz3A}), 7.77(\mathrm{~d}, J=1.9,1 \mathrm{H}, \mathrm{Pz} 3 \mathrm{~B}), 7.74$ (d, $J=2.2,1 \mathrm{H}, \mathrm{Pz} 5 \mathrm{C}), 7.58(\mathrm{~d}, J=2.3,1 \mathrm{H}, \mathrm{Pz5B}), 7.55(\mathrm{~d}, J=2.3,1 \mathrm{H}, \mathrm{Pz} 5 \mathrm{~A}), 7.34(\mathrm{~d}, J=$ 2.0, 1H, Pz3C), $6.95(\mathrm{~m}, 3 \mathrm{H}, \mathrm{H6}, \mathrm{H7}$, and $\mathrm{H} 8), 6.25(\mathrm{t}, \mathrm{J}=2.2,1 \mathrm{H}, \mathrm{Pz} 4 \mathrm{C}), 6.16(\mathrm{t}, J=2.1,1 \mathrm{H}$, Pz4A), $6.13(t, J=2.2,1 \mathrm{H}, \mathrm{Pz} 4 \mathrm{~B}), 4.11(\mathrm{t}, J=3.0,1 \mathrm{H}, \mathrm{H} 1), 3.35\left(\mathrm{dd}, J=3.2,8.0,1 \mathrm{H}, \mathrm{H} 2^{\prime}\right)$, $2.85\left(\mathrm{~d}, J=8.0,1 \mathrm{H}, \mathrm{H} 3^{\prime}\right), 2.72(\mathrm{~m}, 1 \mathrm{H}, \mathrm{H} 2), 2.59(\mathrm{~s}, 3 \mathrm{H}, \mathrm{H} 12), 2.42\left(\mathrm{~s}, 3 \mathrm{H}, \mathrm{H} 5^{\prime}\right), 2.18(\mathrm{~s}, 3 \mathrm{H}$, $\mathrm{H} 11), 1.35(\mathrm{dd}, J=2.5,10.7,1 \mathrm{H}, \mathrm{H} 3), 1.26\left(\mathrm{~d}, J=8.2,9 \mathrm{H}, \mathrm{PMe}_{3}\right) .{ }^{13} \mathrm{C}\left(\mathrm{CDCl}_{3}\right): \delta$ (major isomer) 178.96 (C4'), 178.50 (C1'), 146.44 (Pz3A), 142.65 (Pz3B), 140.41 (Pz3C), 139.05 
(C9 or C10), 138.51 (C9 or C10), 136.77 (Pz5C), 135.78 (Tp), 135.73 (Tp), 131.79 (C5 and C7), 126.33 (C6), 124.21 (C8), 106.11 (Tp), 105.96 (Tp), 105.39 (Pz4A), 64.47 (C3), 57.83 $\left(C 3^{\prime}\right), 57.54(d, J=15.8, C 2), 53.71\left(C 2^{\prime}\right), 50.37(C 4), 47.99(C 1), 26.11(C 11), 23.90\left(C^{\prime}\right)$, $22.82(\mathrm{C} 12), 13.98\left(\mathrm{~d}, J=28.0, \mathrm{PMe}_{3}\right) .{ }^{31} \mathrm{P}\left(\mathrm{CDCl}_{3}\right): \delta-11.08\left(J_{\mathrm{P}-\mathrm{W}}=263\right),-13.67$ (minor). CV: $E_{p, a}=+0.642$ V. IR: $v_{N O}=1568 \mathrm{~cm}^{-1}, v_{C O}=1688 \mathrm{~cm}^{-1}$. HRMS obs'd (\%), calc'd (\%), ppm, $(\mathrm{M}+\mathrm{Na})^{+}: 791.2157$ (88.8), 791.2126 (80.3), 3.8; 792.2155 (74.7), 792.2151 (81.5), 0.4; 793.213 (73.4), 793.2151 (100), 2.7; 794.2151 (58.4), 794.219 (48.3), 4.9; 795.2199 (100), 795.2183 (82.2), 2.

\section{5,8-dimethoxy-2-methyl-3a,4,9,9a-tetrahydro-1H-4,9-ethenobenzo[f]isoindole-}

\section{1,3(2H)-dione (14)}

In a fume hood, a solution of ceric ammonium nitrate (CAN) $(8.5 \mathrm{mg}, 0.016 \mathrm{mmol})$ in water $(501 \mathrm{mg})$ was added to a stirring solution of $10(12.2 \mathrm{mg}, 0.016 \mathrm{mmol})$ in acetone (508 mg). The red, heterogeneous solution was stirred for 1.25 hours. The solution was diluted with $5 \mathrm{~mL} \mathrm{Et}{ }_{2} \mathrm{O}$ and extracted with $1 \mathrm{~mL}$ water. The water layer was extracted with two more $5 \mathrm{~mL}$ portions of $\mathrm{Et}_{2} \mathrm{O}$. The $\mathrm{Et}_{2} \mathrm{O}$ layers were combined and dried with anhydrous $\mathrm{MgSO}_{4}$, filtered, and evaporated in vacuo. Characterization data was collected on the crude product.

${ }^{1} \mathrm{H}$ NMR $\left(\mathrm{CDCl}_{3}\right): \delta 6.66(\mathrm{dd}, J=3.1,4.5,2 \mathrm{H}, \mathrm{H} 1), 6.61(\mathrm{~s}, 2 \mathrm{H}, \mathrm{H} 5), 4.89(\mathrm{~m}, 2 \mathrm{H}, \mathrm{H} 2), 3.77$ $(\mathrm{s}, 6 \mathrm{H}, \mathrm{H} 6), 3.06(\mathrm{t}, J=1.1,2 \mathrm{H}, \mathrm{H} 7), 2.48(\mathrm{~s}, 3 \mathrm{H}, \mathrm{H} 9) .{ }^{13} \mathrm{C} \mathrm{NMR}\left(\mathrm{CDCl}_{3}\right): 177.18(\mathrm{C} 8)$, 149.15, 142.46, 136.36 (C1), 110.06, 56.80 (C6), 46.56 (C7), 35.31 (C2), 24.37 (C9). IR: $v_{C O}$ $=1698 \mathrm{~cm}^{-1}$. 


\section{2,5,8-trimethyl-3a,4,9,9a-tetrahydro-1H-4,9-ethenobenzo[f]isoindole-1,3(2H)-dione} (15)

In a fume hood, a solution of CAN $(63.0 \mathrm{mg}, 0.115 \mathrm{mmol})$ in water $(3.5 \mathrm{~g})$ was added to a stirring solution of $\mathbf{1 1 b}(80.0 \mathrm{mg}, 0.104 \mathrm{mmol})$ in acetone $(3.5 \mathrm{~g})$. The dark reddish brown solution was stirred for 90 minutes. The solution was extracted with $2 \times 25 \mathrm{~mL}$ $\mathrm{Et}_{2} \mathrm{O}$. The $\mathrm{Et}_{2} \mathrm{O}$ fraction was dried with anhydrous $\mathrm{MgSO}_{4}$ and evaporated to a yellowbrown oil. This oil was redissolved in small portions of DCM and loaded onto a $250 \mu \mathrm{m}$ silica preparatory TLC plate and run with $100 \mathrm{~mL} 70 \%$ hexanes/30\% EtOAc. The shortwave UV-absorbing band ( $\mathrm{Rf} \sim 0.4$ ) was scraped into a test tube with $20 \mathrm{~mL} \mathrm{HPLC}$ grade EtOAc and sonicated. The silica was filtered off and the EtOAC evaporated to give 15 as a colorless, crystalline solid (17.4 mg, 63\%).

${ }^{1} \mathrm{H}\left(\mathrm{CDCl}_{3}\right): \delta 6.81(\mathrm{~s}, 2 \mathrm{H}, \mathrm{H} 5), 6.65(\mathrm{dd}, J=3.0,4.5,2 \mathrm{H}, \mathrm{H} 1), 4.67(\mathrm{~m}, 2 \mathrm{H}, \mathrm{H} 2), 3.06(\mathrm{t}, J=$ 1.8, 2H, H7), $2.41(\mathrm{~s}, 3 \mathrm{H}, \mathrm{H} 9), 2.30(\mathrm{~s}, 6 \mathrm{H}, \mathrm{H} 6) .{ }^{13} \mathrm{C}\left(\mathrm{CDCl}_{3}\right): \delta 177.34(\mathrm{C} 8), 137.29$ (C3), 136.04 (C1), 130.41 (C4), 127.59 (C5), 46.41 (C7), 38.20 (C2), 24.12 (C9), 18.19 (C6). IR: $\mathrm{v}_{\mathrm{CO}}=1697 \mathrm{~cm}^{-1}$.

\section{$\mathrm{TpW}(\mathrm{NO})\left(\mathrm{PMe}_{3}\right)\left(3,4-\eta^{2}\right.$-anthracene) (16)}

In a glove box, sodium dispersion in paraffin $(7.25 \mathrm{~g}, \sim 95 \mathrm{mmol} \mathrm{Na})$ was washed with $3 \mathrm{x}$ $200 \mathrm{ml}$ portions of hexanes for $15 \mathrm{~min}$ each, and $1 \times 150 \mathrm{~mL}$ of benzene, which was discarded. Dry benzene $(600 \mathrm{~mL})$ was used to wash the cleaned sodium into a $1 \mathrm{~L}$ roundbottom containing $\mathrm{TpW}(\mathrm{NO})\left(\mathrm{PMe}_{3}\right) \mathrm{Br}(8.03 \mathrm{~g}, 13.8 \mathrm{mmol})$. The flask was capped 
with a rubber septum and the reaction was vigorously stirred for 19 hours. The reaction mixture was filtered with a $150 \mathrm{~mL}$ medium-porosity fritted funnel containing Celite, into a $1 \mathrm{~L}$ roundbottom flask containing anthracene $(19.40 \mathrm{~g}, 109 \mathrm{mmol})$. The sodium was washed with $2 \times 50 \mathrm{~mL}$ portions benzene, into the reaction flask. The flask was capped with a rubber septum and the reaction was vigorously stirred for 6 days. The anthracene was filtered with a $150 \mathrm{~mL}$ medium-porosity fritted funnel, and washed with $70 \mathrm{~mL}$ benzene. A silica slurry was prepared with ether and a column was set up over a $600 \mathrm{~mL}$ medium porosity fritted funnel, such that the column was about $6 \mathrm{~cm}$ high. The column was primed with $30 \mathrm{~mL}$ benzene, and the reaction solution was eluted onto the column ( $650 \mathrm{~mL}$ reaction volume). Added $200 \mathrm{~mL} \mathrm{4:1} \mathrm{benzene:ether} \mathrm{to} \mathrm{column.}$ Added $800 \mathrm{~mL}$ 1:1 benzene:ether to column, a yellow band started to elute, discarded. Added 1 L 3:2 ether:benzene to column, began collecting the orange band that eluted. Added $250 \mathrm{~mL} \mathrm{4:1} \mathrm{ether:benzene} \mathrm{and} 200 \mathrm{~mL}$ ether to pull off last of orange band. Evaporated the orange band eluent to $500 \mathrm{~mL}$, added $200 \mathrm{~mL}$ hexanes and continued evaporating. Evaporated to $300 \mathrm{~mL}$, added $200 \mathrm{~mL}$ hexanes and continued evaporating. Evaporated to $150 \mathrm{~mL}$ and filtered the yellow-orange solid with a $60 \mathrm{~mL}$ fine porosity fritted funnel. Dried in vacuo overnight (2.298 g, 26\%).

${ }^{1} \mathrm{H}\left(\mathrm{CDCl}_{3}\right): \delta 7.88(\mathrm{~d}, J=2.0,1 \mathrm{H}, \mathrm{pz} 3 \mathrm{~B}), 7.81(\mathrm{~d}, J=2.0,1 \mathrm{H}, \mathrm{pz} 5 \mathrm{C}), 7.77(\mathrm{~d}, J=8.2,1 \mathrm{H}$, H8), $7.72(\mathrm{~d}, J=2.0,1 \mathrm{H}, \mathrm{pz} 5 \mathrm{~A}), 7.70(\mathrm{~d}, J=2.0,1 \mathrm{H}, \mathrm{pz5B}), 7.63(\mathrm{~s}, 1 \mathrm{H}, \mathrm{H} 10), 7.56(\mathrm{~d}, J=$ 8.30, 1H, H5), $7.29(\mathrm{~d}, J=2.0,1 \mathrm{H}, \mathrm{pz} 3 \mathrm{C}), 7.27(\mathrm{~m}, 1 \mathrm{H}, \mathrm{H} 6), 7.22(\mathrm{~m}, 1 \mathrm{H}, \mathrm{H} 7), 6.99(\mathrm{~d}, J=$ 2.0, 1H, pz3A), 6.95 (s, 1H, H9), $6.92(\mathrm{dd}, J=9.3,4.3,1 \mathrm{H}, \mathrm{H} 2), 6.42(\mathrm{~d}, J=9.3,1 \mathrm{H}, \mathrm{H} 1$ ), $6.26(t, J=2.0,1 \mathrm{H}, \mathrm{pz} 4 \mathrm{C}), 6.21(\mathrm{t}, J=2.0,1 \mathrm{H}, \mathrm{pz} 4 \mathrm{~B}), 6.08(\mathrm{t}, J=2.0,1 \mathrm{H}, \mathrm{pz} 4 \mathrm{~A}), 3.74(\mathrm{ddd}$, 
$J=13.6,9.0,4.8,1 \mathrm{H}, \mathrm{H} 3), 2.58(\mathrm{~d}, J=9.0,1 \mathrm{H}, \mathrm{H} 4), 1.34\left(\mathrm{~d}, J=8.1,9 \mathrm{H}, \mathrm{PMe}_{3}\right) .{ }^{13} \mathrm{C}\left(\mathrm{CDCl}_{3}\right)$ : $\delta 144.1$ (1C, pz3B), 143.0 (1C, pz3A), 141.9 (1C, C12), 140.2 (1C, pz3C), 136.6 (1C, pz5C), 136.1 (1C, pz5B), 135.9 (1C, pz5A), 132.7 (1C, C2), 132.0 (1C, C13), 131.8 (1C, C14), 131.3 (1C, C11), 127.5 (1C, C8), 126.1 (1C, C5), 125.4 (1C, C9), 124.2 (1C, C6), 123.5 (1C, C7), 123.4 (1C, C10), 119.0 (1C, C1), 106.3 (1C, pz4C), 106.3 (1C, pz4B), 104.9 (1C, pz4A), 59.4 (1C, C4), $57.5(\mathrm{~d}, J=8.4,1 \mathrm{C}, \mathrm{C} 3), 13.7\left(\mathrm{~d}, J=27.6,3 \mathrm{C}, \mathrm{PMe}_{3}\right) \cdot{ }^{31} \mathrm{P}\left(\mathrm{CDCl}_{3}\right): \delta-13.00\left(J_{\mathrm{W}-\mathrm{P}}=\right.$ 292). CV: $E_{p, a}=+0.258$ V. IR: $v_{N O}=1568 \mathrm{~cm}^{-1}$

\section{References.}

(1) He, Y.; Funk, R. L. Organic Letters 2006, 8, 3689.

(2) Segura, J. L.; Martín, N. Chemical Reviews 1999, 99, 3199.

(3) Biermann, D.; Schmidt, W. Journal of the American Chemical Society 1980, 102, 3163.

(4) Chin, R. M.; Dong, L.; Duckett, S. B.; Jones, W. D. Organometallics 1992, $11,871$.

(5) Winemiller, M. D.; Harman, W. D. J. Org. Chem. 2000, 65, 1249.

(6) Brooks, B. C.; Meiere, S. H.; Friedman, L. A.; Carrig, E. H.; Gunnoe, T. B.; Harman, W. D. Journal of the American Chemical Society 2001, 123, 3541.

(7) Tagge, C. D.; Bergman, R. G. J. Am. Chem. Soc. 1996, 118, 6908. 
(8) Ding, F.; Valahovic, M. T.; Keane, J. M.; Anstey, M. R.; Sabat, M.; Trindle, C. O.; Harman, W. D. The Journal of Organic Chemistry 2004, 69, 2257.

(9) Keane, J. M.; Harman, W. D. Organometallics 2005, 24, 1786.

(10) characterization techniques included COSY, NOESY, HSQC, HMBC

(11) Welch, K. D.; Harrison, D. P.; Lis, E. C.; Liu, W.; Salomon, R. J.; Harman, W. D.; Myers, W. H. Organometallics 2007, 26, 2791.

(12) A co-solvent of n-butanol was found to be optimal for the reaction of 1 and $\mathrm{N}$-phenylmaleimide. Methanol and ethanol increased the rate of the cycloaddition, but also increased levels of decomposition in the reaction. We would have liked to complete the cycloaddition in pure n-butanol, but were prevented due to poor solubility of 1 in alcohol solvents.

(13) characterization techniques included COSY, NOESY, HSQC, HMBC

(14) Given that the concentration of N-methylmaleimide is kept much higher than that of the complexes, the reactions were expected to conform to pseudo-first order kinetics, and this was verified by a series of NMR spectra taken over several days. Once the rate constant, kobs, was obtained from linear regression of the integrated rate law, it was divided by the concentration of $\mathrm{N}$-methylmaleimide to give the second order rate constant, $\mathrm{k}$.

(15) The structures of $17 \mathrm{~b}$ and $17 \mathrm{c}$ were undistinguishable using the data available. Thus, values for k1 and k-1 may be reversed.

(16) Brooks, B. C.; Meiere, S. H.; Friedman, L. A.; Carrig, E. H.; Gunnoe, T. B.; Harman, W. D. J. Am. Chem. Soc. 2001, 123, 3541. 
(17) Winemiller, M. D.; Kelsch, B. A.; Sabat, M.; Harman, W. D. Organometallics 1997, 16, 3672.

(18) These calculations were performed in accordance with the methods described in the experimental section in chapter 2.

(19) Salomon, R. J.; Lis, E. C.; Kasbekar, M. U.; Bassett, K. C.; Myers, W. H.; Trindle, C. O.; Sabat, M.; Harman, W. D. Organometallics 2009, 28, 4724.

(20) Graham, P. M.; Meiere, M. S.; Sabat, M.; Harman, W. D. Organometalics 2003, 22, 4364 .

(21) Jones, W. H.; Mangold, D.; Plieninger, H. Tetrahedron 1962, 18, 267.

(22) Oku, A.; Ohnishi, Y.; Mashio, F. The Journal of Organic Chemistry 1972, 37,4264 .

(23) Kiselev, V. D. K., E. A.; Potapova, L. N.; Iskhakova, G. G. Russian Chemical Bulletin, International Edition 2004, 53, 51. 
Chapter 4

Tandem Electrophile-Nucleophile Additions to Naphthalene and Anthracene Complexes 
Metal complexes have been used to facilitate a variety of transformations on arenes. Some metals form stable bonds with the arene being functionalized, such as the metaltricarbonyl complexes. These complexes withdraw electron density from the arene $\pi$ system, making them susceptible to deprotonation or nucleophilic attack. ${ }^{1-3}$ In other cases the metal complex functions as a catalyst, binding the arene in question only long enough to complete the desired transformation. One transformation for which catalytic transition metal-arene chemistry is well developed is cross-coupling reactions of arenes. The Suzuki, ${ }^{4} \mathrm{Negishi}^{5-7}$ and Heck $^{8-10}$ reactions have become valuable methods for the coupling of two aromatic rings. Such cross-coupling reactions typically result in the formation of a new bond between two $\mathrm{sp}^{2}$ carbons. ${ }^{11,12}$ Catalytic cross-coupling reactions that form an $\mathrm{C}_{\mathrm{sp2} 2}-\mathrm{C}_{\mathrm{sp} 3}$ bond are also known, but can be more difficult to perform, ${ }^{13,14,15}$ owing to undesired eliminations and hydrodehalogenation reactions. ${ }^{16}$ The Harman group envisioned a complementary $\mathrm{C}_{\mathrm{sp} 2}-\mathrm{C}_{\mathrm{sp} 3}$ bond forming reaction in which $\left\{\mathrm{TpW}(\mathrm{NO})\left(\mathrm{PMe}_{3}\right)\right\}$ was used to activate an arene via dihapto coordination. Protonation of such an arene complex would create an electrophilic arenium species that could react with a second aromatic molecule through a Friedel-Crafts type reaction mechanism, and a subsequent deprotonation would regenerate the acid. As shown below, the product, after removal of the metal, would be a hydroarylated arene (Scheme 1).

\section{Scheme 1}
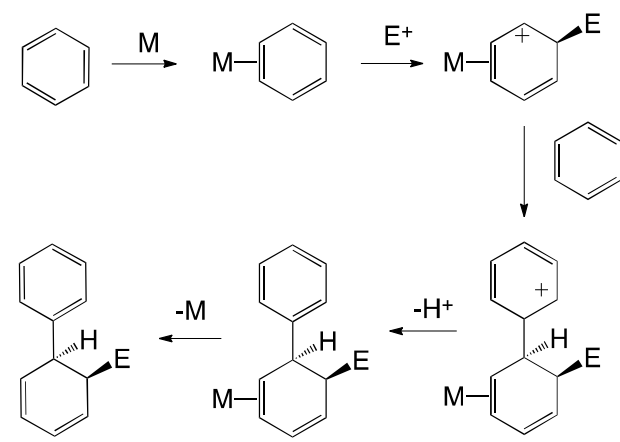


\section{Results and Discussion}

The naphthalene complex, $\mathrm{TpW}(\mathrm{NO})\left(\mathrm{PMe}_{3}\right)\left(3,4-\eta^{2}\right.$-naphthalene), 1, was synthesized as the precursor of the electrophilic reagent in our Friedel-Crafts reaction. This complex could be protonated to give $\mathbf{2}$, a transiently stable cation that could be isolated at ambient temperature. Proton NMR data for the naphthalenium ligand of complex $\mathbf{2}$ generally matched that of the $\operatorname{Re}$ analog, ${ }^{17}$ with the exception of $\mathrm{H} 4$, whose peak appears considerably more downfield for the W system owing to its " $\eta$-allyl" character. ${ }^{18}$ When naphthalene complex 1 was stirred in a $\mathrm{CHCl}_{3}$ solution of indole along with 0.1 equiv of the acid catalyst $\left[\mathrm{Ph}_{2} \mathrm{NH}_{2}\right] \mathrm{OTf}$ (DPhAT), the addition product 3 was obtained (Scheme 2). Similar results were observed with pyrrole to yield compound 4. While furan failed to react with naphthalene complex 1 under the conditions tested, 2-methyl- and 2,3-dimethylfuran were both sufficiently nucleophilic to undergo ringcoupling. The 2,3-dimethylfuran-derived product $\mathbf{5}$ was chosen as an example for full characterization. Parallel reactions with nucleophilic benzenes such as 1,3,5-trimethoxybenzene and resorcinol were not observed. These nucleophiles must not be sufficiently nucleophilic to react with $\mathbf{2}$ on the timescale for which $\mathbf{2}$ can survive. ${ }^{19}$ 
Scheme 2

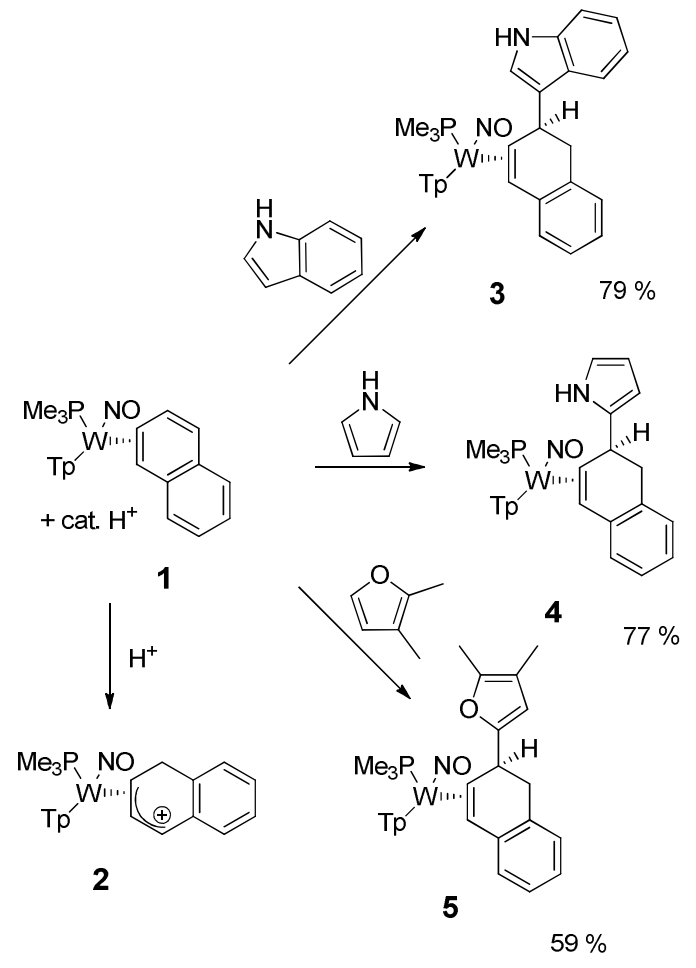

With regard to characterization of $\mathbf{3 - 5}$, the $\mathrm{H} 2$ signal showed a strong NOE interaction with the $\mathrm{PMe}_{3}$ ligand, which supports the assignment of nucleophilic addition anti to the metal fragment. Data from two dimensional NMR experiments indicated that the addition reactions to naphthalene 2 occurred in a 1,2-fashion, rather than the 1,4-addition occasionally observed with rhenium complexes. ${ }^{20}$ In the case of the pyrrole-derived product 4 , as well as the dimethylfuran analog 5, HMBC and NOE data, along with chemical shifts of the aromatic protons, confirm that the electrophilic addition occurs at the alpha-carbon of these heterocycles. $\mathrm{HMBC}, \mathrm{COSY}$, and $\mathrm{NOE}$ data further support the given structural and stereochemical assignments. ${ }^{21}$ A solid-state molecular structure determination for the indolyldihydronaphthalene $\mathbf{3}$ confirms that the addition of the indole occurs anti to the tungsten metal fragment (Figure 1). 
Figure 1 - Solid-state molecular structure of the indolyldihydronapthalene product 3.

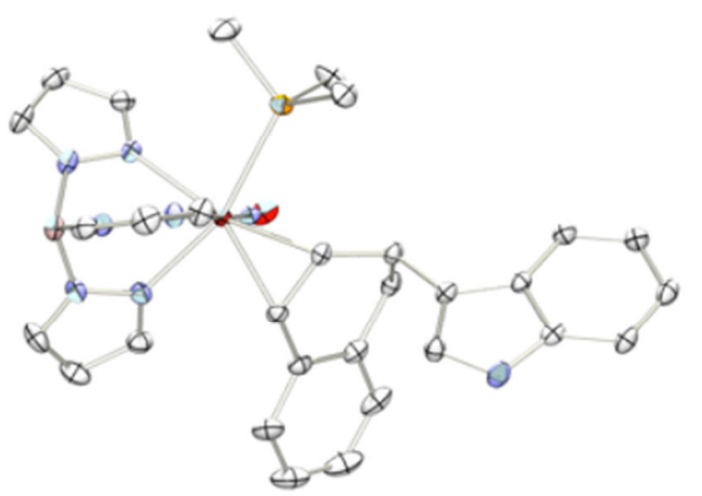

These dihydronaphthalene derivatives 3-5, can be readily oxidized in the presence of CAN. Treating 3-5 with one equivalent of CAN produced the organic products $6-8$ with yields of $61 \%, 28 \%$, and $47 \%$, respectively. NOE and COSY interactions between $\mathrm{H} 1$ and $\mathrm{H} 2$ of compounds 6-8 confirmed 1,2 addition in the liberated dihydronapthalenes (Scheme 3).

\section{Scheme 3}

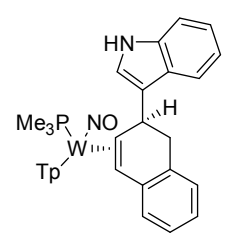

3

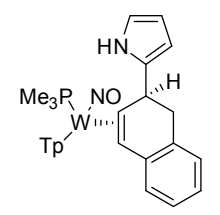

4

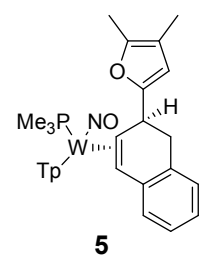

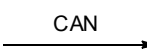

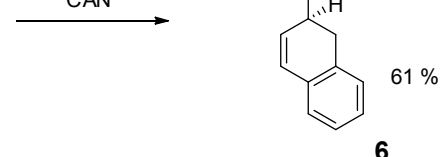

6

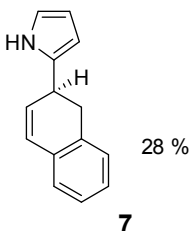

7

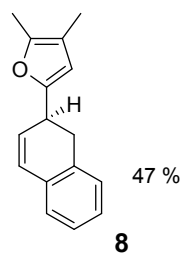

These products stand in contrast to the typical behavior of naphthalene. In most cases, naphthalene undergoes electrophilic substitution reactions preferentially at the 1-position. 
However, under thermodynamic control or in the presence of a bulky electrophile, 2substitution is preferred. ${ }^{22} \eta^{2}$-coordination of naphthalene with the $\mathrm{TpW}(\mathrm{NO})\left(\mathrm{PMe}_{3}\right)$ metal fragment allows for selective protonation at the 1-position, followed by nucleophilic addition to the 2-position. Of the organic complexes made through this strategy, only $\mathbf{7}$ has been previously synthesized: under photochemical conditions, pyrrole and naphthalene are reported to combine to produce 7 as one component of a complex mixture of products. ${ }^{23}$

Given the limited scope of aromatic nucleophiles that were found to react with $\mathbf{2}$, we sought to generalize the 1,2-tandem edition to 1 using a proton and other classes of nucleophiles. One nucleophile that was an appealing target was 1-methoxy-2-methyl-(1trimethylsilyloxy)propene (MMTP). An empirical model developed by Mayr and coworkers suggests that this nucleophile is significantly more nucleophilic than the aromatic nucleophiles tested. ${ }^{19}$ When 1 was quantitatively protonated and combined with MMTP, however, the starting material was recovered after work-up, indicating that deprotonation was favored over addition of the nucleophile (Scheme 4).

Scheme 4

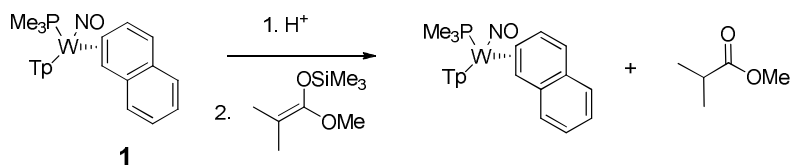

1

Similar results were observed upon the addition of nitrogen nucleophiles such as morpholine and aniline, where deprotonation occurred over addition. As deprotonation leads directly to a rearomatized arene in the case of $\operatorname{TpW}(\mathrm{NO})\left(\mathrm{PMe}_{3}\right)\left(3,4-\eta^{2}\right.$-naphthalene), we might expect that this complex is more susceptible to such a reaction pathway as compared with tungsten complexes of phenol or $\mathrm{N}, \mathrm{N}$-dimethylaniline, which exist as their $2 \mathrm{H}$-tautomers (the $N, N$-dimethylaniline being protonated). ${ }^{24}$ 
These two complexes, $\mathrm{TpW}(\mathrm{NO})\left(\mathrm{PMe}_{3}\right)\left(5,6-\eta^{2}-\right.$ phenol), 9, and $\left[\mathrm{TpW}(\mathrm{NO})\left(\mathrm{PMe}_{3}\right)\left(5,6-\eta^{2}-\right.\right.$ $\mathrm{N}, \mathrm{N}$-dimethylanilinium)] triflate, $\mathbf{1 0}$, also react with halogen electrophiles, including $\mathrm{N}$ chlorosuccinimide (NCS) and Selectfluor ${ }^{\mathrm{TM}}$, a mild fluorinating reagent, to generate electrophilic complexes that can undergo Friedel-Crafts reactions with aromatic nucleophiles. These complexes are interesting in that the introduction of a heteroatom to C3 in each complex creates a new stereocenter in which the halogen adds anti to the metal complex (for steric reasons). ${ }^{24}$ The aromatic nucleophile also adds anti to the metal for steric reasons, yielding a complex with $c i s-\gamma, \delta$-disubstitution patterns (Scheme 5). For 9, the nucleophile can be added directly following the completion of the halogen addition. For $\mathbf{1 0}$, the halogenated complex can be isolated via the addition of a methoxy group at C4. The addition of an acid would eliminate methanol, generating the electrophilic complex that reacted with the aromatic nucleophile. The chemistry of $\mathbf{9}$ was developed by Michael Todd and Victor Zottig. The chemistry of $\mathbf{1 0}$ was developed by Rebecca Salomon and Jared Pienkos.

\section{Scheme 5}

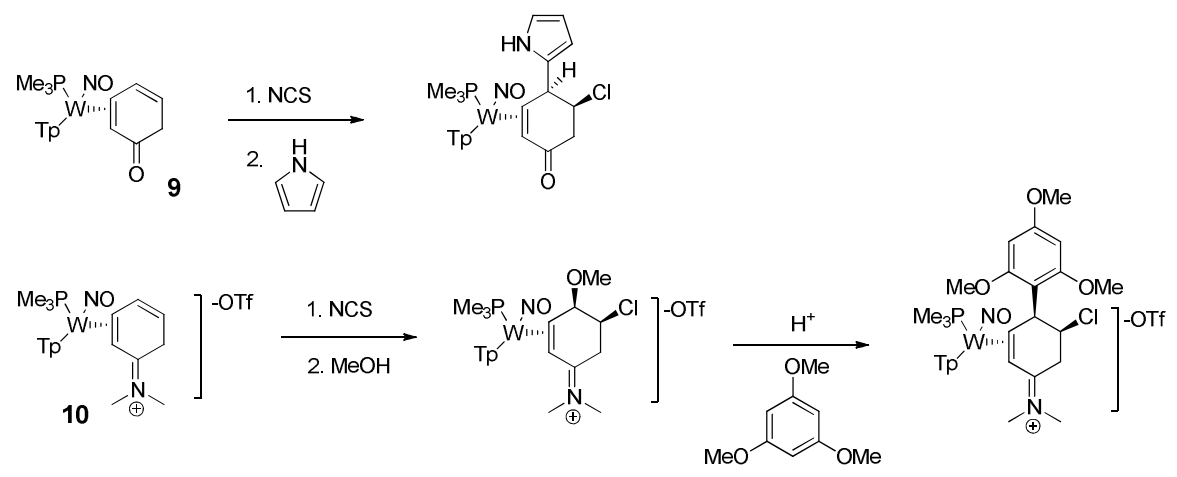

It was hoped that Selectfluor ${ }^{\mathrm{TM}}$, NCS, and other heteroatom electrophiles such as $\mathrm{N}$ bromosuccinimide (NBS) and epoxidizing reagents would react with 1 to expand its scope of possible functionalization. The initial reaction tried was a halogenation followed by addition of methanol to give a 1-halo-2-methoxydihydronaphthalene complex. When Selectfluor ${ }^{\mathrm{TM}}$ was used as the halogen source, no reaction was observed with $\mathbf{1}$. When NCS or NBS were used, 1 
decomposed rapidly. The reaction of $\mathbf{1}$ with $m$-chloroperoxybenzoic acid (mCPBA), an epoxidizing reagent, also gave decomposition of 1 . As NCS, NBS, and $m$ CPBA may all be thought of as oxidizing reagents, it is possible that the oxidation of $\mathbf{1}$ is competitive with or even preferred to the addition of the heteroatom electrophile.

\section{Tandem Additions to $\mathrm{TpW}(\mathrm{NO})\left(\mathrm{PMe}_{3}\right)\left(3,4-\eta^{2}\right.$-anthracene)}

We surveyed the scope of reactivity for $\operatorname{TpW}(\mathrm{NO})\left(\mathrm{PMe}_{3}\right)\left(3,4-\eta^{2}\right.$-anthracene), 11, with respect to protonation and addition of heteroatom electrophiles and found that it largely mirrored the reactivity observed for the naphthalene complex, $\mathbf{1}$. Complex $\mathbf{1 1}$ can be protonated quantitatively to give $\mathbf{1 2}$, which is not indefinitely stable, but is more stable than its naphthalenium analogue (Scheme 6). Aromatic nucleophiles which add to $\mathbf{2}$ could be added to 12, but unfortunately weaker nucleophiles would not react with 12 .

\section{Scheme 6}

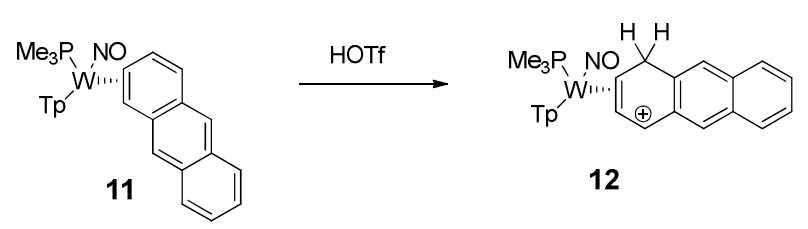

Reactions of $\mathbf{1 1}$ with Selectfluor ${ }^{\mathrm{TM}}$ and NCS did not yield promising results, with each reagent causing decomposition of 11. Surprisingly, when NBS (dissolved in acetonitrile) was added to $\mathbf{1 1}$ (dissolved in chloroform) a new complex formed, signaled by the formation of a dark red solution. This new species was first characterized by ${ }^{31} \mathrm{P}$ NMR spectroscopy, where it presented a signal at $-6.06 \mathrm{ppm}$ and ${ }^{31} \mathrm{P}-{ }^{183} \mathrm{~W}$ coupling constant of $262 \mathrm{~Hz}$. While the chemical shift of the new peak is close to that of $12(-6.52 \mathrm{ppm})$, the ${ }^{31} \mathrm{P}_{-}{ }^{183} \mathrm{~W}$ coupling constant of $\mathbf{1 2}$ is $271 \mathrm{~Hz}$. These coupling constants were sufficiently different for us to conclude that the product resulting from the mixture of $\mathbf{1 1}$ and NBS was not merely 12, which could have been formed from trace acid present. In order to collect ${ }^{1} \mathrm{H}$ NMR data an equivalent of NBS was added to 11 
in deuterated solvent. The product was tentatively assigned as $\mathbf{1 3}$ (Scheme 7). The upbound proton, $\mathrm{H} 2$, was identified at $4.52 \mathrm{ppm}$ and coupled to the downbound proton, $\mathrm{H} 3$, at $5.28 \mathrm{ppm}$. $\mathrm{H} 3$ coupled to $\mathrm{H} 4$, at $7.45 \mathrm{ppm}$, which is slightly downfield of the $\mathrm{H} 4$ signal for $\mathbf{1 2}$ (7.30 ppm). H2 showed a weak correlation to a singlet at $6.07 \mathrm{ppm}$, which is likely $\mathrm{H} 1$.

Scheme 7
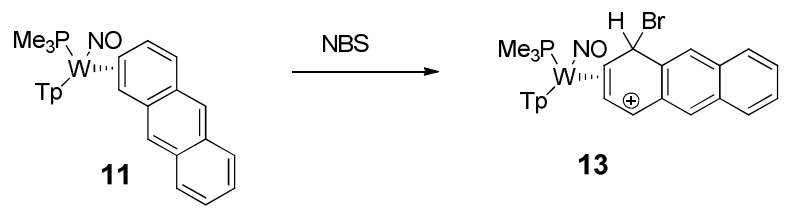

13

While NBS and 11 reacted at room temp, running the reaction at low temperature (-30 $-35^{\circ} \mathrm{C}$ ) yielded cleaner addition of the nucleophile. When the enolate of dimethylmalonate was prepared via deprotonation with lithium methoxide in methanol and added to a solution of 13 , a light yellow solution resulted. The new complex was isolated via an extraction and its identity determined using two dimensional ${ }^{1} \mathrm{H}$ NMR techniques. This complex had four doublets between $4.0 \mathrm{ppm}$ and $5.0 \mathrm{ppm}$ in its ${ }^{1} \mathrm{H}$ NMR spectrum. The proton of the bound double bond proximal to the $\mathrm{PMe}_{3}$ (upbound) was identified at $2.51 \mathrm{ppm}$. It coupled to the other proton of the bound double bond (distal to the $\mathrm{PMe}_{3}$ ligand, downbound), and had a weak correlation to one of the doublets, at $4.83 \mathrm{ppm}$. This doublet coupled to one other doublet, at $4.26 \mathrm{ppm}$. The downbound proton, at $1.50 \mathrm{ppm}$ had a weak correlation to another doublet, at $5.02 \mathrm{ppm}$. This doublet coupled to only one other signal, the last doublet, at $4.34 \mathrm{ppm}$. Additionally, there are four singlets between $3.3 \mathrm{ppm}$ and $3.9 \mathrm{ppm}$, which is consistent with the presence of four diastereotopic methoxy groups. The product was thus assigned as 14, a 1,4-addition of two equivalents of dimethylmalonate to $\mathbf{1 1}$ (Scheme 8). 
Scheme 8

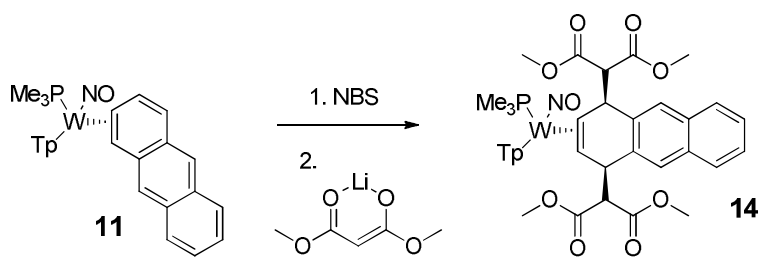

Other carbon nucleophiles were tried under these and similar conditions. Enolates of acetone and acetophenone gave decomposition and a mixture of products. Aromatic nucleophiles such as 1,3,5-trimethoxybenzene, 2,3-dimethylfuran, pyrrole, indole, and their $N$ methyl counterparts did not form appreciable amounts of product before decomposing. Triethylamine was added to help scavenge protons, but this did not improve the outcomes of carbon nucleophile additions.

While the isolation and characterization of products derived from other nucleophiles proved problematic due to the presence of multiple isomers or decomposition of the product, we found we were able to observe examples of additions similar to 14 with a variety of noncarbon nucleophiles. Lithium methoxide dissolved in methanol was added to 13 at $-30{ }^{\circ} \mathrm{C}$ and yielded one major product observable by ${ }^{31} \mathrm{P}$ NMR. Although an extraction caused isomerization or decomposition of some product, enough of the ${ }^{1} \mathrm{H}$ resonances of the major product could be identified using two dimensional NMR techniques to suggest that the product was a 1,4-dihydroanthracene. The upbound and downbound proton resonances on the bound double bond were located at $3.10 \mathrm{ppm}$ and $1.88 \mathrm{ppm}$, respectively. Each of these signals had a COSY interaction with a signal at $5.01 \mathrm{ppm}$ and $5.03 \mathrm{ppm}$, respectively. The locations of these methine protons' signals are similar to those observed for $\mathbf{1 4}$, and consistent with the downfield location of methine protons in $\left\{\mathrm{TpW}(\mathrm{NO})\left(\mathrm{PMe}_{3}\right)\right\}$ complexes of dihydronaphthalenes relative to free dihydronaphthalenes. Additionally, each methine signal displayed an NOE interaction with a singlet at $3.49 \mathrm{ppm}$ and $3.39 \mathrm{ppm}$, respectively, which is consistent with the incorporation of 
two methoxy groups into the complex, which may be assigned as 15 (Scheme 9). A similar procedure was followed in which imidazole added to 13 . COSY was used to assign the major product as a 1,4-dihydronaphthalene, 16, where the upbound and downbound proton resonances were located at $3.14 \mathrm{ppm}$ and $2.01 \mathrm{ppm}$, respectively. Each of these resonances had a COSY interaction with a methine proton at $6.26 \mathrm{ppm}$ and $6.28 \mathrm{ppm}$, respectively. Two unique sets of imidazole protons could be identified (at 6.77, 6.97, and 7.63 ppm; and 6.72, 6.91, and $7.35 \mathrm{ppm})$, although there was not enough information to place the rings in either position on the anthracene ring. These methine protons may appear downfield of those for $\mathbf{1 5}$ due to anisotropic effects from the imidazole rings. It should be noted that imidazole does not add to the complex 13 without the addition of triethylamine to the reaction. Triethylamine itself does not react with 13, making it an ideal base to assist in generating nucleophiles.

\section{Scheme 9}
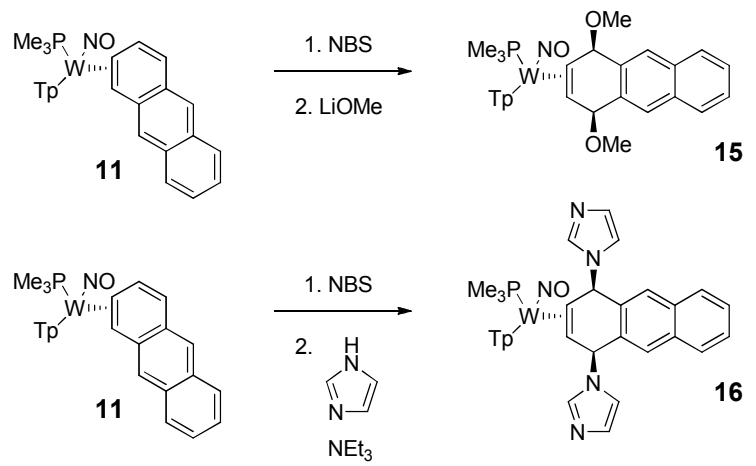

When propylamine was added to 13 a ${ }^{31} \mathrm{P}$ NMR signal for a major product was observed at $-10.72 \mathrm{ppm}$ with a ${ }^{31} \mathrm{P}_{-}{ }^{183} \mathrm{~W}$ coupling constant of $269 \mathrm{~Hz}$. When allowed to stand at room temperature, however, a new signal grew in at $-11.29 \mathrm{ppm}$ with a ${ }^{31} \mathrm{P}_{-}{ }^{183} \mathrm{~W}$ coupling constant of $278 \mathrm{~Hz}$. In an attempt to observe the initial product, an extraction was performed on a fresh sample very soon after the addition of propylamine to 13 . The major product comprised about $75 \%$ of the mixture observed in the ${ }^{1} \mathrm{H}$ NMR spectrum. The connectivity of the product as determined from COSY is most consistent with a 1,2-addition product. The upbound and 
downbound protons were identified at $3.23 \mathrm{ppm}$ and $2.25 \mathrm{ppm}$, respectively. The upbound proton displayed a weak interaction with a doublet at $5.61 \mathrm{ppm}$, which displayed a strong interaction with a doublet at $5.13 \mathrm{ppm}$. A pair of multiplets, each of which integrated to one proton, at 3.60 and $3.88 \mathrm{ppm}$, displayed a strong correlation with each other, as well as a multiplet at $1.89 \mathrm{ppm}$, which integrated to two protons. This multiplet signal correlated to a triplet at $1.06 \mathrm{ppm}$. Significantly, only one propyl group could be identified in the ${ }^{1} \mathrm{H}$ NMR spectrum. This propyl group did not display any correlation to any of the peaks identified on the anthracene ring, but the presence of diastereotopic protons indicates that the group is near an asymmetric feature, consistent with an attachment to the tungsten complex. This complex was not further pursued due to the instability of the product during the workup, but might be tentatively identified as 17 (Scheme 10).

Scheme 10

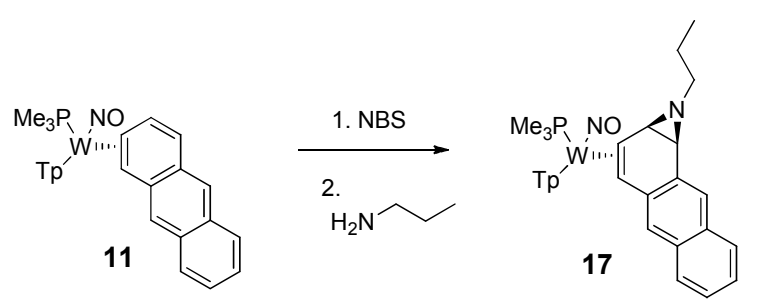

Aniline reacted with 13 to yield a product that was consistent with a 1,2-addition product that incorporated two equivalents of aniline. Interestingly, the ${ }^{31} \mathrm{P} N \mathrm{NM}$ spectrum taken soon after the addition of aniline and triethylamine showed two major products and a small amount of decomposition. Over the course of a week one of the products disappeared, giving a single product peak. An extraction yielded $\mathbf{1 8}$ in approximately $85 \%$ purity (Scheme 11). The upbound and downbound proton signals were identified at $3.02 \mathrm{ppm}$ and $2.30 \mathrm{ppm}$. The upbound proton displayed a small NMR correlation with a doublet at $4.63 \mathrm{ppm}$, which displays a strong interaction with a doublet at $5.50 \mathrm{ppm}$. Each of these doublets correlated to a signal at $3.67 \mathrm{ppm}$ and $4.69 \mathrm{ppm}$, respectively, and each of these signals correlated to NMR signals in the 
aromatic region of the spectrum. These correlations are consistent with the 1,2-addition of two equivalents of aniline where the aniline rings are syn to each other. The methine protons on the anthracene ring both display NOE interactions to the $\mathrm{PMe}_{3}$ signal, indicating that the additions are anti to the metal complex.

\section{Scheme 11}
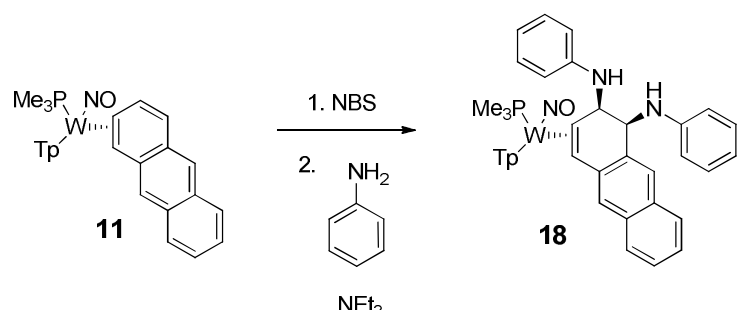

$\mathrm{NEt}_{3}$

The reaction of 13 with thiophenol followed the pattern observed with aniline. The ${ }^{31} \mathrm{P}$ NMR spectrum of the reaction solution taken after the sample was removed from the cold bath showed a number of signals. After several days at room temperature, however, one major signal predominated and the reaction was extracted. The mixture contained $\sim 13 \% 11$ by NMR as well as a product that was spectroscopically similar to $\mathbf{1 8}$. Free anthracene and free thiophenol could also be seen in the ${ }^{1} \mathrm{H}$ NMR spectrum, however, these did not obscure the complex peaks (excepting those of any bound thiophenol rings). The upbound and downbound protons were identified at $3.10 \mathrm{ppm}$ and $2.31 \mathrm{ppm}$, respectively. The upbound proton displayed a slight correlation to a signal at $4.60 \mathrm{ppm}$, which had a strong correlation to a proton at 5.53 ppm, consistent with a 1,2-addition product, 19 (Scheme 12). All the anthracene ring protons and thiophenol protons could be identified using two dimensional NMR analysis, and an integration of aromatic region is consistent with the presence of two thiophenol rings in the complex. We expected that the methine protons $\mathrm{H} 1$ and $\mathrm{H} 2$ would display NOE interactions with the ortho protons of the thiophenol rings. Indeed, the $\mathrm{H} 1$ signal, at $5.53 \mathrm{ppm}$, displayed an interaction to a signal at $7.76 \mathrm{ppm}$, while the $\mathrm{H} 2$ signal, at $4.60 \mathrm{ppm}$, displayed an NOE interaction to a signal at $7.71 \mathrm{ppm}$. The rest of the thiophenol ring protons appeared to be at 
$7.28 \mathrm{ppm}$ (meta) and $7.15 \mathrm{ppm}$ (para), according to COSY interactions, but individual resonances were impossible to identify due to signal overlap and an excess of thiophenol.

Scheme 12

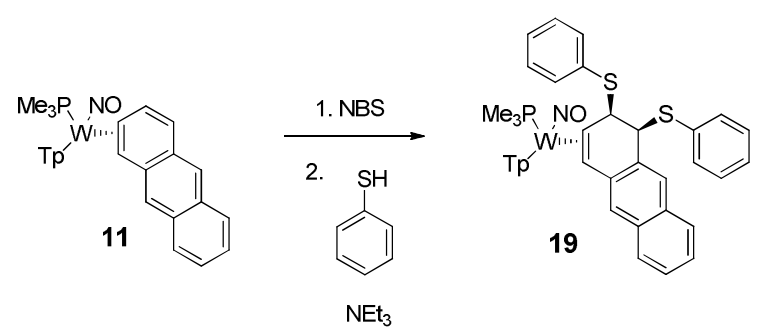

What is interesting about products $14-19$ is that for all of them except 17 , two dimensional NMR spectroscopy allows us to distinguish two unique equivalents of the nucleophile that was added to 13 as functional groups now present in the product. Given that the reaction is designed as a tandem electrophile-nucleophile addition to $\mathbf{1 1}$, where the electrophile is $N$-bromosuccinimide and the nucleophile is variable, the obvious and compelling question that arises from these observations is "where did the bromide go?" We considered that in adding a nucleophile to 13 , we should generate a neutral species with a bromide at C1. This bromide could be susceptible to being lost, as its loss would generate a cation, 20, with both allylic and benzylic stabilization provided from the metal complex and anthracene ring, respectively (Scheme 13). Once the bromide is lost, a second nucleophile could add to the complex to generate a neutral species.

\section{Scheme 13}

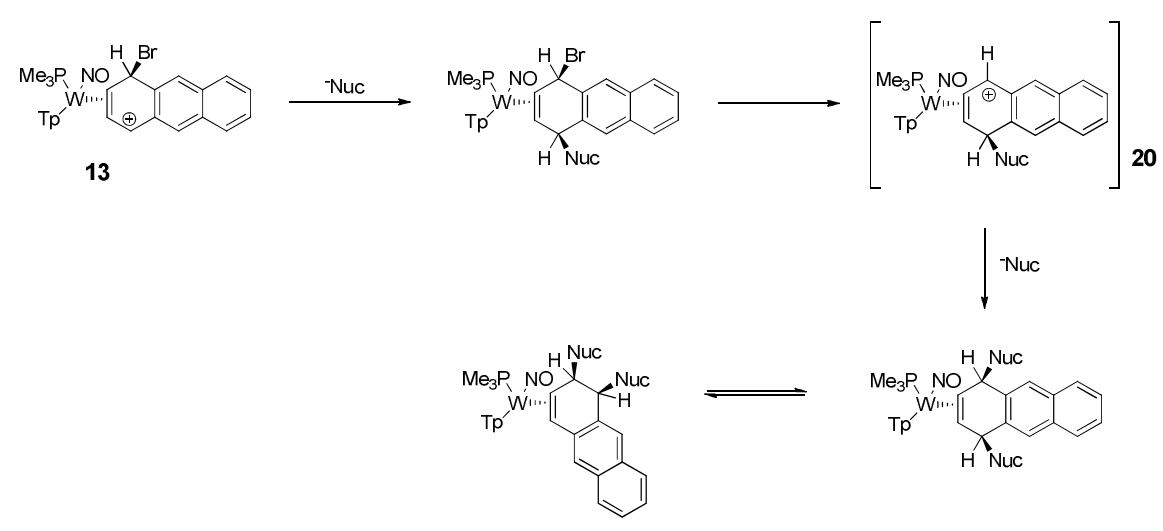


To explore the feasibility of losing bromide from a neutral complex, we used Gaussian to calculate an optimized structure of 21, Figure 2, the 1,4-bromo intermediate through which 16 must be obtained under this mechanism (Scheme 14).

Scheme 14

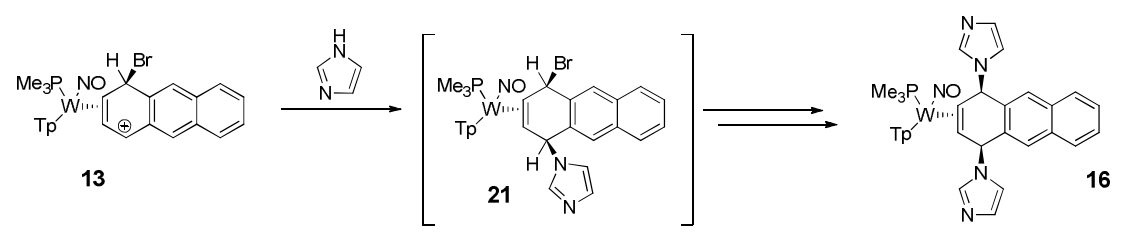

The carbon-bromide bond length in the optimized structure was calculated as $2.32 \AA$, which is significantly longer than a typical carbon-bromide bond (1.9 $)$. This suggests that the bond is weaker in $\mathbf{2 1}$ relative to the typical $\mathrm{C}-\mathrm{Br}$ bond, which is consistent with our proposed mechanism, where the bromide is easily lost.

Figure 2 - Gaussian structure of $\mathrm{TpW}(\mathrm{NO})\left(\mathrm{PMe}_{3}\right)\left(2,3-\eta^{2}-1-((1 \mathrm{~S}, 4 \mathrm{R})-4-\right.$ bromo-1,4-dihydroanthracen-1-yl)-1H-imidazole, 21. Rendered in Gaussview.

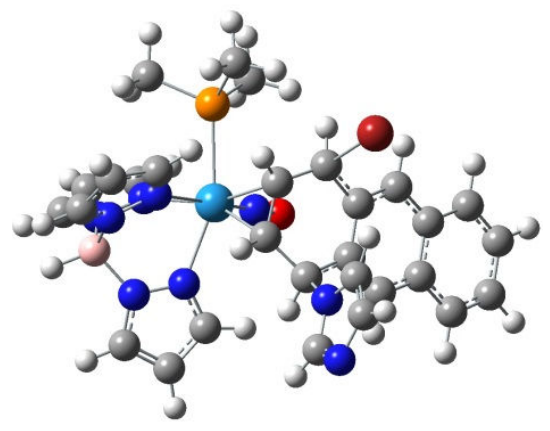

We hoped that $\mathbf{2 0}$ would be sufficiently stable such that if exactly one equivalent of a nucleophile was added to a solution of $\mathbf{1 3}$, a different nucleophile could be added afterward to give us a variety of dihydroanthracenes with mix-and-matched nucleophiles. Unfortunately, all reactions run with pairs of nucleophiles gave product mixtures, regardless of which nucleophile was added first. Indeed, our inability to isolate many of $\mathbf{1 4}-\mathbf{1 9}$ cleanly suggests that $\mathbf{2 0}$ is not stable enough to be resistant to side reactions. 
In the case of 17, the NMR data suggests there is only one equivalent of propylamine present. The addition product was assigned as $\mathbf{1 7}$ as opposed to a product that still contains a bromide, as our observation over the range of nucleophiles added to 13 was that the bromide was labile. While we can't rule out a bromo/amine product such as $\mathbf{2 2}$, if an amine proton was present (Scheme 15), it should couple to its neighbors ( $\mathrm{H} 2$ or the propyl protons), and no such coupling is observed. This observation does not support the existence of a structure like 22.

Scheme 15
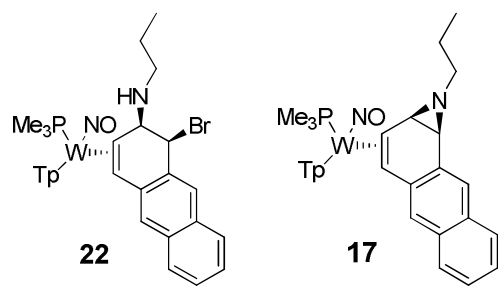

One peculiarity of this tandem reaction is that some nucleophiles added to $\mathbf{1 3}$ lead to 1,4-dihydroanthracene products, while others lead to 1,2-dihydroanthracene products. Of note, the reactions for which 1,2-addition is observed usually begin as mixtures, as observed by ${ }^{31} \mathrm{P}$ NMR, which are allowed to stand at room temperature for a couple of days, after which they contain one major product. Attempts to isolate the product of $\mathbf{1 3}$ and aniline very soon after the aniline was added to the reaction resulted in isolation of the 1,2-addition product. The work described in chapter 2 showed that for cationic allylic complexes of $\left\{\mathrm{TpW}(\mathrm{NO})\left(\mathrm{PMe}_{3}\right)\right\}$ the allylic terminus opposite the $\mathrm{PMe}_{3}$ ligand bears much more of the positive charge than the terminus proximal to the $\mathrm{PMe}_{3}$, making it the kinetically-preferred location for nucleophilic attack. Attack at this carbon would generate a 1,4-addition product (Scheme 16). 


\section{Scheme 16}
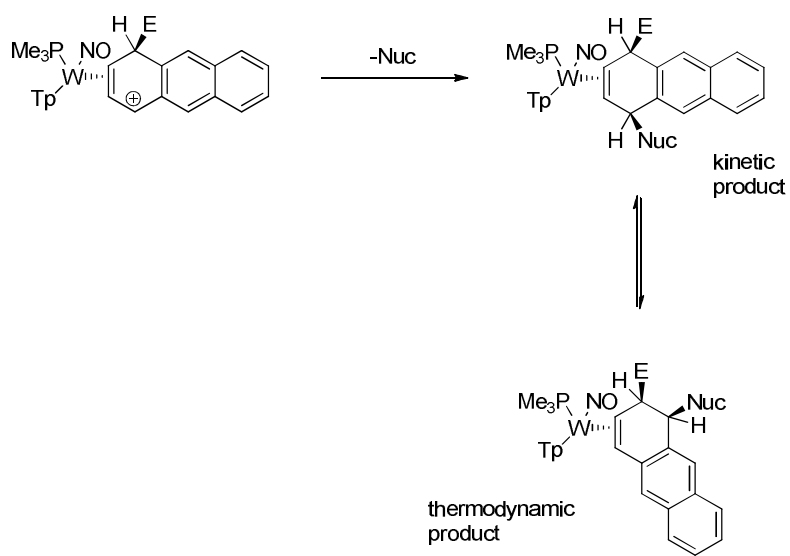

However, for the Friedel-Crafts types additions described in the previous section, only 1,2addition products are observed for $\operatorname{TpW}(\mathrm{NO})\left(\mathrm{PMe}_{3}\right)\left(3,4-\eta^{2}\right.$-naphthalene), indicating that the thermodynamic product is accessible at room temperature for this system. It seems reasonable that once the $\mathrm{N}$-bromosuccinimide/nucleophile reactions are completed at $-30{ }^{\circ} \mathrm{C}$, warming them up to room temperature would make isomerization from a 1,4-addition product to a 1,2addition product possible. In our attempts to isolate a 1,4-addition product from $\mathbf{1 3}$ and aniline, an aqueous extraction was performed, providing acidic protons that could quickly facilitate an isomerization that would otherwise take a couple of days.

A few examples exist in the literature in which a transition metal complex is used to facilitate a double nucleophilic addition to an arene. Manganese tricarbonyl complexes of arenes are sufficiently electrophilic to undergo successive nucleophilic additions (although the choice of the first nucleophile is limited) to yield substituted 1,3 -cyclohexadienes. ${ }^{25}$ Another technique by which two nucleophiles can be added to an arene- $\mathrm{Mn}(\mathrm{CO})_{3}$ complex involves adding nitrosonium hexafluorophosphate to 'reactivate' the manganese complex after the first nucleophile is added. ${ }^{26}$ One report from Pearson et al., found that an amine-substituted $\eta^{6}$ benzene complex of $\mathrm{Mn}(\mathrm{CO})_{3}$ could undergo a nucleophilic addition of organomagnesium or organolithium reagents to give an $\eta^{5}$-arene complex. ${ }^{2} \quad \mathrm{NOPF}_{6}$ was added to give the 
corresponding $\mathrm{Mn}(\mathrm{CO})_{2}(\mathrm{NO})$ reagent, after which sodium borohydride could be added as the second nucleophile. The organic was recovered as a 5-substituted cyclohex-2-en-1-one (Scheme 17).

Scheme 17

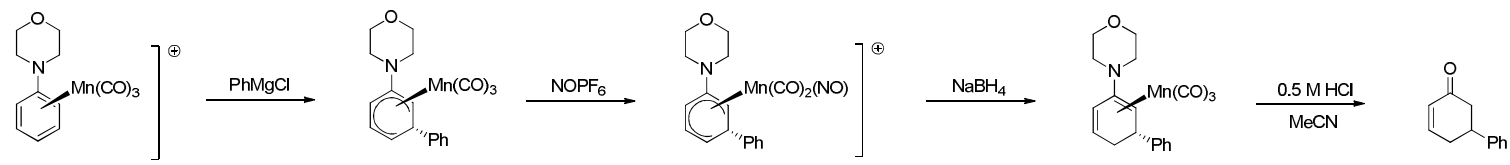

In another example, Pigge et al. developed CpRu(II) complexes of arenes with $\beta$-amido phosphonate groups which could undergo spirocyclizations upon deprotonation. ${ }^{27}$ After further functionalization, the ruthenium complex could be oxidized in a nucleophilic solvent (water or methanol) to release the spirocycle as part of a cyclohexadiene incorporating a methoxy or hydroxyl group (Scheme 18).

\section{Scheme 18}

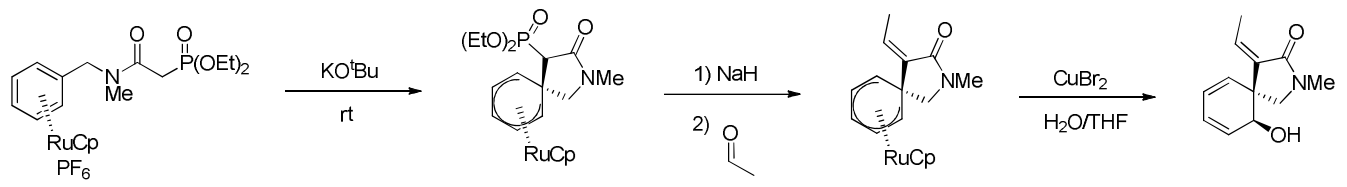

The double nucleophilic additions observed from $\operatorname{TpW}(\mathrm{NO})\left(\mathrm{PMe}_{3}\right)\left(3,4-\eta^{2}\right.$-anthracene) and $\mathrm{N}$-bromosuccinimide stands apart from these examples because the "activating group" adds to the arene before the first nucleophile, and its loss generates the active complex which can react with the second nucleophile. Reactions on anthracene, especially on the terminal ring, are possible when it is complexed to $\left\{\mathrm{TpW}(\mathrm{NO})\left(\mathrm{PMe}_{3}\right)\right\}$, unlike when it is complexed to $\mathrm{Mn}(\mathrm{CO})_{3}$, on which the anthracene is prone to slipping off. ${ }^{28,29}$ While the double nucleophilic addition appears promising for a nice range of nucleophiles, the fact that the first and second nucleophiles must be the same is a limitation of this method for anthracene functionalization. 


\section{Conclusion}

The exploration of the reactivity of $\operatorname{TpW}(\mathrm{NO})\left(\mathrm{PMe}_{3}\right)\left(3,4-\eta^{2}\right.$-naphthalene) and $\operatorname{TpW}(\mathrm{NO})\left(\mathrm{PMe}_{3}\right)\left(3,4-\eta^{2}\right.$-anthracene) with noncarbon electrophiles yielded insight into the range of products possible, as well as insight into the reaction pathways those reactions must follow. $\operatorname{TpW}(\mathrm{NO})\left(\mathrm{PMe}_{3}\right)\left(3,4-\eta^{2}\right.$-naphthalene) reacted with a proton to generate a transiently stable allylic complex to which a narrow range of aromatic nucleophiles could be added. The organic fragments could be easily decomplexed to give 1,2-dihydronaphthalenes in modest yields. The naphthalene complex decomposed or did not react with other noncarbon electrophiles such as $\mathrm{N}$-chlorosuccinimide and $\mathrm{N}$-bromosuccinimide. Its anthracene analog was found to react with $\mathrm{N}$-bromosuccinimide in a surprising fashion, where it facilitated a net double nucleophilic addition to generate 1,2- or 1,4-dihydroanthracene complexes. The instability of the majority of these complexes hindered their full characterization.

\section{Experimental Section.}

General Methods. NMR spectra were obtained on either a 300, 500, or $600 \mathrm{MHz}$ spectrometer (Varian INOVA or Bruker Avance). All chemical shifts are reported in ppm. Proton and carbon shifts are referenced to tetramethylsilane (TMS) utilizing residual $1 \mathrm{H}$ or $13 \mathrm{C}$ signals of the deuterated solvent as an internal standard. Phosphorus NMR signals are referenced to $85 \%$ H3PO4 ( $\delta) 0.00$ ppm using a triphenylphosphate external standard in acetone ( $\delta=-16.58 \mathrm{ppm})$. Coupling constants $(\mathrm{J})$ are reported in hertz $(\mathrm{Hz})$. Infrared (IR) spectra were recorded on a MIDAC Prospect Series (model PRS) spectrometer as a glaze on a horizontal attenuated total reflectance (HATR) accessory (Pike Industries). Electrochemical experiments were performed under a dinitrogen atmosphere using a BAS Epsilon EC-2000 potentiostat. Cyclic voltammetry data were taken at ambient temperature at $100 \mathrm{mV} / \mathrm{s}$ in a standard three-electrode cell with a 
glassy carbon working electrode using tetrabutylammonium hexafluorophosphate (TBAH) as an electrolyte [approximately $0.5 \mathrm{M}$ in dimethylacetamide (DMA)] unless otherwise noted. All potentials are reported versus the normal hydrogen electrode (NHE) using cobaltocenium hexafluorophosphate $(E 1 / 2=-0.78 \mathrm{~V})$, ferrocene $(E 1 / 2=+0.55 \mathrm{~V})$, or decamethylferrocene $(\mathrm{E} 1 / 2$ $=+0.04 \mathrm{~V})$ as an internal standard. The peak-to-peak separation was $100 \mathrm{mV}$ or less for all reversible couples. High-resolution electrospray ionization mass spectrometry (ESI-MS) analyses were obtained on a Bruker BioTOF-Q instrument running in ESI mode from samples dissolved in 1:3 water/acetonitrile solution containing sodium trifluoroacetate ( $\mathrm{NaTFA}$ ), and using $[\mathrm{Na}(\mathrm{NaTFA}) \mathrm{x}]+$ clusters as an internal standard. For metal complexes, these data are reported using the five most intense peaks from the isotopic envelope for either $M+$ (for monocationic complexes) or for $[\mathrm{M}+\mathrm{H}]+$ or $[\mathrm{M}+\mathrm{Na}]+$ (for neutral complexes). The data are listed as $\mathrm{m} / \mathrm{z}$ with the intensity relative to the most abundant peak of the isotopic envelope given in parentheses for both the calculated and observed peaks. The difference between calculated and observed peaks is reported in ppm. For organic species, the calculated and observed peaks for $[M+H]+$ or $[\mathrm{M}+\mathrm{Na}]+$ are reported, with the difference between them reported in ppm. LRMS data was acquired on a Shimadzu G-17A/QP-5050 GC-MS instrument operating either in GC-MS or in direct inlet/MS mode. Mass spectra are reported as $M+$ for neutral or monocationic samples. In all cases, observed isotopic envelopes were consistent with the molecular composition reported. The data are listed as $\mathrm{m} / \mathrm{z}$ with the intensity relative to the most abundant peak of the isotopic envelope given in parentheses for both the calculated and observed peaks. 


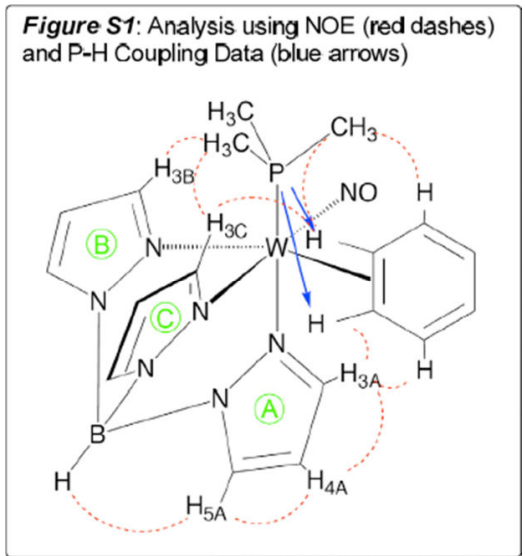

DFT Calculations. Initial structures were built in GAUSSVIEW (5.0.8) with the PM6 semi-empirical method in GAUSSIAN 09. These structures were refined stepwise in Gaussian using B3LYP and a series of basis functions incorporating LANL2 pseudopotentials and associated basis functions provided in those packages or directly from the PM6 structures. The most demanding calculations reported here put the LANL2DZ pseudopotential and its basis only on the W atom, and used the 6-31G(d) basis for all other atoms.

For transition state structures, vibrational analysis revealed the presence of a single imaginary frequency. In all other cases, vibrational analyses verified that optimized structures were located at local minima, with the presence of only real frequencies.

Synthesis and characterization for $\operatorname{TpW}(\mathrm{NO})\left(\mathrm{PMe}_{3}\right)\left(\eta^{2}\right.$-naphthalene) $\quad(\mathbf{1})^{21}$ and $\operatorname{TpW}(\mathrm{NO})\left(\mathrm{PMe}_{3}\right)\left(\eta^{2}\right.$-anthracene) $(\mathbf{1 1})^{30}$ have been previously reported.

$\mathrm{TpW}(\mathrm{NO})\left(\mathrm{PMe}_{3}\right)\left(2,3-\eta^{2}-1 H\right.$-naphthalenium) triflate (2)

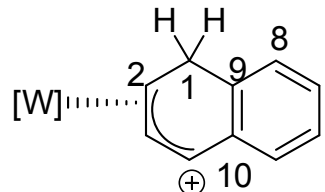


Triflic acid (22 mg, $0.147 \mathrm{mmol})$ in $\mathrm{CHCl}_{3}(1.52 \mathrm{~g})$ was added to $1(51 \mathrm{mg}, 0.081 \mathrm{mmol})$. The resulting orange solution was precipitated over stirring ether $(16 \mathrm{~mL})$ and filtered through a 15 $\mathrm{mL}$ medium porosity fritted funnel to give 2 as an orange solid (53 $\mathrm{mg}, 84 \%$ ).

${ }^{1} \mathrm{H}$ NMR $\left(\mathrm{CDCl}_{3}\right): \delta 8.24(\mathrm{~d}, J=2.0,1 \mathrm{H}, \mathrm{Pz} 3 \mathrm{C}), 8.06(\mathrm{~d}, J=2.0,1 \mathrm{H}, \mathrm{Pz} 3 \mathrm{~B}), 8.04(\mathrm{~d}, J=2.0,1 \mathrm{H}$, Pz3A), 7.89 (d, $J=2.0,1 \mathrm{H}, \mathrm{Pz} 5 \mathrm{C}), 7.81(\mathrm{~d}, J=2.0,1 \mathrm{H}, \mathrm{Pz5B}), 7.72(\mathrm{~d}, J=2.0,1 \mathrm{H}, \mathrm{Pz} 5 \mathrm{~A}), 7.39$ (d, $J=$ 7.5, 1H, H5), $7.29(\mathrm{~m}, 1 \mathrm{H}, \mathrm{H} 6), 7.17(\mathrm{~m}, 1 \mathrm{H}, \mathrm{H} 7), 7.16(\mathrm{~m}, 1 \mathrm{H}, \mathrm{H} 8), 6.71(\mathrm{~d}, \mathrm{~J}=7.2,1 \mathrm{H}, \mathrm{H} 4), 6.59(\mathrm{t}$, $J=2.0,1 \mathrm{H}, \mathrm{Pz} 4 \mathrm{C}), 6.39(\mathrm{t}, J=2.0,1 \mathrm{H}, \mathrm{Pz} 4 \mathrm{~B}), 6.34(\mathrm{t}, J=2.0,1 \mathrm{H}, \mathrm{Pz} 4 \mathrm{~A}), 5.12(\mathrm{dd}, J=20.7,5.6,1 \mathrm{H}$, H1), $5.04(\mathrm{~m}, 1 \mathrm{H}, \mathrm{H} 2), 4.95(\mathrm{t}, J=7.3,1 \mathrm{H}, \mathrm{H} 3), 3.83\left(\mathrm{~d}, J=20.5,1 \mathrm{H}, \mathrm{H} 1^{\prime}\right), 1.23(\mathrm{~d}, J=9.4,9 \mathrm{H}$, $\left.\mathrm{PMe}_{3}\right) .{ }^{13} \mathrm{C}$ NMR $\left(\mathrm{CDCl}_{3}\right): \delta 147.09$ (Pz3A), 144.56 (Pz3B), 143.20 (Pz3C), 138.38 (Pz5C), 138.35 (Pz5A), 138.17 (Pz5B), 136.61 (C9 or C10), 132.86 (C9 or C10), 131.25 (C7), 130.46 (C4), 129.73 (C5), 128.10 (C8), 126.88 (C6), 109.02 (Pz4C), 108.47 (Pz4B), 107.39 (Pz4A), 96.55 (C3), 72.15 (C2), 33.17 (C1), 12.85 (d, J = 32, $\mathrm{PMe}_{3}$ ).

\section{$\operatorname{TpW}(\mathrm{NO})\left(\mathrm{PMe}_{3}\right)\left(3,4-\eta^{2}\right.$-(3-(1,2-dihydronaphthalen-2-yl)-1H-indole)) (3)}

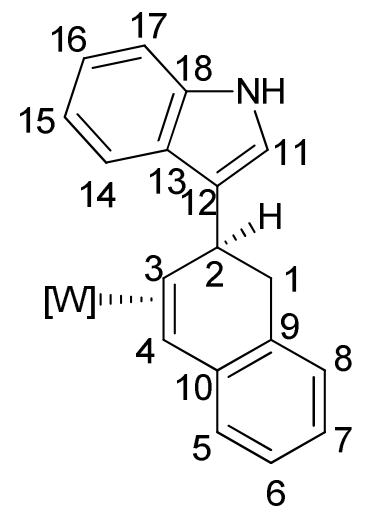

1 (201 mg, $0.319 \mathrm{mmol}$ ), indole (178 mg, $1.521 \mathrm{mmol}$ ), and diphenylammonium triflate (DPhAT) (10 $\mathrm{mg}, 0.031 \mathrm{mmol})$ were weighed into a 4-dram vial. $\mathrm{CHCl}_{3}(4.982 \mathrm{~g})$ was added to the vial, and the reaction mixture was stirred for a week. $\mathrm{Et}_{2} \mathrm{O}(5 \mathrm{~mL})$ was added to precipitate a light beige precipitate, which was filtered on a $15 \mathrm{~mL}$ fine-porosity fritted funnel as 3 (188 $\mathrm{mg}, 0.252$ $\mathrm{mmol}, 79 \%)$. 
${ }^{1} \mathrm{H}$ NMR (d $\mathrm{d}^{6}$-acetone): $\delta 9.67(\mathrm{~s}, 1 \mathrm{H}, \mathrm{NH}), 8.15(\mathrm{~d}, 1 \mathrm{H}, J=2.0, \mathrm{Pz} 3 \mathrm{~B}), 8.01(\mathrm{~d}, 1 \mathrm{H}, J=2.0, \mathrm{Pz} 5 \mathrm{C})$, $7.96(\mathrm{~d}, 1 \mathrm{H}, J=2.0, \mathrm{Pz} 5 \mathrm{~B}), 7.86(\mathrm{~d}, 1 \mathrm{H}, J=2.0, \mathrm{Pz} 5 \mathrm{~A}), 7.79(\mathrm{~d}, 1 \mathrm{H}, J=7.6, \mathrm{H} 11), 7.71(\mathrm{~d}, 1 \mathrm{H}, J=$ 2.0, Pz3C), $7.39(\mathrm{~d}, 1 \mathrm{H}, J=\mathrm{Pz} 3 \mathrm{~A}), 7.35(\mathrm{~d}, 1 \mathrm{H}, \mathrm{J}=7.9, \mathrm{H} 14), 7.08(\mathrm{~m}, 1 \mathrm{H}, \mathrm{H} 15), 7.05(\mathrm{~m}, 1 \mathrm{H}, \mathrm{H} 17)$, $7.02(\mathrm{~m}, 1 \mathrm{H}, \mathrm{H} 16), 6.95(\mathrm{t}, 1 \mathrm{H}, J=7.5, \mathrm{H} 6), 6.76(\mathrm{~d}, 1 \mathrm{H}, J=7.3, \mathrm{H} 8), 6.68(\mathrm{t}, 1 \mathrm{H}, J=7.3, \mathrm{H} 7), 6.61$ $(\mathrm{d}, 1 \mathrm{H}, J=7.6, \mathrm{H} 5), 6.41(\mathrm{t}, 1 \mathrm{H}, J=2.0, \mathrm{Pz} 4 \mathrm{~B}), 6.36(\mathrm{t}, 1 \mathrm{H}, J=2.0, \mathrm{Pz} 4 \mathrm{C}), 6.21(\mathrm{t}, 1 \mathrm{H}, J=2.0, \mathrm{Pz} 4 \mathrm{~A})$, $4.59(\mathrm{~d}, 1 \mathrm{H}, J=6.1, \mathrm{H} 2), 3.74(\mathrm{dd}, 1 \mathrm{H}, J=6.2,15.4 \mathrm{H} 1), 3.30(\mathrm{dd}, 1 \mathrm{H}, J=10.2,12.6, \mathrm{H} 3), 2.66$ (d, $\left.1 \mathrm{H}, J=15.8, H 1^{\prime}\right), 2.24(\mathrm{dd}, 1 \mathrm{H}, J=1.8,10.2, \mathrm{H} 4), 1.39\left(\mathrm{~d}, 9 \mathrm{H}, J=8.3, \mathrm{PMe}_{3}\right) .{ }^{13} \mathrm{C} \mathrm{NMR}\left(\mathrm{d}^{6}-\right.$ acetone): $\delta 146.7$ (s, C10), 144.5 (s, Pz3A), 144.3 (s, Pz3B), 142.0 (s, Pz3C), 138.0 (s, Pz5C), 137.8 (s, C18), 137.6 (s, C13), 137.2 (s, Pz5B), 136.7 (s, Pz5A), 133.9 (s, C9), 129.6 (s, C12), 129.6 (s, C8), 129.5 (s, C5), 124.7 (s, C6), 123.4 (s, C16), 123.2 (s, C7), 121.8 (s, C15), 119.5 (s, C11), 119.4 (s, C17), 112.2 (s, C14), 107.4 (s, Pz4B), 107.2 (s, Pz4C), 106.0 (s, Pz4A), 63.9 (s, C3), 55.1 (s, C4), $37.9(\mathrm{~s}, \mathrm{C} 2), 36.0(\mathrm{~s}, \mathrm{C} 1), 13.5\left(\mathrm{~d}, J=28, \mathrm{PMe}_{3}\right) .{ }^{31} \mathrm{P} \mathrm{NMR}\left(\mathrm{d}^{6}\right.$-acetone): $\delta-8.62\left(J_{\mathrm{P}-\mathrm{w}}=281 \mathrm{~Hz}\right) . \mathrm{CV}$ $(D M A): E_{p, a}=+0.488$ V. IR: $v_{\text {NO }}=1550 \mathrm{~cm}^{-1}$. HRMS $(M+N a)^{+}$obs'd $(\%)$, calc'd (\%), ppm: 769.20481 (83.1), 769.20715 (80.1), -3; 770.2098 (81.3), 770.20965 (81.8), 0.2; 771.20731 (100), 771.20967 (100), -3.1; 772.21193 (50.7), 772.21345 (48.7), -2; 773.20883 (76.2), 773.21287 (81.9), -5.2.

\section{$\operatorname{TpW}(\mathrm{NO})\left(\mathrm{PMe}_{3}\right)\left(3,4-\eta^{2}-(2-(1,2-\right.$ dihydronaphthalen-2-yl)-1H-pyrrole)) (4)}

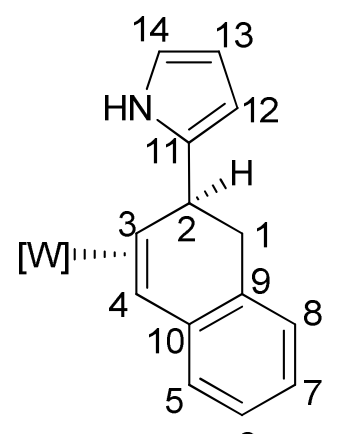

6

1 (150 mg, $0.238 \mathrm{mmol}$ ) and camphorsulfonic acid (15 mg, $0.065 \mathrm{mmol}$ ) were weighed into a 4dram vial. $\mathrm{CHCl}_{3}(1.531 \mathrm{~g})$ and pyrrole $(102 \mathrm{mg}, 1.52 \mathrm{mmol})$ were added and after stirring, the solution was allowed to stand 2.5 hours. The vial was removed from the glovebox, and the 
solution was diluted with $30 \mathrm{~mL} \mathrm{DCM}$ and extracted with $10 \mathrm{~mL}$ of sat. aq. $\mathrm{NaHCO}_{3}$ solution. The aqueous layer was back-extracted with $5 \mathrm{~mL}$ DCM. The organic layer was extracted twice with $10 \mathrm{~mL}$ portions of water, each of which was back-extracted with DCM $(5 \mathrm{~mL})$. The combined organic layer was dried over anhydrous $\mathrm{MgSO}_{4}$ and filtered on a $60 \mathrm{~mL}$ medium-porosity fritted funnel. The filtrate was concentrated in vacuo. The brown oil was re-dissolved in minimal DCM and added to a stirring solution of hexanes $(30 \mathrm{~mL})$. The pale tan solid that precipitated was collected on a $15 \mathrm{~mL}$ fine-porosity fritted funnel to give 4 (128 mg, $0.183 \mathrm{mmol}, 77 \%$ ).

${ }^{1} \mathrm{H} \mathrm{NMR}\left(\mathrm{CDCl}_{3}\right): \delta 8.05$ (d, 1H, J = 2.0, Pz3B), 7.87 (broad s, 1H, NH), $7.75(\mathrm{~d}, 1 \mathrm{H}, J=2.0, \mathrm{Pz} 5 \mathrm{C})$, $7.71(\mathrm{~d}, 1 \mathrm{H}, J=2.0, \mathrm{pz} 5 \mathrm{~B}), 7.63(\mathrm{~d}, 1 \mathrm{H}, J=2.0, \mathrm{Pz} 5 \mathrm{~A}), 7.39(\mathrm{~d}, 1 \mathrm{H}, J=2.0, \mathrm{Pz} 3 \mathrm{C}), 7.33(\mathrm{~d}, 1 \mathrm{H}, J=$ 2.0, pz3A), $7.07(\mathrm{~m}, 2 \mathrm{H}, \mathrm{H} 6$ and $\mathrm{H} 8), 6.88(\mathrm{t}, 1 \mathrm{H}, J=7.5, \mathrm{H} 7), 6.66(\mathrm{~d}, 1 \mathrm{H}, J=7.5, \mathrm{H} 5), 6.46(\mathrm{~m}, 1 \mathrm{H}$, H14), $6.29(\mathrm{t}, 1 \mathrm{H}, J=2.0, \mathrm{Pz} 4 \mathrm{~B}), 6.20(\mathrm{t}, 1 \mathrm{H}, J=2.0, \mathrm{Pz} 4 \mathrm{C}), 6.12(\mathrm{t}, 1 \mathrm{H}, J=2.0, \mathrm{Pz} 4 \mathrm{~A}), 6.08(\mathrm{~m}, 1 \mathrm{H}$, H13), $5.99(\mathrm{~m}, 1 \mathrm{H}, \mathrm{H} 12), 4.17(\mathrm{~d}, 1 \mathrm{H}, J=6.5, \mathrm{H} 2), 3.59(\mathrm{dd}, 1 \mathrm{H}, \mathrm{J}=6.7,15.9, \mathrm{H} 1), 3.03(\mathrm{dd}, 1 \mathrm{H}, J=$ $10.3,12.5, H 3), 2.71\left(d d, 1 H, J=6.7,15.9, H 1^{\prime}\right), 2.14(d d, 1 H, J=1.8,10.3, H 4), 1.33(d, 9 H, J=$ 8.1, $\left.\mathrm{PMe}_{3}\right) .{ }^{13} \mathrm{C} \mathrm{NMR}\left(\mathrm{CDCl}_{3}\right): \delta 144.7$ (s, C10), 144.0 (s, pz3A), 143.4 (s, C11), 143.1 (s, Pz3B), 140.6 (s, Pz3C), 136.7 (s, Pz5C), 136.1 (s, Pz5B), 135.4 (s, Pz5A), 132.4 (s, C9), 129.1 (s, C5), 129.1 (s, C8), 124.5 (s, C6), 123.5 (s, C7), 116.6 (s, C14), 106.9 (s, C13), 106.9 (s, Pz4B), 106.0 (s, Pz4C), 105.4 (s, Pz4A), 102.5 (s, C12), 62.5 (s, C3), 53.5 (s, C4), 39.3 (s, C2), 34.5 (s, C1), 13.6 (d, J = 28, $\left.\mathrm{PMe}_{3}\right) .{ }^{31} \mathrm{P}$ NMR $\left(\mathrm{CDCl}_{3}\right): \delta-9.36\left(J_{\mathrm{P}-\mathrm{W}}=280 \mathrm{~Hz}\right) . \mathrm{CV}(\mathrm{DMA}): \mathrm{E}_{\mathrm{p}, \mathrm{a}}=+0.533 \mathrm{~V} . \mathrm{IR}: \mathrm{V}_{\mathrm{NO}}=1535 \mathrm{~cm}^{-1}$ HRMS (M+Na) ${ }^{+}$obs'd (\%), calc'd (\%), ppm: 719.19051 (65.1), 719.19144 (82.2), -1.3; 720.19459 (67.6), 720.19397 (81.2), 0.9; 721.19485 (100), 721.1939 (100), 1.3; 722.19681 (47.3), 722.19785 (46), -1.4; 723.19812 (81.5), 723.19712 (82.7), 1.4.

$\mathrm{TpW}(\mathrm{NO})\left(\mathrm{PMe}_{3}\right)\left(3,4-\eta^{2}-(5-(1,2-\right.$ dihydronaphthalen-2-yl)-2,3-dimethylfuran)) (5) 


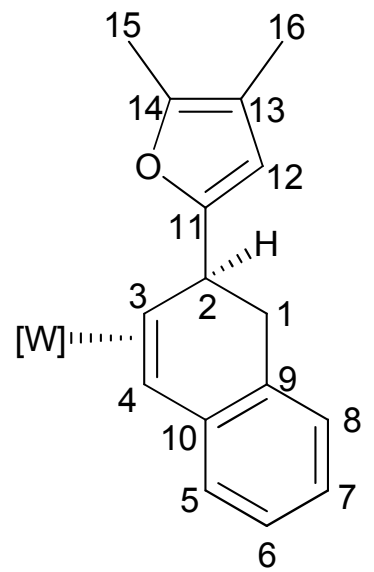

1 (101 mg, $0.160 \mathrm{mmol}$ ) and camphorsulfonic acid (16 mg, $0.070 \mathrm{mmol}$ ) were weighed into a vial and dissolved in $\mathrm{CHCl}_{3}(1.006 \mathrm{~g})$. 2,3-dimethylfuran (59 $\mathrm{mg}, 0.615 \mathrm{mmol}$ ) was added to the solution and the reaction was allowed to stand for 3 hours. The vial was removed from the glovebox, and the solution was diluted with DCM $(20 \mathrm{~mL})$ and extracted with $5 \mathrm{~mL}$ of a sat. aq. $\mathrm{NaHCO}_{3}$ solution. The aqueous layer was back-extracted with $5 \mathrm{~mL} \mathrm{DCM}$. The DCM solution was extracted twice with $10 \mathrm{~mL}$ portions of water, each of which was back-extracted with DCM $(5 \mathrm{~mL})$. The combined organic layers were dried over anhydrous $\mathrm{MgSO}_{4}$, filtered on a $60 \mathrm{~mL}$ medium-porosity fritted funnel, and concentrated in vacuo. The brown residue was taken into a glovebox, dissolved in minimal DCM and precipitated in stirring hexanes $(30 \mathrm{~mL})$. The mixture was filtered on a $15 \mathrm{~mL}$ fine-porosity fritted funnel to give 5 as a light tan solid $(68 \mathrm{mg}, 0.094$ $\mathrm{mmol}, 59 \%)$.

${ }^{1} \mathrm{H} N M R\left(\mathrm{CDCl}_{3}\right): \delta 8.09(\mathrm{~d}, 1 \mathrm{H}, J=2.0, \mathrm{Pz} 3 \mathrm{~B}), 8.00(\mathrm{~d}, 1 \mathrm{H}, J=2.0, \mathrm{Pz} 5 \mathrm{C}), 7.94(\mathrm{~d}, 1 \mathrm{H}, J=2.0, \mathrm{Pz} 5 \mathrm{~B})$, $7.82(\mathrm{~d}, 1 \mathrm{H}, J=2.0, \mathrm{Pz} 5 \mathrm{~A}), 7.68(\mathrm{~d}, 1 \mathrm{H}, J=2.0, \mathrm{Pz} 3 \mathrm{C}), 7.27(\mathrm{~d}, 1 \mathrm{H}, J=2.0, \mathrm{Pz} 3 \mathrm{~A}), 6.96(\mathrm{~d}, 1 \mathrm{H}, J=$ 7.5, H8), $6.93(\mathrm{dd}, 1 \mathrm{H}, J=7.5,8.4, \mathrm{H} 6), 6.75(\mathrm{dd}, 1 \mathrm{H}, J=7.5,8.4, \mathrm{H7}), 6.48(\mathrm{~d}, 1 \mathrm{H}, J=7.6, \mathrm{H} 5)$, $6.38(\mathrm{t}, 1 \mathrm{H}, J=2.0, \mathrm{Pz} 4 \mathrm{~B}), 6.37(\mathrm{t}, 1 \mathrm{H}, J=2.0, \mathrm{Pz} 4 \mathrm{C}), 6.17(\mathrm{t}, 1 \mathrm{H}, J=2.0, \mathrm{Pz} 4 \mathrm{~A}), 5.66(\mathrm{~s}, 1 \mathrm{H}, \mathrm{H} 12)$, $4.05(\mathrm{~d}, 1 \mathrm{H}, J=6.7, \mathrm{H} 2), 3.57(\mathrm{dd}, 1 \mathrm{H}, J=7.1,16.0, \mathrm{H} 1), 3.22(\mathrm{dd}, 1 \mathrm{H}, J=10.4,11.5, \mathrm{H} 3), 2.73$ (d, 
$\left.1 \mathrm{H}, J=16.0, \mathrm{H} 1^{\prime}\right), 2.13(\mathrm{~s}, 3 \mathrm{H}, \mathrm{H} 15), 2.00(\mathrm{dd}, 1 \mathrm{H}, J=1.9,10.3, \mathrm{H} 4), 1.75(\mathrm{~s}, 3 \mathrm{H}, \mathrm{H} 16), 1.36$ (d, 9H, $\left.J=8.3, \mathrm{PMe}_{3}\right) .{ }^{13} \mathrm{C} \mathrm{NMR}\left(\mathrm{CDCl}_{3}\right): \delta 162.9(\mathrm{~s}, \mathrm{C} 11), 146.0(\mathrm{~s}, \mathrm{C} 10), 144.8(\mathrm{~s}, \mathrm{C} 14), 144.2(\mathrm{~s}, \mathrm{Pz} 3 \mathrm{~B})$, 144.2 (s, Pz3A), 141.8 (s, Pz3C), 138.0 (s, Pz5C), 137.2 (s, Pz5B), 136.7 (s, Pz5A), 133.1 (s, C9), 129.6 (s, C5), 128.9 (s, C8), 124.6 (s, C6), 123.2 (s, C7), 115.0 (s, C13), 108.3 (s, C12), 107.3 (s, Pz4B), 107.2 (s, Pz4C), 105.9 (s, Pz4A), 60.1 (s, C3), 54.2 (s, C4), 40.4 (s, C2), 33.1 (s, C1), 13.2 (d, J $\left.=28.1, \mathrm{PMe}_{3}\right), 11.5(\mathrm{~s}, \mathrm{C} 15), 10.1(\mathrm{~s}, \mathrm{C} 16) .{ }^{31} \mathrm{P} \mathrm{NMR}\left(\mathrm{CDCl}_{3}\right): \delta-9.36\left(J_{\mathrm{p}-\mathrm{w}}=280 \mathrm{~Hz}\right) . \mathrm{CV}(\mathrm{DMA}): \mathrm{E}_{\mathrm{p}, \mathrm{a}}$ $=+0.543$ V. IR: $V_{\text {NO }}=1552 \mathrm{~cm}^{-1}$. HRMS $(M+N a)^{+}$obs'd (\%), calc'd (\%), ppm: $748.20465(100)$, 748.20679 (81.2), -2.9; 749.21042 (95.3), 749.20933 (81.4), 1.5; 750.20893 (95.9), 750.20929 (100), -0.5; 751.2124 (54.6), 751.21319 (47.3), -1.1; 752.21027 (86.6), 752.2125 (82.4), -3.

\section{3-(1,2-dihydronaphthalen-2-yl)-1H-indole (6)}

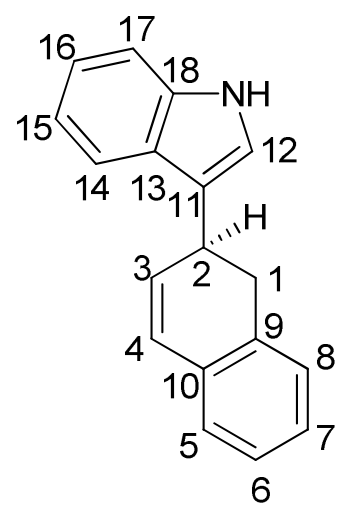

In a fume hood, $3(60 \mathrm{mg}, 0.080 \mathrm{mmol})$ was mixed with acetone $(1.986 \mathrm{~g})$. A solution of ceric ammonium nitrate (CAN) $(44 \mathrm{mg}, 0.080 \mathrm{mmol})$ in water $(1.496 \mathrm{~g})$ was added to give a heterogeneous solution, which was stirred rapidly for 1.5 hours. The slurry was diluted with 20 $\mathrm{mL}$ of $\mathrm{Et}_{2} \mathrm{O}$ and washed with water $(3 \times 10 \mathrm{~mL})$. The water layers were combined and backextracted with $\mathrm{Et}_{2} \mathrm{O}(2 \times 10 \mathrm{~mL})$. The $\mathrm{Et}_{2} \mathrm{O}$ layers were combined and dried over anhydrous $\mathrm{MgSO}_{4}$. The $\mathrm{MgSO}_{4}$ was filtered on a $30 \mathrm{~mL}$ medium-porosity fritted funnel and the $\mathrm{Et}_{2} \mathrm{O}$ filtrate was concentrated in vacuo to yield an orange solid. The solid was redissolved in small portions of dichloromethane (DCM) $(2 \times 0.3 \mathrm{~mL})$ and loaded onto a $250 \mu \mathrm{m}$ silica preparatory plate. The 
plate was eluted with $100 \mathrm{~mL}$ of 70:30 hexanes: ethyl acetate (EtOAc). A large band with $R_{f}=0.8$ was scraped into a test tube, to which $20 \mathrm{~mL}$ of EtOAc was added. The test tube was sonicated for 20 minutes and the slurry was filtered on a $60 \mathrm{~mL}$ medium-porosity fritted funnel and washed with $39 \mathrm{~mL}$ EtOAc. The filtrate was concentrated in vacuo to give 6 (12 mg, $0.048 \mathrm{mmol}$, $61 \%)$ as an oil.

${ }^{1} \mathrm{H}$ NMR $\left(\mathrm{CDCl}_{3}\right): \delta 7.88(\mathrm{~s}, \mathrm{NH}), 7.70(\mathrm{~d}, 1 \mathrm{H}, J=8.0, \mathrm{H} 17), 7.36(\mathrm{~d}, 1 \mathrm{H}, J=8.1, \mathrm{H} 14), 7.23(\mathrm{~m}, 1 \mathrm{H}$, H15), $7.20(m, 1 H, H 6$ or $H 7), 7.16(m, 1 H, H 6$ or $H 7), 7.16(m, 1 H, H 16), 7.13(m, 1 H, H 5), 7.09$ $(\mathrm{d}, 1 \mathrm{H}, J=7.3, \mathrm{H} 8), 6.97(\mathrm{~d}, 1 \mathrm{H}, J=2.2, \mathrm{H} 12), 6.64(\mathrm{dd}, 1 \mathrm{H}, J=2.1,9.5, \mathrm{H} 4), 6.25$ (dd, $1 \mathrm{H}, J=3.8$, 9.6, H3), 4.09 (ddd, $1 \mathrm{H}, J=3.1,6.8,10.1, \mathrm{H} 2), 3.22(\mathrm{dd}, 1 \mathrm{H}, J=7.2,15.5, \mathrm{H} 1), 3.19$ (dd, $1 \mathrm{H}, J=$ 10.3, 15.5, H1). $\left.{ }^{13} \mathrm{C} \mathrm{NMR} \mathrm{(CDCl} 3\right): \delta 136.8$ (s, C18), 135.2 (s, C9), 133.9 (s, C10), 132.8 (s, C3), $128.2(\mathrm{~s}, \mathrm{C} 8), 127.7$ (s, C4), 127.3 (s, C6 or C7), 126.8 (s, C6 or C7), 126.8 (s, C13), 126.2 (s, C5), 122.3 (s, C16), 121.5 (s, C12), 119.5 (s, C15), 119.4 (s, C17), 118.8 (s, C11), 111.5 (s, C14), 35.6 (s, C1), $31.9(\mathrm{~s}, \mathrm{C} 2)$.

\section{2-(1,2-dihydronaphthalen-2-yl)-1H-pyrrole (7)}

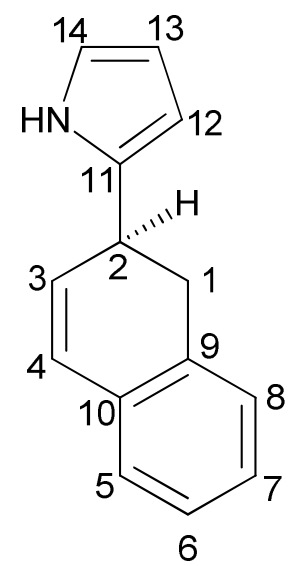

In a fume hood, CAN (101 mg, $0.184 \mathrm{mmol})$ was weighed into a vial and dissolved in water (2.02 g). $4(120 \mathrm{mg}, 0.172 \mathrm{mmol})$ was added to a vial and dissolved in $\mathrm{CHCl}_{3}(2.79 \mathrm{~g})$. The two solutions were combined and the mixture was vigorously stirred for 5 hours. The mixture was 
diluted with $\mathrm{Et}_{2} \mathrm{O}(40 \mathrm{~mL})$ and extracted with water $(2 \times 15 \mathrm{~mL})$. The water layers were combined and extracted with four $20 \mathrm{~mL}$ portions of $\mathrm{Et}_{2} \mathrm{O}$ and the combined organic layers were dried over anhydrous $\mathrm{MgSO}_{4}$. The $\mathrm{MgSO}_{4}$ was filtered on a $60 \mathrm{~mL}$ medium-porosity fritted funnel and rinsed with $\mathrm{Et}_{2} \mathrm{O}(60 \mathrm{~mL})$. The $\mathrm{Et}_{2} \mathrm{O}$ solution was concentrated in vacuo giving a brown oil, which was redissolved in minimal DCM and precipitated by addition to $70 \mathrm{~mL}$ stirring hexanes. The precipitate was filtered over a $60 \mathrm{~mL}$ fine-porosity fritted funnel, washed with 30 $\mathrm{mL}$ hexanes, and discarded. The filtrate was concentrated in vacuo to give an orange solid. The solid was dissolved in small portions of DCM $(2 \times .3 \mathrm{~mL})$ and loaded onto a $500 \mu \mathrm{m}$ silica preparatory plate. The plate was eluted with $100 \mathrm{~mL}$ 3:1 hexanes: EtOAc. A fluorescent band at $R_{f}=0.7$ was scraped off the plate and added into a test tube with $20 \mathrm{~mL}$ EtOAc. The test tube was sonicated for 20 minutes and the silica was filtered over a $60 \mathrm{~mL}$ medium-porosity fritted funnel and washed with $25 \mathrm{~mL}$ and $10 \mathrm{~mL}$ portions of EtOAc. The EtOAc was concentrated in vacuo to give 7 (9 mg, $0.108 \mathrm{mmol}, 28 \%$ ) as a light green oil.

${ }^{1} \mathrm{H} \mathrm{NMR}\left(\mathrm{CDCl}_{3}\right): \delta 7.93(\mathrm{~s}, 1 \mathrm{H}, \mathrm{NH}), 7.20(\mathrm{~m}, 1 \mathrm{H}, J=7.1, \mathrm{H} 6), 7.16(\mathrm{~m}, 1 \mathrm{H}, J=7.5, \mathrm{H} 7), 7.12(\mathrm{~m}$, $1 \mathrm{H}, \mathrm{H} 8), 7.10(\mathrm{~m}, 1 \mathrm{H}, \mathrm{H} 5), 6.61(\mathrm{~m}, 1 \mathrm{H}, \mathrm{H} 14), 6.60(\mathrm{~d}, 1 \mathrm{H}, \mathrm{J}=9.5, \mathrm{H} 4), 6.13(\mathrm{~m}, 1 \mathrm{H}, \mathrm{H} 12), 6.10$ (dd, $1 \mathrm{H}, J=4.4,9.4, H 3), 6.01(\mathrm{~m}, 1 \mathrm{H}, \mathrm{H} 13), 3.81(\mathrm{~m}, 1 \mathrm{H}, \mathrm{H} 2), 3.19(\mathrm{dd}, 1 \mathrm{H}, J=8.2,15.5, \mathrm{H} 1), 3.00(\mathrm{dd}$, $\left.1 \mathrm{H}, J=15.5,7.2, \mathrm{H}^{\prime}\right) .{ }^{13} \mathrm{C} \mathrm{NMR}\left(\mathrm{CDCl}_{3}\right): \delta 134.1$ (s, C11), 134.0 (s, C10), 133.4 (s, C9), 130.7 (s, C3), 128.2 (s, C8), 128.0 (s, C4), 127.7 (s, C7), 127.0 (s, C6), 126.3 (s, C5), 116.9 (s, C14), 108.3 (s, C12), 105.0 (s, C13), 35.5 (s, C1), 33.5 (s C2).

\section{5-(1,2-dihydronaphthalen-2-yl)-2,3-dimethylfuran (8)}




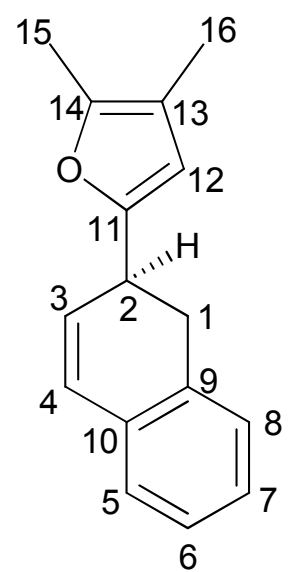

In a fume hood, CAN (47 mg, $0.086 \mathrm{mmol})$ was added to a vial and dissolved in water (1.416 g). 5 (62 $\mathrm{mg}, 0.085 \mathrm{mmol}$ ) was added to a second vial and dissolved in acetone (1.511 g). The two solutions were combined and the mixture stirred for 30 minutes before being diluted with $\mathrm{Et}_{2} \mathrm{O}$ $(30 \mathrm{~mL})$ and extracted with water $(2 \times 15 \mathrm{~mL})$. The water layers were combined and extracted with $\mathrm{Et}_{2} \mathrm{O}(4 \times 10 \mathrm{~mL})$. The $\mathrm{Et}_{2} \mathrm{O}$ layers were combined and dried over anhydrous $\mathrm{MgSO}_{4}$. The $\mathrm{MgSO}_{4}$ was filtered on a $60 \mathrm{~mL}$ medium-porosity fritted funnel and the filtrate was concentrated in vacuo to give a light orange solid. The solid was dissolved in DCM and precipitated over 30 $\mathrm{mL}$ of stirring hexanes. The resulting solid was filtered on $30 \mathrm{~mL}$ fine-porosity fritted funnel and discarded. The filtrate was concentrated in vacuo and dissolved in small portions of DCM $(2 \mathrm{x}$ $0.3 \mathrm{~mL}$ ) and loaded onto a $250 \mu \mathrm{m}$ silica preparatory plate. The preparatory plate was eluted with $100 \mathrm{~mL}$ of 3:1 hexanes: EtOAc. A large band at $R_{f}=0.8-0.9$ was scraped off the plate into a test tube. EtOAc $(20 \mathrm{~mL})$ was added to the test tube and the slurry was sonicated for 25 minutes. The silica was filtered on $60 \mathrm{~mL}$ medium-porosity fritted funnel and washed with 20 $\mathrm{mL}$ of EtOAc. The EtOAc was concentrated in vacuo to give 8 as an oil (9 mg, $0.0399 \mathrm{mmol}, 47 \%)$ with a small amount of substituted naphthalene as an impurity.

${ }^{1} \mathrm{H}$ NMR $\left(\mathrm{CDCl}_{3}\right): \delta 7.13-7.17(\mathrm{~m}, 3 \mathrm{H}, \mathrm{H} 6, \mathrm{H} 7$, and $\mathrm{H} 8), 7.09(\mathrm{~d}, 1 \mathrm{H}, J=7.3, \mathrm{H} 5), 6.58(\mathrm{dd}, 1 \mathrm{H}, J=$ 2.2, 9.6, H4), 6.07 (dd, $1 \mathrm{H}, J=3.8,9.5, \mathrm{H} 3$ ), $5.84(\mathrm{~s}, 1 \mathrm{H}, \mathrm{H} 12$ ), 3.69 (ddd, $1 \mathrm{H}, \mathrm{J}=3.0,6.5,10.2 \mathrm{H} 2$ ), 
$3.07(\mathrm{dd}, 1 \mathrm{H}, J=7.0,15.4,1 \mathrm{H}), 2.97\left(\mathrm{dd}, 1 \mathrm{H}, J=10.4,15.4 \mathrm{H} 1^{\prime}\right), 2.13(\mathrm{~s}, 1 \mathrm{H}, \mathrm{H} 15), 1.85(\mathrm{~s}, 1 \mathrm{H}$, H16). ${ }^{13} \mathrm{CNMR}\left(\mathrm{CDCl}_{3}\right): \delta 155.0$ (s, C11), 146.6 (s, C14), 135.1 (s, C9), 134.3 (s, C10), 130.1 (s, C3), 129.0 (s, C6, C7, or C8), 128.7 (s, C4), 128.3 (s, C6, C7, or C8), 127.7 (s, C6, C7, or C8), 127.1 (s, C5), 115.2 (s, C13), 109.0 (s, C12), 34.8 (s, C2), 33.9 (s, C1), 11.4 (s, C15), 10.0 (s, C16).

$\mathrm{TpW}(\mathrm{NO})\left(\mathrm{PMe}_{3}\right)\left(2,3-\eta^{2}-1 H\right.$-anthracenium) triflate (12)

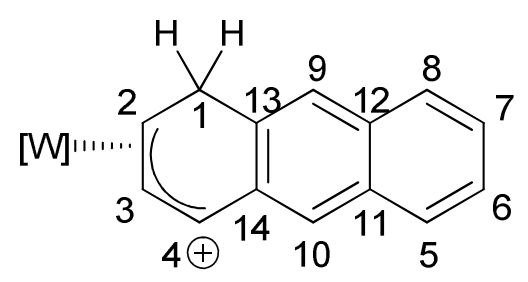

11 (16 mg, $0.023 \mathrm{mmol}$ ) was dissolved in deuterated MeCN (518 mg) and HOTf (3.5 mg (0.023 $\mathrm{mmol}$ ) in $36 \mathrm{mg} \mathrm{d}-\mathrm{MeCN}$ ) was added to produce 12. NMR spectroscopy data was collected on this sample.

${ }^{1} \mathrm{H}$ NMR $(\mathrm{d}-\mathrm{MeCN}): \delta 8.28(\mathrm{~d}, J=2.0,1 \mathrm{H}, \mathrm{pz} 3 \mathrm{~A}), 8.14(\mathrm{~d}, J=2.0,1 \mathrm{H}, \mathrm{pz} 3 \mathrm{~B}), 8.13(\mathrm{~m}, 1 \mathrm{H}, \mathrm{H} 5), 8.07$ $(\mathrm{d}, J=2.0,1 \mathrm{H}, \mathrm{pz} 3 \mathrm{C}), 8.05(\mathrm{~d}, J=2.0,1 \mathrm{H}, \mathrm{pz} 5 \mathrm{C}), 7.92(\mathrm{~m}, 2 \mathrm{H}, \mathrm{pz} 5 \mathrm{~B}$ and $\mathrm{H} 6), 7.86(\mathrm{~d}, J=2.0,1 \mathrm{H}$, pz5A), $7.78(s, 1 H, H 10), 7.62(s, 1 H, H 9), 7.49(m, 1 H, H 7), 7.48(m, 1 H, H 8), 7.30(d, J=7.6,1 H$, $\mathrm{H} 4), 6.58(\mathrm{t}, J=2.0,1 \mathrm{H}, \mathrm{pz} 4 \mathrm{C}), 6.43(\mathrm{t}, J=2.0,1 \mathrm{H}, \mathrm{pz} 4 \mathrm{~B}), 6.42(\mathrm{t}, J=2.0,1 \mathrm{H}, \mathrm{pz} 4 \mathrm{~A}), 5.22(\mathrm{dd}, J=$ 5.5, 20.3, 1H, H1), $5.05(\mathrm{t}, J=7.6,1 \mathrm{H}, \mathrm{H} 3), 4.69(\mathrm{~m}, 1 \mathrm{H}, \mathrm{H} 2), 4.02\left(\mathrm{~d}, J=20.3,1 \mathrm{H}, \mathrm{H} 1^{\prime}\right), 1.19(\mathrm{~d}, J=$ 9.7, 9H, $\left.\mathrm{PMe}_{3}\right) .{ }^{13} \mathrm{C}$ NMR (d-MeCN): $\delta 148.7$ (pz3A), 145.9 (pz3B), 143.2 (pz3C), 139.7 (pz5A and pz5C), 139.5 (pz5B), 137.1 (C4), 135.9 (C12), 132.3 (C14), 131.7 (C14), 131.2 (C13), 129.5 (C6), 128.9 (C8), 128.0 (C11), 125.7 (C10), 127.2 (C9), 126.6 (C7), 109.4 (pz4B), 109.1 (pz4C), 108.2 (pz4A), $97.7(\mathrm{C} 3), 69.1(\mathrm{C} 2), 33.6(\mathrm{C} 1), 12.9\left(\mathrm{~d}, J=32, \mathrm{PMe}_{3}\right) .{ }^{31} \mathrm{P} \mathrm{NMR}(\mathrm{d}-\mathrm{MeCN}): \delta-6.52\left(J_{\mathrm{P}-\mathrm{w}}=\right.$ $271 \mathrm{~Hz})$.

$\mathrm{TpW}(\mathrm{NO})\left(\mathrm{PMe}_{3}\right)\left(2,3-\eta^{2}-1 H-1-\right.$ bromoanthracenium) succinimide (13) 


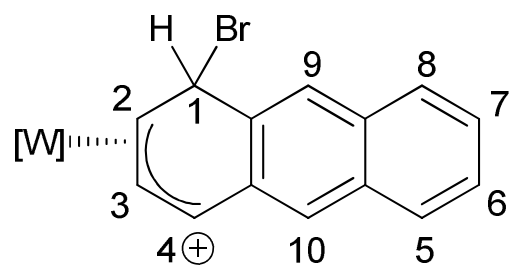

11 (16 mg, $0.023 \mathrm{mmol}$ ) was dissolved in $\mathrm{CDCl}_{3}(612 \mathrm{mg})$ and $\mathrm{N}$-bromosuccinimide $(4.3 \mathrm{mg}$, $0.024 \mathrm{mmol}$; in $169 \mathrm{mg} \mathrm{d}-\mathrm{MeCN}$ ) were placed in a $-30{ }^{\circ} \mathrm{C}$ cold bath for $50 \mathrm{~min}$. The $\mathrm{N}$ bromosuccinimide solution was added to the complex solution to give $\mathbf{1 3}$ as a red-orange solution (along with $\sim 25 \%$ of a second complex, perhaps a 13 -succinimide adduct), on which NMR spectroscopy data was collected.

Selected ${ }^{1} \mathrm{H}$ NMR $\left(\mathrm{CDCl}_{3}\right): \delta 8.15(\mathrm{~s}, 1 \mathrm{H}, \mathrm{H} 10), 7.65(\mathrm{~s}, 1 \mathrm{H}, \mathrm{H} 9), 7.55(\mathrm{~m}, 1 \mathrm{H}, \mathrm{H} 5), 7.45(\mathrm{~m}, 1 \mathrm{H}, \mathrm{H} 4)$, $6.07(\mathrm{~s}, 1 \mathrm{H}, \mathrm{H} 1), 5.27(\mathrm{t}, J=7.4,1 \mathrm{H}, \mathrm{H} 3), 4.52(\mathrm{dd}, J=7.2,13.8,1 \mathrm{H}, \mathrm{H} 2), 2.68-2.84(\mathrm{~m}, 4 \mathrm{H}$, succinimide), $1.23\left(\mathrm{~d}, J=9.7,9 \mathrm{H}, \mathrm{PMe}_{3}\right) .{ }^{31} \mathrm{P} \mathrm{NMR}\left(\mathrm{CDCl}_{3}\right): \delta-6.06\left(J_{\mathrm{P}-\mathrm{w}}=263 \mathrm{~Hz}\right),-9.74\left(J_{\mathrm{P}-\mathrm{w}}=264\right.$ $\mathrm{Hz})$.

\section{$\operatorname{TpW}(\mathrm{NO})\left(\mathrm{PMe}_{3}\right)\left(2,3-\eta^{2}\right.$-tetramethyl-2,2'-((1R,4S)-1,4-dihydroanthracene-1,4-diyl)dimalonate))} (14)<smiles>COC(=O)C(C(=O)OC)[C@@H]1C=C[C@H](C(C(=O)OC)C(=O)OC)c2cc3ccccc3cc21</smiles>

11 (30 mg, $0.044 \mathrm{mmol}$ ) was dissolved in $\mathrm{CDCl}_{3}(1.22 \mathrm{~g})$ and $\mathrm{N}$-bromosuccinimide $(9.6 \mathrm{mg}, 0.054$ mmol) was dissolved in $\mathrm{MeCN}\left(507 \mathrm{mg}\right.$ ). Both solutions were placed in a $-30{ }^{\circ} \mathrm{C}$ cold bath for at least $30 \mathrm{~min}$. Dimethylmalonate (33 $\mathrm{mg}, 0.250 \mathrm{mmol}$ ) and LiOMe $(6 \mathrm{mg}, 0.158 \mathrm{mmol})$ were dissolved in $\mathrm{MeOH}(256 \mathrm{mg})$. The $\mathrm{N}$-bromosuccinimide solution was added to the complex 
solution, followed directly by the dimethylmalonate solution. The reaction was left in the cold bath overnight. In the fume hood, the reaction was extracted $3 x$ with $2 \mathrm{~mL}$ portions of saturated $\mathrm{NaHCO}_{3}$. The organic layer was dried with $\mathrm{MgSO}_{4}$, filtered over a Celite plug, and evaporated in vacuo to give 14, from which NMR spectroscopy data was collected.

${ }^{1} \mathrm{H}$ NMR $\left(\mathrm{CDCl}_{3}\right)$ (free anthracene omitted): $\delta 8.31(\mathrm{~d}, J=2.0,1 \mathrm{H}, \mathrm{Tp}), 7.80(\mathrm{~d}, J=2.0,1 \mathrm{H}, \mathrm{Tp})$, $7.74(\mathrm{~s}, 1 \mathrm{H}, \mathrm{H} 10), 7.70$ (d, $J=2.0,1 \mathrm{H}, \mathrm{Tp}), 7.70(\mathrm{~m}, 2 \mathrm{H}, \mathrm{H} 6$ and $\mathrm{H} 7), 7.61(\mathrm{~d}, J=2.0,1 \mathrm{H}, \mathrm{Tp}), 7.59$ (s, 1H, H9), $7.57(\mathrm{~d}, J=2.0,1 \mathrm{H}, \mathrm{Tp}), 7.29(\mathrm{~m}, 2 \mathrm{H}, \mathrm{H} 5$ and H8), $7.28(\mathrm{~d}, J=2.0,1 \mathrm{H}, \mathrm{Tp}), 6.28(\mathrm{t}, J=$ 2.0, $1 \mathrm{H}, \mathrm{Tp}), 6.21(\mathrm{t}, J=2.0,1 \mathrm{H}, \mathrm{Tp}), 6.12(\mathrm{t}, J=2.0,1 \mathrm{H}, \mathrm{Tp}), 5.02(\mathrm{~d}, J=10.3,1 \mathrm{H}, \mathrm{H} 4), 4.83(\mathrm{~d}, J=$ $10.0,1 \mathrm{H}, \mathrm{H} 1), 4.34(\mathrm{~d}, J=10.4,1 \mathrm{H}, \mathrm{H} 12), 4.25$ (d, J = 9.9, 1H, H11), 3.87 (s, 3H, OMe), 3.59 (s, 3H, OMe), 3.55 (s, 3H, OMe), 3.30 (s, 3H, OMe), 2.51 (dd, $J=10.3,11.2,1 \mathrm{H}, \mathrm{H} 2$ ), 1.50 (ddd, $J=1.6$, $2.9,10.6,1 \mathrm{H}, \mathrm{H} 3), 1.18\left(\mathrm{~d}, J=8.2,9 \mathrm{H}, \mathrm{PMe}_{3}\right) .{ }^{31} \mathrm{P} \mathrm{NMR}\left(\mathrm{CDCl}_{3}\right): \delta-10.46\left(J_{\mathrm{P}-\mathrm{W}}=280 \mathrm{~Hz}\right) . \quad \mathrm{IR}: \mathrm{v}_{\mathrm{NO}}=$ $1556 \mathrm{~cm}^{-1}, \mathrm{v}_{\mathrm{CO}}=1732 \mathrm{~cm}^{-1}, \mathrm{v}_{\mathrm{BH}}=2490 \mathrm{~cm}^{-1}$.

\section{$\mathrm{TpW}(\mathrm{NO})\left(\mathrm{PMe}_{3}\right)\left(2,3-\eta^{2}-(1 \mathrm{R}, 4 \mathrm{~S})-1,4-d i m e t h o x y-1,4-d i h y d r o a n t h r a c e n e\right)(15)$}

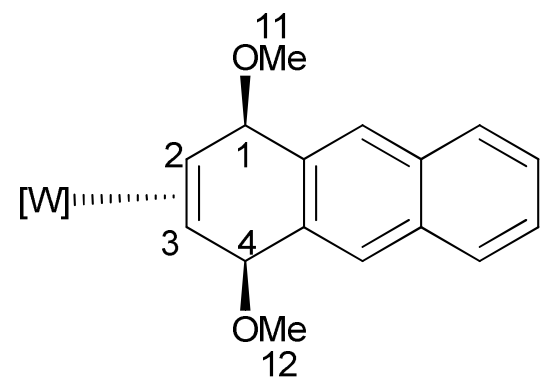

11 (30 mg, $0.044 \mathrm{mmol}$ ) was dissolved in $\mathrm{CDCl}_{3}(989 \mathrm{mg}$ ) and $\mathrm{N}$-bromosuccinimide $(8 \mathrm{mg}, 0.045$ $\mathrm{mmol}$ ) was dissolved in $\mathrm{MeCN}\left(504 \mathrm{mg}\right.$ ). Both solutions were placed in a $-30{ }^{\circ} \mathrm{C}$ cold bath for at least $30 \mathrm{~min}$. LiOMe $(6 \mathrm{mg}, 0.158 \mathrm{mmol}$ ) was dissolved in $\mathrm{MeOH}(206 \mathrm{mg}$ ). The $\mathrm{N}$ bromosuccinimide solution was added to the complex solution, followed directly by the $\mathrm{MeOH}$ solution. The reaction was left in the cold bath overnight. The reaction was extracted $3 x$ with 2 $\mathrm{mL}$ portions of water, which were backextracted with $0.5 \mathrm{~mL}$ DCM. The organic layers were 
dried with $\mathrm{MgSO}_{4}$, filtered over a Celite plug, and evaporated in vacuo to give 15, in 75\% purity, from which NMR spectroscopy data was collected.

Selected ${ }^{1} \mathrm{H}$ NMR $\left(\mathrm{CDCl}_{3}\right): \delta 5.03(\mathrm{~m}, 1 \mathrm{H}, \mathrm{H} 4), 5.01(\mathrm{~m}, 1 \mathrm{H}, \mathrm{H} 1), 3.49(\mathrm{~s}, 3 \mathrm{H}, \mathrm{H} 11), 3.39(\mathrm{~s}, 3 \mathrm{H}$, $\mathrm{H} 12$ ), 3.10 (ddd, $J=1.6,10.3,12.2,1 \mathrm{H}, \mathrm{H} 2), 1.88(\mathrm{dt}, J=1.9,10.1,1 \mathrm{H}, \mathrm{H} 3), 1.20(\mathrm{~d}, J=8.2,9 \mathrm{H}$, $\left.\mathrm{PMe}_{3}\right) .{ }^{31} \mathrm{P} \mathrm{NMR}\left(\mathrm{CDCl}_{3}\right): \delta-10.90\left(J_{\mathrm{P}-\mathrm{W}}=273 \mathrm{~Hz}\right)$.

$\operatorname{TpW}(\mathrm{NO})\left(\mathrm{PMe}_{3}\right)\left(2,3-\eta^{2}-(1 \mathrm{R}, 4 \mathrm{~S})-1,4-d i(1 \mathrm{H}-\mathrm{imidazol}-1-\mathrm{yl})-1,4-d i h y d r o a n t h r a c e n e\right)(16)$

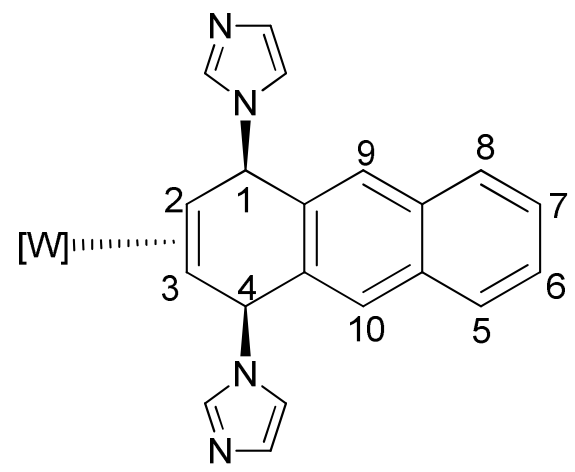

11 (30 mg, $0.044 \mathrm{mmol}$ ) was dissolved in $\mathrm{CDCl}_{3}(1.011 \mathrm{~g}$ ) and $\mathrm{N}$-bromosuccinimide $(9 \mathrm{mg}, 0.051$ mmol) was dissolved in $\mathrm{MeCN}\left(520 \mathrm{mg}\right.$ ). Both solutions were placed in a $-30{ }^{\circ} \mathrm{C}$ cold bath for at least $30 \mathrm{~min}$. Imidazole $(30 \mathrm{mg}, 0.448 \mathrm{mmol})$ and triethylamine $(25 \mathrm{mg}, 0.248 \mathrm{mmol})$ were dissolved in $\mathrm{CDCl}_{3}$ (400 mg). The $\mathrm{N}$-bromosuccinimide solution was added to the complex solution, followed directly by the imidazole solution. The reaction was left in the cold bath for 1.5 hours. The reaction was extracted $1 x$ with $2 \mathrm{~mL}$ of saturated $\mathrm{Na}_{2} \mathrm{CO}_{3}$, followed by and extraction with water $(2 \mathrm{~mL})$, which were backextracted with $0.5 \mathrm{~mL}$ DCM. The organic layers were dried with $\mathrm{MgSO}_{4}$, filtered over a Celite plug, and evaporated in vacuo to give 16, in 95 \% purity, from which NMR spectroscopy data was collected.

${ }^{1} \mathrm{H} \mathrm{NMR}\left(\mathrm{CDCl}_{3}\right): \delta 7.86(\mathrm{~d}, J=2.0,1 \mathrm{H}, \mathrm{Tp}), 7.85(\mathrm{~d}, J=2.0,1 \mathrm{H}, \mathrm{Tp}), 7.82(\mathrm{~m}, 2 \mathrm{H}, \mathrm{H} 6$ and H7), 7.79 (d, J = 2.0,1H, Tp), $7.77(\mathrm{~s}, 1 \mathrm{H}, \mathrm{H} 9$ or $\mathrm{H} 10), 7.75(\mathrm{~s}, 1 \mathrm{H}, \mathrm{H} 9$ or $\mathrm{H} 10), 7.70(\mathrm{~d}, J=2.0,1 \mathrm{H}, \mathrm{Tp}), 7.64$ (d, J = 2.0, 1H, Tp), $7.63(\mathrm{~m}, 1 \mathrm{H}, \mathrm{imid}), 7.45(\mathrm{~m}, 2 \mathrm{H}, \mathrm{H} 5$ and $\mathrm{H} 8), 7.39(\mathrm{~d}, J=2.0,1 \mathrm{H}, \mathrm{Tp}), 7.35$ (m, 1H, imid), 6.97 (m, 1H, imid), $6.91(\mathrm{~m}, 1 \mathrm{H}, \mathrm{imid}), 6.77(\mathrm{~m}, 1 \mathrm{H}, \mathrm{imid}), 6.72(\mathrm{~m}, 1 \mathrm{H}, \mathrm{imid}), 6.32(\mathrm{t}, J$ 
$=2.0,1 \mathrm{H}, \mathrm{Tp}), 6.28($ broad $\mathrm{s}, 1 \mathrm{H}, \mathrm{H} 4), 6.26($ broad $\mathrm{s}, 1 \mathrm{H}, \mathrm{H} 1), 6.23(\mathrm{t}, J=2.0,1 \mathrm{H}, \mathrm{Tp}), 6.22(\mathrm{t}, J=$ 2.0, $1 \mathrm{H}, \mathrm{Tp}), 3.14(\mathrm{dd}, J=2.5,10.4,1 \mathrm{H}, \mathrm{H} 2), 2.01$ (ddd, $J=1.6,3.1,10.3,1 \mathrm{H}, \mathrm{H} 3), 1.15(\mathrm{~d}, J=8.1$, $\left.9 \mathrm{H}, \mathrm{PMe}_{3}\right) .{ }^{31} \mathrm{P} \mathrm{NMR}\left(\mathrm{CDCl}_{3}\right): \delta-11.89\left(J_{\mathrm{P}-\mathrm{W}}=271 \mathrm{~Hz}\right) . \quad \mathrm{IR}: \mathrm{V}_{\mathrm{NO}}=1568 \mathrm{~cm}^{-1}, \mathrm{~V}_{\mathrm{BH}}=2489 \mathrm{~cm}^{-1}$

TpW(NO)(PMe $)\left(3,4-\eta^{2}\right.$-1-propyl-1a,9b-dihydro-1H-anthra[1,2-b]azirine) (17)

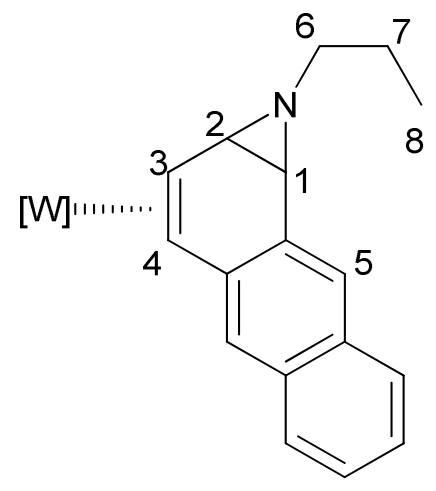

11 (30 mg, $0.044 \mathrm{mmol}$ ) was dissolved in $\mathrm{CDCl}_{3}(1.222 \mathrm{~g}$ ) and $\mathrm{N}$-bromosuccinimide $(8 \mathrm{mg}, 0.045$ mmol) was dissolved in $\mathrm{MeCN}(474 \mathrm{mg})$. Both solutions were placed in a $-30{ }^{\circ} \mathrm{C}$ cold bath for at least $30 \mathrm{~min}$. The $\mathrm{N}$-bromosuccinimide solution was added to the complex solution, followed directly by propylamine $(40 \mathrm{mg}, 0.678 \mathrm{mmol})$. The reaction was left in the cold bath overnight. The reaction was extracted $3 x$ with $2 \mathrm{~mL}$ portions of saturated $\mathrm{NaHCO}_{3}$. The organic layers were dried with $\mathrm{MgSO}_{4}$, filtered over a Celite plug, and evaporated in vacuo to give 17, in 75\% purity, from which NMR spectroscopy data was collected.

Selected ${ }^{1} \mathrm{H}$ NMR $\left(\mathrm{CDCl}_{3}\right): \delta 7.78(\mathrm{~s}, 1 \mathrm{H}, \mathrm{H} 5), 5.61(\mathrm{~d}, J=6.6,1 \mathrm{H}, \mathrm{H} 1), 5.13(\mathrm{~d}, J=6.9,1 \mathrm{H}, \mathrm{H} 2)$, $3.88(\mathrm{dt}, J=7.6,14.0,1 \mathrm{H}, \mathrm{H} 6), 3.60\left(\mathrm{~m}, 1 \mathrm{H}, \mathrm{H} 6^{\prime}\right), 3.23(\mathrm{dd}, J=9.9,11.6,1 \mathrm{H}, \mathrm{H} 3), 2.25(\mathrm{~d}, J=10.0$, $1 \mathrm{H}, \mathrm{H} 4), 1.89(\mathrm{~m}, 2 \mathrm{H}, \mathrm{H} 7), 1.23(\mathrm{~d}, J=8.2,9 \mathrm{H}, \mathrm{PMe} 3), 1.06(\mathrm{t}, J=7.5,3 \mathrm{H}, \mathrm{H} 8) .{ }^{31} \mathrm{P} \mathrm{NMR}\left(\mathrm{CDCl}_{3}\right): \delta$ $-12.32\left(J_{\mathrm{P}-\mathrm{W}}=270 \mathrm{~Hz}\right)$.

$\mathrm{TpW}(\mathrm{NO})\left(\mathrm{PMe}_{3}\right)\left(3,4-\eta^{2}-(1 \mathrm{~S}, 2 \mathrm{R})-\mathrm{N1}, \mathrm{N2}\right.$-diphenyl-1,2-dihydroanthracene-1,2-diamine) (18) 


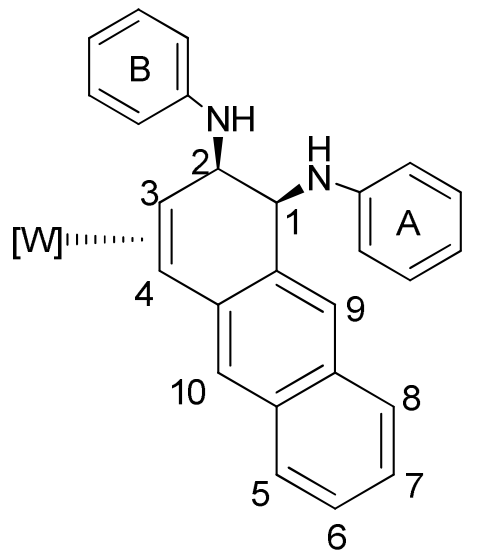

11 (90 mg, $0.132 \mathrm{mmol}$ ) was dissolved in $\mathrm{CDCl}_{3}(2.46 \mathrm{~g})$ and $\mathrm{N}$-bromosuccinimide (24 mg, 0.135 mmol) was dissolved in $\mathrm{MeCN}$ (1.009 g). Both solutions were placed in a $-30{ }^{\circ} \mathrm{C}$ cold bath for at least $30 \mathrm{~min}$. The $\mathrm{N}$-bromosuccinimide solution was added to the complex solution, followed directly by aniline (100 mg, $1.075 \mathrm{mmol}$ ) and triethylamine (30 mg, $0.297 \mathrm{mmol})$. The reaction was left in the cold bath overnight. The reaction was extracted $4 \mathrm{x}$ with $3 \mathrm{~mL}$ portions of water, which were backextracted with $0.5 \mathrm{~mL} \mathrm{DCM}$. The organic layers were dried with $\mathrm{MgSO}_{4}$, filtered over a Celite plug, and concentrated to $\sim 1.5 \mathrm{~mL}$. The solution was added to $17 \mathrm{~mL}$ stirring hexanes. The yellow solid was filtered over a $15 \mathrm{~mL}$ fine-porosity fritted funnel to give $\mathbf{1 8}$ (60 $\mathrm{mg}, 53 \%$, 85\% purity).

${ }^{1} \mathrm{H} \mathrm{NMR}\left(\mathrm{CDCl}_{3}\right): \delta 8.09(\mathrm{~s}, 1 \mathrm{H}, \mathrm{H} 9), 8.07(\mathrm{~d}, J=2.0,1 \mathrm{H}, \mathrm{pz} 3 \mathrm{~B}), 7.78(\mathrm{~d}, J=2.0,1 \mathrm{H}, \mathrm{pz} 5 \mathrm{C}), 7.73(\mathrm{~d}, J$ $=2.0,1 \mathrm{H}, \mathrm{pz} 5 \mathrm{~B}), 7.72(\mathrm{~m}, 1 \mathrm{H}, \mathrm{H} 8), 7.67(\mathrm{~d}, J=2.0,1 \mathrm{H}, \mathrm{pz} 5 \mathrm{~A}), 7.59(\mathrm{~d}, J=8.2,1 \mathrm{H}, \mathrm{H} 5), 7.36(\mathrm{~m}$, $1 \mathrm{H}, \mathrm{H} 6), 7.36(\mathrm{~d}, J=2.0,1 \mathrm{H}, \mathrm{pz} 3 \mathrm{C}), 7.30(\mathrm{~d}, J=2.0,1 \mathrm{H}, \mathrm{pz} 3 \mathrm{~A}), 7.23(\mathrm{~m}, 1 \mathrm{H}, \mathrm{H} 7), 7.22(\mathrm{~m}, 2 \mathrm{H}, \mathrm{A}-$ meta), 7.15 (m, 2H, B-meta), 7.04 (d, J = 8.2, 2H, A-ortho), 7.01 (s, 1H, H10), 6.72 (m, 1H, Apara), $6.70(\mathrm{~m}, 2 \mathrm{H}, \mathrm{B}$-ortho), $6.67(\mathrm{~m}, 2 \mathrm{H}, \mathrm{B}-$ para) $, 6.29(\mathrm{t}, J=2.0,1 \mathrm{H}, \mathrm{pz} 4 \mathrm{~B}), 6.23(\mathrm{t}, J=2.0,1 \mathrm{H}$, pz5B), $6.10(\mathrm{t}, J=2.0,1 \mathrm{H}, \mathrm{pz} 3 \mathrm{~B}), 5.50(\mathrm{ddd}, J=1.2,4.2,10.5,1 \mathrm{H}, \mathrm{H} 1), 4.69(\mathrm{~d}, J=10.5,1 \mathrm{H}, \mathrm{A}-\mathrm{NH})$, $4.63(\mathrm{dd}, J=3.3,9.3,1 \mathrm{H}, \mathrm{H} 2), 3.67(\mathrm{~d}, J=10.1,1 \mathrm{H}, \mathrm{B}-\mathrm{NH}), 3.02(\mathrm{ddd}, J=2.0,10.3,11.9,1 \mathrm{H}, \mathrm{H} 3$ ), $2.30(\mathrm{dd}, J=1.6,10.3,1 \mathrm{H}, \mathrm{H} 4), 1.23\left(\mathrm{~d}, J=8.0,9 \mathrm{H}, \mathrm{PMe}_{3}\right) .{ }^{13} \mathrm{C} \mathrm{NMR}\left(\mathrm{CDCl}_{3}\right): \delta 149.36,148.85$, $143.58,143.46,143.28,140.35,136.92,136.32,135.69,134.25,132.23,131.55,129.58,129.47$, 
$127.84,126.16,125.79,125.44,125.11,123.76,117.73,116.72,115.22,114.21,106.63$ (pz4C), 106.18 (pz4B), 105.33 (pz4A), 60.85 (C2), 59.32 (d, $J=12.3, C 3), 53.07$ (C1 or C4), 53.01 (C1 or C4), $13.45\left(\mathrm{~d}, J=28.1, \mathrm{PMe}_{3}\right) .{ }^{31} \mathrm{P}$ NMR $\left(\mathrm{CDCl}_{3}\right): \delta-12.78\left(J_{\mathrm{P}-\mathrm{W}}=276 \mathrm{~Hz}\right) . \quad \mathrm{IR}: \mathrm{v}_{\mathrm{NO}}=1556 \mathrm{~cm}^{-1}, \mathrm{v}_{\mathrm{BH}}=$ $2489 \mathrm{~cm}^{-1}$.

$\mathrm{TpW}(\mathrm{NO})\left(\mathrm{PMe}_{3}\right)\left(3,4-\eta^{2}-((1 \mathrm{~S}, 2 \mathrm{R})-1,2-\right.$ dihydroanthracene-1,2-diyl)bis(phenylsulfane)) (19)<smiles>[X]c1ccc2cc3cc(C)c(Sc4ccccc4)c(Sc4ccccc4)c3cc2c1</smiles>

11 (30 mg, $0.044 \mathrm{mmol}$ ) was dissolved in $\mathrm{CDCl}_{3}(999 \mathrm{mg}$ ) and $\mathrm{N}$-bromosuccinimide $(7.5 \mathrm{mg}$, $0.042 \mathrm{mmol}$ ) was dissolved in $\mathrm{MeCN}(4.93 \mathrm{mg})$. Both solutions were placed in a $-30^{\circ} \mathrm{C}$ cold bath for at least $30 \mathrm{~min}$. The $\mathrm{N}$-bromosuccinimide solution was added to the complex solution, followed directly by thiophenol $(30 \mathrm{mg}, 0.273 \mathrm{mmol}$ ) and triethylamine $(20 \mathrm{mg}, 0.198 \mathrm{mmol})$. The reaction was left in the cold bath overnight. The reaction was extracted $3 x$ with $2 \mathrm{~mL}$ portions of water, which were backextracted with $0.5 \mathrm{~mL}$ DCM. The organic layers were dried with $\mathrm{MgSO}_{4}$, filtered over a Celite plug, and evaporated in vacuo to a dark red film as 19 , on which NMR spectroscopy data was collected.

${ }^{1} \mathrm{H} \mathrm{NMR}\left(\mathrm{CDCl}_{3}\right): \delta 8.22(\mathrm{~s}, 1 \mathrm{H}, \mathrm{H} 9), 7.94(\mathrm{~d}, J=2.0,1 \mathrm{H}, \mathrm{pz} 3 \mathrm{~B}), 7.77(\mathrm{~m}, 1 \mathrm{H}, \mathrm{H} 8), 7.76(\mathrm{~m}, 3 \mathrm{H}, \mathrm{A}-$ ortho and pz5C), 7.71 (m, 2H, B-ortho), 7.69 (d, J = 2.0, 1H, pz5B), $7.64(\mathrm{~d}, J=2.0,1 \mathrm{H}, \mathrm{pz} 5 \mathrm{~A}), 7.57$ (m, 1H, H5), $7.36(\mathrm{~m}, 1 \mathrm{H}, \mathrm{H} 6), 7.31(\mathrm{~d}, J=2.0,1 \mathrm{H}, \mathrm{pz} 3 \mathrm{~A}), 7.28$ (m, 4H, A-meta and B-meta), 7.24 (m, 1H, H7), $7.16(\mathrm{~m}, 2 \mathrm{H}, \mathrm{A}$-para and B-para), $7.07(\mathrm{~d}, J=2.0,1 \mathrm{H}, \mathrm{pz} 3 \mathrm{C}), 6.96(\mathrm{~s}, 1 \mathrm{H}, \mathrm{H} 10), 6.24(\mathrm{t}$, $J=2.0,1 \mathrm{H}, \mathrm{pz} 4 \mathrm{~B}), 6.23(\mathrm{t}, J=2.0,1 \mathrm{H}, \mathrm{pz} 4 \mathrm{C}), 6.09(\mathrm{t}, J=2.0,1 \mathrm{H}, \mathrm{pz} 4 \mathrm{~A}), 5.53(\mathrm{dd}, J=1.4,4.2,1 \mathrm{H}$, 
$\mathrm{H} 1$ ), 4.60 (dd, $J=1.6,4.0,1 \mathrm{H}, \mathrm{H} 2$ ), 3.10 (ddd, $J=1.9,10.3,12.0,1 \mathrm{H}, \mathrm{H} 3$ ), 2.31 (dd, $J=1.4,10.3$, $1 \mathrm{H}, \mathrm{H} 4), 0.93\left(\mathrm{~d}, J=8.1,9 \mathrm{H}, \mathrm{PMe}_{3}\right) .{ }^{31} \mathrm{P} \mathrm{NMR}\left(\mathrm{CDCl}_{3}\right): \delta-12.08\left(J_{\mathrm{P}-\mathrm{W}}=276 \mathrm{~Hz}\right)$.

\section{References}

(1) Kuendig, E. P.; Ripa, A.; Liu, R.; Bernardinelli, G. The Journal of Organic Chemistry $1994,59,4773$.

(2) Pearson, A. J.; Vickerman, R. J. Tetrahedron Letters 1998, 39, 5931.

(3) Morley, J. A.; Woolsey, N. F. The Journal of Organic Chemistry 1992, 57, 6487.

(4) Suzuki, A. Chemical Communications 2005, 38, 4759.

(5) Negishi, E.-i.; Wang, G.; Rao, H.; Xu, Z. The Journal of Organic Chemistry 2010, $75,3151$.

(6) Negishi, E.-i.; Anastasia, L. Chemical Reviews 2003, 103, 1979.

(7) Jana, R.; Pathak, T. P.; Sigman, M. S. Chemical Reviews 2011, 111, 1417.

(8) Barnard, C. F. J. Organometallics 2008, 27, 5402.

(9) Dounay, A. B.; Overman, L. E. Chemical Reviews 2003, 103, 2945.

(10) Beletskaya, I. P.; Cheprakov, A. V. Chemical Reviews 2000, 100, 3009.

(11) Suzuki, A. Angewandte Chemie International Edition 2011, 50, 6722.

(12) Negishi, E.-i. Angewandte Chemie International Edition 2011, 50, 6738.

(13) Luh, T.-Y.; Leung, M.-k.; Wong, K.-T. Chemical Reviews 2000, 100, 3187.

(14) Rousseaux, S.; García-Fortanet, J.; Del Aguila Sanchez, M. A.; Buchwald, S. L. Journal of the American Chemical Society 2011, 133, 9282.

(15) Lee, C.-W.; Oh, K. S.; Kim, K. S.; Ahn, K. H. Org. Lett. 2000, 2, 1213.

(16) Frisch, A. C.; Beller, M. Angewandte Chemie International Edition 2005, 44, 674.

(17) Valahovic, M. T.; Gunnoe, T. B.; Sabat, M.; Harman, W. D. J. Am. Chem. Soc. 2002, 124, 3309.

(18) Harrison, D. P.; Nichols-Nielander, A. C.; Zottig, V. E.; Strausberg, L.; Salomon, R. J.; Trindle, C. O.; Sabat, M.; Gunnoe, T. B.; Iovan, D. A.; Myers, W. H.; Harman, W. D. Organometallics 2011, 30, 2587.

(19) Mayr, H.; Bug, T.; Gotta, M. F.; Hering, N.; Irrgang, B.; Janker, B.; Kempf, B.; Loos, R.; Ofial, A. R.; Remennikov, G.; Schimmel, H. Journal of the American Chemical Society 2001, $123,9500$.

(20) Ding, F.; Valahovic, M. T.; Keane, J. M.; Anstey, M. R.; Sabat, M.; Trindle, C. O.; Harman, W. D. J. Org. Chem. 2003, 69, 2257.

(21) Welch, K. D.; Harrison, D. P.; Lis, E. C.; Liu, W.; Salomon, R. J.; Harman, W. D.; Myers, W. H. Organometallics 2007, 26, 2791.

(22) Carey, F. A. S., Richard J. Advanced Organic Chemistry, Part A: Structure and Mechanisms; Springer: New York, 2007; Vol. 5.

(23) McCullough, J. J.; Wu, W. S.; Huang, C. W. Journal of the Chemical Society, Perkin Transactions 2 1972, 370.

(24) Pienkos, J. A.; Zottig, V. E.; lovan, D. A.; Li, M.; Harrison, D. P.; Sabat, M.; Salomon, R. J.; Strausberg, L.; Teran, V. A.; Myers, W. H.; Harman, W. D. Organometallics 2013, 32,691 .

(25) Roell, B. C.; McDaniel, K. F.; Vaughan, W. S.; Macy, T. S. Organometallics 1993, $12,224$. 
(26) Chung, Y. K.; Sweigart, D. A.; Connelly, N. G.; Sheridan, J. B. Journal of the American Chemical Society 1985, 107, 2388.

(27) Pigge, F. C.; Dalvi, R. Tetrahedron 2008, 64, 10123.

(28) Sun, S.; Yeung, L. K.; Sweigart, D. A.; Lee, T.-Y.; Lee, S. S.; Chung, Y. K.; Switzer, S. R.; Pike, R. D. Organometallics 1995, 14, 2613.

(29) McNair, A. M.; Mann, K. R. Inorganic Chemistry 1986, 25, 2519.

(30) Strausberg, L.; Li, M.; Harrison, D. P.; Myers, W. H.; Sabat, M.; Harman, W. D. Organometallics 2013, 32, 915. 
Chapter 5

Acetal Reactions of Anthracene Complexes 
A great variety of natural products, medicines, and materials contain two or three fused ring structures as their backbones. Given the wide availability and low cost of the fused ring aromatics naphthalene and anthracene, synthetic methods to elaborate these small molecules into functionalized products are highly desirable. However, producing functionalized small molecules from naphthalene and anthracene remains a synthetic challenge in organic chemistry, especially if the transformation will destroy the aromaticity of one of the rings. Alkylation conditions involve the use of strong Lewis acids and can result in product mixtures for naphthalene, or middle ring alkylation for anthracene. Birch conditions can be used to dearomatize both molecules, but are incompatible with many functional groups in addition to exhibiting poor selectivity.

We believed coordination of naphthalene and anthracene to the $\pi$-base $\left\{\mathrm{TpW}(\mathrm{NO})\left(\mathrm{PMe}_{3}\right)\right\}$ would drastically alter the observed chemistry of these two molecules, making them amenable to selective additions under mild conditions to yield functionalized, dearomatized small molecules. Precedents exist for the functionalization of naphthalene using similar $\pi$-bases. 1,4-Dihydronaphthalenes have been generated in moderate yields from a variety of carbon-based electrophiles and nucleophiles and $\left[\mathrm{Os}\left(\mathrm{NH}_{3}\right)_{5}\left(\eta^{2}\right.\right.$-naphthalene $\left.)\right](\mathrm{OTf})_{2}{ }^{1}$ A more electron rich rhenium complex, $\operatorname{TpRe}(\mathrm{CO})(\mathrm{L})\left(\mathrm{n}^{2}\right.$-naphthalene $)(\mathrm{L}=$ pyridine, $N$ methylimidazole, or $\mathrm{PMe}_{3}$ ) afforded 1,2- or 1,4-dihydronaphthalenes from carbon-based electrophiles and nucleophiles, depending on the ancillary ligand. ${ }^{2}$ As described in chapter 3 , work on $\mathrm{TpW}(\mathrm{NO})\left(\mathrm{PMe}_{3}\right)\left(\eta^{2}\right.$-naphthalene) has yielded exclusively 1,2-dihydronaphthalenes from the addition of a proton and aromatic carbon-based nucleophiles. ${ }^{3}$ However, we wished to explore the scope of reactions of $\operatorname{TpW}(\mathrm{NO})\left(\mathrm{PMe}_{3}\right)\left(\eta^{2}\right.$-naphthalene) with carbon-based electrophiles. 


\section{Results}

A variety of carbon electrophiles were introduced to $\mathrm{TpW}(\mathrm{NO})\left(\mathrm{PMe}_{3}\right)\left(\eta^{2}\right.$-naphthalene) (1) during an initial screening of reaction conditions. Acylation was attempted using acyl chloride and acetic anhydride, neither of which was found to react with 1 , while benzoyl chloride led to decomposition of 1 at $25^{\circ} \mathrm{C}$. Addition of Michael acceptors to 1 led to a variety of outcomes, depending on the identity of the Michael acceptor, the solvent, and the acid used (Scheme 1). Acrolein, methacrolein, and methyl acrylate all gave decomposition of $\mathbf{1}$, while cinnamaldehyde and 3-penten-2-one caused decomposition or gave no reaction, depending on the solvent and acid used. Methyl vinyl ketone (MVK) initially formed a major product when combined with 1 and one equivalent of diphenylammonium triflate (DPhAT) in DMF. Comparison of the product's ${ }^{31} \mathrm{P}$ NMR chemical shift and ${ }^{31} \mathrm{P}_{-}{ }^{183} \mathrm{~W}$ coupling constant to both the starting material, 1, and to other $\left\{\mathrm{TpW}(\mathrm{NO})\left(\mathrm{PMe}_{3}\right)\right\}$ complexes of naphthalenes, suggests that the product observed is $\mathbf{2}$, formed from the initial addition of MVK to the 1-carbon of naphthalene, followed by proton transfer from the ipso carbon on the naphthalene ring to the alpha carbon of MVK, resulting in the net alkylation of the 1-position. This reaction did not proceed cleanly, however, giving both decomposition and multiple products by the time $\mathbf{1}$ had been completely consumed. The reaction of $\mathbf{1}$ with tosyl isocyanate follows a similar pathway to generate the amide, $\mathbf{2} \mathbf{b}$, which can be isolated. The ${ }^{1} \mathbf{H}$ spectrum of $\mathbf{2} \mathbf{b}$ is similar to that of $\mathbf{1}$, where the protons on the bound carbons are at 3.67 and $2.55 \mathrm{ppm}$. All other protons are downfield of $6.5 \mathrm{ppm}$, as we would expect for aromatic resonances (excepting the tosyl methyl, at $2.44 \mathrm{ppm}$ ). Significantly, the doublet at $6.4 \mathrm{ppm}$ in a spectrum of 1 , which represents $\mathrm{H} 1$, is absent in the spectrum of $\mathbf{2} \mathbf{b}$. There is also a singlet at $9.98 \mathrm{ppm}$ in $\mathbf{2} \mathbf{b}$, which is consistent with an amide signal. 
Scheme 1
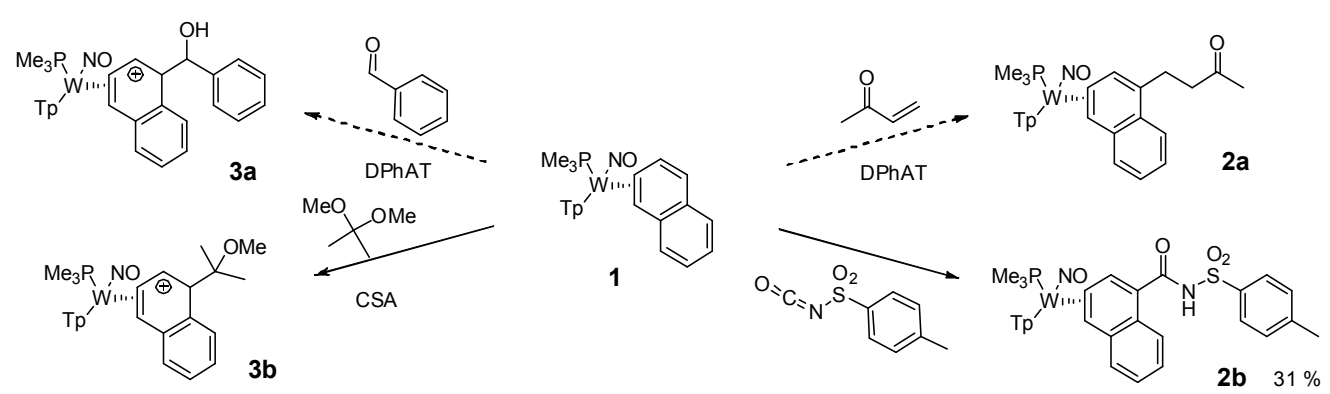

The reaction of benzaldehyde with $\mathbf{1}$ was explored under many sets of conditions. Using DPhAT and chloroform, a new product was initially generated have a ${ }^{31} \mathrm{P}_{-}{ }^{183} \mathrm{~W}$ coupling constant suggestive of a cationic species, possibly 3a. However, this species decomposed before all of $\mathbf{1}$ had reacted, and attempts to trap the product failed. Previous success with dimethoxymethane $e^{2}$ lead us to explore the reactivity of 1 with acetal reagents. When 1 was combined with dimethoxymethane and an equivalent of DPhAT in chloroform or DMF, decomposition resulted. A transiently stable species was formed from the combination of $\mathbf{1}$ and dimethoxypropane, with camphorsulfonic acid (CSA) as the proton source. We hoped that the switch from DPhAT to CSA would simplify the workup of neutral products, as CSA could be easily removed by extraction. The reaction was complete in two hours and the cationic product, possibly $\mathbf{3 b}$, could be trapped with a nucleophile. However, the neutral complex decomposed during the workup. We concluded that the reaction of 1 with dimethoxypropane was not an ideal reaction for study. Due to greater stability of the intermediate and greater ease of work up of the products, the use of dimethoxypropane as a carbon electrophile was developed more fully for the complementary complex, $\mathrm{TpW}(\mathrm{NO})\left(\mathrm{PMe}_{3}\right)\left(\eta^{2}\right.$-anthracene), 4. $\mathrm{TpW}(\mathrm{NO})\left(\mathrm{PMe}_{3}\right)\left(\eta^{2}-\right.$ anthracene) was combined with two equivalents of CSA in a $2.4 \mathrm{M}$ solution of dimethoxypropane in chloroform, resulting in a reaction which was complete in two to three hours. The new product had a ${ }^{31} \mathrm{P}$ NMR chemical shift of -11.09 , and a ${ }^{31} \mathrm{P}_{-}{ }^{183} \mathrm{~W}$ coupling constant of $265 \mathrm{~Hz}$, which is significantly upfield of the value for the cationic product resulting 
from the protonation of 4 , where $\delta=-6.52$ and $J=271 \mathrm{~Hz}$. An analysis of the 2D NMR spectra of the isolated intermediate showed that the three protons on the allylic carbons coupled to each other, with each shifted downfield from where it is expected for a neutral species. H2, or the allyl terminus proximal to the phosphine ligand, had a chemical shift of 8.68 and did not show any coupling to any protons in the COSY spectrum other than $\mathrm{H} 3$, at 5.90 . $\mathrm{H} 3$ coupled to $\mathrm{H} 4$, at 3.87. No proton was identified as belonging to $\mathrm{C} 1$. The proton spectrum of the intermediate showed two singlets, at 2.46 and 2.79 , each of which integrated to approximately three protons. The singlet at 2.46 displayed an NOE interaction with $\mathrm{H} 2$, while the other displayed an NOE to another singlet, at 7.96, which corresponds to H9. Taken together, these data indicate the product is $\mathbf{5}$, an ylidene species formed by the elimination of methanol after the initial addition of the acetal electrophile (Scheme 2).

Scheme 2

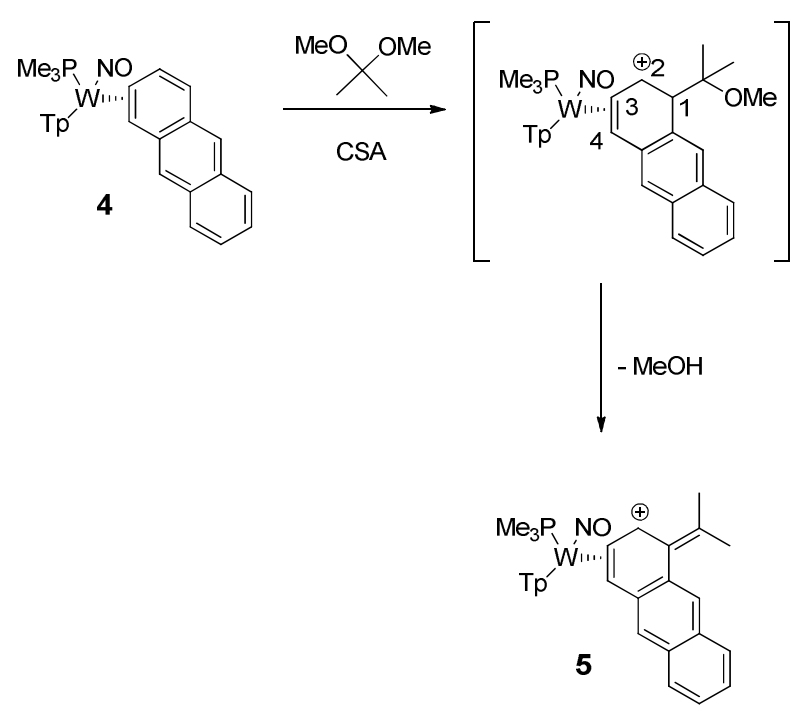

As was the case for the naphthalene product, 5 was only transiently stable. However, a nucleophile could be added to generate a neutral species that, in many cases, could be isolated cleanly. When the nucleophile was nitrogen-based, the observed product had a ${ }^{31} \mathrm{P} N M R$ chemical shift upfield of -12.00 , and a ${ }^{31} \mathrm{P}_{-}{ }^{183} \mathrm{~W}$ coupling constant between 289 and $295 \mathrm{~Hz}$. 
These are characteristic of 4, suggesting what was confirmed by analysis of the 2D NMR data for these complexes, that the products were substituted anthracenes. Thus, $\mathbf{6 ,} \mathbf{7}$, and $\mathbf{8}$ could be generated from the combination of 5 with propylamine, morpholine, and imidazole, respectively (Scheme 3). These complexes have ${ }^{1} \mathrm{H}$ NMR resonances for $\mathrm{H} 2-\mathrm{H} 4$ that are very similar to those for 4, where the $\mathrm{H} 2$ resonance is approximately $7.05 \mathrm{ppm}, \mathrm{H} 3$ is approximately $3.7 \mathrm{ppm}$, and H4 is approximately $2.6 \mathrm{ppm}$. Interestingly, the resonance for H9 appears downfield of 9 ppm for $\mathbf{6}$ and $\mathbf{7}$, while it is at 7.90 ppm for $\mathbf{8}$.

\section{Scheme 3}

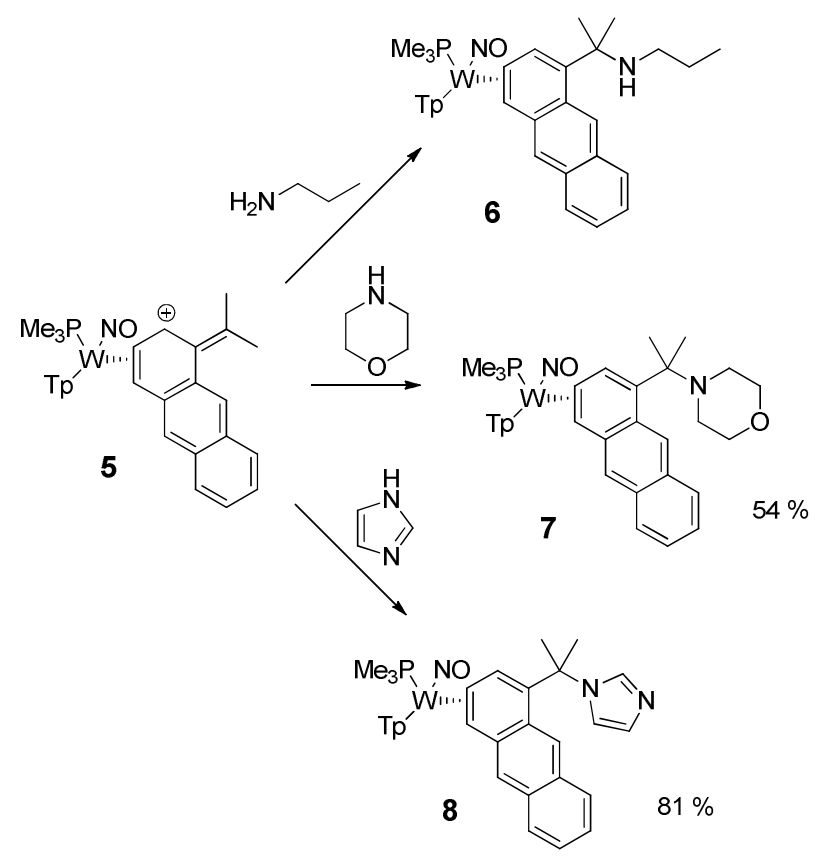

Interestingly, a different product was observed when carbon-based nucleophiles were used. In cases where a clean nucleophile addition was observed, the initial product observed had a ${ }^{31} \mathrm{P}$ NMR chemical shift downfield of -12.00 and $a{ }^{31} \mathrm{P}_{-}{ }^{183} \mathrm{~W}$ coupling constant between 270 and $280 \mathrm{~Hz}$. This is comparable to the ${ }^{31} \mathrm{P}$ NMR spectra observed for complexes of 1,2dihydronaphthalenes, suggesting that the complexes formed were structurally different from 6 - 8. Carbon nucleophiles generated via deprotonation of nitromethane, dimethylmalonate, and malononitrile added to 5 to give one major product. The ${ }^{1} \mathrm{H}$ NMR spectrum of each product 
showed a doublet of doublets at $4.5-4.7 \mathrm{ppm}$ which coupled to the tungsten-bound proton proximal to the $\mathrm{PMe}_{3}$ ligand and displayed an $\mathrm{NOE}$ interaction with the $\mathrm{PMe}_{3}$ ligand and with a singlet at approximately $2 \mathrm{ppm}$. This doublet of doublets also coupled to a proton on the nucleophile. Taken together, the two dimensional NMR analysis supports product assignment of nucleophilic addition of nitromethane, dimethylmalonate, and malononitrile to 5 as the 1,2ylidene species, $\mathbf{9}$ - $\mathbf{1 1}$ (Scheme 4).

Scheme 4

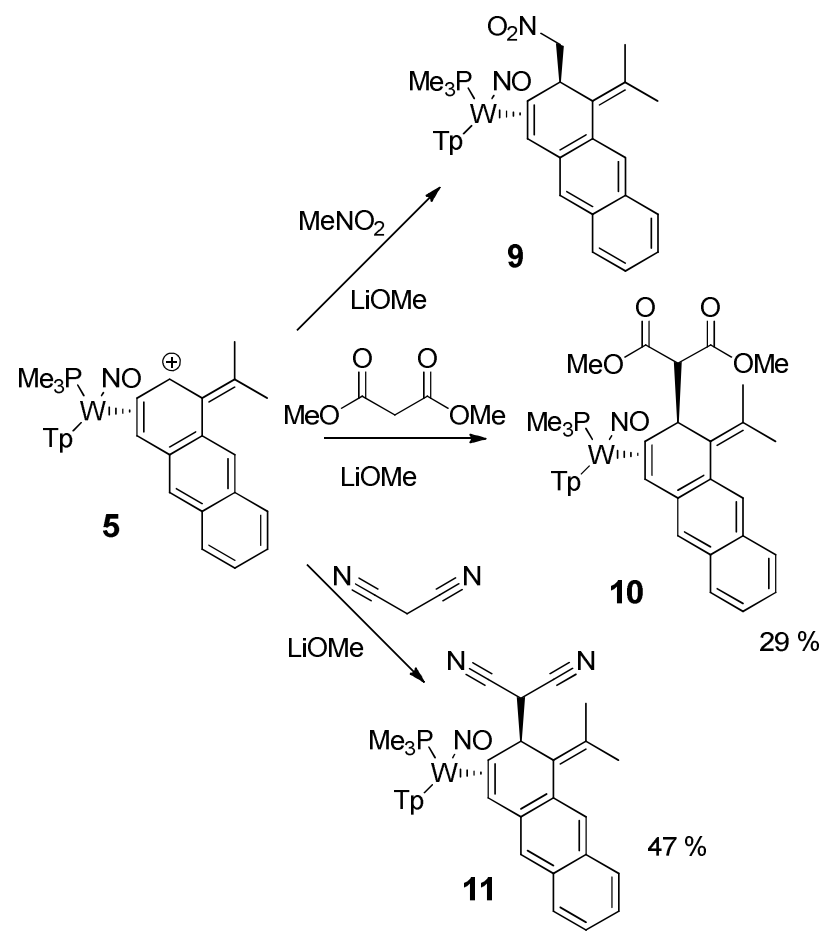

When 11 was allowed to stand in solution, its resonance signal in the ${ }^{31} \mathrm{P}$ NMR spectrum disappeared over time and a new peak grew in which had a larger coupling constant. Working with a new solution of $\mathbf{5}$, addition of malononitrile and lithium methoxide in methanol followed by stirring overnight resulted in the precipitation of a bright orange solid. This solid was collected, but was found to be nearly insoluble in organic solvents. Two dimensional NMR data was collected for a d-MeCN solution of the solid, but the product's structure was unambiguously confirmed from a crystal structure obtained of the product, 12 (Figure 1). 
Figure 1 - ORTEP of 12.

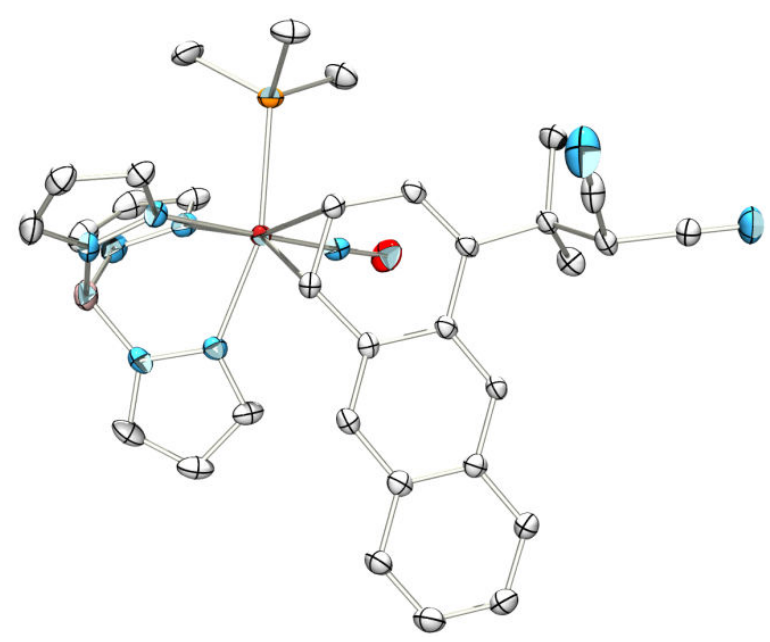

The isomerization of a 1,2-ylidene product to give a benzyl addition product of a rearomatized anthracene was observed for other carbon nucleophiles, as well. Enolates generated from acetone and acetophenone added to 5 to give a product that initially looked like a 1,2-ylidene product in the ${ }^{31} \mathrm{P}$ NMR spectrum. However, when these were worked up via extraction, they yielded multiple products. Allowing them to stand before work-up led to the generation of a benzyl product, which could be isolated cleanly. These products, $\mathbf{1 2}$ from malononitrile and $\mathbf{1 3}$ from acetone, are spectroscopically more similar to 4 than to the 1,2dihydroanthracenes (Scheme 5). The $\mathrm{PMe}_{3}$ signals in the ${ }^{1} \mathrm{H}$ spectra are $1.42 \mathrm{ppm}$ and $1.36 \mathrm{ppm}$, respectively, while signals for $\mathbf{9 - 1 1}$ are slightly upfield of that. The bound proton proximal to the $\mathrm{PMe}_{3}$ ligand has a signal at $3.7-3.9 \mathrm{ppm}$, and couples to $\mathrm{H} 2$ at approximately $7 \mathrm{ppm}$, in each case. Other nucleophiles that were attempted with $\mathbf{5}$ but did not react or yield clean products include trimethylsilyl cyanide, pyrrole, and N-methylpyrrole. 
Scheme 5

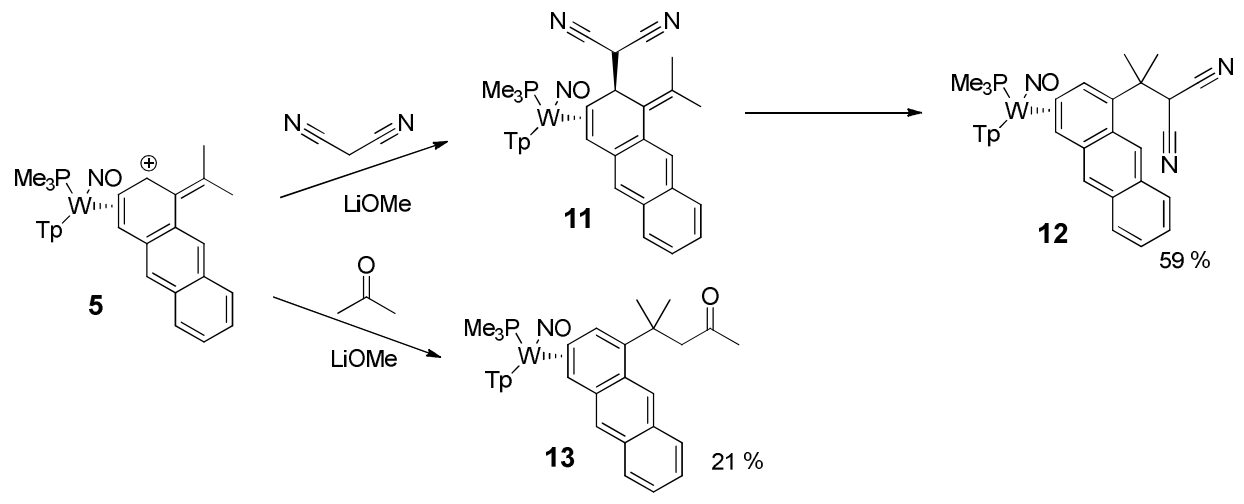

Other heteroatom nucleophiles were added to 5, including lithium methoxide, thiophenol, and tetrabutylammonium fluoride $\left(\mathrm{TBAF} \bullet \mathrm{nH}_{2} \mathrm{O}\right)$. When lithium methoxide was dissolved in methanol and added to a solution of $\mathbf{5}$, a product was initially observed which had a ${ }^{31} \mathrm{P}-{ }^{183} \mathrm{~W}$ coupling constant of $272 \mathrm{~Hz}$, which is similar to coupling constants of 1,2-ylidenes, 9 11. However, an extraction with saturated $\mathrm{K}_{2} \mathrm{CO}_{3}$ solution yielded an intractable mixture. Given the observation that some carbon nucleophiles isomerize over time from an ylidene product to a benzyl product, the methoxide addition product was allowed to stand for a week, generating a new product observable in the ${ }^{31} \mathrm{P}$ NMR spectrum $(-12.77 \mathrm{ppm}, 292 \mathrm{~Hz})$, before a work-up by extraction with saturated $\mathrm{Na}_{2} \mathrm{CO}_{3}$ solution was performed. This modification did not prevent the formation of multiple new products and decomposition, suggesting that if the benzyl product, 14, is formed, it is not stable under any aqueous work up conditions (Scheme 6). Thiophenol did not react with $\mathbf{5}$ under neutral conditions, however, once deprotonated with LiOMe it was sufficiently nucleophilic to react. This generated a product bearing spectroscopic similarities to the amine addition products, and was assigned as 15 . This complex features a proton that decouples at $3.66 \mathrm{ppm}$ (the bound proton proximal to the $\mathrm{PMe}_{3}$ ligand, or $\mathrm{H} 3$ ) which couples to a doublet at $6.75 \mathrm{ppm}(\mathrm{H} 2)$. Both these protons show an NOE interaction with the $\mathrm{PMe}_{3}$ ligand, and $\mathrm{H} 2$ also shows an NOE interaction with a slightly broadened singlet at $1.72 \mathrm{ppm}$. This singlet shows an NOE interaction with another singlet at $2.07 \mathrm{ppm}$, which has an NOE interaction with a 
singlet at $8.98 \mathrm{ppm}$. This severely downshifted singlet likely results from $\mathrm{H} 9$, the proton on the $\mathrm{B}$ ring of anthracene. H9 likely experiences an anisotropic effect from the aromatic thiophenol ring, causing its ${ }^{1} \mathrm{H}$ NMR signal to appear downfield of such signals in comparable complexes. That $\mathrm{H} 9$ has NOE interactions with two doublets, at $7.94 \mathrm{ppm}$ and $7.39 \mathrm{ppm}$ ( $\mathrm{H} 13$ and $\mathrm{H} 8$, respectively), makes its downfield shift due to anisotropy plausible. When the reaction of $\mathbf{5}$ and thiophenol was scaled up, an interesting byproduct was observed. A new signal appeared at $5.24 \mathrm{ppm}$ that coupled to a singlet at $2.25 \mathrm{ppm}$. Both of these signals had NOE interactions with $\mathrm{H} 2$, at $6.82 \mathrm{ppm}$, leading to a structure assignment as $\mathbf{1 6}$, where thiophenol is eliminated to form a propenyl group at $\mathrm{C} 1$ of anthracene. Complex $\mathbf{1 6}$ could also be generated directly from $\mathbf{5}$ by the addition of pyridine, which acts as a base. Addition of TBAF to 5 resulted in the formation of one major complex, believed to be $\mathbf{1 7}$, as observed by ${ }^{31} \mathrm{P}$ NMR spectroscopy. However, attempts to isolate this product through extraction resulted in its decomposition.

Scheme 6

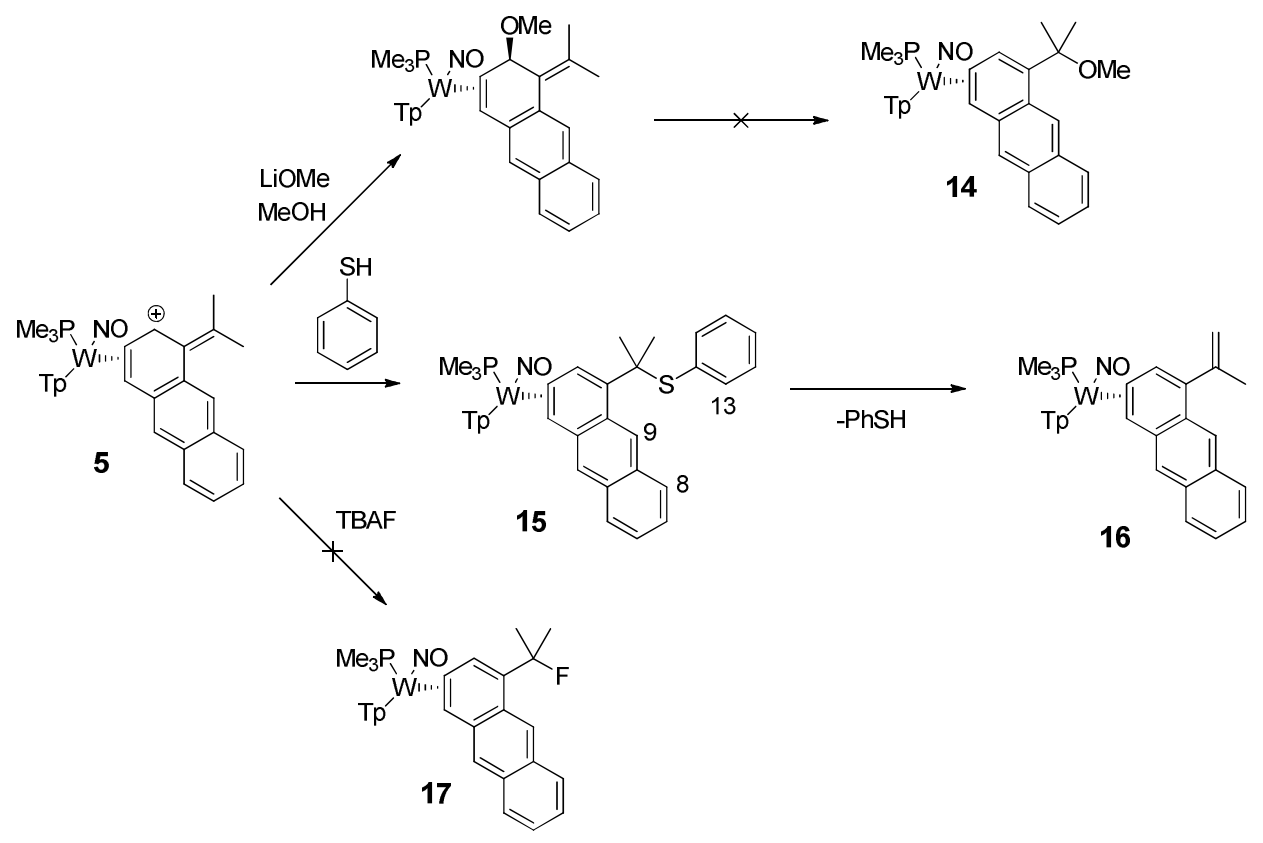

We hoped to make this reaction-type general for a variety of acetal reagents. Reagents with unsaturated moieties were of particular interest given that their successful addition would 
generate extended $\pi$ systems that could be stabilized by the metal complex (Figure 2 ). Acrolein dimethyl acetal resulted in rapid decomposition of 4, while 1,1-dimethoxyacetone did not react, resulting in slow decomposition of $\mathbf{4}$ due to the presence of the acid. Dimethoxymethane and acetal reagents bearing nitrogen atoms (e.g., $\mathrm{N}, \mathrm{N}$-dimethylformamide diethyl acetal and methylaminoacetaldehyde dimethyl acetal) did not react with 4, while acetalaldehyde dimethyl acetal with $\mathbf{4}$ led to rapid decomposition. Cyclopentanone ethylene ketal did not react with $\mathbf{4}$, but 1,1-dimethoxycyclohexane did react slowly to generate a new intermediate. Benzaldehyde dimethyl acetal also reacted rapidly with $\mathbf{4}$ to generate a new intermediate complex.

Figure 2 - Acetal reagents added to 4.<smiles>C=CC(C)OC(C)OC</smiles><smiles>CCOC(OCC)N(C)C</smiles><smiles>COC(OC)c1ccccc1</smiles>

The reaction of 1,1-dimethoxycyclohexane with 4 was slow, and did not go to completion before the cationic intermediate began to decompose. In order to get a better sense of what the intermediate was, it was trapped by the addition of propylamine, at which point a product could be isolated. The crude product contained approximately $30 \%$ starting material, 4, and excess 1,1-dimethoxycyclohexane. However, two interesting features could be distinguished in the ${ }^{1} \mathrm{H}$ NMR spectrum. First, a proton that decouples in the ${ }^{31} \mathrm{P}$-decoupled ${ }^{1} \mathrm{H}$ spectrum can be observed at $3.1 \mathrm{ppm}$. Second, a doublet of doublets appears at $4.36 \mathrm{ppm}$. 
These features, along with a ${ }^{31} \mathrm{P}$ NMR signal at approximately $-10.2 \mathrm{ppm}$ with ${ }^{31} \mathrm{P}_{-}{ }^{183} \mathrm{~W}$ coupling of $280 \mathrm{~Hz}$, are consistent with 1,2-ylidene products of dimethoxypropane, described above, and suggest a product, $\mathbf{1 8}$, shown below.

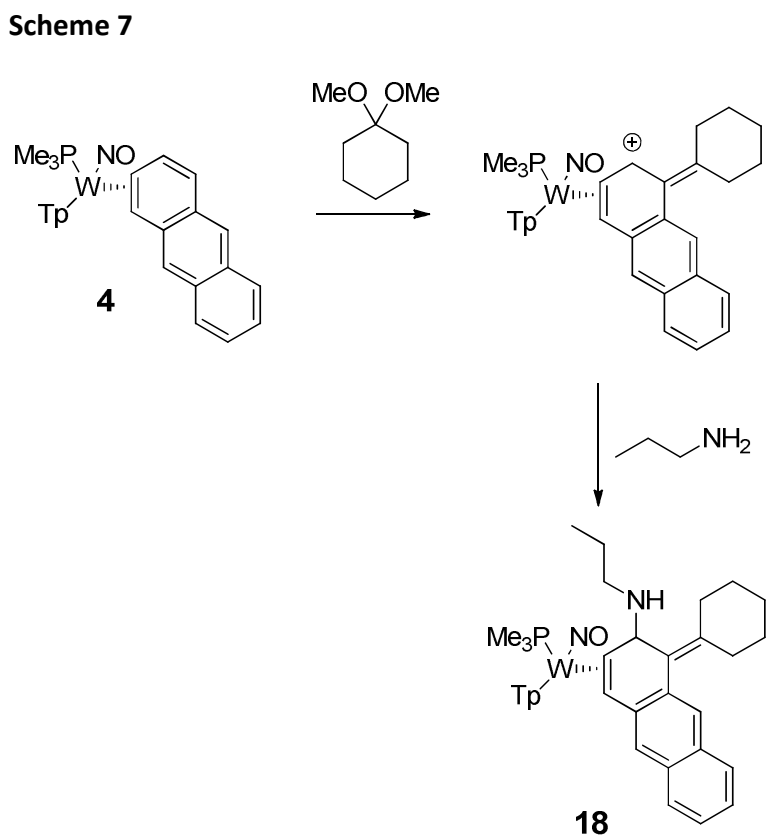

A scale-up of this reaction was attempted so as to remove the excess dimethoxycyclohexane and make NMR analysis easier to perform. Unfortunately, multiple products and decomposition resulted from the work-up conditions. A similar reaction using dimethylmalonate as the nucleophile also underwent decomposition during the work-up.

Benzaldehyde dimethyl acetal also reacted with $\mathbf{4}$ to give a transiently stable intermediate that could be trapped with a nucleophile or isolated via precipitation. Because benzaldehyde dimethyl acetal does not generate a symmetrical acetal, two potential diastereomers of this ylidene could be formed, 19a and 19b (Scheme 8). 
Scheme 8

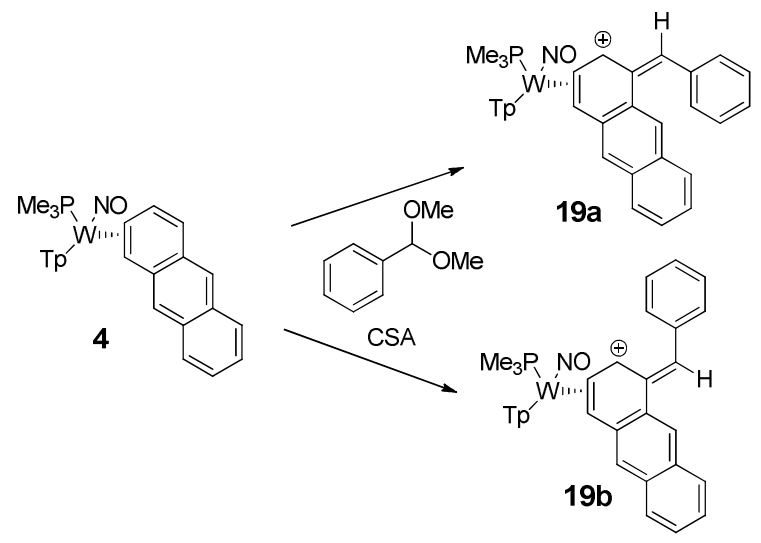

A two dimensional NMR analysis was performed on the isolated intermediate. The three protons on the carbons making up the metal-ligand bond were identified at $8.24 \mathrm{ppm}(\mathrm{H} 2), 6.42$ ppm (H3), and $4.02 \mathrm{ppm}(\mathrm{H} 4) . \mathrm{H} 2$ and $\mathrm{H} 3$ displayed an NOE interaction with the $\mathrm{PMe}_{3}$ signal, and $\mathrm{H} 2$ also had an NOE interaction with a doublet at $7.63 \mathrm{ppm}$. This doublet has an NOE interaction with a doublet of doublets at $7.50 \mathrm{ppm}$, and also with a singlet at $8.21 \mathrm{ppm}$. This set of correlations is consistent with an assignment of the ylidene as $19 \mathrm{~b}$, in which the ortho protons on the phenyl ring have NOE interactions with both $\mathrm{H} 2$ and the vinyl proton of the acetal reagent. No NOE interaction could be unambiguously identified between the vinyl proton and $\mathrm{H} 10$.

A number of nucleophiles were tested with $19 \mathrm{~b}$. Carbon nucleophiles pyrrole and trimethylsilyl cyanide did not react with $\mathbf{1 9 b}$, or gave multiple products, respectively, while dimethylmalonate and nitromethane each gave a major product that could be assigned by two dimensional NMR analysis as 1,2-ylidene products, $\mathbf{2 0}$ and $\mathbf{2 1}$, respectively (Scheme 9). Each featured a signal at about $5.0 \mathrm{ppm}(\mathrm{H} 2)$ that coupled to protons on the added nucleophile, while displaying weak coupling to H3. Additionally, each $\mathrm{H} 2$ proton displayed an NOE interaction with a doublet at 7.5-7.7 ppm, which is likely the signal for the ortho protons on the phenyl ring in each product. In each product, the vinyl proton generated from the acetal addition had an NOE 
interaction with $\mathrm{H} 9$, the proton on the middle ring of the anthracene. Nitrogen nucleophiles propylamine and imidazole gave benzyl addition products, $\mathbf{2 2}$ and $\mathbf{2 3}$, while lithium methoxide generated multiple products. Major features of the benzyl products were the presence of $\mathrm{H} 2$ 's signal at $6.83 \mathrm{ppm}(22)$ and $6.50 \mathrm{ppm}(\mathbf{2 3})$, along with ${ }^{31} \mathrm{P}$ NMR signals upfield of -12.00 and ${ }^{31} \mathrm{P}$ ${ }^{183} \mathrm{~W}$ coupling constants greater than $290 \mathrm{~Hz}$.

\section{Scheme 9}

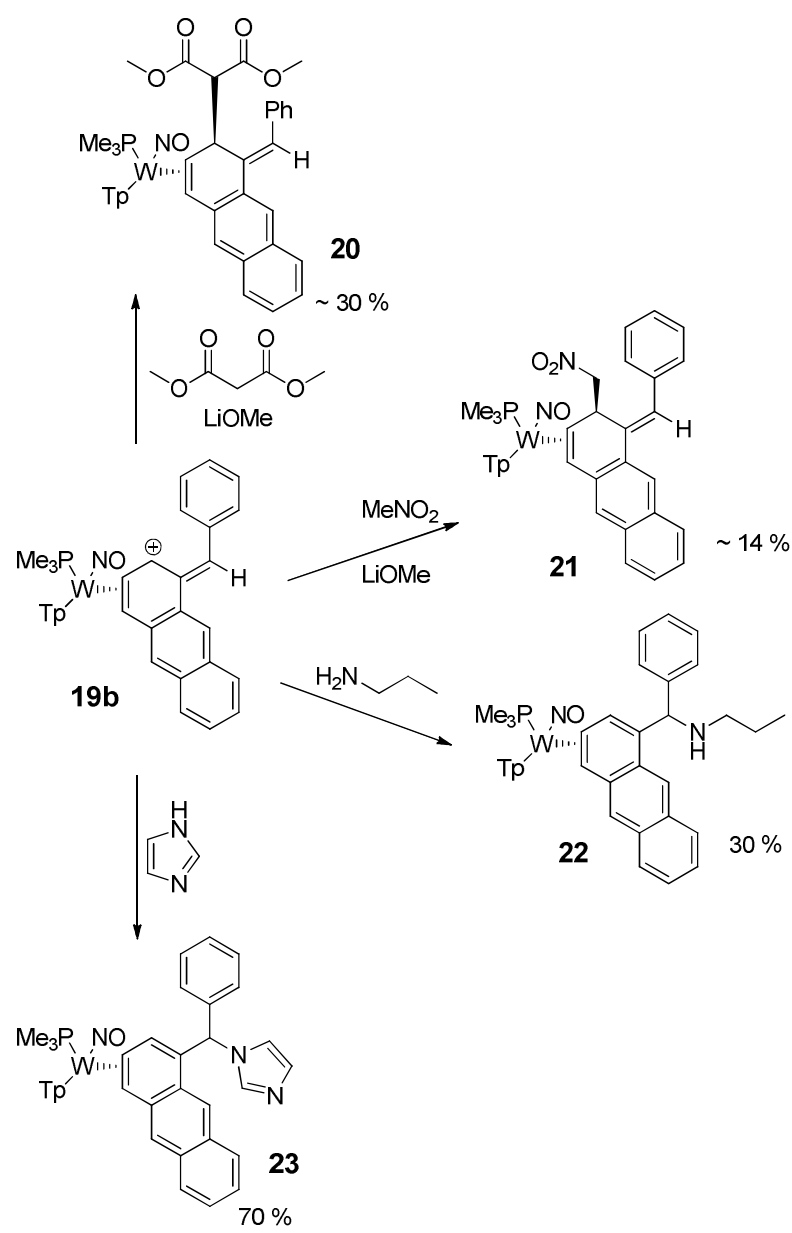

Having generated a collection of 1,2-ylidene and 1-substituted anthracene complexes, we, next turned our attention to finding conditions for the removal of the metal and isolation of the new organic. Demetallation for several of the complexes was achieved via oxidation of the metal using ceric ammonium nitrate (CAN) (Scheme 10). Following decomplexation, the organic 
was isolated via an extraction, and, if necessary, chromatography. Complex $\mathbf{8}$ was decomplexed using $300 \mathrm{~nm}$ light in a photoreactor and isolated via an extraction.

Scheme 10
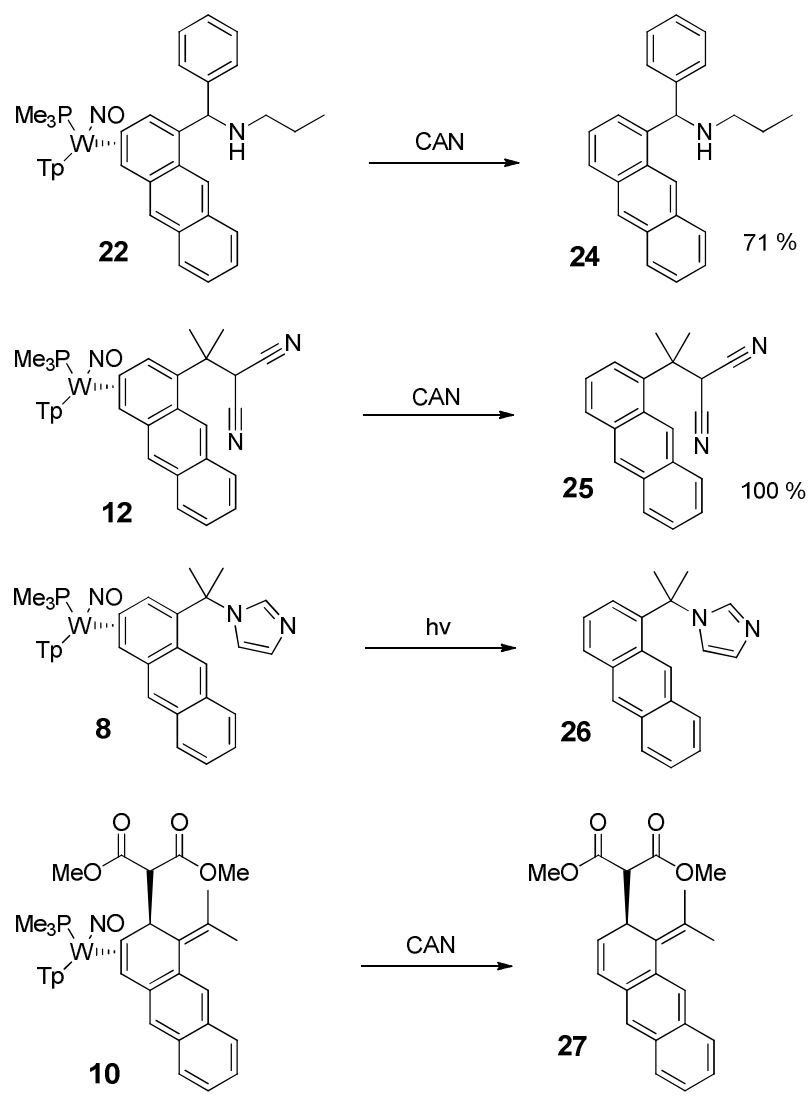

We were interested in further elaborating our 1-substituted anthracenes and sought conditions for their oxidation to anthraquinones. Using $\mathbf{2 5}$ as a model, four equivalents of CAN in water was sufficient to generate a new species that could be studied after an extraction. The new organic had unique spectroscopic features, but these do not support its assignment as a 1substituted anthraquinone (Scheme 11). The singlet at $8.55 \mathrm{ppm}$ which correlates to the middle ring protons in $\mathbf{2 5}$ is not present in the new organic, $\mathbf{2 8}$, while the IR spectrum of $\mathbf{2 8}$ has a large stretch at $1667 \mathrm{~cm}^{-1}$, which is consistent with the carbonyl stretch of anthraquinone. The IR spectrum of 28 also contains a smaller stretch at $2254 \mathrm{~cm}^{-1}$, which is consistent with the nitrile stretch of malononitrile. However, the methyl groups in a purported anthraquinone product 
should be identical, and we observe two singlets at 1.95 and $1.97 \mathrm{ppm}$ in the product. One possible explanation is that one of the nitriles was hydrolyzed to an amide. This would create asymmetry in the molecule. A similar procedure was attempted with $\mathbf{2 4}$, but led to decomposition of the anthracene.

\section{Scheme 11}
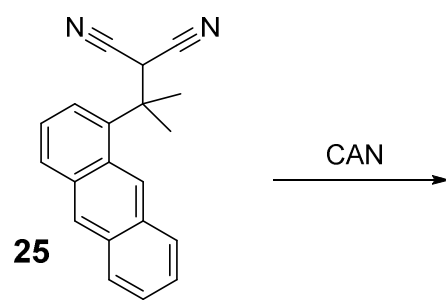

Discussion

The difficulties we experienced in cleanly adding a carbon electrophile to naphthalene complex, 1, led us to explore the feasibility of adding carbon electrophiles to anthracene complex, 4. We anticipated that the larger ring system of $\mathbf{4}$ would offer greater charge stabilization through delocalization upon addition of the electrophile. This effect was observed in the protonations of $\mathbf{1}$ and $\mathbf{4}$, in which the protonated $\mathbf{4}$ survived much longer in solution compared to protonated 1 . This trend was also observed in $\left\{\left[\mathrm{Os}\left(\mathrm{NH}_{3}\right)_{5}\right](\mathrm{OTf})_{2}\right\}$ complexes of naphthalene and anthracene, which were found to be much more stable when protonated than $\left[\mathrm{Os}\left(\mathrm{NH}_{3}\right)_{5}\left(\eta^{2}\right.\right.$-benzene $\left.)\right](\mathrm{OTf})_{2}{ }^{4}$ 
Scheme 12
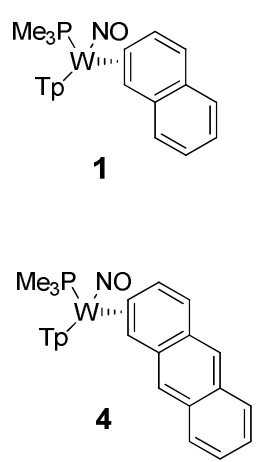
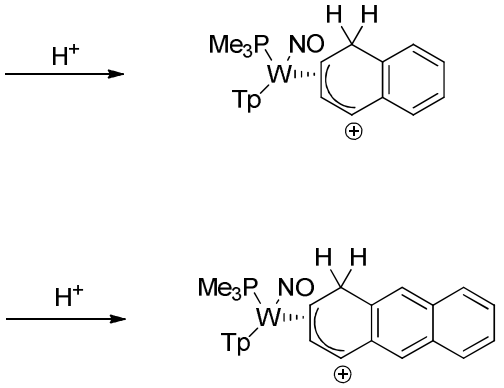

In addition to the greater stability we expected for cationic elaborations of $\mathbf{4}$, we also expected that electrophilic and nucleophilic additions to 4 would occur in the ring to which the metal was bound, or the A ring. This is a departure from the observed chemistry of anthracene under organic methods, in which reactivity is greatest in the middle, or B, ring.

While the chemistry of anthracene complexes of $\left\{\mathrm{TpW}(\mathrm{NO})\left(\mathrm{PMe}_{3}\right)\right\}$ precursors $\left\{\left[\mathrm{Os}\left(\mathrm{NH}_{3}\right)_{5}\right](\mathrm{OTf})_{2}\right\}$ and $\{\mathrm{TpRe}(\mathrm{CO})(\mathrm{L})\}\left(\mathrm{L}=\right.$ pyridine, $\mathrm{N}$-methylimidazole, or $\mathrm{PMe}_{3}$ ) has not been studied in great detail, some parallels can be drawn from the chemistry of naphthalene complexes of these metals. Addition of dimethoxymethane and triflic acid to $\left[\mathrm{Os}\left(\mathrm{NH}_{3}\right)_{5}\left(\mathrm{n}^{2}-\right.\right.$ naphthalene)](OTf $)_{2}$ yielded an isolable allylic intermediate with a methoxymethyl group at $\mathrm{C} 1 .^{1}$ A variety of nucleophiles, including dimethyl malonate, could then be added to the allylic species at $-40{ }^{\circ} \mathrm{C}$ to generate 1,4 -dihydronaphthalenes which were oxidized in situ to isolate the new organic (Scheme 13). 
Scheme 13

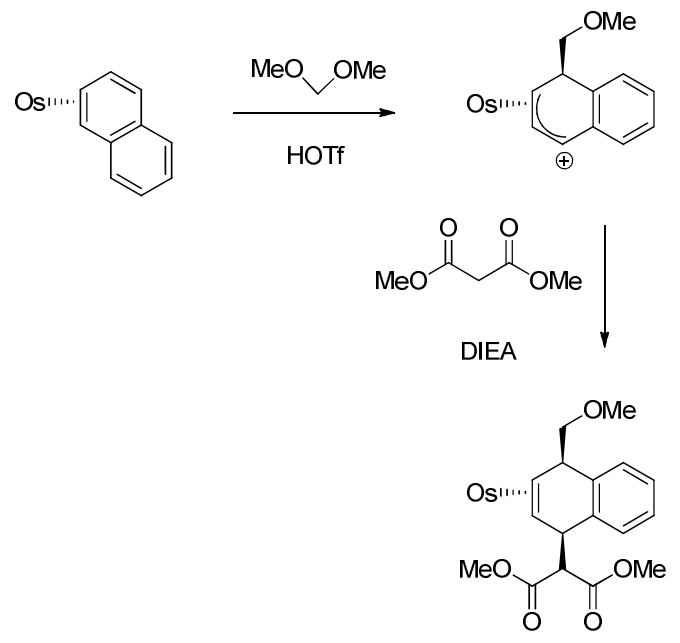

When dimethoxymethane was combined with TBSOTf, DBU, and $\operatorname{TpRe}(\mathrm{CO})(\mathrm{L})\left(\eta^{2}\right.$-naphthalene), where $\mathrm{L}$ is one of pyridine, $\mathrm{N}$-methylimidazole, or $\mathrm{PMe}_{3}$, a similar allylic species was generated, to which dimethylmalonate also added 1,4. Unlike $\left[\mathrm{Os}\left(\mathrm{NH}_{3}\right)_{5}\left(\eta^{2}\right.\right.$-naphthalene $\left.)\right](\mathrm{OTf})_{2}$, the rhenium complexes are chiral, and two isomers are observed when naphthalene binds to the metal. Depending on the preference of the complex for one isomer or the other, the product of the tandem electrophilic/nucleophilic addition was reversed (Scheme 14). ${ }^{2}$

\section{Scheme 14}

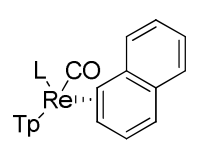

29A
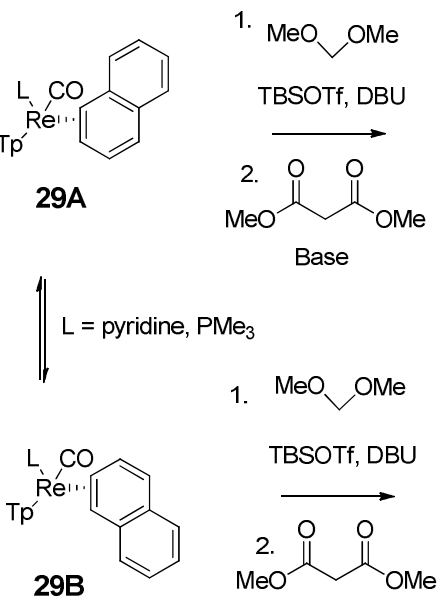

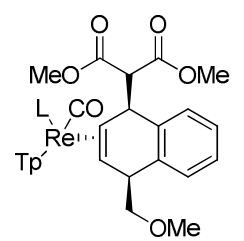

$\mathrm{L}=$ pyridine

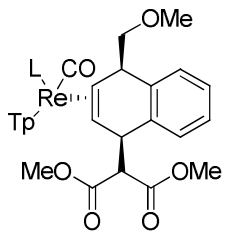

$\mathrm{L}=\mathrm{PMe}_{3}$ 
Michael acceptors also reacted with $\operatorname{TpRe}(\mathrm{CO})(\mathrm{L})\left(\mathrm{n}^{2}\right.$-naphthalene), where $\mathrm{L}$ was pyridine or $\mathrm{N}$ methylimidazole, to form tricyclic species in which the cycloaddition occurred $1,2 .^{2}$ The FriedelCrafts chemistry explored with $\operatorname{TpW}(\mathrm{NO})\left(\mathrm{PMe}_{3}\right)\left(\eta^{2}\right.$-naphthalene) yielded 1,2dihydronaphthalenes, although in these cases a proton served as the electrophile. ${ }^{3}$

Where nucleophilic addition occurs on the ring of $\mathrm{TpW}(\mathrm{NO})\left(\mathrm{PMe}_{3}\right)\left(\eta^{2}\right.$-anthracene) when combined with dimethoxypropane and acid, that addition is 1,2. This makes sense from a thermodynamic standpoint, as 1,2-addition allows continued interaction of the $\pi$ system of the unbound rings with the metal complex, which would not be possible for a 1,4-addition product. Indeed, a computational investigation with Gaussian (performed according to the methods described in chapter 2 experimental section) found that $\operatorname{TpW}(\mathrm{NO})\left(\mathrm{PMe}_{3}\right)\left(3,4-\eta^{2}-1,2-\right.$ dihydronaphthalene) was approximately $5.5 \mathrm{kcal} / \mathrm{mol}$ more stable than $\mathrm{TpW}(\mathrm{NO})\left(\mathrm{PMe}_{3}\right)\left(2,3-\eta^{2}-\right.$ 1,4-dihydronaphthalene). 1,4-addition for the osmium complex was justified by kinetic arguments: the metal was shown to bind more closely to the $\mathrm{C} 2$ terminus of the allyl, such that C4 was better exposed to the $\pi$ system of the unbound ring and to the incoming nucleophile. ${ }^{1}$ The rhenium complexes also exhibited 1,4-addition of dimethoxymethane and a nucleophile, despite being performed at room temperature. ${ }^{2}$ However, when tandem additions were performed with a proton and a nucleophile, 1,2-addition was observed for complexes in which the ancillary ligand, L, was pyridine or $\mathrm{N}$-methylimidazole. When the ancillary ligand was $\mathrm{PMe}_{3}$, 1,4-addition was observed. Multiple isomers of the rhenium naphthalene complexes are observed at room temperature for each ancillary ligand. When the ancillary ligand is pyridine or $\mathrm{N}$-methylimidazole, isomer 29A dominates; with $\mathrm{PMe}_{3}$, isomer 29B dominates. This preference for the naphthalene ring to lie away from the ancillary ligand when it is $\mathrm{PMe}_{3}$ is justified by the relative steric bulk of that ligand. Thus, when $\operatorname{TpRe}(\mathrm{CO})\left(\mathrm{PMe}_{3}\right)\left(\eta^{2}\right.$-naphthalene) is protonated, the 1,4-addition of the nucleophile leads to a less sterically congested configuration in the 
product. When $\operatorname{TpRe}(\mathrm{CO})($ pyridine $)\left(\eta^{2}\right.$-naphthalene) or $\operatorname{TpRe}(\mathrm{CO})(\mathrm{MeIm})\left(\eta^{2}\right.$-naphthalene) are protonated, the allyl configuration leading to 1,2-addition is most stable and accessible, making 1,2-dihydronaphthalene the preferred product. When the electrophile was dimethoxymethane, however, all the rhenium complexes generated 1,4-dihydronaphthalenes upon addition of a nucleophile. This suggests that the methoxymethyl group was sufficiently bulky to make 1,2addition unfavorable.

We have observed a trend in which osmium-naphthalene complexes yield exclusively 1,4-addition products, rhenium-naphthalene complexes yield either 1,2- or 1,4-addition products depending on the identity (and steric profile) of the ancillary ligand and the identity of the electrophile used, and tungsten-naphthalene and anthracene complexes yield 1,2-addition under the reaction conditions described. One important insight to explaining this trend may lie in the fact that the tungsten metal complex is the most electron-rich of the three complexes. ${ }^{5}$ Since it is best able of the three to stabilize a cationic species, it offers 1,4-addition products pathways by which they might isomerize to 1,2 -addition products, namely, by loss of the nucleophile from C4 followed by its addition at C2.

\section{Ylidene Formation}

One interesting feature of the combination of 4 with dimethoxypropane and acid that is not discussed above is the observation that, following the initial loss of methanol to generate the active species, a second equivalent of methanol is lost once the electrophile adds to 4 . This generates the ylidene, $\mathbf{5}$, with an extended delocalized $\pi$ system which can stabilize the positive charge of the allyl. This kind of elimination to give an exocyclic alkene is not observed in other cases of acetal addition to naphthalene complexes of osmium and rhenium, but it has a couple of complements in work with complexes of other ligands. Its most recent comparable is a reaction between $\operatorname{TpW}(\mathrm{NO})\left(\mathrm{PMe}_{3}\right)\left(\eta^{2}\right.$-phenol) and benzaldehyde. The phenol is deprotonated 
to generate an active species, which then attacks the benzaldehyde and condenses to give an alkene with E configuration (Scheme 15). ${ }^{6}$ This reaction yields an o-quinone methide structure which was found to be unreactive over a range of dienophiles. Its reactivity with other electrophiles was not reported.

\section{Scheme 15}

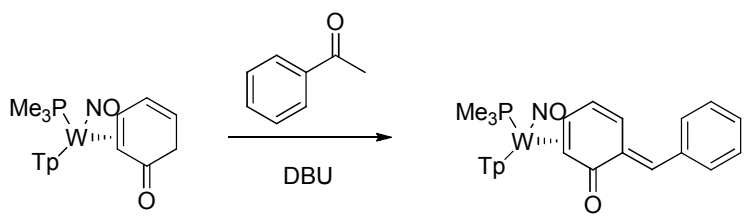

Osmium dearomatization chemistry also bears examples of condensation reactions between acetals and complexes of anisole and pyrrole. In these cases, the ylidene formed was deprotonated to give a diene, which reacted with dienophiles to give bi- and tri-cyclic systems. ${ }^{7,8}$ The reaction of $\mathbf{4}$ with dimethoxypropane provides an interesting contrast to the reaction of acetaldehyde diethyl acetal with $\left[\mathrm{Os}\left(\mathrm{NH}_{3}\right)_{5}\left(\eta^{2}\right.\right.$-anisole $\left.)\right](\mathrm{OTf})_{2}$. Despite the excess of acid used, a second equivalent of ethanol is not eliminated during the reaction, and instead a base is added to deprotonate the ipso carbon. Once this occurs, acid can be added to eliminate ethanol, generating the ylidene. ${ }^{7}$ This species was found to be highly reactive towards nucleophiles, but the chemistry most fully explored with it occurred after its deprotonation to give a vinyl group. It is possible that nucleophilic additions were not explored in great detail for this system because diastereoselectivity for the ylidene was poor $(\mathrm{de}=0)$ and because the expected product from those additions would be a 4-substituted anisole, not a dearomatized product (Scheme 16). 


\section{Scheme 16}
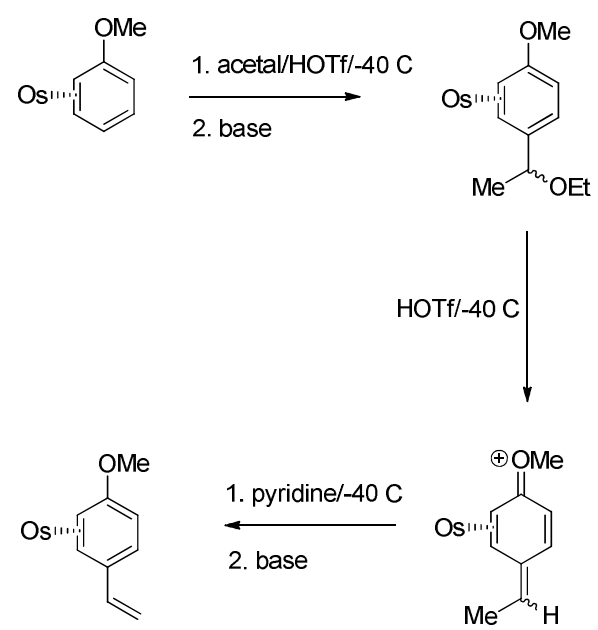

Another observation made about the anisole chemistry was that when 1,1dimethoxycyclopentane was used as the acetal, the ylidene generated was more stable than the ylidene from acetaldehyde diethyl acetal. No explanation was offered for this observation, but the larger alkyl substituent of cyclopentane may have helped to stabilize the positively charged ylidene. This trend might also explain why dimethoxypropane worked well as an acetal in $\mathrm{TpW}(\mathrm{NO})\left(\mathrm{PMe}_{3}\right)\left(\eta^{2}\right.$-anthracene) chemistry, but why other acetals with fewer than two alkyl groups attached did not. Cyclopentanone ethylene ketal may not have worked due to the tethered nature of the ketal, while 1,1-dimethoxycyclohexane may have been slow to react with 4 because its active site was sterically hindered by the ring. Benzaldehyde dimethyl acetal may have been a good choice despite having just one functional group because its condensation reaction with 4 generates an extended $\pi$ system which may provide extra stabilization to the positive charge.

\section{Benzyl vs. Ring Addition}

Benzyl cations or radicals are significantly more stable than other primary cations or radicals due to the multiple resonance structures that can contribute to the stabilization. Complexation with chromium tricarbonyl fragments can further stabilize a benzyl cation due to 
the overlap of the chromium d-orbitals with the p-orbital of the benzylic carbon, facilitating cyclizations at the benzyl carbon, among other reaction types. ${ }^{9}$ Given the emphasis of dearomatization chemistry on effecting reactions that lead to elaborated, nonaromatic organic molecules, it is not surprising that there are not examples in previous work of reactions yielding benzyl addition products. In the acetal reactions of osmium complexes given above, the ylidene is deprotonated to give a vinyl group. ${ }^{7,8}$ A benzyl addition could be achieved by the addition of a proton and then a nucleophile, but as this reaction would give a substituted aromatic product, it is not likely that this reaction type was actively explored. Michael addition reactions to rhenium anisole complexes did lead to 4-substituted anisole products that displayed significant stereochemical selectivity at the benzyl carbon. ${ }^{10}$ This stereochemical control was thought to arise from an ordered transition state in which the Michael acceptor aligned with the anisole ring, resembling an endo-Diels-Alder transition state. In the osmium and rhenium complexes of naphthalene, no ylidene was formed from an acetal, making rearomatization by benzyl addition impossible. Working from 5, however, a nucleophile can attack the ylidene to generate a 1substituted anthracene product. Given the thermodynamic stability of a 1-substituted anthracene product over a 1,2-dihydroanthracene product, it is not surprising that we can observe addition to the benzyl carbon over $\mathrm{C} 2$ in the ring. It might be more surprising that we can observe any 1,2-addition products at all.

To explore the feasibility of addition at C2, we used DFT calculations to generate an optimized structure and orbital coefficients for $\mathbf{5}$, using the B3LYP method with a hybrid basis set (LANL2DZ pseudopotential and basis set on W and 6-31G(d) on all other atoms. The LUMO for $\mathbf{5}$ generated from this calculation is shown below. It features prominent orbital coefficients on both the benzyl carbon of the ylidene and on $\mathrm{C} 2$ in the ring, indicating that entering nucleophiles could attack at either position. 
Figure 3 - LUMO of 5, optimized in Gaussian.

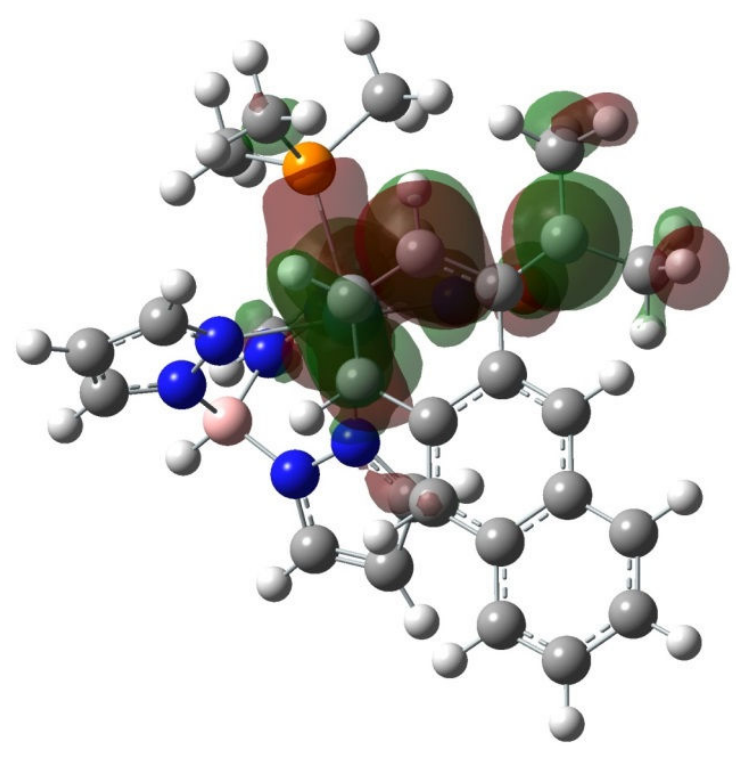

This result is consistent with the observation of both 1-substituted anthracene products and 1,2dihydroanthracene products. Since 1,2-dihydroanthracene products are most often achieved from carbon nucleophiles, even when those products can't be isolated, we can generally conclude that softer nucleophiles, such as the enolates, are more amenable to C2 addition, even though they can isomerize over time to the benzyl products. That the products of softer nucleophiles can isomerize from ring addition to benzyl addition shows that ring addition is reversible and suggests that if harder nucleophiles added to $\mathrm{C} 2$, their addition would also be reversible. That we do not observe ring addition from nucleophiles like morpholine implies that the isomerization from ring to benzyl addition is much faster for these nucleophiles.

One observation that must be noted is that while there is little evidence of 19a (the minor isomer of the reaction of benzaldehyde dimethyl acetal with 4 , where the phenyl is proximal to the $\mathrm{PMe}_{3}$ ligand) resulting from the isolation of $\mathbf{1 9}$ for two dimensional NMR analysis, minor product isomers can be seen in some product spectra, particularly 23. While they are in sufficiently low concentration that they cannot be unambiguously identified, they are possibly 1-substituted anthracenes in which the stereochemistry at the benzyl carbon is 
reversed. In order for this to occur, 19a must be accessible. It seems unlikely that the stereochemistry can change at the benzyl carbon while in ylidene form, ${ }^{8}$ but if the nucleophilic addition is reversible, it would be possible to form 19a from 19b, leading to reversed stereochemistry in the product. It is improbable that epimerization could occur via attack of the nucleophile at the face syn to the metal, as access to this face is hindered by the nitrosyl ligand. There are no examples of such syn attack in any tungsten arene complexes.

\section{Irradiation}

Our search for an efficient, mild procedure for the isolation of new organics from the metal complex led us to explore the feasibility of light as a decomplexation tool. Using the computational methods described above, we generated figures of the HOMO and LUMO of 4, shown below in Figure 4. Significantly, the HOMO of $\mathbf{4}$ shows a strong bonding interaction between the tungsten center and the two bound carbons of the anthracene ring. The LUMO of 4 shows an antibonding interaction between the tungsten and anthracene, as well as a slight bonding interaction between the tungsten and phosphine. From this information, we predicted that if a 1-substituted anthracene complex was irradiated with light of sufficient energy, an electron could be promoted from the HOMO to the LUMO, breaking the bond between the metal and ligand. In the case of $\mathbf{4}$, the energy difference between the HOMO and LUMO corresponds in energy to light with a wavelength of $340 \mathrm{~nm}$. This is not necessarily equal to the excitation energy of the electron, nor is it an exact match for other anthracene complexes, but it does provide a general idea of the wavelength of light needed to make decomplexation via irradiation possible. 
Figure 4-HOMO and LUMO of 1.
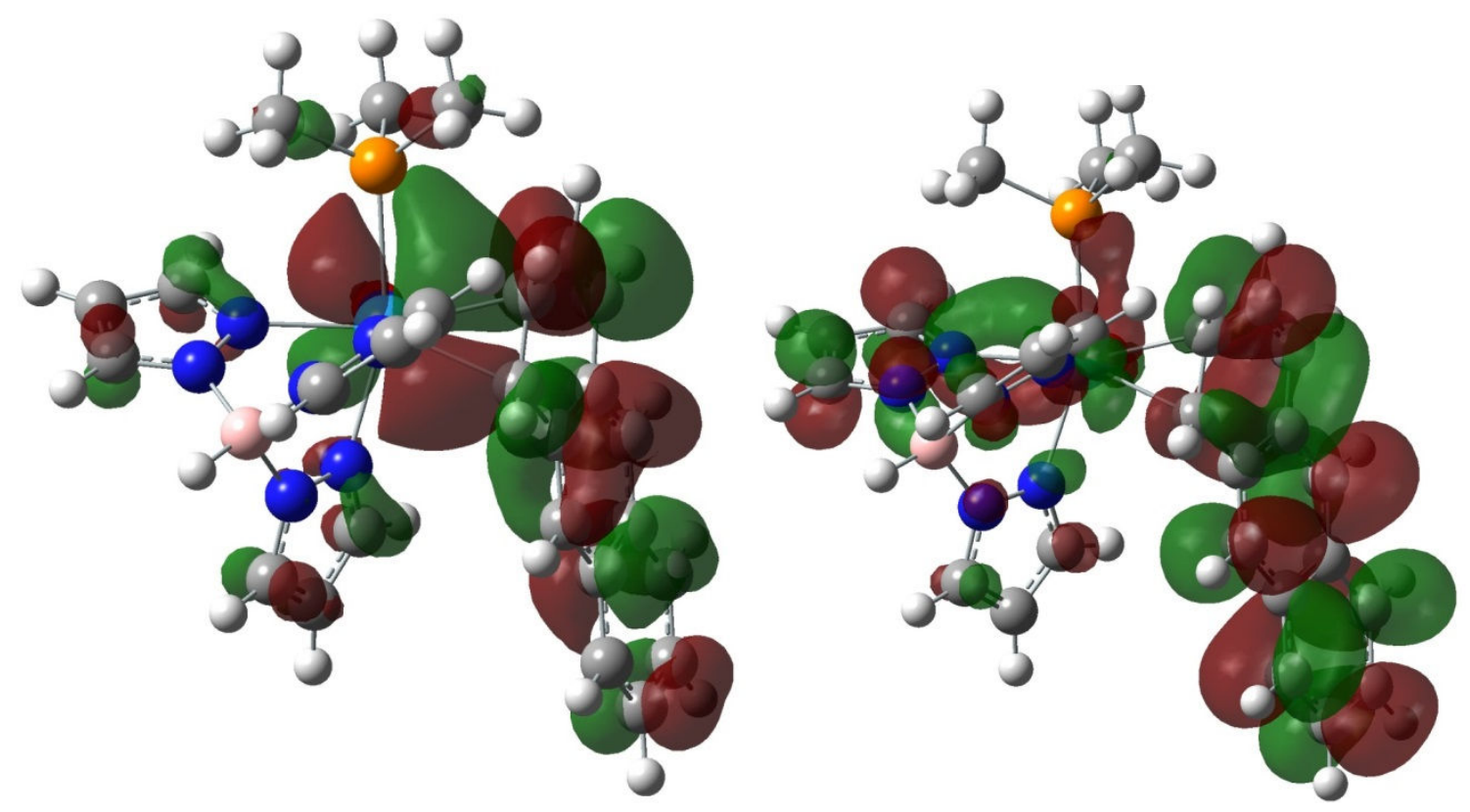

Using 8 as a model we found that 90 minutes in a photoreactor equipped with $300 \mathrm{~nm}$ light bulbs was sufficient to release the ligand, which was then isolated via an extraction. This oxidation method was not rigorously explored with other complexes and may not be generalizable across all $\left\{\mathrm{TpW}(\mathrm{NO})\left(\mathrm{PMe}_{3}\right)\right\}$ complexes. For example, a phenol complex, below, was subjected to $300 \mathrm{~nm}$ light as well, but decomposed to give an intractable mixture from which the organic cyclic enone could not be isolated (Scheme 17). An examination of the HOMO and LUMO for this complex reveal bonding interactions between the tungsten and bound double bond in each of these orbitals, thus it need not be surprising that decomplexation via irradiation failed (Figure 5). HOMO-LUMO analysis using computational methods could help us identify candidate complexes for which irradiation is likely to be a successful decomplexation method. 
Scheme 17

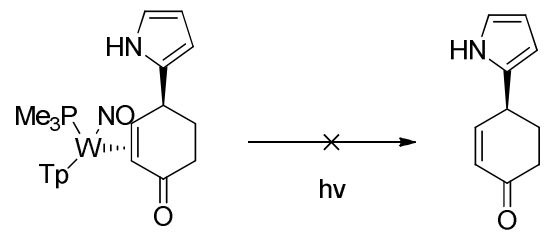

Figure 5 - HOMO and LUMO of phenol-derivative complex.
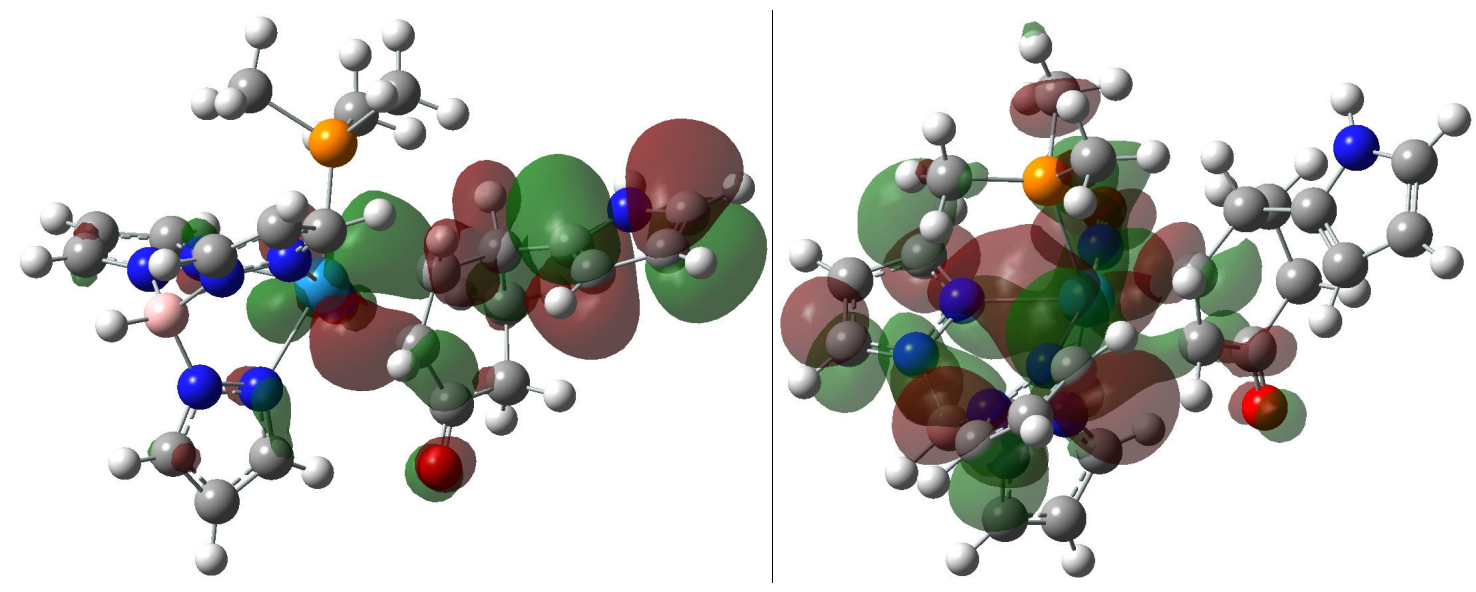

Anthraquinones

The preference for benzyl addition in many of the reactions described above yields products that are 1-substituted anthracenes. While 1-substituted anthracenes have generated some interest in medicine, in one context as anti-tumor agents, ${ }^{11,12}$ we anticipated that anthraquinones would be of greater interest due to the variety of known anthraquinone derivatives that are useful in both medicine, where there is interest in them as Alzheimer's treatments, and industry. ${ }^{13,14}$ There are several methods which are commonly used to make anthraquinones. These include the Diels-Alder reaction of butadiene with 1,4-naphthaquinone, followed by oxidation to anthraquinone, the reaction of benzene with phthalic anhydride and aluminum chloride, and the direct oxidation of anthracene to anthraquinone ${ }^{15}$. We hoped to exploit this latter method with our new 1-substituted anthracenes. We chose $\mathbf{2 5}$ as a test case because its functional groups were unlikely to be affected by the oxidation conditions chosen. As described above, the spectroscopic evidence collected on the product is consistent with $\mathbf{2 8}$, 
but not the targeted anthraquinone. Alternatively, $\mathbf{2 4}$ is susceptible to oxidation at the amine group, making it more likely to breakdown under the conditions employed.

\section{Conclusion}

The work summarized in this chapter demonstrates the successful addition of carbon electrophiles to the anthracene complex of $\left\{\mathrm{TpW}(\mathrm{NO})\left(\mathrm{PMe}_{3}\right)\right\}$, where addition was observed exclusively at C1. Carbon and nitrogen nucleophiles could be added to the ylidene resulting from acetal addition, leading to 1-substituted anthracenes or 1,2-dihydroanthracenes, depending on the nature of the nucleophile. Decomplexation methods were developed to recover some of these new organics from their metal complexes. Initial explorations of methods to make anthraquinones from 1-substituted anthracenes have yielded promising results.

\section{Experimental Section.}

General Methods. NMR spectra were obtained on a 300 (Varian INOVA), or 500, 600, or 800 $\mathrm{MHz}$ spectrometer (Bruker Avance). All chemical shifts are reported in ppm and proton and carbon shifts are referenced to tetramethylsilane (TMS) utilizing residual ${ }^{1} \mathrm{H}$ or ${ }^{13} \mathrm{C}$ signals of the deuterated solvents as an internal standard. Phosphorus NMR signals are referenced to $85 \%$ $\mathrm{H}_{3} \mathrm{PO}_{4}(\delta=0.00)$ using a triphenylphosphate external standard $(\delta=-16.58)$. Coupling constants

$(J)$ are reported in hertz $(\mathrm{Hz})$. Infrared spectra $(\mathrm{IR})$ were recorded on a MIDAC Prospect Series (Model PRS) spectrometer or a Nicolet Avatar 330 FT-IR spectrometer as a glaze on a Horizontal Attenuated Total Reflectance (HATR) accessory (Pike Industries). Electrochemical experiments were performed under a dinitrogen atmosphere using a BAS Epsilon EC-2000 potentiostat. Cyclic voltammetry data was taken at ambient temperature at $100 \mathrm{mV} / \mathrm{s}\left(25^{\circ} \mathrm{C}\right)$ in a standard 
three-electrode cell with a glassy carbon working electrode, $N, N$-dimethylacetamide (DMA) or acetonitrile (MeCN) solvent (unless otherwise specified), and tetrabutylammonium hexaflurophosphate (TBAH) electrolyte (approx. $0.5 \mathrm{M}$ ). All potentials are reported versus NHE (Normal Hydrogen Electrode) using cobaltocenium hexafluorophosphate $\left(E_{1 / 2}=-0.78 \mathrm{~V}\right)$, ferrocene $\left(E_{1 / 2}=+0.55 \mathrm{~V}\right)$, or decamethylferrocene $\left(E_{1 / 2}=+0.04 \mathrm{~V}\right)$ as an internal standard. The peak-to-peak separation was less than $100 \mathrm{mV}$ for all reversible couples. Elemental analyses (EA) were obtained from Atlantic Microlabs. High resolution electrospray ionization mass spectrometry (ESI-MS) analyses were obtained from the University of Illinois at UrbanaChampaign Mass Spectrometry Laboratory or the University of Richmond on a Bruker BioTOF-Q running in ESI mode, from samples dissolved in water/acetonitrile solution containing $0.1 \mathrm{M}$ trifluoroacetic acid then mixed with $0.1 \mathrm{M}$ aqueous sodium trifluoroacetate (NaTFA), using $[\mathrm{Na}(\mathrm{NaTFA}) \mathrm{x}]^{+}$clusters as an internal standard. Unless otherwise noted, all synthetic reactions were performed in a glovebox under a dry nitrogen atmosphere. Solvents and liquid reagents were thoroughly purged with nitrogen prior to use. Deuterated solvents were used as received from Cambridge Isotopes. General Proton Assignments are in accordance with the Figure S1. Protons of $\mathrm{Tp}$ and other ligands were assigned using a combination of 2-dimensional NMR experiments and phosphorus-proton coupling when unambiguous assignments were possible. When unambiguous assignments were not possible the Pz protons are labeled as Tp protons. Coordination diastereomers are described by the defining feature's proximity to the $\mathrm{PMe}_{3}$ ligand relative to the $\mathrm{W}-\mathrm{PMe}_{3}$ bond (e.g. the fewer number of bonds from the $\mathrm{PMe}_{3}$ passing through the upper portion of the coordinated ring system to the defining feature dictates the proximal (P) ligand). 


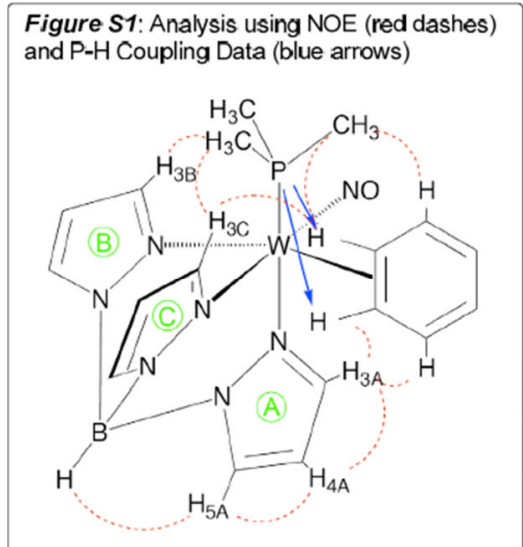

DFT Calculations. Initial structures were built in GAUSSVIEW (5.0.8) with the PM6 semi-empirical method in GAUSSIAN 09. These structures were refined stepwise in Gaussian using B3LYP and a series of basis functions incorporating LANL2 pseudopotentials and associated basis functions provided in those packages or directly from the PM6 structures. The most demanding calculations reported here put the LANL2DZ pseudopotential and its basis only on the W atom, and used the 6-31G(d) basis for all other atoms.

For transition state structures, vibrational analysis revealed the presence of a single imaginary frequency. In all other cases, vibrational analyses verified that optimized structures were located at local minima, with the presence of only real frequencies.

Synthesis and characterization for $\operatorname{TpW}(\mathrm{NO})\left(\mathrm{PMe}_{3}\right)\left(\eta^{2}\right.$-naphthalene) $\quad(\mathbf{1})^{16}$ and $\operatorname{TpW}(\mathrm{NO})\left(\mathrm{PMe}_{3}\right)\left(\eta^{2}\right.$-anthracene) $(4)^{17}$ have been previously reported.

\section{$\mathrm{TpW}(\mathrm{NO})\left(\mathrm{PMe}_{3}\right)\left(3,4-\eta^{2}-N\right.$-tosyl-1-naphthamide) (2b)}<smiles>Cc1ccc(S(=O)NC(=O)c2cccc3ccccc23)cc1</smiles> 
$P$-tosyl isocyanate $(115 \mathrm{mg}, 0.584 \mathrm{mmol})$ was added to a vial containing 1 (75 $\mathrm{mg}, 0.119 \mathrm{mmol})$ in $\mathrm{CHCl}_{3}(1.553 \mathrm{~g})$ and the reaction was allowed to stand for 48 hours. The reaction was extracted $3 x$ with $2 \mathrm{~mL}$ portions of water, and the water layers were backextracted with DCM. The organic layer was dried with $\mathrm{MgSO}_{4}$, which was filtered off over a Celite plug. The organic layer was evaporated to a film. The film was scraped into a vial, $1 \mathrm{~mL}$ ether was added, and the solid was stirred vigorously for a week. The ether was carefully pipeted out and discarded, and $1 \mathrm{~mL}$ cold ether $\left(-30^{\circ} \mathrm{C}\right)$ was added. This solution was quickly pipeted over a $15 \mathrm{~mL}$ mediumporosity fritted funnel, and two more $1 \mathrm{~mL}$ portions of cold ether were added and pipeted over the funnel. The light brown solid was dried to give $\mathbf{2 b}$ ( $30 \mathrm{mg}, 31 \%$ ).

${ }^{1} \mathrm{H}\left(\mathrm{CDCl}_{3}\right): \delta 9.98(\mathrm{~s}, 1 \mathrm{H}$, amide), $7.96(\mathrm{~d}, J=8.3,2 \mathrm{H}, \mathrm{H} 9), 7.85(\mathrm{~d}, J=2.0,1 \mathrm{H}, \mathrm{pz}), 7.79(\mathrm{~d}, J=2.0$, $1 \mathrm{H}, \mathrm{pz}), 7.74(\mathrm{~d}, J=2.0,1 \mathrm{H}, \mathrm{pz}), 7.66(\mathrm{~d}, J=2.0,1 \mathrm{H}, \mathrm{pz}), 7.31(\mathrm{~d}, J=8.1,2 \mathrm{H}, \mathrm{H} 10), 7.21(\mathrm{~m}, 1 \mathrm{H}$, H8), $7.19(\mathrm{~m}, 1 \mathrm{H}, \mathrm{H} 2), 7.10(\mathrm{~d}, J=2.0,1 \mathrm{H}, \mathrm{pz}), 7.06(\mathrm{~m}, 1 \mathrm{H}, \mathrm{H} 6$ or H7), 6.99 (d, J = 2.0, $1 \mathrm{H}, \mathrm{pz})$, $6.94(\mathrm{~m}, 1 \mathrm{H}, \mathrm{H} 6$ or H7), 6.69 (d, J = 7.6, 1H, H5), $6.26(\mathrm{t}, J=2.0,2 \mathrm{H}, \mathrm{pz}), 6.11(\mathrm{t}, J=2.0,1 \mathrm{H}, \mathrm{pz})$, 3.67 (ddd, $J=4.5,8.9,13.2,1 \mathrm{H}, \mathrm{H} 3$ ), 2.55 (d, $J=9.0,1 \mathrm{H}, \mathrm{H} 4), 2.44$ (s, 3H, Me), 1.22 (d, J = 7.9, $\left.9 \mathrm{H}, \mathrm{PMe}_{3}\right) .{ }^{31} \mathrm{P}\left(\mathrm{CDCl}_{3}\right): \delta-14.06\left(J_{\mathrm{W}-\mathrm{P}}=290\right)$.

\section{$\mathrm{TpW}(\mathrm{NO})\left(\mathrm{PMe}_{3}\right)\left(3,4-\eta^{2}\right.$-1-(propan-2-ylidene)-1,2-dihydroanthracenium) p-toluenesulfonate (5)}

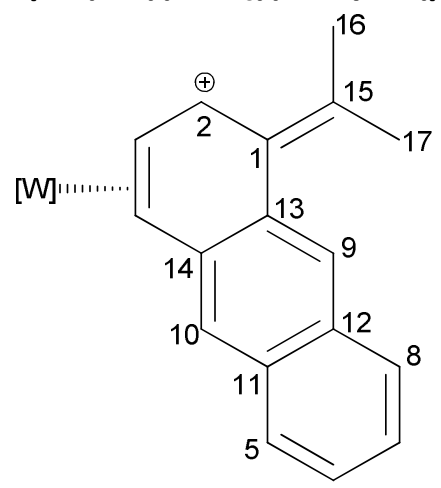

Dimethoxypropane (308 mg, $2.96 \mathrm{mmol}$ ) was added to a vial containing 4 (51 mg, $0.075 \mathrm{mmol}$ ) and $p$-toluenesulfonic acid $(25 \mathrm{mg}, 0.145 \mathrm{mmol})$ dissolved in $\mathrm{CHCl}_{3}(1.53 \mathrm{~g})$. The reaction was allowed to stand for 3 hours, and was then precipitated over $15 \mathrm{~mL}$ stirring ether. The red solid 
was collected on a $15 \mathrm{~mL}$ fine-porosity fritted funnel and dried in vacuo for several minutes to give 5 (59 mg, including excess acid).

${ }^{1} \mathrm{H}\left(\mathrm{CDCl}_{3}\right)$ (p-toluenesulfonate peaks omitted): $\delta 8.66$ (br s, $\left.1 \mathrm{H}, \mathrm{H} 2\right), 8.44(\mathrm{~d}, J=2.0,1 \mathrm{H}, \mathrm{pz} 3 \mathrm{C}$ ), $7.96(\mathrm{~s}, 1 \mathrm{H}, \mathrm{H} 9), 7.91$ (d, J = 2.0, 1H, pz5C), 7.86 (d, J=2.0, 1H, Tp), 7.84 (d, J=2.0, $1 \mathrm{H}, \mathrm{Tp}), 7.83$ (d, $1 \mathrm{H}, \mathrm{H} 5$ or H8), 7.74 (d, J=2.0, 1H, pz3B), 7.55 (d, J=8.4, 1H, H5 or H8), 7.45 (t, J= 7.0, 1H, H6 or $\mathrm{H7}$ ), $7.33(\mathrm{t}, J=7.0,1 \mathrm{H}, \mathrm{H} 6$ or $\mathrm{H7}), 6.94(\mathrm{~d}, J=2.0,1 \mathrm{H}, \mathrm{pz} 5 \mathrm{~B}), 6.82(\mathrm{~s}, 1 \mathrm{H}, \mathrm{H} 10), 6.56(\mathrm{t}, J=2.0$, $1 \mathrm{H}, \mathrm{pz} 4 \mathrm{C}), 6.38(\mathrm{t}, J=2.0,1 \mathrm{H}, \mathrm{pz} 4 \mathrm{~A}), 6.08(\mathrm{t}, J=2.0,1 \mathrm{H}, \mathrm{pz} 4 \mathrm{~B}), 5.91(\mathrm{~m}, 1 \mathrm{H}, \mathrm{H} 3), 3.86(\mathrm{~d}, J=6.5$, $1 \mathrm{H}, \mathrm{H} 4), 2.78(\mathrm{~s}, 3 \mathrm{H}, \mathrm{H} 17), 2.45(\mathrm{~s}, 3 \mathrm{H}, \mathrm{H} 16), 1.28\left(\mathrm{~d}, J=9.1,9 \mathrm{H}, \mathrm{PMe}_{3}\right) .{ }^{13} \mathrm{C}\left(\mathrm{CDCl}_{3}\right): \delta 155.98$ (C15), 144.35 (pz3C), 143.61 (C2), 142.98 (Tp), 142.08 (pz5B), 140.56, 139.46, 138.26 (pz5C), 137.82 (Tp), 137.07 (pz3B), 132.47, 131.22, 130.17 (C1), 127.92 (C5 or C8), 127.73 (C9), 127.00 (C5 or C8), 126.49 (C6, C7, or C10), 126.46 (C6, C7, or C10), 125.63 (C6 or C7), 108.74 (pz4C), 108.21 (pz4A), 105.98 (pz4B), 88.67 (d, $J=10.2, C 3), 75.20$ (d, J = 15.2, C4), 28.45 (d, J = 9.9, C17), $25.02(\mathrm{C} 16), 13.61\left(\mathrm{~d}, J=31.0, \mathrm{PMe}_{3}\right) .{ }^{31} \mathrm{P}\left(\mathrm{CDCl}_{3}\right): \delta-11.09\left(J_{\mathrm{W}-\mathrm{P}}=265\right) . \quad \mathrm{IR}: \mathrm{v}_{\mathrm{NO}}=1634 \mathrm{~cm}^{-1}$, $\mathrm{V}_{\mathrm{BH}}=2514 \mathrm{~cm}^{-1}$.

\section{$\operatorname{TpW}(\mathrm{NO})\left(\mathrm{PMe}_{3}\right)\left(3,4-\eta^{2}-N-(2-(\right.$ anthracen-1-yl)propan-2-yl)propan-1-amine) (6)}

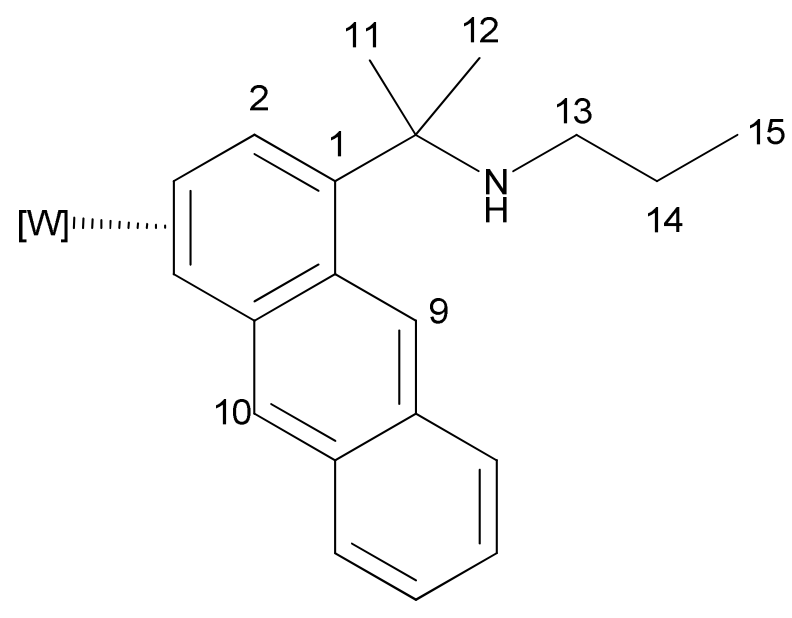

Dimethoxypropane (192 mg, $1.85 \mathrm{mmol}$ ) was added to a vial containing 4 (30 mg, $0.044 \mathrm{mmol}$ ) and camphorsulfonic acid $\left(24 \mathrm{mg}, 0.103 \mathrm{mmol}\right.$ ) dissolved in $\mathrm{CHCl}_{3}(968 \mathrm{mg})$. The reaction was 
allowed to stand for 3 hours, and then four drops of propylamine were added. The reaction was extracted $3 x$ with $2 \mathrm{~mL}$ portions of water, and the water layers were backextracted with DCM. The organic layer was dried with $\mathrm{CaSO}_{4}$, which was filtered off over a Celite plug. The organic layer was evaporated to a yellow film on which ${ }^{1} \mathrm{H}$ NMR data was collected.

${ }^{1} \mathrm{H}\left(\mathrm{CDCl}_{3}\right): \delta 9.11(\mathrm{~s}, 1 \mathrm{H}, \mathrm{H} 9), 7.84(\mathrm{~d}, J=2.0,1 \mathrm{H}, \mathrm{Tp}), 7.83(\mathrm{~m}, 1 \mathrm{H}, \mathrm{H} 5), 7.78(\mathrm{~d}, J=2.0,1 \mathrm{H}, \mathrm{Tp})$, $7.71(\mathrm{~d}, J=2.0,1 \mathrm{H}, \mathrm{Tp}), 7.68(\mathrm{~d}, J=2.0,1 \mathrm{H}, \mathrm{Tp}), 7.52(\mathrm{~m}, 1 \mathrm{H}, \mathrm{H} 8), 7.27(\mathrm{~m}, 1 \mathrm{H}, \mathrm{H} 7), 7.25(\mathrm{~d}, J=$ 2.0, $1 \mathrm{H}, \mathrm{Tp}), 7.22(\mathrm{~m}, 1 \mathrm{H}, \mathrm{H} 6), 7.05(\mathrm{~d}, J=2.0,1 \mathrm{H}, \mathrm{Tp}), 7.03(\mathrm{~d}, J=5.0,1 \mathrm{H}, \mathrm{H} 2), 6.98(\mathrm{~s}, 1 \mathrm{H}, \mathrm{H} 10)$, $6.24(\mathrm{t}, J=2.0,1 \mathrm{H}, \mathrm{Tp}), 6.20(\mathrm{t}, J=2.0,1 \mathrm{H}, \mathrm{Tp}), 6.08(\mathrm{t}, J=2.0,1 \mathrm{H}, \mathrm{Tp}), 3.77$ (ddd, $J=5.2,9.0$, 14.1, 1H, H3), $2.70\left(m, 1 \mathrm{H}, \mathrm{H} 13^{\prime}\right), 2.60(\mathrm{~d}, J=9.0,1 \mathrm{H}, \mathrm{H} 4), 2.38(\mathrm{~m}, 1 \mathrm{H}, \mathrm{H} 13), 1.76(\mathrm{~s}, 3 \mathrm{H}, \mathrm{H} 11$ or H12), 1.63 (s, 3H, H11 or H12), 1.42 (m, 2H, H14), 1.34 (d, J = 7.90, 9H, PMe 3 ), 0.85 (t, J = 7.4, 3H, H15). ${ }^{31} \mathrm{P}\left(\mathrm{CDCl}_{3}\right): \delta-12.39\left(J_{\mathrm{W}-\mathrm{P}}=295\right)$.

\section{$\operatorname{TpW}(\mathrm{NO})\left(\mathrm{PMe}_{3}\right)\left(3,4-\eta^{2}-4-(2-(\right.$ anthracen-1-yl)propan-2-yl)morpholine) (7)}

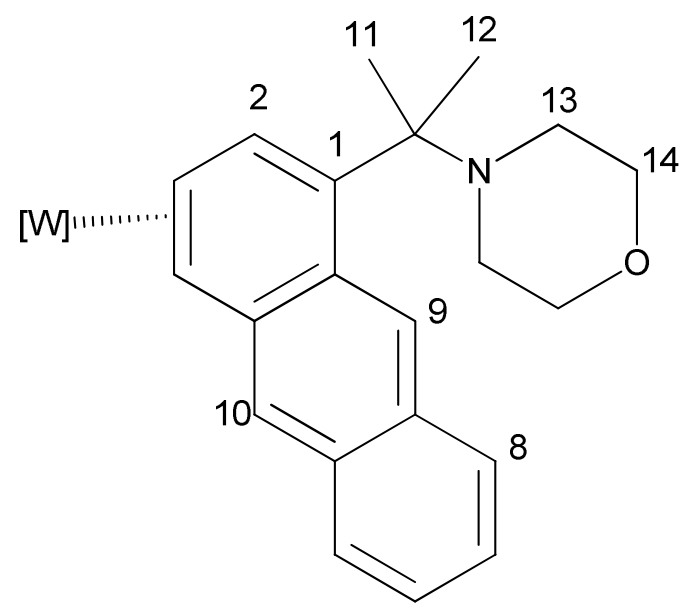

Dimethoxypropane $(606 \mathrm{mg}, 5.83 \mathrm{mmol}$ ) was added to a vial containing 4 (91 mg, $0.134 \mathrm{mmol}$ ) and camphorsulfonic acid $(80 \mathrm{mg}, 0.345 \mathrm{mmol})$ dissolved in $\mathrm{CHCl}_{3}(3.605 \mathrm{~g})$. The reaction was allowed to stand for 3.25 hours, then 10 drops of morpholine was added. The reaction was allowed to stand for 1.25 hours, then was extracted $4 x$ with $3 \mathrm{~mL}$ portions of water. The water layers were backextracted with $1 \mathrm{~mL}$ portions of DCM. The combined organic layers were dried 
with $\mathrm{MgSO}_{4}$, which was filtered off over a Celite plug. The solution was concentrated to $2 \mathrm{~mL}$ and precipitated over $15 \mathrm{~mL}$ stirring hexanes. The yellow precipitate was filtered over a $15 \mathrm{~mL}$ fine-porosity fritted funnel and dried in vacuo to give 7 (58 $\mathrm{mg}, 54 \%$ ).

${ }^{1} \mathrm{H}\left(\mathrm{CDCl}_{3}\right): \delta 9.51(\mathrm{~s}, 1 \mathrm{H}, \mathrm{H} 9), 7.85(\mathrm{~d}, J=2.0,1 \mathrm{H}, \mathrm{pz} 3 \mathrm{~B}), 7.82(\mathrm{~d}, J=8.2,1 \mathrm{H}, \mathrm{H} 8), 7.79(\mathrm{~d}, J=2.0$, $1 \mathrm{H}, \mathrm{pz} 5 \mathrm{C}), 7.71(\mathrm{~d}, J=2.0,1 \mathrm{H}, \mathrm{pz} 5 \mathrm{~B}), 7.69(\mathrm{~d}, J=2.0,1 \mathrm{H}, \mathrm{pz} 5 \mathrm{~A}), 7.54(\mathrm{~d}, J=8.2,1 \mathrm{H}, \mathrm{H} 5), 7.30(\mathrm{~m}$, $J=1.2,6.7,8.0,1 \mathrm{H}, \mathrm{H} 6), 7.25(\mathrm{~d}, J=2.0,1 \mathrm{H}, \mathrm{pz} 3 \mathrm{C}), 7.24(\mathrm{~m}, J=1.2,6.8,8.1,1 \mathrm{H}, \mathrm{H} 7), 7.18(\mathrm{~d}, J=$ 2.0, 1H, pz3A), $7.07(\mathrm{~d}, J=5.0,1 \mathrm{H}, \mathrm{H} 2), 6.99(\mathrm{~s}, 1 \mathrm{H}, \mathrm{H} 10), 6.25(\mathrm{t}, J=2.0,1 \mathrm{H}, \mathrm{pz} 4 \mathrm{C}), 6.20(\mathrm{t}, J=$ 2.0, $1 \mathrm{H}, \mathrm{pz} 4 \mathrm{~B}$ ), $6.11(\mathrm{t}, J=2.0,1 \mathrm{H}, \mathrm{pz} 4 \mathrm{~A}), 3.70$ (under morpholine peaks, $1 \mathrm{H}, \mathrm{H} 3), 3.69(\mathrm{~m}, 4 \mathrm{H}$, H14), 3.67 (m, 1H, H13), 2.88 (m, 1H, H13'), 2.68 (m 2H, H13"), 2.60 (d, J = 9.1, 1H, H4), 1.74 (s, $3 \mathrm{H}, \mathrm{H} 11$ or $\mathrm{H} 12), 1.49(\mathrm{~s}, 3 \mathrm{H}, \mathrm{H} 11$ or $\mathrm{H} 12), 1.37\left(\mathrm{~d}, J=8.0,9 \mathrm{H}, \mathrm{PMe}_{3}\right) \cdot{ }^{31} \mathrm{P}\left(\mathrm{CDCl}_{3}\right): \delta-12.21\left(\mathrm{JW}_{\mathrm{-P}}=\right.$ 295).

$\mathrm{TpW}(\mathrm{NO})\left(\mathrm{PMe}_{3}\right)\left(3,4-\eta^{2}-1-(2-(\right.$ anthracen-1-yl)propan-2-yl)-1H-imidazole) (8)

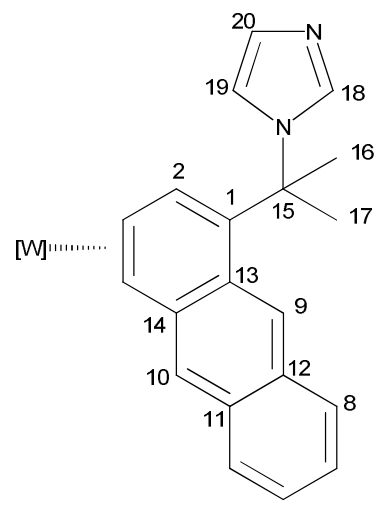

Dimethoxypropane $(598 \mathrm{mg}, 5.75 \mathrm{mmol}$ ) was added to a vial containing 4 (91 mg, $0.134 \mathrm{mmol}$ ) and camphorsulfonic acid $\left(77 \mathrm{mg}, 0.332 \mathrm{mmol}\right.$ ) dissolved in $\mathrm{CHCl}_{3}(3.62 \mathrm{~g})$. The reaction was allowed to stand for 3 hours and then added to a vial containing imidazole (146 mg, $2.15 \mathrm{mmol}$ ). The reaction was allowed to stand for 2 hours before being extracted $3 x$ with $3 \mathrm{~mL}$ portions of saturated aqueous $\mathrm{Na}_{2} \mathrm{CO}_{3}$. The water layers were backextracted with $1 \mathrm{~mL}$ portions of DCM. The combined organic layers were dried with $\mathrm{MgSO}_{4}$, which was filtered off over a Celite plug. 
The solution was concentrated to $\sim 2 \mathrm{~mL}$ and precipitated over $21 \mathrm{~mL}$ stirring hexanes. The yellow solid was filtered over a $15 \mathrm{~mL}$ fine-porosity fritted funnel and dried in vacuo for 30 minutes to give 8 ( $86 \mathrm{mg}, 81 \%)$.

${ }^{1} \mathrm{H}\left(\mathrm{CDCl}_{3}\right): \delta 7.90(\mathrm{~s}, 1 \mathrm{H}, \mathrm{H} 9), 7.87(\mathrm{~d}, J=2.0,1 \mathrm{H}, \mathrm{pz} 3 \mathrm{~B}), 7.82(\mathrm{~d}, J=2.0,1 \mathrm{H}, \mathrm{pz} 5 \mathrm{C}), 7.74(\mathrm{~d}, J=$ 2.0, $1 \mathrm{H}, \mathrm{pz} 5 \mathrm{~B}$ ), $7.70(\mathrm{~d}, J=2.0,1 \mathrm{H}, \mathrm{pz} 5 \mathrm{~A}), 7.48$ (d, $J=9.0,1 \mathrm{H} \mathrm{H7}), 7.46$ (d, $J=9.0,1 \mathrm{H}, \mathrm{H} 5), 7.37(\mathrm{~s}$, $1 \mathrm{H}, \mathrm{H} 19), 7.27(\mathrm{~d}, J=2.0, \mathrm{pz} 3 \mathrm{C}), 7.25(\mathrm{~m}, 1 \mathrm{H}, \mathrm{H} 6), 7.15(\mathrm{~m}, 1 \mathrm{H}, \mathrm{H} 2), 7.14(\mathrm{~m}, 1 \mathrm{H}, \mathrm{H} 8), 7.13(\mathrm{~s}, 1 \mathrm{H}$, $\mathrm{H} 18), 7.03(\mathrm{~s}, 1 \mathrm{H}, \mathrm{H} 20), 7.02(\mathrm{~d}, J=2.0,1 \mathrm{H}, \mathrm{pz} 3 \mathrm{~A}), 6.96(\mathrm{~s}, 1 \mathrm{H}, \mathrm{H} 10), 6.29(\mathrm{t}, J=2.0,1 \mathrm{H}, \mathrm{pz} 4 \mathrm{C})$, $6.24(\mathrm{t}, J=2.0,1 \mathrm{H}, \mathrm{pz} 4 \mathrm{~B}), 6.08(\mathrm{t}, J=2.0,1 \mathrm{H}, \mathrm{pz} 4 \mathrm{~A}), 3.72(\mathrm{ddd}, J=13.8,9.1,5.3,1 \mathrm{H}, \mathrm{H3}), 2.60(\mathrm{~d}$, $J=9.2,1 \mathrm{H}, \mathrm{H} 4), 2.21(\mathrm{~s}, 3 \mathrm{H}, \mathrm{H} 16), 2.05(\mathrm{~s}, 3 \mathrm{H}, \mathrm{H} 17), 1.35\left(\mathrm{~d}, J=8.1,9 \mathrm{H}, \mathrm{PMe}_{3}\right) \cdot{ }^{13} \mathrm{C}\left(\mathrm{CDCl}_{3}\right): \delta$ 143.53 (pz3B), 143.28 (pz3A), 143.04 (pz3C), 139.96 (C14), 136.73 (pz5C), 136.28 (pz5A), 136.03 (pz5B), 135.28 (C9), 133.62 (C2), 131.16 (C11), 130.99 (C12), 130.12 (C13), 129.16 (C1), 128.43 (C20), 127.96 (C7), 126.44 (C10), 126.42 (C5), 124.96 (C6), 123.55 (C8), 122.86 (C18), 118.60 (C19), 106.56 (pz4B), 106.43 (pz4C), 104.95 (pz4A), 60.40 (C15), 60.23 (C4), 56.23 (d, J = 8.0, C3), 32.56 (C17), 31.95 (C16), 13.67 (d, $\left.J=28, \mathrm{PMe}_{3}\right) .{ }^{31} \mathrm{P}\left(\mathrm{CDCl}_{3}\right): \delta-12.91\left(J_{\mathrm{W}-\mathrm{P}}=289\right) . \mathrm{CV}: \mathrm{E}_{\mathrm{p}, \mathrm{a}}=$ +0.305 V. IR: $V_{\mathrm{NO}}=1561 \mathrm{~cm}^{-1}, \mathrm{~V}_{\mathrm{BH}}=2488 \mathrm{~cm}^{-1}$.

$\mathrm{TpW}(\mathrm{NO})\left(\mathrm{PMe}_{3}\right)\left(3,4-\eta^{2}-(\mathrm{R})-2-(\right.$ nitromethyl)-1-(propan-2-ylidene)-1,2-dihydroanthracene) (9)

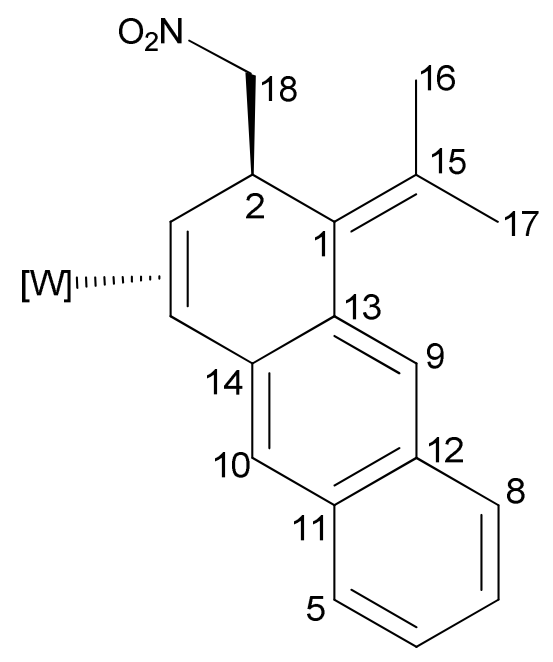


Dimethoxypropane $(386 \mathrm{mg}, 3.71 \mathrm{mmol}$ ) was added to a vial containing 4 (51 mg, $0.075 \mathrm{mmol}$ ) and camphorsulfonic acid (40 mg, $0.172 \mathrm{mmol})$ dissolved in $\mathrm{CHCl}_{3}(1.514 \mathrm{~g})$. The reaction was allowed to stand for 2 hours. LiOMe $(10 \mathrm{mg}, 0.263 \mathrm{mmol})$ and nitromethane $(121 \mathrm{mg}, 1.98$ mmol) were dissolved in $\mathrm{MeOH}(258 \mathrm{mg}$ ) and added to the reaction. The solvent was evaporated in vacuo and the film redissolved in DCM. The solution was precipitated over $11 \mathrm{~mL}$ stirring hexanes, and the solid filtered over a $15 \mathrm{~mL}$ fine-porosity fritted funnel and discarded. The filtrate was evaporated to a yellow powdery film as $\mathbf{9}$, one which NMR data was collected. ${ }^{1} \mathrm{H}\left(\mathrm{CDCl}_{3}\right): \delta 7.74(\mathrm{~d}, J=8.1,1 \mathrm{H}, \mathrm{H} 8), 7.69(\mathrm{~s}, 1 \mathrm{H}, \mathrm{H} 9), 7.52(\mathrm{~d}, J=8.2,1 \mathrm{H}, \mathrm{H} 5), 7.32$ (ddd, J = 1.1, $6.8,8.0,1 \mathrm{H}, \mathrm{H} 6$ ), 7.23 (ddd, $J=1.1,6.8,8.0,1 \mathrm{H}, \mathrm{H} 7$ ), 6.89 (s, 1H, H10), 4.70 (dd, $J=6.7,8.1,1 \mathrm{H}$, H2), $4.46(d d, J=6.6,10.8,1 H, H 18), 4.40$ (dd, $\left.J=8.4,10.9,1 H, H 18^{\prime}\right), 2.94$ (ddd, $J=1.2,10.0$, 12.5, 1H, H3), 2.16 (dd, $J=1.4,10.2,1 \mathrm{H}, \mathrm{H} 4), 2.05(\mathrm{~s}, 3 \mathrm{H}, \mathrm{H} 16), 2.03(\mathrm{~s}, 3 \mathrm{H}, \mathrm{H} 17), 1.24$ (d, J = 8.2, 9H, $\left.\mathrm{PMe}_{3}\right) .{ }^{13} \mathrm{C}\left(\mathrm{CDCl}_{3}\right): \delta 145.01$ (C14), 143.75 (pz3A), 142.37 (pz3B), 139.97 (pz3C), 136.69, 136.29 (рz5B), 135.54 (pz5A), 132.70, 132.29, 131.23, 130.89, 128.98 (с9), 128.89, 127.67 (C8), 126.12 (C5), 126.05 (C10), 125.12 (C6), 123.57 (C7), 106.65 (pz4C), 106.31 (pz4B), 105.23 (pz4A), 85.17 (C18), 56.58 (d, J = 12.9, C3), 56.05 (C4), 43.58 (C2), 25.37 (C17), 22.31 (C16), 13.24 (d, J = 28.3, $\left.\mathrm{PMe}_{3}\right) \cdot{ }^{31} \mathrm{P}\left(\mathrm{CDCl}_{3}\right): \delta-11.72\left(J_{\mathrm{W}-\mathrm{P}}=276\right)$.

$\mathrm{TpW}(\mathrm{NO})\left(\mathrm{PMe}_{3}\right)\left(3,4-\eta^{2}\right.$-dimethyl 2-(1-(propan-2-ylidene)-1,2-dihydroanthracen-2-yl)malonate) (10)

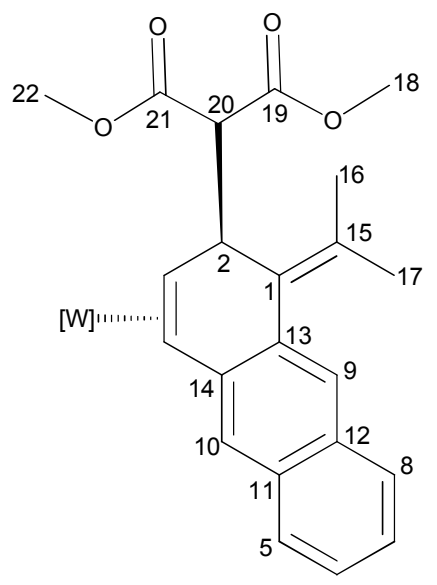


Dimethoxypropane (503 mg, $4.84 \mathrm{mmol}$ ) was added to a vial containing 4 (75 mg, $0.110 \mathrm{mmol}$ ) and camphorsulfonic acid (61 $\mathrm{mg}, 0.263 \mathrm{mmol}$ ) dissolved in $\mathrm{CHCl}_{3}(3.00 \mathrm{~g})$. The reaction was allowed to stand for 2.75 hours. LiOMe $(8 \mathrm{mg}, 0.211 \mathrm{mmol})$ and dimethylmalonate $(33 \mathrm{mg}, 0.25$ mmol) were dissolved in $\mathrm{MeOH}(413 \mathrm{mg}$ ) and added to the reaction. The reaction was allowed to stand for 2 hours, then extracted $3 x$ with $3 \mathrm{~mL}$ portions of water. The water layers were backextracted with $0.5 \mathrm{~mL} \mathrm{DCM}$ portions. The combined organic layers were dried with $\mathrm{MgSO}_{4}$, which was filtered over a Celite plug. The solution was concentrated to $\sim 1 \mathrm{~mL}$ and precipitated over $20 \mathrm{~mL}$ stirring hexanes, and dried in vacuo for an hour to give 10 (27 mg, $29 \%$ ).

${ }^{1} \mathrm{H}\left(\mathrm{CDCl}_{3}\right): \delta 7.99(\mathrm{~d}, J=2.0,1 \mathrm{H}, \mathrm{pz} 3 \mathrm{~B}), 7.76(\mathrm{~d}, J=2.0,1 \mathrm{H}, \mathrm{pz} 5 \mathrm{C}), 7.74(\mathrm{~d}, J=8.3,1 \mathrm{H}, \mathrm{H} 8), 7.70$ $(\mathrm{d}, J=2.0,1 \mathrm{H}, \mathrm{pz} 5 \mathrm{~B}), 7.61(\mathrm{~d}, J=2.0,1 \mathrm{H}, \mathrm{pz} 5 \mathrm{~A}), 7.59(\mathrm{~s}, 1 \mathrm{H}, \mathrm{H} 10), 7.51(\mathrm{~d}, J=8.3,1 \mathrm{H}, \mathrm{H} 5), 7.32$ $(\mathrm{d}, J=2.0,1 \mathrm{H}, \mathrm{pz} 3 \mathrm{C}), 7.31(\mathrm{~m}, 1 \mathrm{H}, \mathrm{H} 6), 7.22(\mathrm{~m}, 1 \mathrm{H}, \mathrm{H} 7), 7.21(\mathrm{~d}, J=2.0,1 \mathrm{H}, \mathrm{pz} 3 \mathrm{~A}), 6.86(\mathrm{~s}, 1 \mathrm{H}$, H9), $6.25(\mathrm{t}, J=2.0,1 \mathrm{H}, \mathrm{pz} 4 \mathrm{C}), 6.24(\mathrm{t}, J=2.01 \mathrm{H}, \mathrm{pz} 4 \mathrm{~B}), 6.02(\mathrm{t}, J=2.0,1 \mathrm{H}, \mathrm{pz} 4 \mathrm{~A}), 4.62(\mathrm{~d}, J=$ 10.5, 1H, H2), 3.73 (s, 3H, H22), 3.65 (s, 3H, H18), 3.46 (d, J = 10.5, 1H, H20), 3.10 (ddd, J = 11.9, 10.1, 1.4, 1H, H3), $2.12(\mathrm{dd}, J=10.1,1.4,1 \mathrm{H}, \mathrm{H} 4), 2.02(\mathrm{~s}, 3 \mathrm{H}, \mathrm{H} 16), 2.00(\mathrm{~s}, 3 \mathrm{H}, \mathrm{H} 17), 1.29$ (d, J = 8.3, 9H, $\left.\mathrm{PMe}_{3}\right) .{ }^{13} \mathrm{C}\left(\mathrm{CDCl}_{3}\right): \delta 170.46$ (C21), 169.30 (C19), 145.25 (C14), 143.66 (pz3A), 142.20 (pz3B), 139.91 (pz3C), 136.59 (pz5C), 136.17 (pz5B), 135.44 (pz5A), 132.10 (C12 or C13), 132.06 (C12 or C13), 131.59 (C11), 130.77 (C15), 129.89 (C1), 129.20 (C10), 127.67 (C8), 126.09 (C5), 125.60 (C9), 124.74 (C6), 123.19 (C7), 106.54 (pz4C), 106.11 (pz4B), 105.09 (pz4A), 61.66 (C20), $58.58(d, J=11.9, C 3), 56.18$ (C4), 52.19 (C22), 51.91 (C18), 43.65 (C2), 25.36 (C17), 22.57 (C16), $13.70\left(\mathrm{~d}, J=27.8, \mathrm{PMe}_{3}\right) \cdot{ }^{31} \mathrm{P}\left(\mathrm{CDCl}_{3}\right): \delta-11.37\left(J_{\mathrm{W}-\mathrm{p}}=280\right)$. HRMS: obs'd $(\%)$, calc'd $(\%), \mathrm{ppm}$ $\left(\mathrm{M}+\mathrm{Na}^{+}\right): 874.2388$ (74.9), 874.2386 (77.6), 0.2; 875.2415 (77.5), 875.2411 (81.9), 0.5; 876.2408 (100), 876.2412 (100), -0.5; 877.2448 (48.8), 877.2448 (81.4), 0; 878.2440 (78.8), 878.2444 (81.4), -0.5. 


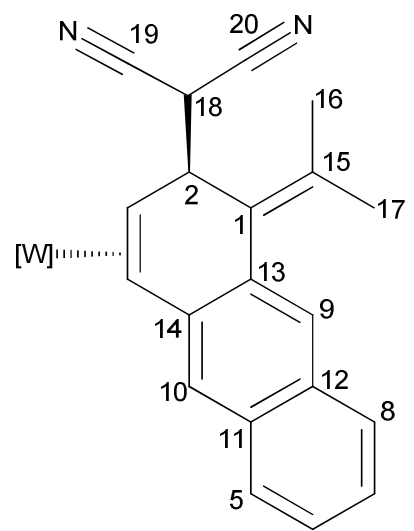

Dimethoxypropane $(610 \mathrm{mg}, 5.87 \mathrm{mmol}$ ) was added to a vial containing 4 (92 $\mathrm{mg}, 0.135 \mathrm{mmol}$ ) and camphorsulfonic acid $(74 \mathrm{mg}, 0.319 \mathrm{mmol})$ dissolved in $\mathrm{CHCl}_{3}(3.604 \mathrm{~g})$. The reaction was allowed to stand for 2.85 hours. LiOMe $(15 \mathrm{mg}, 0.395 \mathrm{mmol})$ and malononitrile $(85 \mathrm{mg}, 1.29$ mmol) were dissolved in $\mathrm{MeOH}(971 \mathrm{mg}$ ) and added to the reaction. Extracted $4 \mathrm{x}$ with $3 \mathrm{~mL}$ portions of water. The water layers were backextracted with $0.5 \mathrm{~mL}$ DCM portions. The combined organic layers were dried with $\mathrm{MgSO}_{4}$, which was filtered over a Celite plug. The solution was concentrated to $\sim 2 \mathrm{~mL}$ and precipitated over $15 \mathrm{~mL}$ stirring hexanes, and dried in vacuo for several minutes to give 11 (50 mg, $47 \%$ ).

${ }^{1} \mathrm{H}\left(\mathrm{CDCl}_{3}\right): \delta 7.99(\mathrm{~d}, J=2.0,1 \mathrm{H}, \mathrm{pz} 3 \mathrm{~B}), 7.80(\mathrm{~d}, J=2.0,1 \mathrm{H}, \mathrm{pz} 5 \mathrm{C}), 7.78(\mathrm{~s}, 1 \mathrm{H}, \mathrm{H} 9), 7.76(\mathrm{~d}, J=$ $8.2,1 \mathrm{H}, \mathrm{H} 8), 7.74(\mathrm{~d}, J=2.0,1 \mathrm{H}, \mathrm{pz} 5 \mathrm{~B}), 7.64(\mathrm{~d}, J=2.0,1 \mathrm{H}, \mathrm{pz} 5 \mathrm{~A}), 7.53(\mathrm{~d}, J=8.2,1 \mathrm{H}, \mathrm{H} 5), 7.44$ $(\mathrm{d}, J=2.0,1 \mathrm{H}, \mathrm{pz} 3 \mathrm{C}), 7.36(\mathrm{dd}, J=8.2,6.7,1 \mathrm{H}, \mathrm{H6}), 7.27$ (dd, $J=8.3,6.8,1 \mathrm{H}, \mathrm{H} 7), 7.23$ (d, $J=2.0$ $1 \mathrm{H}, \mathrm{pz} 3 \mathrm{~A}), 6.92(\mathrm{~s}, 1 \mathrm{H}, \mathrm{H} 10), 6.31(\mathrm{t}, J=2.0,1 \mathrm{H}, \mathrm{pz} 4 \mathrm{C}), 6.29(\mathrm{t}, J=2.0,1 \mathrm{H}, \mathrm{pz} 4 \mathrm{~B}), 6.06(\mathrm{t}, J=2.0$, $1 \mathrm{H}, \mathrm{pz} 4 \mathrm{~A}$ ), 4.54 (d, $J=10.3,1 \mathrm{H}, \mathrm{H} 2$ ), 3.25 (ddd, $J=11.8,10.0,1.3,1 \mathrm{H}, \mathrm{H} 3$ ), 2.14 (dd, $J=9.9,1,3$, $1 \mathrm{H}, \mathrm{H} 4), 2.12(\mathrm{~s}, 3 \mathrm{H}, \mathrm{H} 16), 2.10(\mathrm{~s}, 3 \mathrm{H}, \mathrm{H} 17), 1.27\left(\mathrm{~d}, J=8.1,9 \mathrm{H}, \mathrm{PMe}_{3}\right) .{ }^{13} \mathrm{C}\left(\mathrm{CDCl}_{3}\right): \delta 144.04$ (C14), 143.65 (pz3A), 142.30 (pz3B), 140.06 (pz3C), 136.80 (pz5C), 136.52 (pz5B), 135.85 (pz5A), 135.68 (C15), 132.45 (C11), 130.90 (C12), 129.64 (C9), 129.38 (C13), 127.77 (C8), 127.07 (C1), 126.26 (C5), 126.20 (C10), 125.56 (C6), 123.96 (C7), 114.85 (C19 or C20), 113.19 (C19 or C20), 106.84 (pz4C), 106.58 (pz4B), 105.36 (pz4A), 55.99 (d, $J=12.7, C 3), 55.38$ (C4), 46.34 (C2), 33.21 
(C18), 25.46 (C17), 22.73 (C16), $13.37\left(\mathrm{~d}, J=28.0, \mathrm{PMe}_{3}\right) .{ }^{31} \mathrm{P}\left(\mathrm{CDCl}_{3}\right): \delta-12.44\left(J_{\mathrm{W}-\mathrm{P}}=272\right)$. IR: $\mathrm{v}_{\mathrm{NO}}$ $=1565 \mathrm{~cm}^{-1}, \mathrm{v}_{\mathrm{CN}}=2248 \mathrm{~cm}^{-1}, \mathrm{v}_{\mathrm{BH}}=2489 \mathrm{~cm}^{-1}$.

$\mathrm{TpW}(\mathrm{NO})\left(\mathrm{PMe}_{3}\right)\left(3,4-\eta^{2}-2-(2-(\right.$ anthracen-1-yl)propan-2-yl)malononitrile) (12)

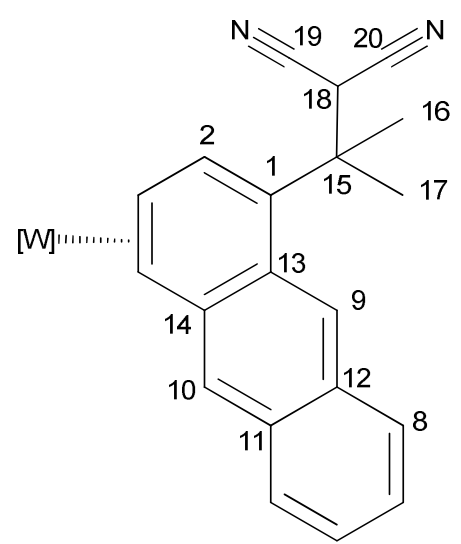

Dimethoxypropane $(716 \mathrm{mg}, 6.88 \mathrm{mmol}$ ) was added to a vial containing 4 (124 mg, $0.182 \mathrm{mmol}$ ) and camphorsulfonic acid (95 mg, $0.409 \mathrm{mmol}$ ) dissolved in $\mathrm{CHCl}_{3}(4.33 \mathrm{~g})$. The reaction was allowed to stand for 2.5 hours. LiOMe $(24 \mathrm{mg}, 0.632 \mathrm{mmol}$ ) and malononitrile $(305 \mathrm{mg}, 4.62$ $\mathrm{mmol})$ were dissolved in $\mathrm{MeOH}(1.47 \mathrm{mg})$ and added to the reaction. The reaction was capped and allowed to stir for 3 days. Ether $(2 \mathrm{~mL})$ was added to the vial, and the bright orange solid was filtered over a $15 \mathrm{~mL}$ fine-porosity fritted funnel. The solid was dried in vacuo for an hour to give 12 (85 mg, $59 \%$ ).

${ }^{1} \mathrm{H}$ (d-acetone): $\delta 8.05(\mathrm{~d}, J=2.0,1 \mathrm{H}, \mathrm{pz} 5 \mathrm{C}), 8.02(\mathrm{~s}, 1 \mathrm{H}, \mathrm{H9}), 7.96(\mathrm{~d}, J=2.0,1 \mathrm{H}, \mathrm{pz} 5 \mathrm{~B}), 7.94(\mathrm{~d}, J$ $=2.0,1 \mathrm{H}, \mathrm{pz} 3 \mathrm{~B}), 7.90(\mathrm{~d}, J=2.0,1 \mathrm{H}, \mathrm{pz} 5 \mathrm{~A}), 7.87(\mathrm{~d}, J=8.2,1 \mathrm{H}, \mathrm{H} 8), 7.64(\mathrm{~d}, J=2.0,1 \mathrm{H}, \mathrm{pz} 3 \mathrm{C})$, $7.50(\mathrm{~d}, J=8.2,1 \mathrm{H}, \mathrm{H} 5), 7.36(\mathrm{~d}, J=5.3,1 \mathrm{H}, \mathrm{H} 2), 7.30$ (ddd, $J=8.1,6.7,1.2,1 \mathrm{H}, \mathrm{H} 6), 7.20$ (ddd, $J$ $=8.0,6.7,1.2,1 \mathrm{H}, \mathrm{H} 7), 6.87(\mathrm{~s}, 1 \mathrm{H}, \mathrm{H} 10), 6.66(\mathrm{~d}, J=2.0,1 \mathrm{H}, \mathrm{pz} 3 \mathrm{~A}), 6.42(\mathrm{t}, J=2.0,1 \mathrm{H}, \mathrm{pz} 4 \mathrm{C})$, $6.34(\mathrm{t}, J=2.0,1 \mathrm{H}, \mathrm{pz} 4 \mathrm{~B}), 6.09(\mathrm{t}, J=2.0,1 \mathrm{H}, \mathrm{pz} 4 \mathrm{~A}), 5.64(\mathrm{~s}, 1 \mathrm{H}, \mathrm{H} 18), 3.90$ (ddd, $J=13.3,8.7$, 5.4, 1H, H3), 2.52 (d, J = 8.7, 1H, H4), $2.04(\mathrm{~s}, 3 \mathrm{H}, \mathrm{H} 16), 1.84(\mathrm{~s}, 3 \mathrm{H}, \mathrm{H} 17), 1.42(\mathrm{~d}, J=8.2,9 \mathrm{H}$, $\left.\mathrm{PMe}_{3}\right) .{ }^{13} \mathrm{C}$ (d-acetone): $\delta$ 145.45, 145.08 (pz3B), 143.00 (pz3A), 142.11 (pz3C), 138.08 (C2), 137.91 (pz5C), 137.68 (pz5B), 137.42 (pz5A), 132.18, 132.08, 130.08, 129.35, 129.19 (C8), 127.30 
(C10), 126.30 (C5), 125.97 (C6), 124.49 (C7), 122.39 (C9), 115.29 (C19), 114.54 (C20), 107.66

(pz4C), 107.58 (pz4B), 105.61 (pz4A), 61.77 (C4), 57.69 (d, J = 7.9, C3), 42.39 (C15 or C18), 42.29

(C15 or C18), 35.30 (C17), 29.78 (C16), 13.95 (d, J = 28, $\left.\mathrm{PMe}_{3}\right) \cdot{ }^{31} \mathrm{P}\left(\mathrm{CDCl}_{3}\right): \delta-12.21\left(J_{\mathrm{W}-\mathrm{P}}=289\right)$.

$C V: E_{p, a}=+0.305$ V. IR: $v_{N O}=1561 \mathrm{~cm}^{-1}, v_{C N}=2214 \mathrm{~cm}^{-1}, v_{B H}=2488 \mathrm{~cm}^{-1}$.

\section{$\mathrm{TpW}(\mathrm{NO})\left(\mathrm{PMe}_{3}\right)\left(3,4-\eta^{2}-4-(\right.$ anthracen-1-yl)-4-methylpentan-2-one) (13)}

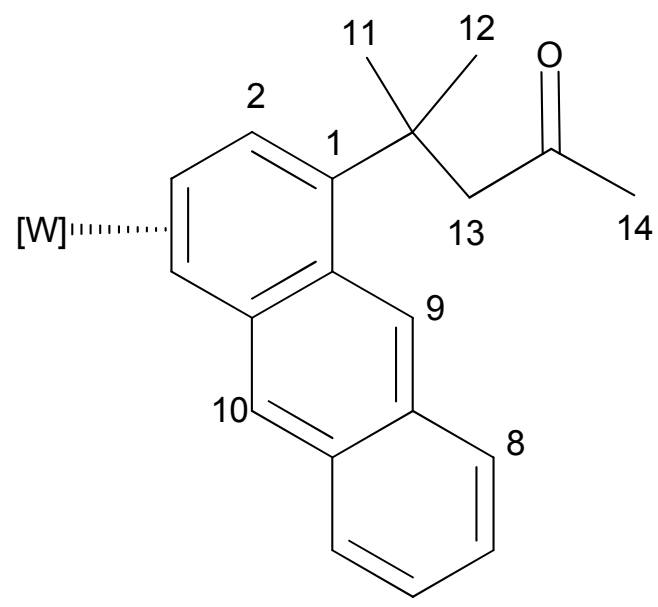

Dimethoxypropane $(612 \mathrm{mg}, 5.88 \mathrm{mmol}$ ) was added to a vial containing 4 (91 mg, $0.134 \mathrm{mmol}$ ) and camphorsulfonic acid $(74 \mathrm{mg}, 0.319 \mathrm{mmol})$ dissolved in $\mathrm{CHCl}_{3}(3.58 \mathrm{~g})$. The reaction was allowed to stand for 2.7 hours. LiOMe $(18 \mathrm{mg}, 0.474 \mathrm{mmol}$ ) and acetone (195 mg, $3.36 \mathrm{mmol}$ ) were dissolved in $\mathrm{MeOH}$ (1.038 g) and added to the reaction. Let stand for 24 hours. Extracted $3 x$ with $3 \mathrm{~mL}$ portions of water. Backextracted water layers with $0.5 \mathrm{~mL}$ portions of DCM. Combined organic layers were dried with $\mathrm{MgSO}_{4}$, which was filtered over a Celite plug. The solution was concentrated to $\sim 2 \mathrm{~mL}$ and precipitated over $20 \mathrm{~mL}$ stirring hexanes. The yellow solid was filtered over a $15 \mathrm{~mL}$ fine-porosity fritted funnel and dried in vacuo to give 13 at 85\% purity (22 mg, 21\%).

${ }^{1} \mathrm{H}\left(\mathrm{CDCl}_{3}\right): \delta 8.76(\mathrm{~s}, 1 \mathrm{H}, \mathrm{H} 9), 7.86(\mathrm{~m}, 1 \mathrm{H}, \mathrm{H} 8), 7.84(\mathrm{~d}, J=2.0,1 \mathrm{H}, \mathrm{pz} 3 \mathrm{~B}), 7.81(\mathrm{~d}, J=2.0,1 \mathrm{H}$, pz5C), $7.72(\mathrm{~d}, J=2.0,1 \mathrm{H}, \mathrm{pz} 5 \mathrm{~B}), 7.70(\mathrm{~d}, J=2.0,1 \mathrm{H}, \mathrm{pz} 5 \mathrm{~A}), 7.53(\mathrm{~m}, 1 \mathrm{H}, \mathrm{H} 5), 7.29(\mathrm{~m}, 1 \mathrm{H}, \mathrm{H} 6)$, 
$7.27(\mathrm{~d}, J=2.0,1 \mathrm{H}, \mathrm{pz} 3 \mathrm{C}), 7.22(\mathrm{~m}, 1 \mathrm{H}, \mathrm{H} 7), 7.12(\mathrm{~d}, J=2.0,1 \mathrm{H}, \mathrm{pz} 3 \mathrm{~A}), 7.05(\mathrm{~d}, J=5.0,1 \mathrm{H}, \mathrm{H} 2)$, $7.01(\mathrm{~s}, 1 \mathrm{H}, \mathrm{H} 10), 6.27(\mathrm{t}, J=2.0,1 \mathrm{H}, \mathrm{pz} 4 \mathrm{C}), 6.22(\mathrm{t}, J=2.0,1 \mathrm{H}, \mathrm{pz} 4 \mathrm{~B}), 6.11(\mathrm{t}, J=2.0,1 \mathrm{H}, \mathrm{pz} 4 \mathrm{~A})$, $3.70(\mathrm{~m}, 1 \mathrm{H}, \mathrm{H} 3), 3.24(\mathrm{~s}, 3 \mathrm{H}, \mathrm{H} 14), 2.59(\mathrm{~d}, J=9.0,1 \mathrm{H}, \mathrm{H} 4), 1.86(\mathrm{~s}, 3 \mathrm{H}, \mathrm{H} 11$ or H12), 1.84 (d, J = 13.5, $2 \mathrm{H}, \mathrm{H} 13), 1.70(\mathrm{~s}, 3 \mathrm{H}, \mathrm{H} 11$ or $\mathrm{H} 12), 1.36\left(\mathrm{~d}, J=8.0,9 \mathrm{H}, \mathrm{PMe}_{3}\right) \cdot{ }^{31} \mathrm{P}\left(\mathrm{CDCl}_{3}\right): \delta-12.34\left(J_{\mathrm{W}-\mathrm{P}}=\right.$ 293).

$\mathrm{TpW}(\mathrm{NO})\left(\mathrm{PMe}_{3}\right)\left(3,4-\eta^{2}-(2-(\right.$ anthracen-1-yl)propan-2-yl)(phenyl)sulfane)

\section{$\operatorname{TpW}(\mathrm{NO})\left(\mathrm{PMe}_{3}\right)\left(3,4-\eta^{2}-1-(\right.$ prop-1-en-2-yl)anthracene) (16)}
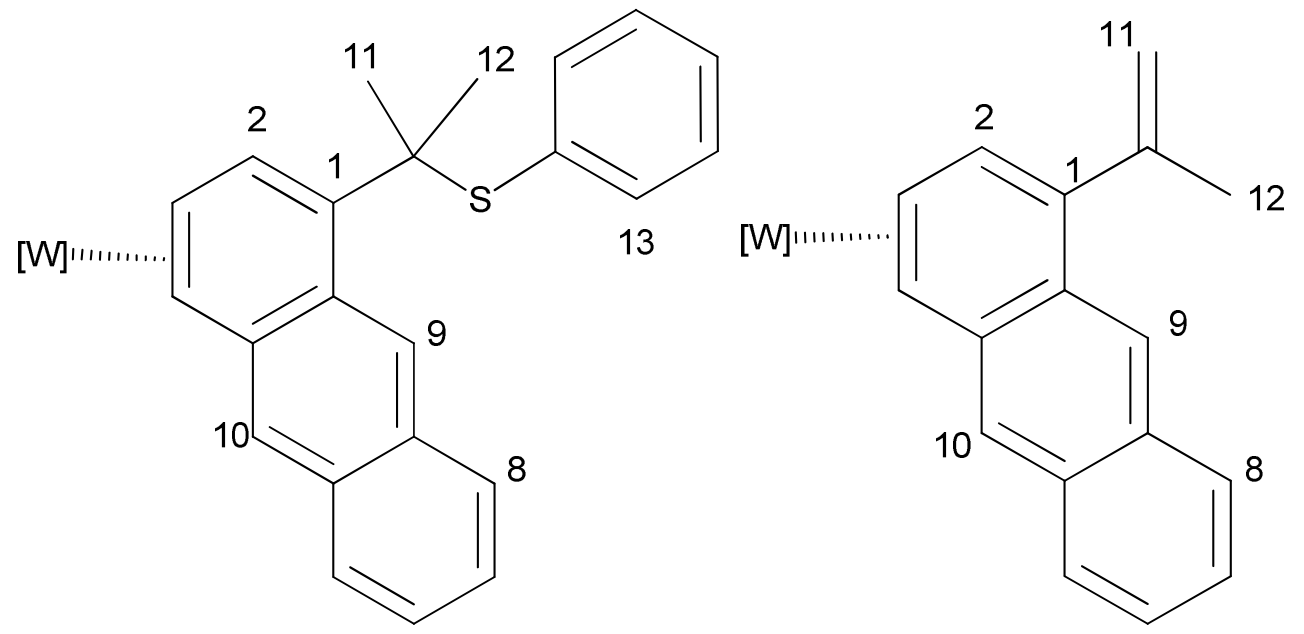

Dimethoxypropane (605 mg, $5.82 \mathrm{mmol}$ ) was added to a vial containing 4 (90 mg, $0.132 \mathrm{mmol}$ ) and camphorsulfonic acid $\left(78 \mathrm{mg}, 0.336 \mathrm{mmol}\right.$ ) dissolved in $\mathrm{CHCl}_{3}(3.62 \mathrm{~g})$. The reaction was allowed to stand for 2.75 hours and then thiophenol $(88 \mathrm{mg}, 0.800 \mathrm{mmol}$ ) was added to the reaction. The solution was then added to a vial containing LiOMe (12 $\mathrm{mg}, 0.316 \mathrm{mmol})$, and the reaction was allowed to stand $40 \mathrm{~min}$. The reaction was extracted $4 x$ with $3 \mathrm{~mL}$ portions of water, which were backextracted with $0.5 \mathrm{~mL}$ portions of DCM. The combined organic layers were dried with $\mathrm{MgSO}_{4}$, then filtered over a Celite plug. The solution was concentrated to 1.5 $\mathrm{mL}$ and precipitated over $15 \mathrm{~mL}$ stirring hexanes. The yellow-orange sold was filtered over a 15 $\mathrm{mL}$ fine-porosity fritted funnel and dried in vacuo for $10 \mathrm{~min}$ to give 15 and 16 (60 mg, including 10 \% impurity). 
Selected NMR data for $15:{ }^{1} \mathrm{H}\left(\mathrm{CDCl}_{3}\right): \delta 8.98(\mathrm{~s}, 1 \mathrm{H}, 9 \mathrm{H}), 7.94(\mathrm{~d}, J=8.1,2 \mathrm{H}, \mathrm{H} 13), 6.75(\mathrm{~d}, J=$ 4.8, 1H, H2), $3.65(\mathrm{~m}, 1 \mathrm{H}, \mathrm{H} 3), 2.63(\mathrm{~d}, 1 \mathrm{H}, \mathrm{H} 4), 2.07(\mathrm{~s}, 3 \mathrm{H}, \mathrm{H} 11$ or H12), $1.72(\mathrm{~s}, 3 \mathrm{H}, \mathrm{H} 11$ or H12), $1.25\left(\mathrm{~d}, J=8.0,9 \mathrm{H}, \mathrm{PMe}_{3}\right) \cdot{ }^{31} \mathrm{P}\left(\mathrm{CDCl}_{3}\right): \delta-12.67\left(J_{\mathrm{W}-\mathrm{P}}=294\right)$.

Selected NMR data for $16:{ }^{1} \mathrm{H}\left(\mathrm{CDCl}_{3}\right): \delta 6.82(\mathrm{~d}, \mathrm{~J}=4.8,1 \mathrm{H}, \mathrm{H} 2), 5.24(\mathrm{~m}, 2 \mathrm{H}, \mathrm{H} 11), 3.71(\mathrm{~m}, 1 \mathrm{H}$, $\mathrm{H} 3), 2.62(\mathrm{~d}, 1 \mathrm{H}, \mathrm{H} 4), 2.25(\mathrm{~s}, 3 \mathrm{H}, \mathrm{H} 12), 1.37\left(\mathrm{~d}, J=8.1,9 \mathrm{H}, \mathrm{PMe}_{3}\right) \cdot{ }^{31} \mathrm{P}\left(\mathrm{CDCl}_{3}\right): \delta-12.40\left(J_{\mathrm{W}-\mathrm{P}}=\right.$ 295).

$\mathrm{TpW}(\mathrm{NO})\left(\mathrm{PMe}_{3}\right)\left(3,4-\eta^{2}\right.$-(E)-1-benzylidene-1,2-dihydroanthracen-2-ylium) p-toluenesulfonate (19b)

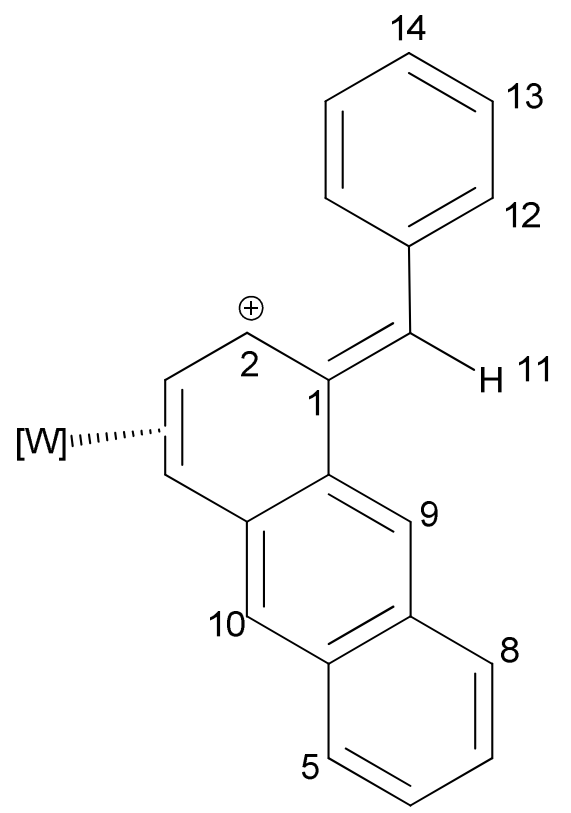

Benzaldehyde dimethyl acetal $(130 \mathrm{mg}, 0.855 \mathrm{mmol})$ was added to a vial containing 4 (50 mg, $0.073 \mathrm{mmol}$ ) and $p$-toluenesulfonic acid $(29 \mathrm{mg}, 0.169 \mathrm{mmol})$ dissolved in $\mathrm{CHCl}_{3}(1.696 \mathrm{~g})$. The reaction was allowed to stand for $40 \mathrm{~min}$, and was then precipitated over $19 \mathrm{~mL}$ stirring ether. The brown solid was collected on a $15 \mathrm{~mL}$ fine-porosity fritted funnel and dried in vacuo for several minutes to give $19 \mathrm{a}$ ( $83 \mathrm{mg}$, including excess acid).

${ }^{1} \mathrm{H}\left(\mathrm{CDCl}_{3}\right)$ (p-toluenesulfonate peaks omitted): $\delta 8.65(\mathrm{pz} 3 \mathrm{C}), 8.24(\mathrm{H} 2), 8.21(\mathrm{H} 11), 8.17(\mathrm{H} 9)$, 7.86 (pz5C), $7.85(\mathrm{H} 8), 7.79(\mathrm{pz} 5 \mathrm{~B}), 7.74$ (pz5A), 7.69 (pz3B), 7.63 (H12), $7.58(\mathrm{H} 5), 7.50$ (H13), 
7.46 (H6), 7.35 (H7), 7.34 (H14), 6.84 (H10), 6.78 (pz3A), 6.52 (pz4C), 6.42 (H3), 6.30 (pz4B), 6.08 (pz4A), $4.02(\mathrm{H} 4), 0.71\left(\mathrm{~d}, J=9.1,9 \mathrm{H}, \mathrm{PMe}_{3}\right) .{ }^{31} \mathrm{P}\left(\mathrm{CDCl}_{3}\right): \delta-11.52\left(J_{\mathrm{W}-\mathrm{P}}=264\right)$.

$\mathrm{TpW}(\mathrm{NO})\left(\mathrm{PMe}_{3}\right)\left(3,4-\eta^{2}\right.$-(S,E)-dimethyl 2-(1-benzylidene-1,2-dihydroanthracen-2-yl)malonate) (20)

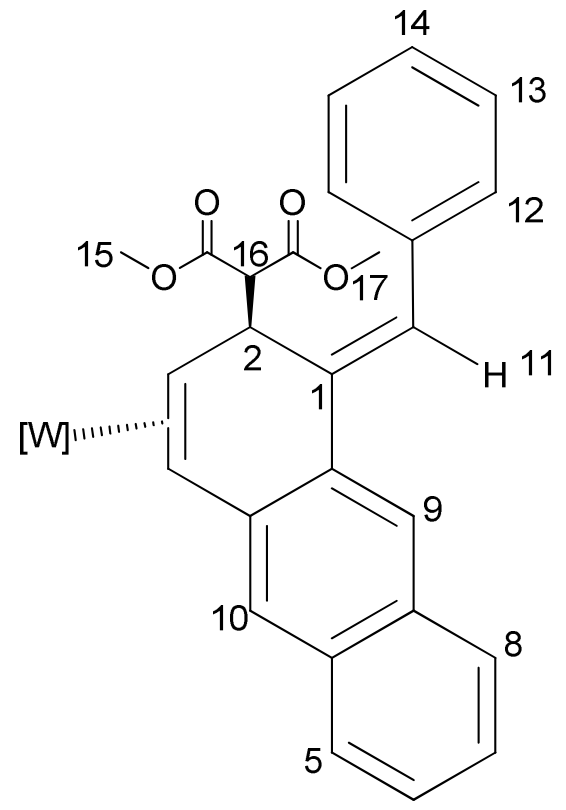

Benzaldehyde dimethyl acetal (199 mg, $1.31 \mathrm{mmol}$ ) was added to a vial containing 4 (61 mg, $0.090 \mathrm{mmol}$ ) and camphorsulfonic acid (48 $\mathrm{mg}, 0.207 \mathrm{mmol}$ ) dissolved in $\mathrm{CHCl}_{3}(2.587 \mathrm{~g})$. The reaction was allowed to stand for $30 \mathrm{~min}$. LiOMe $(8 \mathrm{mg}, 0.211 \mathrm{mmol})$ and dimethylmalonate (33 $\mathrm{mg}, 0.25 \mathrm{mmol})$ were dissolved in $\mathrm{MeOH}(326 \mathrm{mg})$ and added to the reaction. The reaction was allowed to stand for two hours, then extracted $3 x$ with $3 \mathrm{~mL}$ portions of water. The combined organic layers were dried with $\mathrm{MgSO}_{4}$, and filtered over a Celite plug. The solution was concentrated $1 \mathrm{~mL}$ and precipitated over $17 \mathrm{~mL}$ stirring hexanes. The light yellow solid was filtered over a $15 \mathrm{~mL}$ fine-porosity fritted funnel and dried in vacuo for $15 \mathrm{~min}$ to give $\mathbf{2 0}$ (28 mg, including 10 \% minor isomer, $30 \%)$.

${ }^{1} \mathrm{H}\left(\mathrm{CDCl}_{3}\right): \delta 7.81(\mathrm{~m}, 2 \mathrm{H}, \mathrm{H} 9$ and pz3B), $7.80(\mathrm{~m}, 1 \mathrm{H}, \mathrm{H} 8), 7.74(\mathrm{~m}, 3 \mathrm{H}, \mathrm{H} 12$ and pz5C), $7.64(\mathrm{~d}, J$ $=2.0,1 \mathrm{H}, \mathrm{pz} 5 \mathrm{~B}), 7.62(\mathrm{~d}, J=2.0,1 \mathrm{H}, \mathrm{pz} 5 \mathrm{~A}), 7.55(\mathrm{~d}, J=8.2,1 \mathrm{H}, \mathrm{H} 5), 7.39(\mathrm{dd}, J=7.7,2 \mathrm{H}, \mathrm{H} 13)$, 
$7.34(\mathrm{~d}, J=2.0,1 \mathrm{H}, \mathrm{pz} 3 \mathrm{C}), 7.33(\mathrm{~m}, 1 \mathrm{H}, \mathrm{H} 6), 7.25(\mathrm{~m}, 1 \mathrm{H}, \mathrm{H} 7), 7.23(\mathrm{~m}, 1 \mathrm{H}, \mathrm{H} 14), 7.15(\mathrm{~s}, 1 \mathrm{H}$, H11), $7.05(\mathrm{~d}, J=2.0,1 \mathrm{H}, \mathrm{pz} 3 \mathrm{~A}), 6.86(\mathrm{~s}, 1 \mathrm{H}, \mathrm{H} 10) 6.24(\mathrm{t}, J=2.0,1 \mathrm{H}, \mathrm{pz} 4 \mathrm{C}), 6.17(\mathrm{t}, J=2.0,1 \mathrm{H}$, pz4B), $6.05(\mathrm{t}, J=2.0,1 \mathrm{H}, \mathrm{pz} 4 \mathrm{~A}), 5.07(\mathrm{~d}, J=9.4,1 \mathrm{H}, \mathrm{H} 2), 3.90(\mathrm{~d}, J=9.51 \mathrm{H}, \mathrm{H} 16), 3.75(\mathrm{~s}, 3 \mathrm{H}$, $\mathrm{H} 15$ or H17), 3.61 (s, 3H, H15 or H17), 3.02 (ddd, $J=1.7,10.2,11.6,1 \mathrm{H}, \mathrm{H} 3$ ), 2.20 (dd, $J=1.7,9.9$, $1 \mathrm{H}, \mathrm{H} 4), 0.82\left(\mathrm{~d}, J=8.2,9 \mathrm{H}, \mathrm{PMe}_{3}\right) .{ }^{31} \mathrm{P}\left(\mathrm{CDCl}_{3}\right): \delta-12.20\left(J_{\mathrm{W}-\mathrm{P}}=277\right) . \quad \mathrm{IR}: \mathrm{v}_{\mathrm{NO}}=1567 \mathrm{~cm}^{-1}, \mathrm{v}_{\mathrm{CO}}=$ $1731 \mathrm{~cm}^{-1}, \mathrm{~V}_{\mathrm{BH}}=2486 \mathrm{~cm}^{-1}$.

$\mathrm{TpW}(\mathrm{NO})\left(\mathrm{PMe}_{3}\right)\left(3,4-\eta^{2}-(\mathrm{R}, \mathrm{E})-1-\right.$-benzylidene-2-(nitromethyl)-1,2-dihydroanthracene) (21)

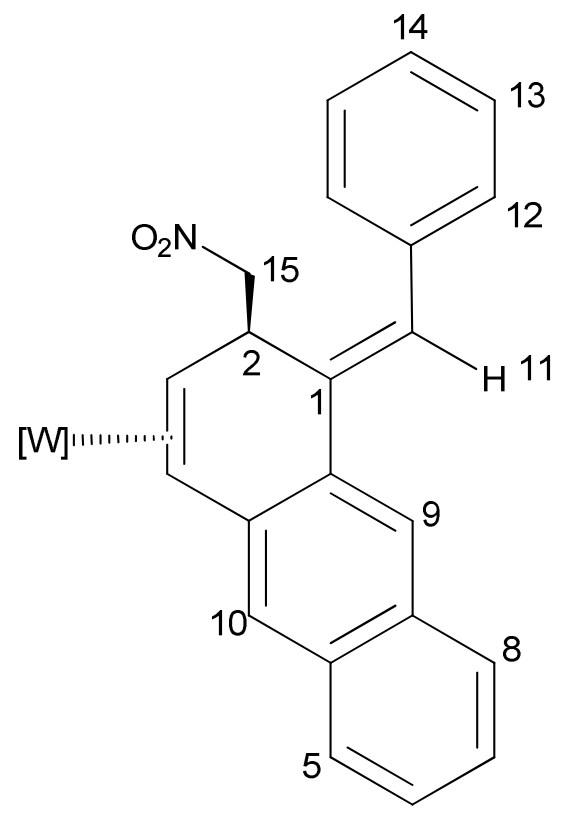

Benzaldehyde dimethyl acetal (198 mg, $1.31 \mathrm{mmol}$ ) was added to a vial containing 4 (60 mg, $0.088 \mathrm{mmol}$ ) and camphorsulfonic acid (48 $\mathrm{mg}, 0.207 \mathrm{mmol}$ ) dissolved in $\mathrm{CHCl}_{3}$ (2.607 g). The reaction was allowed to stand for $35 \mathrm{~min}$. LiOMe $(8 \mathrm{mg}, 0.211 \mathrm{mmol})$ and nitromethane $(18 \mathrm{mg}$, $0.295 \mathrm{mmol}$ ) were dissolved in $\mathrm{MeOH}(333 \mathrm{mg}$ ) and added to the reaction. The reaction was allowed to stand for 1.5 hours, then extracted $3 x$ with $3 \mathrm{~mL}$ portions of water. The combined organic layers were dried with $\mathrm{MgSO}_{4}$, and filtered over a Celite plug. The solution was concentrated $1 \mathrm{~mL}$ and precipitated over $16 \mathrm{~mL}$ stirring hexanes. The light yellow solid was 
filtered over a $15 \mathrm{~mL}$ fine-porosity fritted funnel and dried in vacuo for $15 \mathrm{~min}$ to give $\mathbf{2 1}$ (11 mg, including 10 \% impurity, 14 \%).

${ }^{1} \mathrm{H}\left(\mathrm{CDCl}_{3}\right): \delta 7.88(\mathrm{~s}, 1 \mathrm{H}, \mathrm{H} 9), 7.83(\mathrm{pz} 3 \mathrm{~B}), 7.78(\mathrm{~d}, \mathrm{~J}=8.2,1 \mathrm{H}, \mathrm{H} 8), 7.75(\mathrm{~d}, \mathrm{~J}=2.0,1 \mathrm{H}, \mathrm{pz} 5 \mathrm{C}), 7.66$ $(d, J=2.0,1 H, p z 5 B), 7.62(d, J=2.0,1 H, p z 5 A), 7.57(d, J=7.7,2 H, H 12), 7.54(d, J=8.8,1 H$, $\mathrm{H} 5), 7.44(\mathrm{t}, \mathrm{J}=7.9,2 \mathrm{H}, \mathrm{H} 13), 7.36(\mathrm{~m}, 2 \mathrm{H}, \mathrm{H} 6$ and $\mathrm{H} 14), 7.34(\mathrm{~d}, \mathrm{~J}=2.0,1 \mathrm{H}, \mathrm{pz} 3 \mathrm{C}), 7.28(\mathrm{~s}, 1 \mathrm{H}$, $\mathrm{H} 11), 7.25(\mathrm{~m}, 1 \mathrm{H}, \mathrm{H7}), 7.03(\mathrm{~d}, \mathrm{~J}=2.0,1 \mathrm{H}, \mathrm{pz} 3 \mathrm{~A}), 6.91(\mathrm{~s}, 1 \mathrm{H}, \mathrm{H} 10), 6.24(\mathrm{t}, \mathrm{J}=2.0,1 \mathrm{H}, \mathrm{pz} 4 \mathrm{C})$, $6.20(\mathrm{t}, \mathrm{J}=2.0,1 \mathrm{H}, \mathrm{pz} 4 \mathrm{~B}), 6.05(\mathrm{t}, \mathrm{J}=2.0,1 \mathrm{H}, \mathrm{pz} 4 \mathrm{~A}), 5.00(\mathrm{dd}, \mathrm{J}=3.0,10.6,1 \mathrm{H}, \mathrm{H} 2), 4.94(\mathrm{dd}, \mathrm{J}=$ 3.5, 11.2, 1H, H15), $4.64\left(\mathrm{t}, \mathrm{J}=10.8,1 \mathrm{H}, \mathrm{H} 15^{\prime}\right), 2.89(\mathrm{ddd}, \mathrm{J}=0.9,9.8,11.3,1 \mathrm{H}, 3 \mathrm{H}), 2.25$ (dd, J = $1.3,9.9,1 \mathrm{H}, \mathrm{H} 4), 0.76\left(\mathrm{~d}, \mathrm{~J}=8.2,9 \mathrm{H}, \mathrm{PMe}_{3}\right) .{ }^{31} \mathrm{P}\left(\mathrm{CDCl}_{3}\right): \delta-12.62\left(J_{\mathrm{W}-\mathrm{P}}=276\right)$.

\section{$\operatorname{TpW}(\mathrm{NO})\left(\mathrm{PMe}_{3}\right)\left(3,4-\eta^{2}-\mathrm{N}\right.$-(anthracen-1-yl(phenyl)methyl)propan-1-amine) (22)}

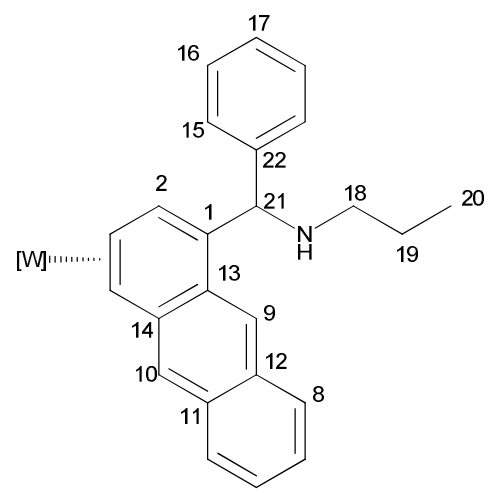

Benzaldehyde dimethyl acetal $(603 \mathrm{mg}, 3.97 \mathrm{mmol})$ was added to a vial containing 4 (208 mg, $0.305 \mathrm{mmol}$ ) and camphorsulfonic acid (172 $\mathrm{mg}, 0.741 \mathrm{mmol}$ ) dissolved in $\mathrm{CHCl}_{3}(6.61 \mathrm{~g})$. The reaction was allowed to stand for $40 \mathrm{~min}$ and propylamine $(511 \mathrm{mg}, 8.66 \mathrm{mmol}$ ) was added to the reaction. The reaction was capped and stirred for 3 days. The bright orange solid was filtered over a $15 \mathrm{~mL}$ fine-porosity fritted funnel and washed with $5 \mathrm{~mL}$ ether to give $\mathbf{2 2}(76 \mathrm{mg}$, $30 \%)$.

${ }^{1} \mathrm{H}$ (d-acetone): $\delta 8.10(\mathrm{~s}, 1 \mathrm{H}, \mathrm{H} 9), 8.02(\mathrm{~d}, J=2.0,1 \mathrm{H}, \mathrm{pz} 5 \mathrm{C}), 7.95(\mathrm{~d}, J=2.0,1 \mathrm{H}, \mathrm{pz} 3 \mathrm{~B}), 7.94(\mathrm{~d}, J$ $=2.0,1 \mathrm{H}, \mathrm{pz} 5 \mathrm{~B}), 7.88(\mathrm{~d}, J=2.0,1 \mathrm{H}, \mathrm{pz} 5 \mathrm{~A}), 7.83(\mathrm{~d}, J=8.1,1 \mathrm{H}, \mathrm{H} 8), 7.69(\mathrm{~d}, J=8.1,2 \mathrm{H}, \mathrm{H} 15)$, 
$7.55(\mathrm{~d}, J=2.0,1 \mathrm{H}, \mathrm{pz} 3 \mathrm{C}), 7.53(\mathrm{~d}, J=8.1,1 \mathrm{H}, \mathrm{H} 5), 7.35(\mathrm{t}, J=7.4,2 \mathrm{H}, \mathrm{H} 16), 7.26(\mathrm{~m}, 1 \mathrm{H}, \mathrm{H} 6)$, $7.22(\mathrm{~m}, 1 \mathrm{H}, \mathrm{H} 17), 7.21(\mathrm{~m}, 1 \mathrm{H}, \mathrm{H} 7), 6.90(\mathrm{~s}, 1 \mathrm{H}, \mathrm{H} 10), 6.87(\mathrm{~d}, J=2.0,1 \mathrm{H}, \mathrm{pz} 3 \mathrm{~A}), 6.83(\mathrm{~d}, J=5.2$, $1 \mathrm{H}, \mathrm{H} 2), 6.37(\mathrm{t}, J=2.0,1 \mathrm{H}, \mathrm{pz} 4 \mathrm{C}), 6.33(\mathrm{t}, J=2.0,1 \mathrm{H}, \mathrm{pz} 4 \mathrm{~B}), 6.13(\mathrm{t}, J=2.0,1 \mathrm{H}, \mathrm{pz} 4 \mathrm{~A}), 5.37(\mathrm{~s}$, $1 \mathrm{H}, \mathrm{H} 21$ ), 3.73 (ddd, $J=13.5,8.7,5.2,1 \mathrm{H}, \mathrm{H} 3), 2.77$ (dt, $J=11.3,6.7,1 \mathrm{H}, \mathrm{H} 18), 2.60(\mathrm{dt}, J=11.3$, 6.7, $\left.1 \mathrm{H}, \mathrm{H} 18^{\prime}\right), 2.49(\mathrm{~d}, J=8.8,1 \mathrm{H}, \mathrm{H} 4), 1.50(\mathrm{~m}, 2 \mathrm{H}, \mathrm{H} 19), 1.38\left(\mathrm{~d}, J=8.8,9 \mathrm{H}, \mathrm{PMe}_{3}\right) 0.91(\mathrm{t}, J=$ 7.3, 3H, H20). ${ }^{13} \mathrm{C}$ (d-acetone): $\delta 146.99$ (C22), 145.15 (C14), 142.76 (pz3A), 141.90 (pz3C), 138.68, 138.03 (pz5C), 137.57 (pz3B), 137.19 (C2), 135.94 (C13), 132.65, 132.44, 132.29, 132.23, 129.24 (C15), 128.82 (C8), 128.76 (C16), 127.17 (C17), 126.51 (C5), 126.28 (C10), 125.33 (C6), 124.38 (C7), 119.57 (C9), 107.65 (pz4C), 107.59 (pz4B), 105.75 (pz4A), 63.14 (C21), 60.96 (C4), $58.24(\mathrm{~d}, J=8, \mathrm{C} 3), 52.25$ (C18), 24.54 (C19), 13.75 (d, J = 28, PMe $), 12.52$ (C20). ${ }^{31} \mathrm{P}$ (d-acetone): $\delta-12.21\left(J_{W-p}=293\right) . C V: E_{p, a}=+0.229$ V. IR: $v_{N O}=1548 \mathrm{~cm}^{-1}$. HRMS: obs'd (\%), calc'd (\%), ppm $\left(\mathrm{M}+\mathrm{Na}^{+}\right): 849.2685(78), 849.2698(78),-1.6 ; 850.2715$ (80), 850.2723 (84), -1.0; 851.2715 (100), 851.2725 (100), -1.1; 852.2747 (42), 852.2760 (53), -1.5; 853.2746 (76), 853.2756 (80), -1.2.

$\operatorname{TpW}(\mathrm{NO})\left(\mathrm{PMe}_{3}\right)\left(3,4-\eta^{2}\right.$ - 1-(anthracen-1-yl(phenyl)methyl)-1H-imidazole) (23)

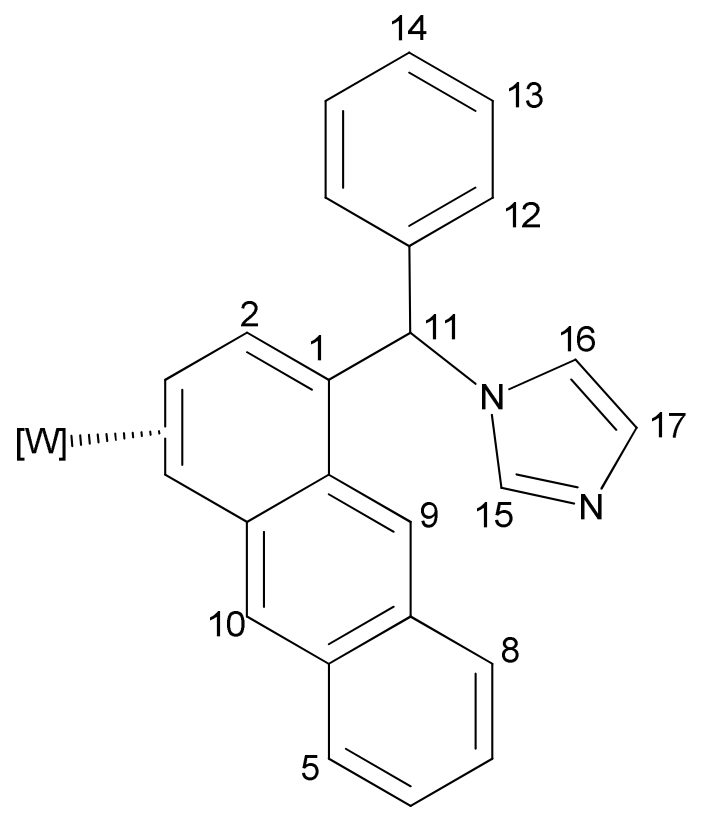


Benzaldehyde dimethyl acetal (202 mg, $1.33 \mathrm{mmol}$ ) was added to a vial containing 4 (60 mg, $0.088 \mathrm{mmol}$ ) and camphorsulfonic acid (48 $\mathrm{mg}, 0.207 \mathrm{mmol}$ ) dissolved in $\mathrm{CHCl}_{3}$ (2.602 g). The reaction was allowed to stand for $35 \mathrm{~min}$ and then added to a vial containing imidazole $(146 \mathrm{mg}$, $2.15 \mathrm{mmol}$ ). The reaction was allowed to stand for 3 hours before being extracted $3 \mathrm{x}$ with $3 \mathrm{~mL}$ portions of water. The water layers were backextracted with $0.5 \mathrm{~mL}$ portions of DCM. The combined organic layers were dried with $\mathrm{MgSO}_{4}$, which was filtered off over a Celite plug. The solution was concentrated to $\sim 1 \mathrm{~mL}$ and precipitated over $17 \mathrm{~mL}$ stirring hexanes. The yellow solid was filtered over a $15 \mathrm{~mL}$ fine-porosity fritted funnel and dried in vacuo for 15 minutes to give 23 with a 10\% impurity (52 mg, $70 \%$ ).

${ }^{1} \mathrm{H}\left(\mathrm{CDCl}_{3}\right): \delta 7.83(\mathrm{~d}, J=2.0,1 \mathrm{H}, \mathrm{pz} 3 \mathrm{~B}), 7.79(\mathrm{~d}, J=2.0,1 \mathrm{H}, \mathrm{pz} 5 \mathrm{C}), 7.73(\mathrm{~m}, 1 \mathrm{H}, \mathrm{H} 16), 7.72(\mathrm{~d}, J=$ 2.0, $1 \mathrm{H}, \mathrm{pz} 5 \mathrm{~B}), 7.70(\mathrm{~d}, J=2.0,1 \mathrm{H}, \mathrm{pz} 5 \mathrm{~A}), 7.63(\mathrm{~d}, J=8.2,1 \mathrm{H}, \mathrm{H} 8), 7.54(\mathrm{~m}, 1 \mathrm{H}, \mathrm{H} 15), 7.50(\mathrm{~d}, J=$ 8.2, $1 \mathrm{H}, \mathrm{H} 5), 7.36(\mathrm{~m}, 2 \mathrm{H}, \mathrm{H} 12), 7.30-7.35(\mathrm{~m}, 3 \mathrm{H}, \mathrm{H} 13$ and $\mathrm{H} 14), 7.28(\mathrm{~m}, 1 \mathrm{H}, \mathrm{H} 17), 7.27$ (m, $2 \mathrm{H}, \mathrm{H} 6$ and $\mathrm{H} 9), 7.21(\mathrm{~d}, J=2.0,1 \mathrm{H}, \mathrm{pz} 3 \mathrm{C}), 7.18(\mathrm{~m}, 1 \mathrm{H}, \mathrm{H} 7), 7.13(\mathrm{t}, J=1.2,1 \mathrm{H}, \mathrm{H} 11), 6.92(\mathrm{~s}, 1 \mathrm{H}$, $\mathrm{H} 10), 6.84(\mathrm{~d}, J=2.0,1 \mathrm{H}, \mathrm{pz} 3 \mathrm{~A}), 6.51(\mathrm{~d}, J=5.1,1 \mathrm{H}, \mathrm{H} 2), 6.26(\mathrm{t}, J=2.0,1 \mathrm{H}, \mathrm{pz} 4 \mathrm{C}), 6.22(\mathrm{t}, J=$ 2.0, $1 \mathrm{H}, \mathrm{pz} 4 \mathrm{~B}), 6.08(\mathrm{t}, J=2.0,1 \mathrm{H}, \mathrm{pz} 4 \mathrm{~A}), 3.61(\mathrm{ddd}, J=5.2,8.8,13.5,1 \mathrm{H}, \mathrm{H} 3), 2.55(\mathrm{~d}, J=8.8,1 \mathrm{H}$, $\mathrm{H} 4), 0.98\left(\mathrm{~d}, J=8.0,9 \mathrm{H}, \mathrm{PMe}_{3}\right) \cdot{ }^{31} \mathrm{P}\left(\mathrm{CDCl}_{3}\right): \delta-13.13\left(J_{\mathrm{W}-\mathrm{P}}=291\right)$.

$\mathrm{N}$-(anthracen-1-yl(phenyl)methyl)propan-1-amine (24) 


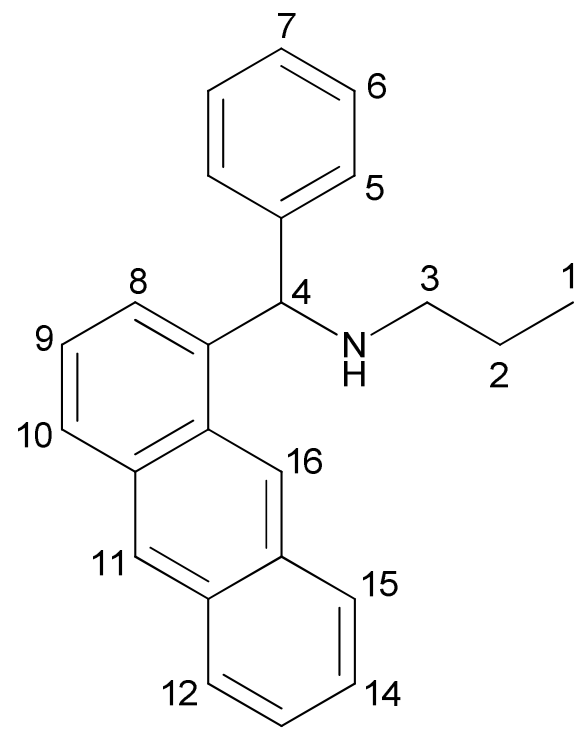

13

$22(36 \mathrm{mg}, 0.043 \mathrm{mmol})$ was dissolved in acetone $(3.077 \mathrm{~g})$ and CAN (25 mg, $0.046 \mathrm{mmol})$ was dissolved in water $(3.026 \mathrm{~g})$. The CAN solution was added to the rapidly stirring acetone solution and allowed to react for 1.5 hours. The solution was transferred to a $125 \mathrm{~mL}$ seperatory funnel containing $20 \mathrm{~mL}$ ether and $10 \mathrm{~mL}$ water and extracted. The water layer was extracted again with a $20 \mathrm{~mL}$ portion of ether, then a $10 \mathrm{~mL}$ portion of ether. The ether layers were combined and extracted with $10 \mathrm{~mL}$ water, which was backextracted with $10 \mathrm{~mL}$ ether. The ether layers were dried with $\mathrm{MgSO}_{4}$, which was filtered over a 60 medium-porosity fritted funnel. The ether was evaporated in vacuo to a film. The film was redissolved in DCM and precipitated over $24 \mathrm{~mL}$ HPLC-grade hexanes. The brown solid was filtered over a $15 \mathrm{~mL}$ fineporosity fritted funnel and discarded. The solution was evaporated in vacuo to a film. The film was dissolved in minimal DCM and loaded onto a $500 \mu \mathrm{m}$ silica preparatory plate. The plate was run under 70:30 HPLC grade hexanes:HPLC grade ethyl acetate. The band at $R_{f}=0.7-0.8$ was scraped off and added to a test tube with $22 \mathrm{~mL} \mathrm{HPLC}$ grade ethyl acetate. The test tube was sonicated for $20 \mathrm{~min}$. The silica was filtered over a $60 \mathrm{~mL}$ fine-porosity fritted funnel and 
washed with $20 \mathrm{~mL}$ HPLC grade ethyl acetate. The ethyl acetate was removed in vacuo to give 24 as a film (10 mg, $71 \%$, with a 7 \% impurity, possibly 2-substituted anthracene).

${ }^{1} \mathrm{H}\left(\mathrm{CDCl}_{3}\right): \delta 8.72(\mathrm{~s}, 1 \mathrm{H}, \mathrm{H} 11$ or $\mathrm{H} 16), 8.42(\mathrm{~s}, 1 \mathrm{H}, \mathrm{H} 11$ or $\mathrm{H} 16), 7.97(\mathrm{~m}, 2 \mathrm{H}, \mathrm{H} 12$ and $\mathrm{H} 15), 7.92$ (d, $J=8.6,1 \mathrm{H}, \mathrm{H} 8$ or $\mathrm{H} 10), 7.69$ (d, $J=6.9,1 \mathrm{H}, \mathrm{H} 8$ or $\mathrm{H} 10), 7.52$ (d, $J=7.7,2 \mathrm{H}, \mathrm{H} 5), 7.46$ (dd, $J=$ 6.9, 8.3, $1 \mathrm{H}, \mathrm{H} 9), 7.44(\mathrm{~m}, 2 \mathrm{H}, \mathrm{H} 13$ and $\mathrm{H} 14), 7.30(\mathrm{t}, J=7.7,2 \mathrm{H}, \mathrm{H} 6), 7.21(\mathrm{t}, J=7.4,1 \mathrm{H}, \mathrm{H} 7), 5.74$ $(\mathrm{s}, 1 \mathrm{H}, \mathrm{H} 4), 2.74(\mathrm{~m}, 2 \mathrm{H}, \mathrm{H} 3), 1.61(\mathrm{~m}, 2 \mathrm{H}, \mathrm{H} 2), 0.96(\mathrm{t}, J=7.6,3 \mathrm{H}, \mathrm{H} 1) .{ }^{13} \mathrm{C}\left(\mathrm{CDCl}_{3}\right): \delta 132.62$, 131.74, 131.37, 130.14, 128.82, 128.62 (2C), 128.09 (3C), 127.97, 127.24, 127.19, 125.59, $125.49,124.44,122.57,63.83,50.98,23.66,12.05$.

\section{2-(2-(anthracen-1-yl)propan-2-yl)malononitrile (25)}

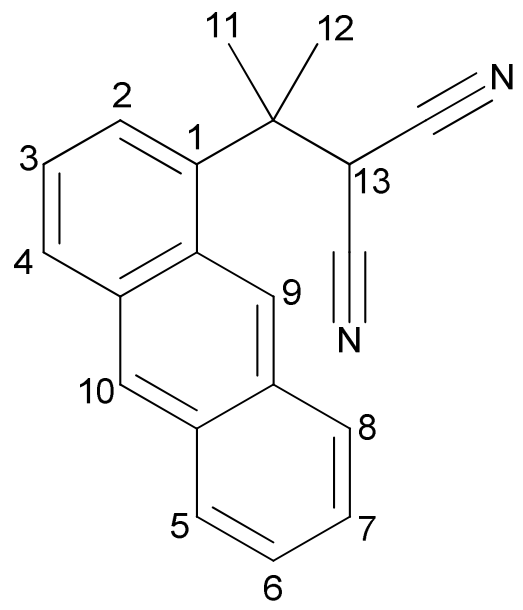

$12(66 \mathrm{mg}, 0.084 \mathrm{mmol})$ was dissolved in acetone $(3.63 \mathrm{~g})$ and CAN (47 mg, $0.086 \mathrm{mmol})$ was dissolved in water $(3.05 \mathrm{~g})$. The CAN solution was added to the rapidly stirring acetone solution and allowed to react for 1 hour. The solution was transferred to a $125 \mathrm{~mL}$ seperatory funnel containing $20 \mathrm{~mL}$ ether and $10 \mathrm{~mL}$ water and extracted. The water layer was extracted again with $2 \times 20 \mathrm{~mL}$ portions of ether. The ether layers were combined and extracted with $10 \mathrm{~mL}$ water. The ether layers were dried with $\mathrm{MgSO}_{4}$, which was filtered over a 60 medium-porosity fritted funnel. The ether was evaporated in vacuo to a film. The film was redissolved in DCM and precipitated over $50 \mathrm{~mL} \mathrm{HPLC}$-grade hexanes. The brown solid was filtered over a $15 \mathrm{~mL}$ 
fine-porosity fritted funnel and discarded. The solution was evaporated in vacuo to a white powder as 25 (24 mg, $100 \%)$.

${ }^{1} \mathrm{H}\left(\mathrm{CDCl}_{3}\right): \delta 8.55(\mathrm{~s}, 2 \mathrm{H}, \mathrm{H} 9$ and $\mathrm{H} 10), 8.04(\mathrm{~m}, 3 \mathrm{H}, \mathrm{H} 4, \mathrm{H} 5$, and $\mathrm{H} 8), 7.64(\mathrm{~d}, J=7.1,1 \mathrm{H}, \mathrm{H} 2), 7.56$ (m, 2H, H6 and H7), 7.45 (dd, $J=8.0,1 \mathrm{H}, \mathrm{H} 3$ ), 5.25 (s, 1H, H13), 2.07 (s, 6H, H11 and H12). ${ }^{13} \mathrm{C}$ $\left(\mathrm{CDCl}_{3}\right): \delta 135.84,133.44,131.59,131.05,131.00$ (C5 or C8), 129.67 (C9 or C10), 128.63 (C4), 128.28, 127.92 (C5 or C8), 126.77 (C6 and C7), 126.53 (C2), 124.60 (C3), 122.81 (C9 or C10), 112.26, 43.38, 34.30 (C13), 27.49 (C11 and C12).

\section{1-(2-(anthracen-1-yl)propan-2-yl)-1H-imidazole (26)}<smiles>CC(C)(c1cccc2cc3ccccc3cc12)n1ccnc1</smiles>

8 (15 mg, $0.019 \mathrm{mmol})$ was dissolved in $\mathrm{CDCl}_{3}(1.5 \mathrm{~g})$ and placed in a photoreactor fitted with $300 \mathrm{~nm}$ lamps and irradiated for $90 \mathrm{~min}$. The reaction was extracted with $5 \mathrm{~mL}$ ether and $4 \mathrm{~mL}$ water. The water layer was backextracted $5 \mathrm{~mL}$ and the ether layer was extracted with $4 \mathrm{~mL}$ water. The ether layers were dried MgSO4, filtered over a Celite plug and evaporated in vacuo to a film. NMR data was collected on the film.

${ }^{1} \mathrm{H}\left(\mathrm{CDCl}_{3}\right): \delta 8.49(\mathrm{~s}, 1 \mathrm{H}), 8.30(\mathrm{~s}, 1 \mathrm{H}), 8.09(\mathrm{~d}, \mathrm{~J}=8.4,1 \mathrm{H}), 7.96(\mathrm{~d}, \mathrm{~J}=8.5,1 \mathrm{H}), 7.74-7.66(\mathrm{~m}$, $4 H), 7.51-7.43(m, 3 H), 7.07(m, 1 H), 2.26(s, 6 H)$.

Dimethyl 2-(1-(propan-2-ylidene)-1,2-dihydroanthracen-2-yl)malonate (27) 


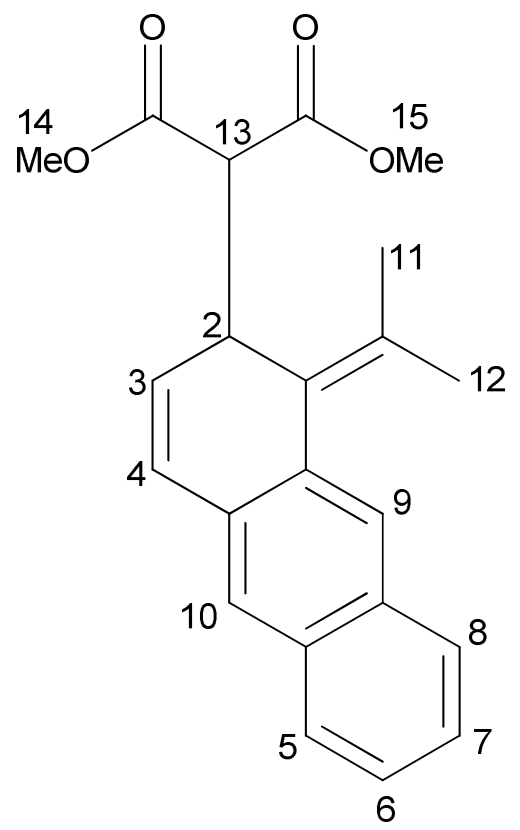

10 (12 mg, $0.014 \mathrm{mmol}$ ) was dissolved in acetone $(551 \mathrm{mg})$ and CAN (9 mg, $0.016 \mathrm{mmol})$ in water (508 mg). The CAN solution was added to the rapidly stirring acetone solution and allowed to react for 1 hour. DCM $(5 \mathrm{~mL})$ and water $(2 \mathrm{~mL})$ were added to the reaction and extracted. The water layer was extracted $2 x$ with $2 \mathrm{~mL}$ portions of water. The combined organic layers were dried with $\mathrm{MgSO}_{4}$, which was filtered over a Celite plug. The solvent was removed in vacuo and NMR data was collected on the crude product.

${ }^{1} \mathrm{H}\left(\mathrm{CDCl}_{3}\right): \delta 7.77(\mathrm{~m}, 2 \mathrm{H}, \mathrm{H} 5$ and $\mathrm{H} 8), 7.61(\mathrm{~s}, 1 \mathrm{H}, \mathrm{H} 9$ or $\mathrm{H} 10), 7.49$ (s, 1H, H9 or H10), 7.42 (dd, J $=3.3,6.3,2 \mathrm{H}, \mathrm{H} 6$ and $\mathrm{H} 7), 6.68(\mathrm{~d}, J=9.4,1 \mathrm{H}, \mathrm{H} 4), 6.32(\mathrm{dd}, J=6.3,9.4,1 \mathrm{H}, \mathrm{H} 3), 4.23(\mathrm{dd}, J=$ 6.3, 10.7, 1H, H2), 3.68 (s, 3H, OMe), 3.61 (s, 3H, OMe), 3.24 (d, J = 10.8, 1H, H13), 1.97 (s, 6H, $\mathrm{H} 11$ and $\mathrm{H} 12)$.

2-cyano-3-(9,10-dioxo-9,10-dihydroanthracen-1-yl)-3-methylbutanamide (28) 
<smiles>CC(C)(C(N)=O)C(C#N)c1cccc2c1C(=O)c1ccccc1C2=O</smiles>

25 (24 mg, $0.085 \mathrm{mmol}$ ) was dissolved in THF (576 mg) and CAN (182 mg, $0.332 \mathrm{mmol}$ ) in water (182 mg). The CAN solution was added to the rapidly stirring acetone solution and allowed to react for $25 \mathrm{~min}$. DCM $(2 \mathrm{~mL})$ and water $(2 \mathrm{~mL})$ were added and the solution extracted. The water layer was extracted $2 \mathrm{x}$ with $2 \mathrm{~mL}$ portions DCM. The combined organic layers were dried with $\mathrm{MgSO}_{4}$, which was filtered over a Celite plug. The solution was evaporated in vacuo and NMR data collected on the crude product.

${ }^{1} \mathrm{H}\left(\mathrm{CDCl}_{3}\right): \delta 8.04(\mathrm{~d}, \mathrm{~J}=7.5), 7.93(\mathrm{~d}, \mathrm{~J}=7.7), 7.83(\mathrm{~m}, 2 \mathrm{H}), 7.70(\mathrm{~m}, 2 \mathrm{H}), 7.44(\mathrm{~m}, 2 \mathrm{H}), 6.10(\mathrm{~m}$, 1H), $5.93(\mathrm{~s}), 1.97(\mathrm{~s}, 3 \mathrm{H}), 1.95(\mathrm{~s}, 3 \mathrm{H})$. IR: $\mathrm{v}_{\mathrm{CO}}=1667 \mathrm{~cm}^{-1}, \mathrm{v}_{\mathrm{CN}}=2254 \mathrm{~cm}^{-1}$.

\section{References}

(1) Winemiller, M. D.; Harman, W. D. Journal of the American Chemical Society 1998, 120, 7835.

(2) Ding, F.; Valahovic, M. T.; Keane, J. M.; Anstey, M. R.; Sabat, M.; Trindle, C. O.; Harman, W. D. The Journal of Organic Chemistry 2004, 69, 2257.

(3) Pienkos, J. A.; Zottig, V. E.; lovan, D. A.; Li, M.; Harrison, D. P.; Sabat, M.; Salomon, R. J.; Strausberg, L.; Teran, V. A.; Myers, W. H.; Harman, W. D. Organometallics 2013, $32,691$.

(4) Winemiller, M. D.; Kopach, M. E.; Harman, W. D. Journal of the American Chemical Society 1997, 119, 2096.

(5) Keane, J. M.; Harman, W. D. Organometallics 2005, 24, 1786.

(6) Todd, M. A.; Grachan, M. L.; Sabat, M.; Myers, W. H.; Harman, W. D. Organometallics 2006, 25, 3948.

(7) Kolis, S. P.; Chordia, M. D.; Liu, R.; Kopach, M. E.; Harman, W. D. Journal of the American Chemical Society 1998, 120, 2218.

(8) Hodges, L. M.; Spera, M. L.; Moody, M. W.; Harman, W. D. Journal of the American Chemical Society 1996, 118, 7117.

(9) Uemura, M. In Transition Metal Arene pi-Complexes in Organic Synthesis and Catalysts; Springer Berlin Heidelberg: 2004; Vol. 7, p 129. 
(10) Smith, P. L.; Keane, J. M.; Shankman, S. E.; Chordia, M. D.; Harman, W. D. Journal of the American Chemical Society 2004, 126, 15543.

(11) Butts, C. A.; Xi, J.; Brannigan, G.; Saad, A. A.; Venkatachalan, S. P.; Pearce, R. A.; Klein, M. L.; Eckenhoff, R. G.; Dmochowski, I. J. Proceedings of the National Academy of Sciences 2009, 106, 6501.

(12) Bair, K. W.; Office, U. S. P., Ed. 1988; Vol. 4,719,049.

(13) Bulic, B.; Pickhardt, M.; Mandelkow, E. Journal of Medicinal Chemistry 2013.

(14) Cai; Sun, M.; Xing, J.; Corke, H. Journal of Agricultural and Food Chemistry 2004, $52,7884$.

(15) Ho, T.-L. Synthesis 1973, 206.

(16) Welch, K. D.; Harrison, D. P.; Lis, E. C.; Liu, W.; Salomon, R. J.; Harman, W. D.; Myers, W. H. Organometallics 2007, 26, 2791.

(17) Strausberg, L.; Li, M.; Harrison, D. P.; Myers, W. H.; Sabat, M.; Harman, W. D. Organometallics 2013, 32, 915. 
Chapter 6

Concluding Remarks 
As of July 2013, naphthalene could be acquired from Acros Organics for as little as $\$ 0.03$ per gram; anthracene could be acquired at $\$ 0.20$ per gram. These molecules provide many potential sites of functionalization, making them a cheap scaffold for synthetic elaboration, provided reaction conditions can be designed to give selective products. Complexation of naphthalene and anthracene with the $\pi$ basic metal fragment $\left\{\mathrm{TpW}(\mathrm{NO})\left(\mathrm{PMe}_{3}\right)\right\}$ provides a strategy for the functionalization of these molecules that is complementary to what exists in the literature.

Naphthalene, through its $\left\{\mathrm{TpW}(\mathrm{NO})\left(\mathrm{PMe}_{3}\right)\right\}$ complex, was made amenable to addition of a proton at $\mathrm{C} 1$ to form an allylic complex where the allyl showed a large degree of asymmetry. Spectroscopic information showed $\mathrm{C} 4$ to be the most cationic cite on the naphthalenium ring, but nucleophiles added at C2 instead, generating a product in which the remaining alkene was conjugated to the unbound ring. We speculate that the greater electron richness of $\left\{\mathrm{TpW}(\mathrm{NO})\left(\mathrm{PMe}_{3}\right)\right\}$ compared to its rhenium and osmium analogs is better able to stabilize the cationic complex, making isomerization from a 1,4-addition product to a 1,2addition product more favorable. The small scope of nucleophiles that would successfully add to naphthalene under these conditions is an unfortunate limitation of this reaction type.

The tungsten naphthalene complex was also found to undergo a Diels-Alder cycloaddition with $\mathrm{N}$-methylmaleimide, a reaction which is nearly impossible for free naphthalene to perform. A study of reactions with methylated naphthalene complexes yielded insight into the mechanism by which this reaction proceeds. One point of interest is that the analogous anthracene complex does not undergo this reaction, a reversal of chemistry seen in free anthracene.

Metal complexes of anthracene typically exhibit reactivity in the terminal ring, wear the metal is bound, and $\left\{\mathrm{TpW}(\mathrm{NO})\left(\mathrm{PMe}_{3}\right)\right\}$ is no different. In addition to protonation, the 
anthracene complex was found to react with acetal reagents to introduce a carbon electrophile at C1. In a departure from the reactivity of acetal reagents with rhenium and osmium complexes, the electrophile eliminated two equivalents of methanol to form an ylidene moiety on the anthracene. Nucleophiles added to both $\mathrm{C} 2$ and the benzyl carbon of this cationic intermediate, generating 1,2-dihydroanthracenes and 1-substituted anthracenes, respectively. The anthracene complex also underwent addition of electrophilic bromine from $\mathrm{N}$ bromosuccinimide at $\mathrm{C} 1$ to form a complex to which a nucleophile could add at C4. The bromide was then lost to allow the addition of a second equivalent of nucleophile. Both of these reactions provided relatively mild conditions to dearomatized products with predictable stereochemistry.

Oxidative decomplexation conditions were developed for some of the organics described in this work. Isolation of the new organic fragment is the ultimate goal of dearomatization chemistry, as this organic will likely not be easily synthesized by traditional organic methods, and may exhibit biological activity and have biomedical uses.

One of the major limitations of $\left\{\mathrm{TpW}(\mathrm{NO})\left(\mathrm{PMe}_{3}\right)\right\}$ chemistry is that while reactions are stereoselective for particular products, they do not yield enantioselective products. Any future biomedical application of these products will likely require an enantiopure sample of the organic. A strategy that was successfully used on the rhenium system to isolate enantioenriched complexes was the addition of $\alpha$ pinene to $\operatorname{TpRe}(\mathrm{CO})(\mathrm{Melm})\left(\eta^{2}\right.$-benzene). ${ }^{1} \quad(\mathrm{R})-\alpha$ pinene can form a strong bond to the (S) hand of the rhenium complex, but forms a weak bond to the $(R)$ hand, being easily replaced with another $\pi$ ligand. Once this happens, the two complexes can be easily separated and the (R) hand can be used in functionalization reactions.

Another strategy to separate the enantiomers of the metal would be to replace the ancillary ligand of $\left\{\mathrm{TpW}(\mathrm{NO})\left(\mathrm{PMe}_{3}\right)\right\}$ with a chiral small molecule such as (S)-nicotine. This 
would generate diastereomers with the $(\mathrm{R})$ and $(\mathrm{S})$ hands of the metal complex which should be seperable by chromatography. I feel sorry for the person who gets stuck with that project.

(1) Meiere, S. H.; Valahovic, M. T.; Harman, W. D. Journal of the American Chemical Society 2002, 124, 15099. 
Appendix 
$1,{ }^{1} \mathrm{H}, \mathrm{CD}_{3} \mathrm{CN}$
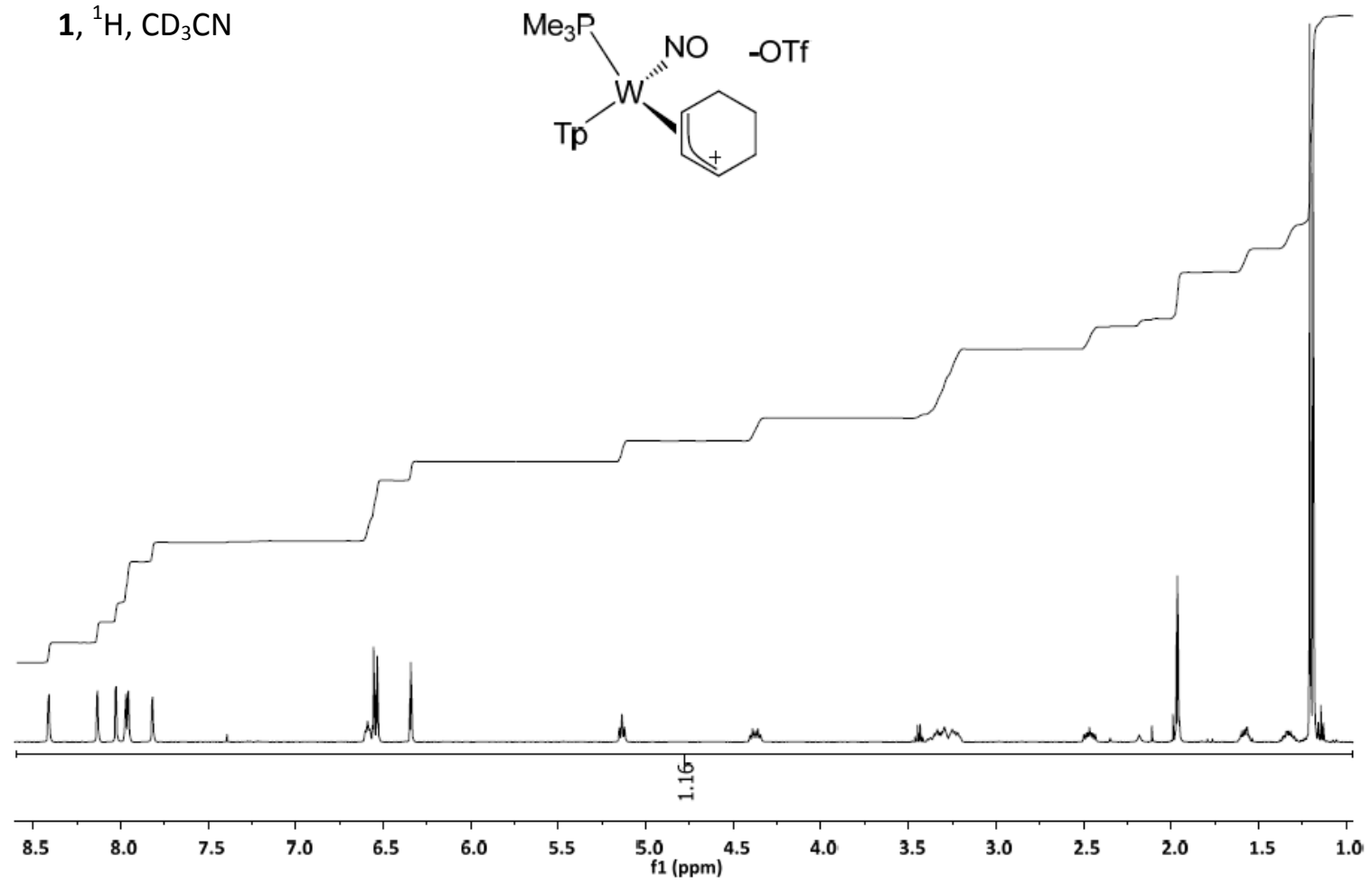

$1,{ }^{13} \mathrm{C}, \mathrm{CD}_{3} \mathrm{CN}$
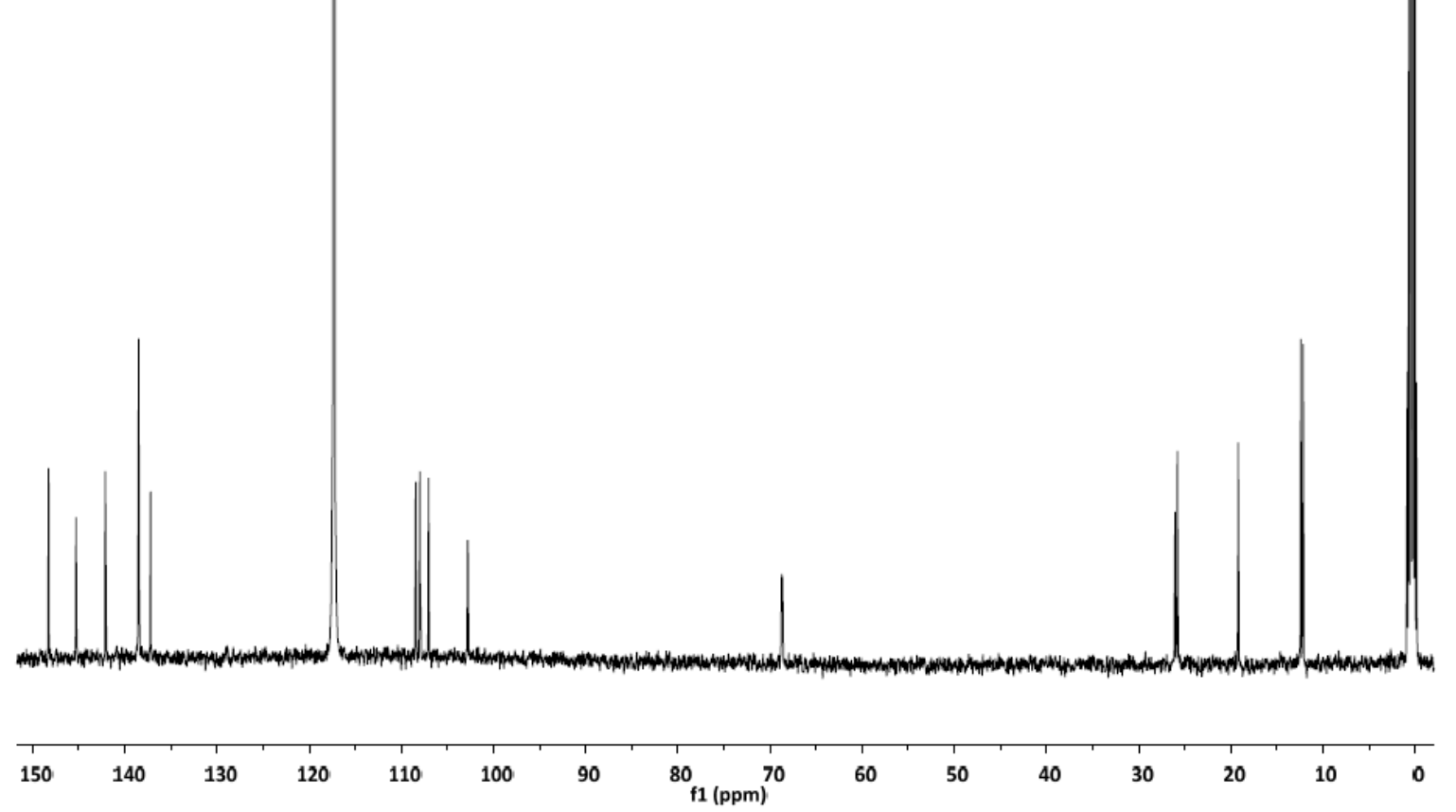

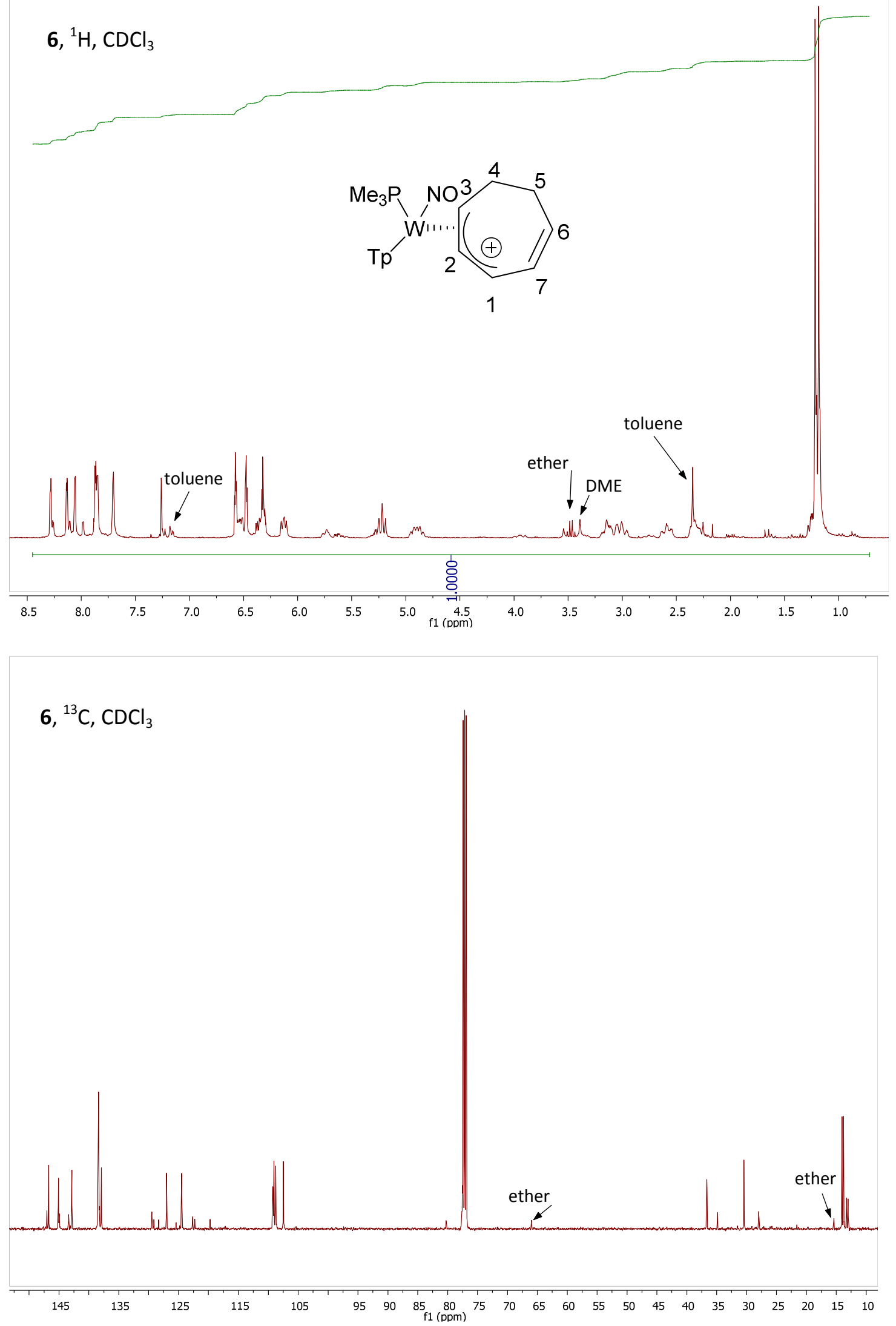


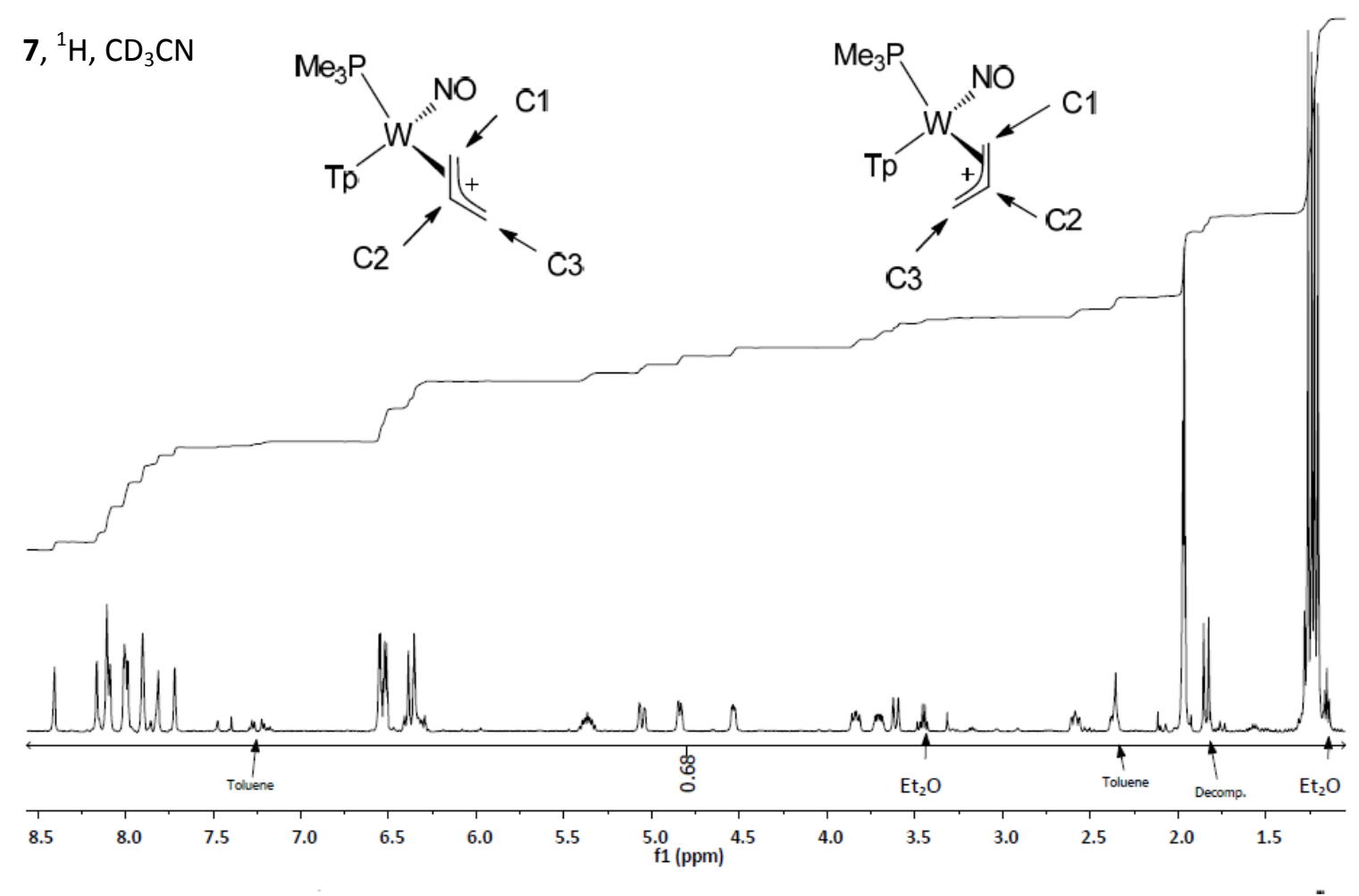

7, ${ }^{13} \mathrm{C}, \mathrm{CD}_{3} \mathrm{CN}$

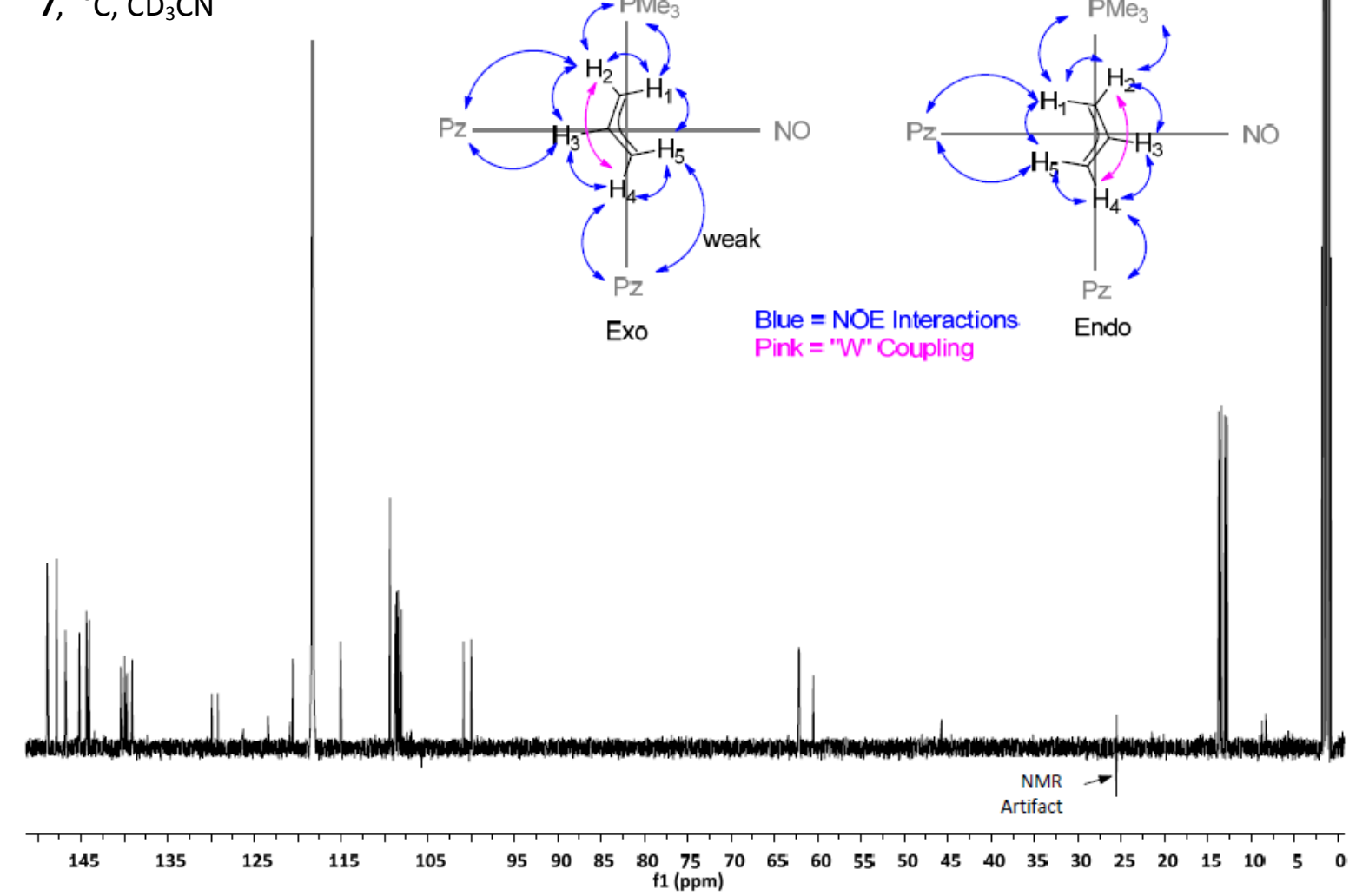


8, ${ }^{1} \mathrm{H}, \mathrm{CD}_{3} \mathrm{CN}$
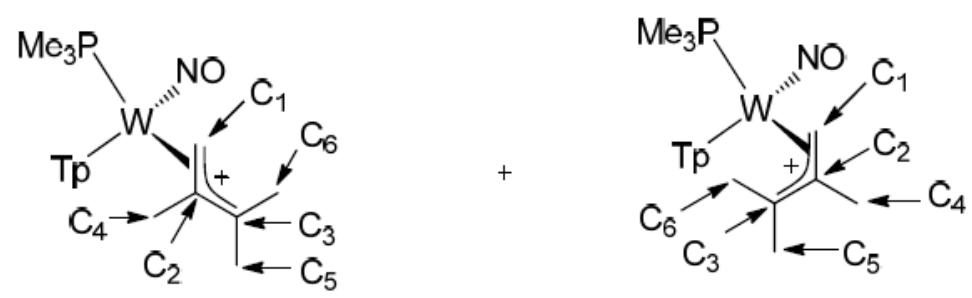

Exo

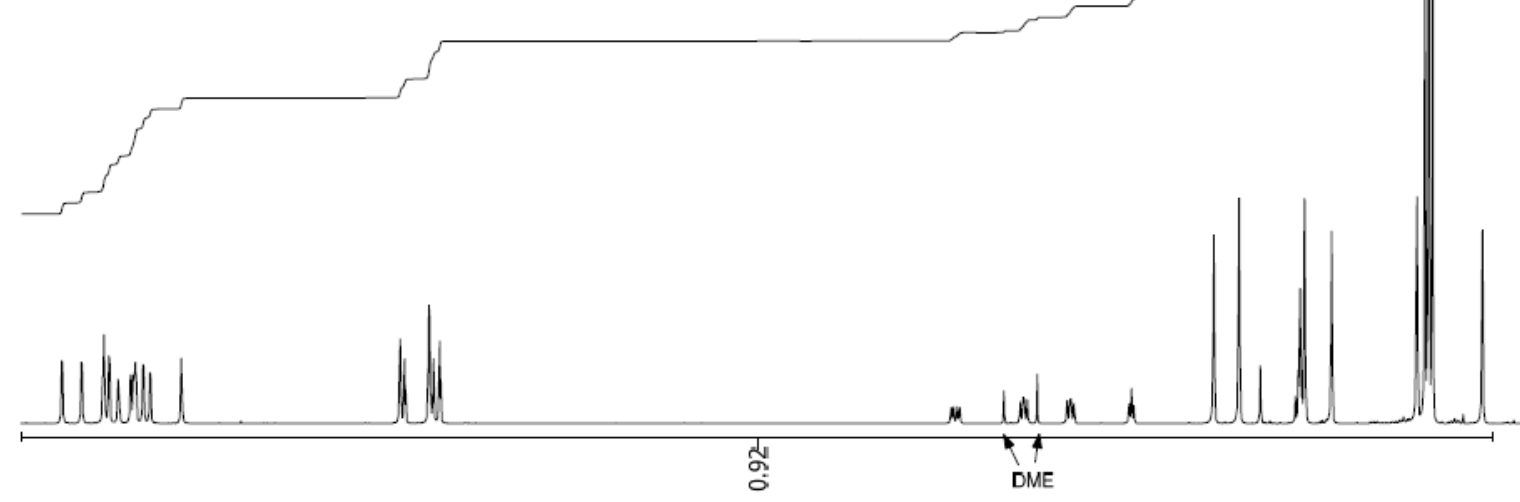

nelith

Endo

8, ${ }^{1} \mathrm{H}, \mathrm{CD}_{3} \mathrm{CN}$

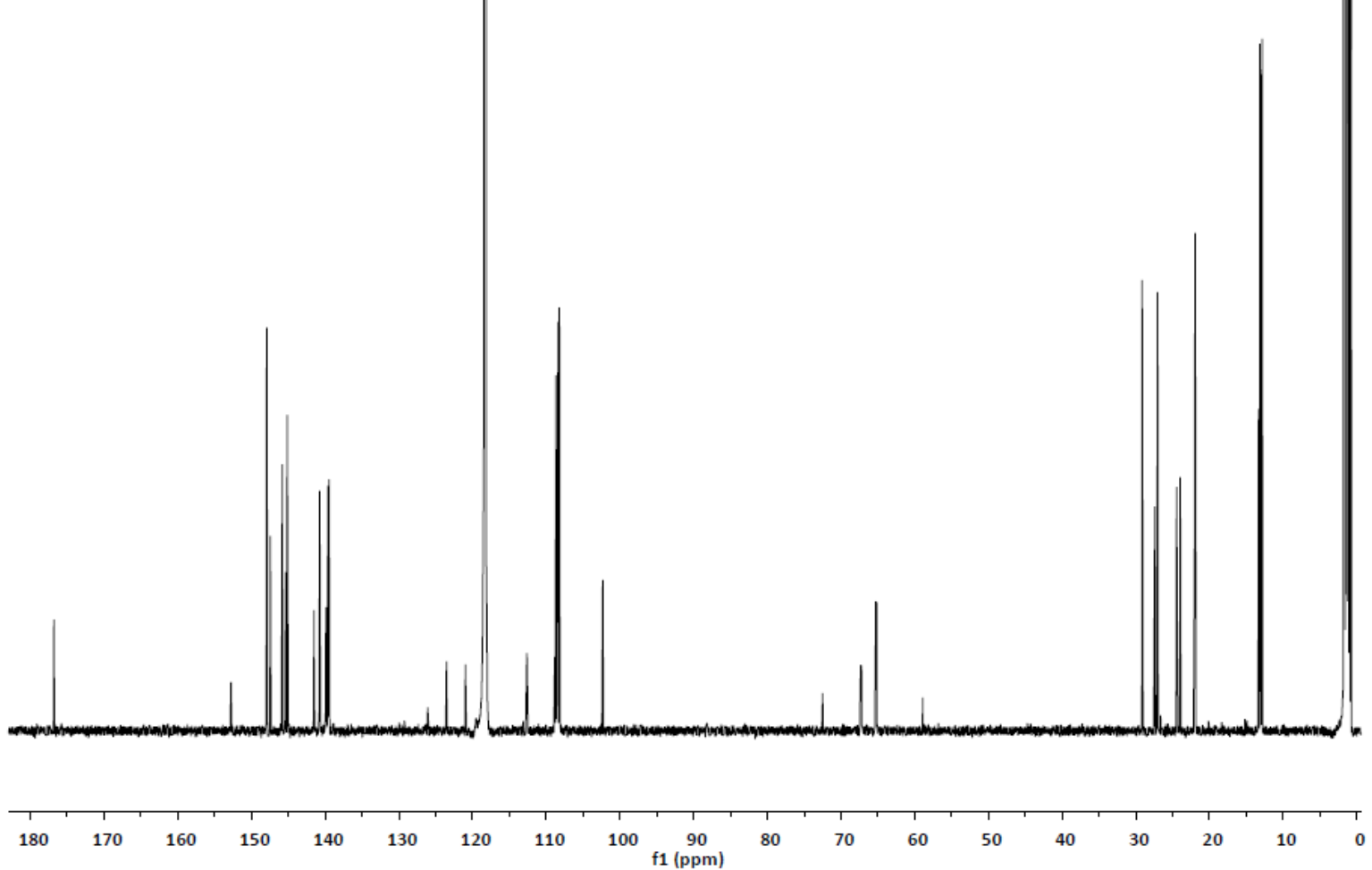




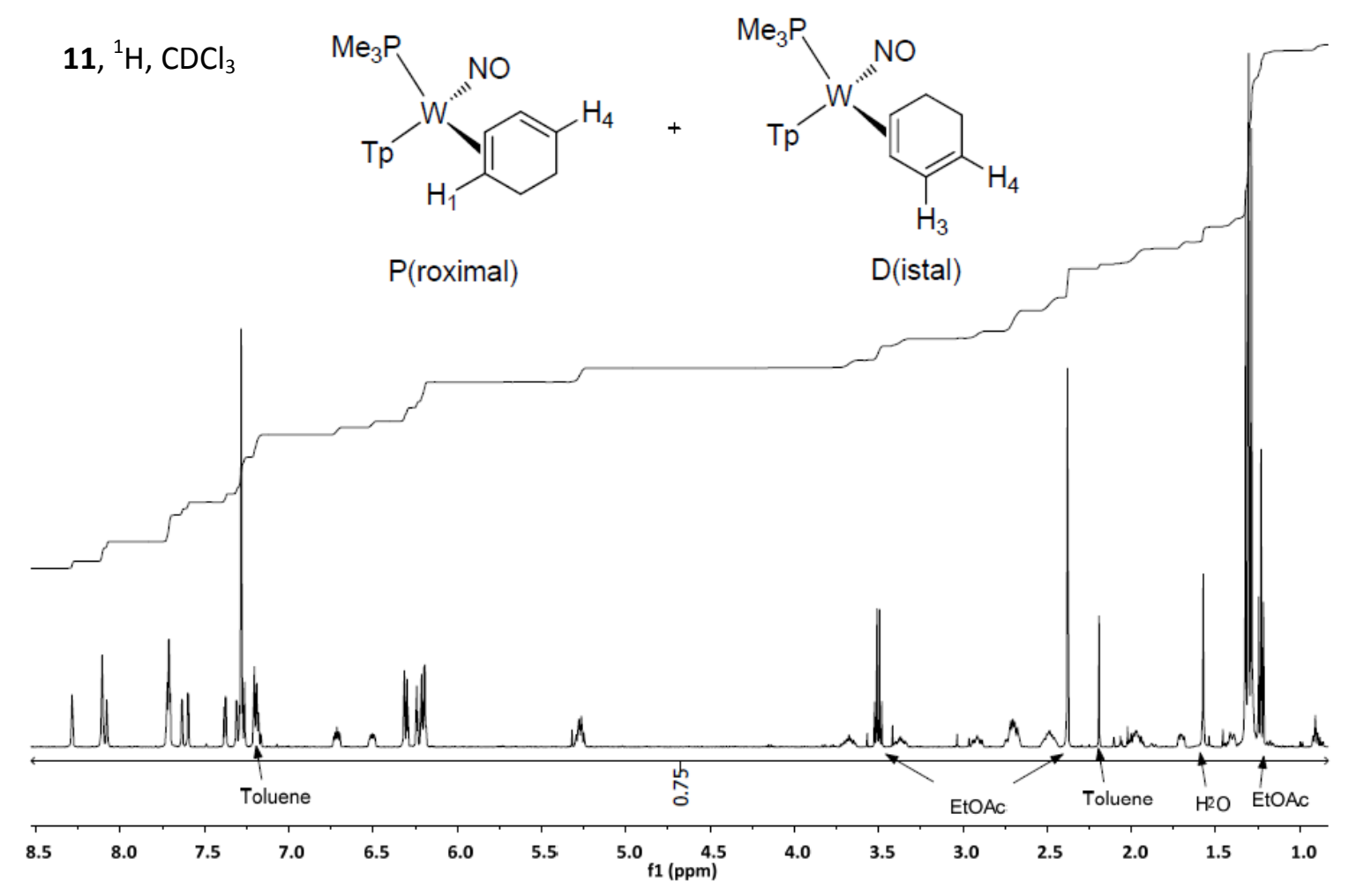

11, ${ }^{13} \mathrm{C}, \mathrm{CDCl}_{3}$
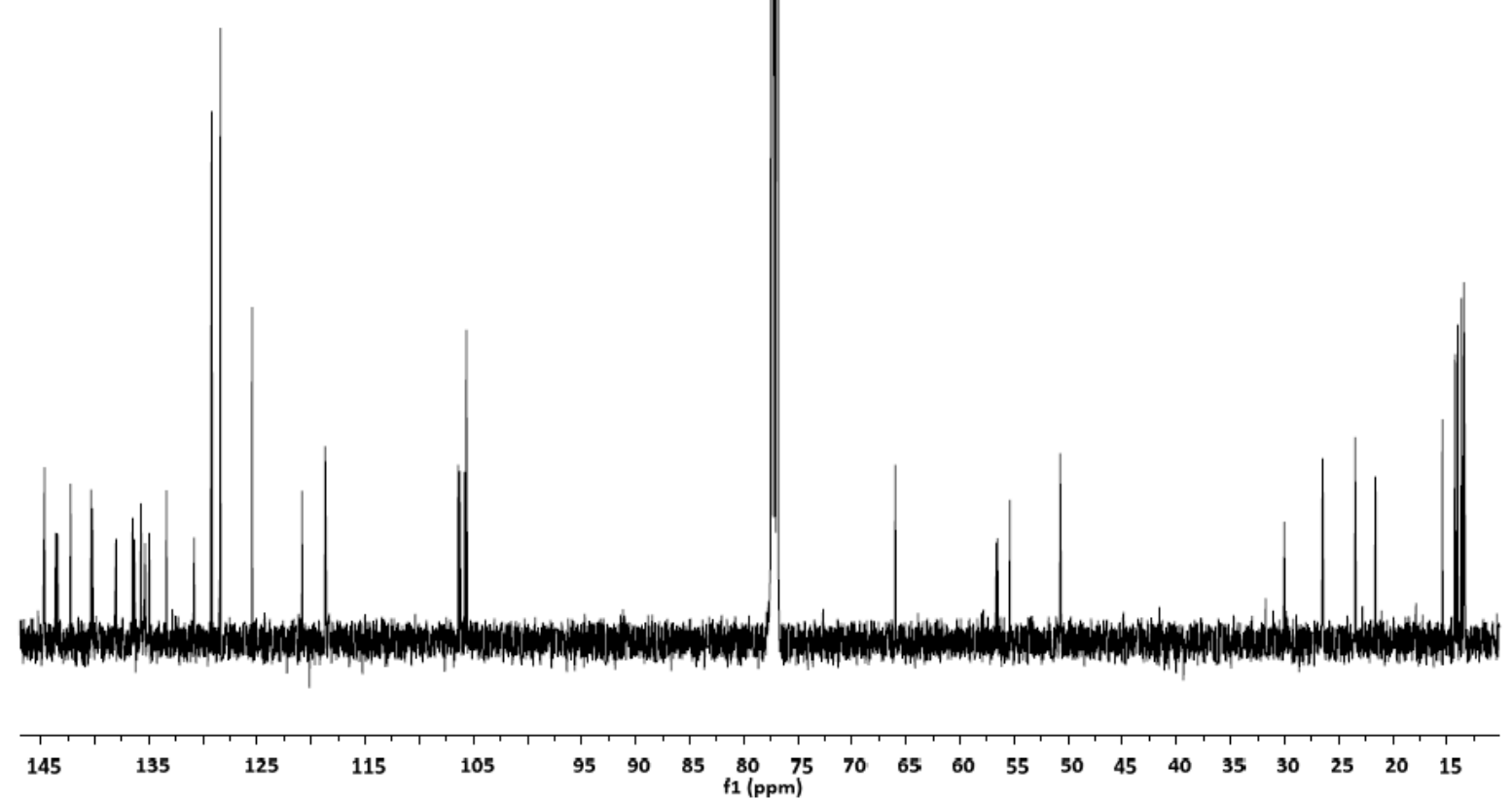


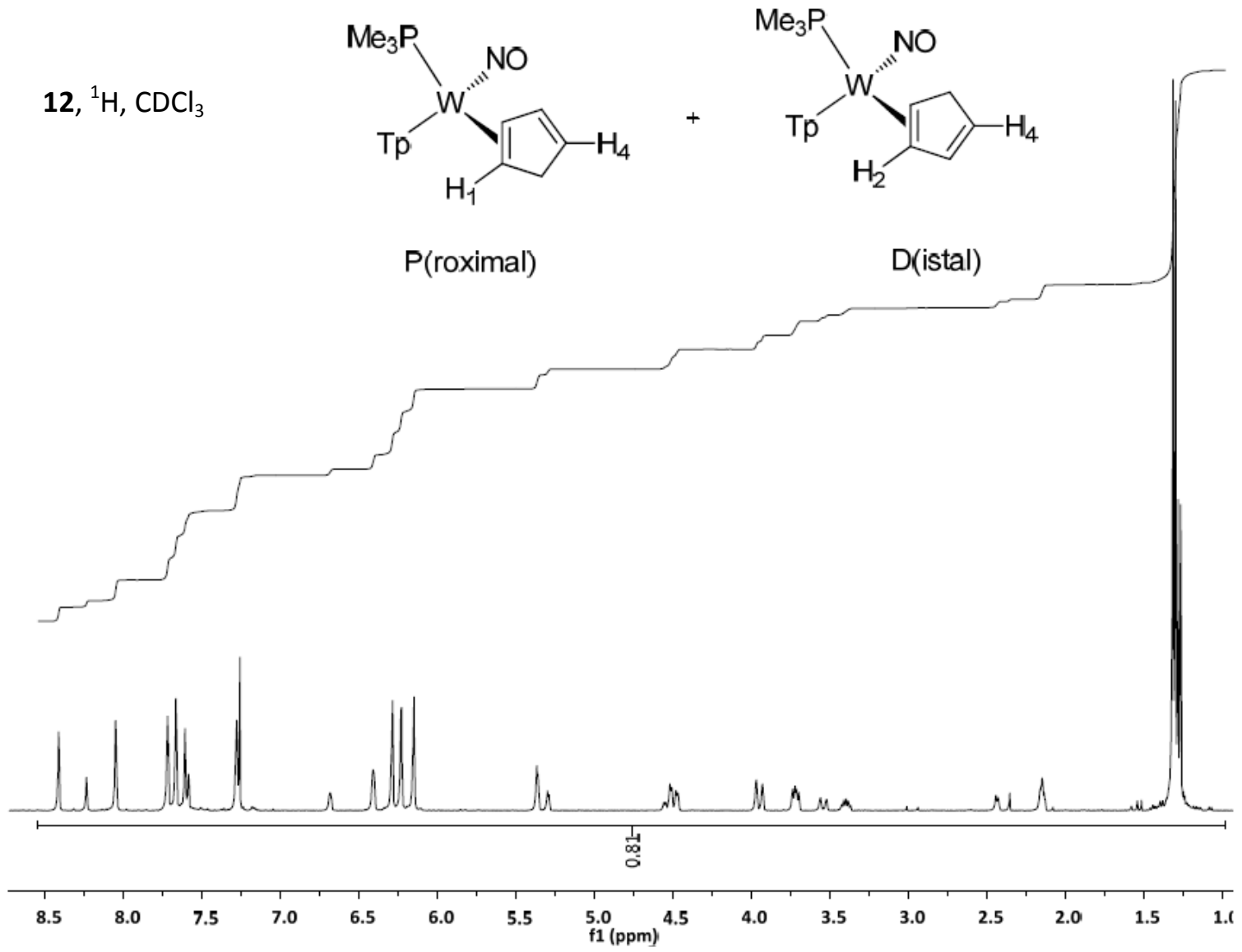

12, ${ }^{13} \mathrm{C}, \mathrm{CDCl}_{3}$
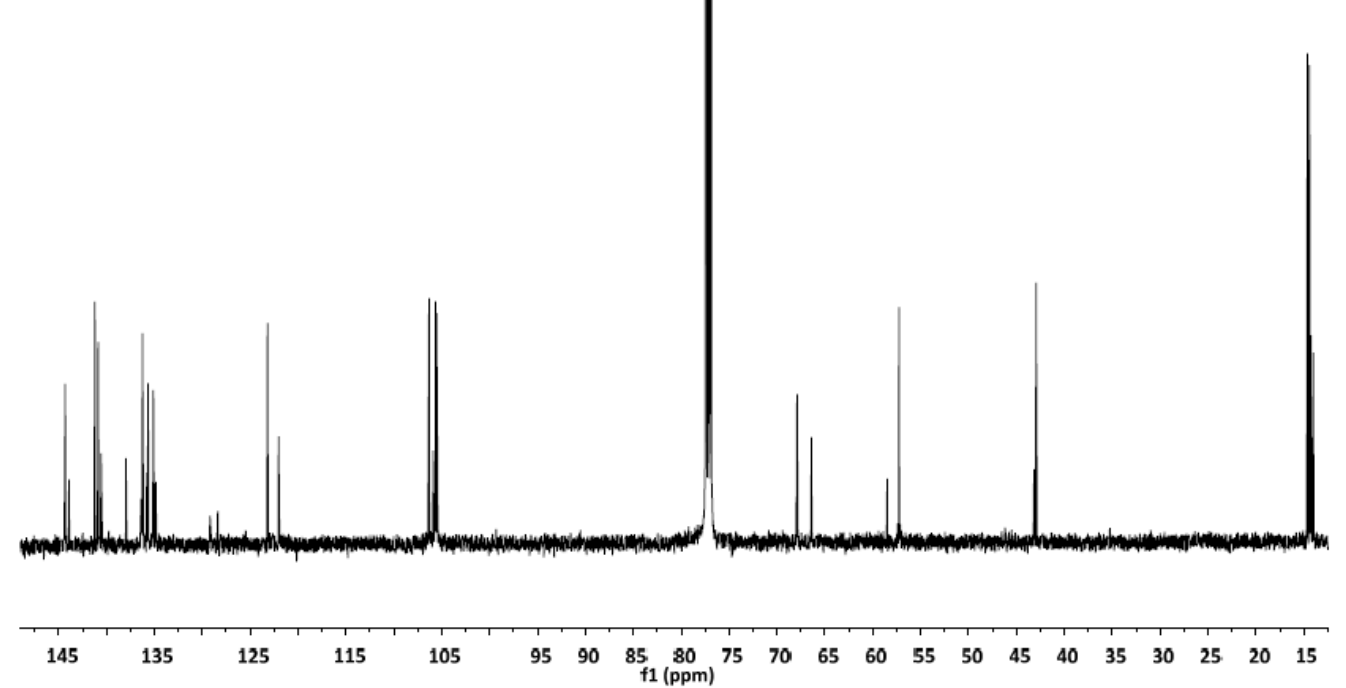


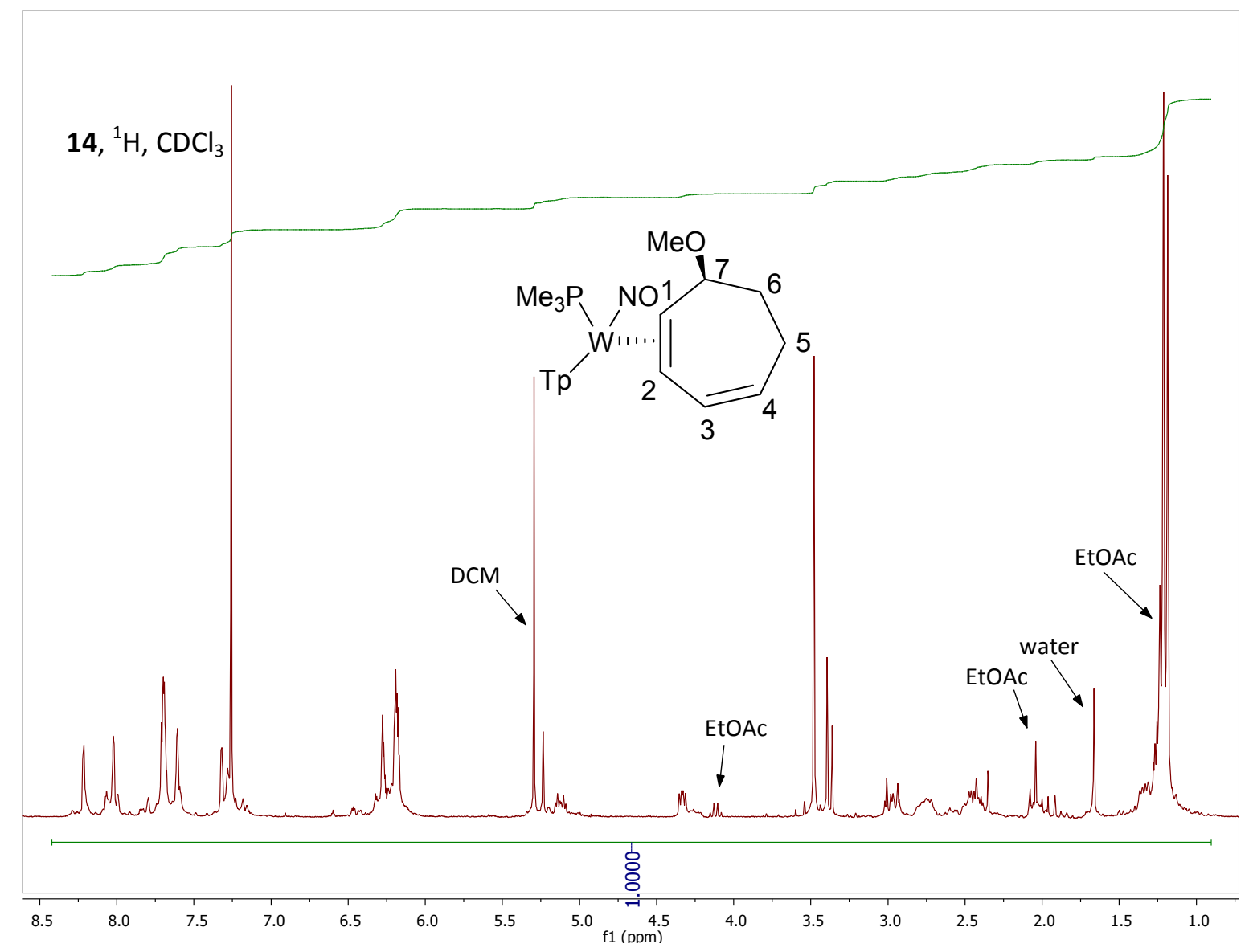




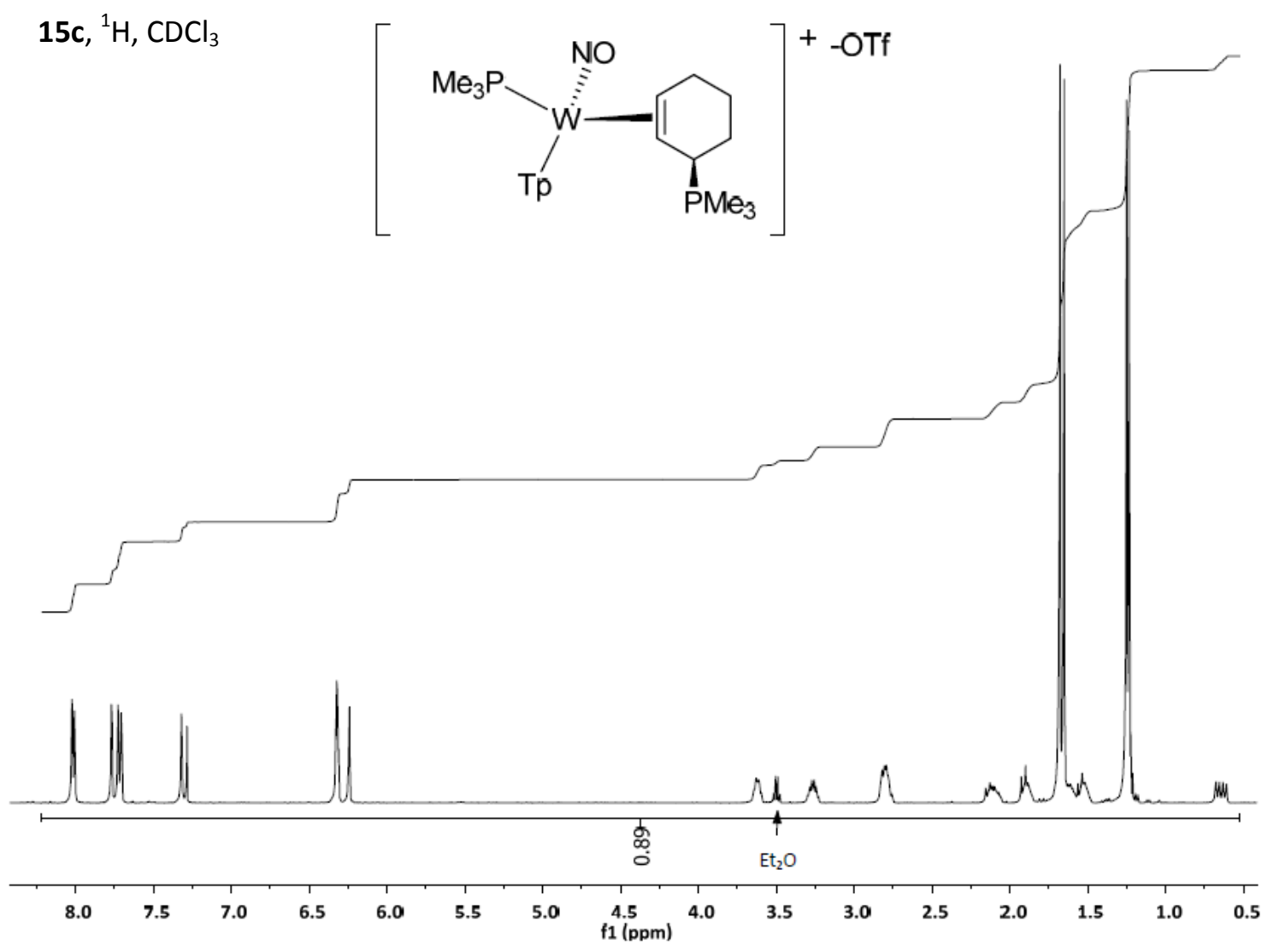

15c, ${ }^{13} \mathrm{C}, \mathrm{CDCl}_{3}$

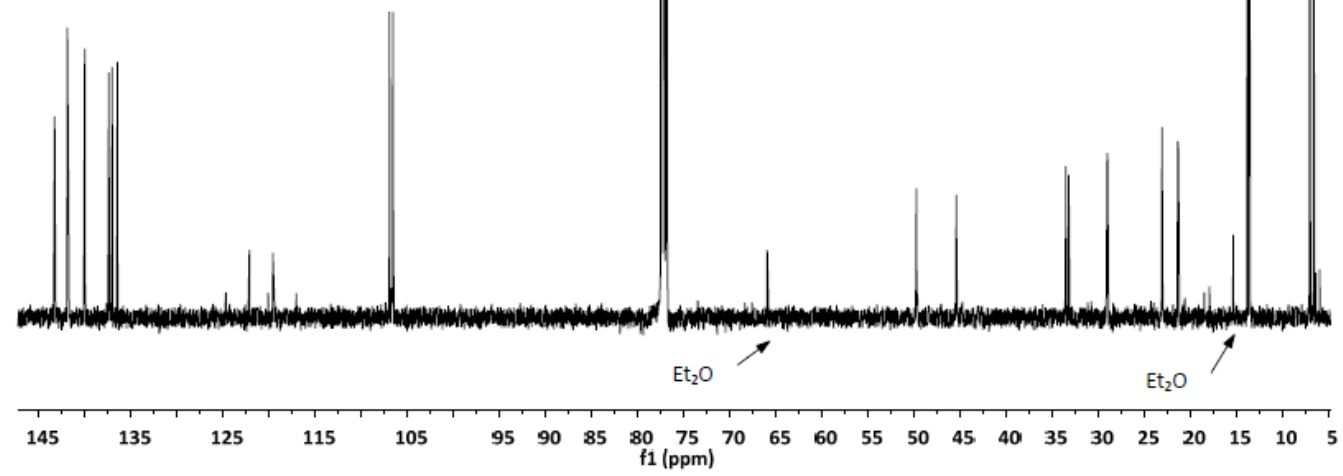



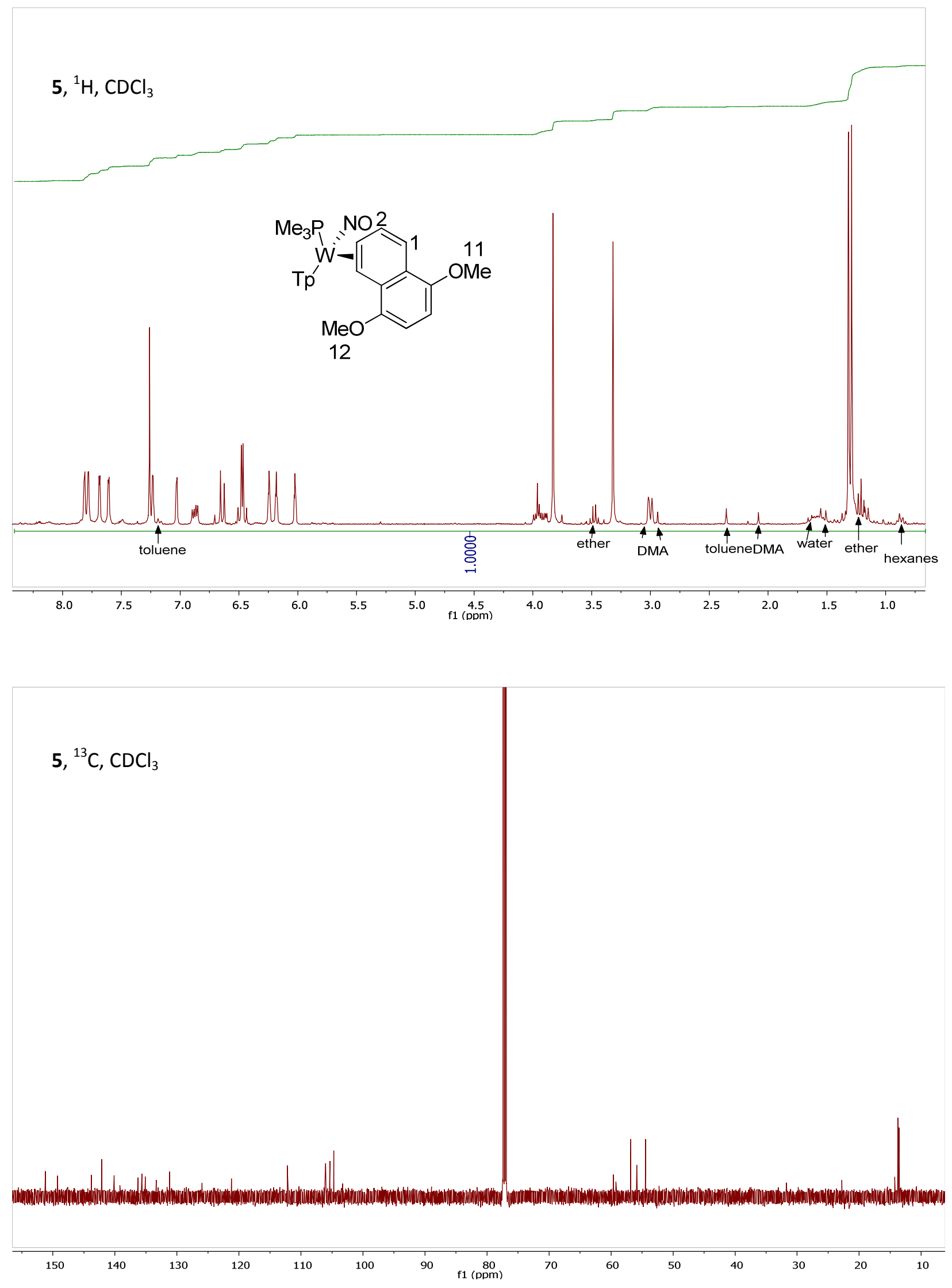


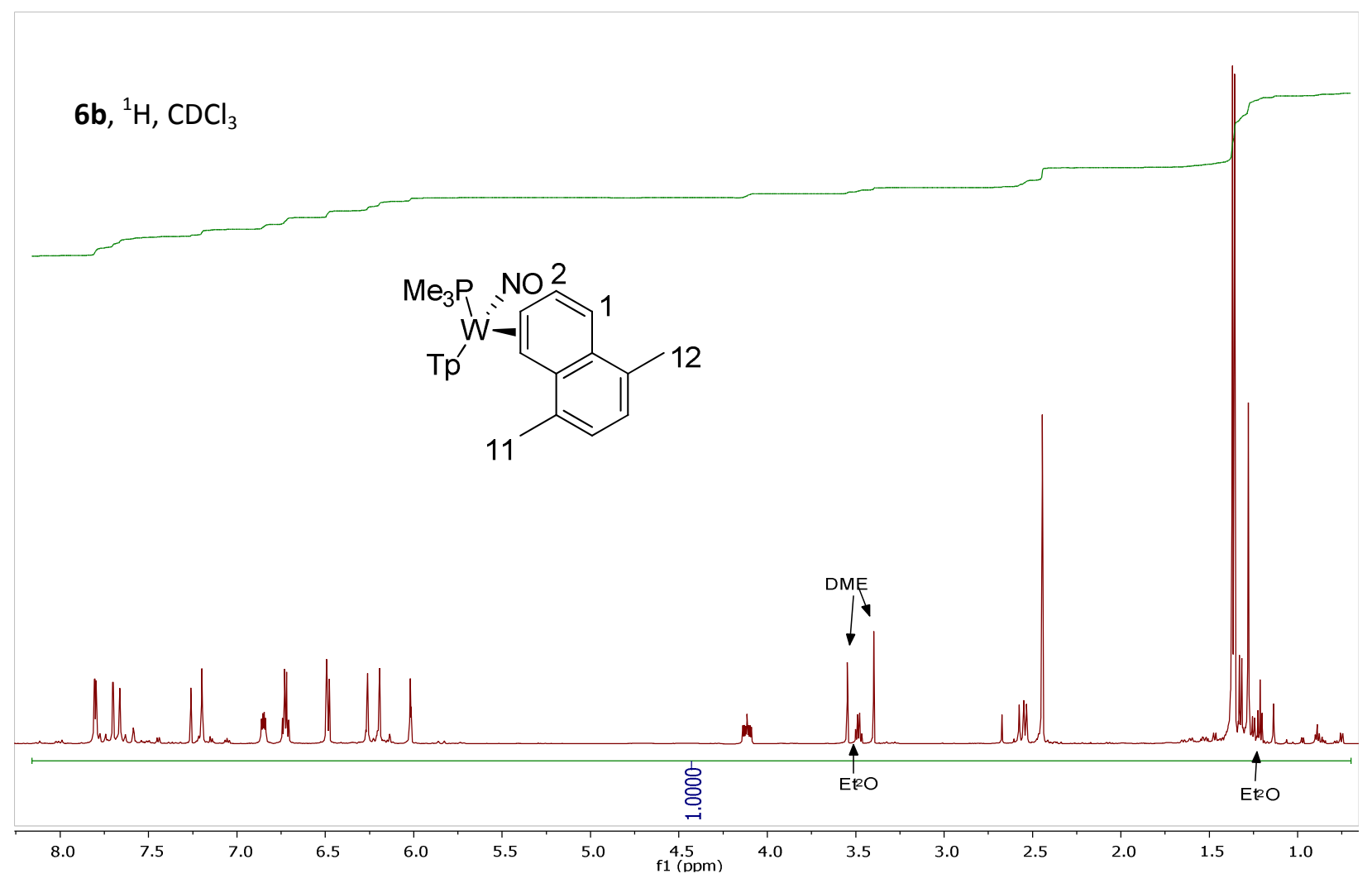

6b, ${ }^{13} \mathrm{C}, \mathrm{CDCl}_{3}$

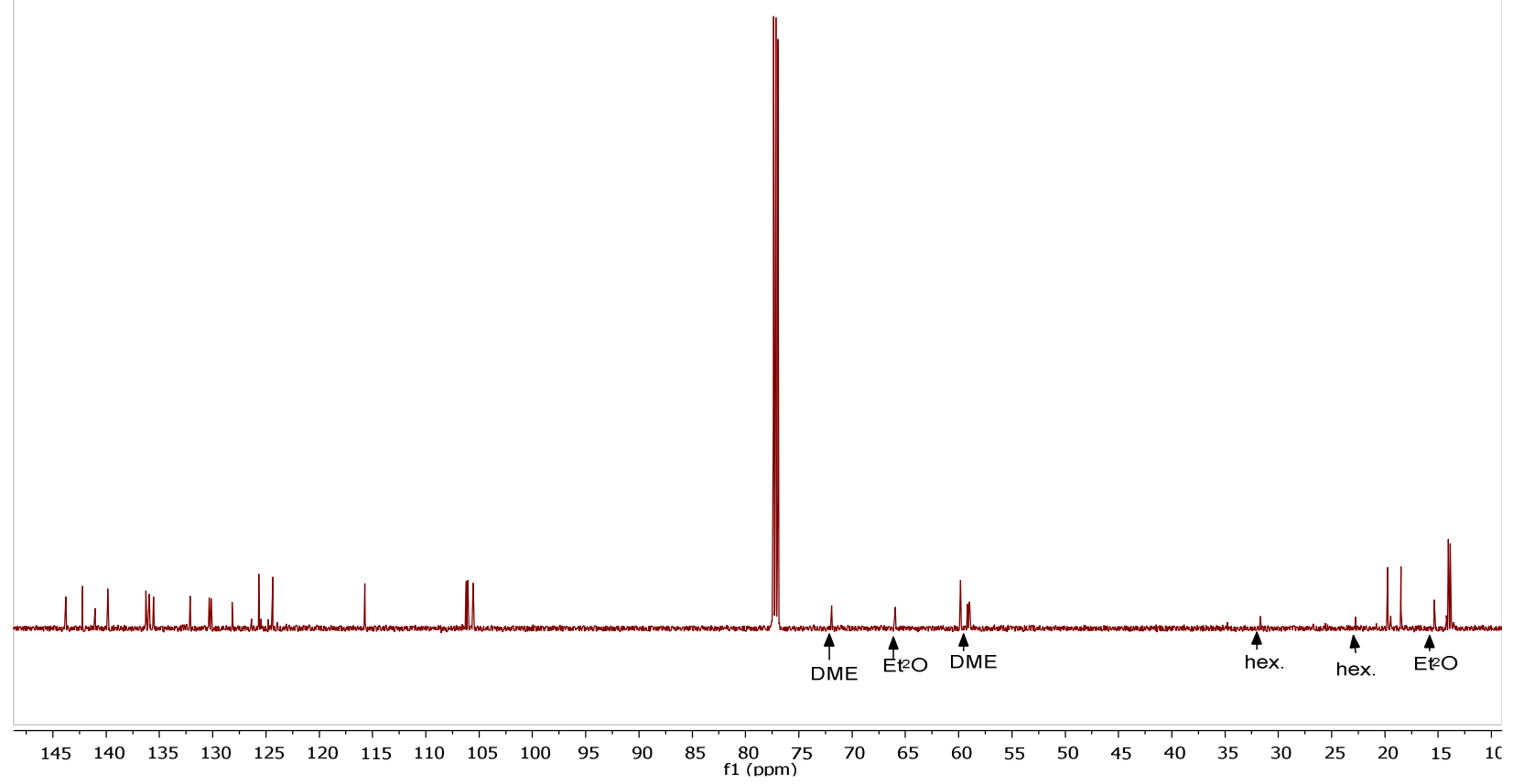



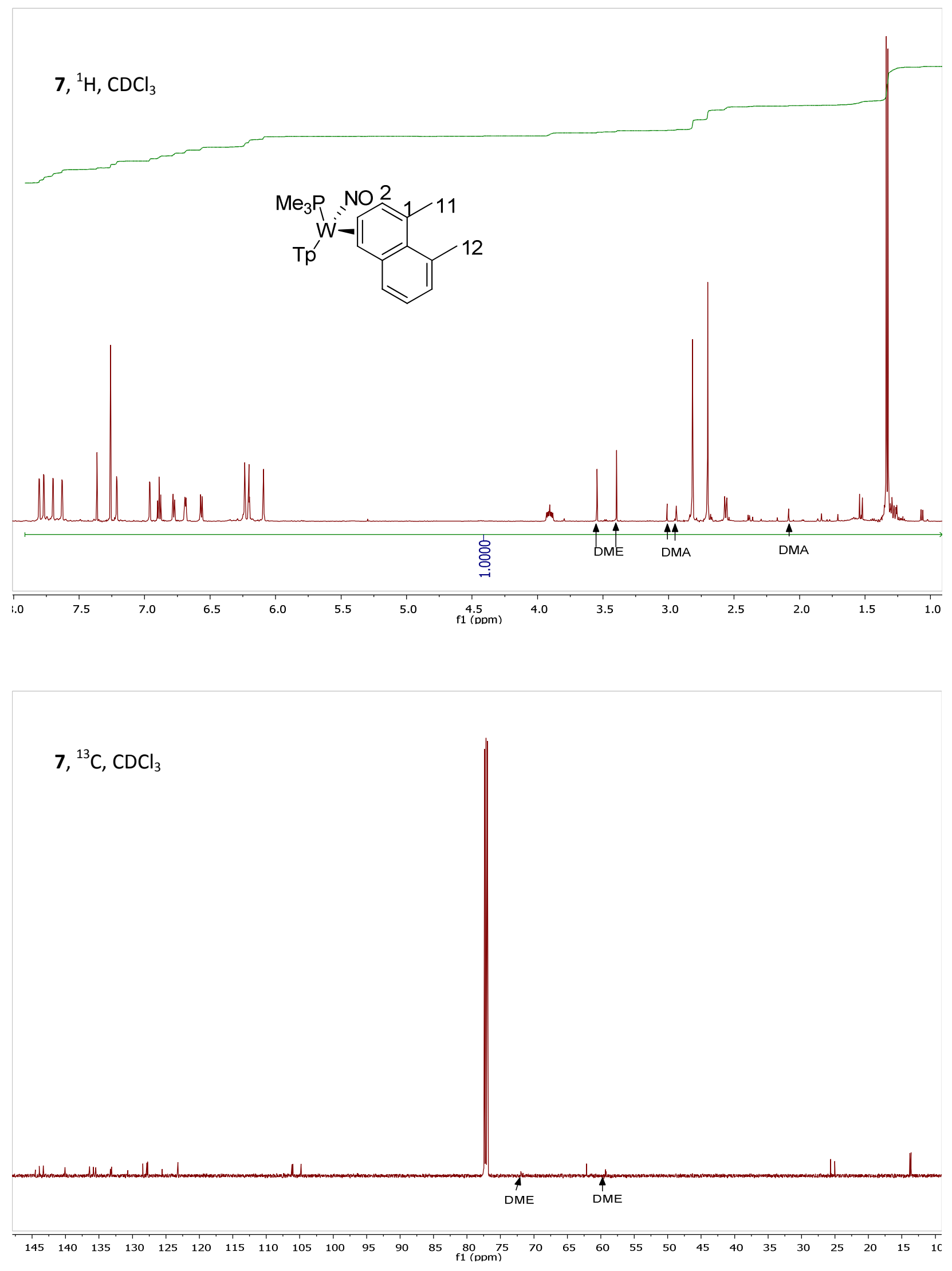


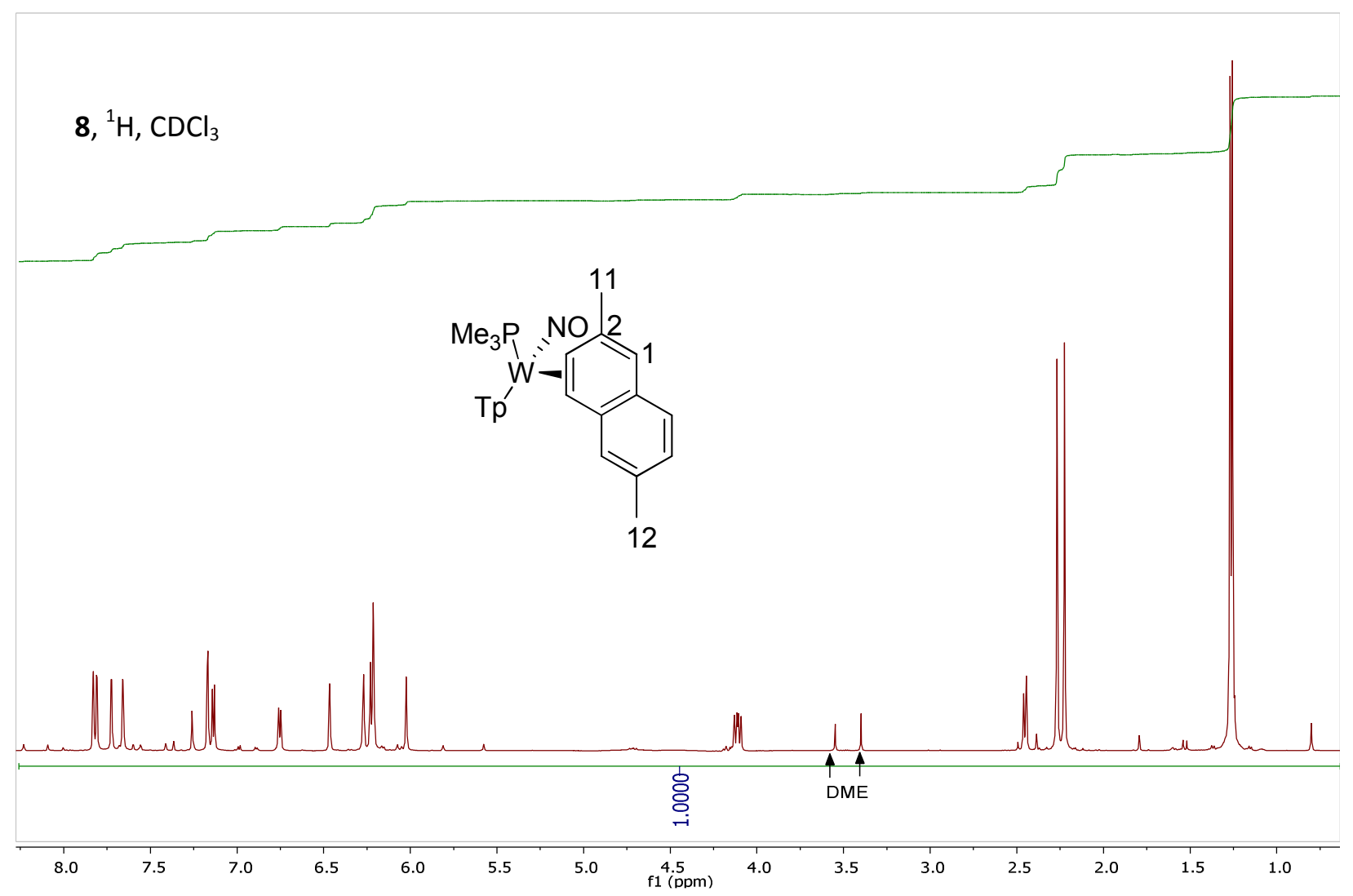

$8,{ }^{13} \mathrm{C}, \mathrm{CDCl}_{3}$

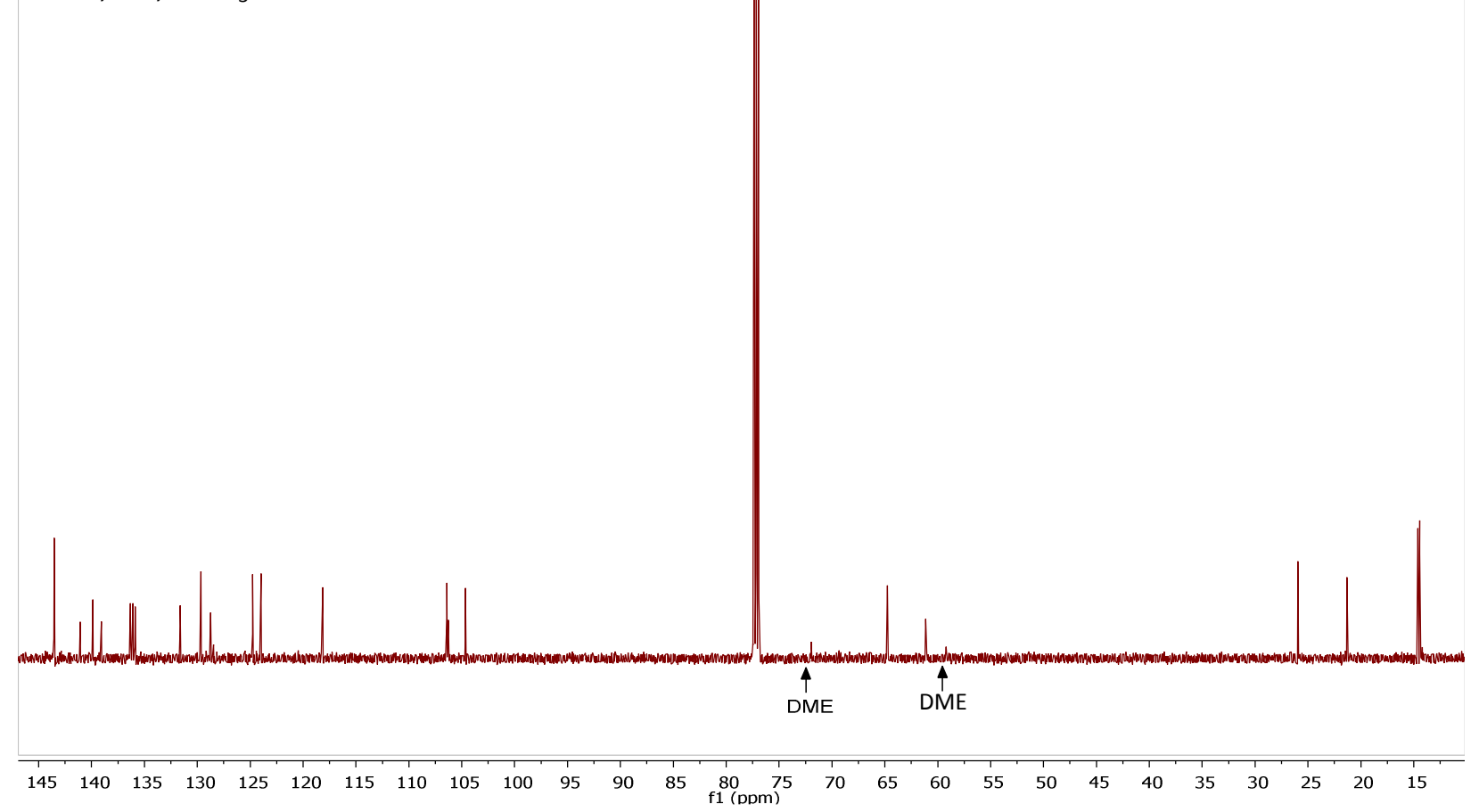



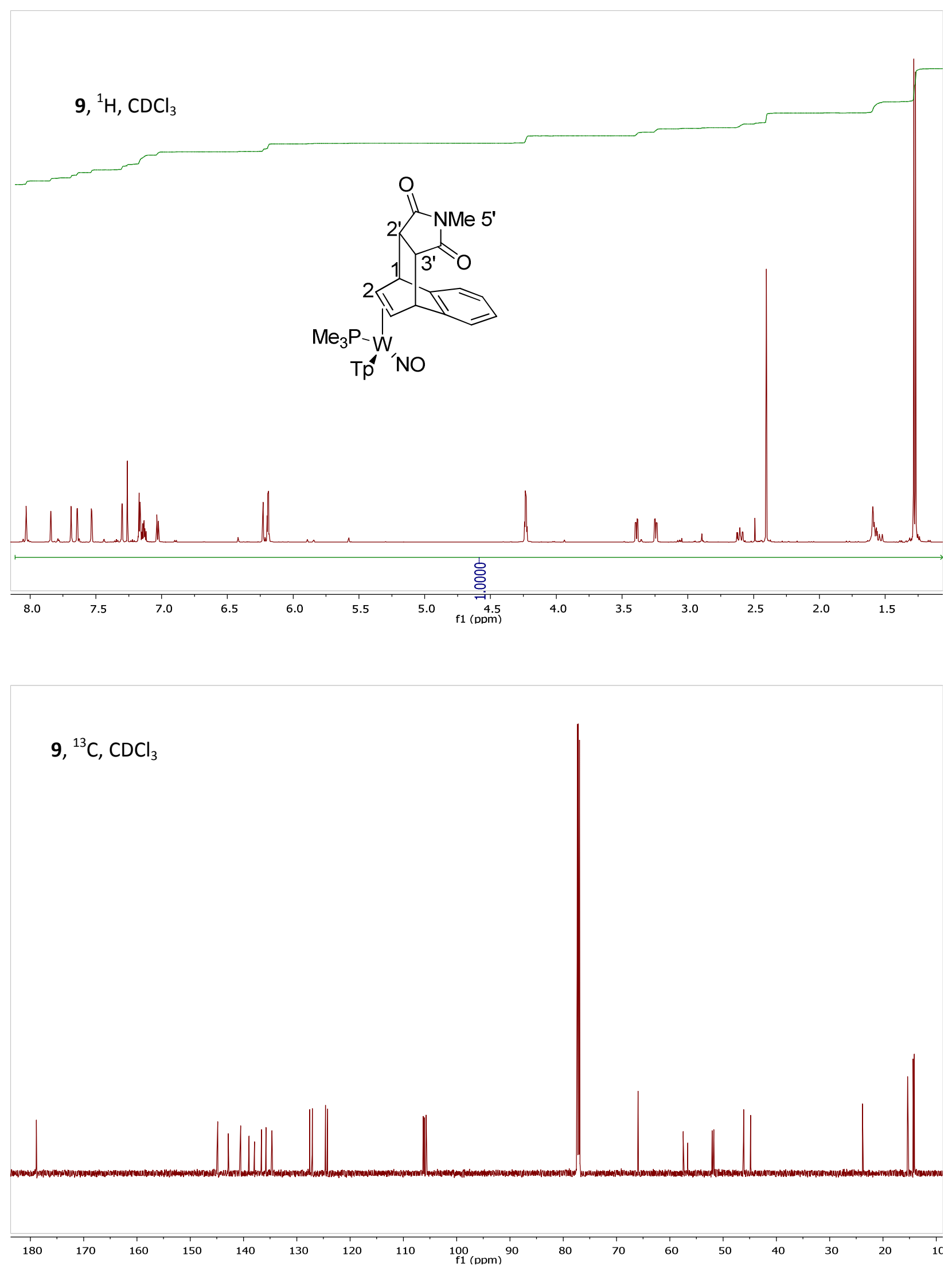

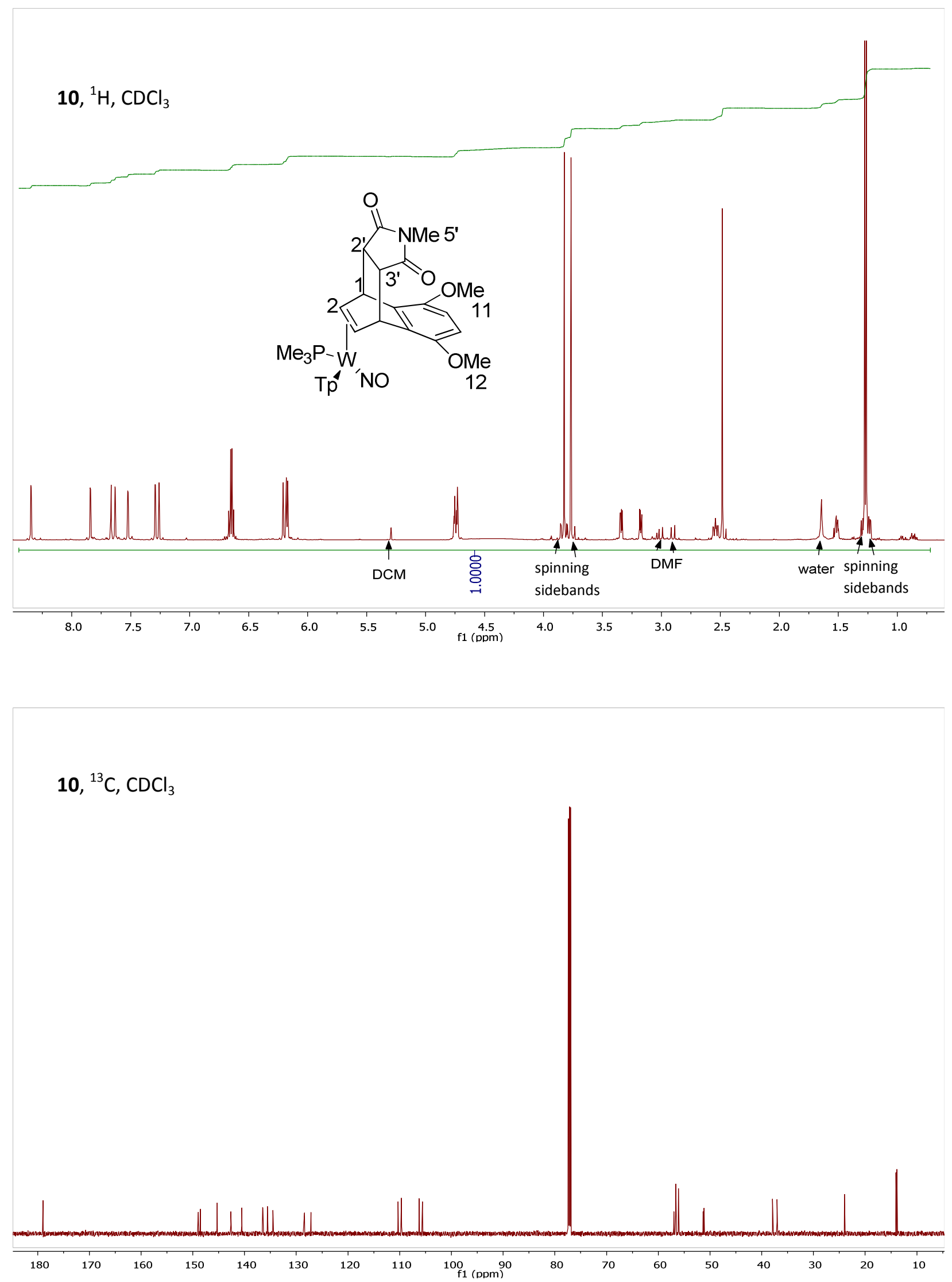

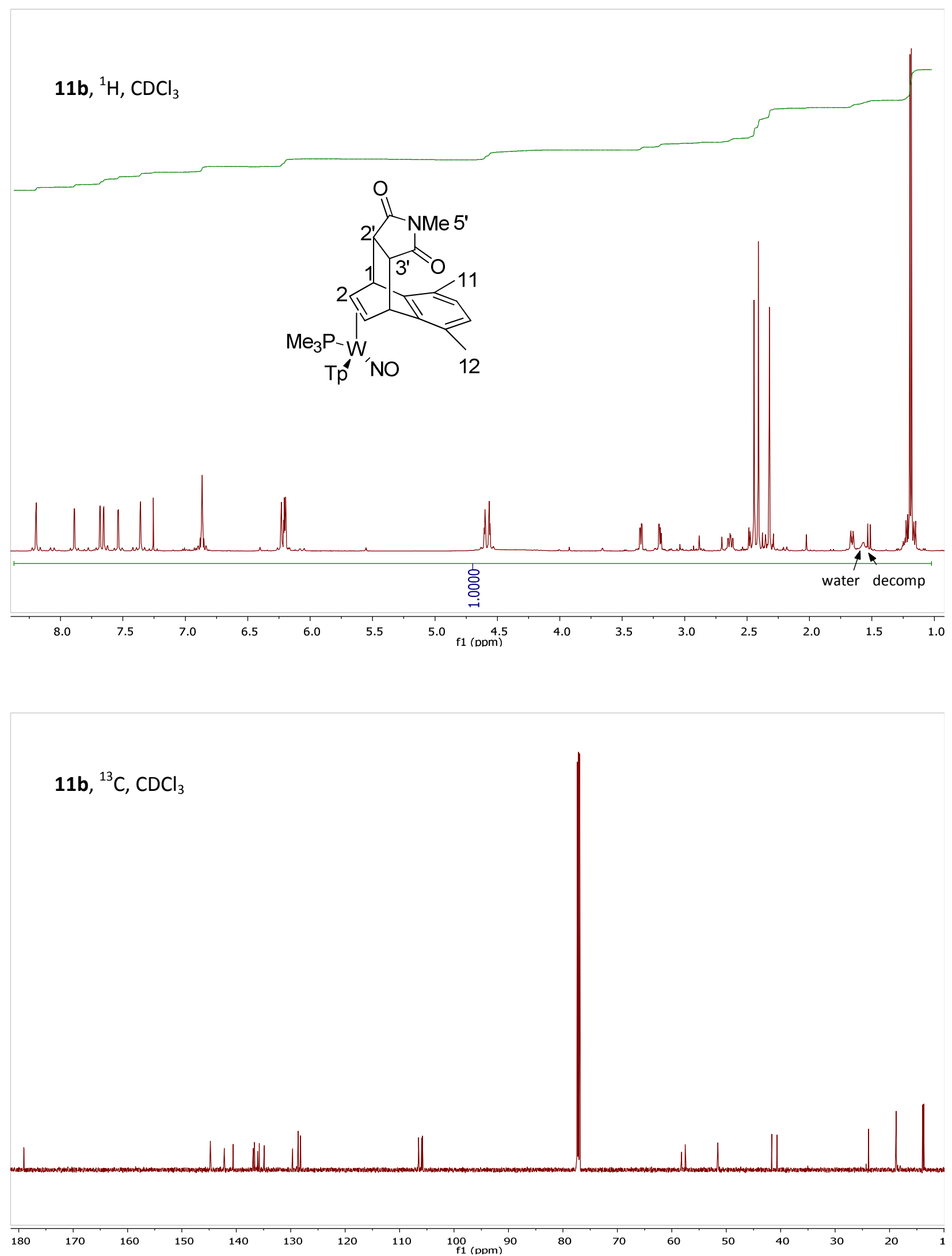


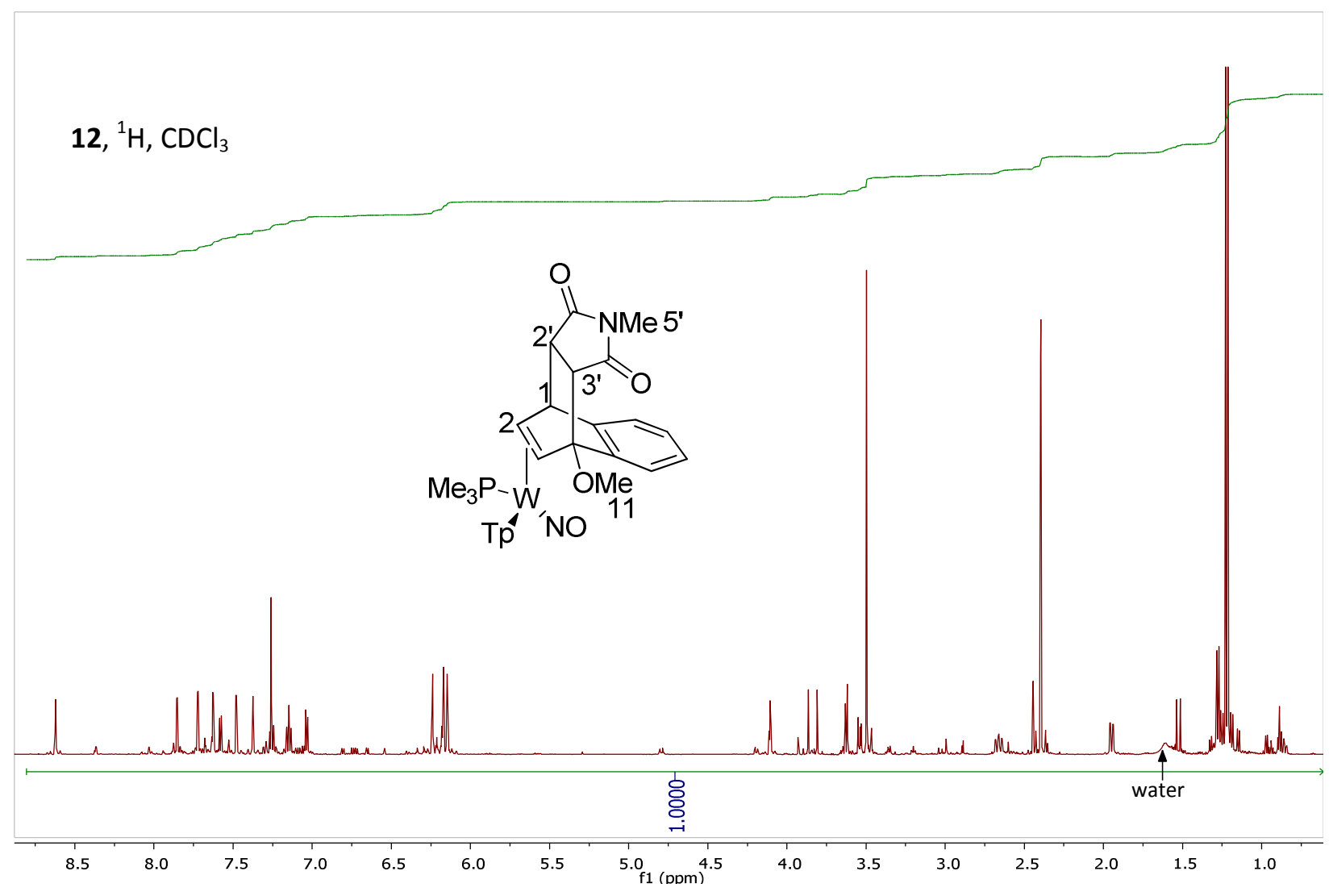

12, ${ }^{13} \mathrm{C}, \mathrm{CDCl}_{3}$

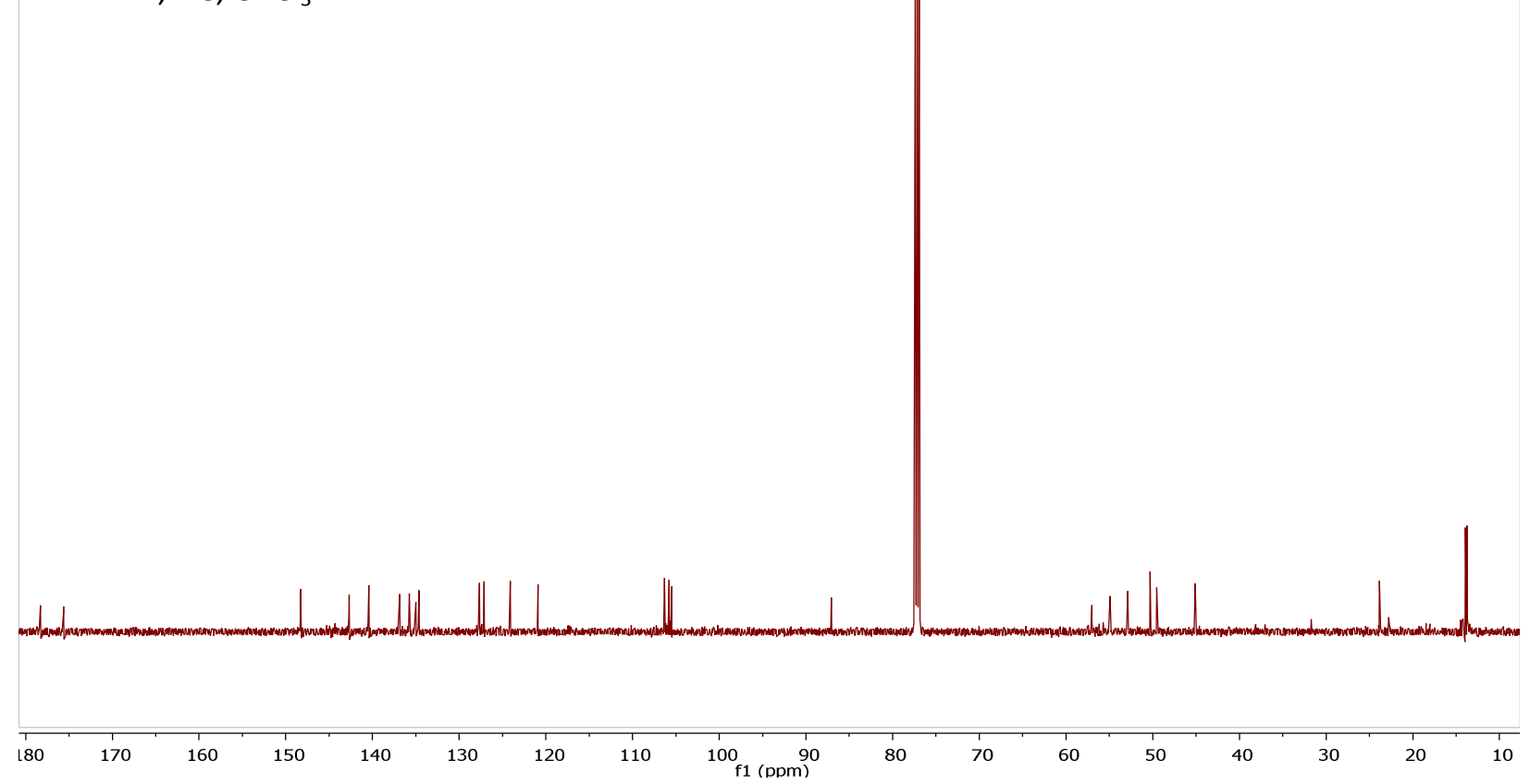


13, ${ }^{1} \mathrm{H}, \mathrm{CDCl}_{3}$
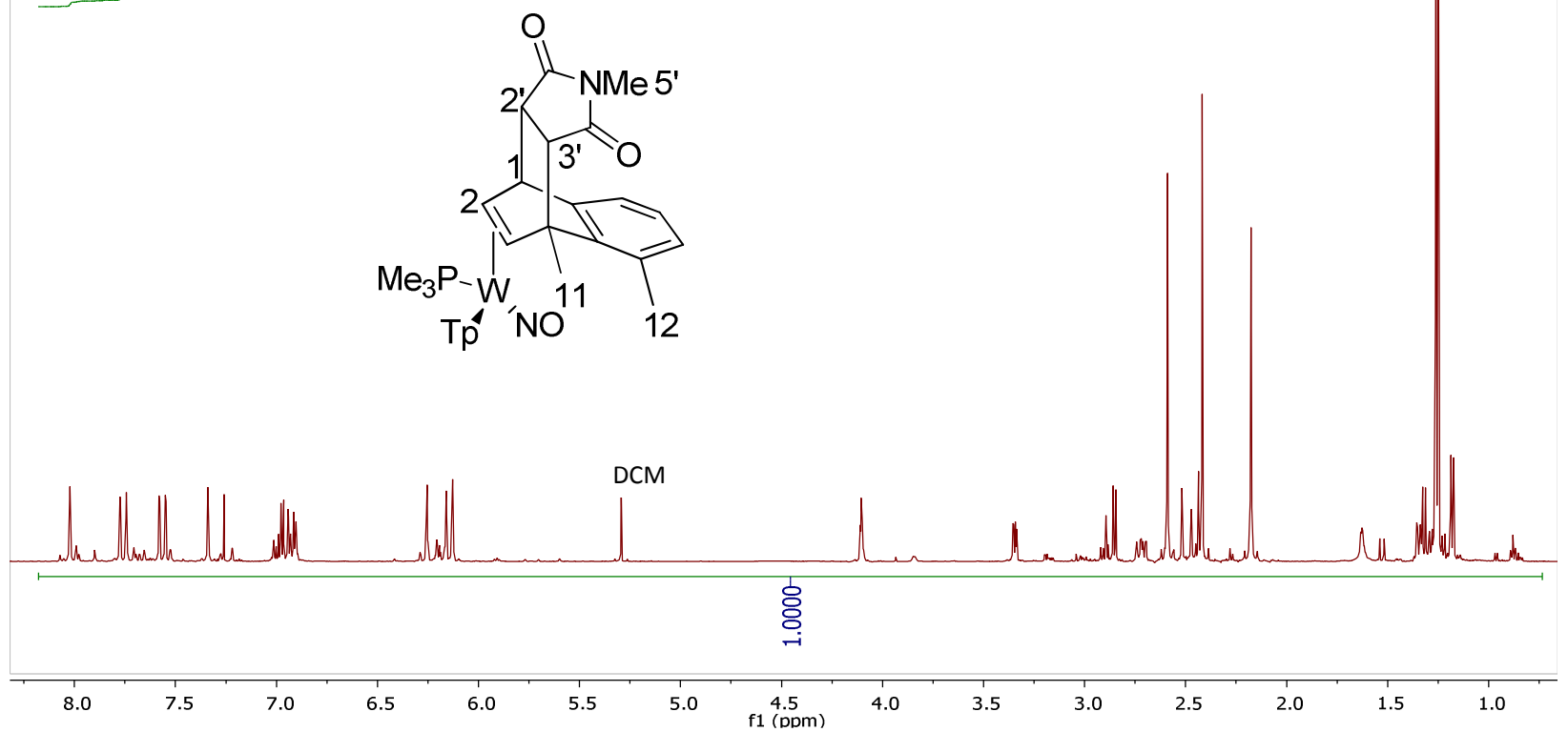

13, ${ }^{13} \mathrm{C}, \mathrm{CDCl}_{3}$

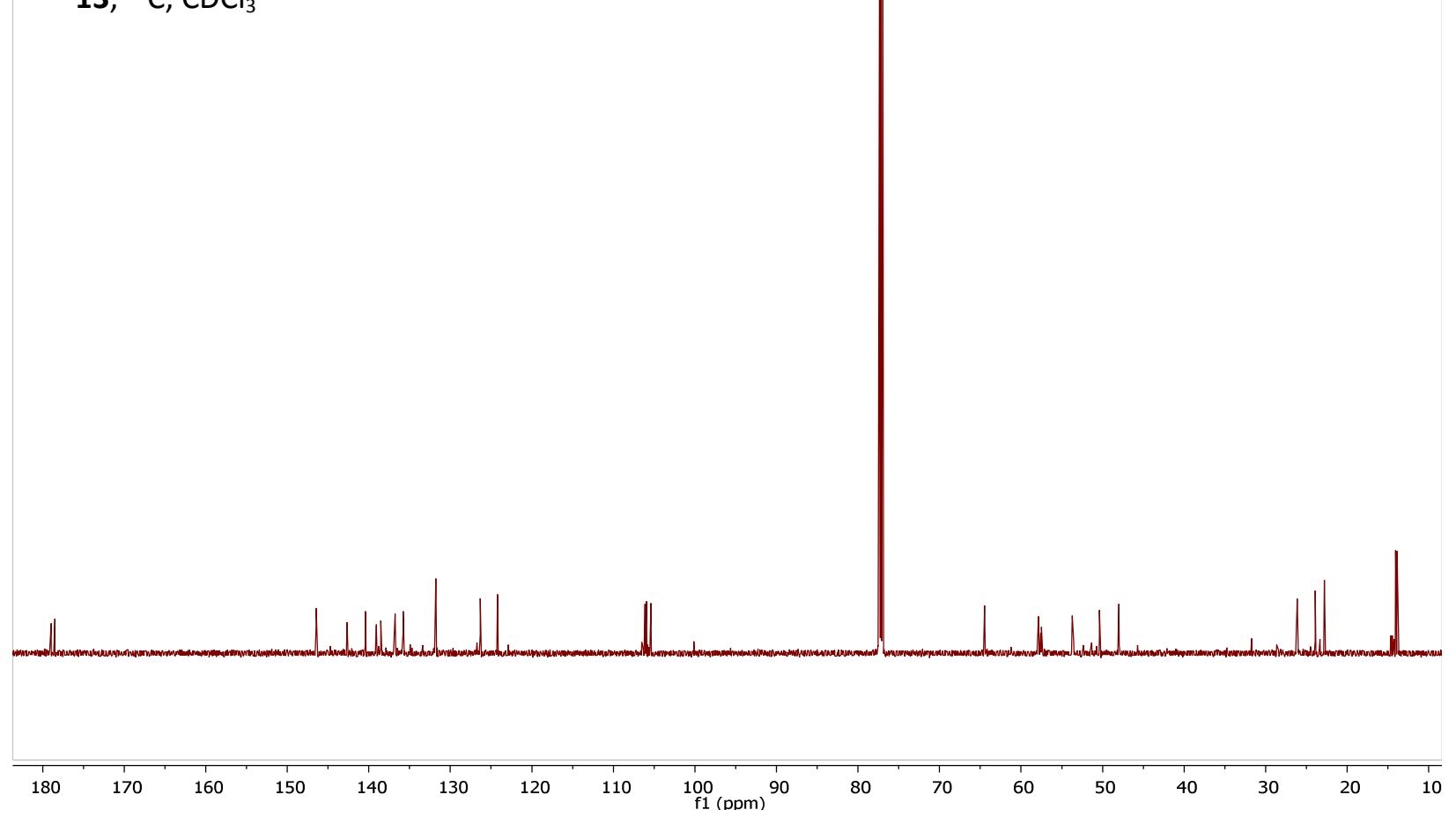




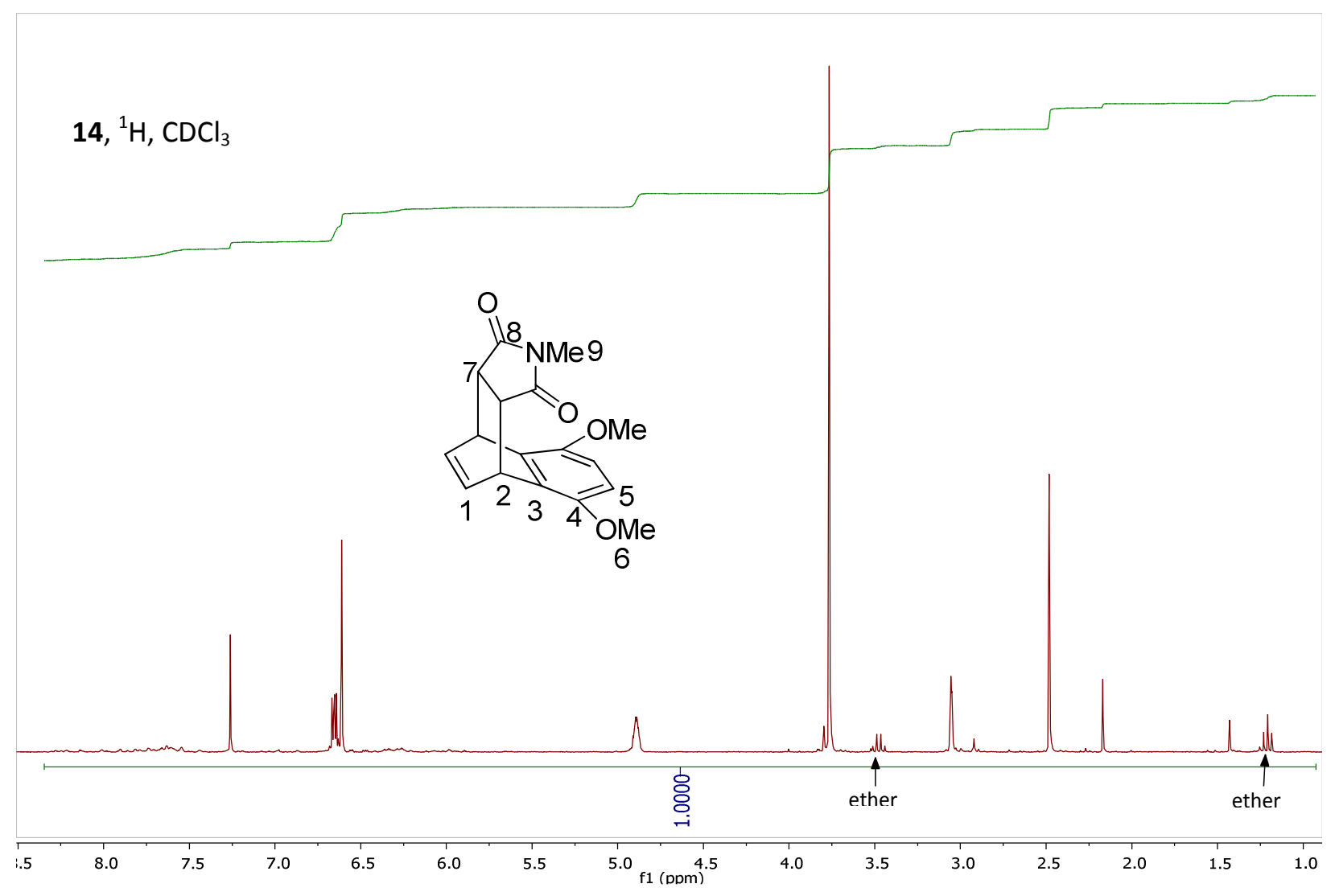

14, ${ }^{13} \mathrm{C}, \mathrm{CDCl}_{3}$

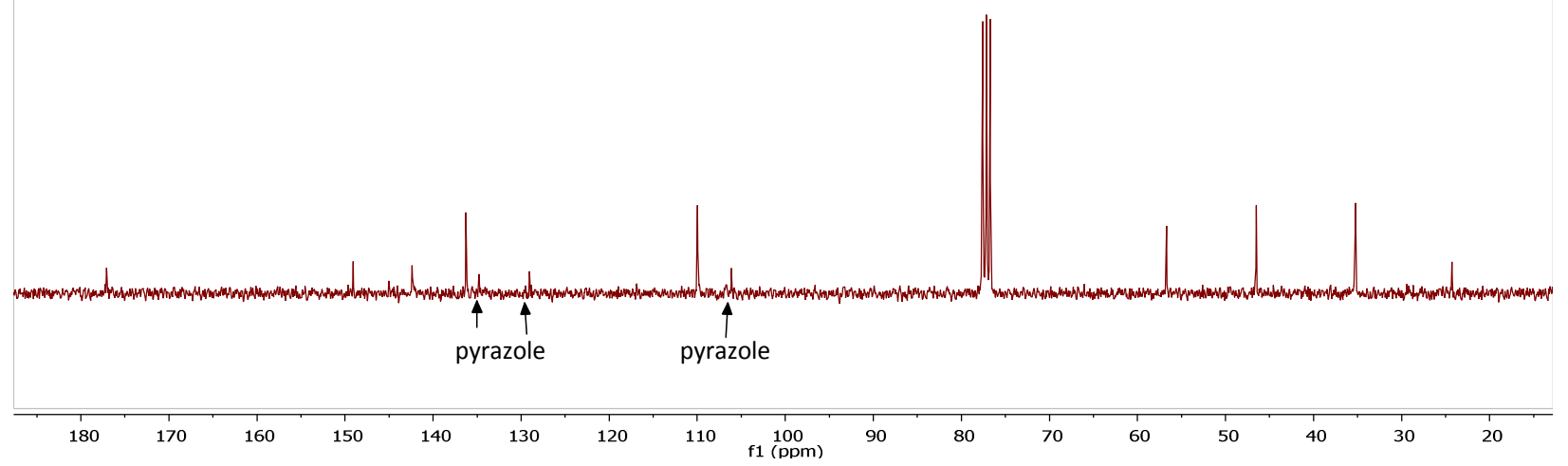




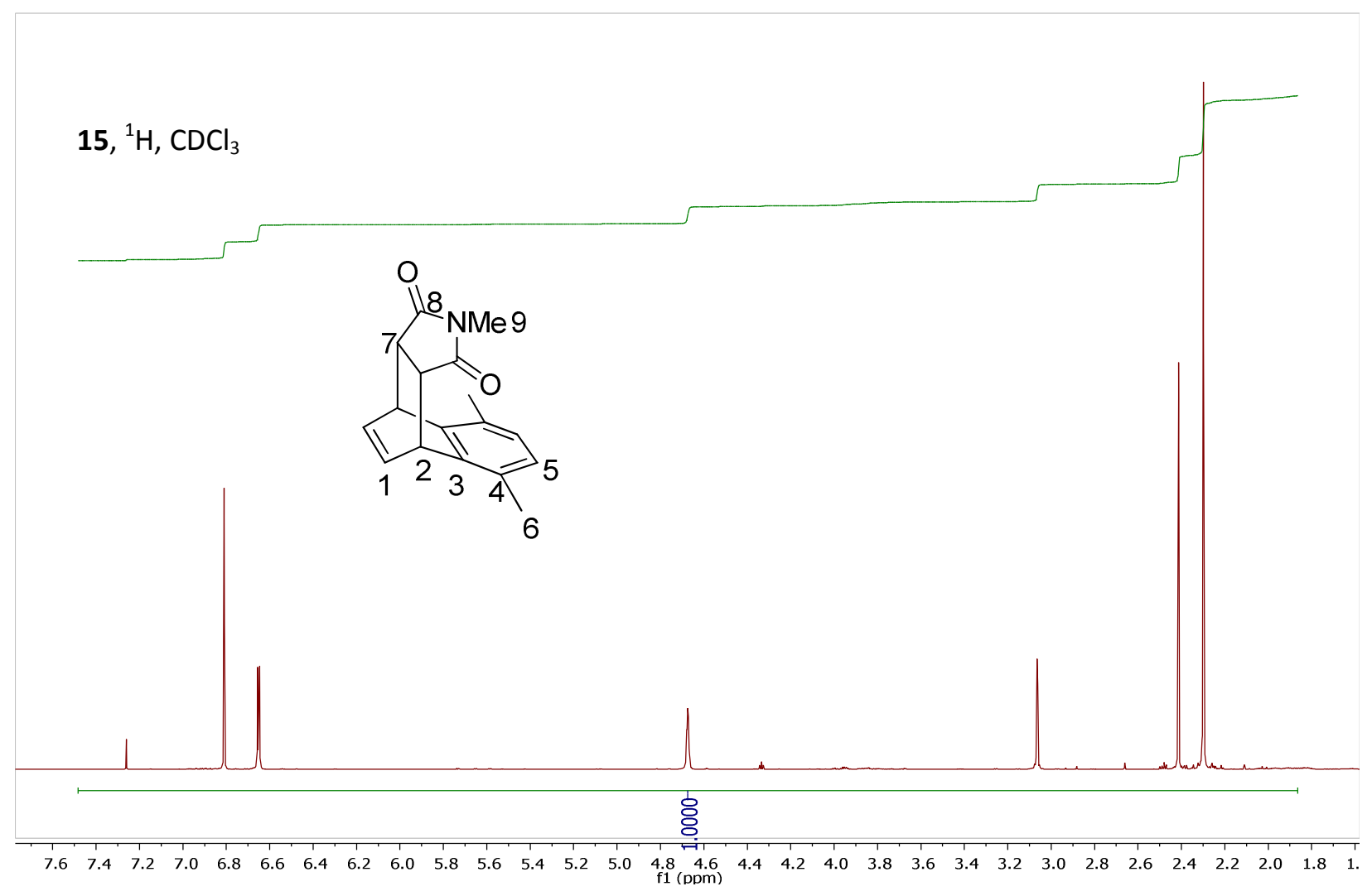

15, ${ }^{13} \mathrm{C}, \mathrm{CDCl}_{3}$
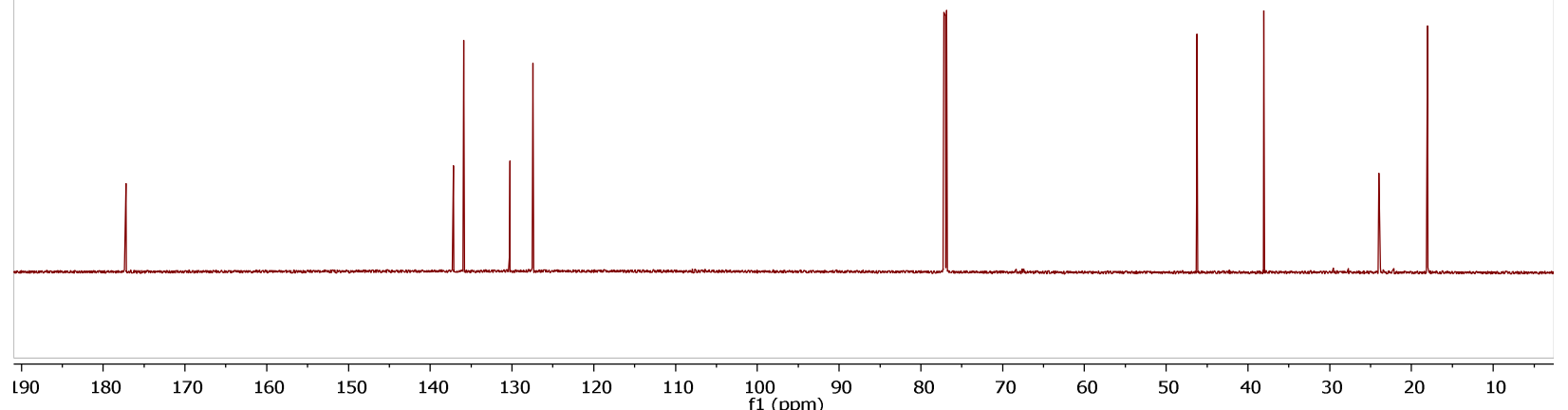


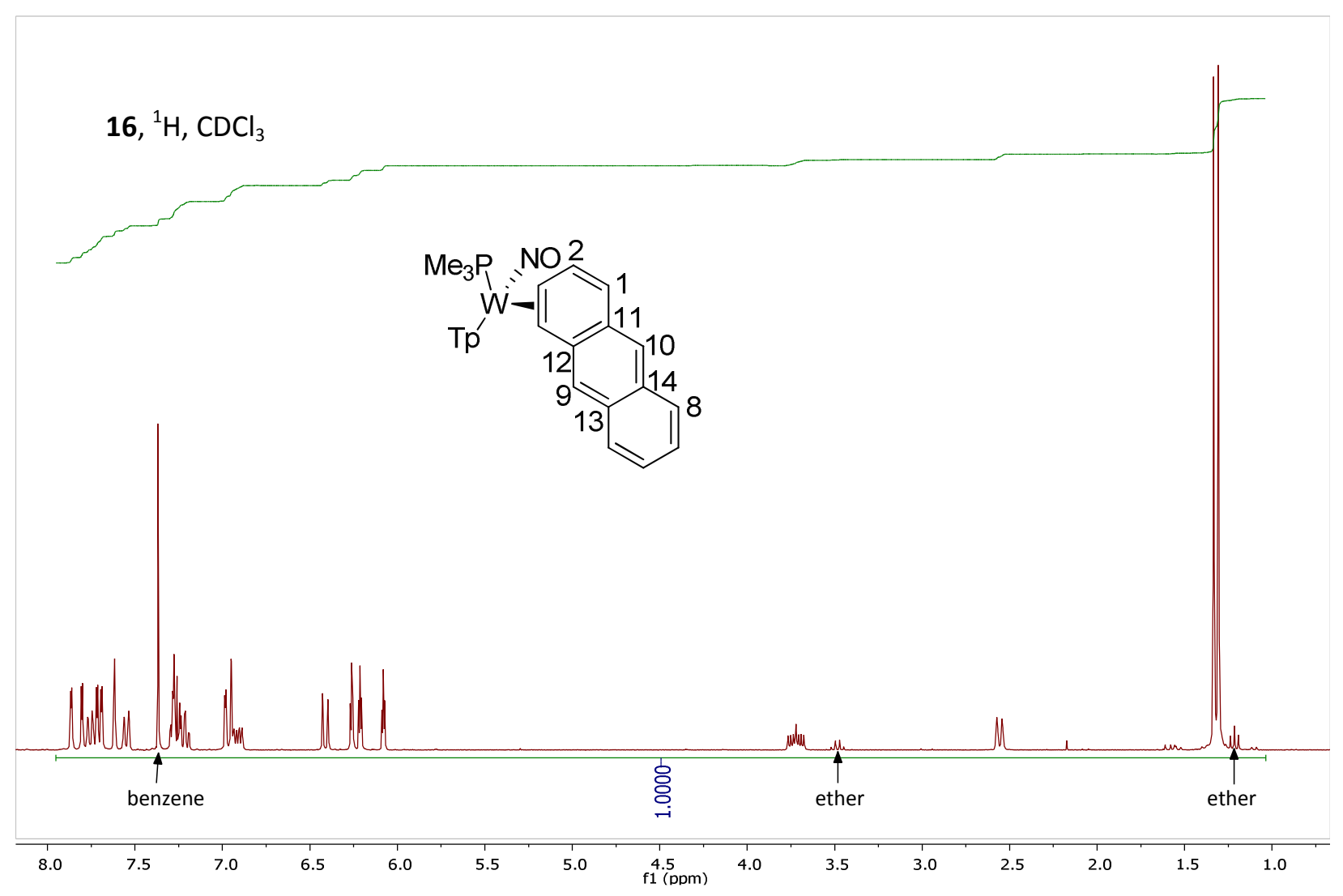

$16,{ }^{13} \mathrm{C}, \mathrm{CDCl}_{3}$

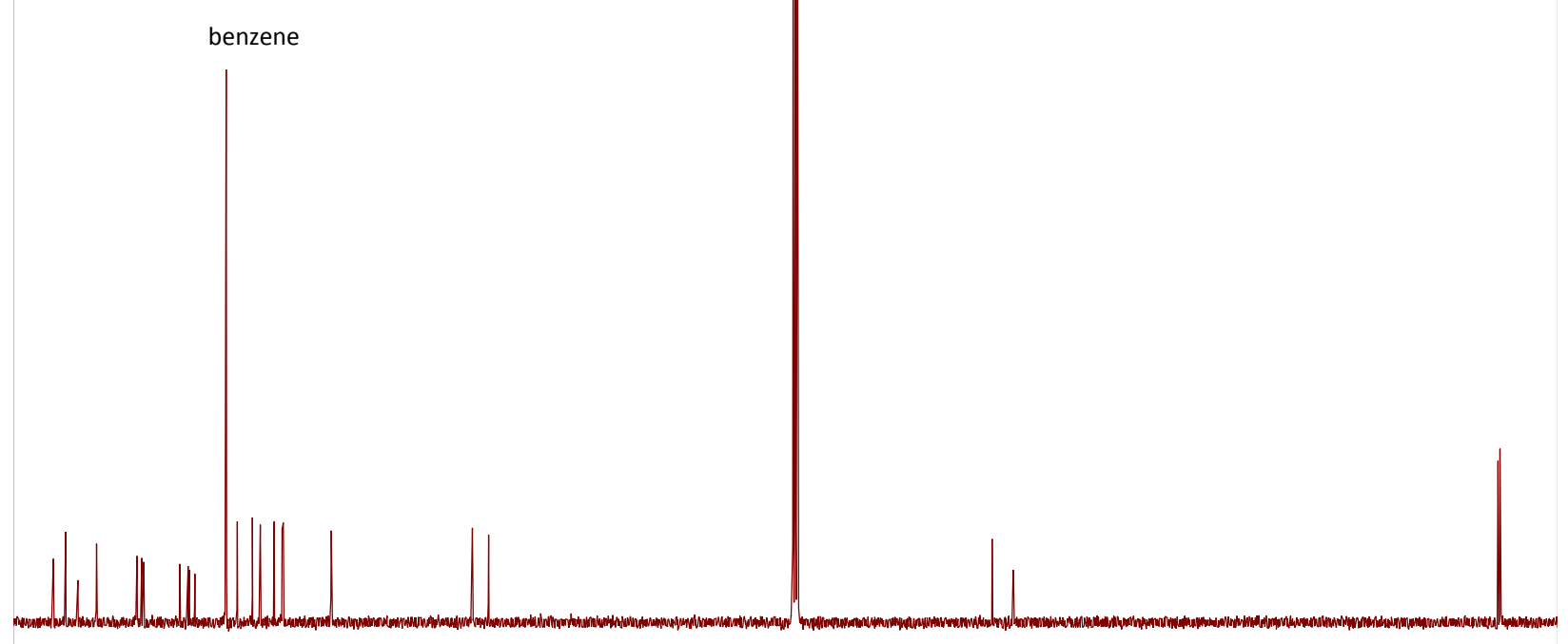

$\begin{array}{llllllllllllllllllllllllllll}145 & 140 & 135 & 130 & 125 & 120 & 115 & 110 & 105 & 100 & 95 & 90 & 85 & 80 & 75 & 70 & 65 & 60 & 55 & 50 & 45 & 40 & 35 & 30 & 25 & 20 & 15 & 10\end{array}$ 

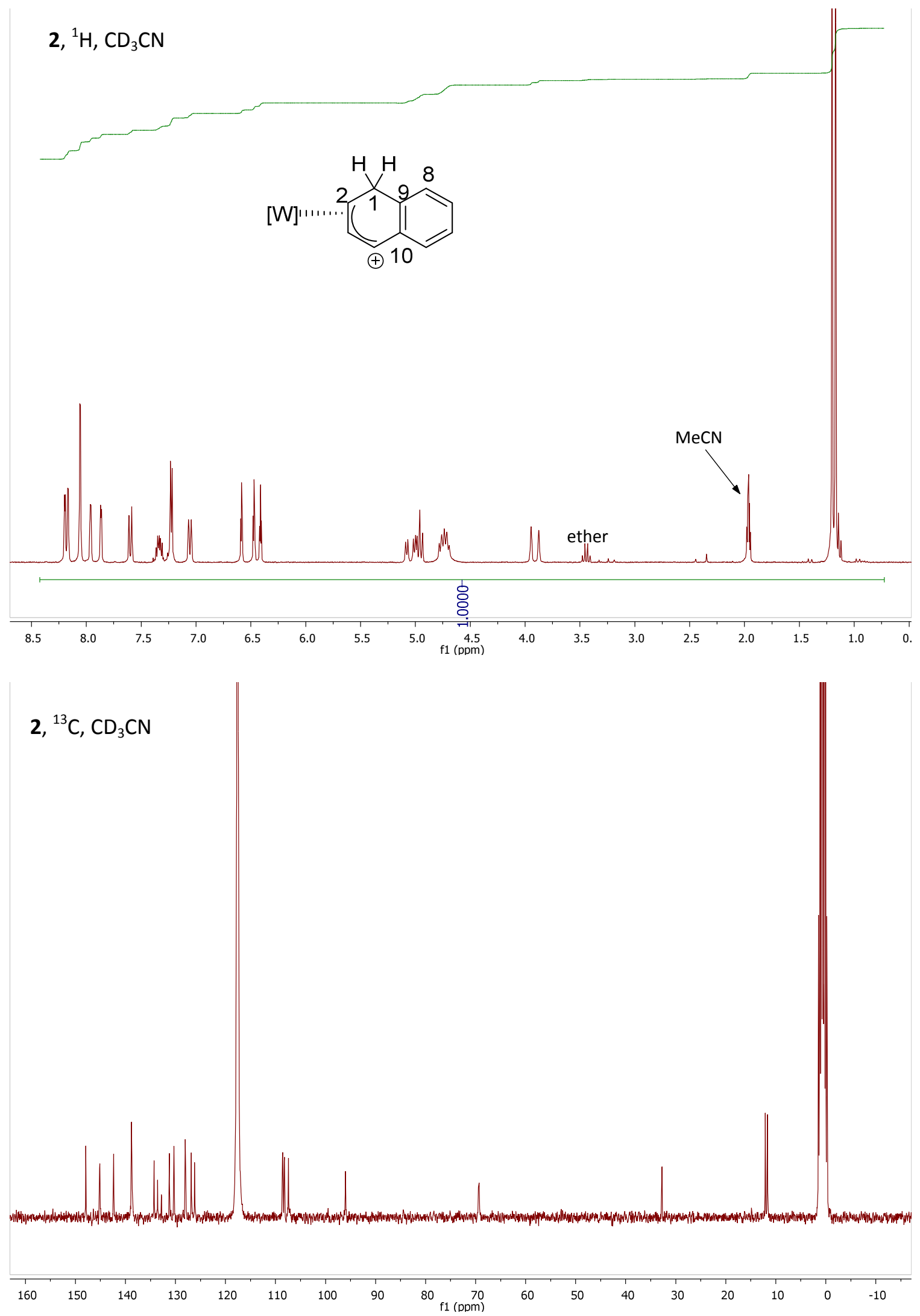

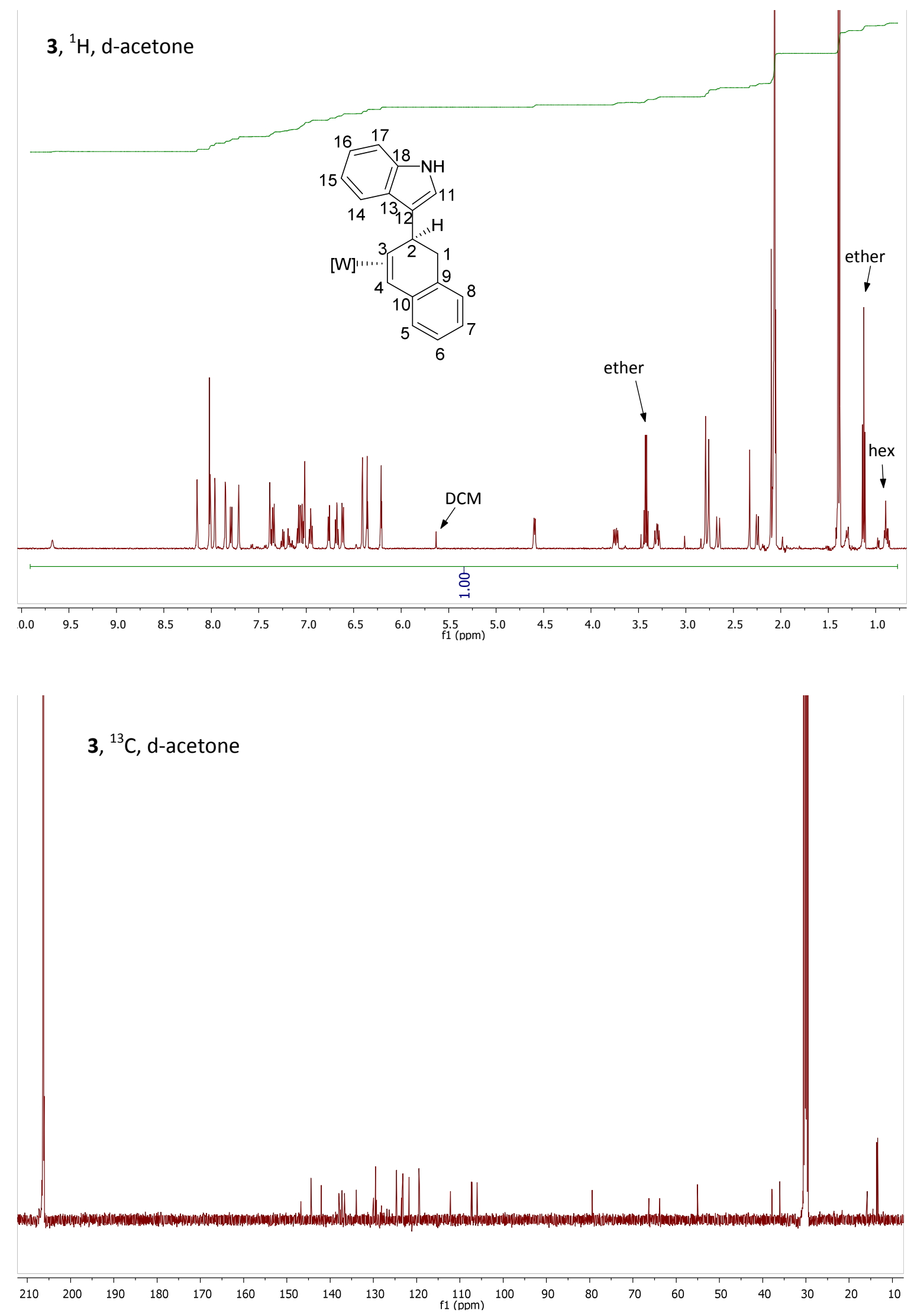

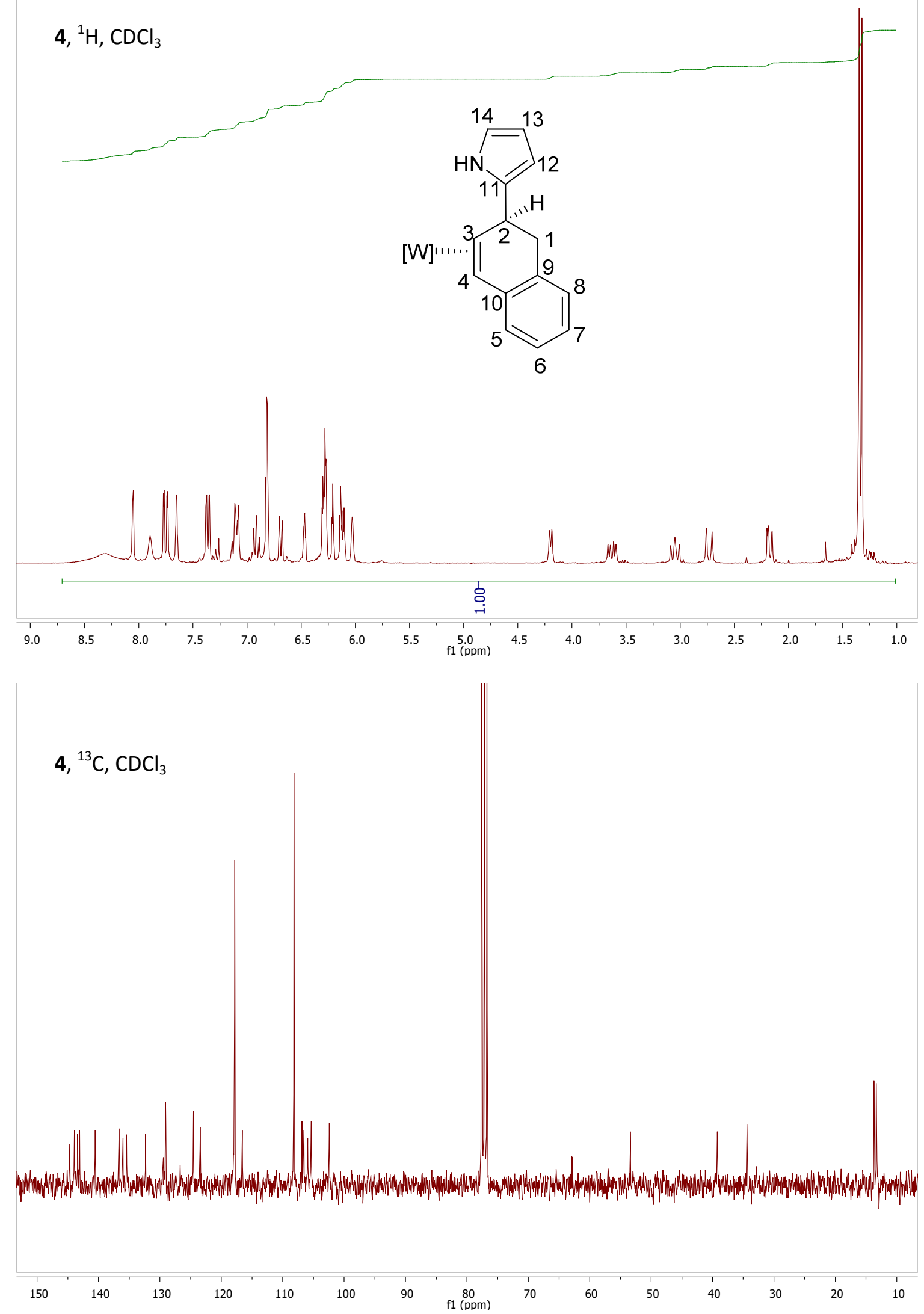


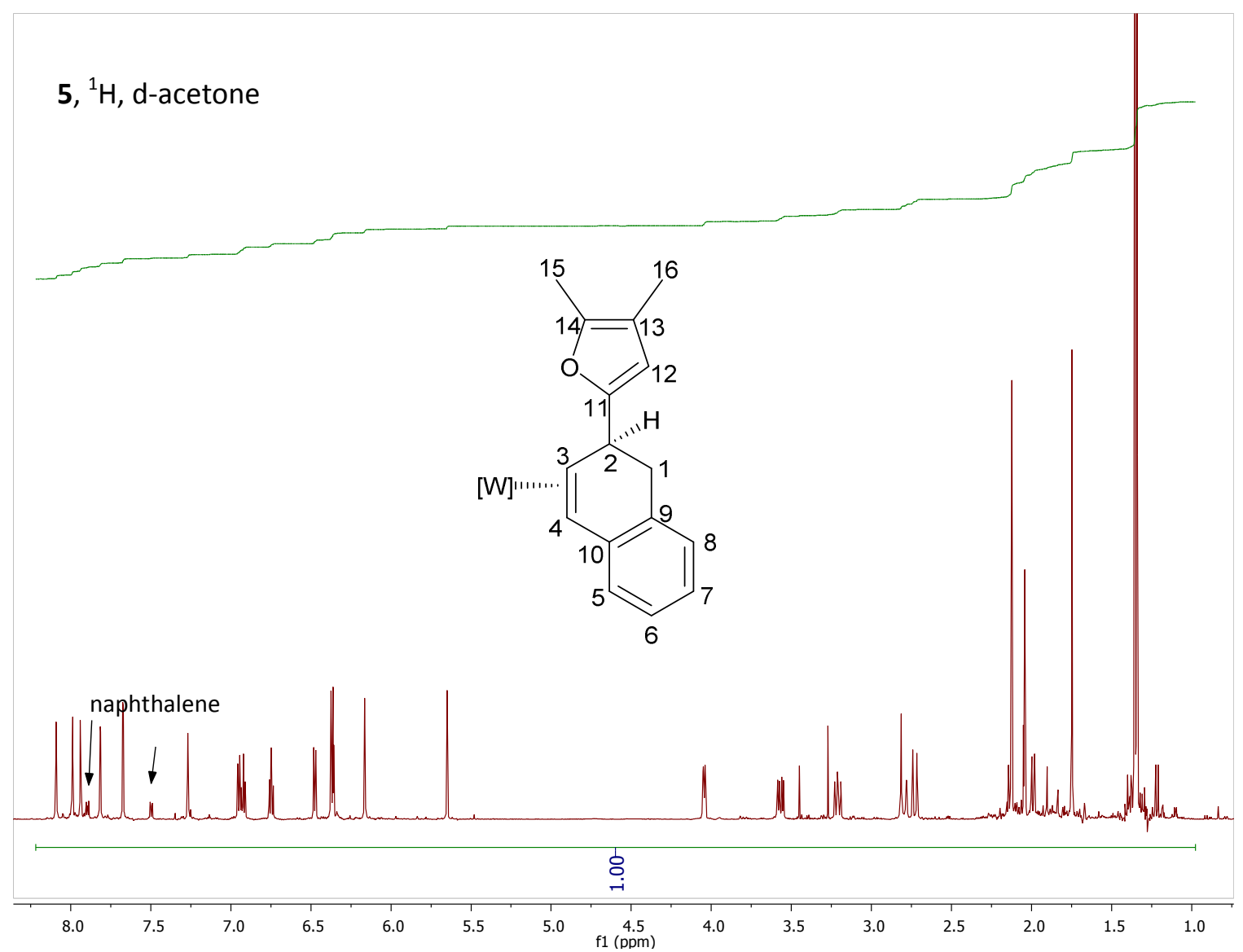

5, ${ }^{13} \mathrm{C}$, d-acetone

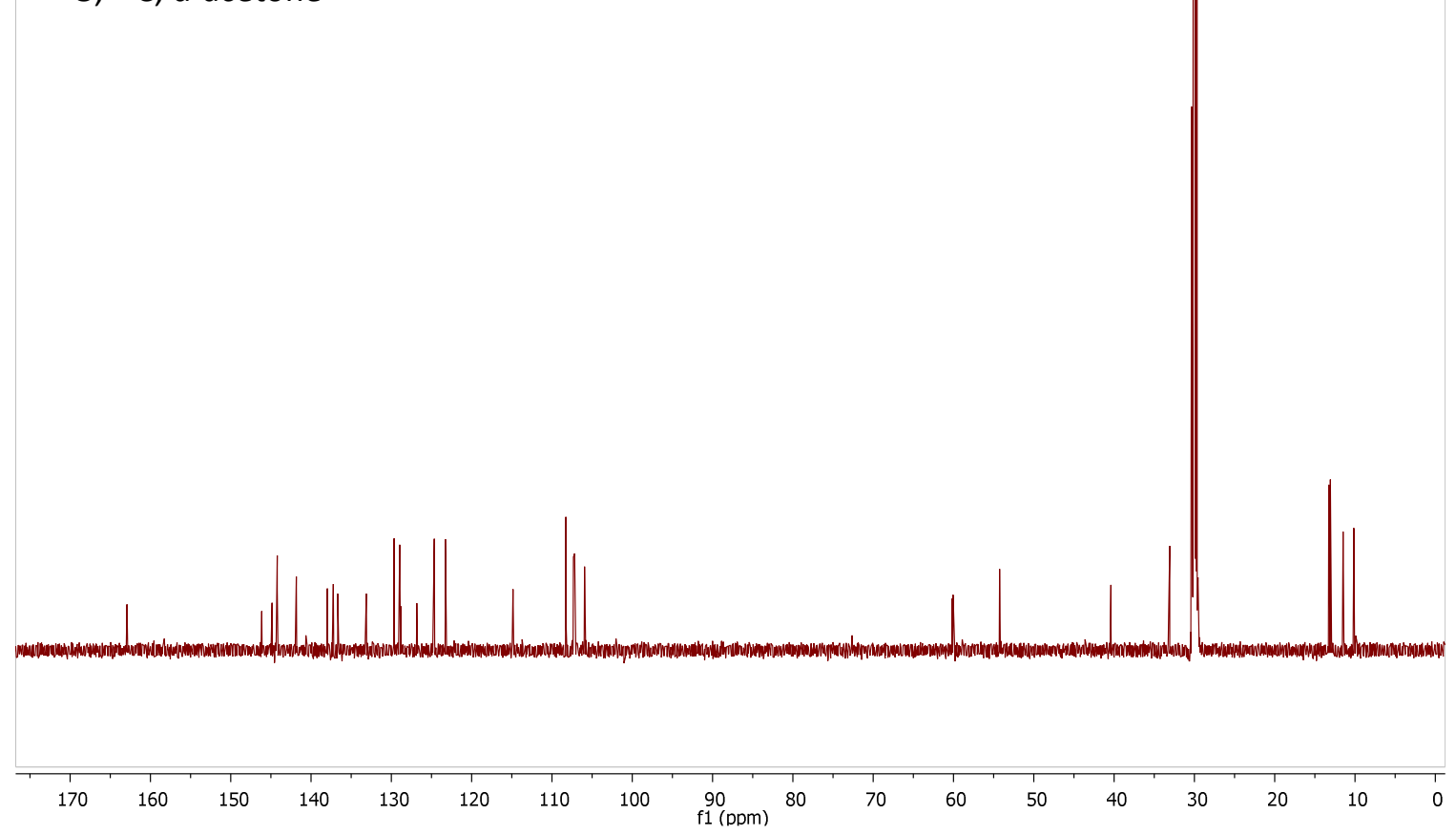


6, ${ }^{1} \mathrm{H}, \mathrm{CDCl}_{3}$

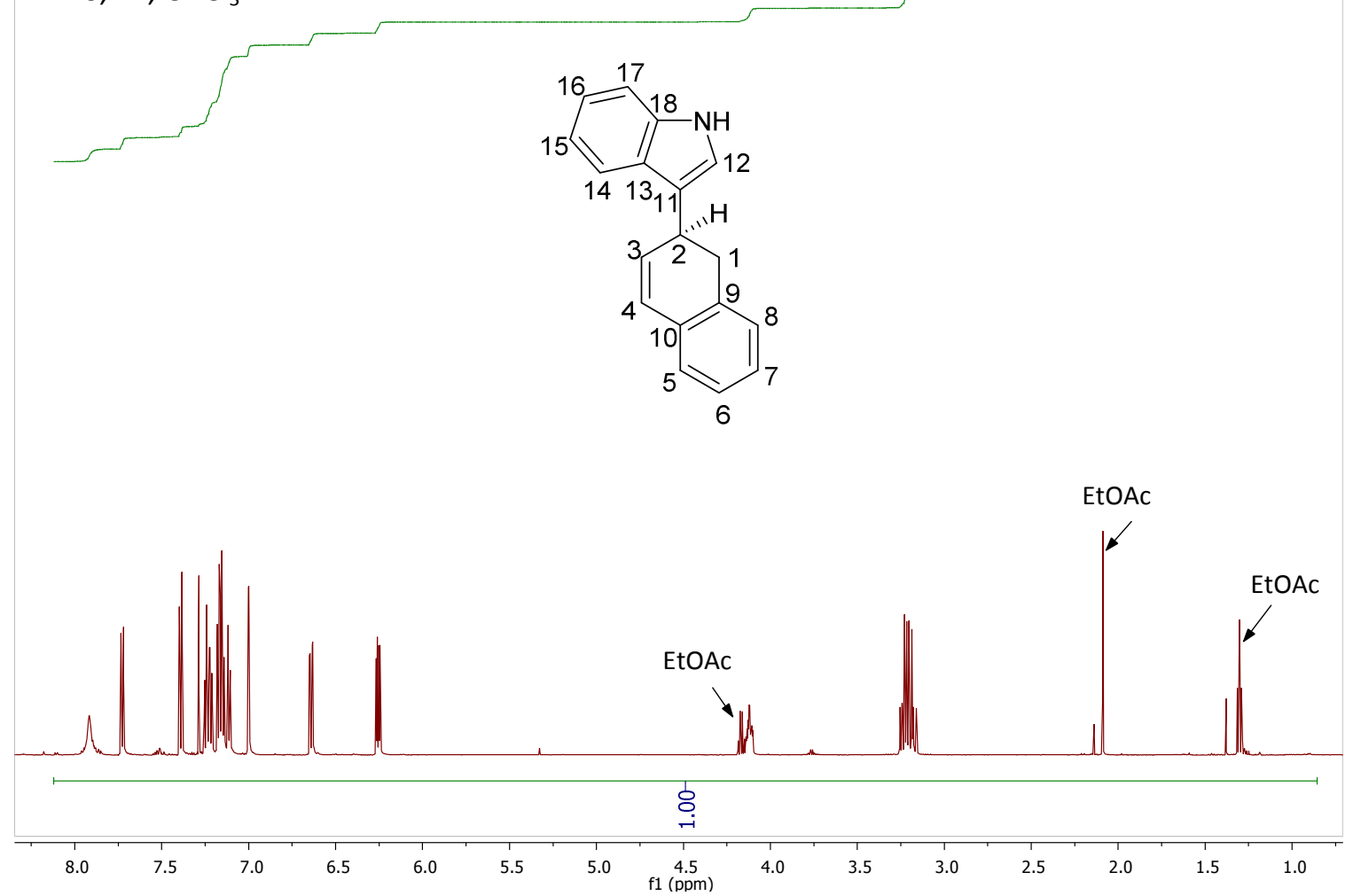

6, ${ }^{13} \mathrm{C}, \mathrm{CDCl}_{3}$

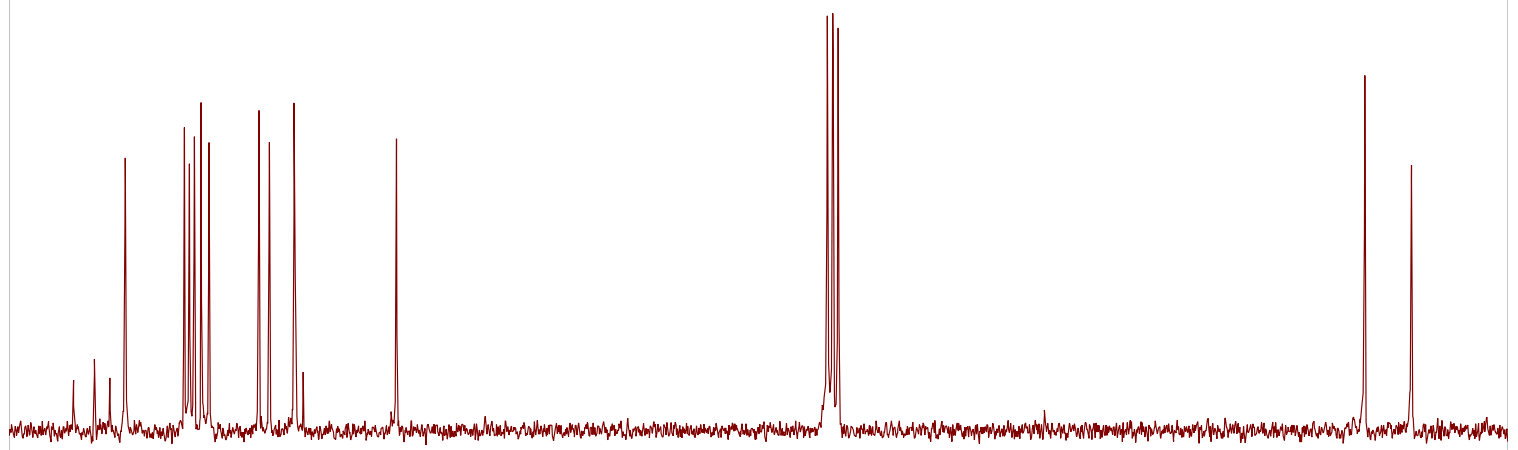

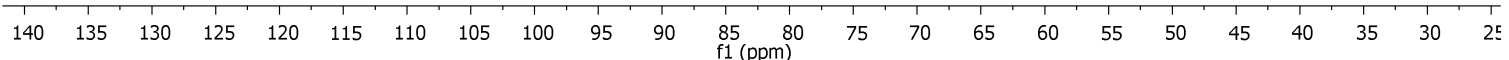




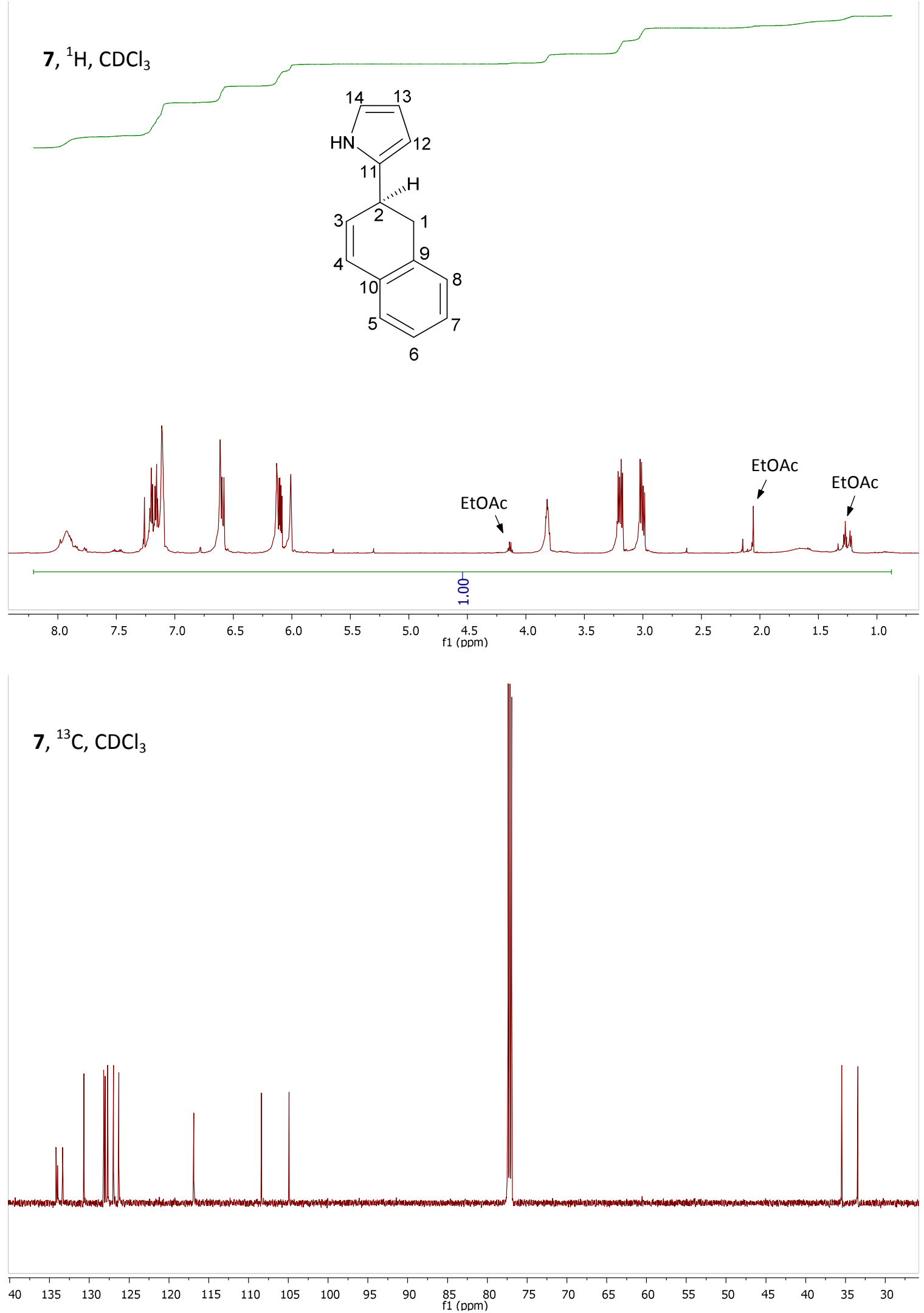




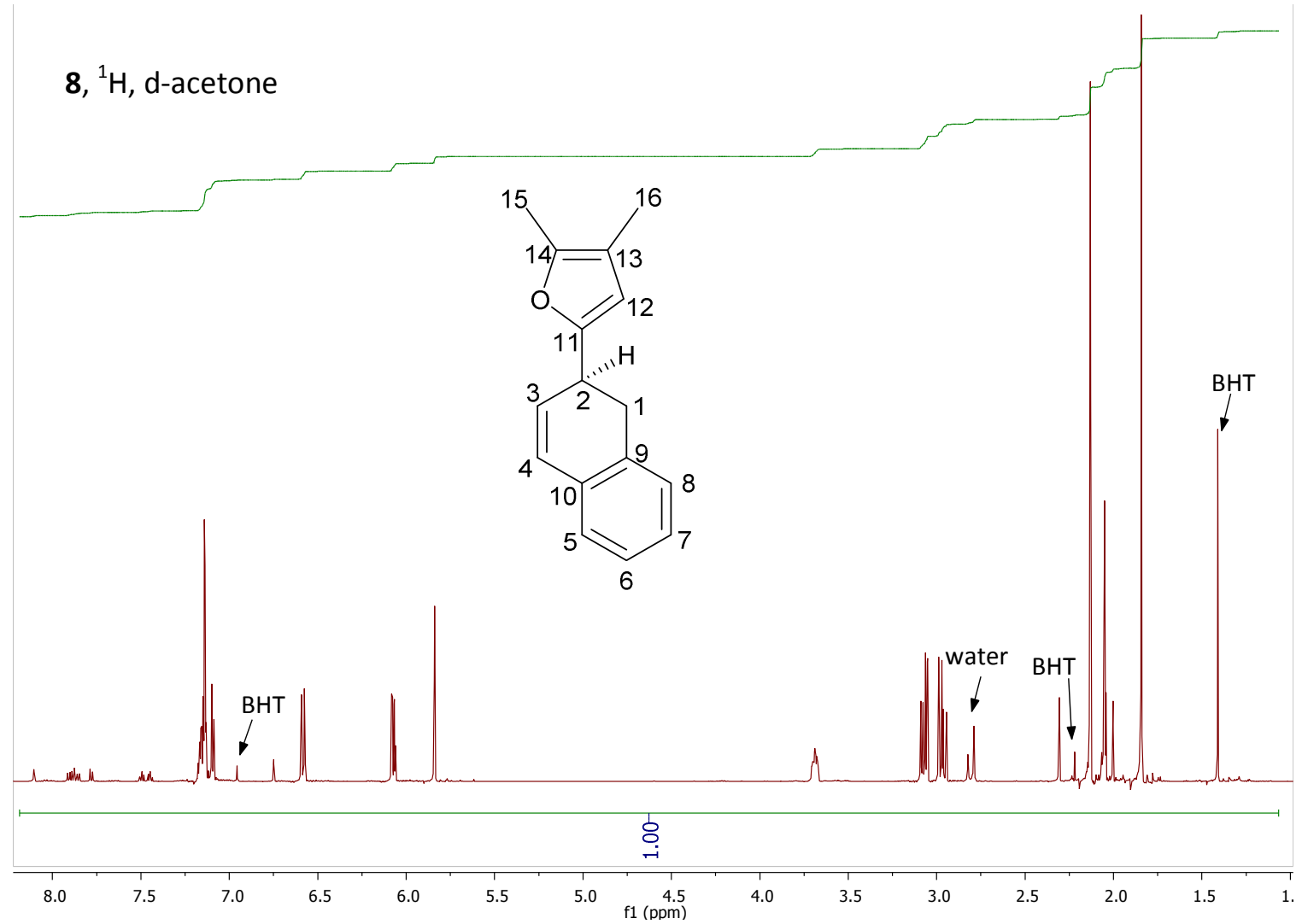

8, ${ }^{1} \mathrm{H}, \mathrm{d}$-acetone

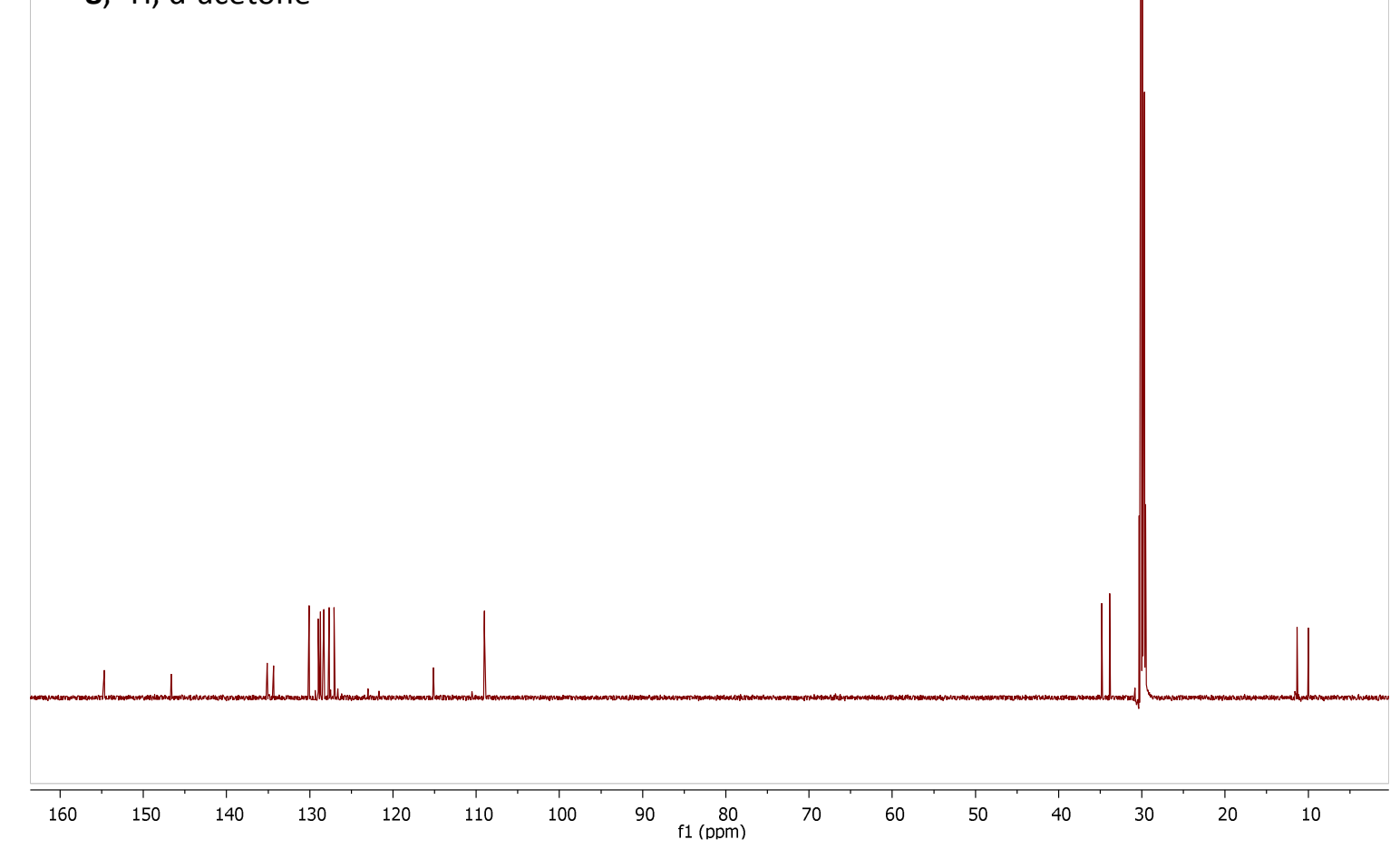



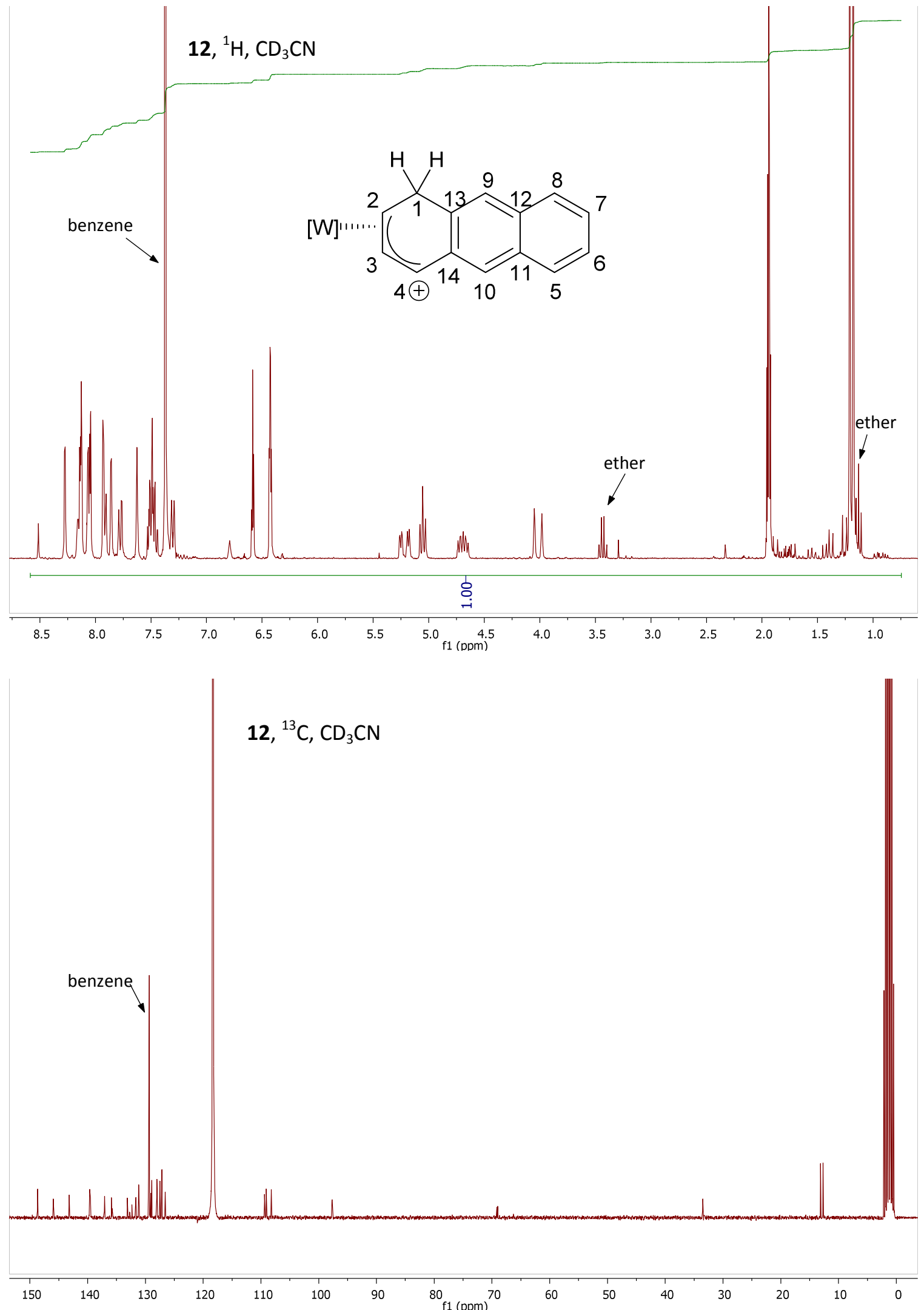

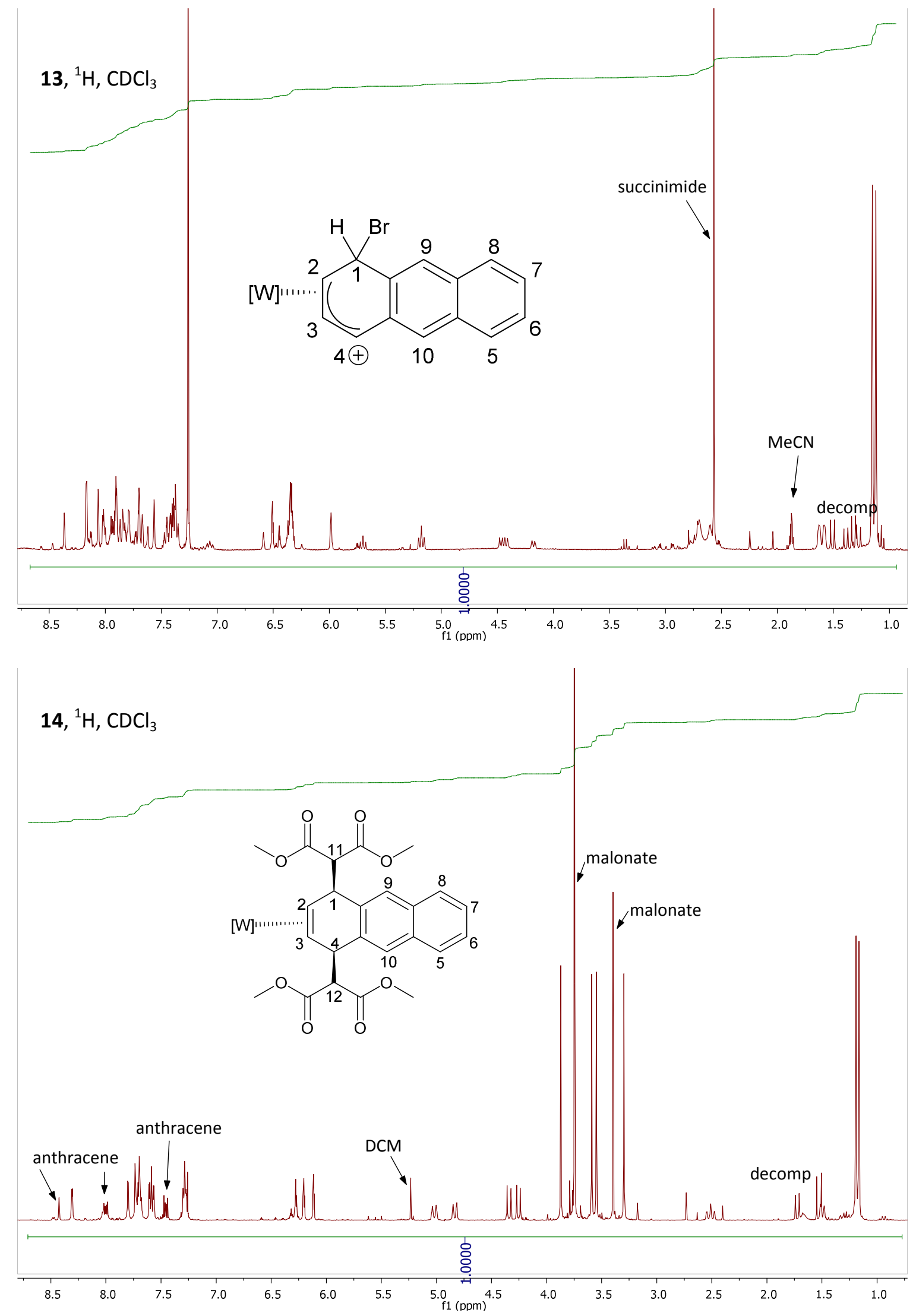
15, ${ }^{1} \mathrm{H}, \mathrm{CDCl}_{3}$

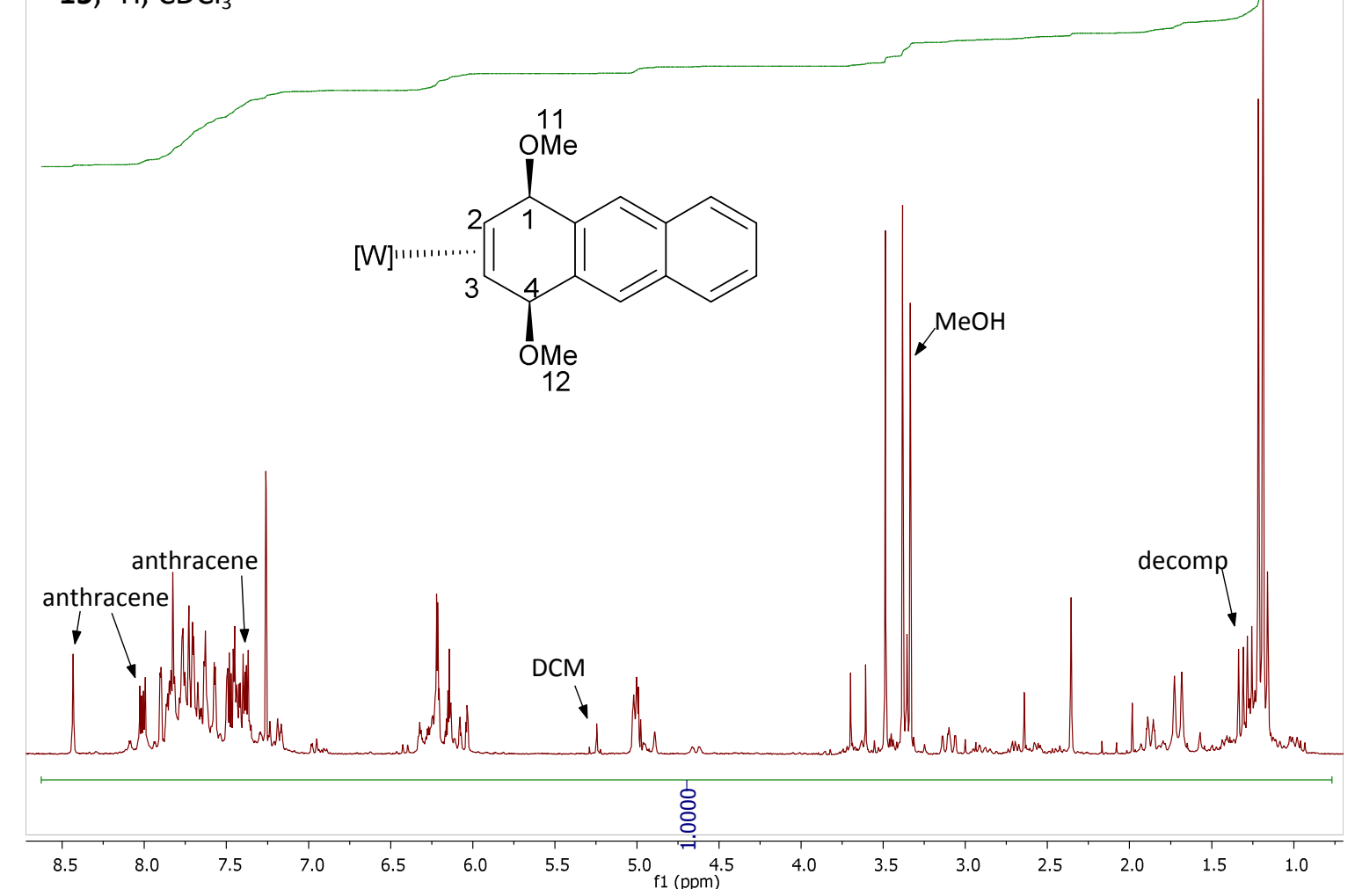

16, ${ }^{1} \mathrm{H}, \mathrm{CDCl}_{3}$<smiles>N#CC1=CC(n2ccnc2)c2cc3ccccc3cc2[C@H]1n1ccnc1</smiles>

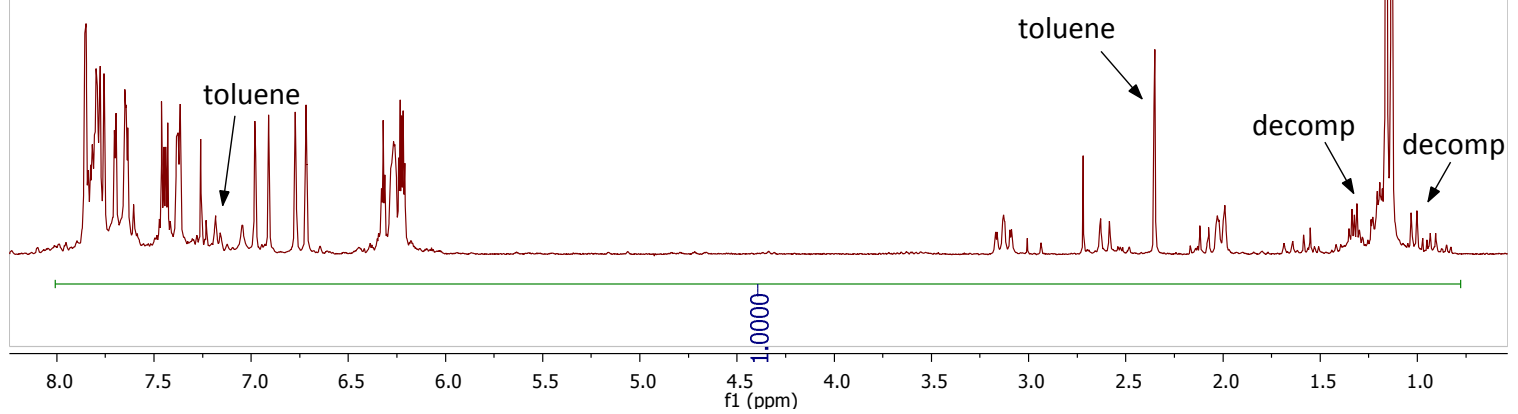



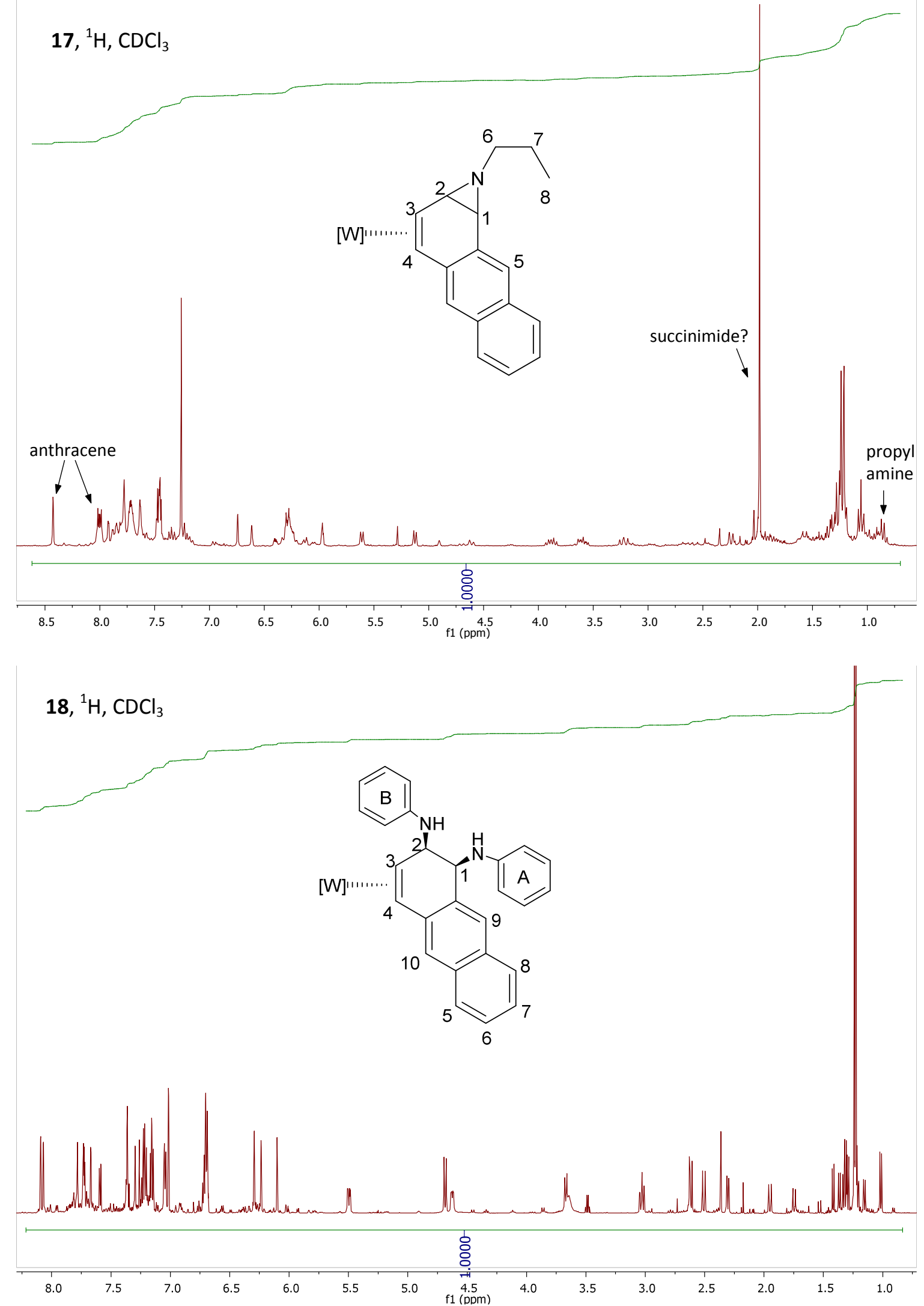
$18,{ }^{13} \mathrm{C}, \mathrm{CDCl}_{3}$
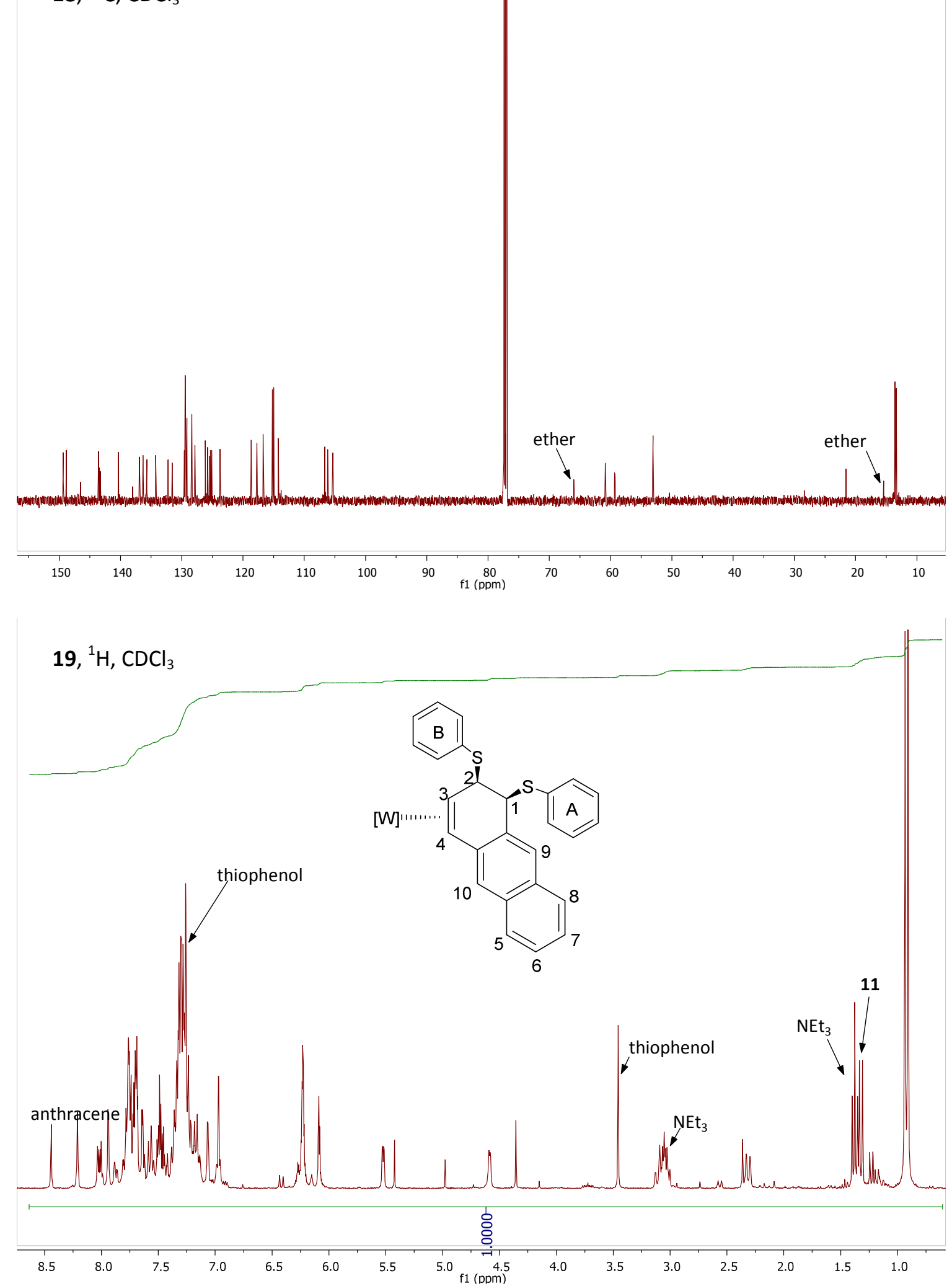


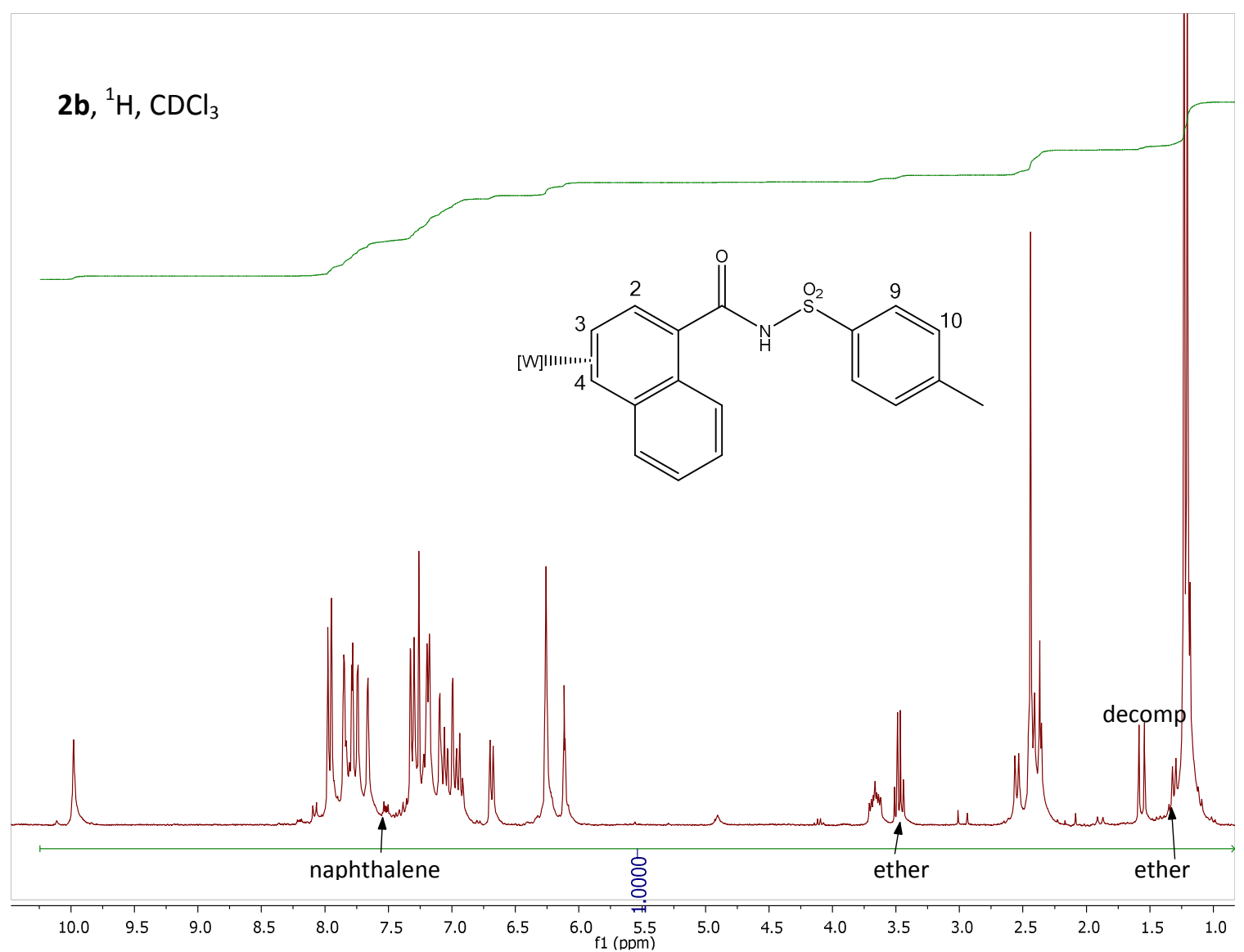




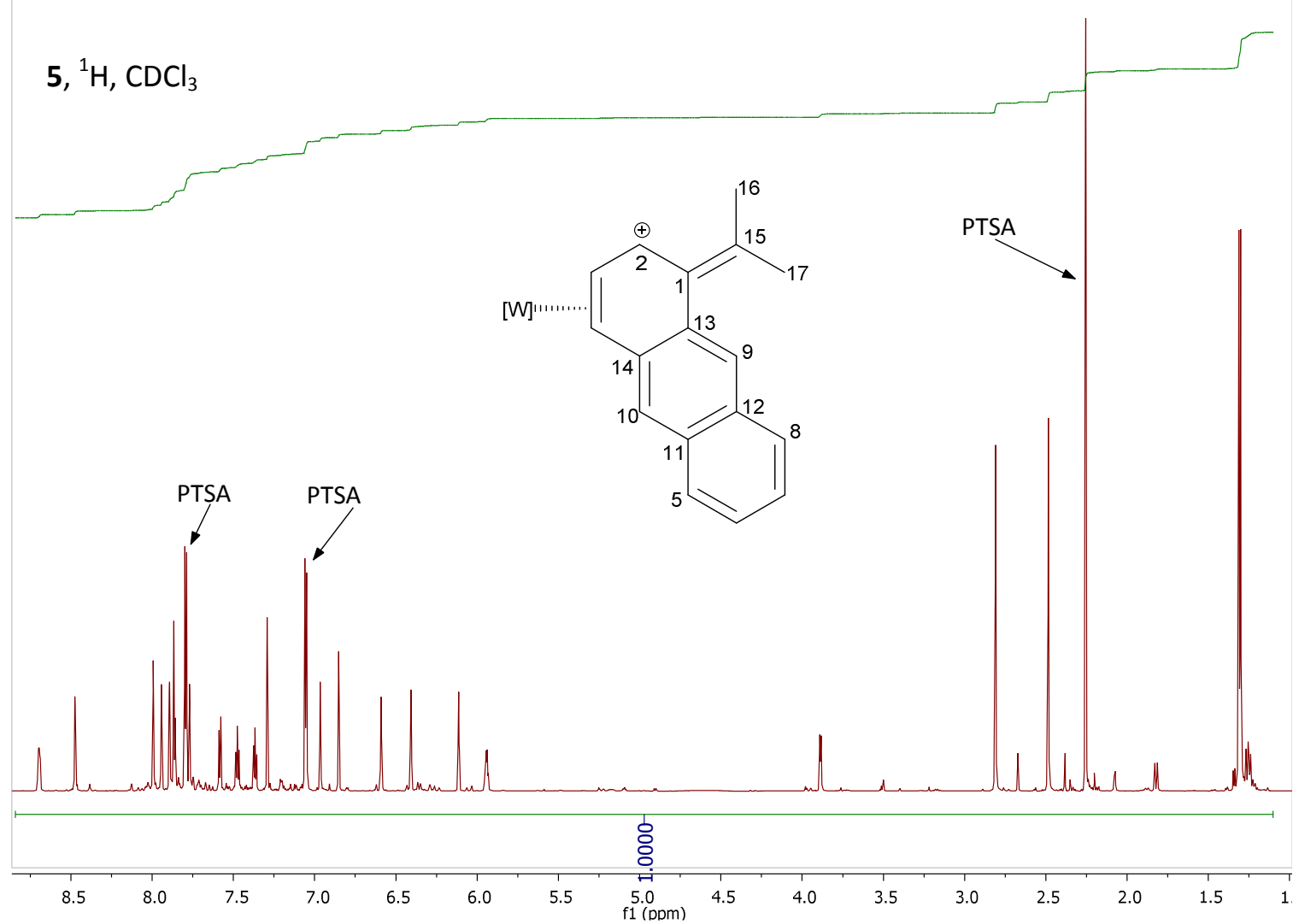

5, ${ }^{13} \mathrm{C}, \mathrm{CDCl}_{3}$

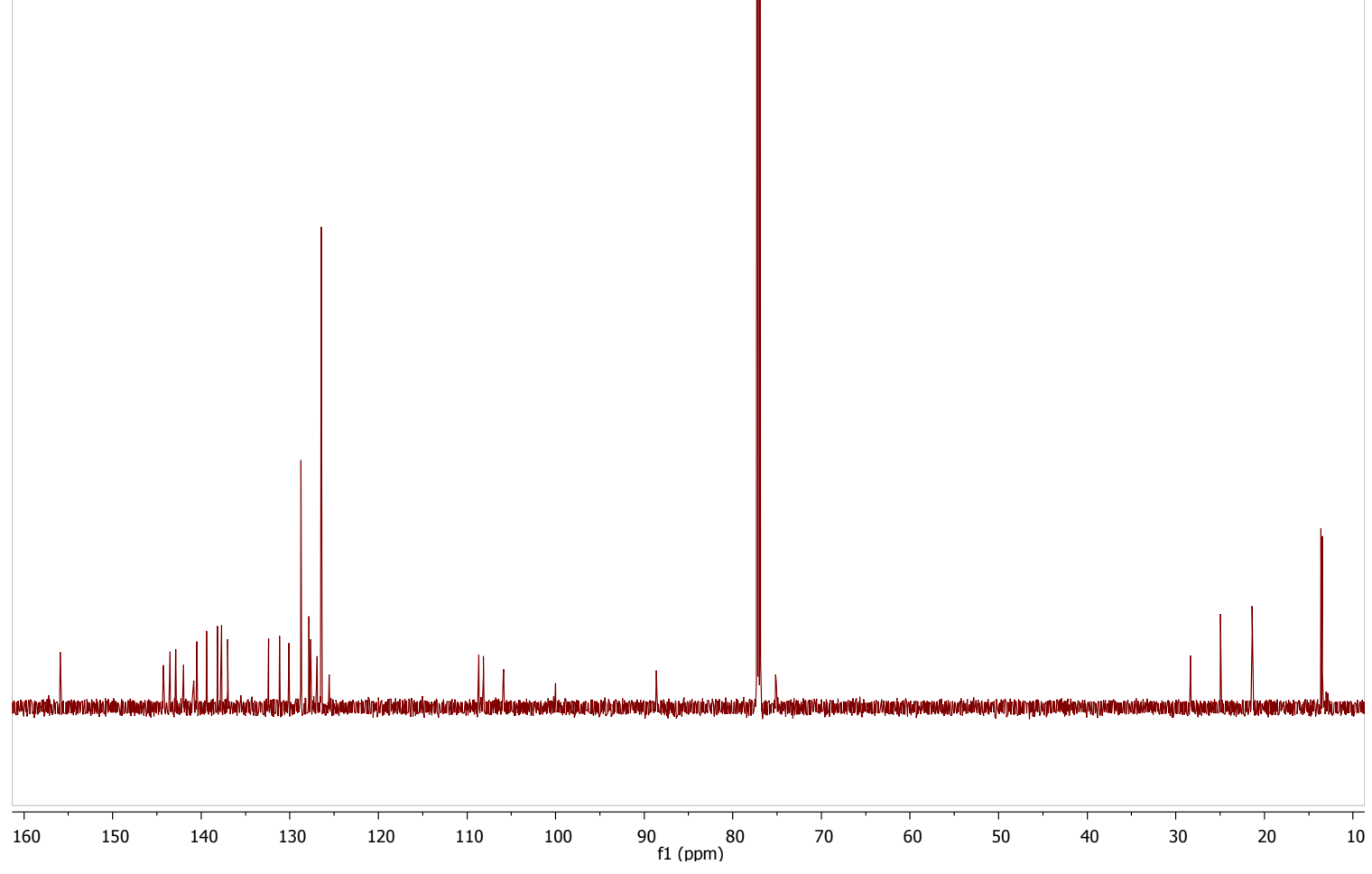



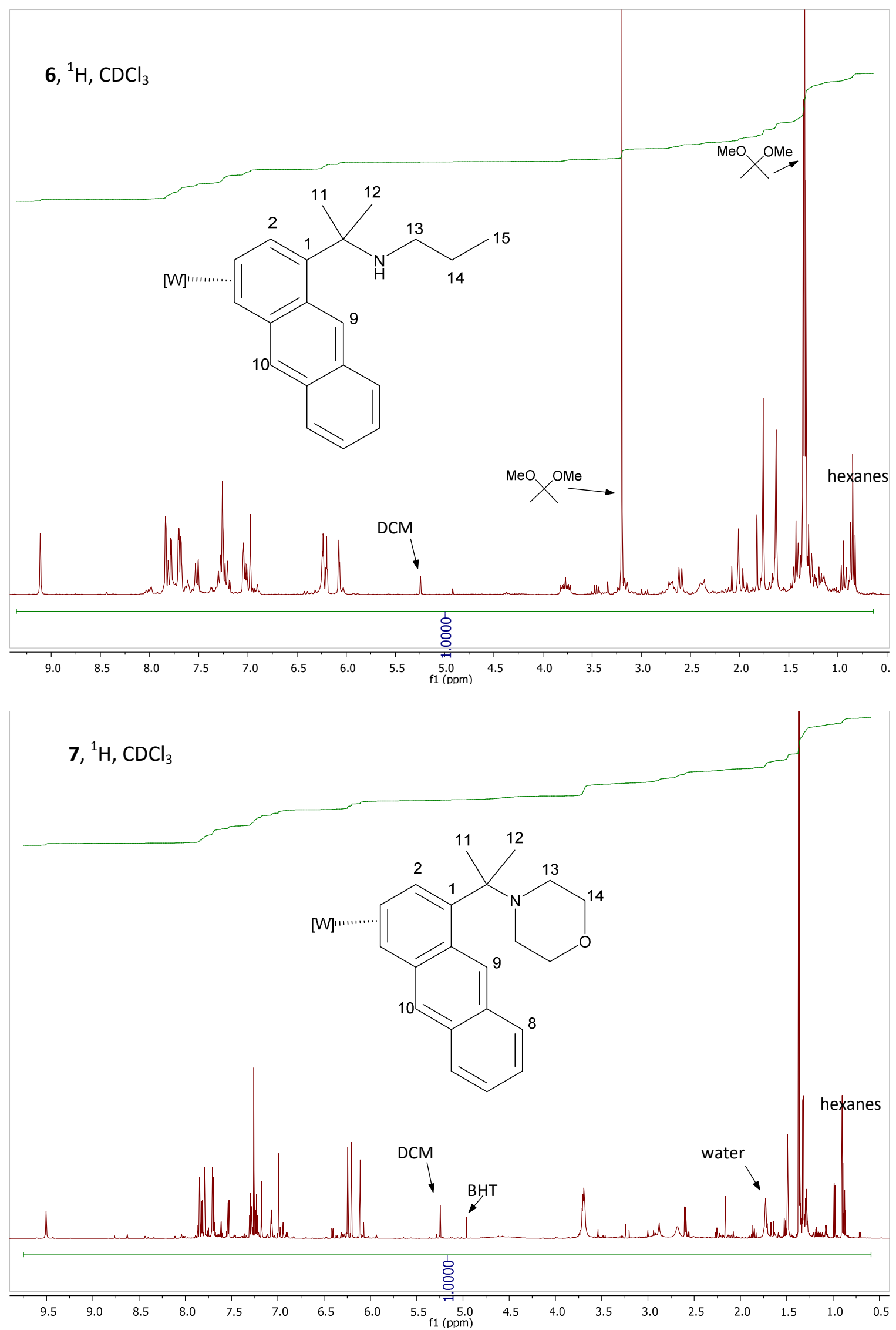


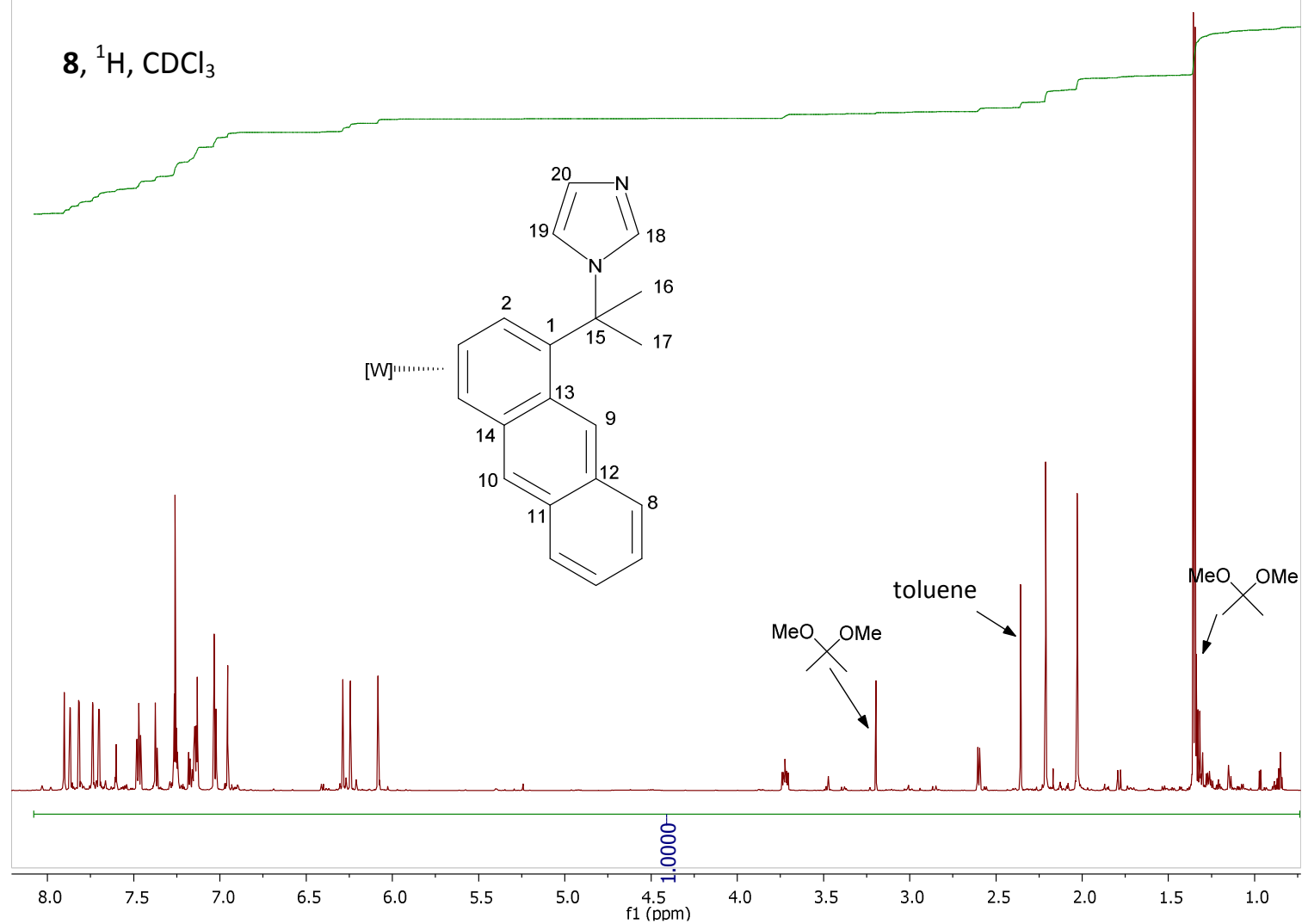

8, ${ }^{13} \mathrm{C}, \mathrm{CDCl}_{3}$

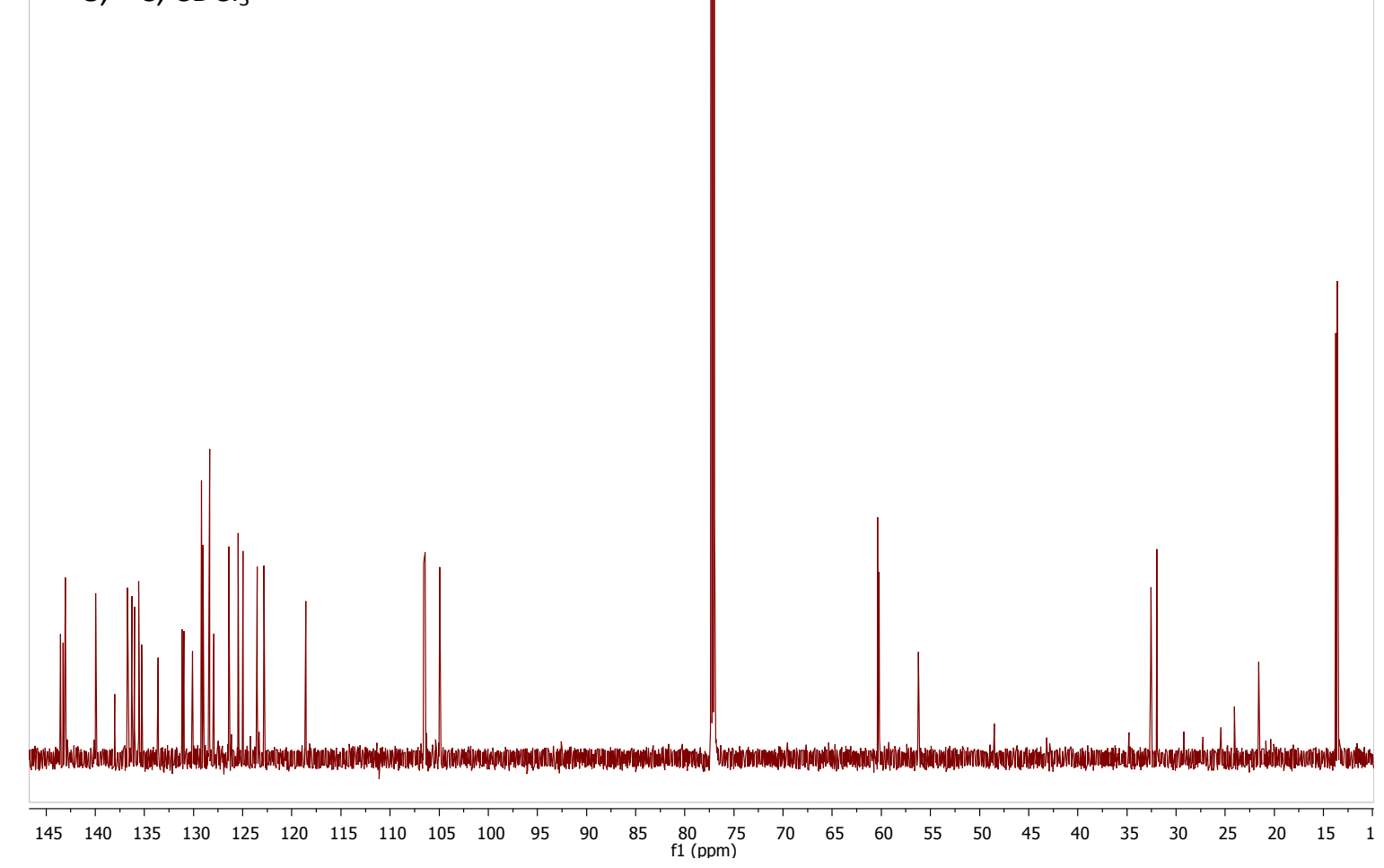




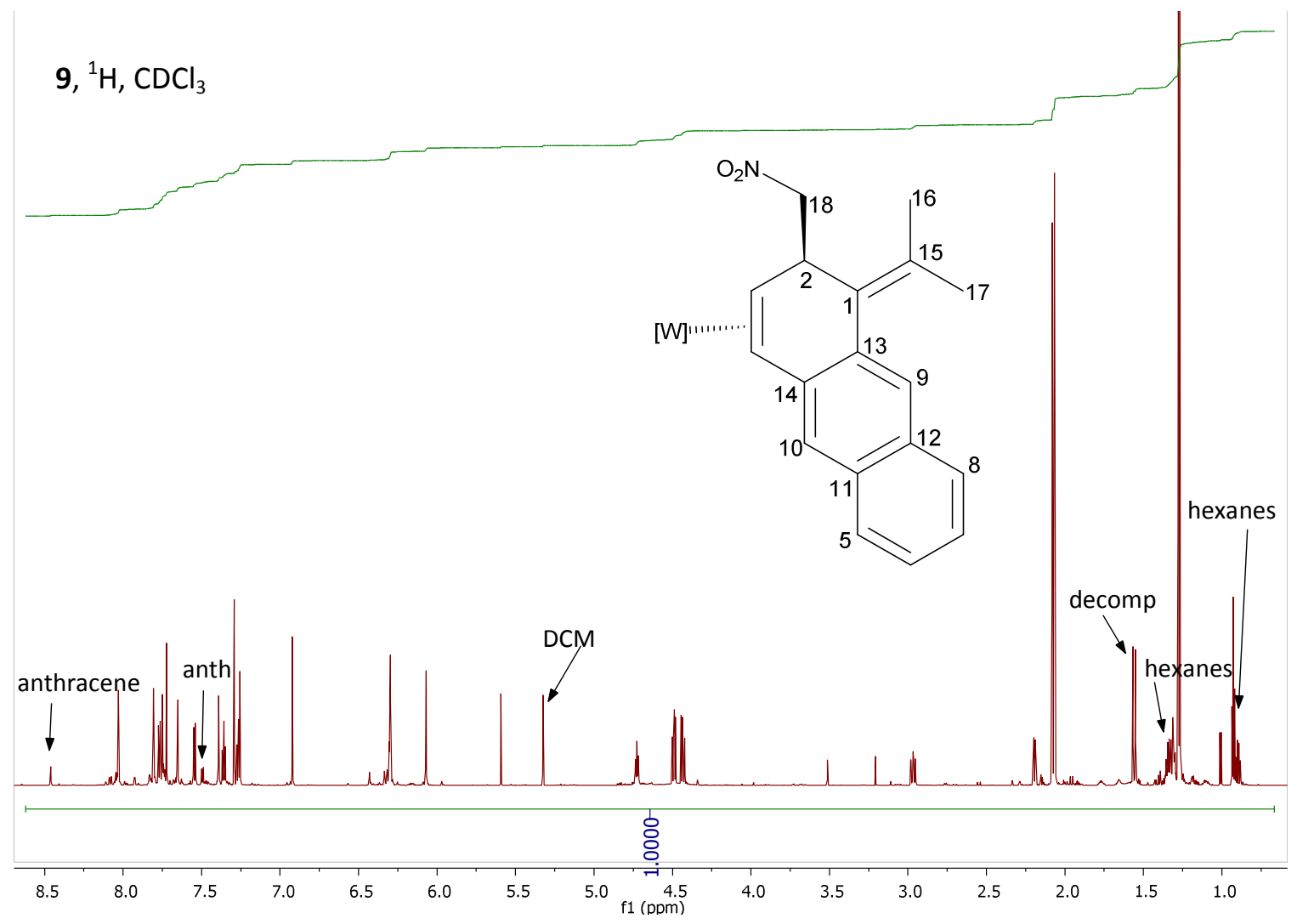

9, ${ }^{13} \mathrm{C}, \mathrm{CDCl}_{3}$
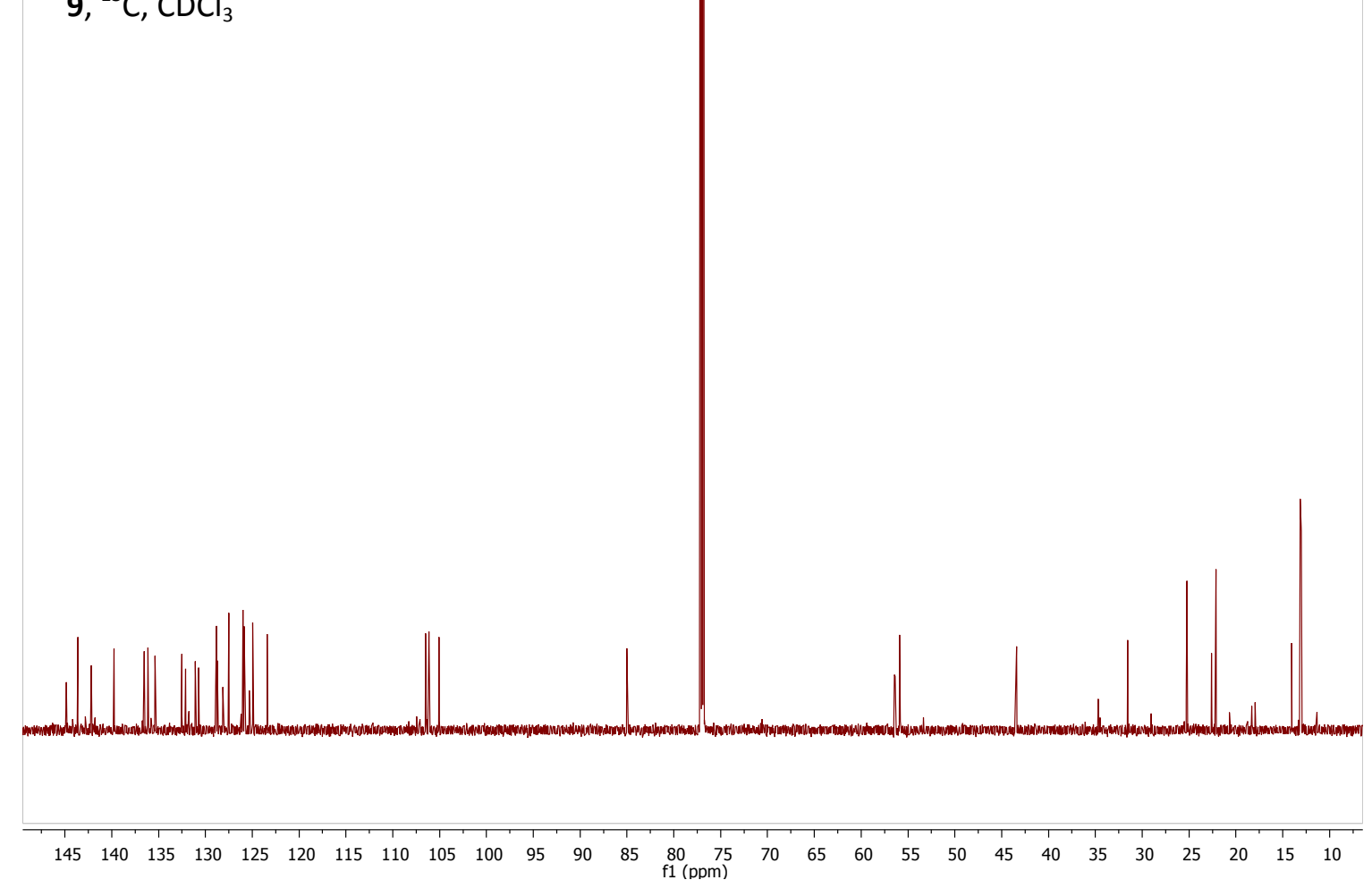


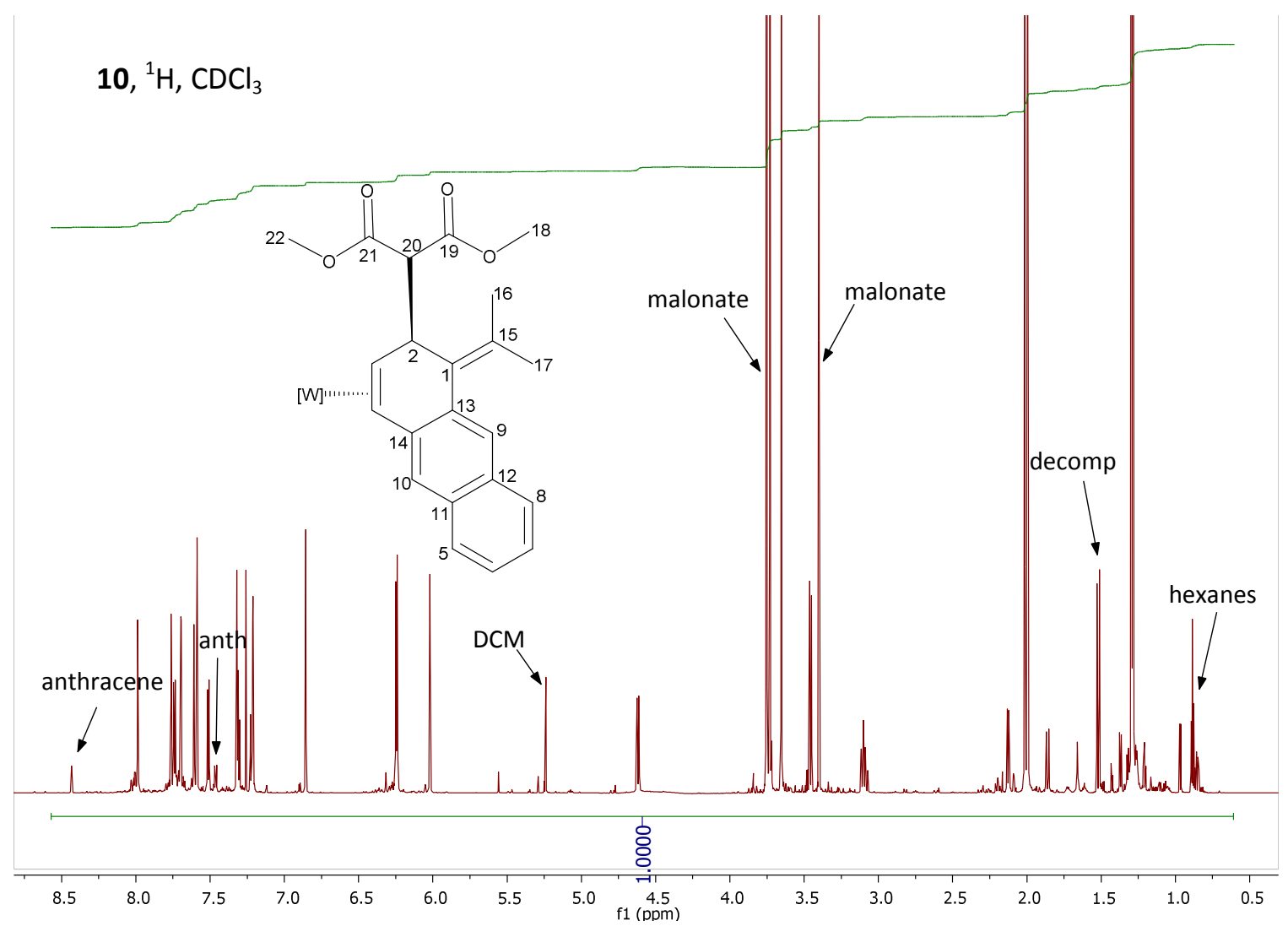

10, ${ }^{13} \mathrm{C}, \mathrm{CDCl}_{3}$

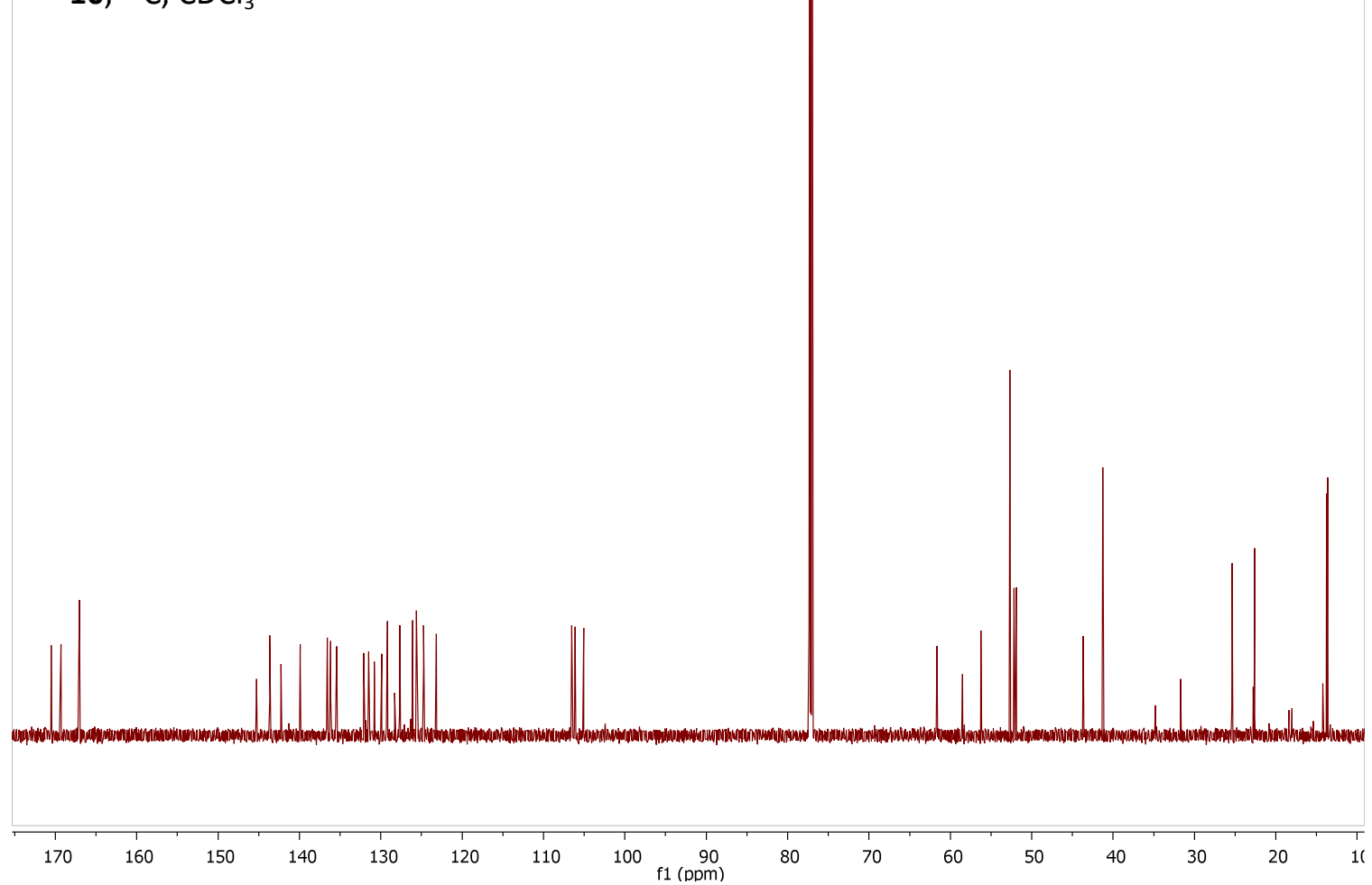




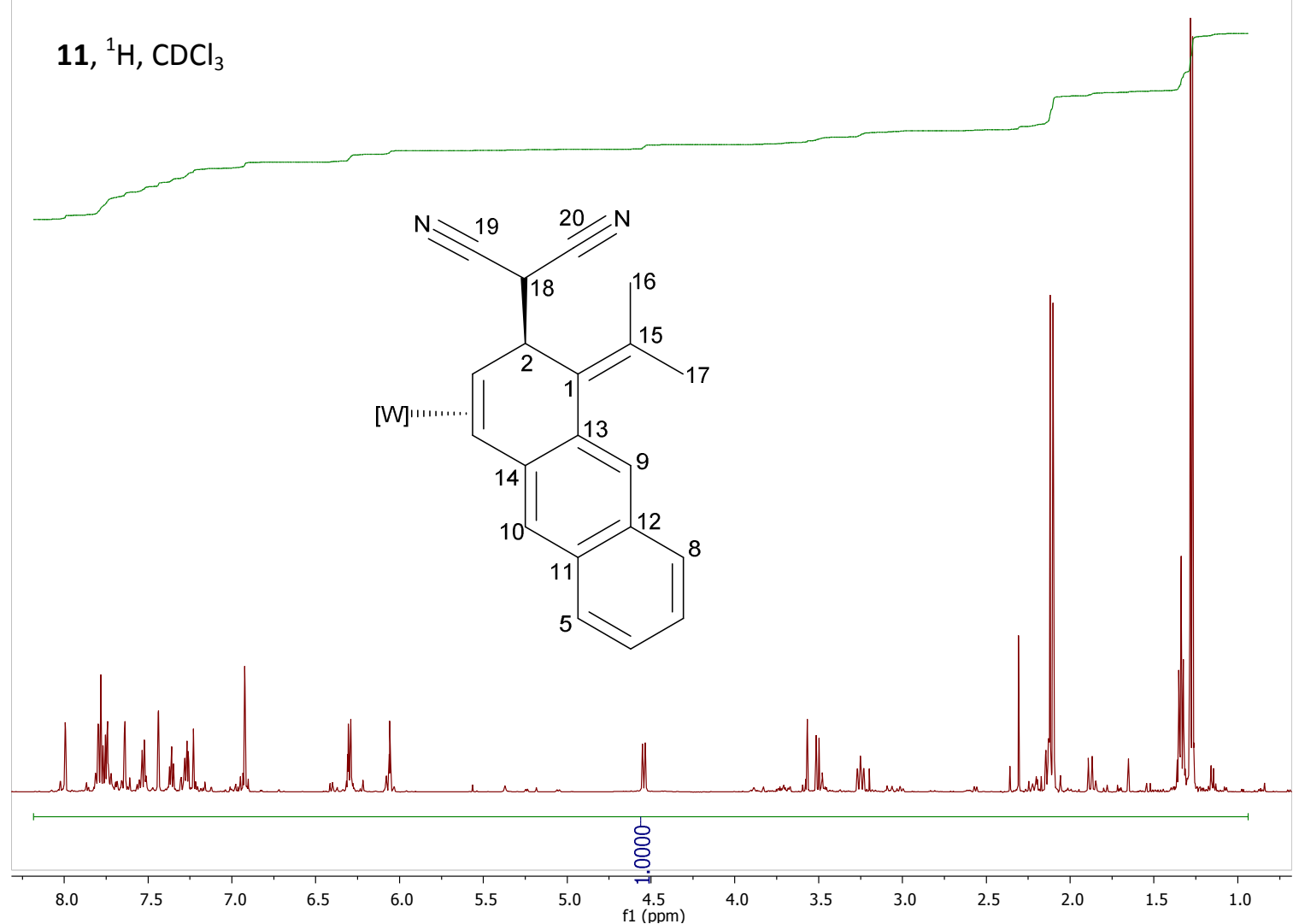

$11,{ }^{13} \mathrm{C}, \mathrm{CDCl}_{3}$

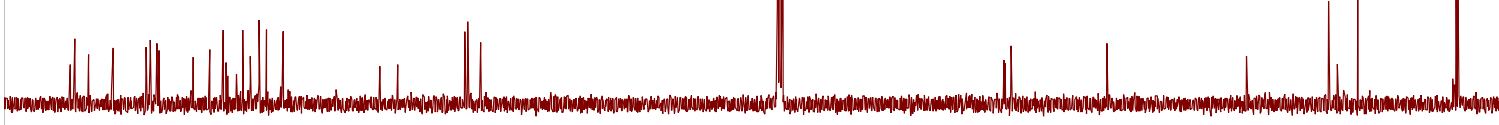

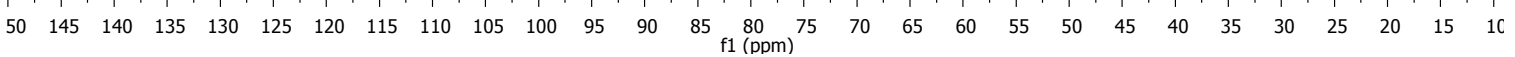



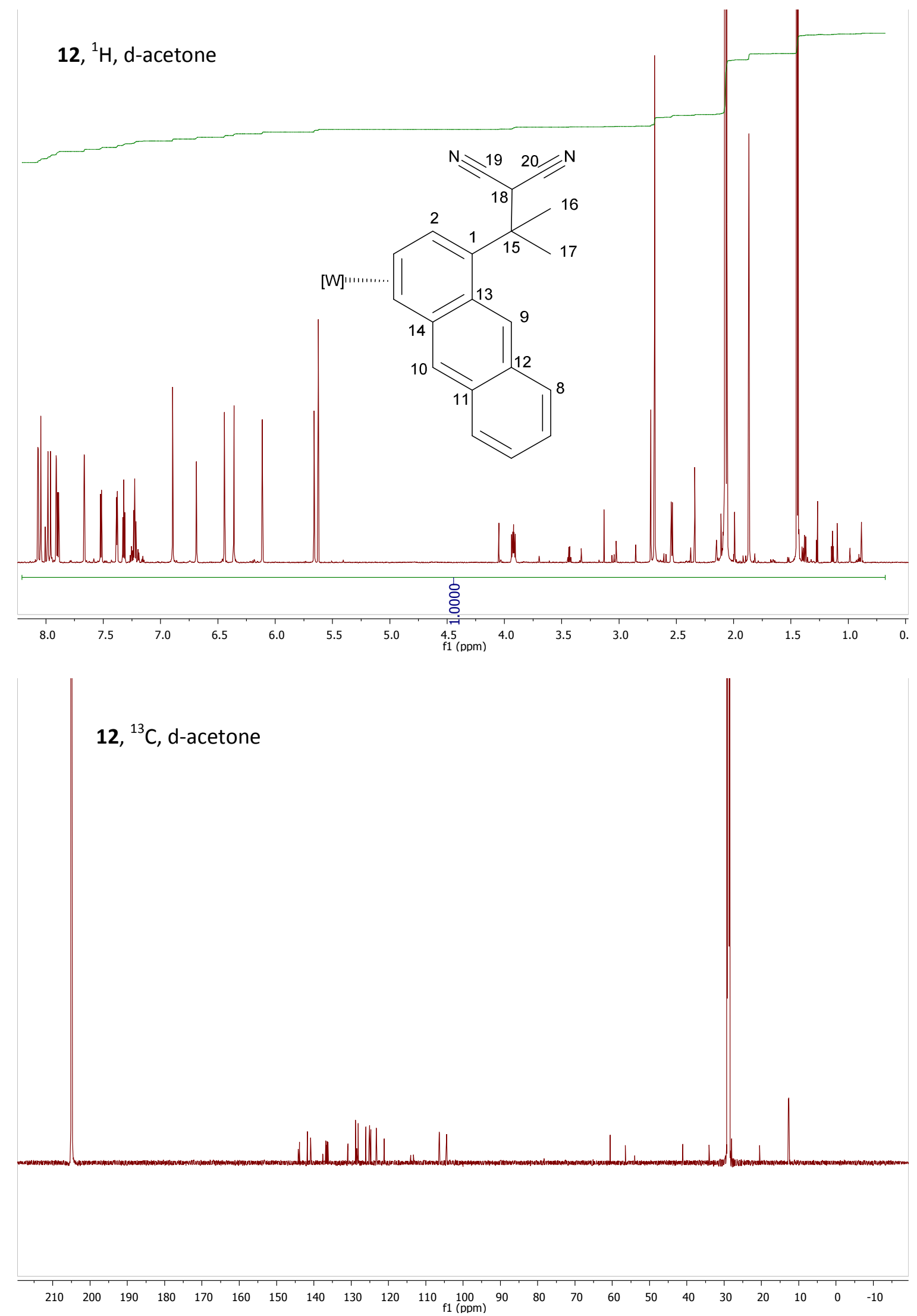

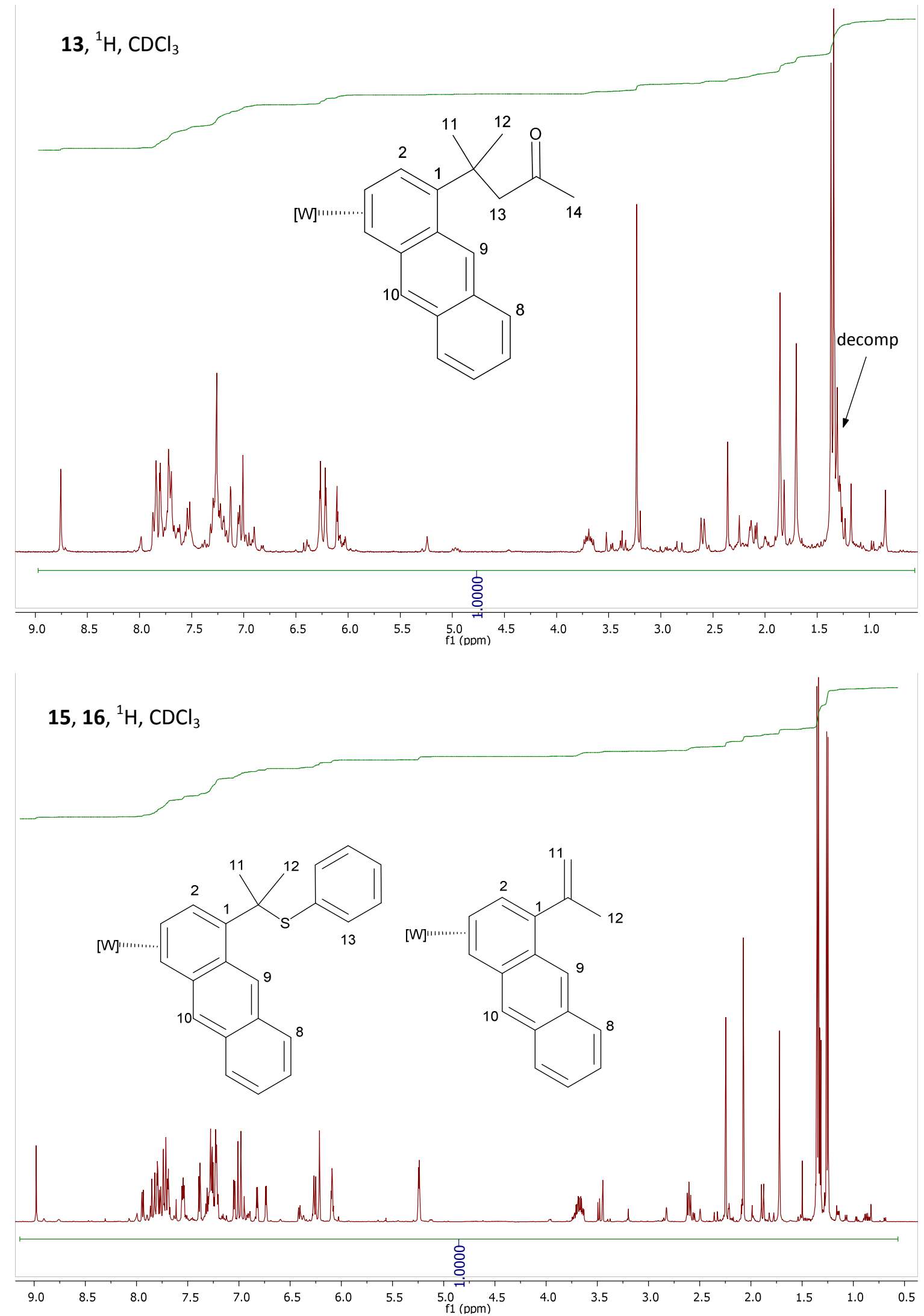

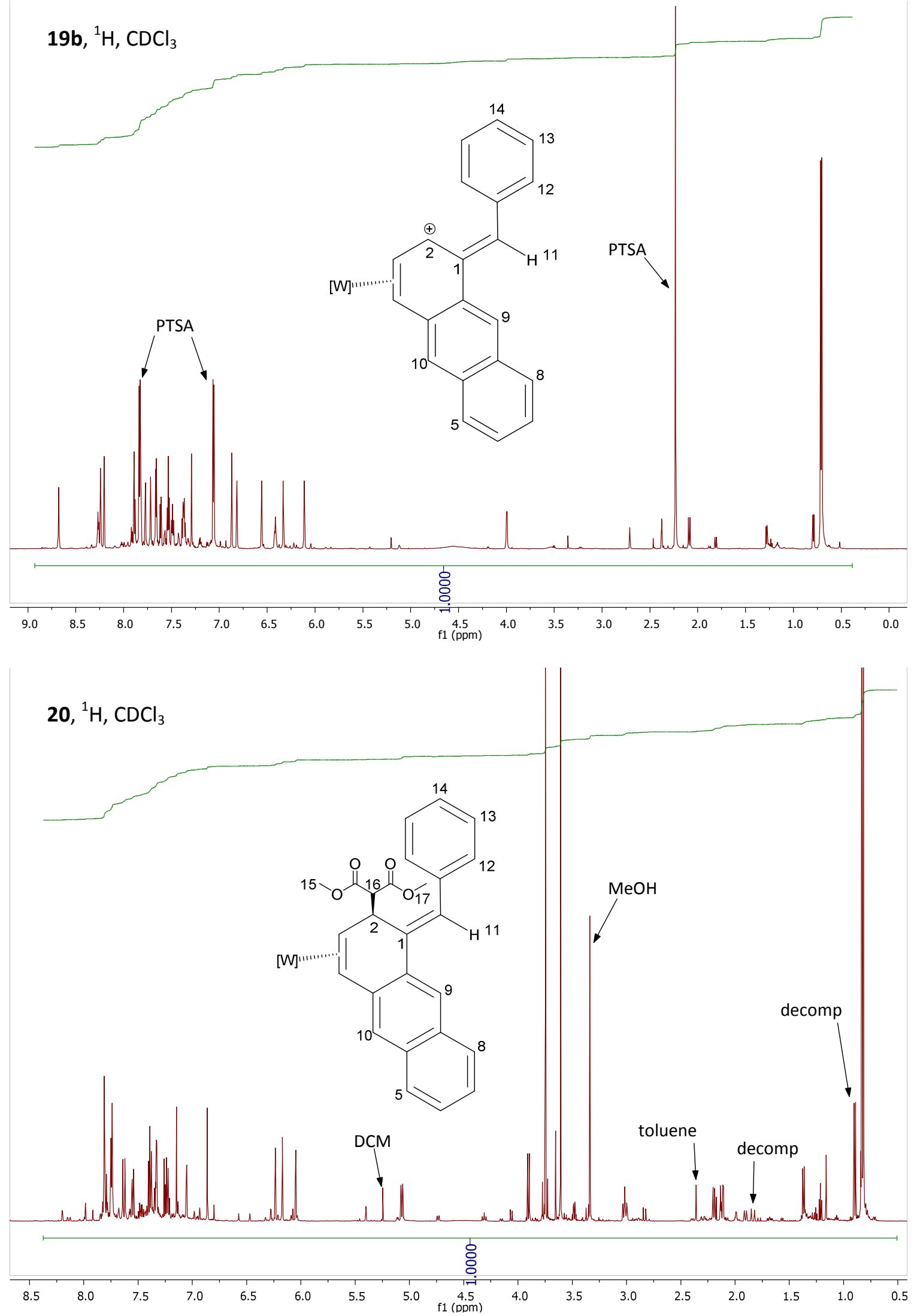
21, ${ }^{1} \mathrm{H}, \mathrm{CDCl}_{3}$

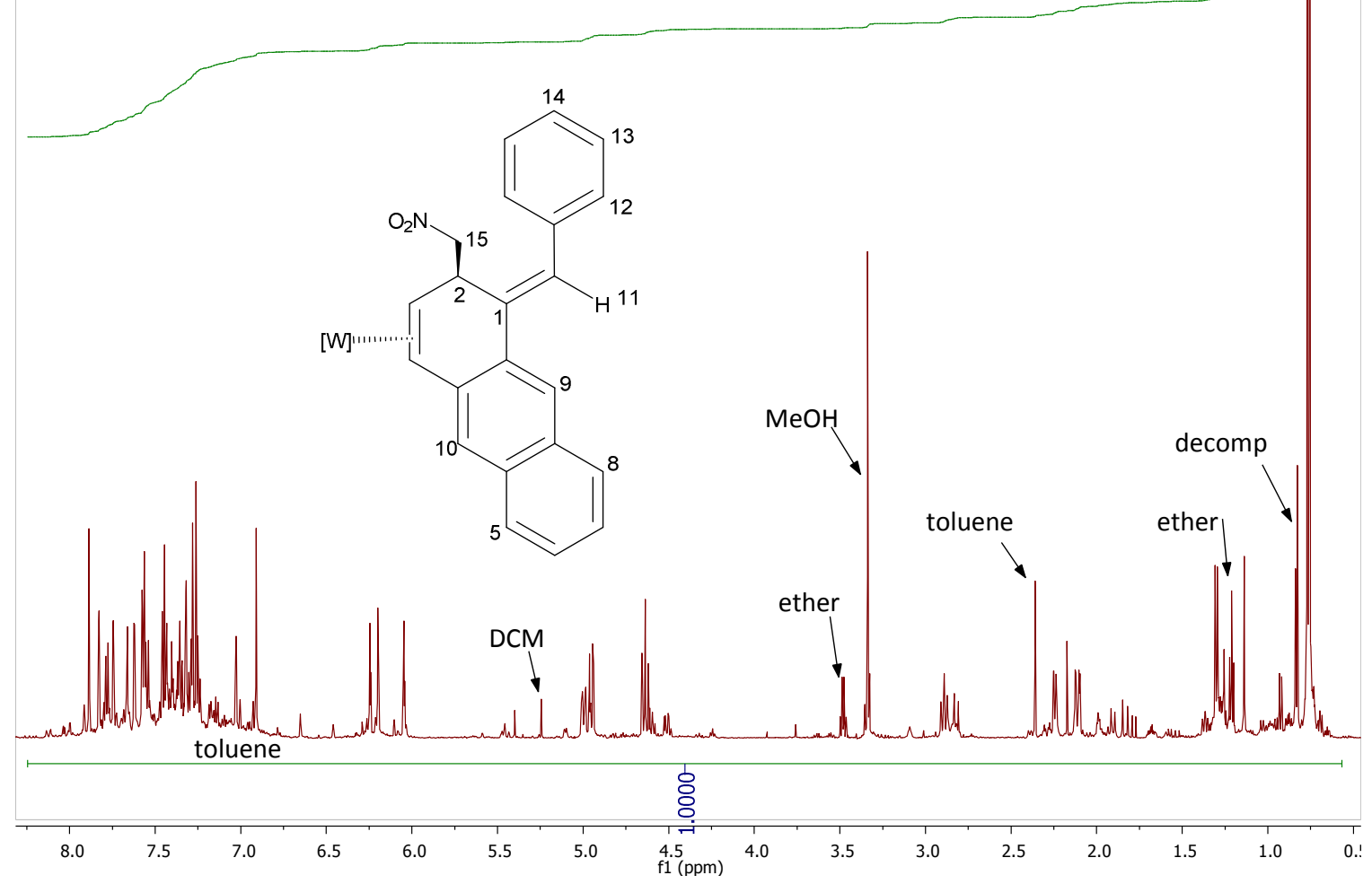

22, ${ }^{1} \mathrm{H}, \mathrm{CDCl}_{3}$

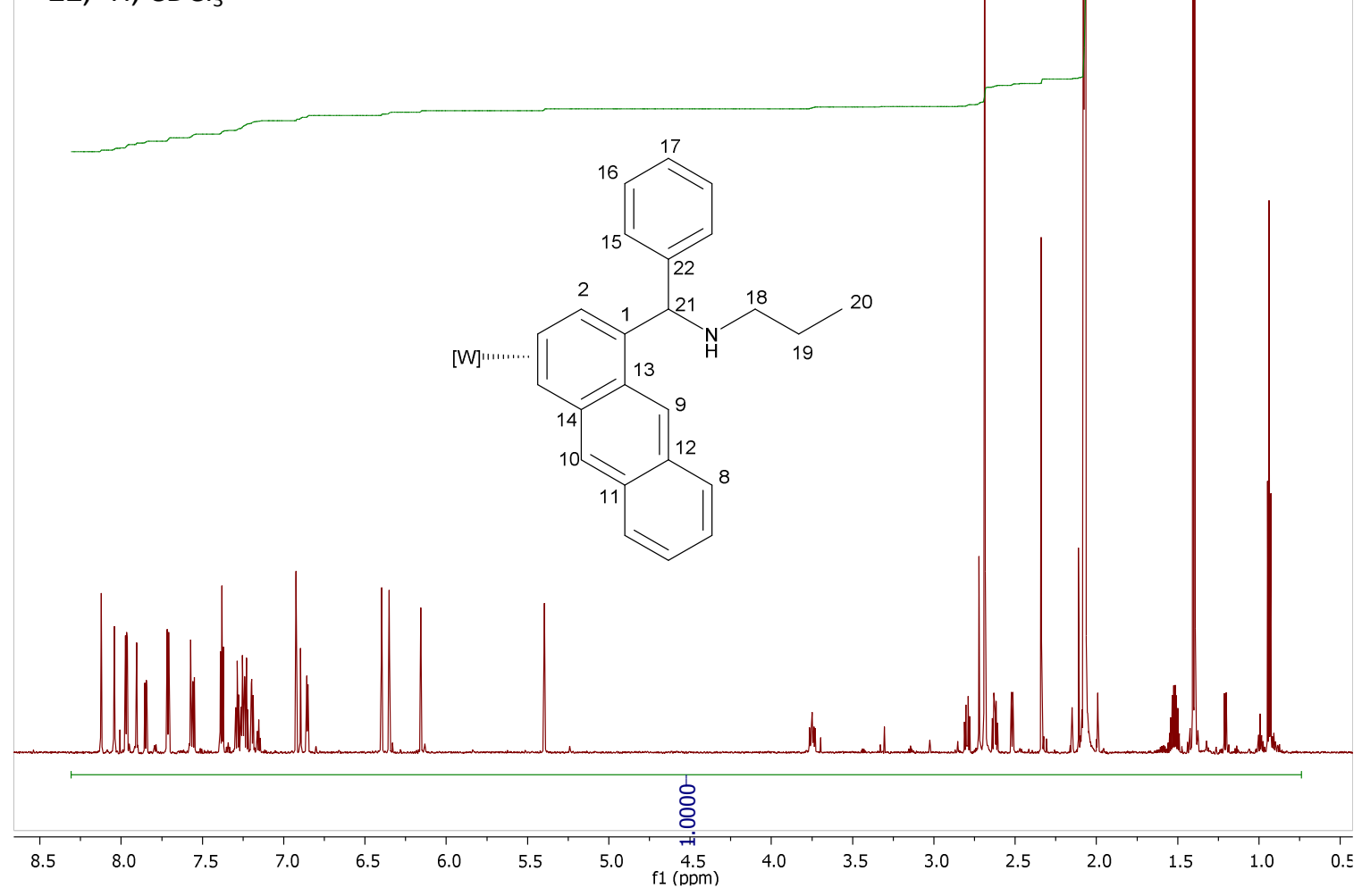


22, ${ }^{13} \mathrm{C}, \mathrm{CDCl}_{3}$

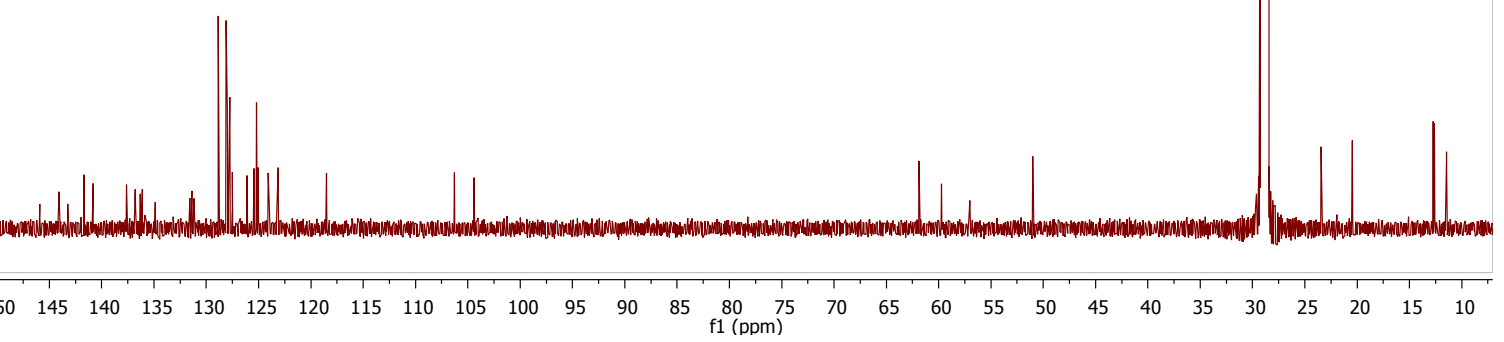

23, ${ }^{1} \mathrm{H}, \mathrm{CDCl}_{3}$

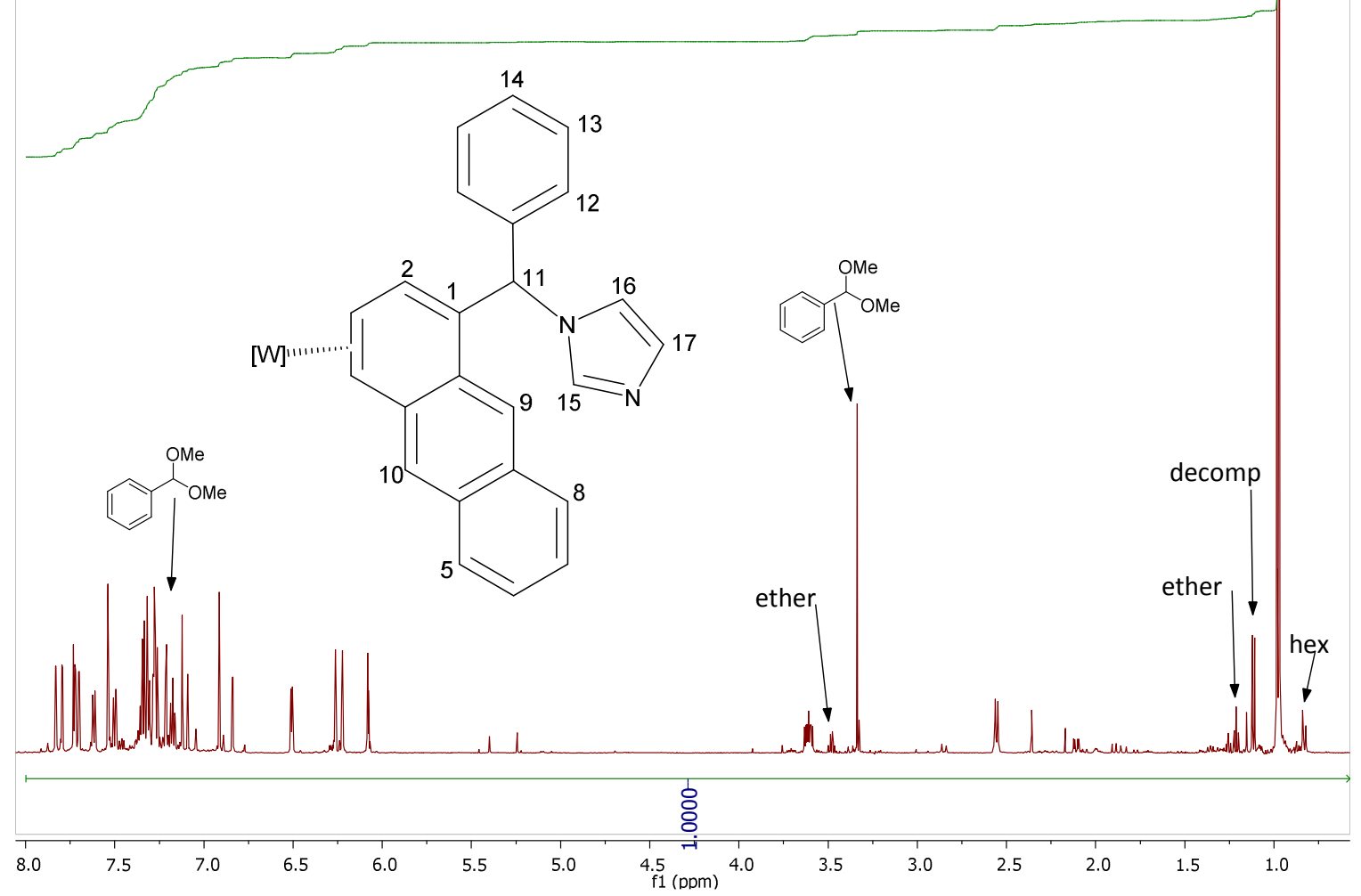




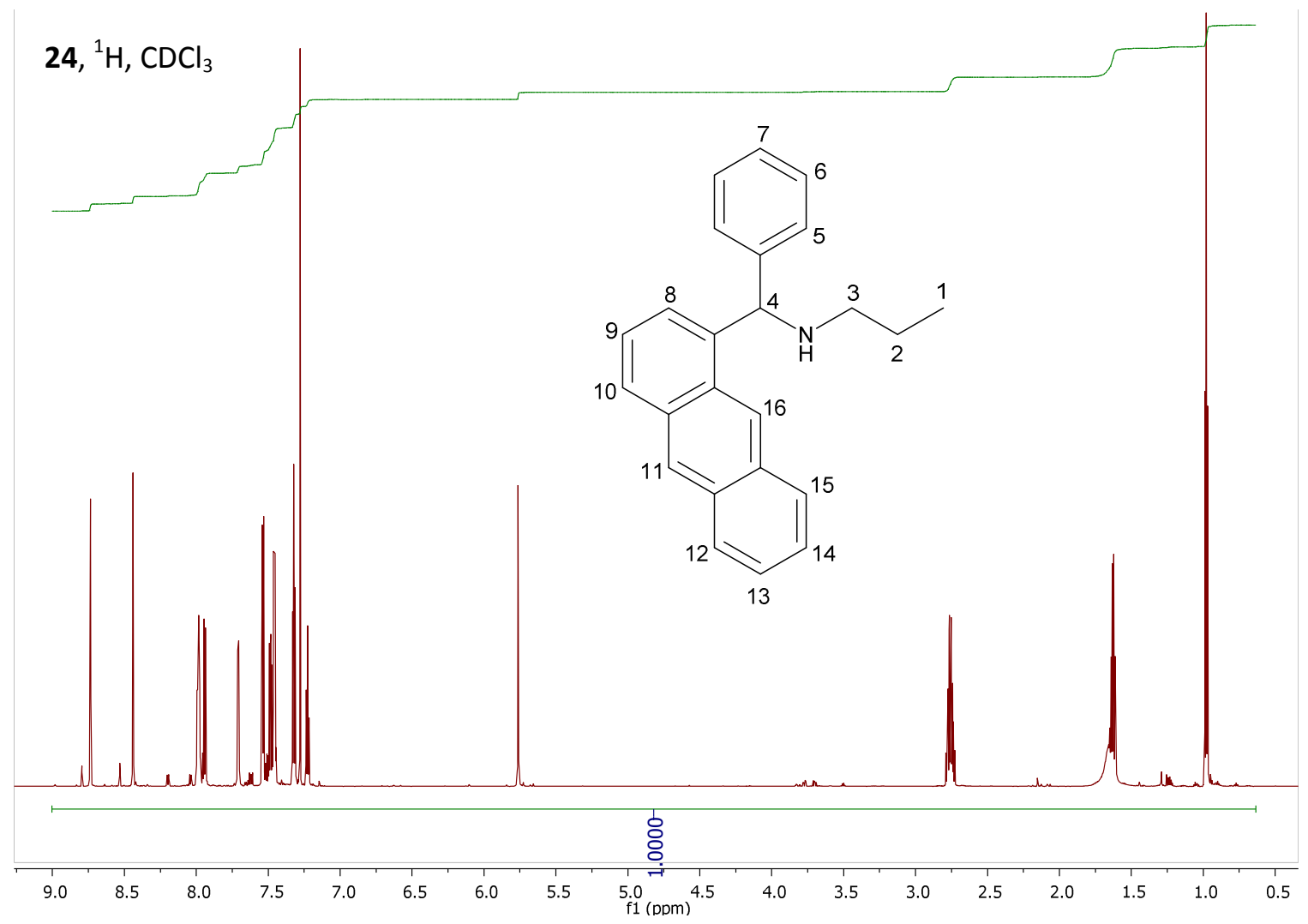

24, ${ }^{13} \mathrm{C}, \mathrm{CDCl}_{3}$

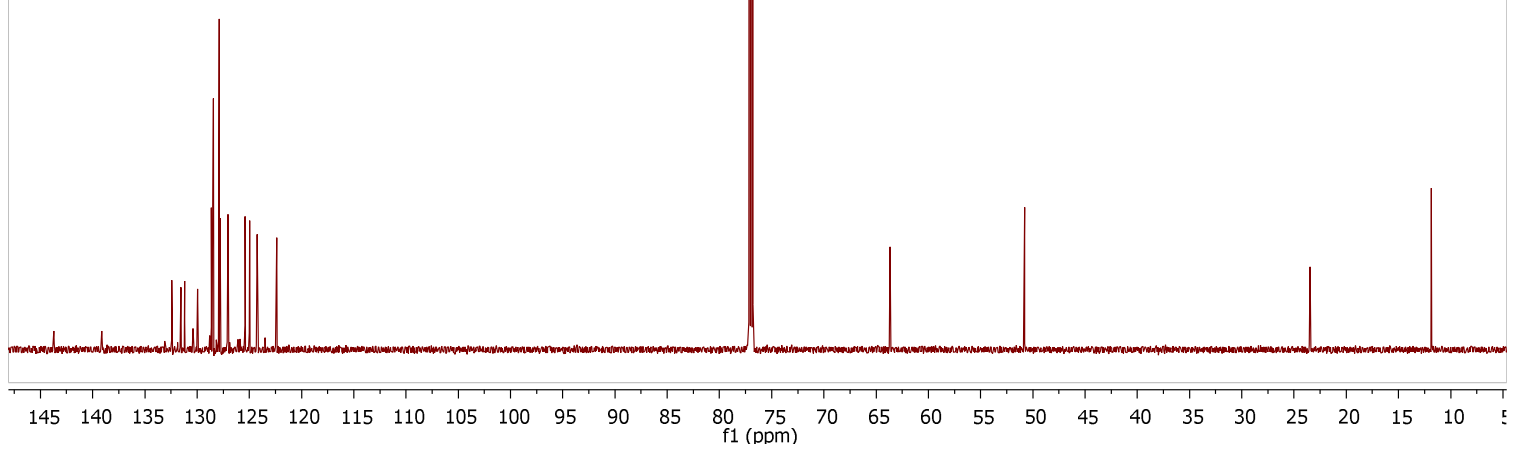




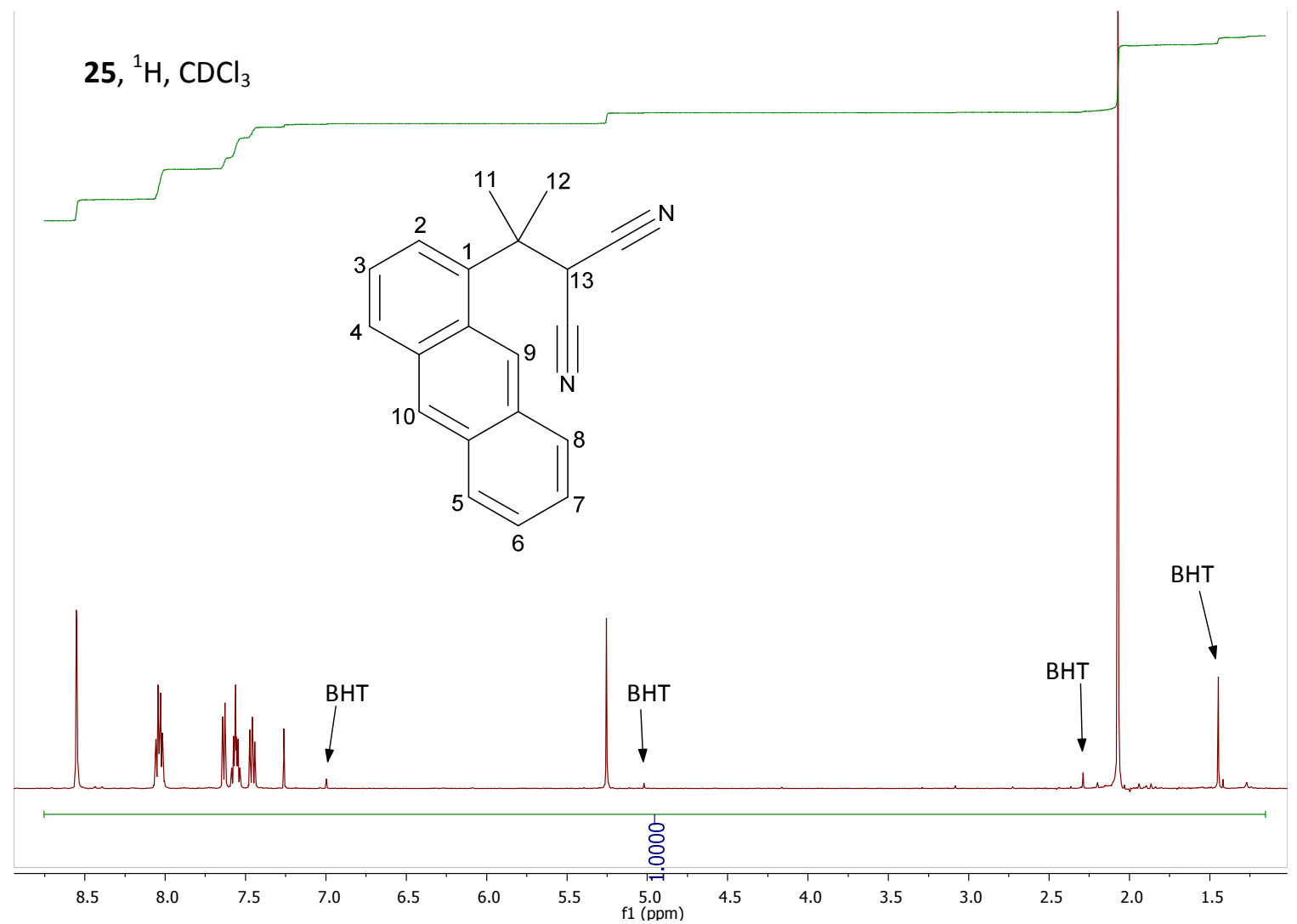

25, ${ }^{13} \mathrm{C}, \mathrm{CDCl}_{3}$

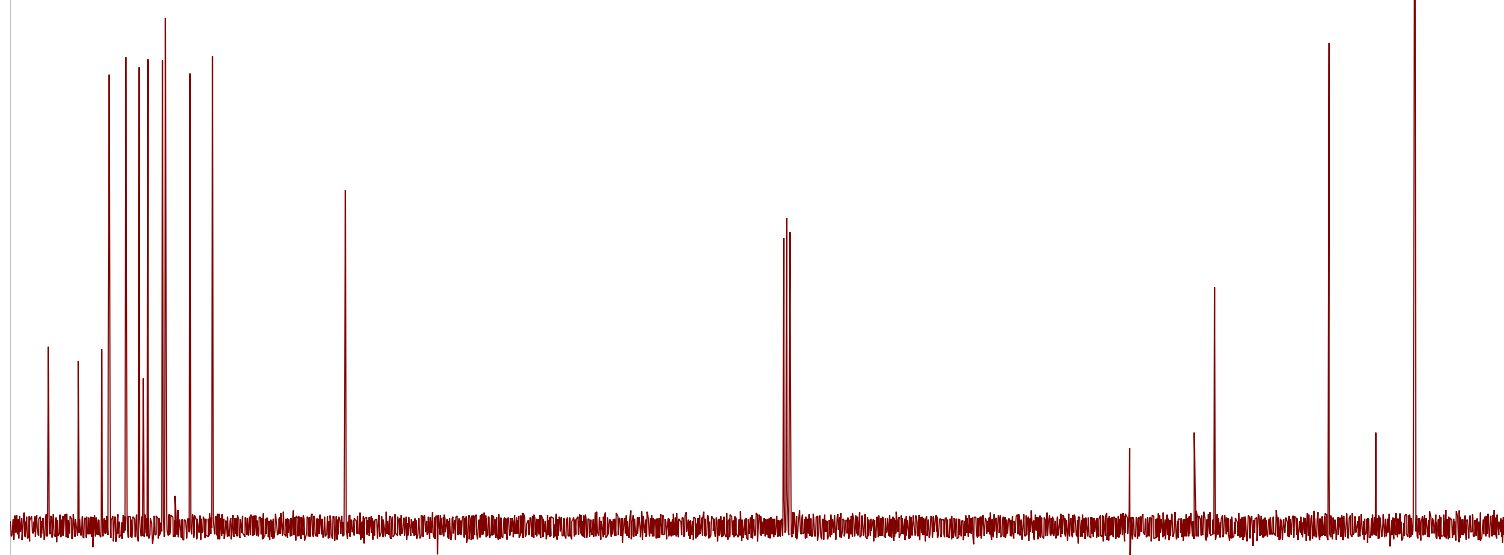

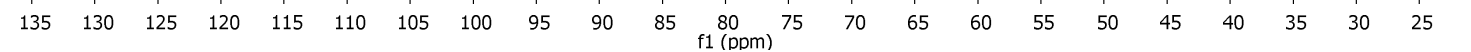




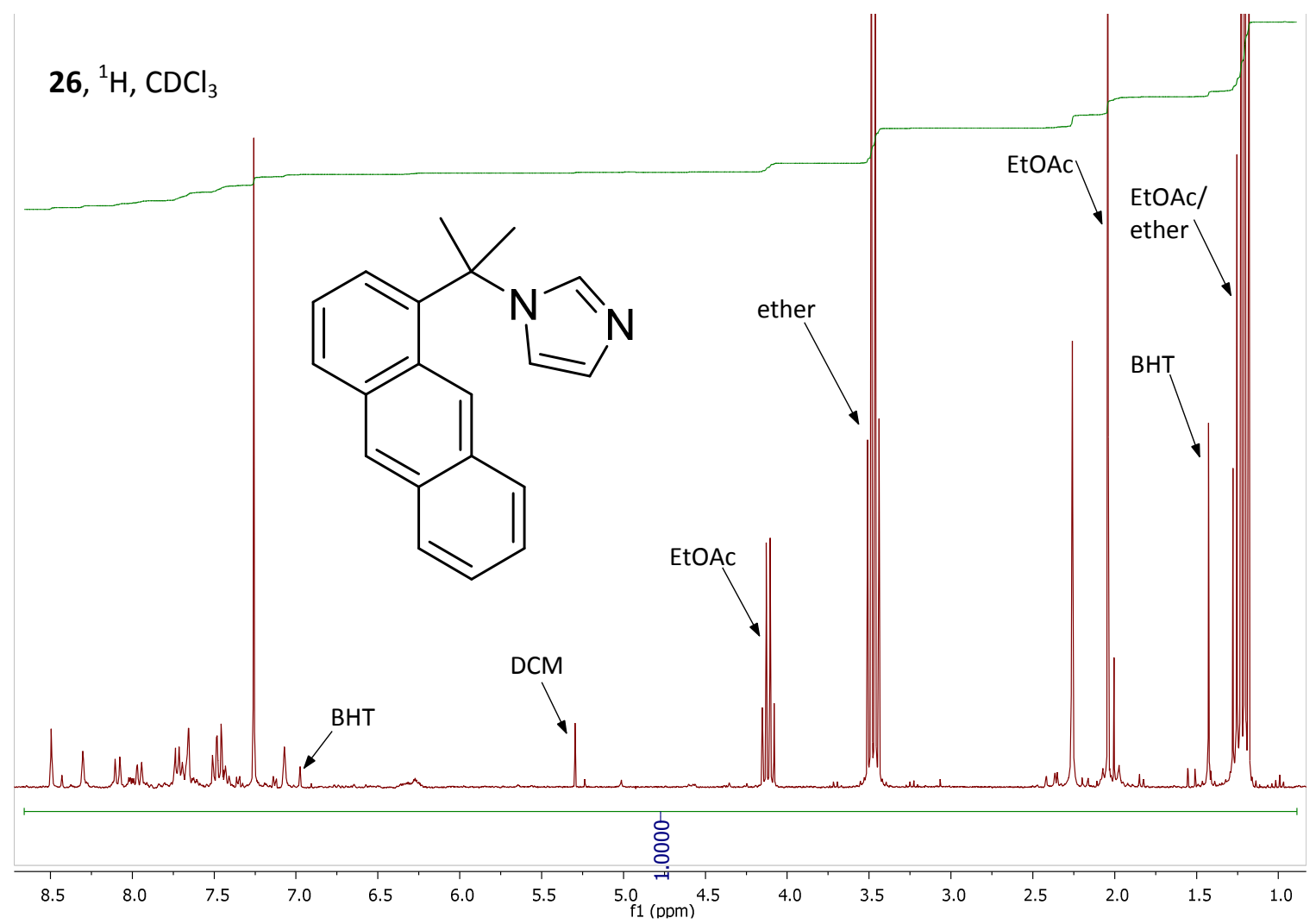

27, ${ }^{1} \mathrm{H}, \mathrm{CDCl}_{3}$
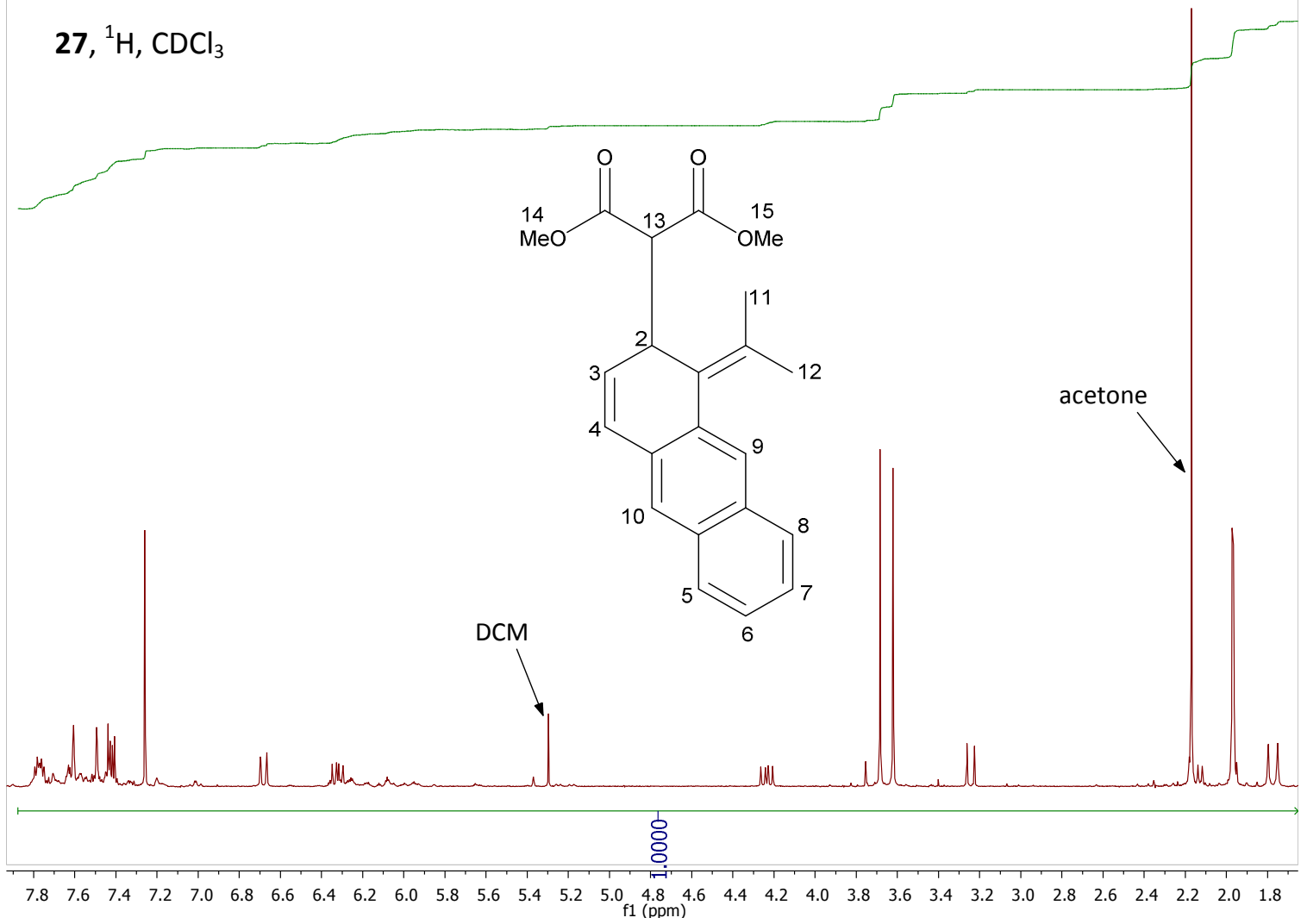


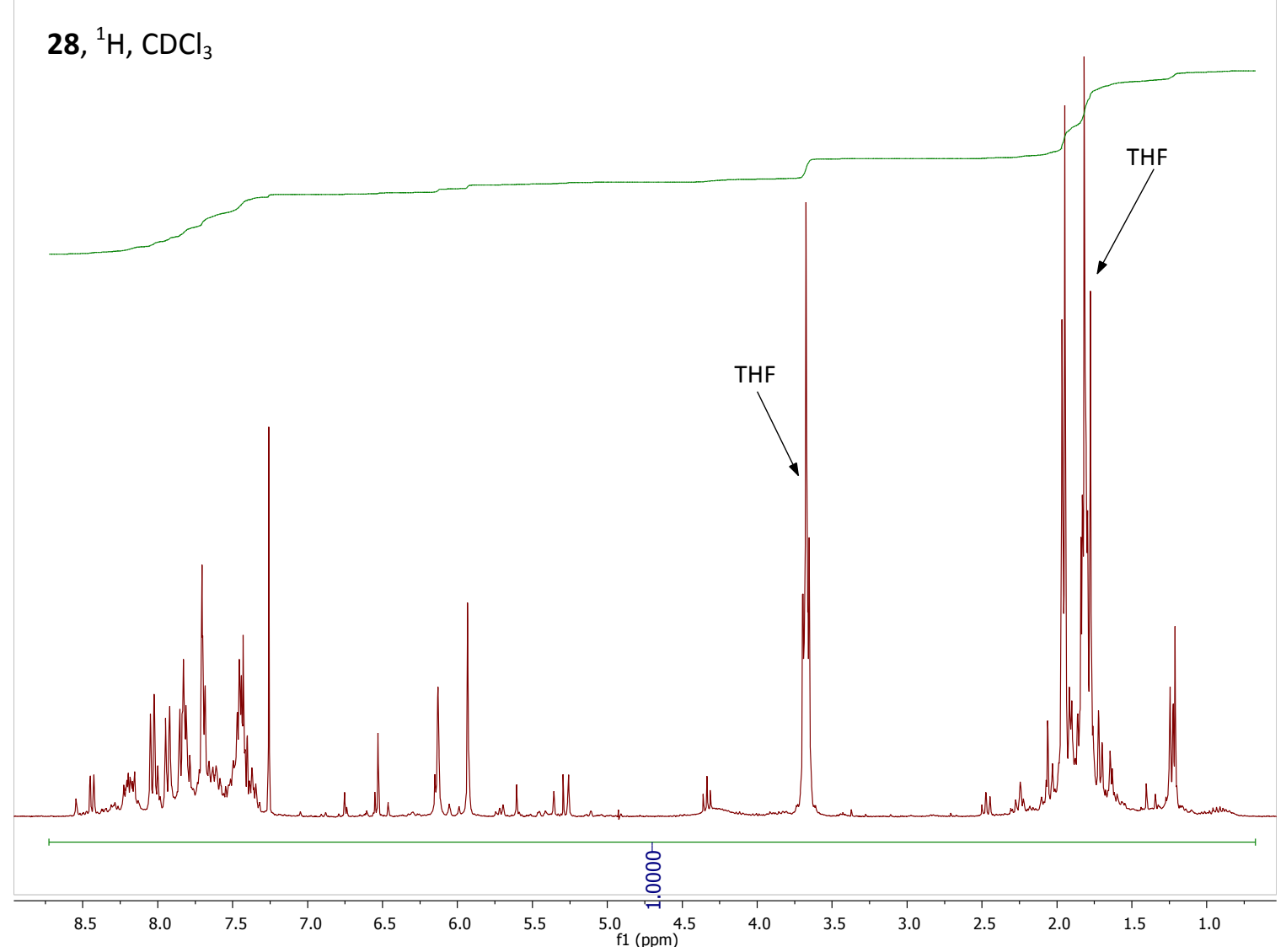

28, from crystal, ${ }^{1} \mathrm{H}, \mathrm{CDCl}_{3}$

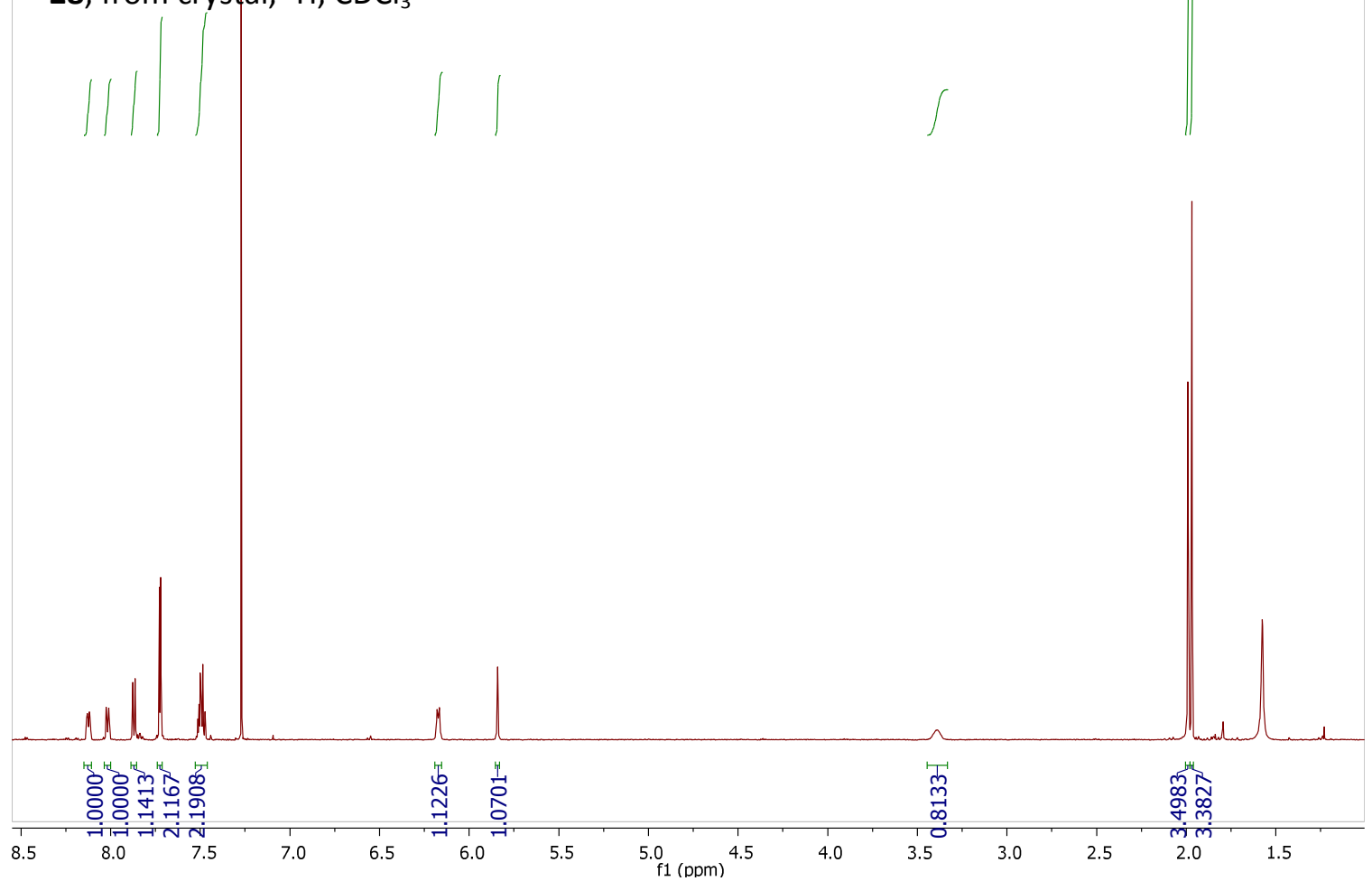




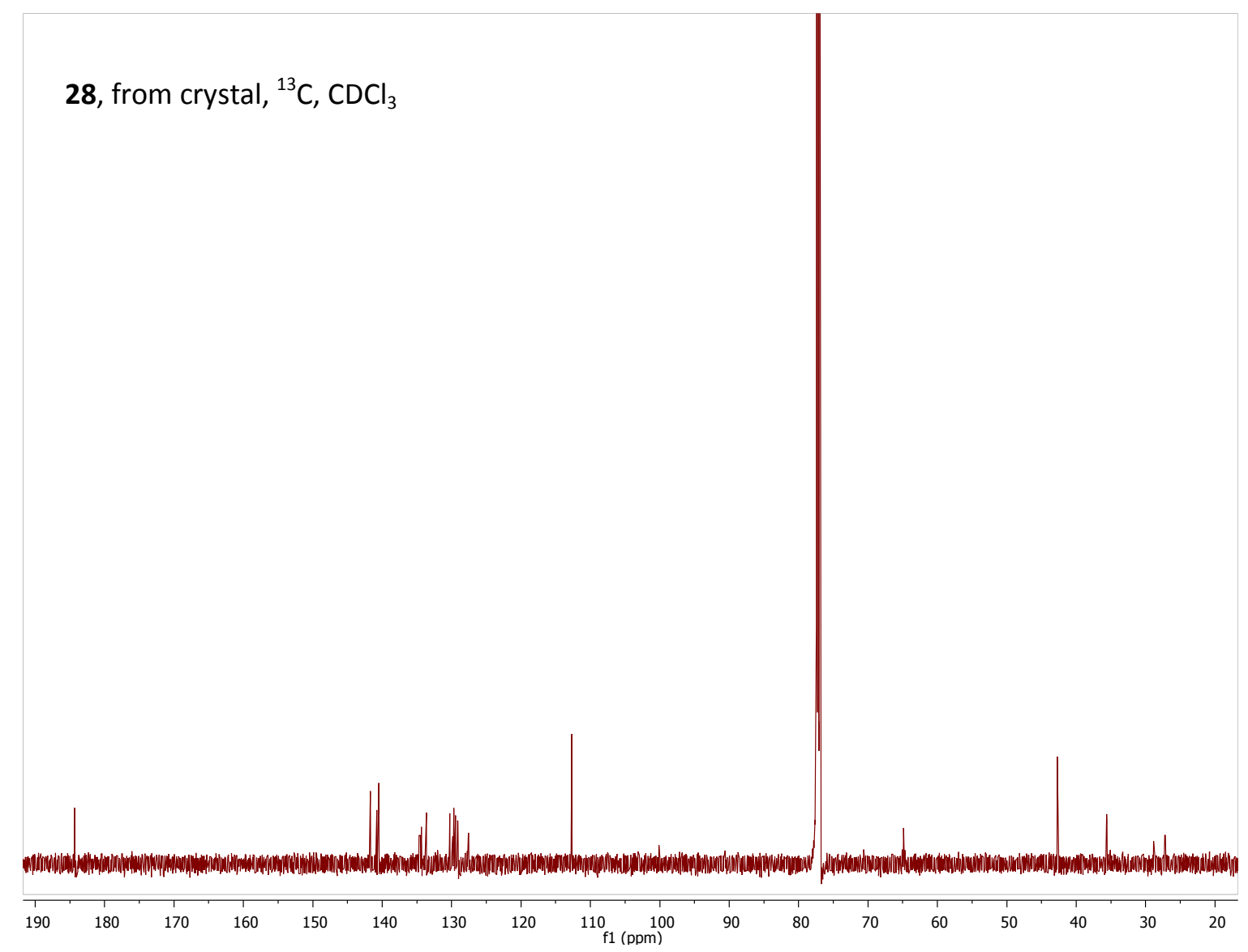

